\title{
Embodiment in \\ Evolution and Culture
}

\author{
Edited by \\ GREGOR ETZELMÜLLER \\ and CHRISTIAN TEWES
}

Mohr Siebeck 
Embodiment in Evolution

and Culture

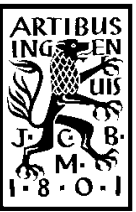





\title{
Embodiment in Evolution and Culture
}

\author{
Edited by \\ Gregor Etzelmüller and Christian Tewes
}

Mohr Siebeck 
Gregor Etzelmüller, born 1971; Professor for Systematic Theology at Osnabrück University and Principal Investigator of the Heidelberg Marsilius Project "Embodiment as Paradigm for an Evolutionary Cultural Anthropology”.

Christian Tewes, born 1972; adjunct Professor (Privatdozent) for Philosophy at the University of Jena and Principal Investigator of the Heidelberg Marsilius Project "Embodiment as Paradigm for an Evolutionary Cultural Anthropology".

e-ISBN PDF 978-3-16-154901-4

ISBN 978-3-16-154736-2

Die Deutsche Nationalbibliothek lists this publication in the Deutsche Nationalbibliographie; detailed bibliographic data are available on the Internet at $h t t p: / / d n b . d n b$.de.

(C) 2016 by Mohr Siebeck, Tübingen, Germany www.mohr.de

This book may not be reproduced, in whole or in part, in any form (beyond that permitted by copyright law) without the publisher's written permission. This applies particularly to reproductions, translations, microfilms and storage and processing in electronic systems.

The book was typeset by Laupp \& Göbel in Gomaringen using Garamond typeface, printed by Laupp \& Göbel in Gomaringen on non-aging paper and bound by Buchbinderei Nädele in Nehren.

Printed in Germany. 


\section{Acknowledgements}

To answer the question of how evolution brings forth an embodied human being that is able to transcend itself by shaping itself culturally, the Heidelberg Marsilius-Project "Embodiment as Paradigm for an Evolutionary Cultural Anthropology” (specifically, Gregor Etzelmüller, Thomas Fuchs, Grit Schwarzkopf and Christian Tewes) organised an international symposium on "Embodiment in Evolution and Culture" which was held in December 2014 at the International Academic Forum Heidelberg (IWH). 22 outstanding international scholars (neurologists, physicians, paleoanthropologists, philosophers, theologians, and educational scientists) from four countries (England, Italy, the USA and Germany) came together to explore and debate the diverse ways in which communication and brain development, body and language, tool use and hominization, embodiment and transcendence are coupled and shape one another. To understand the human being as a symbol-using creature, such interdisciplinary collaboration between evolutionary and historical anthropologies is vital, and the Heidelberg symposium showed such collaboration at its best.

After the symposium we continued work on the findings in our Heidelberg Marsilius project while linking up with external researchers and academics. The present book documents the results of this extended interdisciplinary and international dialogue.

We thank the Deutsche Forschungsgemeinschaft and the Marsilius-Kolleg of the University of Heidelberg for supporting our symposium. We are very grateful to the directors of the Heidelberg Marsilius College, Prof. Dr. Thomas Rausch and Prof. Dr. Bernd Schneidmüller, and the Directing Manager of the Marsilius-Kolleg, Tobias Just, M. A. We also thank the International Academic Forum Heidelberg for the inclusion of our symposium in the IWH's symposia program. We are also grateful to the Director of the IWH, Prof. Dr. Peter Comba, and the Managing Director of the IWH, Dr. Ellen Peerenboom.

We thank our co-organizers, Thomas Fuchs and Grit Schwarzkopf, for helping us organize the Heidelberg symposium.

Finally, we thank Dr. Adrian Wilding, Christina Gallinat, Mailin Hebell, Damian Peikert, Tilman Rivinius, Daniel Vespermann, and the publisher Mohr Siebeck for their invaluable cooperation in preparing the manuscript for publication. We owe a special debt of gratitude to Dr. Stephanie Warnke-De Nobili.

Gregor Etzelmüller and Christian Tewes 



\section{Table of Contents}

Acknowledgements $\ldots \ldots \ldots \ldots \ldots \ldots \ldots \ldots \ldots \ldots \ldots \ldots \ldots \ldots \ldots \ldots, \quad \mathrm{V}$

Gregor Etzelmüller/Christian Tewes

Introduction ................................ 1

1. Philosophical Concepts and Perspectives of Embodiment

Christian Tewes

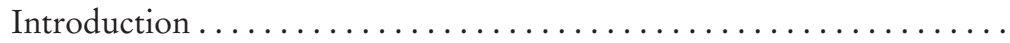

Mog Stapleton

Leaky Levels and the Case for Proper Embodiment ............

Christian Tewes

Embodied Habitual Memory Formation: Enacted or Extended? ......

Karim Zabidi/Erik Myin

Radically Enactive Numerical Cognition . . . . . . . . . . . . . 57

Christian Spabn

Beyond Dualism? The Implications of Evolutionary Theory

for an Anthropological Determination of Human Being ...........

2. The Embodied Evolution of Symbolic Competence

Magnus Schlette

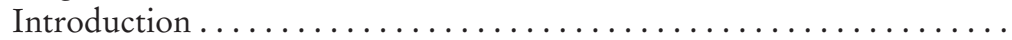

Thomas Fuchs

The Embodied Development of Language . . . . . . . . . . . . . . 107

Terrence Deacon

On Human (Symbolic) Nature: How the Word Became Flesh

Jordan Zlatev

Preconditions in Human Embodiment for the Evolution

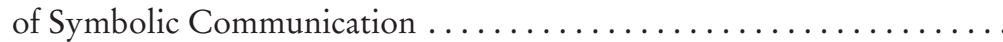


Matthias Jung

Stages of Embodied Articulation

3. Embodiment as a Bridging Concept for Evolutionary and Historical Anthropology

Alexander Massmann

Introduction

Gregor Etzelmüller

The Lived Body as the Tipping Point Between an Evolutionary and a Historical Anthropology $\ldots \ldots \ldots \ldots \ldots \ldots \ldots \ldots \ldots$

Eve-Marie Engels

The Roots of Human Morals and Culture in Pre-Human Sympathy.

Charles Darwin's Natural and Cultural History of Morals ..........

Christoph Wulf

The Creation of Body Knowledge in Mimetic Processes . . . . . . . . . .

Annette Weissenrieder

"It Proceeded from the Entrance of a Demon into the Man".

Epileptic Seizures in Ancient Medical Texts and the New Testament ...

4. The Mutual Intertwinement of Nature and Culture

Miriam Haidle

Introduction . .

Lambros Malafouris

On Human Becoming and Incompleteness: A Material Engagement Approach to the Study of Embodiment in Evolution and Culture .....

Duilio Garofoli

Metaplasticit-ies: Material Engagement Meets Mutational Enhancement

Shaun Gallagher/Tailer G. Ransom

Artifacting Minds: Material Engagement Theory and Joint Action .....

Wolfgang Welsch

Bodily Changes during the Protocultural Period and Their Ongoing Impact on Culture . . . . . . . . . . . . . . . . . . . . . 


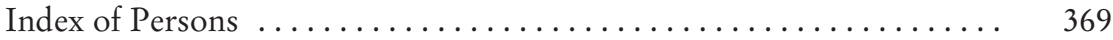

Index of Subjects $\ldots \ldots \ldots \ldots \ldots \ldots \ldots \ldots \ldots \ldots \ldots \ldots \ldots \ldots \ldots$ 



\section{Introduction \\ Gregor Etzelmüller/Christian Tewes}

Since the 19th century, the theory of evolution has unsettled and shaken traditional and fundamental anthropological assumptions about the place of human beings in nature. The Darwinian integration of human evolution into natural history was countered by the philosophical and theological anthropologies of the 20th century (Scheler, Plessner, Gehlen, Portmann, Pannenberg) with their attempts to hold on to the special status of humans in their intrinsically openness to the world and their spirit-endowed nature. Today, evolutionary anthropology, as well as the more recent philosophical anthropology, are increasingly based on the paradigm of embodied cognition (e.g., Varela, Thompson and Rosch, Clark, Gallagher, Thompson, Deacon, Donald, Tomasello, and Jung). Building on "embodied and enactive cognitive science", this volume aims at answering the question to what extent the human mind and human cultural cognition are attributable to the structures of human existence, structures that have emerged in the course of evolution and have in turn been affected by cultural evolution. The paradigm of embodiment shows why the traditional dualistic opposition of nature and culture, body and mind is unjustified and how it can be overcome by an enactive framework of research on embodiment.

\section{Current Research on Embodied Cognition}

If one surveys the landscape of ever-growing research into embodied cognition, one sees not only commonalities but also important differences in the interpretation of this paradigm's theoretical commitments. With Richard Menary, one can distinguish between a moderate and a strong embodied mind thesis. The proponents of the former view think that some mental states depend on non-neural bodily processes or states. The latter position, by contrast, emphasizes that at least some mental states are constituted by those non-neural bodily processes (see Menary 2015). It is worth noting that the moderate approach even encompasses certain varieties of internalism. According to the latter positon, cognition can be located within the brain (it is brain bound) without involving any constitutive processes that exceed the boundaries of the skull. Frederick Adams and Kenneth Aizawa, for instance, do not deny that the nervous system is connected to the body and the environment in multifarious causal ways. They also acknowledge the claim that cognitive vehicles in the lifeworld such as mathematical notations, 
or writing tools such as pencils or graphic characters, enable and support the accomplishment of cognitive tasks and operations (see Adams and Aizawa 2001). However, they are strongly critical of the claim that those states are constitutive for cognition. They stick to the more traditional view in the cognitive sciences that the human mind supervenes exclusively on brain states.

Both enactivism and the extended mind thesis reject this internalist reading of cognition in favour of strong approaches to the embodiment paradigm. Proponents of enactivism have pointed out that there is a mutual and constant circulation between body, brain and their environment (Thompson and Varela 2001, 424). The explication of this circular process in sensorimotor and autopoietic terms reveals that the strong embodied mind thesis is not solely justified by the almost trivial insight that perceptual content plays a foundational role in the human mind. The crucial point is rather that cognition is not something that happens inside the skull but is constituted as a "relational domain" by the incessant interaction between body, mind, and world (see Thompson and Stapleton 2009).

To understand this kind of interaction in more detail, it is important to note that the living organism enacts the world in which it exists. This means that organisms actively constitute their environment and are simultaneously constituted by it. Thus, the strong embodied mind thesis is justified in the case of enactivism by means of explicating the relational co-constitution of cognitive and mental processes.

Moreover, this approach leads to the conclusion that there is not simply a pregiven world that an animal with a central nervous system matches or represents by means of its neural states (Stewart 2014, 3). Rather, when animals build nests, dams, or burrows, they reshape and structure the developmental environment for subsequent generations (Sterelny 2010, 470).

Accordingly, the cognitive and cultural evolution of human beings is not to be seen as a constant process of adaptation to an independent environment existing identically for every living being; it is instead to be seen as a process of co-evolution of interrelated systems. Over the course of human development, it is particularly the historical-cultural dimension of cognition and the culturally shaped environment that - contingent on each other - expand in scope. When one approaches the theory of embodiment from such an evolutionary perspective, one begins to see its potential for overcoming the still implicitly present Cartesian divide between mind (res cogitans) and matter (res extensa) which continues to cast its shadow on the scientific exploration of man.

This dichotomy expresses itself in the fact that the natural roots of human beings are traditionally explored by means of the natural sciences and related disciplines, whereas the varieties of cultural practices in the lifeworld frequently fall into the domain of the humanities. This division of labor is always in danger of merely giving the dualism a new name, for instance a contrast between nature and mind or nature and nurture. Especially in anthropology, there is a strong tendency to uphold dichotomous thinking, even today (Thompson 2007, 410-411).

Evolutionary anthropology has tended to emphasize the continuity between animals and human beings by pointing to recent findings from evolutionary psy- 
chology, palaeoanthropology, molecular biology, comparative neurosciences, etc. Most properties or skills that have been considered exclusive human features in the past are rooted in capacities that can be found in other species too (see de Waal 2009, Welsch, Singer, and Wunder 2011). The strict naturalistic reinterpretation of those findings in a Neo-Darwinian framework sometimes leads to the claim that it may be possible to explain cultural entities or institutions such as religion or art purely in terms of biological functions (see Dennett 2006, Dawkins 2006). Additionally, evolutionary anthropology assumes that there is, in a realistic sense, a given human nature due to the environmental constraints on human adaptation.

Some cultural anthropologists, on the other hand, basing their tenets on historical studies, respond to these encroachments with a rather radical view. They favor a constructivist stance, correctly pointing out that the natural sciences and the interpretation of experiments and findings are also produced within the cultural realm of the lifeworld (Schnegg 2015, 42). A more radical interpretation of this fact is the conviction that scientific findings need to be conceived as cultural constructs (von Glasersfeld 1996). From this point of view, science does not reveal forms or aspects of reality (laws, structures, and entities) that exist as the result of a relational co-constitution of human practices with the environment. To take one example, not only gender but even the ostensibly "natural" distinction of the sexes has been conceptualized in this research tradition as an ideological construct (see Butler 2014). Here the impact of postmodernist and radical constructivist thinking on cultural anthropology is obvious (Schnegg 2015, 39).

Unsurprisingly, such tendencies sometimes result in a clash between different research traditions, and dualistic implications sometimes reappear in modern guise. This has led to a chasm opening up between evolutionary and historical anthropology, hampering scientific attempts to bridge the natural and cultural realm with a coherent conceptual framework.

\section{Evolutionary Continuity and Discontinuity}

As the above considerations have already indicated, it is the central thesis of this book that enactivism has the potential to contribute in significant ways to overcome this unfruitful divide in contemporary anthropological research. This is because enactivism allows for a genuine and coherent explication of the strong embodied mind thesis. Evan Thompson, for instance, has worked out in detail the thesis that there is a deep continuity between mind and life (Thompson 2007).

His key idea is that even the simplest organism enacts its environment in such a way that, via a sense-making process, an environment emerges that is meaningful for the organism. "In observing other creatures struggling to continue their existence - starting with bacteria that actively swim away from a chemical repellent - we can, through the evidence of our own experience and the Darwinian evidence of the continuity of life, view inwardness and purposiveness as proper to living being" (Thompson 2007, 163). To take another example, physicochem- 
ical macromolecules may manifest themselves within the milieu of a bacterium as nutrition or poison, that is, as meaningful affordances that either support or threaten the organism's life. These are relational emergent properties which cannot be reduced to a purely physicochemical description of reality.

This is entirely in line with Hans Jonas' concept of life as something manifested in the peculiar organization of organisms, which he sees as coextensive with mindful, self-sustaining processes. The fact that mental life is always embodied does not only mean "that the mind even on its highest reach remains part of the organism" but that the organism, even in its lowest form, prefigures mind (Jonas 1966, 1; Sheets-Johnstone 1999, 52). Especially the autopoietic explication of organisms, their adaptivity and teleological structure, may be regarded as the attempt to operationalize those bio-philosophical insights and make them available for further empirical research (see Di Paolo 2005).

However, it would be wrong to suppose that cultural processes are simply built upon biological processes as higher layers of organization. It is one thing to elaborate convincing arguments and refer to empirical evidence for the evolutionary continuity of mind and life. It is quite another matter to give explanations for how the obvious discontinuities among non-human primates and humans have evolved. One explanation given for the distinctiveness of the latter is in terms of plasticity. Learning capacities and creativity, such as the ability to adapt to novel situations in multifarious ways or to create new problem-solving strategies, are important properties of plasticity. It has frequently been pointed out that plasticity is a design feature of the brain with specific modifications among the species. To a certain extent, this feature is present in every brain system.

However, in such a comparative approach, a distinctive feature of the human brain is its superplasticity. Human beings are remarkably quick at evaluating a wide range of strategies for surviving in, and adapting to, a rapidly changing environment (Donald 2001, 210). Compared with other animals, "what is different about human beings is that they are not just adapted for specific pre-existent structures in their environment such as pheromone trails and larvae, but rather that they are adapted for acquiring totally new skills and knowledge from their social-cultural environments" (Tomasello 2003, 238f.).

As studies of niche construction have shown, the co-constitution of organisms and environment generally results from continuous bottom-up and topdown processes. This is true a fortiori with processes of enculturation, which are key factors in creating niches for enabling, developing, and stabilizing new learning strategies and flexible behavior. Those cultural niches are based on the acquisition and transfer of the cultural practices of social groups in a way that modifies both the biological and informational environment in mutually determining ways (Sterelny 2010, 470; Menary 2015, 4). Thus, from a phylogenetic and ontogenetic perspective culture plays a paramount role in explaining significant aspects of the evolutionary discontinuity between non-human and human primates mentioned above. 


\section{Evolutionary Specificities of the Human Body}

Michael Tomasello's research on great apes and infants can be of help in deepening our understanding of these relations between nature and culture. Humans differ from great apes in at least two important respects: by means of intergenerational learning processes and a complex understanding of actors as intentional and cooperative agents. Already by the age of six months, infants develop the ability to follow the direction of a parent's gaze. This enables them to predict people's action in familiar surroundings. This is an important prerequisite to understanding fully-fledged intentional actions at a later age. An important intermediate state for understanding decision-making processes and action plans is the so called "nine month revolution" (Tomasello 2001, 61). At this age children start to understand other human beings as goal-directed and they begin to engage in triadic interactions (Tomasello et al. 2005, 682).

This leads to powerful cultural learning strategies, such as imitative learning, whereby the observer must conduct a complex means-end analysis of actions (Tomasello et al. 2005, 680). As these findings indicate, it is wrong to suppose that the ability for triadic interaction could be acquired in an atomistic fashion. It is obvious from an evolutionary viewpoint that culture functions as an ontogenetic niche for children. From the very beginning of their lives, children are engaged and immersed in ongoing social practices and a world of cultural artefacts, which enable their participation in social interactions (Portmann 1944; Tomasello 2001, 78-79).

It is important to emphasize, however, that a fundamental cognitive ability such as following the gaze of conspecifics requires a special shape and setup of the body for realizing such intersubjective cognitive processes in the first place. The latter is the upshot of synchronic cultural and biological evolutionary processes. In support of these thesis, research points to the fact that the human eye has passed through an evolutionary process of adaptation that enables a gaze to be followed. There is evidence that "in humans the widely exposed sclera (the white of the eye surrounding the darker coloured iris) make it easy for others to discern the gaze direction" (Kobayashi and Kohshima 2001, 419). This is an adaptation that distinguishes us, among other things, from other primates.

Furthermore, it is possible to show that the contribution made by eyeball movement (compared to head movement) to the change in gaze direction is very high in humans (Kobayashi and Kohshima 2001, 434). In evolutionary terms, this might be due to mutations that changed the phenotypes in such a way that it supported the generation of new forms of social interaction. At the same time, those properties are positively selected due to their functional effectiveness within the socio-cultural realm. Of course, such changes and adaptations occur in the context of specific constraints upon the entire morphological structure of the body and its embeddedness in the cultural environment.

To give an example, the larger the size of the body becomes, the more effective is eyeball movement for controlling gaze direction compared to head or body movement (Kobayashi and Kohshima 2001, 426-427). This underlines on the 
one hand the significance of the shape, composition and makeup of the individual body for the emergence of cultural niches. On the other hand, it indicates how cultural constraints can be simultaneously co-constitutive for the human body when we may assume that eyeball movement for controlling gaze direction is positively selected in the emerging cultural niche. This is then an example how cultural and biological factors in evolution are combined an inseparable synergetic process (Malafouris 2013, 39).

These aspects of the the phylo- and ontogenetic setup of social interactions get a further justification in a phenomenological approach to intercorporeality that is an integral part of the enactive research program. Here we refer to the thesis that social interactions are based on a form of direct perception. At a pre-reflective level, we have direct access, for instance, to the emotional states of other persons, an access which is not mediated by sub-personal or personal simulation processes or inferences (Gallagher 2008, 539).

One can explicate and complement this account with the concept of interbodily resonance. A person's feelings are expressed in various bodily reactions (facial, gestural, or interoceptive) which function as a resonance platform. Thomas Fuchs has termed this process intra-bodily resonance (Froese and Fuchs $2013,212)$. When those expressions become visible by means of intra-bodily-resonance processes (blushing and frowning as expressions of anger), they also become accessible for other people and serve as inter-personal resonance platforms of the feeling-bodies, intertwined and mutually shaped by an ongoing interactive process (a succinct example is contagious laughter). These basic interbodily expressive patterns are directly accessible in perception.

The remarks above on gaze direction already indicate that this capacity allows us to take the perspective of conspecifics and to direct our mutual attention to a third entity. This leads to an important extension of inter-bodily relationships. Building on the capacity for direct perception, it is possible to grasp how the evolutionary development of triadic communication and the ability to symbolize and reconstruct the perspective (beliefs, desires, plans) of other human beings ("theory of mind") could occur in the first place.

However, even the evolution of human language has only been possible because of a further evolutionary variation of the human body, in particular the supralaryngeal vocal tract:

The human larynx or 'voice box' (containing the vocal folds or vocal cords) differs significantly in position from the larynx of other primates such as monkeys ... One unfortunate consequence of this development is that the lower position of the human larynx makes it much more possible for the humans to choke on pieces of food ... In evolutionary terms, there must have been a big advantage in getting this extra vocal power (i. e. larger range of sounds) ... (Yule 2014, 5).

The advantage of a differentiated language outweighed its potentially fatal disadvantage (Fuchs 2013, 23 f.). As Donald summarizes research on that matter, this must be due to a positive selection pressure at the cultural level (Donald 1991, 237). 
If we consider evolutionary development and the examples of the human eye and vocal tract we can see that the intertwining of natural and cultural evolutionary processes generate bodily forms which allow for richer forms of intersubjectivity (and in this sense further forms of cultural developments) and which are positively selected due to a feedback loop of selection pressure within cultural niches. In this sense we can speak of a mutual causality of nature and culture.

The multidirectional and circular interactions of physiological, psychological, and social processes come to the fore once one resists those dualistic tendencies in the humanities and natural sciences mentioned earlier. What is needed is an interdisciplinary focus on the exploration of the embodied mind. The enactive probing and shaping of the environment by the organism leads to an emergence of mental processes at different levels of complexity with an immediate feedback upon how the organism guides its current and prospective action cycles in its habitat (Stewart [2010] 2014, 4-5). This implies that any understanding of the nature of human beings needs to take into account their mental, cognitive, and social operations as well as the physical embeddedness of those mental processes.

\section{Aims and Structure of the Volume}

It is the aim of this anthology to examine, unfold, and substantiate the thesis that the paradigm of embodiment can bridge the gap between cultural and evolutionary anthropology, thereby enabling a fruitful exchange between the two different research perspectives. Further, the paradigm of embodiment can offer new answers to the question of how evolutionary processes could give rise to a living being with a specific mental constitution capable of accelerating, stabilizing, and influencing its own cultural development in such a remarkable way (Tomasello's famous "ratchet effect", Tomasello 2001, 5).

It is likely that a coherent answer to this question will also include a new account of nature which is different from any model of nature that reductive naturalism or physicalism offer (McDowell 1994, 77). Because human beings are to a certain extent responsive to reason-giving processes that are indispensable in a complete description and explanation of human actions in action theory and anthropological research, an evolutionary approach must explain how natural evolutionary processes could bring forth human beings who interact with and are responsive to the "space of reason" within the socio-cultural realm. Taking these aspects together, this points to the continuity of mind and life in nature whereby basic sense-making processes prefigure at least some aspects of the normative and meaningful dimension of full-fledged reasons. This is especially important with regard to ethical reflections and how reasons could, to put it in Davidson's terms, be “causes" or play at least a vital role in the development of self-governance and moral commitments of persons (see Davidson [1963] 1980b, [1970] 1980c, [1971] 1980d). The self-differentiation of values and the enactive mind within the evolutionary lifemind continuum is therefore important in that it sheds light on the explication of reason and normativity (Di Paolo Rohde, and De Jaegher [2010] 2014, 50-51). 
With regard to these questions, the volume starts with an elaboration of the concept of embodiment (Section One). As already indicated, the literature on embodiment explicates this paradigmatic concept in diverse ways. Certainly there is general agreement between classical enactivism, radical enactivism, and the extended mind theory that cognitive states not only depend on bodily processes but that bodily processes play a constitutive role for the mental formation and effectiveness of a person. However, there are also important differences in detail in how these shared assumptions are developed. The significance of these details for the entire embodied approach to evolution and culture is the central topic of the first chapter and is analyzed in the papers that follow. The authors go on to explore how, in the structural coupling of the organism with its habitat, not only new forms of significance emerge but also mental and cognitive abilities that cannot be reduced to the physio-chemical realm.

This question is explored further in Section Two: how, by means of their intercorporeal constitution in the lifeworld, do human beings develop cognitive and linguistic capacities which transcend the bere and now of their immediate bodily-based egocentric perspective, such as in declarative memory and prospective plans of action? At the cultural level, the embodied mind manifests itself as a "cultural bodily-based power materialized in countless cultural products that enables individual as well as intersubjective conducts of life in dense memories and imaginations" (Welker 2015, 310). Using insights from different fields, such as cognitive semiotics, biological anthropology, developmental psychology, and philosophy of language, the contributors to this section develop an evolutionary explanation of language and symbol use.

Section Three explores to what extent the paradigm of embodiment can overcome the widening chasm between evolutionary and historical anthropology. Discussing the ideas of Charles Darwin, the section shows how human evolution brings forth a being, which is able to develop forms of sympathy and empathy. In the co-evolution of human cognition and empathy, specific human forms of joint attention and mimesis play a crucial role. They lead to powerful cultural learning strategies. Consequently, on the one side, evolutionary anthropology conceives of humans as beings whose variety can only be described by historical means. On the other side, historical anthropology makes it clear that even premodern cultures were familiar with the fact that human beings are embodied.

The insights of Sections Two and Three are used to shed light on the issue of human phylogenesis in Section Four. To explain the origins of contemporary human cultural behavior, evolutionary-biological processes such as genetic mutation and selection are widely seen as - to some extent - necessary but not sufficient. The contributors to this section follow "bottom-up" approaches to get closer to the central question of how the specific combination of natural and cultural characters expressed by contemporary humans could have developed in physical and cognitive interaction with the material world. This last part of the book focuses on the significance of participatory interaction with artefacts and materiality for our development as a species. In this regard, material-engagement theory and its insights are of primary interest in the volume's final section. 


\section{Bibliography}

Adams, F. and K. Aizawa. 2001. The Bounds of Cognition. Philosophical Psychology 14: 43-64.

Arendt, H. 1958. The Human Condition. Chicago: University of Chicago Press.

Bayertz, K. 2014. Der aufrechte Gang. Eine Geschichte des anthropologischen Denkens. München: Beck.

Butler, J. 2004. Undoing Gender. New York, NY: Routledge.

Campbell, R. and M. H. Bickhard. 2011. Physicalism, Emergence and Downward Causation. Axiomathes 21: 33-56.

Darwin, C. 1966. On the Origin of Species By Means of Natural Selection (1859). With an introduction by Ernst Mayr. Cambridge, MA: Harvard University Press.

-. 1981. The Descent of Man, and Selection in Relation to Sex (1871). With an introduction by J. T. Bonner. Photoreproduction of the 1871 ed., London, Princeton, NJ: Princeton University Press.

Davidson, D. 1980a. Essays on Actions and Events. Oxford: Clarendon Press.

-. (1963) 1980b. Actions, Reasons and Causes. In D. Davidson 1980a, 3-19.

-. (1970) 1980c. How is Weakness of the Will Possible? In D. Davidson 1980a, 21-42.

-. (1971) 1980d. Agency. In D. Davidson 1980a, 43-61.

Dennett, D. 2006. Breaking the Spell. Religion as a Natural Phenomenon. New York: Viking Press.

de Waal, F. 2009. The Age of Empathy. Nature's Lessons for a Kinder Society, New York: Harmony Books.

Donald, M. 1991. Origins of the Modern Mind. Three Stages in the Evolution of Culture and Cognition. Cambridge, MA, London, UK: Harvard University Press.

Di Paolo, E. 2005. Autopoiesis, Adaptivity, Teleology, Agency. Phenomenology and the Cognitive Sciences 4: 429-452.

Di Paolo, E., M. Rohde, and H. De Jaegher. [2010] 2014. Horizons for the Enactive Mind: Values, Social Interaction and Play. In Toward a New Paradigm for Cognitive Science, ed. J. Stewart, O. Gapenne, and E. A. Di Paolo, 33-87. Cambridge, MA, London: MIT Press.

Dawkins, R. 2006. The God Delusion. New York: Bantam Books.

Freeman, W. J. 1995. Societies of Brains. A Study in the Neuroscience of Love and Hate. New Jersey: Lawrence Erlbaum Associates.

Froese, T. and T. Fuchs. 2012. The Extended Body: A Case Study in the Neurophenomenology of Social Interaction. Phenomenology and the Cognitive Sciences 11: 205235.

Fuchs, T. 2013. Verkörperung, Sozialität und Kultur. In Interdisziplinäre Anthropologie. Leib - Geist - Kultur, ed. T. Breyer and G. Etzelmüller, T. Fuchs, and Grit Schwarzkopf, 11-33. Heidelberg: Winter.

Gallagher, S. 2008. Direct Perception in the Intersubjective Context. Consciousness and Cognition 17: 535-543.

Jonas, H. 1966. The Phenomenon of Life. Toward a Philosophical Biology. New York: Harper \& Row.

Jung, M. 2009. Der bewusste Ausdruck. Anthropologie der Artikulation. Berlin, New: York: de Gruyter.

Keil, G. 2003. Homunkulismus in den Kognitionswissenschaften. In Ist der Geist berechenbar? Philosophische Reflexionen, ed. W. R. Köhler, H. D. Mutschler, 77-112. Darmstadt: Wissenschaftliche Buchgesellschaft.

Kelso, J. A.S. 1995. Dynamic Patterns. The Self-Organization of Brain and Behavior. Cambridge, MA, London, UK: The MIT Press. 
Kobayashi, H. and S. Kohshima, S. 2001. Unique Morphology of the Human Eye and its Adaptive Meaning. Comparative Studies on External Morphology of the Primate Eye. Journal of Human Evolution 40: 419-435.

Malafouris, L. 2013. How Things Shape the Mind. A Theory of Material Engagement, Cambridge, MA, London, UK: The MIT Press.

McDowell, J. 1994. Mind and World. With a New Introduction by the Author. Cambridge, MA, London, UK: Harvard University Press.

Menary, R. 2015. Mathematical Cognition - A Case of Enculturation. In T. Metzinger and J. M. Windt. Open MIND 25(T): 1-20. Frankfurt a. M.

Portmann, A. 1944. Biologische Fragmente. Zu einer Lehre vom Menschen. Basel: Benno Schwabe \& Co.

Schnegg, M. 2015. Epistemology: The Nature and Validation of Knowledge. In Handbook of Methods in Cultural Anthropology, H. R. Bernhard and C. Gravlee, 21-54. 2nd Edition, 1st Edition 1998. Lanham, Boulder, New York: Rowman \& Littlefield.

Sheets-Johnstone, M. 1999. The Primacy of Movement. Philadelphia: John Benjamin.

Stewart, J. [2010] 2014. Foundational Issues in Enaction as a Paradigm for Cognitive Science. In Toward a New Paradigm for Cognitive Science, ed. J. Stewart, O. Gapenne, E. Di Paolo and A. Ezequiel, 1-31. Cambridge MA, London UK: MIT Press.

Sterelny, K. 2010. Minds: Extended or Scaffolded? Phenomenology and The Cognitive Sciences 9: 465-481.

Thompson, E. 2007. Mind in Life. Biology, Phenomenology, and the Sciences of Mind. Cambridge MA, London UK: The Belknap Press of Harvard University Press.

Thompson, E. and F. J. Varela. 2001. Radical Embodiment: Neural Dynamics and Consciousness. Trends in Cognitive Sciences 5: 418-425.

Tomasello, M. 2001. The Cultural Origins of Human Cognition. Cambridge MA, London UK: Harvard University Press.

Tomasello, M., M. Carpenter, J. Call, T. Behne, and H. Moll. 2005. Understanding and Sharing Intentions: The Origins of Cultural Cognition. Behavioral and Brain Sciences 28: 675-691.

van Gelder, T. 1998. The Dynamical Hypothesis in Cognitive Science. Behavioral and Brain Sciences 21: 615-665.

von Glasersfeld, E. 1996. Radical Constructivism. A Way of Knowing and Learning. London: Falmer Press.

Weber, A. and F. J. Varela. 2002. Life After Kant: Natural Purposes and the Autopoietic Foundations of Biological Individuality. Phenomenology and the Cognitive Sciences 1: 97-125.

Welker, M. 2015. Was kann theologische Rede von Inkarnation und Auferstehung zur Anthropologie Beitragen? In Verkörperung als Paradigma theologischer Anthropologie, ed. G. Etzelmüller and A. Weissenrieder, 317-325. Berlin, New York: de Gruyter.

Welsch, W., W. Singer, and A. Wunder, eds. 2011. Interdisciplinary Anthropology. Continuing Evolution of Man. Berlin, Heidelberg: Springer.

Welsch, W. 2012. Homo Mundanus. Jenseits der anthropischen Denkform der Moderne. Weilerswist: Velbrück Wissenschaft.

Yule, G. 2014. The Study of Language. Fifth Edition. Cambridge: Cambridge University Press. 


\section{Philosophical Concepts}

and Perspectives of Embodiment 



\section{Introduction}

\section{Christian Terwes}

At first sight, it might seem surprising that the concept of embodiment is playing an ever-increasing role in the philosophy of mind and anthropology. Is it not evident from many empirical findings that the body "shapes the mind" in important respects, as Shaun Gallagher set out in detail years ago (see Gallagher 2005)? Only very few philosophers defend the argument in the current bodymind debate that there is a strict dichotomy between mental and physical events, let alone entirely different substances (see Meixner 2004). And most theorists in the field today accept that humans are the outcome of an evolutionary process and that Darwin's insight (not to be confused with Neo-Darwinism) in the Origin of Species (Darwin 1859) has significant explanatory value not only with regard to the biological heritage of the human mind but also the cultural condition (cultural niches) of its existence.

Though these issues are often agreed upon, the explanatory role of the body and the metaphysical and anthropological implications of embodiment have still to find broad consensus within the cognitive sciences and anthropology. There are several reasons for this, as the contributions in the first chapter explain. The most obvious is that embodied cognition is frequently viewed as an alternative to standard cognitive sciences, or at least as a challenge to some of their basic assumptions (see Shapiro 2011). The challenges concern such questions as the relationship of the brain and body to consciousness and cognition, the question of whether basic minds have representational, content-involving access to the world or the role of emotions and affections for cognitive processes.

The first contribution of this chapter analyzes in greater depth the relation between brain and body with regard to cognition and consciousness. Mog Stapleton introduces and defends the thesis of proper embodiment. This concept of embodiment is intimately related to Thompson and Cosmelli's view, developed in recent years, that the entire body and not only the brain plays a constitutive role in the emergence of consciousness (see Cosmelli and Thompson [2010] 2014). According to the thesis of proper embodiment, our physiology is paramount to our cognition: the underlying "mechanisms" of cognition and consciousness are so finely grained that the specification of the algorithm for cognition would entail aspects of the "internal body" and not only the configurations and processes of the brain.

The reference to mechanisms indicates that there might be functional states that determine how the implementation of sub-personal processes gives rise to 
cognitive and mental states. Stapleton coins this approach "orthodox embodied cognitive sciences” (see Stapleton 2013). This refers to one important research tradition in current embodied cognitive science that shares the traditional functionalist approach to mind and cognition but does not confine the implementation of cognitive and mental states to the working mechanisms of the brain. The latter approach thus includes the position of extended functionalism (see Wheeler 2010).

But how is the concept of proper embodiment related to orthodox embodied cognitive science? To answer this question, it is important to note that the thesis consists of two sub-theses that Stapleton develops in the course of her paper. She terms the first one "internal embodiment" and defines it as the thesis that "the internal 'gooey' body matters to cognition and consciousness in a fundamental way" (Stapleton, this volume). She refers here in particular to the sense of our internal body in interoception and the constitutive role it plays in consciousness and cognition. The second sub-thesis is termed "particular embodiment." This is the view that the fine-grained details of functional implementation matter when it comes to the emergence of cognitive states. Taking both sub-theses and their implications together, Stapleton argues that the seemingly clear-cut distinction between (functional) algorithms and their implementation - a key feature of orthodox cognitive science - is undermined by proper embodiment.

The topic of the second paper, by Christian Tewes, concerns the question of whether the extended mind hypothesis (still as part of the orthodox embodied cognitive sciences) or the enactivist research program in embodied cognition is better suited to deal with the research field of embodied memory formation. Enactivism involves, among other things, the thesis that even basic organisms have sense-making capacities and that there is a strong relation between actions and perceptions at different levels of explanation that are not fully captured in the traditional cognitive sciences.

In the inaugural paper of the "extended mind hypothesis" (henceforth, EM) the extension of memory capacities already plays an important role (see Clark and Chalmers [1998] 2010). The extension of cognitive and mental states and capacities is usually justified by means of a dynamical systems approach in order to show in what cases the mind actually extends the skull and the body and integrates items of the environment into the cognitive machinery.

Proponents of the enactivist camp have challenged this picture und provided some distinctions and criteria to differentiate between new emerging (autonomous) systems and the mere extension or enhancement of mental capacities. Besides the more formal concepts of autonomous systems and sense-making processes, enactivism also relies on phenomenological insights and concepts to argue for these distinctions. It is Tewes' thesis that these basic distinctions are also of major significance for exploring the constitution of memory. He elaborates this point with particular regard to the question of how enactivism can contribute to research on habitual embodied memory formation and how memory research undertaken within the framework of EM can profit from the enactive research program and its insights. 
In the paper which follows, Karim Zahidi and Erik Myin apply the radical enactivist approach to the phylo- and ontogenetic emergence of numerical cognition. According to "Radically Enactive/Embodied Cognition" (henceforth REC), basic cognition is constituted by concrete items of environmentally situated organismic activity (Hutto and Myin 2013,11). What is "radical" with regard to the REC approach is the conviction that basic forms of perception and interaction are mindful - such experiences have phenomenal character and are intentionally directed - but do not involve content and the use of representation. Thus, as Zahidi and Myin point out in their paper, sensorimotor cognition such as chasing a rabbit or grasping a leaf are directed towards objects but do not represent them (see Zahidi and Myin, this volume). However, this does not imply that the existence of representation and contentful states should be abandoned. Quite the contrary, these shape the human mind in important respects even if they only emerge at later phylo- and ontogenetic stages.

Proponents of REC also hold the view that computational abilities - in strict analogy with representational states - require sociocultural language contexts for their evolutionary emergence. Zahidi and Myin's aim is to develop an REC account of the phylo- and ontogenetic development of numerical cognition, one that implies the inversion of the explanans and the explanandum of the traditional cognitive sciences. In this sense "REC aims to truly account for computation in contrast to assuming computation as a basic, and fundamentally unaccounted for, ingredient in nature" (Zahidi and Myin, this volume).

It is the aim of their paper to show that descriptions of certain types of behavior displayed by animals or pre-verbal infants does not warrant the ascription to them of arithmetical competence. In their discussion, the authors focus on a proposal that monkeys already possess number concepts. If this claim were to be true, it would apparently refute certain basic tenets of the REC approach to cognition.

Zahidi and Myin develop conceptual arguments against such a "rich interpretation" of numerical abilities in monkeys and infants, namely "that to treat similar things or situations as perceptually similar" already involves representational and computational abilities. Furthermore, they point to possible empirically-grounded "lean interpretations" that could explain the same behavioral evidence without the assumption of basic computational abilities.

In the final contribution of this chapter, Christian Spahn explores the wider reasons that have motivated the "turn to embodiment" in the first place. In the first part of his paper, he analyzes some deeply rooted motives in Western culture that have led to dualistic concepts. We measure ourselves with regard to values and self-governance that are connected with our ability to "distance ourselves from mere biological impulses and instincts” (Spahn, this volume). This has contributed to the emergence of basic distinctions such as "is" (factual reality) and "ought" (ideal realm) and the idea of mental powers shaping our nature (impulses and instincts).

Spahn then shows how enactivism as a theory of embodied cognition tries to overcome these dualisms by focusing on the philosophy of organic life (see 
Thompson 2007). The differences between the physical and the organic body and the attempt to elaborate a concept of nature that does not coincide with reductive physicalism guide Spahn's discussion. He also evaluates Thompson and Cosmelli's critique of neurocentric intuitions that consciousness supervenes locally on brain states, drawing upon their conceptual and empirically-based arguments for why one cannot isolate brain states and their corresponding conscious states from the entire body, even in a thought experiment (see Cosmelli and Thompson [2010] 2014).

However, as he points out in the last part of the paper, Spahn does not think that dualistic implications are entirely solved within the enactivist research program and in its findings. What remains to be explained are such basic questions as how the interiority of the organism is related to consciousness. Is it possible to answer this question within a naturalistic framework or do we need to look at alternative ontological options? And can the sense-making processes of organismic life be built upon to deliver a justification for values and ethical judgements (facts versus justification procedures) as well?

Each of these issues delineates fields which embodiment-based research can fruitfully explore in future.

\section{Bibliography}

Clark, A. and D. Chalmers. (1998) 2010. The Extended Mind. In The Extended Mind, ed. R. Menary, 27-42. Cambridge, MA: The MIT Press.

Cosmelli, D. and E. Thompson. (2010) 2014. Embodiment or Envatment? Reflections on the Bodily Basis of Consciousness. In Enaction: Toward a New Paradigm for Cognitive Science, ed. J. Stewart, O. Gapenne, and E. A. Di Paolo, 361-385. Cambridge, MA: The MIT Press.

Darwin, C. 1859. On the Origin of Species. London: John Murray.

Gallagher, S. 2005. How the body shapes the mind. New York: Oxford University Press.

Hutto, D. D. and E. Myin. 2013. Radicalizing Enactivism. Basic Minds Without Content. Cambridge, MA: The MIT Press.

Meixner, U. 2004. The Two Sides of Being. A Reassessment of Psycho-Physical Dualism. Paderborn: Mentis.

Shapiro, L. 2011. Embodied Cognition. London: Routledge.

Spahn, C. 2016. Beyond Dualism? The Implications of Evolutionary Theory for an Anthropological Determination of Human Being. In this volume.

Stapleton, M. 2013. Steps to a "Properly Embodied" Cognitive Science. Cognitive Systems Research 22-23: 1-11.

Stapleton, M. 2016. Leaky Levels and the Case for Proper Embodiment. In this volume.

Tewes, C. 2016. Embodied Habitual Memory Formation: Enacted or Extended? In this volume.

Thompson, E. 2007. Mind in Life. Biology, Phenomenology, and the Sciences of Mind. Cambridge MA, London UK: The Belknap Press of Harvard University Press

Wheeler, M. 2010. In Defense of Extended Functionalism. In The Extended Mind, ed. R. Menary, 245-270. Cambridge, MA: The MIT Press.

Zahidi, K. and E. Myin. 2016. Radically Enactive Numerical Cognition. In this volume. 


\title{
Leaky Levels and the Case for Proper Embodiment
}

\author{
Mog Stapleton
}

\begin{abstract}
In this chapter I present the thesis of Proper Embodiment: the claim that (at least some of) the details of our physiology matter to cognition and consciousness in a fundamental way. This thesis is composed of two sub-claims: (1) if we are to design, build, or evolve artificial systems that are cognitive in the way that we are, these systems will have to be internally embodied, and (2) the exploitation of the particular internal embodiment that allows systems to evolve solutions with greatly decreased computational complexity - and thus to be flexible and adaptive in the ways that are characteristic of cognitive systems - means that the orthodox distinction between algorithm and implementation is undermined. Evolved cognitive systems are therefore likely not to instantiate the distinction between phenomenology, algorithm, and implementation. The leaky levels evident in evolved cognitive systems motivate an extension of orthodox embodied cognitive science to the internal, affective, "gooey" realm that has so far only been embraced by those in the enactive tradition. This suggests that if we are to build artificial systems that will be genuinely cognitive they will have to incorporate parts of the gooey realm currently considered to be "mere implementation."
\end{abstract}

\section{Orthodox Embodied Cognitive Science}

While there are many conceptions of embodiment that are relevant to philosophy of mind and cognitive science (see for example, Clark 1999; Wilson 2002; Anderson 2003; Ziemke 2003; Shapiro 2007) a broad overarching characterization used both by philosophers and those in the other cognitive sciences, including but not limited to robotics, is that our problem solving abilities are much less a matter of internal processing of information that comes into the system than orthodox cognitive science used to assume. Susan Hurley characterized the way of thinking about how minds work in the pre-embodiment revolution era as "the classical sandwich" (Hurley 1998): the thing that is doing the important work - the thinking or cognizing - is sandwiched between perception, bringing the information in, and acting according to the results of the information processing. On this model, the body is there to do the sensing and acting. Other than this the non-neural body is there just to keep the parts of the brain that these cognitive processes supervene on/are identical with (depending on your philosophical take) alive. Embodied cognitive science rejected this picture arguing that cognition cannot be cleanly separated from perception and action, and that many of our problem solving abilities are more a matter of adjusting the system itself, phylogenetically, 
ontogenetically, or in occurrent action, such that the information processing that we would assume to be required if we were to set about programming these abilities into artificial systems, is actually "offloaded" onto the system's morphology.

While the term "offloading" in this respect is useful to see the difference from the pre-embodiment approach it is somewhat misleading because it implies that the standard case is that computations are done by the brain, but that they can be done by other structures instead. It rather seems to be the opposite. For many abilities the standard case is that we utilize the morphology of the body, the possibilities for action that it gives to us, and other structures in the environment so that we never have to do it all in our head (see Clark 1997, 2008b, [2001] 2013) indeed most of us could not even do the calculating required for most of the things we do purely in our head. The result of taking embodiment seriously in robotics, is that when designing artificial cognitive systems, instead of deciding what function was to be implemented and then designing a program to implement it in a particular robot body (a top-down approach) one could rather consider what kinds of creatures achieve these tasks, consider how their bodies allow them to do it, emulate parts of that embodiment in the artificial system, and then program minimally to enable the system to engage in the bodily behavior (a bottom-up approach) (see Pfeifer and Bongard 2006, for a detailed examination of these principles at work in artificial cognitive systems and robotics).

The lesson for philosophy of mind should be clear from considering this case: the mind is both simpler and more complex than we previously imagined. On the one hand, we do not do as much of the information crunching that - on the orthodox approach - we assumed we must to support the ways that we perceive, think, and act. On the other hand, parts of what we had categorized as the mental when we assumed that minds were/supervened on neural information processing, seem now to be located in places that we are not intuitively comfortable in thinking of providing the supervenience/realizing base for mindedness (Clark 1997, 2008b; Clark and Chalmers 1998).

The choice is then to say that those are not parts of cognitive processes after all (the real cognition goes on in the head) in which case one needs to come up with a "mark of the mental" to distinguish real cognitive processes from processes that play a merely causal (rather than constitutive) role in cognitive processing (Adams and Aizawa 2008). Or, to bite the bullet and acknowledge that if we accept functionalism as the orthodox approach did, not only is mind not identical with the brain, but it is also perfectly consistent that mind is realized not merely by the brain. Andy Clark expresses this view in terms of what he calls the Larger Mechanism Story (LMS) as follows:

Aspects of body and world can, at times, be proper parts of larger mechanisms whose states and overall operating profile determine (or minimally, help determine) our mental states and properties. (Clark 2008a, 39)

Functionalism about the mind of course implies multiple realizability, which entails that, provided that there are other materials which can implement the necessary processes, the functions that give rise to mind need not be limited to 
our biological brains. This means that not only might artificial brains be created, but that there is no principled reason for confining minded processes to brain processes (biological or artificial). Thus, work on embodied (and extended) cognition that comes through this tradition is - in principle - entailed by the functionalism that orthodox cognitive science also assumed (see Wheeler 2010). I therefore refer to this approach to embodied cognition (of which Andy Clark [e. g., Clark 1997, 2008b] is a paradigm propagator) as "orthodox embodied cognitive science."1

Hence, while it might seem at first surprising that orthodox embodied cognitive science says very little about the role of the physiological - and homeostatic - body in cognition we can see that this is because (1) orthodox assumptions about cognition held that minded processes supervened in some way on brain processes, and (2) functionalism entailed that not only could the brain processes that it was assumed that minded processes supervene on be multiply realized in different implementations but could also be partially realized by other structures of the brain in the human case (hence "offloading"). The very term "extended" (in addition to "offloading") indicates that this kind of embodiment is still working within the orthodox "brainbound" tradition (see Clark 2008b) but extending it outwards by following the implications of functionalism to their logical conclusion.

We can therefore see why even though fans of orthodox embodied cognition recognize that the body is important to cognition and mindedness, they are nevertheless loathe to think that physiological processes in the body proper make a contribution other than a causal or modulatory one to cognition. Any contribution that they do think is made e.g., by emotions, is made in virtue of those processes being represented in the brain. So while extended emotions fit in to this orthodox embodiment picture, this is not in virtue of the bodily contribution to cognition but rather once again following the implications of functionalism for the brainbound world view: i. e., taking emotions as represented in the brain as the standard case, and then showing that the processes we assumed were done in the brain are actually done (or can actually be partially done) through e. g., body posture, gesture, interpersonal engagement, or coupling with or structuring the environment in certain ways.

Let us then assume the basic tenet of orthodox embodiment: that cognition is not (at least not always) brainbound. Is there any more philosophical work to be done by asking the following question: if we are to build an artificial system that is genuinely cognitive, will implementing all of the processes that LMS throws light upon, be enough?

My hypothesis is that it will not be enough. This position, in and of itself, is not original; it is the position taken by many in the radical embodied ${ }^{2}$ and

\footnotetext{
1 I previously termed this "traditional embodied cognitive science" (Stapleton 2013).

2 I use the term 'radical embodiment' here following Clark's (1999) distinction between simple and radical embodiment. Enactivism is therefore one (but not the only) version of radical embodiment.
} 
enactive camps (see in particular Cosmelli and Thompson 2010; Thompson and Cosmelli 2011, which my project is heavily influenced by). However, these positions often put themselves, or are put, at odds with the orthodox embodiment approach because they build on assumptions which are not shared by all camps, e. g., the rejection of the representational/computational theory of mind, or the premise that a particular kind of self-organizing and self-creating organization underpins the development of cognition in key ways (see Thompson 2007; Di Paolo 2005, 2009; Di Paolo and Thompson 2014). These approaches may be thought of as biological rather than computational for a number of reasons but at the very least because they take as their standard case biologically cognitive creatures and seek to extrapolate from there to what is required for cognition, rather than taking the standard case to be the analogy of the mind to the computer.

I am sympathetic to both camps (though my publication history reveals that my intuitions mesh rather more with those of the biologically inspired approaches). Of course, as each side holds assumptions that contradict those of the other, one cannot be a full member of both camps at the same time (see Thompson and Stapleton 2009, for a discussion of why enactivism is not the same as externalism). What I then want to do is to take the spirit (rather than the details) of both camps and consider what insights it gives us. How can we do this, when orthodox embodied cognitive science is built upon functionalism, and enactive approaches reject functionalism about the mind?

I suggest that the spirit of orthodox embodiment is expressed by Clark (2008a) in LMS. Although this is a functionalist principle it is minimally functionalist: it does not entail a representational or computational view of the mind, it merely points to a mechanistic supervenience/realizing base for the mind. That this kind of mechanistic approach to the mind is still a kind of functionalism can be seen in Clark's work on "microfunctionalism” where he argues that functionalism does not need to be identified with high level formal descriptions such as beliefs and desires, rather what is essential to functionalism is that the "structure not the stuff counts" (Clark 1989, 31).

That cognitive creatures are mechanistic in this minimal sense is generally accepted in cognitive science - by both the orthodox and the radical. Where sides differ is in answering the question of what the minimal set of mechanisms is that enables/realizes cognition i. e., which are the ones we need to implement in order to build a cognitive system. The orthodox embodiment story clearly pushes the boundaries of the Marrian algorithmic level towards - and into - the implementational level for morphological features (Clark 2013). Yet, as explained above in virtue of its roots in the orthodox (brainbound) tradition, this minimal base does not include the internal goings-on in the physiological body. The intuition behind this is presumably that anything that is important that goes on in the physiological body is represented in the brain and so a functionalization of the relevant processes in the brain will include any relevant information from the body proper.

This is where I argue that the orthodox embodiment story errs. Let us talk in the mechanistic terms that are accepted by both them and the radicals, and 
argue that the minimal realizing system is not quite big enough yet: That it must include at least some mechanisms that go on in the biological body (both the non-neural body and parts of the neural body that are typically functionalized out) as proposed by Cosmelli and Thompson (2010) and Thompson and Cosmelli (2011) with their thesis of "dynamic entanglement" (see also Clark 2013, for a discussion of dynamic entanglement from the orthodox embodied perspective).

Here I outline a story ${ }^{3}$ which I propose should be accepted by both orthodox and radical embodimenters. While those in the enactive traditions will not think the story presented here complete as a minimal base for cognition, they should accept that it is at least part of what they consider the minimal mechanistic base and not reject it as externalist rather than embodied (Thompson and Stapleton 2009). And, because the story does not rest upon the assumptions of the radical approaches that orthodox embodiment rejects, and because it is presented as an extension of the mechanistic story and the fluidity of the algorithmic/implementational distinction that lies at the heart of the orthodox embodiment approach, without contradicting any of its own assumptions, orthodox embodimenters should also accept this story.

\section{Introducing Proper Embodiment}

The thesis of "Proper Embodiment" presented here is that (at least some of) the details of our physiology matter to cognition and consciousness in a fundamental way such that (at least some of) the mechanisms of cognition are so fine-grained that specifying the algorithm for cognition would entail specifying parts of the internal body normally considered to be background or enabling conditions for cognition.

I argue for this thesis through two independent theses: internal embodiment and particular embodiment. "Internal embodiment" is the thesis that the internal "gooey" body matters to cognition and consciousness in a fundamental way. "Particular embodiment" is the thesis that the particular details of our implementation matter to cognition. Taken together, these generate what I think is a compelling case that cognition is not merely embodied in the sense of orthodox embodied cognitive science, but Properly Embodied.

\footnotetext{
3 The work presented here is a "big picture" view of the project developed in detail in my doctoral thesis (Stapleton 2012) situating this in respect to traditional and radical embodied cognitive science.
} 


\section{Internal Embodiment}

\section{Internal embodiment: the internal "gooey" body matters to cognition and consciousness in a fundamental way}

In arguing for internal embodiment I focus on the role that interoception, the sense of the internal body, plays in cognition and consciousness. The term 'interoception' was originally used by Sherrington (1948) to refer to the sense of the visceral body (e.g., afferent information from smooth muscles and exocrine glands). A. D. Craig has since argued that due to sharing a common pathway through the spinal cord and processing areas in the brain, pain, temperature, and light touch should also come under the category of interoceptive senses and so "interoception should be redefined as the sense of the physiological condition of the entire body not just the viscera" (Craig 2002, 655). This sense of the physiological condition of the body gives a broad sense of how the body is faring. Although much of this information does not necessarily make it to conscious awareness, indeed Craig proposes that it is only in primates that this information is represented ${ }^{4}$ in the right anterior insula, which is correlated with the sense of subjective feelings and emotions, it is nevertheless typically co-activated with the limbic motor cortex and so may underpin the motivational and valenced aspect of affective feelings as distinct from mere feelings of sensations. Interoception is therefore plausibly the basis for at least a minimal sense of value and thus intrinsic motivation, key parts of the cognitive apparatus that are underspecified by the orthodox embodiment paradigm but which a properly embodied story should give us an account of.

Furthermore, recent work in affective neuroscience and predictive coding gives us reason to think that this interoceptive information may be involved in perceptual phenomenology. One such model, proposed by Barrett and Bar (2009) argues that when we perceive an object the brain makes a quick initial prediction about that object providing the gist of the situation but this does not yet correspond to our perception of the world. Rather, given this gist, the brain is left to predict the details of the situation based on previous knowledge, where "knowledge" is cashed out in terms of sensory-motor patterns that involve internal sensations including autonomic and endocrine information. On this model these predictions, and the filling out of the predictions, are recurrent and continue until the predictions at macro- and micro-levels no longer generate error signals when they are compared to incoming information. Information about internal bodily changes feeds in throughout this recurrency embedding affectivity into perception right from low level vision and including into the dorsal "where" visual stream.

This model may initially seem unintuitive, influenced as we are by the Marrian framework of visual processing upon which, if affect plays any role it comes

${ }^{4}$ I use this term in the (non-philosophically loaded) minimal sense understood in neuroscience. 
in as an addition to fully formed perceptual contents. But consider an intuition pump from William James:

Conceive yourself, if possible, suddenly stripped of all the emotion with which your world now inspires you, and try to imagine it as it exists, purely by itself, without your favourable or unfavourable, hopeful or apprehensive comment. It will be almost impossible for you to realize such a condition of negativity and deadness. No one portion of the universe would then have importance beyond another; and the whole collection of things and series of its events would be without significance, character, expression, or perspective. Whatever of value, interest, or meaning our respective worlds may appear embued with are thus pure gifts of the spectator's mind. (James 1902, 150)

While James appeals to emotions here, for him emotions are perceptions of bodily feelings, and so by definition available to conscious awareness, Barrett and Bar's model proposes that affect is playing an even more fundamental role in perception which they call "unconscious affect." They argue that:

"Unconscious affect" (as it is called) is why a drink tastes delicious or is unappetizing ... why we experience some people as nice and others as mean ... and why some paintings are beautiful while others are ugly. (Barrett and Bar 2009, 1328)

This idea of "unconscious" contributions to experience that nevertheless shape the phenomenality of our experience is not unprecedented in philosophy. The phenomenological tradition has given us the concepts of pre-reflective and pre-intentional experience which gives all experience its characteristic 'colour' (see e. g., Ratcliffe 2010) but also contributes to the very structure of cognition. Ratcliffe (2005) for example draws on the phenomenological tradition to propose a reading of James' emotion theory that goes beyond emotions structuring our perceptual phenomenology to their being constituents of cognition. Understanding intentionality in the traditional phenomenological sense, as not merely the "aboutness" of a mental state but rather "conceptualized in practical terms, as an orientation that does not merely reveal but also differently configures the experienced world" (Ratcliffe 2005, 192) allows us to understand James as arguing that emotions/feelings are not only perceptions of bodily feelings but rather are constituted by/through both the perception of these bodily feelings and the feelings themselves.

While at first glance there might seem to be a tension here between on the one hand a part of an objective environment being revealed to one in virtue of one's senses and, on the other hand, one's world being a subjective construct, this tension is illusory. The claim is that there is an external world but we have access to only the parts of that world that are made available to us through our senses. What the phenomenological approach brings out - that the more biological approach may leave implicit - is that the senses do not make parts of that world available to us "as is" but rather the world is translated through our particular sensory mechanisms and possibilities for interaction such that experience is structured by these in a way we cannot eliminate. Thus, given that affect is intimately bound with our sensory capacities, it also shapes how we experience the world - "our world," and how we can act in that world. And, it is this claim that 
if affect shapes how we experience the world then it also shapes how we can act in that world, that I take to be the heart of Ratcliffe's Jamesian/phenomenological claim that affect is constitutive of cognition. I have argued elsewhere in detail that drawing on the biological details of the interoceptive underpinnings of affectivity can give us good reason to think of affect as constitutive of cognition also in a non-phenomenological sense (Stapleton 2012). For the purposes of this chapter however the work outlined so far should be enough to motivate the plausibility of the weaker claim that in natural cognitive systems like ourselves, having an internal body shapes consciousness and cognition even when the interoceptive/affective information is unconscious/pre-reflective. And, because the information that feeds into cognition and consciousness is imbued with a natural value, in terms of value to the physiological system, to create an artificial system that is genuinely cognitive - and therefore has its own intrinsic values as a basis for motivation we may need to implement some kind of functionally equivalent "internal body."

Internal embodiment - that the internal "gooey" body matters to cognition and consciousness in a fundamental way - on its own does not require a modification of Clark's LMS so much as it is an extension of it inwards. It contributes to the story something that was lacking in the standard functionalist framework: value and motivation, and begins to reintegrate the phenomenological with the functional to more properly address our actual explanandum in cognitive science: natural cognitive systems. Does Internal Embodiment on its own however actually require an internal body, even a functionalized version of one? It is not immediately obvious that the functions that the internal body plays in contributing to value and consciousness couldn't be implemented in the brain (or externally). After all, the orthodox embodimenter would argue, even in the biological case, the real contribution that they give to cognition and consciousness is in virtue of their representations in the brain. If this is the case, then it is not that the gooey body matters to cognition and consciousness in a fundamental way but rather that our gooey bodies implement functions that matter to cognition and consciousness in a fundamental way. While this may be an important addition to the orthodox embodiment story, it is nevertheless a trivial kind of internal embodiment, because the internality is not what is playing the key functional role.

Do we have reason for thinking that the internal, gooey body that has evolved as part of us, has a fundamentally more important role than a mere functional one at this Larger Mechanistic level? Or, to phrase this in different terms, are our physiological processes a mere happenstance of our evolution the essential functions of which can be happily implemented in a variety of materials and locations? I propose that this is not the case. Rather, natural cognitive systems are not only internally embodied but also embodied in a particular way that means large mechanistic functionalization of these processes just may not suffice ${ }^{5}$. This is the thesis of particular embodiment.

\footnotetext{
${ }^{5}$ For an extended meditation upon this theme in respect to creature consciousness that explicates the tight "entanglement" of the neurophysiological details, see Cosmelli and Thompson (2010); Thompson and Cosmelli (2011).
} 


\section{Particular Embodiment}

\section{Particular Embodiment: the particular details of our implementation matter to cognition}

Orthodox embodied cognitive science rests upon a version of functionalism that is expressed by Clark's LMS discussed above (see Wheeler 2010). Like most versions of functionalism this abstracts from the details of implementation because, as Clark puts it, the "structure not the stuff counts" (Clark 1989, 31). Any mechanistic view of the mind will of course endorse the principle that it is the structure and not the stuff that counts when it comes to bringing about cognitive processes. That there really is a distinction between structure and stuff, however, may not be as obvious as it first appears. ${ }^{6}$

The thesis of particular embodiment is that the particular details of our implementation matter to cognition and hence any functionalization of the substructure of cognition would need to be at a fineness of grain that functionalizes these details. In order to motivate this thesis I will here put forward two "proofs of concept” drawn from evolutionary robotics: GasNets and evolved hardware.

\section{GasNets}

The principle behind evolutionary robotics is that, by emulating variation, heritability and natural selection one can artificially "evolve" robotic (or simulated) agents with complex behavior, gaining the standard advantages of neural nets, such as graceful degradation, as well as the targeted behavioral outcomes normally achieved through traditional programming. This is done by hooking up a group of neural networks to a task environment, or a simulation thereof, and selecting the most successful ones based on whatever fitness function you are using (i. e., those that are most successful - or least bad - at the task assigned). In order to increase variation of "genes" a few of those who were not most successful, but close by, are added and this group allowed to multiply while the rest are culled and recombinations of these "genotypes" and mutations are introduced. These steps are then repeated over and over, through many generations until networks evolve that can solve the task (the amount of generations needed to evolve a successful solution means that simulations are more practical than evolving networks using physical robotic agents at each stage).

It has been known for some time that communication in the brain is not only mediated by electrical and chemical signaling but also gasotransmission, such

${ }^{6}$ The violation of this distinction is especially evident in autopoietic organization (Varela, Maturana, and Uribe 1974; Maturana and Varela 1980; Thompson 2007; for an accessible introduction, see Di Paolo and Thompson 2014). However, here I am concerned with putting forward a position that does not require a commitment to grounding cognition in autopoietic organization. 
as through gases like nitric oxide (NO), carbon monoxide (CO), and hydrogen sulfide $\left(\mathrm{H}_{2} \mathrm{~S}\right)$. The assumption has always been, I take it, that in natural cognitive systems our implementation is gooey and complex as a result of our evolution but that this messy natural "design" could be abstracted away from and functionalized, and perhaps even improved upon. In short, there has reigned a culture of "electrical chauvinism" where it has been assumed that all of the important properties of cognition are represented at this level and molecular signaling and other gooey implementation can be factored out.

Smith and colleagues (2002) set up an experiment to compare the evolvability and adaptivity of solutions in standard artificial neural network models designed to model electrical transmission between nodes (NoGas) and an adaptation of the standard artificial neural network that was designed to also model gasotransmission (GasNet). The difference between the NoGas and the GasNet is that in the GasNet activations of nodes are not only a function of the inputs of the connected nodes (as with standard neural networks) but is also a function of the concentration of gas at that node.

The task that Smith and colleagues set was for robotic agents starting from an arbitrary position in a black-walled arena to find and navigate towards a white triangle while avoiding a white square. They show that basing their evolution of solutions on the GasNet class consistently produced successful solutions in fewer generations than evolution of solutions on the NoGas class. They argue that the GasNet solutions seem to be more evolvable because they are more amenable to being tuned to the particular characteristics of the environment, which is to say that the solutions are more flexibly adaptive. This adaptivity seems to arise from particular features of the gas diffusion mechanism which enable functions to be based on input patterns over time, which in turn allows noisy input to be filtered out.

This example from evolutionary robotics shows that a particular (gooey) feature of our embodiment plays a key role in evolvability leading to populations that can quickly adapt to a learning task and a particular environment. This is interesting in and of itself, but what is of particular relevance to us here is that both the GasNet and the NoGas controllers evolved functionally equivalent timing mechanisms. And yet, despite the functional equivalence in terms of the success criterion - the particular implementations resulted in a quite different amenability to being tuned to a particular environment. The moral for us to take from GasNets is that just because both GasNet and NoGas are successful solutions to the environment that they have evolved for (once they have evolved and reached $100 \%$ fitness) and are therefore functionally equivalent in regard to the success criterion, this does not mean that the level of explanation at which we see the functional equivalence is the correct one to understand what is really key to the ability of each controller to succeed. That is to say what is key to a controller's being flexible and adaptive - the qualities that we are interested in if natural cognition is our explanandum - is not the same as what is key to the mere successful implementation of a function. By looking at the ease of evolvability and the mechanisms which underpin this amenability to being tuned to a 
particular environment we can see that the relevant level of explanation for the adaptive behavior of the controllers is that which specifies the interaction of the gas and the nodes. The key point is this: In evolved systems this is not just the implementation, but rather it is the relevant level for the algorithm of an adaptive system. This is relevant not only over evolutionary timescales but, as Philippedes and colleagues (2005) note, also at the time scale of the (neurally plastic) changes themselves so that the biology of gas diffusion in real brains, and their subsequent modelling of GasNets parallels the embodied cognition approach to cognitive science, but internally. They state:

In highlighting the functional importance of brain morphology, these phenomena take us increasingly further away from connectionist ideas and suggest that Pfeifer's notion of ecological balance, which requires a harmonious relationship between an agents' morphology, materials and control, can perhaps be taken inside the head. (Philippedes et al. 2005, 145)

This suggests that when it comes to cognition that functional equivalence may have to be at a much lower level than that specified by the LMS (and other functionalist approaches). ${ }^{7}$

\section{Evolved Hardware}

A second line of evidence for the thesis of particular embodiment also comes from one of the authors of the GasNet study: Adrian Thompson. While typically in evolutionary robotics algorithms are evolved in simulation and then transferred to hardware, this study used evolutionary algorithms to configure the switches on a Field-Programmable Gate Array evaluating the circuit based on its performance in the real-world (Thompson 1997). The aim of the experiment was to evolve a recurrent network of logic gates, and the Field-Programmable Gate Array is a digital chip which should therefore be ideally suited to this task. The surprising solution which evolved however was not based on logic gates. That is, the gates in the chip were not used to do logic. The solution that evolved exploited physical characteristics of the chip and behaviors that emerged. For example, a quarter of the cells in the array were clearly contributing to the target behavior as disabling them resulted in loss of the solution, but some of these cells were not even connected to the main part of the circuit. This defies the standard separation of algorithm and implementation. In this case the exploitation of physical characteristics of the chip enabled the system to evolve solutions which had greatly decreased computational complexity compared to traditionally designed algorithms. In this respect this example corroborates and strengthens the conclusions from the Gas-

\footnotetext{
7 Note that this is not the same claim as is used against the parity principle in the extended mind debate. I am not here concerned with whether mental states such as beliefs or memories can be specified at a high or low functional level or whether implementational differences in these would violate their claim to instantiate these mental kinds. Rather, I am concerned with the substructure of the flexible adaptive behavior that enables/realizes cognition.
} 
Net study. But this example from evolved hardware does even more than this: it gives us a real case where we can see that in evolved systems the line between algorithm and implementation is blurred so that it is no longer a trivial matter to implement an algorithm evolved on one particular piece of hardware on another piece of hardware in the way that functionalism assumes that one must.

\section{The Case for Proper Embodiment}

I have argued for two theses: internal embodiment and particular embodiment:

1. Internal embodiment: the internal "gooey" body matters to cognition and consciousness in a fundamental way

2. Particular Embodiment: the particular details of our implementation matter to cognition

The examples I have outlined in support of the thesis of particular embodiment give us good reason to think that the solutions that have evolved to make us the flexible, adaptive, neurally plastic cognitive systems that we are, are likely a result of the exploitation of our particular embodiment, both over evolutionary and developmental time, but very plausibly also over the time scale of the plastic changes that underpin new learning in hour-to-hour and day-to-day contexts. From this perspective the clean levels inherited from orthodox cognitive science and which remain implicit in orthodox embodied cognitive science - algorithmic and implementational - are revealed to be leaky in evolved systems.

While on its own the thesis of particular embodiment could be considered a mere extension to orthodox embodied cognition (see for example Clark's considerations of A. Thompson's work in Clark 2013), in combination with the thesis of internal embodiment it packs a much heftier punch: the internal physiological realm that interoception brings information from is a complex, dynamic system, and the lessons that we gain from the evolutionary robotics and hardware examples give us reason to think that it will not be easy to separate the algorithm of the relevant processes from their gooey implementation. The thesis of particular embodiment, while consistent with orthodox embodied cognitive science, is that much more radical in combination with the thesis of internal embodiment as together they not only suggest, as Philippedes and colleagues (2005) say, that the balance between morphology, materials and control can be taken inside the head, but that it can also be taken into the body proper.

The combination of internal embodiment and particular embodiment may seem to undermine orthodox embodied cognitive science because by pushing the leakiness of the algorithm/implementation boundary so far it is no longer clear whether there is a boundary at all. ${ }^{8}$ However, while these theses may well

\footnotetext{
${ }^{8}$ Note that this position is not concerned with undermining extended functionalist positions of the kind that take as their explanandum mental states such as beliefs, desires, memories, etc. This is a thesis about the substructure of cognition conceived of as flexible adaptive behavior
} 
contribute to undermining orthodox embodied cognitive science in combination with other assumptions or arguments (as for example is done in the enactive literature), as they are presented here, they do not need to be in conflict with the spirit of orthodox embodiment approaches. Taking this spirit to be accurately expressed by the LMS, the theses presented here motivate the modification of this to a "Smaller Mechanism Story" or a "nanofunctionalist" explanation (Stapleton 2012) where the processes that make up the substructure of cognition are much closer to the implementational details than traditionally envisioned. Being so close to the implementational details means that much of the body that was factored out on the orthodox approach is now going to play a role in the algorithmic substructure of cognitive processes. Nevertheless, a Properly Embodied cognitive science is not a biologically chauvinist position; a "smaller mechanism" or "nanofunctionalist" story implies that these functions could in principle be instantiated in different materials and could therefore in principle be extended or rather external elements could in principle be "incorporated" (Clark 2008b; Thompson and Stapleton 2009) - but this instantiation is going to be at a much finer grain than traditionally assumed. ${ }^{9}$ Proper Embodiment can thus be taken as extending orthodox embodied cognition inwardly, and thereby also extending the explanandum beyond abstract cognitive processes that are the target of much of cognitive science research back to the flexible, adaptive processes at work in evolved cognitive systems.

\section{Bibliography}

Adams, F. and K. Aizawa. 2008. The Bounds of Cognition. New York: John Wiley \& Sons. Anderson, M. L. 2003. Embodied Cognition: A Field Guide. Artificial Intelligence 149 (1): 91-130.

Barrett, L. F. and M. Bar. 2009. See It With Feeling: Affective Predictions During Object Perception. Philosophical Transactions of the Royal Society of London. Series B, Biological Sciences 364 (1521): 1325-1334.

Clark, A. 1989. Microfunctionalism: Connectionism and the Scientific Explanation of Mental States. Retrieved July 17, 2011, from http://www.era.lib.ed.ac.uk/handle/1842/1332.

-. 1997. Being There: Putting Brain, Body and World Together Again. Cambridge, MA: MIT Press.

-. 1999. An Embodied Cognitive Science? Trends in Cognitive Sciences 3 (9): 345-351.

-. 2008a. Pressing the Flesh: A Tension in the Study of the Embodied, Embedded Mind? Philosophy and Phenomenological Research 86 (1): 37-59.

-. 2008b. Supersizing the Mind: Embodiment, Action, and Cognitive Extension. Oxford: Oxford University Press.

-. (2001) 2013. Mindware: An Introduction to the Philosophy of Cognitive Science. Oxford: Oxford University Press.

Clark, A. and D. J. Chalmers. 1998. The Extended Mind. Analysis 58: 7-19.

that gives rise to the kinds of experiences that we then categorize according to these "cognitive" categories.

9 Whether or not the possibility of being instantiated in different materials implies that nanofunctionalism entails multiple realizability is a question for another time. 
Cosmelli, D. and E. Thompson. 2010. Embodiment or Envatment? Reflections on the Bodily Basis of Consciousness. In Enaction: Towards a New Paradigm for Cognitive Science, ed. J. Stewart, O. Gapenne, and E. A. Di Paolo, 361-385. Cambridge, MA: MIT Press.

Craig, A. D. 2002. How Do You Feel? Interoception: The Sense of the Physiological Condition of the Body. Nature Reviews Neuroscience 3 (8): 655-666.

Di Paolo, E. 2005. Autopoiesis, Adaptivity, Teleology, Agency. Phenomenology and the Cognitive Sciences 4: 429-452.

-. 2009. Extended Life. Topoi 28 (1): 9-21.

Di Paolo, E. and E. Thompson. 2014. The Enactive Approach. In The Routledge Handbook of Embodied Cognition, ed. L. Shapiro, 68-78. London: Routledge, Chapman \& Hall.

Hurley, S. 1998. Consciousness in Action. Cambridge, MA: Harvard University Press.

James, W. 1902. The Varieties of Religious Experience: A Study in Human Nature. Bombay: Longmans, Green \& co.

Maturana, H. R. and F. G. Varela. 1980. Autopoiesis and Cognition: The Realization of the Living. Dordrecht: Springer.

Pfeifer, R. and J. Bongard. 2006. How the Body Shapes the Way We Think: A New View of Intelligence. Cambridge, MA: MIT Press.

Philippides, A., P. Husband, T. Smith, and M. O’Shea. 2005. Flexible Couplings: Diffusing Neuromodulators and Adaptive Robotics. Artificial Life 11 (1-2): 139-160.

Ratcliffe, M. 2005. William James on Emotion and Intentionality. International Journal of Philosophical Studies 13 (2): 179-202.

-. 2010. The Phenomenology of Mood and the Meaning of Life. In Handbook of Philosophy of Emotion, ed. P. Goldie, 349-371. Oxford: Oxford University Press.

Shapiro, L. 2007. The Embodied Cognition Research Programme. Philosophy Compass 2 (2): 338-346.

Sherrington, C. S. 1948. The Integrative Action of the Nervous System. Cambridge, UK: Cambridge University Press.

Smith, T., P. Husbands, A.Philippides, and M. O'Shea. 2002. Neuronal Plasticity and Temporal Adaptivity: GasNet Robot Control Networks. Adaptive Behavior 10 (3-4): 161-183.

Stapleton, M. 2013. Steps to a "Properly Embodied" Cognitive Science. Cognitive Systems Research 22-23: 1-11.

Stapleton, M. 2012. "Proper Embodiment: the Role of the Body in Affect and Cognition." Phd Thesis, University of Edinburgh.

Thompson, A. 1997. Artificial Evolution in the Physical World. In Evolutionary Robotics: From Intelligent Robots to Artificial Life (ER'97), ed. T. Gomi, 101-125. Ontario: AAI Books.

Thompson, E. 2007. Mind in Life: Biology, Phenomenology, and the Sciences of Mind. Cambridge, MA: Harvard University Press.

Thompson, E. and D. Cosmelli. 2011. Brain in a Vat or Body in a World?: Brainbound Versus Enactive Views of Experience. Philosophical Topics 39 (1): 163-180.

Thompson, E. and M. Stapleton. 2009. Making Sense of Sense-Making: Reflections on Enactive and Extended Mind Theories. Topoi 28 (1): 23-30.

Varela, F. G., H. R. Maturana, and R. Uribe. 1974. Autopoiesis: The Organization of Living Systems, its Characterization and a Model. Biosystems 5 (4): 187-196.

Wheeler, M. 2010. In Defense of Extended Functionalism. In The Extended Mind, ed. R. Menary, 245-270. Cambridge, MA: MIT Press.

Wilson, M. 2002. Six Views of Embodied Cognition. Psychonomic Bulletin E Review 9 (4): 625-636.

Ziemke, T. 2003. What's That Thing Called Embodiment? In Proceedings of the 25th Annual Meeting of the Cognitive Science Society, ed. R. Alterman and D. Kirsh, 1305-1310. Mahwah, NJ: Lawrence Erlbaum Associates, Inc. 


\title{
Embodied Habitual Memory Formation: Enacted or Extended?
}

\author{
Christian Tewes
}

\begin{abstract}
It is the aim of this paper to explore in more detail what enactivism can contribute to embodied memory research. This is accomplished by a comparative analysis of the formation of memories with regard to the extended mind hypothesis. Proponents of the latter base their views on the supposed "hybrid nature" of the human mind. According to this approach, biological brain-based memories are supplemented by external storage systems, thereby incorporating different forms of cultural technology into the human mind. I argue in this paper that a strong embodied approach to memory formation first needs to develop a phenomenological and autonomous system account of habitual body memory. This lays the foundation for explaining whether memories are integrated into emerging unities or are merely extended.
\end{abstract}

\section{Introduction}

It is the focus of an intense debate over the last couple of years whether enactivism and the extended mind hypothesis mutually exclude each other or follow complementary routes in embodiment research. Proponents of both camps have tended to opt for the first interpretation but often for quite different reasons.

Along these lines, Rowlands has argued in a comparative analysis that the difference between enactivism and the extended mind hypothesis (hereafter, EM) does not lie in functional or non-functionalist accounts of embodied cognition. On the contrary, while enactivism stresses the importance of the body and its embeddedness in the environment, the enactive approach to mind and cognition does not fall outside the paradigm of functionalism. According to Rowlands, the crucial difference between both theories is rather the question of where cognitive processes take place. Whereas proponents of EM hold the view that cognition can extend into the world via external cognitive vehicles such as tools or computers, Rowlands thinks that enactivism lacks the conceptual resources to show how cognitive or mental states could transgress a sensorimotor system or organism in the first place (Rowlands 2009, 61). ${ }^{1}$ Thus enactivism is committed to a kind of internalism.

\footnotetext{
1 There is no clear-cut distinction in the EM debate between cognitive and mental states. At one point in their inaugural paper on the "Extended Mind", Clark and Chalmers define mental states as beliefs, desires, and emotions and contrast them with "cognitive processing" (including
} 
Enactivists are eager to point out that this reconstruction of the enactive approach to cognition and mind misses significant distinctions and therefore misrepresents their position in important respects. Moreover, advocates of enactivism such as Ezequiel Di Paolo and Evan Thompson have shown that EM's tenets are ultimately based on central assumptions of classical cognitivism that enactivism tries to overcome (Di Paolo 2009; Thompson and Stapleton 2009).

I will argue in this paper that enactive-inspired research has offered conceptual distinctions, methods, and findings that are of philosophical significance and which have a striking impact on empirical cognitive research. I will demonstrate this with regard to certain aspects of habitual memory formation. This will be accomplished via a comparative analysis of EM and enactive research. Memory formation is a central topic in the realm of EM-inspired research. There are impressive works in EM that show how the cultural invention of external storage systems enhances and enables types of recollection that constitute the enculturated human mind. However, what is missing in those bybrid accounts of the mind is the co-constitutive role of the body and intercorporeality for those external vehicles and the emergence of new system identities. Furthermore, extended functionalism as a central tenet of EM does not allow a consistent specification of cognition and mental states in the first place. By contrast, proponents of enactivism have developed and refined a notion of sense-making that includes meaning, intentionality and an experiential dimension in relation to the world. This enables the integration of phenomenological insights and research methods that are crucial for the exploration of embodied memory capacities.

In the first section, I will give an outline of the extended mind hypothesis by exploring the question of whether the original EM thesis and its later augmentation ("second-wave EM") are in the position to deliver a convincing framework for memory research. In the second section, major ingredients of enactive research are introduced, including an autonomous system approach to habits that lays the foundation for a conceptually clarified account of memory formation as well as the phenomenological concept of body memory. In the final section, I apply the distinctions developed in the paper to different aspects of EM-inspired memory research. It is the aim of the final section to show that enactivism offers both conceptual clarification of the impact of external memory storage systems on the human mind and a foundation for further embodied memory research.

recognition, discrimination, and searching) (Clark and Chalmers [1998] 2010, 33). I think that these distinctions are inconclusive and are in need of further elaboration. However, it would exceed the scope of this paper to show this in the specific thematic field of embodied memory research. When I introduce some key concepts from the enactive approach in the second section, it will become clear that even basic cognitive processes are not entirely devoid of a sense-making (mental) dimension. 


\section{The Extension of the Mind: Basic Principles and Assumptions}

What is the primary motivation behind the idea that mental and cognitive states,

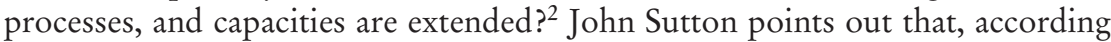
to EM, mental and cognitive properties and processes are "unevenly distributed across biological and non-biological realms" (Sutton 2010, 189). This view of the human mind is by no means self-evident. It is obvious that social rituals, artefacts, technology, and language expand and enhance cognitive abilities. Yet the decisive question is whether external memory devices such as the invention of symbolic storage systems should be treated as genuine parts of the "cognitive architecture" itself (Donald 1991, 309). EM theorists are convinced that the integration of external items into a newly emerging cognitive system is a matter of fact:

What matters most is our obsessive, endless weaving of biotechnological webs: the constant two-way traffic between biological wetware and tools, media, props, and technologies. The very best of these resources are not so much used as incorporated into the user herself. They fall into place as aspects of the thinking process. (Clark 2003, 198)

These considerations make clear what is at stake in EM. The idea of incorporating tools, media, and technology implies an anthropological specification of human beings. The human mind is hybrid in its nature, made up of biological and non-biological parts (Clark 2003, 198).

Seeing it from this perspective, the idea of "extending the human mind" is intimately related to the conviction of the "leaky" nature of human beings, or, to use ananother metaphor, a view of humans as knots in a biotechnological web. In their inaugural paper on "The Extended Mind," Andy Clark and David Chalmers give quite a detailed justification for this view by explicating the term "externalism" so as to answer the question "where the mind stops and the rest of the world begins?" (Clark and Chalmers [1998] 2010, 27). They are not referring to the concept of semantic or content externalism. The primary meaning of the latter consists in the conviction that content is not fixed in the head but by details of the environment that might be opaque to laypersons in specific social or historical contexts (e. g., water $\left.=\mathrm{H}_{2} \mathrm{O}\right)$ (Hurley 2010, 102).

Instead, Clark and Chalmers suggest a concept of "active externalism," where the accomplishment of epistemic actions such as a mathematical calculation includes pencil and paper or an electronical device (Clark and Chalmers [1998] 2010, 28-29). In such circumstances a coupled system emerges that is constituted by a "two-way interaction" between a human organism and an external device (Clark and Chalmers [1998] 2010, 29). The central idea behind this systemic approach is that all components of the system play an indispensable active causal role in establishing and executing a cognitive process.

${ }^{2}$ I presuppose in this paper that states are realized by processes. However, what distinguishes states from other processes is their persistence over a longer period. This depends on the organization of stabilized processes within a system (Campbell 2009, 460). Furthermore, I understand a "capacity" - by ascribing it to an agent - as a disposition or power to act in a specific way (Mumford and Anjum 2011, 5). 
Adams and Aizawa caricature this account of mind and cognition with a rhetorical question: why is the pencil thinking that $2+2=4$ ? They also give a hypothetical answer: because it is coupled to the system during the mathematical calculation (Adams and Aizawa 2010, 67). Adams and Aizawa take this example as a starting point to accuse the friends of EM of committing a "causal-constitution fallacy." Pencil and paper might play an important causal role in accomplishing the cognitive task, but it would be wrong to ascribe to such artefacts any constitutive function in the realization of cognitive operations.

Clark's reply to this caricature already implies a kind of "delocalisation" or distribution of extended cognitive or mental processes over the body and parts of the coupled environment:

The appeal to coupling is not intended to make any external object "cognitive" ...

Rather, it is intended to make some object, which in and of itself is not usefully (or perhaps not even intelligibly) thought as either cognitive or noncognitive, into a proper part of some cognitive system, such as a human agent. (Clark 2010a, 83)

For Clark, this principle holds not only for external parts that are coupled with a formerly existing system; the same is true for "internal" parts or processes such as V4 neurons or groups thereof. He thus rejects the idea that V4 neurons (decoupled parts) have the power to think or are the locus of thinking (Clark 2010a, 83).

These are no trivial clarifications of Clark's own position. One should keep in mind that for the extended mind theorist too, mental or cognitive processes are (a) not intrinsic properties of system parts and (b) do not (at least not necessarily) pre-exist the time of coupling. This implies that such processes are emergent features of the entire system that cannot be reduced to its components (see Campbell and Bickhard 2011). This is also in line with Clark's further remarks on the systemic approach in EM. He refers to works in dynamical systems theory where global features of a system are also specified as emergent properties that have a top-down influence on microphysical components of the system (see Kelso 1995).

\subsection{The Parity Principle and Extended Functionalism}

Considering what has been said so far, it is obvious that these explications, by themselves, are not sufficient to show how mental and cognitive processes could be extended at all. The reason is that the process of "coupling" says nothing about the systemic properties that are emergent products of active causal interrelationships between an organism and specific external items of the environment. An organism is coupled in multifarious causal interrelationships with its environment at the atomic and sub-atomic level of description, such as the permanent reverberation of light waves/particles from a light-source such as the sun. However, this alone does not lead to the emergence of mental and cognitive properties. Hence, constant coupling is not sufficient for the emergence of the 
latter and therefore not appropriate for classifying an emergent property as a cognitive or mental process.

The attempt to answer the question of what justifies the ascription of cognitive properties to an extended coupled system leads us to what the EM literature calls the "parity principle" (henceforth, PP): "If, as we confront some task, a part of the world functions as a process which, were it to go on in the head, we would have no hesitation in accepting as part of the cognitive process, then that part of the world is (for that time) part of the cognitive process" (Clark 2010, 44). This principle has come under attack in the last couple of years, albeit for quite different reasons. The immediate reply concerns the charge that PP simply "externalizes the internal” (Menary 2010, 234). It is not difficult to see why this is so. The decision whether a process $X$ is cognitive or not depends, according to PP, on re-localizing $X$ in a thought experiment into the skull. Such a "test procedure" implies at least that the mark of the mental or cognition is to be found in the head, a position one would normally describe as cognitive internalism.

However, one could view PP as merely a heuristic means for giving an externalist account of cognition without the need to state essential criteria for different cognitive kinds. There is a rich research tradition in the cognitive sciences that explores cognitive processes in relation to the processing capacities of the brain and to the cognitive architecture. From this perspective, the suggested test procedure merely follows the established research tradition in cognitive sciences.

Furthermore, PP seems at least to hint at the circumstances under which one can classify an emergent property of a coupled system as a cognitive or mental process in the first place. It is interesting that Clark stresses further motivational reasons to include PP as a major ingredient in the EM agenda. Important for him are the anti-biochauvinistic implications of the principle. PP should function as a means (a "veil of ignorance") to ignore the "metabolic boundaries of skull and skin." This is supposed to enable a better focus on the computational and functional power of entire systems and the specific functional roles of the material vehicles of mental and cognitive states (Clark [2008] 2011, 77).

This makes clear that proponents of the extended mind hypothesis are not only committed to the view that cognitive states extend the boundaries of skin and skull. They are also committed to a variant of "extended functionalism." Clark's hint about the anti-biochauvinistic intention behind PP points to the functionalist account of mind and cognition. According to this approach, psychological phenomena are identified by their multiply realizable causal functional roles (Horgan [1994] 2000, 476). Psychic types such as fear, trust, or joy have no species-specific boundaries with regard to their concrete (token) instantiation. If physical entities such as Martians, robots, or non-human organisms were to exhibit the suitable functional profile at the systemic level of complexity, then one would be justified in ascribing to them the respective psychological states or processes as well.

Wheeler elucidates why PP is especially apt to bring to the fore the interrelations between EM and extended functionalism. Parity implies functional equivalence between internal and external elements that unfold the same behavior due 
to their causal-functional contributions to the system. The application of PP thus allows that the same type of cognitive and mental states could be realized in quite different "generic formats," namely both biological and non-biological (or an integrated combination) and extended and non-extended ones (Wheeler 2010, 248). Conceived in this way, the thesis that cognitive and mental states are multiply realizable is built into PP itself.

There are two main lines of criticism of PP. The first line stresses that parity leads to untenable consequences but that since it is not an essential part of EM it could be replaced by other heuristic means. These means are elaborated in the so-called "second-wave" EM and center on the "complementary principle." The second line of argumentation comes from an enactive perspective and conceives PP as merely a symptom of deeper problems intrinsic to EM itself. In the next section, I introduce both lines of criticism via the example of the formation and enhancement of memory capacities.

\subsection{The Realization of Memory as a Challenge to PP}

In their inaugural paper, Clark and Chalmers apply PP to a now famous thought experiment that already has to do with the support and extension of memory capacities. This thought experiment involves Inga, a woman who decides to visit an exhibition at the Museum of Modern Art. She remembers that the museum is on 53rd Street and walks into the museum. There is a further person in this thought experiment, Otto, who suffers from early-stage Alzheimer's. He is dependent on a structured environment with "memory prostheses," like many other patients who suffer from dementia. Such patients (or their family and friends) augment their home with notes or similar reminders in order to sustain and further their autonomous lives. In Otto's imagined case, however, he carries around a notebook "that plays the role usually played by a biological memory" (Clark and Chalmers [1998] 2010, 35). He makes the decision to go to the museum, consults his notebook to find out where it is, then goes to 53rd Street and visits the exhibition. The conclusion Clark and Chalmers draw is straightforward: "The moral is that when it comes to belief, there is nothing sacred about skull and skin. What makes some information count as a belief is the role it plays, and there is no reason why the relevant role can be played only from inside the body" (Clark and Chalmers [1998] 2010 35). The point about extending the causal role of beliefs to artefacts that are not a proper part of the body is in need of further clarification. It is not plausible to suppose that every interaction with a computer or other artefacts results in cognitive extensions of the preceding cognitive system.

Consequently, Clark and Chalmers add three criteria to show under what circumstances the conceptual integration of the notebook - or better its causal-functional (memory capacities) integration with Otto's cognitive architecture - is justified. The added source must (i) be "reliably available," which means that Otto always carries his notebook with him and gets the required infor- 
mation more or less whenever he wants it. This points to the second criterion, namely (ii) that the retrieval of stored information is automatically accomplished and is as "truthworthy" as the information Inga receives from her biological memory. This leads to the third criterion (iii) that the stored information should be easily accessible (Clark 2010b, 46).

I think it is not unfair to say that these criteria are merely angmentations of EM that result from the failure to give a plausible account of a cognitive agent at the system level in the first place. I will come back to this point in later sections of the paper.

However, there are already problems with PP and with these additional criteria at the functional level of description, for instance the question of how new material items are incorporated as causal-functional roles into the cognitive architecture of agents. Are external memory storage systems such as Otto's notebook similar enough to biologically based "internal" memory systems to allow their functional integration with the cognitive architecture? Given PP and the three criteria above, such a strong similarity or isomorphism seems at least what is required if EM is to be more than a conceptual possibility and actually be realized in our world. In this context, Rupert (2004) in particular has argued that proponents of EM have failed to show that the use of external memory devices justifies the view of extended (or integrated) cognition. The decisive point, according to him, is that internal acts of remembering and extended memory states are so dissimilar in important respects that one needs to consider them as different cognitive kinds.

Rupert does not deny that culturally invented and accumulated storage systems function as a cognitive scaffold for the human mind and that they affect the structure of internal memory processing (Rupert 2004, 408). But he denies that this justifies the assumption that those internal and external modes of processing can be explained in the same causal terms or that they amount to an overarching natural kind of memory (Rupert 2004, 410).

He substantiates his claim with two examples, the first concerning the functioning of working memory. In order to understand Rupert's example, it is necessary briefly to introduce some interrelations between working memory and other aspects of the process of remembering. "Short term memory" is defined in cognitive psychology as the retention of a small amount of memories for short time periods, whereas "working memory" is defined as a larger integrated system for the manipulation and execution of complex cognitive tasks. What particularly distinguishes the working memory from short-term memory is the conception of a "central executive." This determines the integration and coordination between several "buffers" (the phonological loop of verbal information or the visuospatial sketchpad for visual information). It can be conceived as a control system that guides attention and allocates how the available information should be manipulated; it also has access to long-term memory (Revlin [2012] 2013, 140). With regard to our example, Rupert's question is whether an external memory storage system could sustain the same cognitive memory function as the working memory. 
In a thought-experiment of his own, Rupert imagines a situation where someone tries to keep track of an ongoing conversation by writing down every aspect of the talk on paper. The idea here is to decide whether the storage of the talk on a piece of paper could function in a similar or analogous way to the working memory as described above. In the imaginary scenario this would obviously not work. Any attempt to differentiate important from unimportant information written on the paper and then integrate this into the distinct strands of the conversation would destroy the practice of communication (Rupert 2004, 410). However, one could reply that this example does not convincingly prove a disanalogy between the functioning of the working memory and an external functional surrogate; everything depends in this case on the "micro-configuration" of the thought experiment. In the future, there might be external technological devices that would enable the agent to write down, keep track of and utilize the information in real time. It is not clear why this possibility is excluded and Rupert does little to dispel such counterarguments.

Rupert's second example is more convincing. It concerns the so-called "negative transfer effect": experiments that show how memorized associations between terms (for instance, pairs of names) can block the ability to remember the same terms when placed in new associations (Rupert 2004, 414). If, for instance, a subject learns the combination "Hannah is married to Marcel," then subjects who have previously learned this association need more time to learn a new pairing of one of the terms ("Hannah is married to Richard").

As Rupert points out, a similar negative transfer effect (generation effect) is not to be expected when people write down word combinations on a new list created from word pairs written on an older list. In this case, a subject hears the new association "Hannah is married to Richard" and can immediately write down this new pair. Experiments show that a negative transfer effect only rarely blocks the external recording of this new association on paper or a laptop. The same is likely to be true with later recollections of the newly-paired word association. If an experimenter says "Hannah" and indicates that he is referring to the second list, the subject can simply read off the newly-paired terms "Hannah and Richard" without any interference (Rupert 2004, 414).

Despite the specific problem in the first example, PP faces serious difficulties as a result of these considerations. A thorough analysis of the respective causal-functional roles reveals that items of external memory might not display the right "functional profile" that would allow their equivalent integration (with the same functional effects) into the cognitive architecture of human agents. Even more seriously, extended memory devices such as written books or Otto's laptop do not actually deserve, according to Rupert, the ascription of cognitive states or processes: "We seem forced, then, to recognize two different explanatory kinds, internal memory and external resources used as memory aids, with no reason yet found to think that external aids constitute genuinely cognitive states or processes" (Rupert 2004, 418).

Wheeler's answer to these challenges is straightforward. He rejects the view that Rupert's considerations present a problem for PP and that PP should - 
as adherents of second-wave EM argue - be thereby abandoned. Everything depends, in effect, on the benchmark for PP. Rupert's examples presuppose that an extended functionalist approach should match the fine-grained details of the realization of (internal) cognitive processes. Otherwise, the absence of the negative transfer effect is not a counterargument to the extended realization of memory capacities in the case of externalized records (Wheeler 2010, 252256).

However, why suppose in the first place that an extended functionalist approach should match the fine-grained details of the realization of cognitive processes? Perhaps it is not crucial for a functional specification of memory formation that internal and external processes of recollections are similar in every respect. On the contrary, why not assume that the functional specification must correspond to a high level of description, namely "other things being equal, [to] a system that exhibits some fine-grained functional trait and one that doesn't both count as cognitive" (Wheeler 2010, 257)? In this case, the negative transfer effect does not rule out that external storage systems can function as a constitutive part of recollection processes.

As it stands, this is not entirely convincing. It might be true that Rupert presupposes and favors a chauvinistic form of functionalism that privileges “inner-oriented human cognitive psychology" (Wheeler 2010, 255). But what makes Wheeler so sure that the fine-grained details of the realization of functional-causal roles do not determine whether $X$ is a cognitive process? Rupert's question why we should think that external memory aids such as written symbols on paper or a laptop "constitute genuinely cognitive states" still remains to be answered.

Anticipating this reply, Wheeler concedes that the micro-functional profile (the fine-grained details of the functional realization) could actually turn out to make the decisive difference to whether a process $X$ is cognitive or not. However, he does not think that this concession is devastating for PP. According to him, this would only be the case when the fine-grained details of the micro-functional roles could only be realized internally or by means of a biological system. Nevertheless, these restrictions are not implied by PP or micro-functionalism (Wheeler 2010, 260).

I see another serious challenge for PP when Wheeler asserts that functional specification in the light of Rupert's counter examples should be given at a more general level of description. By doing so, the integration of external states or processes as "cognitive" into a system is guaranteed by definition. Wheeler does not deliver any independent reasons for what could count as cognition in the first place. This leaves us without any criteria to decide whether PP can convincingly be grounded on the idea of a coarse-grained functionalism. Given this, PP's role as a heuristic means for deciding whether an external process is part of the cognitive machinery at all must surely be open to doubt. This is so because PP as such is neutral with regard to the question of whether cognitive kinds such as episodic memories are realized at a fine-grained level of description or a coarsegrained one. 


\subsection{Second-Wave EM and the Complementary Principle}

That the extended functionalist position is in need of a clearer account of cognition as such is not a view held by the proponents of the so-called "second-wave" EM. John Sutton chooses another strategy to meet the challenges faced by PP. According to Sutton, the justification for EM should not be based solely on PP but rather on the independent "complementary principle" (henceforth, $\mathrm{CP}$ ). The key idea behind $\mathrm{CP}$ is the acknowledgement that external resources such as Otto's laptop computer or other stored information are sometimes quite dissimilar to the functioning of the brain. For Sutton, however, it is not out of the question that internal and external mechanisms, media, and processing capacities might result from a coupling in a new cognitive agent (system):

It's just because isolated items aren't stored atomically in the brain that our relatively vulnerable biological memories are supplemented by more stable external scaffolding. Brains like ours need media, objects, and other people to function fully as minds. Seeing the brain as a leaky associative engine ... forces attention to our reliance on external representation in the technological and cultural world. (Sutton 2010, 205)

This passage is revealing for different reasons. Given the supposedly leaky or hybrid nature of human beings (see Stapleton, this volume), it makes sense for Sutton to suggest replacing PP by CP in order to open up an interdisciplinary approach to mind and cognition within the framework of EM. Sutton refers here in particular to Donald's distinction between "exograms" and "engrams," to make the point even clearer. According to Donald, engrams are "impermanent" (they only last a lifetime) and depend on the capacity of the human nervous system and the genetic setup, whereas systems of exograms such as libraries, with their stored and catalogued symbolic records, are expandable, outlive individuals and sometimes outlast their own specific cultural heritage and origin (Donald 1991, 315).

Sutton does not entirely endorse this strict distinction because he thinks that "cognitive artefacts" are not only commodities of the "active mind" but sometimes interact with the brain and body so that they become part of the human mind (Sutton 2010, 190). It is beyond doubt that culture has a major impact on memory formation, via artefacts, symbols, media, recording systems, and the like, and one can explore how this affects the functioning of the brain from a phylo- and ontogenetic perspective. Sutton's own work on this interrelationship within the cultural realm is very useful for understanding the enormous capacity of cultural devices to extend human memory. We will see in the final section what enactivism can contribute to this important line of memory research.

Nevertheless, the quotation from Sutton also indicates that CP is not capable of dealing with the challenges levelled against EM and set out in the previous section. Thus, it is certainly a misleading metaphor that "brains like ours" need media or technology to "function fully as minds." The basic intuition is again the internalist assumption that the brain is almost identical with the human mind but needs a cultural supplement to unfold its ultimate cognitive and mental 
capacities. The quotation mentions the role neither of the body nor the experiential base in the development and preservation of memory capacities. To embrace the complementary aspects of internal and external memory patterns does not explain how these dissimilar causal-functional roles result in a new emerging cognitive system with enhanced or extended memory capacities. If we concede, as Sutton does, that there are significant dissimilarities in causal-functional roles in the case of memory formation, then we are back to Rupert's worry that these roles amount to different explanatory kinds. Sutton tries to disarm this worry by suggesting we resist the tendency in complementary-based EM to "treat the inside and the outside as distinct realms with fixed properties" (Sutton 2010, 206). But on its own this does not solve the problem of explaining how coupling could result in new unities (systems) with emerging memory capacities. Clark himself draws a possible conclusion from this result: "Perhaps the real moral of the story is that the realm of the mental is itself too disunified to count as a scientific kind." But it could be even more serious: "We just don't know a mind when we see one. Could the reason for this be that there simply aren't any there? Might the extended mind debate form part of a reduction of the very notion of mind in cognitive science?” (Clark 2010b, 63).

These are by no means rhetorical questions. Clark is, of course, eager to reassure that EM does not lead to a reductio of its own position; quite the contrary, EM should stand for the biotechnological openness of the "ideas of mind and reason" (Clark 2010b, 64). Even so, it is far from clear whether EM has the conceptual recourses to unify what appears so dissimilar in kind and character in the first place. In the next step, I will explore whether enactivism can provide, at least in principle, convincing criteria for giving a unified account of memory formation.

\section{Enactivism, Incorporation and Extension}

The enactive approach to cognition and embodiment rests upon a variety of concepts, research tools, and methods that mutually support each other (see Thompson 2007). Here I introduce those concepts, research tools, and methods that are important for a unified account of the embodied mind and the emergence of integrated memory capacities.

\subsection{Autonomy, Identity and Sense-Making}

At first sight, enactivism and EM both share and integrate concepts and research methods from dynamical systems theory, such as the constant coupling between organism and environment (Kelso, 1995, 284). Nevertheless, there are important differences between both theories in how the system approach is interpreted and further specified. Proponents of enactivism focus in particular on "autonomous systems" in order to explicate cognition, normativity, and meaning. The para- 
digm case for autonomy in this theory is the organism. In terms of Hans Jonas' philosophy of nature and theory of autopoiesis, the organism is conceived as a self-affirming entity that maintains and regulates its identity and interactions with the environment according to norms drawn from the perspective of the system (see Jonas 1966; Thompson 2007, 152-153).

The relevant identity conditions do not only apply to organisms as autopoietic systems. Proponents of enactivism use these criteria to specify autonomous systems at a more general level with a focus on their unity. More fundamentally, such a system (a) generates and regulates its identity under precarious circumstances; (b) every process in the system recursively depends on another process in the system, thereby forming a closed network; and (c) the system determines a possible scope of interactions with the environment (Di Paolo 2009, 15; Thompson and Stapleton 2009, 24). In what follows I look at these tenets in more detail.

Autonomous systems are, despite the principle of closure, thermodynamically open systems that regulate the external flow and exchange of energy with their environment (Thompson and Stapleton 2009, 24). It is not easy to capture fully this dialectical "double aspect" of systemic openness and closure. Maintaining the conditions of thermodynamic non-equilibrium is (i) necessary for autonomy and (ii) a constitutive relational property of the dynamical system itself. ${ }^{3}$

Let us explain in more detail why this is so. An autonomous system such as an organism is a self-maintaining system that actively contributes to the conditions that enables its far-from-equilibrated processes. An organism such as a frog is able to switch recursively between different types of behavior so as to maintain itself, detecting environmental changes that are related to different behavioral choices of the system. This capacity depends on the specific infrastructure of the system (Campbell 2009, 466). Metabolism and the recursive interdependence of organic components not only generate a biological self and its processual boundary with the environment; they are also simultaneously responsible in important respects for the concrete energetic regulation of the system with the environment, as is obvious in the case of satisfying hunger and thirst. This aspect fits perfectly well with Di Paolo's further elaboration of the notion of "precariousness":

Precarious circumstances are those in which isolated constituent processes will tend to run down or extinguish in the absence of the organization of the system in an otherwise equivalent physical situation. In other words, individual constituent processes are not simply conditioned (e. g., modulated, adjusted, modified, or coupled to other processes) but they also depend for their continuation on the organizational network they sustain. (Di Paolo 2009, 16)

3 Thompson sees clearly that the requirement for autonomy is not only the principle of closure but also the simultaneous thermodynamic openness of the system. Nevertheless, he does not specify the persistent self-maintenance of the system in a far-from equilibrated state as a property of autonomous systems themselves. They are "[necessary] conditions not belonging to the system" (Thompson and Stapleton 2009, 24). I think that this shortcoming prevents leading contemporary theorists of Enactivism from giving a fully-fledged account of autonomy in their dynamical systems approach. 
It is obvious that with this characterization Di Paolo focuses mainly on system constituent processes and the role of precarious circumstances in the operational closure and mutual interdependence of the components. Their dynamical existence depends on the entire organization of the network. Nevertheless, when the entire organism survives by changing and adapting its behavior and satisfying its basic energetic needs such as hunger or thirst, the defining description of precarious circumstances is fulfilled as well.

These aspects of enactivism are of major significance for the extended mind debate. The interactive adaptivity of autonomous systems is related to both the self-generation and self-preservation of the system and to the co-constitution of the environment. As Thompson puts it, an autonomous system "enacts its own identity and makes sense of the world from the perspective of that identity" (Thompson 2007, 153). Furthermore, this perspective is normative in character and is rooted in the autonomous system as the center of activity (Di Paolo, Rhode, and De Jaeger 2010, 39). This sense-making capacity and normative engagement with the world is, according to the enactive approach, the mark of cognition and meaning (Di Paolo 2009, 15).

This implies that the coupling between a system and its environment is not a symmetrical relation. Organisms mediate and enact their thermodynamically far-from-equilibrated states as centers of activity at different levels of complexity (Di Paolo 2009, 15). Humans can deliberate, for example, about the consequences of environment pollution and make plans to ensure their access to clean drinkable water in the distant future. This specification of the asymmetric (autonomous) system-environment relation allows further distinctions and inferences that are missing in the system-environment approach of EM.

Enactivism offers a very basic criterion for cognition and mental processes, namely the notion of sense-making. According to the enactive approach, even the simplest organisms possess cognitive capacities. This emphasizes a strong continuity between life and mind (Thompson 2007, 157 ff.; see Spahn, this volume). What this enactive conviction does not imply - as Richard Menary wrongly supposes - is that every living system also has conscious experiences (Menary $2015,3)$. Whether the latter is the case is still a matter of debate and requires further exploration. Nevertheless, the continuity approach towards mind and life is very promising as a starting point for evaluating the extended mind hypothesis. It offers an alternative to the unsolved problem faced by EM, that "the mental is itself too disunified to count as a scientific kind" (Clark 2010b, 63). I will delineate the general reasons for this assumption in the next two paragraphs before applying the resulting distinctions to the realization of memory formation.

\subsection{Non-Locality and Unified Autonomous Systems}

As we have seen, PP in EM creates the obvious problem of "externalizing the internal," a challenge for which the complementary principle is itself no appropriate remedy. This problem does not occur in the enactive approach because 
cognition is conceived as a relational property or process that emerges in interaction by means of the regulation of coupling with the environment (Di Paolo 2009, 19).

This implies that cognition is neither internalist nor externalist as Thompson and Stapleton have pointed out (Thompson and Stapleton 2009, 25). What brings forth meaning and value is the (mediated) coordination of autonomous systems with their environment (Di Paolo, Rohde, and De Jaegher 2010, 51). ${ }^{4}$

Moreover, with its concept of autonomous systems, enactivism offers a formal model and research tool that has the explanatory resources for identifying a unity that might consist of different components. This is so because the concept of autonomous systems is not bound to organisms conceived as autopoietic systems. In this context, proponents of enactivism have argued that systems like the immune system, or intercorporeal synchronizations in mutual interactions such as dance or dialogue, are emergent autonomous systems (Thompson 2007, 419; see Barandiaran 2016). Such emergent autonomous systems by means of inter-bodily relationships have also been termed participatory sense-making (De Jaegher and Di Paolo 2007; Di Paolo, Rohde, and De Jaegher 2010, 71).

Why are these distinctions important for the extended mind debate and embodied memory formation? I will illustrate this with the example of habitus formation. This concept can already be traced back to Aristotle's use of hexis, which is frequently characterized as the socially acquired dispositions of thinking and acting that become second nature. They include styles of acting within a group, the specific posture of the body but also the normative-based incorporation of values. From the enactive perspective, the formation of recurrent and stabilized habits are emergent autonomous systems. Intentional behavior is conceptualized as a sensorimotor achievement. If we assume that a sensorimotor system has the plasticity to learn new behavioral patterns, then constant recurrent behavior can result in a change of plasticity conceived as the concrete action potential of the agent. The latter in turn enforces the stabilization of these recurrent behavioral pattern types, generating a mutual interplay that finally results in new habits (Di Paolo 2003, 13).

The question is, of course, to what extent the mechanisms of habit formation fulfill the criteria of autonomy. When we learn to play an instrument such as a violin, the learning process results in emergent stabilized behavioral patterns, including the coordination of different limbs and organs of the body (e. g., eyehand coordination), brain activity, the entrenched use of an individual violin, and conscious processes. All these aspects of the habit formation and skill development of playing the violin are mutually dependent and stabilize each other, thereby constituting an operationally closed network. Additionally, dynamically self-maintaining habits are also the result of selective processes (i. e., one can

\footnotetext{
4 Rowlands misses this decisive point when he concludes from his assessment of the enactive approach that sensorimotor enactivism is committed to internalism (see Rowlands 2009, 61).
} 
prefer to learn another instrument instead) and can disintegrate due to invasive perturbations or simply when habits are no longer recurrently realized by the agent (see Di Paolo 2003). This implies that habits unfold in their network due to their adaptive stabilization and self-maintenance. A specific recurrent dynamically achieved form underlies their manifestation. As we will see, such self-maintaining habits already specify essential features of habitual body memory. These aspects enable further distinctions and clarifications that are significant for differentiating between the emergence of new identities such as new memory systems and enhanced cognitive abilities by means of embedded individual and collective cultural means.

\subsection{Phenomenological Considerations and Cognitive Scaffolding}

Recapitulating what has been said so far, one could get the impression that the enactive approach is merely offering a more detailed framework for understanding the identity of coupled systems. Yet the enactive approach to cognition and mentality we have contrasted with EM has already suggested major categorical revisions to notions such as "external" or "internal." Further differences lie in the enactive approach's focus on the phenomenological foundation of the body and its integration of tools or prosthesis in its system of movements that are crucial for understanding the process of incorporation. The (phenomenological) methodological procedure is here to focus not on the body as an intentional object but to redirect oneself to the experience of the body as a (partly) "transparent subject," pre-reflectively perceiving and acting in-the-world (Legrand 2007, 504; Thompson and Stapleton 2009, 29). From a phenomenological point of view, the process of shaping the habitual body is revealed by means of a "kinaesthetic reduction.” Elizabeth A. Behnke describes the results of such an investigation as follows:

I am always and already "making a body" in an ongoing kinaesthetic process that privileges some movement possibilities while others are arrested, excluded, curtailed, forgotten, or rendered irrelevant. And although this deeply sedimented kinaesthetic pattern reflects both my own personal past history and a more pervasive "social shaping" of bodies in a given milieu, all this is being ongoingly "executed" here and now, ongoingly carried out in movements and micromovements whose ongoing "how" I can begin to inhabit from within, and study, and describe. (Behnke 1997, 188)

These remarks capture in a nutshell many important aspects of memory formation that I will explicate in the next section. What is striking is the foundational specification of "kinaesthetic patterns" in relation to the autonomous system approach of habits. Phenomenological investigations enable a qualitatively saturated specification of meaningful habits ("personal past history" and "socially shaped" meaningful habits), whereas the autonomous system approach defines the formal aspects of autonomy. This approach to body, mind, and cognition renders enactivism incompatible with the functional commitments of EM. Pre-reflective conscious processes, sense-making, and kinaesthetic patterns form 
an intertwined unity with regard to the inhabited and experienced habitual body, a unity that remains notoriously undetermined in a purely functional re-description.

The phenomenological account also plays a major role in answering the question of whether previous "external" items (from the perspective of an autonomous system) are incorporated into the kinaesthetic bodily infrastructure (movement possibilities) or merely enhance the capacities of a living body. A famous example of the incorporation or "embodying" of environmental items into the entire kinaesthetic structure of the habitual body is Merleau-Ponty's description of the blind man with his cane (Merleau-Ponty [1945] 2005, 162). The cane is no longer experienced as an object. It is fully integrated into the habitualized sensorimotor patterns of the blind man and is "inhabited" as part of the entire feeling body so that the world is experienced at the end of the cane. Thompson and Stapleton characterize this as the fulfilment of the "transparency constraint" what means that the cane is experienced as part of the perceiving and acting subject (Thompson and Stapleton 2009, 29). This leads to the distinction between merely using a tool and incorporating it. Or to put it in "autonomous" terms, between extending the action potential by means of environmental resources or integrating it in a newly emerging habitualized kinaesthetic pattern system.

It is striking that Kim Sterelny (2010) uses such phenomenologically inspired examples to make a similar distinction between entrenched and individualized external cognitive components and merely environmental resources in the context of his scaffolded mind bypothesis. The latter is based on the idea that cognitive skills depend on the mastery of many external resources conceived as "bodies of information" that are not innate. Sterelny explains this by means of the niche construction model. Human beings actively structure their environment; they adapt to their environment but also adapt the environment to themselves (Sterelny 2010, 470). This idea is entirely in line with central tenets of enactivism, namely the idea of a co-constitution of the autonomous systems and their environment. Humans create tools, social institutions, language, or the externalized memory systems mentioned above. This in turn allows the scaffolding of skills and knowledge by means of intra- and intergenerational transmissions; in this sense, culture provides humans with "a second nongenetic ... inheritance system” (Laland, Odling-Smee, and Feldman 2000, 132).

The case of a batsman with his bat exemplifies, what Sterelny has in mind, when he speaks of an individualized and entrenched tool. An expert batsman will choose an individual bat that best fits his weight, kinaesthetic patterns and balance, etc. After using his bat over a longer period, he can become so attuned to it that a substitute bat will feel unsubtle and unwieldy (Sterelny 2010, 474-475). In this case, the bat is not only individualized but entrenched as well. Sterelny thinks that in those circumstances an extension of the body (in the EM sense of a coupled system) is actually achieved (Sterelny 2010, 476). In enactive terms, a new autonomous system has emerged. By contrast, many external items and tools are not extended in this special way. They are interchangeable and contribute to the enhancement and development of the human mind but are not 
constitutive of the latter. In what follows, I will apply these considerations and distinctions to certain aspects of embodied memory formation.

\section{Embodied Habitual Memory Formation}

As the foregoing elaborations on autonomous systems, incorporation, and scaffolding suggest, the differentiation between engrams and exograms in the formation of memory systems is not only incomplete but in need of major clarification as well. I already criticized Donald's brain-centered idea of engrams as "internal memory records" (Donald 1991, 314-315). Such criticism, of course, does not mean denying the central role of the brain in memory formation. Quite the contrary, there is empirical evidence that different brain systems are involved in realizing distinct functions of the working memory. Gruber and Goschke, for instance, have reviewed and evaluated a large amount of research that suggests language has a major impact on the realization of working memory capacities:

The first of these working memory systems relies on prefronto-parietal and prefronto-temporal cortical networks and appears to be also involved in the top-down modulation of domain-specific sensory association areas towards task-relevant information. The second and probably phylogenetically more recent system comprises mainly lefthemispheric premotor and parietal brain regions which also underlie language functions, including inner speech and verbal rehearsal. We propose that this second system plays a central role in the retrieval and maintenance of verbal representations of goals and task rules during the advance preparation for a novel task. (Gruber and Goschke 2004, 106)

These findings indicate the important role of cortical networks in the realization of memory capacities and their embeddedness in the cultural niche (especially the second working memory); however, they do not justify the further claim that memories are somewhere stored in the brain as engrams, retrieved by autocuing mechanisms or triggered in suitable circumstances. As the previous examples already made clear, the emergence of networks by the acquiring of new skills (learning to play the violin) involves not only the brain but the entire kinaesthetic (re)configuration of the body. This implies that the past is "sedimented" in stabilized movement patterns. An experienced car driver does not require that the process of driving is supplemented by various reflective conscious mental acts of recollection. Nevertheless, the pre-reflectively executed acts are obviously rooted in past experiences.

Taking different aspects of habitual pattern formation together, Edward S. Casey suggests the following characterization of habitual body memory from a phenomenological research perspective, namely that it is "an active immanence of the past in the body that informs present bodily actions in an efficacious, orienting, and regular manner" (Casey [1984] 2000, 149). As Casey further points out, the incessant efficacy of the past also in-forms present bodily actions. It structures current behavior with regard to personal and collectively acquired and transferred traditions and is also very important for orientation in new 
situations. Arriving at new places, we tend to establish very quickly new groups of habits, such as to take the correct seat at a table, etc. (Casey [1984] 2000, 151).

The juxtaposition of exograms and engrams is in danger of missing the strong embodied foundation of memory capacities that is also highlighted by Donald's early specification of the transformative powers of exograms on the human mind. In this regard, it is certainly true that external memory is a "critical feature of modern human cognition” (Donald 1991, 312). The creation of larger memory systems is scaffolded in significant ways by new external symbolic storage systems such as libraries or computer networks. Such devices and storage systems are part of the cultural niche and are part of a very effective cultural inheritance system. But this does not mean that individual humans with their biological memories are "monads" that are merely integrated as nodes in the larger external symbolic memory system (Donald 1991, 313). This picture misses important levels of habitual memory foundation and their consequences at different levels of explanation. I will indicate some of these levels and consequences in the paragraph below.

\subsection{Embodied Memories and the Emergence of Inter-Bodily Habitual Memory Systems}

When Casey refers to the deeply sedimented "active immanence of the past" and its regulating character on the present, it would be a gross misunderstanding to conceive the constitution of these memory patterns as confined to monadic brain processes. Learning to fabricate tools by apprenticeship, or learning to dance or program a computer frequently require the mutual coordination and synchronization of behavior among different agents. Imitative capacities are required to learn such complex skills as playing the piano, using tools to construct technological devices, or learning a language. Donald himself has explained in detail that the refinement and evolutionary development of mimetic capacities are one important step towards the emergence of symbolic skills (see Donald 1991, 2001). Imitating goals and the means to realize them is sometimes regarded as the hallmark of bodily mimesis and involves the understanding of intentions (goals) within the intersubjective realm (Zlatev, Persson, and Gärdenfors 2005, 8; see Zlatev, this volume). These skills are required for tool use and the ability to teach them to conspecifics and to pass on this knowledge to the next generation. This explains why a cumulative cultural evolution depends to a large extent on imitative learning (Tomasello 2001, 138; see Wulf, this volume).

As already indicated, the latter enables the accumulation of modified cultural techniques and their adaptation to a variety of functions which has established an efficient non-genetic intergenerational learning transfer at the cultural level. This does not exclude, however, that cultural and genetic mechanisms of inheritance are mutually intertwined (cf. Deacon, this volume). This process of accumulating procedural capacities and implicit knowledge is nothing other than the emer- 
gence of an embodied self-sustaining collective cultural memory system that has its roots in the expansion and refinement of intersubjectively based bodily capacities. The constitution of the latter is explained in more detail by what Thomas Fuchs and Tom Froese have termed the "inter-bodily memory" or "intercorporeal memory” (Froese and Fuchs 2012):

From early childhood on, patterns of interaction are sedimented in the infant's implicit or bodily memory, resulting in what may be called inter-bodily or "intercorporeal memory" ... This means a pre-reflective, practical knowledge of how to interact with others - e. g. how to share pleasure, elicit attention, avoid rejection, re-establish contact, etc. (ibid., 213)

These reflections on sedimented patterns of former interactions in early childhood show that the habitual body memory is not simply in-formed by intercorporeal relationships. What emerges are rather new intercorporeal autonomous memory systems. Emotional synchronization and imitation are key elements for specifying and exploring how these procedural skills and habits integrate different agents into a new memory system network that comprises shared emotions, mutual perspectives, and social encounters. Already from the second month of life, infants and adults share feelings and affects and attune to each other by means of different action patterns in "protoconversations" (Stern 1985, 217; Trevarthen 1989). These are dyadic forms of social interaction, in which adults and infants touch, smile, move, or gaze in an affectionate, rhythmical, and turn-taking manner (Trevarthen and Aitken 2001; Tomasello et al. 2005, 681). A good example is the rhythmically coupled hand movement of an infant during the speech of an adult (Trevarthen and Aitken 2001, 4). Such interactions lead to an inter-bodily memory that Froese and Fuchs (2012) describe as practical knowledge. These descriptions conform to the enactive criteria of autonomous systems because the creation and maintenance of the (single) habitual body and the intercorporeal memory system depend on the dynamic process of a mutual co-constitution.

As the above quotation makes clear, these informing processes are also accessible to phenomenological explanation. Behnke's analysis of the kinaesthetic reduction, mentioned above, already indicate that the formation of kinaesthetic movements reveals not only skill habits but also the "social shaping" of the body (Behnke 1997, 188). This points to the sociocultural dimension of the intercorporeal memory that is constituted by a culturally based sense-making process, as Bourdieu's famous passage on "body hexis" makes clear:

Body hexis speaks directly to the motor function, in the form of a pattern of postures that is both individual and systematic, because linked to a whole system of techniques involving the body and tools, and charged with a host of social meanings and values. (Bourdieu 1977, 87)

Through the acquired skills and enduring dispositions of intercorporeal interactions, the manifold facets of cultural evolution are ingrained as "second nature" into the human body (Bourdieu 1990, 56). It would be wrong, however, to suppose that there is only a culturally induced top-down shaping of the body 
memory. On the contrary, the constitution of intercorporeal memories also enables the use and further development of external storage symbol systems from the "bottom up." This becomes clearer if one looks at the transformatory effects on cognition of the process of embodied enculturation. The use of symbolic systems such as written language or mathematical symbols results in the transformation of cognitive abilities, abilities which are then sustained by the acquisition of new habitualized skills. To give an example, the practice of using tools such as pencils and paper leads to (i) new sensorimotor abilities to manipulate those items in public and (ii) to a redeployment of neural circuits (Menary 2015 , 9). Hence, exograms do not only shape the habitual body but are also sustained by the reorganization and emergence of embodied abilities and dispositions that facilitate and stabilize the fabrication and maintenance of cultural based technologies.

How do these - admittedly sketchy - considerations compare with an EM approach to embodied habitual memory formation? With the aid of the enactive distinctions discussed in the previous section, one can differentiate between the emergence of new memory systems and the mere extension or enhancement of memory capacities. Intersubjective relations such as the incessantly repeated imitation of the parent's posture, voice, and emotional expression generate a long-lasting intercorporeal habitual memory system, consisting of the shared procedural memories of the child and her parents that are expressed in all kinds of bodily performances.

One could reply that inter-bodily memory systems are not autonomous systems in the sense defined in the previous section. The reason is that the coupling between the child and her parents are - despite their intimate relationship fleeting in character (they are merely an effect of short temporal intervals). However, the decisive point is that in this case the incessantly repeated (mutual) imitations and their modifications lead to sedimented habitual dispositions that are actualized in a variety of encounters in dyadic or larger interactions in the family context. In such circumstances, a closed network is instantiated and determines the scope of possible interaction. This network consists of shared emotions, expressions, and meanings that mutually depend on each other and which maintain the identity of the system. This does not mean that every interaction that enhances the mutual memory capacities of the child and her parents in a given situation (playing a memory game or looking at family photos of holiday scenes) is incorporated in the inter-bodily habitual memory system as defined above.

It is one virtue of enactivism that it offers a criterion for why those newly established autonomous systems are cognitive systems in the first place. Interbodily habitual memory systems are the outcome of sense-making processes. When agents are synchronized in coupled actions, new autonomous systems emerge with socio-culturally meaningful patterns that are the outcome of participatory sense-making processes (De Jaegher and Di Paolo 2007; Di Paolo, Rohde, and De Jaegher 2010, 71). The key question is now how these findings are related to distributed memory research in the realm of EM. 


\subsection{Distributed Memory Research}

It is the merit of second-wave extended mind theorists such as John Sutton to focus their research on socially distributed forms of remembering in multifarious ways (see Sutton et al. 2010; Michaelian and Sutton 2013). This is a very important approach in memory research for a closer understanding of the emergence of the human mind and the social self. The formation of embodied memory is, of course, not exhausted by the habitual memory systems described above. Kourken Michaelian and John Sutton describe the phenomenon of distributed memory in general terms as follows:

Whatever their evolutionary history, activities of remembering in human beings are (as we noted above) notably diverse, often involving not only multiple interconnected neural systems, but also bodily practices and external resources of many distinctive kinds. Such is, at least, the view of theorists who see remembering as "distributed," as spread over, coupled with, situated among, or incorporating heterogeneous resources beyond the brain. (Michaelian and Sutton 2010, 3)

As we have seen, only if one augments this description of distributed memory with a purely functionalist approach to mind and cognition does this concept come into conflict with an enactivist framework of memory research.

What is at stake for a distributed and enactive memory approach is, for example, to explore how the scaffolding of cognitive abilities in cultural niches can extend and result in new identities by means of new cultural inventions such as language. The personal self or personal identity is a striking outcome of cultural evolution. It requires, amongst other things, the phylo- and ontogenetic emergence of different memory systems. In addition to the procedural or habitual memory, the constitution of the personal (narrative) self depends on the development of episodic and semantic memory systems. The former refers to specific events and circumstances that have a source in space and time and are autobiographical in nature (Baars and Gage [2007] 2010, 325). Semantic memories are, by contrast, not related to specific episodes (they are more context independent) but connected with "a feeling of knowing" that is not based on the entire recollections of events (Baars and Gage [2007] 2010, 325).

I want briefly to indicate how the enactive approach can also fruitfully contribute to distributed memory research in this domain of higher-order memory capacities. In their historical sketch of current distributed memory research, Michaelian and Sutton (2013) present Daniel Wegner's theory of a transactive memory systems. The central tenet of this theory is the conviction that transactive memories consist of individuals' memory capacities and their ability to actively communicate recollections between them (Wegner, Giuliano, and Hertel 1985, 256). As Wegner and colleagues put it:

It is common in theorizing about the thoughts and memories of individuals to posit an organizational scheme that allows the person to connect thoughts with one another retrieving one when the other is encountered, and so forth. In a dyad, this scheme is complicated somewhat by the fact that the individual memory stores are physically 
separated. Yet it is perfectly reasonable to say that one partner may know, at least to a degree, what is in the other's memory. Thus, one's memory is "connected" to the other's, and it is possible to consider how information is arranged in the dyadic system as a whole. (ibid.)

The enactive approach offers a theoretical framework for how one can conceptualize and explore this dyadic personal relationship in autonomous terms. It can cope, for instance, with the challenge mentioned in the quotation concerning "individual memory stores" which are "physically separated." In enactivism a dyad or inter-bodily relationship is not conceived as consisting of "two boxes" that contain individual memories. Instead, habitual memories are already collectively shaped at the purely habitual level by means of cultural mechanisms such as emotional attunement or imitations as well as explicit instructions in learning processes (Tomasello 1999, 39). This points to the fact highlighted above that external symbol systems and verbal language also in-form the body memory in important respects (think of Bourdieu's concept of "body hexis”).

Moreover, in the actual inter-bodily encounter between two persons their shared habitual body memories (moods, explicit emotions, skills, meanings, and the like) are emergent features of the entire intercorporeal autonomous system. This indicates two possible interpretations of Wegner's transactive memory systems. He seems to favor a purely causal reading of these autobiographical memories as verbalized during the conversation ("one's memory is 'connected' to the other's"). This impression gets even stronger when we read that "one person may know, at least to a degree, what is in the other's memory." In this case, the shared memories consist merely of already existing individual personal memories which are then more easily retrieved via interaction.

However, it is plausible to suppose that verbalized memories also exist that are constituted during the dual narrative memory discourse. Why may this be so? When formerly pre-reflective shared experiences of a concrete event $X$ (we were both immersed in the experience of an overwhelmingly beautiful landscape and our shared feelings towards the landscape) are verbalized during the process of mutual recollection, the symbolic representation of $X$ can also be an emergent feature of the entire transactive memory discourse. In such circumstances, $X$ is a recollection in a verbalized symbolic format that is the result of (a) shared experiences and (b) a common transformation of this content into a conceptualized representation of $X$.

Wegner and colleagues hesitate to extend this model of dyadic memory systems to larger groups (Wegner, Giuliano, and Hertel 1985, 257). But there seems no logical reason why we could not extend this transactive memory model to collectives and societies. A comparison in an enactive research framework with the concept of collective memories appears especially promising (Halbwachs 1939).

These reflections on the transactive memory model open up further research perspectives and indicate how distributed memory research and an enactive approach to remembering and recollection could fruitfully work together. 


\section{Conclusion}

As the foregoing reflections make clear, an enactive approach to memory formation is not simply complementary to that of EM. Proponents of enactivism do not share the functional approach to mind and cognition that unites the first- and second-wave of EM. This has important implications for memory research.

First, with its autonomous system theory and the concept of sense-making, the enactive approach offers research tools and explanatory resources that are better suited to explain phenomena such as incorporation, the identity of a system or simply the extension or enhancement of skills and action potentials. Whether Otto's notebook is actually part of a new emerging memory system depends on its integration with an operationally closed network and its circular self-sustaining relations with other processes of the system. Furthermore, the enactive approach has developed criteria for the specification of cognitive processes that are missing in EM (both first- and second-wave EM).

Second, an indispensable strand of enactive memory research are phenomenological based studies on embodied and intercorporeal aspects of memory formation. The phenomenological foundation of the body and its integration of tools or prostheses in the kinaesthetic movement-system of the living body are crucial for understanding the process of incorporation by means of a "kinaesthetic reduction" and the criterion of "partial transparency." As already mentioned, these phenomenological approaches stand in sharp contrast to functional externalism. Intentionality, meaning, and the entire qualitative dimension of memories receive a foundation in reflective and pre-reflective conscious experiences. Furthermore, a concept such as the habitual body memory is well-suited as a starting point for a phenomenologically based cultural anthropology (see Csordas 1990).

Third, despite these differences between classical enactivism and EM, there are also significant options for fruitful cooperation in embodied memory research. It is undeniable that, for instance, Donald's and Sutton's research on evolutionary and cultural memory formation (symbolic storage systems and socially distributed forms of remembering) have a high explanatory value for memory research at different levels of explanation. The same is true for theories such as cognitive scaffolding (see Sterelny 2010) or cognitive integration (see Menary 2007). Enactivism needs to integrate many of these research insights and tools in order to augment, for instance, the exploration - from an evolutionary perspective - of the role of participatory sense-making in the constitution of the human mind. The same is true for an interdisciplinary research of memory formation.

\section{Acknowledgements}

I am grateful for helpful comments on earlier versions of the paper by André Wunder, Adrian Wilding, Gregor Etzelmüller, and Daniel Vespermann. 


\section{Bibliography}

Adams, F. and K. Aizawa. 2010. Defending the Bounds of Cognition. In The Extended Mind, ed. R. Menary, 67-80. Cambridge, MA: The MIT Press.

Baars, J. and N. M. Gage. (2007) 2010. Cognition, Brain, and Consciousness: Introduction to Cognitive Neuroscience. Oxford: Elsevier.

Barandiaran, X. E. 2016. Autonomy and Enactivism: Towards a Theory of Sensorimotor Autonomous Agency. Topoi. An International Review of Philosophy. doi: 10.1007/ s11245-016-9365-4.

Behnke, E. A. 1997. Ghost Gestures: Phenomenological Investigations of Bodily Micromovements and Their Intercorporeal Implications. Human Studies 20: 181-201.

Bourdieu, P. 1977. Outline of a Theory of Practice. Cambridge, UK: Cambridge University Press.

-. 1990. The Logic of Practice. Stanford, CA: Stanford University Press.

Campbell, R. 2009. A Process-Based Model for an Interactive Ontology. Synthese 166: 453-477.

Campbell, R. and M. Bickhard. 2011. Physicalism, Emergence and Downward Causation. Axiomathes 21(1): 33-56.

Casey, E. (1984) 2000. Remembering. A Phenomenological Study. Bloomington, IN: Indiana University Press.

Clark, A. 2003. Natural-Born Cyborgs: Minds, Technologies, and the Future of Human Intelligence. Oxford: Oxford University Press.

-. 2010a. Coupling, Constitution, and the Cognitive Kind: A Reply to Adams and Aizawa. In The Extended Mind, ed. R. Menary, 81-99. Cambridge, MA: The MIT Press.

-. 2010b. Memento's Revenge: The Extended Mind, Extended. In: The Extended Mind, ed. R. Menary, 43-66.

-. (2008) 2011. Supersizing the Mind. Embodiment, Action, and Cognitive Extension. Oxford: Oxford University Press.

Clark, A. and D. Chalmers. (1998) 2010. The Extended Mind. In The Extended Mind, ed. R. Menary, 27-42. Cambridge, MA: The MIT Press.

Csordas, T. J. 1990. Embodiment as a Paradigm for Anthropology. Ethos 18, no. 1: 5-47.

Deacon, T. 2016. On Human (Symbolic) Nature: How the Word Became Flesh. In this volume.

De Jaegher, H. and E. A. Di Paolo. 2007. Participatory Sense-Making: An Enactive Approach to Social Cognition. Phenomenology and the Cognitive Sciences 6: 485-507.

Di Paolo, E. A. 2003. Organismically-Inspired Robotics: Homeostatic Adaptation and Natural Teleology Beyond the Closed Sensorimotor Loop. In Dynamical Systems Approach to Embodiment and Sociality, ed. K. Murase and T. Asakura, 19-42., 19-42. Adelaide: Advanced Knowledge International.

-. 2009. Extended Life. Topoi 28: 9-21.

Di Paolo, E. A., M. Rohde, and H. De Jaegher. 2010. Horizons for the Enactive Mind: Values, Social Interaction, and Play. In Enaction: Toward a New Paradigm for Cognitive Science, ed. J. Stewart, O. Gapenne, and E. A. Di Paolo, 33-87. Cambridge, MA: The MIT Press.

Donald, M. 1991. Origins of the Modern Mind: Three Stages in the Evolution of Culture and Cognition. Cambridge, MA: Harvard University Press.

-. 2001. A Mind So Rare. The Evolution of Human Consciousness. New York: W. W. Norton \& Company.

Froese, T. and T. Fuchs. 2012. The Extended Body: A Case Study in the Neurophenomenology of Social Interaction. Phenomenology and the Cognitive Sciences 11: 205-236. 
Gruber, O. and T. Goschke. 2004. Executive Control Emerging From Dynamic Interactions Between Brain Systems Mediating Language, Working Memory and Attentional Processes. Acta Psychologica 115: 105-121.

Halbwachs, M. 1939. La mémoire collective. Paris: PUF.

Horgan, T. (1994) 2000. Physicalism (1). In A Companion to the Philosophy of Mind, ed. S. Gutenplan, 471-479. Oxford: Blackwell Publishers.

Hurley, S. 2010. The Varieties of Externalism. In The Extended Mind, ed. R. Menary, 101-153. Cambridge, MA: The MIT Press.

Jonas, H. 1966. The Phenomenon of Life. Toward a Philosophical Biology. New York: Harper \& Row.

Kelso, J. A.S. 1995. Dynamic Patterns. The Self-Organization of Brain and Behavior. Cambridge, MA: The MIT Press.

Legrand, D. 2007. Pre-Reflective Self-Consciousness: On Being Bodily in the World. Janus Head 9: 493-519.

Laland, K. N., J. Odling-Smee, and M. W. Feldman. 2000. Niche Construction, Biological Construction, Biological Evolution, and Cultural Change. Behavioral and Brain Sciences 23: 131-175.

Menary, R. 2007. Cognitive Integration - Mind and Cognition Unbounded. Hampshire: Palgrave Macmillan.

-. 2010. Cognitive Integration and the Extended Mind. In The Extended Mind, ed. R. Menary, 227-243. Cambridge, MA: The MIT Press.

-. 2015. Mathematical Cognition - A Case of Enculturation. In Open MIND 25, ed. T. Metzinger and J. M. Windt, 1-20. Frankfurt/Main: MIND Group.

Merleau-Ponty, M. (1945) 2005. The Phenomenology of Perception. Trans. C. Smith. Taylor and Francis e-Library.

Michaelian, K. and J. Sutton. 2013. Distributed Cognition and Memory Research: History and Current Directions. Review of Philosophy and Psychology 4:1-24.

Mumford, S. and R. L. Anjum. 2011. Getting Causes from Powers. Oxford: Oxford University Press.

Rowlands, M. 2009. Enactivism and the Extended Mind. Topoi 28: 53-62.

Rupert, D. 2004. Challenges to the Hypothesis of Extended Cognition. The Journal of Philosophy 101(8): 389-428.

Revlin, R. (2012) 2013. Cognition: Theory and Practice. New York: Worth Publishers.

Spahn, C. 2016. Beyond Dualism? The Implications of Evolutionary Theory for an Anthropological Determination of Human Being. In this volume.

Stapleton, M. 2016. Leaky Levels and the Case for Proper Embodiment. In this volume.

Sterelny, K. 2010. Minds: Extended or Scaffolded? Phenomenology and the Cognitive Sciences 9: 465-481.

Stern, D. N. 1985. The Interpersonal World of the IInfant: A View From Psychoanalysis and Developmental Psychology. New York: Basic Books.

Sutton, J. 2010. Exograms and Interdisciplinary: History, the Extended Mind, and the Civilizing Process. In The Extended Mind, ed. R. Menary, 189-225. Cambridge, MA: The MIT Press.

Sutton, J., C. B. Harris, P. G. Keil, and A. J. Barnier. 2010. The Psychology of Memory, Extended Cognition, and Socially Distributed Remembering. Phenomenology and the Cognitive Science 9(4): Sciences 521-560.

Tomasello, M. 1999. The Cultural Origins of Human Cognition. Cambridge, MA: Harvard University Press.

-. 2001. Cultural Transmission. A View from Chimpanzees and Human Infants. Journal of Cross-Cultural Psychology 32 (2): 135-146. 
Tomasello, M., M. Carpenter, J. Call, T. Behne, and H. Moll. 2005. Understanding and sharing intentions: The origins of cultural cognition. Behavioral and Brain Sciences 28: 675-691.

Trevarthen, C. 1989. Development of Early Social Interactions and the Affective Regulation of Brain Growth. In Neurobiology of Early Infant Behaviour, ed. C. von Euler, H. Forssberg, H. Lagercrantz, and V. Landin, 191-216. London: Macmillan Education UK.

Trevarthen, C. and K. J. Aitken. 2001. Infant Intersubjectivity: Research, Theory and Clinical Applications. Journal of Child Psychology and Psychiatry 42 (1): 3-48.

Thompson, E. 2007. Mind in Life. Biology, Phenomenology, and the Sciences of Mind. Cambridge, MAUKMA: The Belknap Press of Harvard University Press.

Thompson, E. and M. Stapleton. 2009. Making Sense of Sense-Making: Reflections on Enactive and Extended Mind Theories. Topoi 28(1): 23-30.

Wheeler, M. 2010. In Defense of Extended Functionalism. In The Extended Mind, ed. R. Menary, 245-270. Cambridge, MA: The MIT Press.

Wegner, D. M., T. Giuliano, and P. Hertel. 1985. Cognitive Interdependence in Close Relationships. In Compatible and Incompatible Relationships, ed. W. J. Ickes, 253-276. New York: Springer.

Wulf, C. 2016. The Creation of Body Knowledge in Mimetic Processes. In this volume.

Zlatev, J. 2016. Preconditions in Human Embodiment for the Evolution of Symbolic Communication. In this volume.

Zlatev, J., T. Persson, and P. Gärdenfors. 2005. Bodily Mimesis as "the Missing Link" in Human Cognitive Evolution. Lund University Cognitive Studies 121. Retrieved from http://lup.lub.lu.se/luur/download?func=downloadFile\&recordOId=531391\&fileOId $=625029$. 


\title{
Radically Enactive Numerical Cognition
}

\author{
Karim Zabidi/Erik Myin
}

\begin{abstract}
We deal with the general question of how culture affects cognition by looking at numerical cognition. After presenting radical enactivism, according to which contentful cognition arises only with the emergence of truth telling practices, we confront recent research about the origins of numerical cognition. We contest readings of some of the empirical data, according to which numerical cognition predates culture. We argue that REC-friendly interpretations of the data are not only possible but preferable, as they avoid the staunch theoretical problems which plague cognitivist readings.
\end{abstract}

\section{Introduction}

How does the emergence of culture affect cognition? What changes when skillful and cognitively flexible creatures engage in the extensive cooperative endeavor called society? Perhaps surprisingly, the view of cognition which has been dominant now for several decades views the transition to culture as having relatively modest effects. Here is why. According to the standard view, whenever there is intelligence, there is both representation and computation. Any kind of cognition-driven behavior beyond the simplest reflex-like reactions to the immediate surroundings requires computational operations on contentful internal representations. ${ }^{1}$ Thus, memory-based navigation in for example both insects (Gallistel 1998) and rodents (Tolman 1948) has been understood as relying on the computational manipulation of internal representational entities, be it symbols or maps. In providing such explanations, the representationalist/computationalist view thus assumes that there is representation and computation at a very early stage. If this view is taken literally, it follows that the emergence of culture does not, in an important sense, fundamentally change the nature of cognition. Cognition was already contentful and computational before culture, so culture does nothing to change that. ${ }^{2}$ Of course, culture can make profound differences with respect to what can be represented, and perhaps also in what can be computed, as well in

${ }^{1}$ By 'contentful,' we mean: being subject to truth or accuracy conditions (see Hutto and Myin 2013, 81). If this requirement does not apply, then the label "contentful" and by implication "representational," does not apply.

${ }^{2}$ Evolutionary psychology as defended by Cosmides and Tooby (2013) can serve as a paradigmatic example of a theory of cognition in which the role of culture is downplayed to the extreme. 
the fact that contents can now be externalized and communicated. Nonetheless, the basic picture of representation plus computation as the core of cognition remains untouched.

It is possible to hold on to the traditional view - or something like it - without being led to such an epiphenomenalist view of the power of culture by either deflating the notions of representation and computation, or by taking a fictionalist stance. Deflationary and fictionalist positions allow one to hold that there is a fundamental difference between the representations and computations in, for example, ants and language-using humans, because representations and computations in the former are less substantial, or even fictional. The deflationary move thus consists in minimizing one's commitments when one uses the words 'representation' or 'computation.' For example, one could hold that representation does not require truth or accuracy conditions. Clearly, deflationism isn't an assertive position, but rather one of retreat. The worry, however, is that after the main, and originally motivating commitments with respect to the core notions of the representationalist/cognitivist view are withdrawn, all that remains is a label and not a substantive and distinctive notion, which can then do genuine explanatory work. Fictionalists, on the other hand, insist that 'representation' can do such explanatory work while being entirely fictional. They hold that explanatory virtue and existence can come apart: fictional entities can be perfectly used for explanations, even if they do not exist at all. Again, this raises important concerns. If, in many cases, the explanations one is after in the sciences of cognition, are causal, it remains to be shown by the fictionalist how purely fictional entities can figure in such explanations. Surely, what does not exist cannot be a cause (Hutto and Myin, forthcoming).

There are other options than going deflationary or fictionalist. However, these positions are still predicated on endorsing the main tenets of the traditional view. A more radical move is to simply deny that representation and computation do form the basis of all intelligent behavior. An approach that does just that is REC, or Radically Enactive/Embodied Cognition (Hutto and Myin 2013). We want to highlight REC here, and, taking arithmetic as our main example, argue that REC allows for a more satisfactory position about basic minds, minds in culture, and their relation.

\section{Basic Minds without Content: The REC View ${ }^{3}$}

According to REC, as presented and defended in Hutto and Myin (2013), cognition is not always representational, and does not always involve computation. REC holds that basic forms of cognition are best understood in terms of dynamically unfolding interactions with the environment, rather than being explained in terms of computational operations on contentful representational entities. In

\footnotetext{
3 This section borrows some material from Myin and Hutto (2015).
} 
denying that basic cognition is contentful, REC does not deny that basic cognition is intentional, or directed at specific aspects of the environment. That is, acts of perceptual, motor, or perceptuomotor cognition - chasing and grasping a swirling leaf for example - are directed towards worldly objects and states of affairs, or aspects thereof, yet without representing them. This is intentionality without intensionality - the latter understood in the traditional sense of "representing as," which does not allow substitution of co-referential expressions.

REC does not claim that cognition never involves content, a position termed "Really Radical Enactivism" in Hutto and Myin (2013). Unlike Really Radical Enactivism, REC does not deny that the idea of content, specified, for example, in terms of truth, or truth conditions, makes sense paradigmatically in the realm of language. The distinction between applying a term correctly or incorrectly, between speaking the truth and speaking falsely is valid at least for some instances of speaking. Other public items like maps or diagrams might involve content as well, on the condition that their use is grounded in appropriate norms connected with language. According to REC, and reasoning by elimination, intelligent or cognitive organismic activities, unless they rely on public representations embedded in established sociocultural practices, are not contentful, and hence not representational.

The arguments in Hutto and Myin (2013) for the existence of basic minds, without content, unfolded along two tracks. First, it was pointed out that, for an expanding range of cognitive phenomena, from motor activities to perception, explanations are forthcoming which do not involve content. Here a "Don't Need" strategy was relied upon, similar to the one followed by our fellow non-representationalist Tony Chemero (2009). Secondly, there was a "Can't Have" strategy, arguing that, currently, and after having tried what seemed to be the best resources, no theoretical motivation could be provided for the existence of content beyond what's established as contentful at the sociocultural level. The idea of contentful, yet basic minds, is unsupported, so Hutto and Myin (2013) claimed. In line with this the foundational problem was identified as "The Hard Problem of Content."

\section{RECkoning ${ }^{4}$}

REC's view on representation is paralleled by its take on computation. That is, just as representation is, in REC, not taken to form a basic ingredient of cognition, so neither is computation. Just like representation, computation is an achievement, arrived at only in a specific sociocultural context. This inverts the place assigned to computation within the standard picture held in cognitive science. Instead of an explanans, computation becomes an explanandum, and the explanation has to be achieved via a natural history that details how computa-

\footnotetext{
${ }^{4}$ This account of computation à la REC is a prolegomenon only to a later fuller treatment.
} 
tional practices arise out of noncomputational precursors. By providing such a genetic story of computation, REC aims to truly account for computation in contrast to assuming computation as a basic, and fundamentally unaccounted for, ingredient in nature.

How could such a story go? Computation only got started, so the REC story goes, with the emergence of practices of counting, and simple arithmetical operations such as, initially adding, subtracting, and multiplying. Flegg (2002) provides an illuminating overview of the main lines along which a natural history of computation unfolds. He makes clear that before arithmetical calculation can develop, simpler practices, including the practice of counting, have to be in place. Counting, as Flegg notes, is in itself "an advanced process; it is by no means an instinctive and innate process as it might appear to be” (Flegg 2002, 10). Indeed, before counting can arise, specific perceptual and motoric processes need to be in place. Perceptual abilities such as distinguishing size and shape are prerequisites for counting. But such perceptual abilities are not enough. Counting seems to have arisen out of the application of motoric abilities to put sets of objects into one-to-one correspondence. A rationale for the emergence of such practices of establishing one-to-one correspondences might be found in the practical necessities of communal life in social groups. Especially in agricultural communities in which cattle were a valuable asset, one can see how the ability to check whether any animals were missing from one's flock is important. While it is plausible to suggest that the pairing of objects could have been first done by using collections that were ready at hand, for example body parts or members of the family, this technique is impractical when the target collections are too large. Hence, the need arises for a technique that works for large collections, such as practices like pairing off objects from the target collection with objects from a dedicated collection of token objects (such as shells). Although this technique allows for the inspection of larger collections of objects, it is also highly impractical, in that one is required to carry around a dedicated set of objects. To overcome these limitations, one thus needs a technique that would allow one to produce such collections on the spot. A possible solution to this problem is the practice of tallying, which arose in the Upper Paleolithic, by which one made inscriptions on a stick or knots in a piece of rope - each mark or knot representing one object of the collection to be surveyed. ${ }^{5}$ Arguably, it was this practice that served as a foundation for and precursor to the symbolic representation of number.

The practice of counting does however require more than the ability to put different collections in a one-to-one correspondence. It also requires an awareness of order. Following Flegg, such an awareness can arise from the practice

${ }^{5}$ Much more can be said about the interplay between the emergence of numerical cognition and the embodied practical engagement with material artefacts. Recent developments in cognitive archeology, exemplified by material engagement theory (Malafouris 2013), are consonant with our story about the development of numerical cognition in that they lay great stress on the engagement with material culture in the emergence of cognition in general. For applications of material engagement theory to the domain of numerical cognition, see Malafouris (2010) and Overmann (2016). 
of pairing off objects with body parts, in particular fingers, since they come in a natural order. The importance of the use of ordered body parts to pair off objects is crucial. There are, as Flegg notes, no examples of cultures where the ability to count has arisen without there being a "history of an earlier stage of body or finger matching” (Flegg 2002, 9). All of this further highlights the role of embodied practices in the origins of counting. Tellingly, specific number words can be seen to be directly related to those parts of the body used to pair off certain collections as in this actual sequence of early number words: "the end one is bent, another is bent, the middle one is bent, one is still left, the hand has died" (Flegg 2002, 10).

Once such linguistic practices were in place, counting, properly understood, had arisen. As language and linguistic practices further evolve, number words can become more and more separated from the embodied practices and body parts that were involved in early counting. However, this separation still does not account for the emergence of abstract number concepts. As Flegg notes:

There is much evidence from linguistics to suggest that for a long time they [number words] were directly associated with the particular objects being counted. At this stage, three sheep could be distinguished from two sheep and three fingers from two fingers, but the abstract appreciation of three and two was yet to be understood. Even after he had devised effective methods of counting, it was a long time before awareness of number in the abstract was achieved. (Flegg 2002,11)

Flegg goes on to document how the embodied nature of counting gives rise to the elementary arithmetical operations such as adding and subtracting. Here again two important points need emphasis. Firstly, these operations were always dependent on the context and the objects: one could, for example, add the number of sheep or the number of sacks of grain, but there initially was no notion of abstract addition. In its most basic form, addition would be performed by physically putting together two collections, say of sacks of grain, of the appropriate cardinality and then counting the number of sacks in the amalgamated collection. At a later stage this was done by associating the number of sacks of grain in each collection with fingers (or tallies) and then counting the total number of fingers (or tallies). Note that we find, once again, that the operations performed were always context- or object-sensitive. Even when addition was performed by counting fingers or tallies, there was still no notion of abstract number or abstract arithmetical operations. A true appreciation of the abstract nature of number, so Flegg argues, only emerged possibly as late as the first millennium B. C.:

We are still faced with the problem of putting some rough date on man's becoming aware of numbers in the abstract, or at least suggesting where this might fit into our history of counting. Obviously, this would have been a gradual process which would have come about at different times in different places. In the case of some of the more undeveloped tribes to be found today, it has still not occurred. It is certainly a later occurrence than the invention of any of the counting systems which we have considered. Apart from evidence of tallying, written mathematical records go back approximately to 3000 B.C. Such ancient records are very largely associated with practical applications of number, though there are occasions where we find calculation carried 
out apparently for its own sake. It is reasonable to suggest that awareness of number in the abstract had its beginnings around this time or fairly soon after, though it did not become an influential part of mathematical thinking until the time of the Pythagoreans, that is, roughly the middle of the first millennium before Christ. (Flegg 2002, 34)

We have only briefly sketched the emergence of arithmetic, leaving out much of the details. However, this overview still provides theoretical and evidential support for the RECish idea that genuinely abstract numerical and arithmetical abilities only arise in a context in which the sociocultural practices of language are well in place. In line with REC's views on content in general, contentful statements about numbers, and the arithmetic operations to which they can be subjected too, require - in order to be made at all - that the truth telling practices, without which there are no contents, pre-exist. Of course, arithmetical abilities don't arise out of nothing. They do have precursors, but the abilities out of which arithmetic arises are not arithmetical abilities themselves.

\section{The "Number Sense" Revisited}

If the above is indeed a plausible sketch of a natural history of the emergence and development of basic arithmetic skills, the emergence of arithmetic goes together with the ability to engage in contentful norm-governed public practices. In particular, conceptual abilities relating to the abstract concept of number can only arise in organisms that thrive in a symbolically rich environment. ${ }^{6}$ Species that are not able to engage in the contentful norm-governed manipulation of symbols cannot be said to be computing or do arithmetic. By the same token, young infants cannot be said to perform numerical tasks. This however seems to fly in the face of much work being done in cognitive psychology. There is a large literature on what is called "animal numerical cognition" (Gallistel 1993; Dehaene 2011; De Cruz, Neth, and Schlimm 2010) or the numerical abilities of pre-verbal infants (Butterworth 1999; Dehaene 2011; Lakoff and Núñez 2000; Devlin 2000; De Cruz and De Smedt 2010). Doesn't the existence of such scientific (sub-) industry indicate that the view on numbers and computation sketched here is wrong? No, it doesn't. We will argue that despite having gained wide currency in scientific practice, describing certain types of behavior as shown by animals or pre-verbal infants as a sign of arithmetical competence is not warranted by the empirical evidence.

We will focus on the proposal that monkeys have an abstract number concept. This claim has been defended by Jordan and collaborators. In a first series of experiments, Jordan et al. (2005) showed that rhesus monkeys are able to match a sequence of two or three auditory stimuli, the sounds of animals vocalizing, with a sequence of two or three visual stimuli, videos showing a chorus of two or three animals vocalizing, according to the cardinality of the sequence of stimuli.

${ }^{6}$ Concrete concepts are concepts referring to spatio-temporal objects (or collection of such objects). Abstract concepts refer to properties of concrete objects or collections. 
This provides evidence for the fact that rhesus monkeys have the ability to match numerically equal series of ecologically relevant stimuli across different modalities. In a follow-up study, Jordan et al. (2008) extended these results in two ways. First, they demonstrated that, after training, the ability to match sequences of stimuli across different modalities extended to sequences containing up to nine stimuli, thus showing that the numerical range of the ability was much larger than the first set of experiments indicated. Secondly, whereas in the first experiments the stimuli were ecologically relevant - voices and images of animals - in the subsequent experiments the stimuli were more abstract - squares on a computer screen and simple tones. In the same set of experiments, the researchers also showed that rhesus monkeys were able to tally auditory and visual stimuli. For example, when presented with two visual and two auditory stimuli, the monkey would be more likely to choose, among a set of different arrays, the array containing four dots. In what follows, we will refer to these abilities as MTA (Modal Transfer Abilities). According to Jordan et al. (2008) their findings

demonstrate that monkeys can sum the number of sounds they hear and the number of sights they see. They do so over a large range of numerical values. Ratio dependence in accuracy and reaction time suggests that they rely on analog magnitude representations of number when performing numerical computations within or between senses. Thus, when humans nonverbally quantify sights and sounds in the world around them, they are likely tapping an evolutionarily primitive system that monkeys and perhaps many other animal species share. (Jordan et al. 2008, 623)

A number of claims are made here. Firstly, it is asserted that the capacities that are displayed in the behavior of the monkeys are numerical abilities. Secondly, it is stated that the exercise of such abilities relies on internal numerical representations, which are analog or approximate in nature. Thirdly and finally, it is claimed that at least one of these representational systems is old, in the sense that they are a product of evolution and thus are likely present from birth.

Taken alone, and in concert, these claims go against the REC view. If true, then the REC view is false. For, contrary to the REC view, representational and computational abilities would not be an achievement gained only in culture. The concepts of number and of summing would pre-date culture, both on a phylogenetic level (since it is present in species that are non-cultural) and on an ontogenetic level (since it is present in very young infants). Possession of the concepts of number and summation would be manifest in simple perceptual capabilities. Those basic capacities would be made possible and would be explained by an underlying abstract numerical concept, or representation of number. A more developed number concept (or numerical representation) derives from simpler number concepts or representations. At least the simplest of number representations are innate.

We agree that the truth of these claims would effectively put the REC view of arithmetic out of business. However, we will argue that it takes a lot more conceptual and experimental work to actually establish that the three core claims are in fact true. In particular, we will question whether the available data actually warrant the description of the MTA as a numerical capability. As such, we will 
question whether the positing of number representations actually explains the MTA. And finally, we will ask whether claims about innateness do anything to address the worries we will raise.

The idea that humans and certain other species come equipped with an "evolutionary primitive [number] system" gained widespread currency following the publication, in 1997, of the first edition of Dehaene's The Number Sense (Dehaene 2011). According to Dehaene, this number system is an innate representational system that uses analog or approximate representation of numbers (Approximate Number System, ANS). ${ }^{7}$ The existence of such a system allegedly accounts for various "numerical" abilities of animals and young infants. It is thus a system that incorporates basic arithmetical knowledge and hence that knowledge is implicitly available from the very early stages of ontogenetic development. We will challenge, not the claim that organisms come equipped from birth with a repertoire of behaviors, but, firstly, that these behaviors are properly numerical, and secondly, that these behaviors are the product of a knowledge or representation driven system that operates unconsciously. The general shape of our more specific concern has been identified by Sampson:

A social and historical process has been translated into a fundamental psychological process. This reification loses the connection between the sociohistorical and the psychological; it raises the psychological to the status of a fundamental property of the human mind. (Sampson 1981, 738)

First, consider the terminology of "numerical abilities" with which the literature is infused. Such turns of phrase suggest that the behavior of certain species of animals and young infants is continuous with the arithmetic abilities of mature humans. Or, in other words, that the difference in cognitive behavior between animals and mature humans is merely one of degree but not one of kind. For example Brannon and Merritt write: “The ability to understand and manipulate numbers is a hallmark of human cognition, but the data reviewed in this chapter show that such abilities are not unique to humans" (Brannon and Merritt 2011, 220).

The idea expressed by Brannon and Merritt, and implicitly endorsed by Jordan et al. (2008) and Dehaene (2011) in their description of experimental work, leaps from the fact that certain animals and young children show behavior that can be described in numerical terms to the conclusion that the behavior is an exercise of the same numerical capacity which mature human beings exercise when they, for example, manipulate numerical symbols according to the socioculturally established norms of arithmetic. In other words, because the animal or infant behavior is analogous to a subset of the behavior which we would designate as numerical in mature humans, it is assumed to be an exercise of numerical abilities.

7 The representations are supposed to be analog or approximate because of the fact that although monkeys might be able to discriminate between two items and three items, they are not able to distinguish a collection of 23 items from one consisting of 25 items. Hence, it is claimed that they are only able to represent numerical information in an approximate sense. 
However, such a conclusion is not warranted. The kind of behavior that is shown by animals and infants could just be a precursor to the acquisition of fully numerical, e. g., arithmetic capabilities - exactly in the way we pointed out above, and as such its presence would be a necessary, but not a sufficient condition for literally having numerical capacities.

Of course, the numerical competence of mature humans includes, besides the ability to manipulate the symbols of arithmetic in the appropriate ways, those recognitional capacities that are also present in certain animals. However, from that observation, it does not follow that the animal's recognitional capacities share some underlying cognitive mechanism with the arithmetic capacities of mature human beings. In order to make this inference, one needs three premises to hold simultaneously. Firstly, that the recognitional and arithmetical capacities of mature human beings share some underlying cognitive mechanism C. Secondly, that $\mathrm{C}$ is also present in monkeys. And finally, that $\mathrm{C}$ is recognizably arithmetical. While it seems plausible to accept the first and second premise on the basis of the available evidence and general evolutionary considerations, the third premise is supported neither by the available evidence, nor by independent general or theoretical considerations. ${ }^{8}$ If the REC line on computation and representation is correct, then simpler capacities at play in the recognitional abilities are just that: simpler capacities. The arithmetical capacities are genuinely different. They build on, but go considerably beyond, the simpler capacities such as those involved in the kinds of recognition at issue. Part of what makes them different is that they are subject to sociocultural norms.

In other words, it is not because we do not hesitate to attribute numerical abilities to mature humans, that we should attribute the same abilities to subjects some of whose behaviors are similar. We attribute the numerical ability to humans not only because their behavior is in accordance with certain norms, but also because they participate in the practices by which those norms are established and maintained. In the case of monkeys and young infants, only one of the conditions for attributing numerical abilities is satisfied.

The researchers concerned go beyond simply calling the abilities in question in an as yet unwarranted way - "numerical." They also claim that the abilities manifested in the experiments call for an explanation in terms of a representational system behind the abilities, and, in the case of Stanislas Dehaene,(2011) in terms of an innate representational system.

These further moves should be seen in the light of the fact that, in the case of the behavior shown in the experiments of Jordan et al. (2008), one needs to account for the fact that monkeys are able to connect numerical cues across different modalities. Call this ability the Modal Transfer Ability, or MTA. In discussing the crossmodal number effect in preverbal children, Dehaene (2011) is

\footnotetext{
${ }^{8}$ By "independent general or theoretical considerations" we mean considerations which do not already presuppose that all cognitive capabilities involve the manipulation of mental representations.
} 
upfront about the need for, and alleged virtues of, a representational explanation for the MTA:

The simplest explanation is that the child really perceives numbers rather than auditory patterns or geometrical configurations of objects. The very same representation of number "three" seems to fire in its brain, whether it sees three objects or hears three sounds. This internal, abstract, and amodal representation enables the child to notice the correspondence between the number of objects on one slide and the number of sounds that are simultaneously heard. (Dehaene 2011, 40)

Dehaene's proposal here is that the observed widening of capacities across modalities shown in the experiments can be explained by the presence of an amodal representation. It is the activation of this amodal, numerical representation - its firing in the brain - that "enables" the child to "notice" the similarity in the patterns across the modalities. As it stands, this alleged explanation contains a number of important gaps, which invalidate its explanatory potential. That is, Dehaene's account leaves a number of crucial questions unanswered. In the first place, it is not specified how the numerical representation is arrived at. The obvious answer would be to assume that the abstract amodal representation derives from contact with "the auditory patterns or geometrical configurations of objects." This solution is not available to Dehaene, however, because his reasoning in the passage cited is driven by the idea that the encounter with such auditory stimuli and geometrical patterns is modality specific, and can become amodal or abstract only if it is aided by the presence of an amodal representation. Applying this reasoning to the question of how one gets from encounters with modality specific stimulation to an amodal representation implies the pre-existence of an amodal representation to get the feat accomplished. Call this the Problem of Origin. ${ }^{9}$ Another key question concerns the efficacy of the (as yet unaccounted for) representation. It is said that "this internal, abstract and amodal representation enables the child to notice the correspondence between the number of objects on one slide and the number of sounds that are simultaneously heard" . (Dehaene 2011, 40) but for this to become a credible story, much more needs to be said both about what amodal representations are and how they bring about this enabling. That is, we are not given any information about what makes amodal representations representational, rather than just neural processes that are causally connected to perceptual systems in different modalities. We have not been told which contents are carried by amodal representation or how those contents got there, what justifies calling them contents at all, and how they play a role in further cognitive processes. Given the absence of any answers to these questions, the only property that is specified of the amodal representation is that it "enables" the "noticing of the correspondence." We are told nothing more

9 What we here term the "Problem of Origins" is also raised by Garofoli (this volume) in his discussion of mental modules. While he does not contest that the mind might be modular, he nonetheless argues that the idea that mental modules, in particular modules that are supposedly responsible for culturally variable cognitive functions, are innate, leaves the emergence of these modules through phylogeny entirely mysterious. 
about the representation over and above the fact that it is the enabler of the capacity to be explained, and hence we are not offered a substantive explanation. Call this the Problem of Substance. Given the fact that Dehaene (2011) does not give any answer to the Problem of Substance, his claim that the representation explains the capacity becomes the claim that the enabler of the capacity explains the capacity. This is obviously true for any enabler, irrespective of whether it is representational or non-representational. Since there is nothing in the account which precludes non-representational neural processes playing the role of enablers, Dehaene's (2011) account turns out to be, without further elaboration, not distinctively representational.

In the absence of answers to the Problems of Origin and Substance, the ANS-hypothesis, instead of bringing us closer to an explanation, takes us farther away from one.

But perhaps this goes too quickly. It might be pointed out that there is an answer to the Problem of Origin, if it is assumed that the representations are innate. If this option is taken, it is recognized that, logically, the reason to invoke abstract representations - namely the cognitive impotence of concrete stimuli-precludes the possibility that abstract representations are ever acquired in a process of interaction with such stimuli. It is only logical, then, that Dehaene (2011) actually takes this step. Consider Dehaene's (2011) justification for the ANS-hypothesis as an explanation for the MTA: if there is an ANS, then MTA is allegedly explained by the fact that modal-specific cues are mapped onto amodal number representations provided by the ANS-system. Modal-specific cues of different modalities thus yield different amodal representations that are then compared to each other. A further reason to assume that the ANS has to be innate is the fact that the MTA is preserved even if the stimuli are ecologically non-relevant or ambiguous:

While waiting for conclusive experiments with younger children, it remains possible to maintain that learning, rather than brain maturation, is responsible for the baby's knowledge of numerical correspondence between sensory modalities. By dint of hearing single objects emit only one sound, pairs of objects emit two sounds, and so on, the baby may discover the nonarbitrary relationship between a number of objects and a number of sounds. Yet, is such a return to constructivism plausible? Some objects generate more than one sound, others no sound at all. Environmental cues are therefore not devoid of ambiguity, and it is highly unclear that they would support any form of learning. I therefore suspect that the babies' preference for a correspondence between sounds and objects stems from an innate, abstract competence for numbers. (Dehaene 2011, 50)

Apparently, it is because contact with "the auditory patterns or geometrical configurations of objects" (Dehaene 2011, 40) does not suffice for acquiring the abstract concept that one has to assume that the abstract concept is already in place before the learning process sets off. But if such reasoning applies to ontogeny, it applies to phylogeny as well. To see how this imperils Dehaene's position, consider that either an innate MTA can be accounted for in a naturalistic, scientific way, or it can not. If the MTA can be explained in a naturalistic way, one's 
hopes would lie with a selective process in evolutionary phylogeny. But surely such process is one of contact with "the auditory patterns or geometrical configurations of objects." If there are principled reasons why such contact is unable to lead to abstract capacities in ontogeny, these must apply to phylogeny as well.

In other words, if we agree with Dehaene that environmental stimulation lacks the necessary quality to account for the development, through learning, in ontogeny, then the same must be true for phylogenetic development. It is difficult to see how stimuli that are irrelevant for learning could become relevant for phylogenetic development. But then if Dehaene's argument is correct, this shows that we cannot give a plausible evolutionary theory of the emergence of the MTA. And given the fact that evolutionary biology is the prime candidate for furnishing a non-psychological theory for the emergence of MTA, it also becomes doubtful whether one can ever give a naturalistic explanation for MTA. Thus MTA remains mysterious.

If all of the above is correct, then there is no explanatory advantage whatsoever in assuming an abstract representation when it comes to accounting for MTA, irrespective of whether it is innate or not innate. In other words, despite claims to the contrary, a cognitivist representational framework offers no explanatory advantages over one that eschews representations as explanatory posits. Note that adequate performance in a context of objects which are ecologically irrelevant or ambiguous does nothing to change this verdict. Even if observations of such behavior might point in the direction of innateness, it does nothing to favor an innate abstract representational system such as the ANS. That is, the REC position does not preclude innate mechanisms. It only opposes innate structures such as the ANS, and in particular the reasoning which leads to the alleged necessity of positing a representational structure of its kind.

But if the explanation of the MTA will not be representational, how will it go? Obviously, it will invoke perceptual capacities, and the ways in which creatures learn to treat similar things or situations as perceptually similar, and as allowing for similar activity with respect to them. There is a rich tradition of non-representational research on this issue, for example in Gibsonian ecological psychology. It describes how acquiring perceptual sensitivities involves becoming sensitive to certain environmental variables, something which does not logically require representing those variables (Gibson 1979).

Perhaps, the development of certain perceptual capacities, such as seeing numerical similarity between two situations, proves only to be possible for organisms if they already possess the capacity to perform certain actions. For example, perhaps the ability to see equinumerosity in different modalities derives from a prior capacity to spatially collect or separate equinumerous amounts of objects - to treat one object differently than a collection of two, three, ... objects, for example by segregating them in space. Having acquired those capacities for action might then explain the capacity for enriched perception.

These are speculations, of course. The crucial point remains that there are strong logical reasons against, and no compelling evidence for, the supposition that the matching abilities of monkeys and young infants can serve as evidence 
for the postulation of a genuinely representational abstract system such as the ANS. The introduction of the ANS is, as it stands, a non-explanation. Contrary to what a cursory look at the findings of the young science of "numerical cognition" might indicate, these findings therefore do not put the REC view, that there are no numerical concepts prior to culture, out of business.

\section{Conclusion}

Our argument shows that the reliance on Dehaene's ANS-hypothesis to account for certain behaviors has no explanatory value, as long as the Problems of Origin and Substance are not met. Repudiating Dehaene's ANS-hypothesis and thus denying the numerical nature of the basic capabilities of certain primates and young infants rescues the general cultural approach to mathematical cognition from foundational problems that plague mental representational accounts.

In joint work by G. Lakoff and R. Núñez (2000), and further developed by R. Núñez (2009), we similarly find a critical approach to the ANS-hypothesis. In particular, in Núñez' later work:

A major problem in most accounts of the concept of number is that scholars often introduce crucial elements of the explanans in the very explanandum. That is, they take number systems as pre-given and introduce them as a part of the explanatory proposal itself (Núñez 2008a). Gallistel et al. (2006, 247), for instance, speak of “mental magnitudes" referring to a "real number system in the brain," where the very real numbers are taken for granted, and put them "in the brain.” (Núñez 2009, 71)

To analyze the emergence of numerical (and more generally) mathematical conceptual abilities, Núñez in collaboration with G. Lakoff (2000), and building on the latter's theory of cognitive metaphor, developed the framework of Mathematical Idea Analysis. This approach to mathematical cognition lays great stress on the embodied origins of our conceptual apparatus. Prima facie, it might seem quite close in spirit to our approach, but on closer inspection it conserves elements of cognitivism which we think should be rejected. First, for all its emphasis on the embodied nature of our mental and cognitive apparatus, the approach remains firmly anchored in the tradition in which the brain is the central locus of cognition:

Ideas do not float abstractly in the world. Ideas can be created only by, and instantiated only, in brains. Particular ideas have to be generated by neural structures in brains, and in order for that to happen, exactly the right kind of neural processes must take place in the brain's neural circuitry. (Lakoff and Núñez 2000, 33)

Although in this, and other similar passages, Lakoff and Núñez avoid the term "mental representation," it is difficult not to interpret this passage as making the same concrete/abstract difference as Dehaene in terms of concrete or modal stimuli and their amodal abstract representation, and as endorsing, though couched in terms of "ideas," the claim that mental representations are the entities that drive cognition. This reading is reinforced by the general line of the 
metaphoric approach by which new and specialized (e.g., numerical) ideas and concepts are created by the application of a number of general cognitive mechanisms such as the metaphorizing capacity and the conceptual blending capacity (Lakoff and Núñez 2000, 52). Again, this is a set of capacities which apparently the mind/brain can perform in isolation and for which no socio-cultural background is needed. This is all the more surprising since metaphors and the capacity to construct metaphors are primarily linguistic and socio-cultural practices, which are the result of a long history of socio-cultural innovations. It would seem that the critique that Núñez levels against the ANS-hypothesis is equally applicable to the basic tenets of MIA: crucial elements of the explanans are introduced in the explanandum.

We thus find that an account that tries to approach mathematical cognition from a perspective that is critical of mainstream approaches still conserves traits of these traditional approaches, namely the reification of social and cultural processes into fundamental properties of the mind or brain (socio-cultural practices such as metaphorizing are transformed into fundamental cognitive mechanisms by Lakoff and Núñez). As such, this account remains to at least some extent vulnerable to the same problems as the more traditional approaches. The way to avoid these problems and to fully appreciate the constitutive role of socio-cultural practices in cognition is to go the way of REC.

\section{Acknowledgements}

The research of both authors is supported by the Research Foundation Flanders (FWO, projects Computation Reconsidered [G0B5312N] and Getting Real about Words and Numbers [G0C7315N]). Special thanks go to Farid Zahnoun, Victor Loughlin, Christian Tewes, and Duilio Garofoli for helpful comments on a previous version.

\section{Bibliography}

Butterworth, B. 1999. The Mathematical Brain. London: Macmillan.

Brannon, E. M., D. J. Merritt. 2011. Evolutionary Foundations of the Approximate Number System. In Space, Time and Number in the Brain. Searching for the Foundations of Mathematical Thought, ed. S. Dehaene and E. M. Brannon, 207-224. London: Academic Press.

Chemero, A. 2009. Radical Embodied Cognitive Science. Cambridge, MA: MIT Press.

Cosmides, L. and J. Tooby. 2013. Evolutionary Psychology: New Perspectives on Cognition and Motivation. Annual Review of Psychology 64: 201-229.

De Cruz, H. and J. de Smedt. 2010. The Innateness Hypothesis and Mathematical Concepts. Topoi 29: 3-13.

De Cruz, H., H. Neth, and D.Schlimm. 2010. The Cognitive Basis of Arithmetic. In PhiMSAMP. Philosophy of Mathematics: Sociological Aspects and Mathematical Practice, ed. B. Löwe and T. Müller, 59-106. London: College Publications. 
Dehaene, S. 2011. The Number Sense. How the Mind Creates Mathematics (Revised and Expanded Edition). Oxford: Oxford University Press.

Devlin, K. 2000. The Math Gene. How Mathematical Thinking Evolved and Why Numbers are Like Gossip. New York: Basic Books.

Flegg, G. 2002. Numbers. Their History and Meaning. New York: Dover Publications.

Gallistel, C. R. 1998. Symbolic Processes in the Brain. The Case of Insect Navigation. In An Invitation to Cognitive Science: Vol. 4: Methods, Models, and Conceptual Issues, ed. S. Sternberg and D. Scarborough, 1-51. Cambridge, MA: MIT Press.

Gallistel, C. R. 1993. A conceptual framework for the study of numerical estimation and arithmetic reasoning in animals. In The development of numerical abilities: Animal and buman models, ed. S. T. Boysen and E. J. Capaldi, 211-224. Hillsdale, NJ: Lawrence Erlbaum Associates.

Gallistel, C. R., R. Gelman, and S. Cordes. 2006. The Cultural and Evolutionary History of the Real Numbers. In Evolution and Culture, ed. S. C. Levinson and P. Jaisson, 247-274. Cambridge, MA: MIT Press.

Gibson, J. J. 1979. The Ecological Approach to Visual Perception. Boston, MA: Houghton Mifflin. Hurley, S. 1998. Consciousness in Action. London: Harvard University Press.

Hutto, D. D. and E. Myin. 2013. Radicalizing Enactivism. Basic Minds Without Content. Cambridge, MA: MIT Press.

Hutto, D. D. and E. Myin. Forthcoming. Going Radical. In Oxford Handbook of $4 E$ Cognition, ed. A. Newen, L. Bruin and S. Gallagher. Oxford: Oxford University Press.

Jordan, K. E. and E. M. Brannon. 2006. The Multisensory Representation of Number in Infancy. Proceedings of the National Academy of Sciences of the United States of America 103 (9): 3486-3489.

Jordan, K.E., E.M. Brannon, N. K. Logothetis, and A. A. Ghazanfar. 2005. Monkeys Match the Number of Voices They Hear to the Number of Faces They See. Current Biology 15: 1-5.

Jordan, K. E., E. L. MacLean, E. M. Brannon. 2008. Monkeys match and tally quantities across senses. Cognition 108: 617-625.

Lakoff, G. and R. E. Núñez. 2000. Where Mathematics Comes From. How the Embodied Mind Brings Mathematics Into Being. New York, NY: Basic Books.

Malafouris, L. 2010. The Brain-Artefact Interface (BAI): A Challenge For Archeology and Cultural Neuroscience. Social Cognitive and Affective Neurosciences 5: 264-273.

-. 2013. How Things Shape the Mind: A Theory of Material Engagement. Cambridge, MA: MIT Press.

Myin, E. and D. D. Hutto. 2015. REC: Just Radical Enough. Studies in Logic, Grammar and Rhetoric 41 (1): 61-71.

Núñez, R. E. 2009. Numbers and Arithmetic. Neither Hardwired Nor Out There. Biological Theory 4 (1): 68-83.

Overmann, K. 2016. The Role of Materiality in Numerical Cognition. Quaternary International 407: 1-10.

Sampson, E. E. 1981. Cognitive Psychology as Ideology. American Psychologist 36 (7): 730-743.

Tolman, E. C. 1948. Cognitive Maps in Rats and Men. Psychological Review55 (4): 189208. 



\title{
Beyond Dualism?
}

\section{The Implications of Evolutionary Theory for an Anthropological Determination of Human Being}

\author{
Christian Spabn
}

\begin{abstract}
This paper critically investigates the aim of post-Darwinian theories of embodiment to offer a non-dualistic theory of the 'natural' and 'mental' aspects of human cognition. Traditionally, the belief in genuine human agency (the ability to distance ourselves from natural instincts in the name of genuine moral and epistemic norms) has led to dualistic views of the relation between humans and nature that supposedly have to be overcome in post-Darwinian approaches. A short typology of options is sketched: pre-Darwinian Optimism is distinguished from Dualistic Naturalistic Pessimism. Against this background, Thompson's theory of embodied cognition is analyzed. Thompson's still unrivaled account puts the organism right 'in the gap' between the 'physical' and the 'mental realm'. Autopoiesis-theories offer, it is argued, the necessary categories to distinguish organic bodies from physical bodies, while at the same time (via the concepts of sense-making and organic agency) establishing a deep continuity and connection between 'life and mind'. Further, a brain-centrist view of 'disembodied cognition' can be rejected. However, three important questions remain. How exactly does organic interiority relate to awareness? How does organic and cultural sense-making relate to genuine epistemic and ethical normativity? What method and philosophical outlook should be adopted to clarify these non-empirical conceptual questions? As long as these three questions remain unanswered, the gap between mind and nature remains open for further investigations.
\end{abstract}

\section{Introduction: Are There Good Reasons for a "Bad Dualism" of Humans and Nature?}

Although Darwin's theory is a biological account of the origin and transformation of species, it has served repeatedly as a starting point for a "Darwinian world view": the expansion of "Darwinian ideas" from biology to human life and human culture as such. ${ }^{1}$

${ }^{1}$ I will use the broad term 'Darwinism' in the following to refer to any such attempt to apply Darwin's theory to the realm of culture without implying that such a 'Darwinism' is necessarily linked to Darwin's own views. Darwin only cautiously mentions possible consequences of his theory for the study of human culture in his Origin of Species (Darwin [1859] 1985, 458). He later elaborates his own views on the evolution of humans and culture in The Descent of Man (Darwin [1871] 1981). I use the term 'Darwinian evolution' for any theory of biological evolution that accepts non-directed mutations, competition under scarcity and inheritance (i. e., natural selection) as the most important factors in explaining the transmutation of species. 
Such a temptation to expand "Darwin's ideas" from biology to culture seems natural for at least three reasons: firstly, it can hardly be denied that humans belong to the realm of organisms and are at least partly determined by biological instincts. If we want to understand buman life, we must understand organic life as such, of which humans are only a part. And it is Darwin's theory that offers the framework for any modern interpretation of "life as such." Secondly, Darwin's theory provides a causal explanation of evolution in terms of natural selection. Such a theory offers, at least implicitly, a general structural explanation of events that unfold under conditions of scarcity, competition, and replication. As such, it seems that it could easily be extended to the realm of economic competition, or even to cultural history: companies may be viewed as competing against each other for customers and profit by modifying their products; cultures, groups and institutions may be said to compete for "survival and influence"; even ideas are thought to compete for attention and "replication" (see the theory of the evolution of 'memes' in Dawkins [(1976) 2006, 189-201], and see Blackmore [1999]). It is noteworthy that this kind of expansion is not necessarily based on a "biologistic view" of culture: the structural aspects of competition, variation and replication seem to give some aspects of Darwin's theory a more than biological universality (see Hösle and Illies 1998). Thus, especially within sociobiology, many insights from economics and game theory are integrated into an evolutionary view of animal and human behavior (Trivers 1971, 1972, Axelrod and Hamilton 1981, Axelrod 1984, Maynard Smith 1982, Wilson 1975, Dawkins [1976] 2006). Thirdly, Darwin offers an explanation of the origin of complexity and adaptation along the lines of "descent with modification." More complex and elaborate organic structures and behaviors are a result of a slow gradual evolution. Such a theory, it seems, stresses continuity over discontinuity and seems to be compatible with a reductive account of reality that explains "higher" or "more complex" phenomena by referring to more simple phenomena and causal mechanisms which are not goal-directed (see Hösle 2001). Such a reduction of complexity and the broad range of explanatory power of the mechanisms of evolution are certainly appealing for anyone who is searching for a unified worldview (see Wilson's emphasis on “consilience” in Wilson [1998]).

It is no wonder then, that ever since Darwin's publication in 1859 an expansion of Darwinian ideas into the study of culture has often been promoted. In this context it has become a standard view to claim that, after Darwin, we have to overcome the traditional Western dualisms of humans and animals, nature and culture. ${ }^{2}$ An extension of Darwinian ideas is thus coupled with a call for a naturalistic monism that should replace the age-old dualism of mind and nature. At

${ }^{2}$ Historically, at least four different phases and variations of this expansion can be distinguished (for a typological overview see Illies 2006, 27-43): (1) Early, more ideological and philosophical (and sometimes pseudo-)Darwinian interpretations of "culture as such": one thinks esp. of Herbert Spencer and Ernst Haeckel, critically see Bowler $(1988,2009)$. (2) Ethological explanations of human behavior (Tinbergen 1952, Lorenz 1963). (3) Sociobiology (Trivers 1971, 1972, Wilson 1975, Dawkins [1976] 2006) and (4) Evolutionary Psychology (Tooby and Cosmides 1992), for an overview see further Spahn (2010). 
the same time, such approaches to cultural studies and the humanities have triggered numerous controversial debates and counter-reactions. Thus, a wide range of different stances towards a "Darwinian worldview" can be distinguished reaching from a complete rejection of Darwin's theory or at least of its relevance for the humanities at one end of the spectrum, up to a biological reductionism that views humans as mere "survival machines" (for this term see Dawkins [1976]/2006, xxi, 19-20, 46-65) or human culture as a mere variation of animal behavior at the other end. ${ }^{3}$

Since it is, however, a tautology to say that anything in nature (understood here as the realm of spatio-temporal events) is a part of nature, one might ask why there is even a controversy when it comes to the application of Darwinian ideas to human culture. In other words, why is there one part of nature that has been, according to the Darwinian view, brought forth and shaped by nature, that finds it hard to accept itself as a mere part of nature? Why is this age-old dualism of man and nature - contrary to the aforementioned tautology - so appealing? ${ }^{4}$ Why was the idea of separating "mind" and "nature" that is so strongly challenged in the recent debate about embodiment ${ }^{5}$ so successful in the first place? It is not enough to point to historical religious or philosophical traditions that were allegedly able to evoke a feeling of dignity merely by denying that humans have a natural origin or are only a part of nature. The question is deeper than that. Why is the very idea of not being merely a part of nature so appealing for self-conscious organisms that are born, live and die in nature?

Regardless of any religious considerations, it seems that almost all traditional Western philosophical views since Socrates and Aristotle more or less accept that it is a defining part of our human nature to have something like a "second nature." We are able to control and distance ourselves from mere biological impulses and instincts. Culture, understood in this way as an act of self-distancing from the "merely natural realm" ("first nature"), seems to be a uniquely human feature. 6 Animals and plants, according to this view, aim at survival and replication, they "aim" at life. Humans search for meaning, higher goals or for an expression of their autonomy: they look for something to "live for." Life is a goal in the first case; it is a means to other goals in the second case. ${ }^{7}$ According to this view, the

3 Recently Thomas Nagel (2012) has attacked evolutionary biological reductionism from an atheistic perspective, while at the same time embracing some arguments from the Creationists.

4 Wolfgang Welsch critically analyses the history of dualistic "anthropical thinking” in modern Western philosophy (“anthropische Denkform”) and embraces an evolutionary perspective in order to overcome this human-centered dualism, see Welsch (2012).

5 See the challenges to the body-mind dualism in Thompson (2007, 221-242), and see in general Varela, Thompson, and Rosch (1991).

6 For the notion of a 'second nature,' see Aristotle, Nicomachian Ethics, 1152a30 ff. In connection with modern biological considerations see famously Gehlen ([1940] 2009, 338-348). For a forceful defense of the notion of rational freedom as the ability to take a self-distancing stance towards one's “first nature,” see recently McDowell (1996, 79-85, 1998, 193 f.).

7 Victor Frankl ([1940] 2006) puts Nietzsche's idea of a "reason to live and survive" at the center of his reflection: the why of surviving makes the how, and sometimes even the if, a secondary concern. 
freedom to distance ourselves from our natural impulses (just as well as from cultural prejudices) makes us search for values that give guidance and orientation to human life. We are measured then by the values we consciously choose to embody. One might therefore picture "mind" as a separated force operating on and hopefully sometimes shaping and controlling our "nature": a dualistic view of two substances or forces interacting and counteracting each other is therefore tempting. ${ }^{8}$ One benevolent interpretation of this traditional dualism is thus to say that it captures our intuition that practical reason and human agency are in their very nature "counter-factual" and normative and thereby different from a blind non-directed causal chain of events. ${ }^{9}$

Following this interpretation, the ultimate underlying reason to evoke a dualism of nature and mankind therefore seems rooted in two ideas: a) implicit and reasonable claims about the difference between "Ought" and "Is," between what is "ideal" and "real" and b) about the difference between agency and passive events, freedom and necessity. Both dualisms are related to our ethical self-understanding. Modern Western philosophy has on the one hand strongly emphasized the distinction between "facts" and "values." It is the basis of Hume's and Moore's insights into the naturalistic fallacy (and for Kant's differentiation between the quid juris and the quid factis question) (see Hume [1740] 1978, III.I., 1; Kant 1781, A84; Moore 1903), and it still underlies "ex negativo" the Logical Positivists' rejection of an ethics based on scientific empirical descriptions of reality. ${ }^{10}$ By contrast, evolutionary naturalism seems to insist upon an integration of mankind into our scientific picture of nature. But by emphasizing such monism the idea of non-empirical ethical objectivity and the idea of free human agency are threatened. ${ }^{11}$ Any attempt to integrate Darwinian ideas into our worldview will therefore depend on our view of the relation between normative ethics and descriptions of reality. ${ }^{12}$ Thus the question of how exactly to deal with a dualism of nature and mind understood in this way will likely remain controversial, at least so long as the ethical assumptions that implicitly underlie our picture of "nature" are not made explicit.

\footnotetext{
32-34).

8 A powerful and poetic sketch of the rise of dualism can be found in Jonas ([1973] 1997,

9 See McDowell (1998, 171, 179f.), for the necessary separation of the "logical space of reasons" from the "logical space of nature."

10 See for example Ayer's influential rejection of rationalism in ethics, Ayer ([1936] 1952, chap. VI). Questions about what is good or bad cannot simply be conflated with questions about what is factually real or not, because we can reasonably think of good things that are not real, and there are certainly real or "natural" things that we can reasonably call bad.

${ }_{11}$ Freedom might thus be an illusion of the observer, see Singer (2005) and Dennett (1991).

${ }^{12}$ For a systematic discussion of possible relations between ethics and evolutionism, see Kitcher (1993), and for a critique see Illies (2006, 172-186).
} 


\section{The Dualistic Playing Field before Embodiment: Pre-Darwinian Ethical Optimism and Darwinian Naturalistic Pessimism}

In comparison with standards of culture or normative reasoning, our view of "nature" is usually not neutral, so that we can now begin to see the outline of a broad matrix of implicit philosophical premises that underlie the debate about any reconciliation of a "Darwinian picture of nature" with our "ethical stance." In its most simple form, nature can be understood as either (I), in the more optimistic traditions, enabling human goodness and reliable objective knowledge about itself or at least as preparing the ground for such goodness and knowledge. Pre-Darwinian Optimistic Naturalism assumes a harmony between the true nature of humans and our "goodness" in ethics and our ability to find truth in theoretical contemplation. These days, such a view is often regarded as either a mere religious hope (if we abstract from the sinful part of our nature) or as a bygone pre-modern teleological metaphysical view. Or (II), as the pessimistic tradition sees it, the conception of nature has to be separated from ontological assumptions about "higher goods," "cultural values," or "objective truth claims." Nature can be understood as the neutral realm of brute facts at best, but at worst it is a reality that is opposed to our ethical ambitions and ideals, such that "in the real world" survival matters more than objective recognition of facts and values. Here, nature is taken to be the seat of our dark selfish desires, a realm of egoistic and brutal competition that needs to be overcome by culture and "self-domestication". Similarly, cognition is understood as a tool for survival, not for accurate representation of objectivity. This Naturalistic Pessimism does justice to the aforementioned difference between what is real on the one hand, and good or true on the other hand, even if this often implies an opposition between "reality" and "goodness" or "objectivity." Given this opposition of nature and human normativity, Naturalistic Pessimism involves two possible interpretations of the relation between (ethical and epistemological) norms and "nature."

(II a) One way to understand Naturalistic Pessimism is to see it as an attack on moral and epistemological realism. In ethics, from the perspective of a "school of suspicion," nobler traces of human nature might be considered to be "against our real nature," whereas in epistemology, cognition is more concerned with survival and thus might even distort reality rather than objectively represent it. ${ }^{13}$ Goodness or "human happiness" seem not to be included in the "plan of the universe," as Freud $(1930,58)$ famously put it. A "call for humility" (see Lorenz 1963, chap. 12), and a downgrading of our epistemological ambitions in the light of evolutionary theory (Maturana 1970, 1980), might thus be obvious consequences of Naturalistic Pessimism. Another - unfortunate - consequence is

13 Thus there are two opposing interpretations of Evolutionary Epistemology: On the one hand there is an optimistic version that argues that evolutionary success guarantees something like a reliability of cognitive patterns that otherwise might have not been selected (see esp. Popper). On the other hand there are those views that argue that viability (survival) and accurate representations do not have to coincide at all (see for example the constructivism of Maturana 1980). On both interpretations see Spahn (2011a). 
that one might proclaim biological "values" such as health and strength, survival, "racial purity," etc., as the only new "real" or "scientific" values, as Social Darwinism did. Here, we not only "face," but embrace the "pessimistic" picture and allow "nature," understood in this way, to be our new "realistic" guide in ethics. We give up "ethical illusions" in the name of a better understanding of empirical reality or even remodel our values (while ignoring the well-known philosophical arguments mentioned above against such a naturalistic fallacy) on that (new interpretation of) "reality."

(IIb) Conversely, and more traditionally, one might counter Naturalistic Pessimism by arguing that "cultural self-domestication" and conceptual scientific thinking must lead us away from nature and help us to overcome the more "brutal" instincts as well as the meso-cosmic distortions in our cognition (see Vollmer 1990, $161 \mathrm{ff}$.): the way to goodness and truth, if such a way there is, must be a way of culture and science, not of embracing "nature." The bigger the gap between animals and humans, the less "nature" we find in "culture," it seems, and the greater are the chances for goodness to prevail and for cognition to be trustworthy. Deconstructing the notion of 'nature' and emphasizing "freedom" and the "human-animal gap," is a tempting ethical and epistemological counter-reaction to Naturalistic Pessimism. Nevertheless, even this attempt to defend our normative cognitive abilities still implicitly accepts Naturalistic Pessimism insofar as it would be considered true that any close connection and strong continuity between human nature and culture could only lead to a humiliation or de-evaluation of mankind's cognitive normativity. I interpret such a reaction to the first version (IIa) of Naturalistic Pessimism as a mere variation of Naturalistic Pessimism, since it subscribes to the basic idea of an opposition between "nature" and "ethics" in the same way that Naturalistic Pessimism does. Also in this view, the idea of a possible reconciliation of the realm of nature and the realm of normativity remains doubtful.

(III) These two variations of Naturalistic Pessimism (simply put, either accepting a disenchanted picture of nature as a sober new guide to ethics and epistemology in order to overcome naïve claims, or conversely emphasizing the difference between man and nature in order to "rescue" our ethical and epistemological stance) are both based on juxtaposing "nature" and "norms." They spell out the aforementioned difference between values and facts by equating nature with "facts" and values with "culture" or with a conception of reason that goes beyond or even against nature. ${ }^{14}$ In both its versions, Naturalistic Pessimism, even if it subscribes to evolutionary ideas, remains deeply dualistic. Superficially and in its proud self-interpretation, it seems to be more modern than Cartesian dualism, because it claims to accept a monistic Darwinism. But it is profoundly traditional in its axiological dualism (Spahn and Tewes 2011, $169 \mathrm{ff}$.).

As with any simplistic binary opposition, we should of course not assume that the whole spectrum of reactions to a Darwinian world view is exhausted by these

${ }^{14}$ For a critical view of this urge to develop a "supernatural concept" of cognition in ethics and epistemology, see McDowell (1996, 83 f., 1998, 167). 
two versions of Naturalistic Pessimism. Rather they should be thought of as representing (however popular) polar ends of a spectrum. Against these implicit or explicit dualistic views, theories of embodiment claim to be beyond the gap and to offer a truly integral perspective that actually reconciles the "natural" and the "mental” aspects of human life.

\section{Hyper Intellectualism (and Hyper Physicalism) in Recent Philosophy and the Turn to Embodiment Theory}

Given this urge to overcome dualism in all its forms, in recent years more and more approaches in the philosophy of mind and in the cognitive sciences are proclaiming that in order to understand cognition, even in its normative aspects, we now have to focus much more on "the body" and its place in nature, and not just on the mind, let alone the brain. Buzzwords like "Enactivism," "Embodied Mind." 15 even the idea of an "extended mind"16 have become popular topics for discussions, books, and conferences. A common line of thought in these new approaches is a deep mistrust towards a "Cartesian" view of the mind. ${ }^{17}$

Tyler Burge, for example, has diagnosed a "byper intellectualism" in philosophical epistemology: ${ }^{18}$ the two "main schools" of recent epistemology focus strongly or even exclusively on human cognition in order to explain what it means to have knowledge of the world (Burge 2010, 14). To be more precise, these approaches emphasize only one particular aspect of human cognition: linguistic (sometimes even only linguistic-scientific) reference to the world has become the standard paradigm for cognition. Simply put, in order to have "true justified beliefs" about the world, higher conceptual abilities (i. e., having a language) are required. Animal cognition cannot be regarded as being knowledgeable in this sense, and sometimes it is even denied to be representational awareness at all, because it lacks the necessary linguistic requirements. ${ }^{19}$ According to this view, the senses and the body give us a mere input (sensations, but not representations), the mind or the brain with its conceptual abilities transforms this into experiences and knowledge. In this paradigm, cognition in the full sense of the world is the linguistic or conceptual computation of given sensual contents and this model of cognition focuses on the human brain.

Tyler Burge's work is of course only one very recent example of many modern approaches which claim that cognition is not starting from scratch with the development of the human brain or of sophisticated language, but that it is essen-

15 For both words, see Varela, Thompson, and Rosch (1991).

16 Andy Clark and David Chalmers' famous paper and many responses are included in Menary (2010); see also Rowlands (2010).

17 See the famous book title of Damasio (1994).

18 For a very short look at the program of an evolutionary epistemology in Tyler Burge, see Spahn (2012).

19 See McDowell's emphasis on the difference between animal thought and human thought in McDowell (1996, chap. III-VI), see also the debate in Perler and Wild (2005). 
tially based upon biological agency. Agency, according to Burge, "is grounded in functioning, coordinated behavior by the whole organism, issuing from the individual's central behavioral capacities, not purely from subsystems" (Burge 2010, 331). ${ }^{20}$ The "Enactive Approach" to cognition fostered by Varela, Thompson, and Rosch (1991), is yet another example: it claims that in order to truly understand even such a complex and seemingly autonomous phenomenon as human cognition one has to go all the way back to the logic of organic agency. Alva Noë stresses in a similar fashion the need to look at "action in perception" (Noë 2004) in order to truly understand cognition and the mind. Ruth Millikan advances a "teleo-semantic" view that wants to understand language and reference as functions of organisms: representation and cognition are to be understood from the perspective of biology (Millikan 1984, 2004). In these ways the organic body plays a key role in modern philosophy of cognition, replacing more intellectualistic views of cognition that merely focused on linguistic and computational views of intelligence (Thompson 2007, 3-15).

This widening of the perspective from the human mind or from human knowledge to its evolutionary preconditions is, however, not completely novel. Of course, our knowledge of animal cognition ${ }^{21}$ and of cognitive systems ${ }^{22}$ is nowadays much more detailed than before, and the recent paradigmatic changes that Noë, Thompson, and others describe are yet to be surpassed. But the very idea of tracing the steps of cognition "from the amoeba to Einstein" and trying to situate cognition within the organic body is itself an approach that already guides the exemplary works on evolutionary epistemology of Konrad Lorenz (1973) and Karl Popper (1972), and it is present in the approaches of Jean Piaget (1967) and Maurice Merleau-Ponty (1942), to name only a few. ${ }^{23}$ Contemporary thinking about the mind is therefore again and again inspired by "philosophy of biology." On the other hand there is a move to emphasize the difference between organic bodies and physical bodies, such that early ideas of a reductive physicalism should be overcome in favor of a theory of nature that understands the crucial difference between merely physical and biological categories. ${ }^{24}$ In this context it also seems that normativity is not a category of physics, but it is crucial in understanding organic entities.

In order to sketch these two main ideas (embodiment of the mind, difference between physical and organic bodies) of theories of embodiment I want to

20 Thus he claims that "perceptual representation that objectively represents the physical world is phylogenetically and developmentally the most primitive type of representation. I argue that human beings share representational mind, exercised in perception, with a breathtakingly wide range of animals. Representation of the physical world begins early in the phylogenetic elaboration of life" (Burge 2010, XI).

21 For a detailed conceptual analysis, see the excellent work of Joëlle Proust $(1997,1999)$.

22 Tyler Burge is inspired by the groundbreaking work on the visual system done by Marr (1982).

${ }^{23}$ For an overview of similarities and differences between "intellectual” and biologically inspired epistemology, see Spahn (2011a, 55-77).

24 Early analytic philosophy favored the language of physics as the fundamental language of science. For a critique, see Mayr $(1997,25)$. 
focus on a very recent work by Evan Thompson, one of the most prominent and extensive studies of the relation between mind and organisms (Thompson 2007). The strength of Thompson's work lies in drawing upon neurobiological and biological studies and diverse philosophical traditions, notably Husserl's phenomenological approach. In his book Mind in Life Thompson elaborates his own "philosophy of the organism," taking inspiration from such authors as Kant, Merleau-Ponty and Hans Jonas. What new anti-dualistic ideas does the school of embodiment offer for a theory of the relation of humans and nature, and thus of mind and world?

While traditionally the body-mind problem was spelled out as a problem of unifying "the physical" with "the mental," embodiment theories argue that the "organic body" lies in the gap between these two. ${ }^{25}$ It is neither a mere material object, animated by a "ghost in the machine" nor is cognition possible without a full organic body. Can such a claim of a deep connection between mind and life help us to overcome the above-sketched age-old dualism, and at the same time do justice to underlying intuitions about agency and normativity? In order to answer this question let us first reconstruct: a) what kind of body the organic body is according to Thompson (and thus according to Kant, Merleau-Ponty, and Jonas); secondly, b) how does Thompson argue for the view that not only the brain but perhaps the organic body as a whole is a precondition for cognition or mind?

\section{The Body is not merely a Body: Thompson's Philosophy of the Organism}

Thompson proclaims at the beginning of the book that he wants to argue for a close connection between mind and organic bodies: "Where there is life there is mind, and mind in its most articulated forms belongs to life" (Thompson 2007, IX).

In order to understand life and organisms, most approaches start by looking at the fundamental difference between organic and inorganic systems. ${ }^{26}$ It is the hallmark of modern approaches to focus on essentially new features of organisms without however postulating new "irreducible" substances or "life forces" (élan vital, nisus formativus, etc.). ${ }^{27}$ To put it briefly, in the old battle between mechanism and vitalism in biology, "mechanism" has won, ${ }^{28}$ but reductionism is nevertheless not an option.

Thompson and others don't subscribe to the view that an organism is merely a complex mechanical or material system. What differs between living and inor-

25 This point is also forcefully made by Jonas (1997).

${ }^{26}$ For a general overview of different philosophies of biology, see Spahn (2011b).

27 In German biology Blumenbach (1781) influentially postulated a power of self-formation, a "Bildungstrieb" in organisms, while Vitalists such as Henri Bergson or the Neo-Vitalist Hans Driesch proclaim an irreducible life-force.

${ }^{28}$ For a fair historical assessment, see Mayr (1997, 3-23). 
ganic systems is the realization of a new structure, a new type of "self-identity," not the existence of new substances or forces. Philosophically speaking, organisms realize a new way of sustaining identity over time. Thompson relies on the influential work of Varela and Maturana $(1980,1992)$ in labeling this new structure an auto-poeitic identity (Thompson 2007, chap. 5). ${ }^{29}$ While all forms of energy and matter are the same throughout nature, the organizational complexity changes. Organic identity is, simply put, defined by metabolism and morphogenesis: organic bodies sustain their "form" by constantly exchanging matter and energy with the environment in a self-regulated fashion: this exchange (or flow) of matter and energy is crucial for preserving an organism's identity. While a stone, for example, is considered to be the "same" object or body as long as its material composition does not change, organisms are essentially characterized by such exchanges, or else they "die" and that means they lose their specific organic identity. Philosophically speaking: they are the same precisely because they change: life has integrated change into its peculiar mode of identity.

But self-preservation through constant change is not enough to sufficiently characterize the living body; other emergent physical structures in nature are defined by realizing stability within material or energetic change, as Thompson points out. ${ }^{30}$ The crucial point is that life - in addition to being constant in and through change - is self-regulating and self-producing: it "creates" the parts of the systems that are needed for its viability (=self-preservation) and it must have the necessary organizational structures for active self-regulation. Thus, organisms are auto-poietic bodies (Thompson 2007, 97-122). While, for instance, a burning flame cannot control the influx of energy or material, nor react upon disturbances in the environment, nor produce parts of a system that would be helpful in regulating and balancing the energy flow, an organism has a certain range of flexible responses to changes in the environment and builds its own parts of this response system. This leads to one of the most obvious features of organisms: their inner "purposiveness," something that was already stressed by Aristotle and lies at the center of Kant and Hegel's philosophy of organisms (see Kant, 1790, A280-295). ${ }^{31}$ The parts of organic systems are tools for the preservation of life, they are functional or teleonomic; this is already visible in the Greek term 'organon.'

This reflexive purposeful structure can indeed be labeled a 'self' and, for Thompson, it constitutes the origin of "agency" (Thompson 2007, $167 \mathrm{ff}$., $201 \mathrm{ff}$.). The ability for self-regulated self-sustaining requires what Bertalanffy in his work on cybernetics has described as "negative feedback loops" (Bertalanffy,

29 Following Ludwig von Bertalanffy (1969) one speaks of an "open system in a steady state.” Hans Jonas and Hegel have very similar ways of making the same point, see Spahn (2011b, $37 \mathrm{ff}$.$) .$

30 On the difference between organic systems and physical dissipative structures, like waves and other examples, see Thompson (2007, 72-75).

31 Thompson (2007, 129-149) follows Kant and says: "I have claimed that the theory of autopoiesis offers a naturalized, biological account of Kant's notion of a natural purpose” (Thompson 2007, 140). 
Beier, and Laue 1977): the "set point" ("Sollwert") of the system for its viability must be represented within the system. It must be compared and equilibrated with the "actual values" ("Ist-Wert") of the environment such that the organism can produce compensating actions, if necessary, in order to sustain its metabolism. An example is hunger, where a certain internal chemical situation signals to the organism that there is an internal lack of available energy and this leads the organism to counteract the hunger by looking for food or taking other appropriate actions. Functionally speaking, this requires the representation of the given inner state of the organism and the outer state of the environment, plus the negative feedback regulation of "actions" or processes guided by these two values: the "Ought Value" (set points) and the "Is value" of the system must be coupled and balanced. It also requires that the organism is able to evaluate its environment. Thompson follows Merleau-Ponty in arguing that the inherent goal of the system to maintain autopoiesis makes the environment relevant. Chemical facts, for example, can now "count as" (be treated as) "food" or "poison" or, in more complex contexts, as "signals" for food and poison etc. The organic goal-directed interaction with the environment constitutes a semantic or proto-semantic relation between the organism and the world, a point that lies at the center of Millikan's approach to semantics (Thompson 2007, $75 \mathrm{ff.,} 147 \mathrm{ff}$.; Millikan 1984). Sense-making is thus crucial for organic agency (Thompson 2007, 152-159).

This "cybernetics" of organisms is ontologically speaking extremely fascinating, because, according to Thompson $(2007,72-75,128-165),{ }^{32}$ it underscores the radical structural difference between such a body and any other object or body in nature. Spelling out the implied philosophical ontology of such systems lies at the center of Hans Jonas' $(1966,1973)$ famous philosophy of life that has profoundly influenced Thompson's approach. Jonas says that in addition to the features we have already mentioned, such an organic system is characterized by "transcendence" and "needy freedom." It represents a very peculiar form of self-identity.

Self-identity, according to Jonas, is at first not a given in such a system: to sustain itself becomes a task for the organism. Stones or planets don't "care” for their existence, but life does: it thus has a goal, and we need to understand this goal in order to understand the functional design of organic systems. Thus Jonas and Thompson stress that "interests" and "desires" emerge for the first time with organisms in the world; these phenomena are lacking in the physical world. ${ }^{33}$

This means, secondly, that such a system is dependent on environmental conditions: it is a fragile entity, it is needy. ${ }^{34}$ At the same time - and this leads us to Jonas' third aspect, "transcendence" - such a system exhibits "something like”

32 Also Wandschneider $(2008,144-148)$ puts the "cybernetics" at the center of his philosophy of biology.

33 Jonas, following Heidegger, hereby generalizes Heideggerian themes about the existential qualities of Dasein. Not only humans have a profound “existential” relation to death: all organisms are mortal.

34 Jonas explores this "needy freedom" of organisms in his philosophy of ecology: caring and values come into existence in the world with the existence of life. Thus, Jonas claims, organisms are a "Sollen," an "Ought" not purely an existing "Is" ("Sein”). 
freedom ${ }^{35}$ and reaches already in its very constitution beyond "the here and now." How can this be so? Jonas also argues that an organic system is strictly speaking not identical with its material conditions at a given time (Jonas 1973, $157 \mathrm{ff}$.). On the contrary, we have said that it will and must exchange its material. Thus, Jonas argues, it is "free from," or not dependent on, this particular given material. It cannot be "identified" with it. Almost any material substance (and energy at any given moment) can be replaced and exchanged. But, of course, while the system does not depend in its identity on the specific material or energy that it is made out of it is however dependent on material and energy as such. The identity of such a system - and this is the crucial point - must therefore be characterized as "immanent-transcendent." Jonas and Thompson argue that the organic body is characterized by "Innerlichkeit" or "Interiority," a point that has also been forcefully made by Merleau-Ponty (1942). ${ }^{36}$ Grasping this functional organic unity that continues over time is more important in understanding organisms than mere physico-chemical analysis. This inner self-regulating organization is therefore neither "outside" of the body, nor is it to be identified with the given material-energetical moment. The unity thus transcends any given material "snapshot" of the organism. ${ }^{37}$ The body is not a thing, or in Merleau-Ponty's words, the Leib is not a body (Merleau-Ponty 1942, chap. III). Jonas even speaks of an "ontological revolution" in nature: The "form" overpowers "matter"; or in the more modern language that Thompson uses: emergent systemic properties and holistic causalities are the key to understand such bodies (Thompson 2007, 60-65). Therefore, while you can "show" and "point at" the material parts of a living object, you cannot show or point at "life" itself.

Let us compare this structure to some clearly very similar ideas in the theory of the self-identity of the human mind. The "I"/ "Ego" or the character of a person is in a very similar fashion something "general," it is transcendent-immanent. Any given "Ego" is neither identical with a given thought at any one time, nor could it be reconstructed as an "empty" entity "behind" or "outside of" all thinking. The I has thoughts, but without thoughts it would not exist. Nevertheless, it is not identical with any given thought; it perpetuates itself and sustains its identity through and only through its different thoughts. The similarity between this dialectical ontological identity of the "Ego" and the aforementioned complex self-identity of the organism is very striking (see Spahn 2007, 2011a). Furthermore, we have seen that a "semantic relation" of relevance

${ }^{35}$ Organismus und Freibeit is the German title that Jonas has chosen for his book The phenomenon of life.

36 Thompson discusses Merleau-Ponty's ideas in his (2007, chap. 4).

${ }^{37}$ Dieter Wandschneider $(2008,14 \mathrm{f}$.), makes a similar claim for inorganic bodies: if we take the laws of physics to be more than just mental re-descriptions of reality we are forced to understand physical reality from the vantage point of "deterministic dispositions": from this perspective the mechanical body would also be in essence more than and in its identity different from any given "here and now" or state that it is in. It has a given law-like potential to act in certain ways under certain possible conditions. These possibilities determine what kind of object it is, even if all these potential states can never be actualized at any given moment in time or space. 
is already implied by the organic interaction with its environment. Meaning, relevance, and information are thus not only mental concepts, but may already be "biological categories." 38 Recently, there have been proposals to reconstruct the human ability to make non-biological value judgments from an enactive perspective: value systems are not fixed by biological needs alone and can be employed to re-create social values and shared norms via the "mutual attunement of individual sense-making” (Di Paolo, Rohde, and De Jaegher 2010, for the theory of participatory sense-making see De Jaegher and Di Paolo 2008).

Thompson claims that the notorious mind-body problem can therefore only be solved if we stop trying to reconcile the huge gap between our conception of the "physical" (understood as "material") and "the mental" and realize that "the organic" lies in the gap between them. The organic body is already characterized by "interiority," it is a complex entity, not merely a physical thing (Thompson 2007, $221 \mathrm{f}$.). It therefore seems tempting to argue that mind and life are profoundly linked to each other, because they share a very similar "mode of self-identity," which at the same time is very different from that of inorganic bodies.

Thus it is not surprising that Thompson has recently used his approach to attack what he calls (with Ned Block) the "standard view of cognition," namely the claim that consciousness is "a product (only) of the brain." Not only the brain is connected to the mind, the whole organic body is.

\section{Thinking is not merely in the Brain: Why the Brain cannot be in a Vat}

To conclude this discussion of the emphasis on "the body" in enactive approaches, let's take a short look at Thompson's and Cosmelli's (2013) view that the whole body should be taken into account when looking for the necessary correlates of mind or consciousness (often labeled NCC [neuronal correlates of consciousness]). Ned Block has criticized Noë's view and claims that the "minimal constitutive supervenience basis" for consciousness is the brain, not the whole body. Block wants to defend the claim that he calls the "orthodox view" "that nothing outside of the brain is part of" (Block 2005, 264) the metaphysically constitutive basis for consciousness. Of course, a brain can only survive if the body survives, but in order to explain consciousness it would be sufficient to look at the changes in the brain.

Thompson and Cosmelli argue that this "orthodox view" is misguided and they present two main counterarguments: a) the brain cannot - not even in a thought experiment - be "uncoupled" from the body; b) we must distinguish (the change in) states of consciousness that might have their basis in changes in brain states from the fact that there is "creature consciousness" in the first place.

38 Again, this is the main point of Millikan's (1987) view. 
Regarding argument a) Thompson and Cosmelli use the famous "brain in a vat" argument to explain their first objection. If the brain is sufficient to functionally explain consciousness then it could hypothetically be isolated, put into a vat, and connected to a machine. Such a brain would, according to the thought experiment, be capable of the same conscious states (even though they are now not about a real world mediated through a real body but coming from a computer) as a "normal brain." Thompson and Cosmelli, however, deny this. While it looks as if the brain could be "sustained and controlled" from a machine outside, they point out that such a "vat" must be something that functionally closely resembles a real organic body. The first reason for this is that the "life" of the brain must be sustained. Therefore, the connected system must be able to sustain the brain's metabolism (exchange of neuronal fluids, a circulatory system), and must pump blood into the system (or an equivalent fluid) (Thompson and Cosmelli 2013, 169-172).$^{39}$ For both functions, it would have to be coupled with the brain's metabolism and regulatory systems in such a way that it in fact can no longer be said to merely externally control the brain but must also respond to the brains' needs, and therefore be influenced by the brains' demands: "thinking carefully about this experiment will reveal that the brain and the body are so deeply entangled, structurally and dynamically that they are explanatorily inseparable" $(2013,168)$. Regulatory loops are needed that make it impossible to explain or understand the brain without these other systems of which it is now an integral part. "Hence the external control perspective is not generally valid. Instead, our life-sustaining system and the brain must be seen as reciprocally coupled and mutually regulating systems” (ibid., 170).

Once we take the next step in the thought experiment this coupling becomes even more obvious. The brain must not only survive, it must receive "fake" environmental stimulations. The authors argue that the complexity of neuronal cognition requires, for example, a perfect synchrony with the brain's exploratory motor efferent signals, and these systems must be controlled by the brains' sensorimotor-loops (ibid., 172). They claim that due to the almost infinite computational complexity no virtual but only an actual peripheral sensorimotor system could produce or mimic the necessary input. Since the brain's computation is functionally deeply entrenched in life-regulation and bodily homeostasis it cannot be functionally decoupled from the brain. Thus, they conclude, from an explanatory perspective, the whole complexity of the body must be taken into account in order to understand brain functions.

Regarding argument b), this is directed against the "band pass" argument. ${ }^{40}$ While one might admit that the existence of the brain is deeply connected to bodily regulations one might argue that most of this regulation is subconscious. Therefore, to explain the changes in the stream of a "conscious consciousness,"

39 After sketching some of the requirements for the "vat" machine, the authors conclude: "The life-sustaining system is starting to look less like a vat and more like a surrogate living body or organism” $(2013,170)$.

40 Thompson and Cosmelli argue against Andy Clark (2009). 
or to explain the changes in aware states of mind one might argue like this: for the fully aware or awake stream of consciousness most computational activities of the brains are "shielded off," and only changes in the brain (or even only in the higher brain) count. Thus, only looking at the brain is sufficient for awake creature consciousness. Thompson and Cosmelli argue that this is a fallacy. Of course, it is true (and perhaps sufficient) to believe that changes in awake states of consciousness are only caused by or correlated to changes "in the brain," not in the body. But it would be a mistake to conclude from this that "conscionsness as such" is only linked to the brain. The argument of a change in a conscious state that correlates with a change in the brain already presupposes that the creature has consciousness (ibid., 177). Given consciousness, it might suffice to look at a change in the brain for a change in the content of a given awake state of mind. But if the first argument is correct, then the emergence of consciousness as such would still depend on the brain and the body.

\section{Are we Beyond Dualism yet?}

Thompson's approach is, as we have seen, one of the most prominent and comprehensive theories of embodied cognition. His theory profits from its combination of conceptual philosophical clarity and an incredible richness of empirical detail. The careful rejection of "brain-centrism” and, even more so, Thompson's rich and detailed analysis of organic agency and organic identity are unrivalled. They are more than just gestures in the direction of post-Cartesian approaches; they can and should be considered as groundbreaking outlines of an already almost complete account of what a post-dualistic theory of mind and body must look like. The close relation that Thompson thus emphasizes between body and mind is more than helpful in overcoming a juxtaposition of nature and mind that puts consciousness outside of nature and looks at nature as a realm that is alien or even "hostile" to the mind (see also Thompson 2015, 105). In this respect his work goes refreshingly beyond the simple dualism and oppositions of Naturalistic Pessimism. In particular his emphasis on "emergence" allows him to evade those above-sketched oppositions that occur if we either "reduce" mind to a non-normative and non-mental conception of nature or "rescue it" from such a conception by stressing a "dualistic" distance between mind and nature. Carefully sketching the essential categorical differences within ontological continuity in the way Thompson does - such as separating the organic body from physical bodies with both conceptual clarity and depth of empirical knowledge - is one of the greatest achievements of embodiment theory so far. Nevertheless, while the emphasis on continuity, without denying novelty, is a big step beyond dualistic Naturalistic Pessimism, at least three questions concerning the general philosophical implications of embodiment theories remain.

1) Is a focus on the "special ontological structure" of organisms and their difference from physical bodies sufficient to fully bridge the gap in the bodymind problem? How exactly does the sketched organic "interiority" relate to 
conscious awareness? While the first one can be described from "the third person perspective" and is still a feature of "things in the world" that we can point to (even if we can't point to it directly we can see organisms), the "interiority of consciousness" is, it seems, of a very different nature: we only know it from the "first person perspective." Are we allowed to conclude that we are speaking about the same thing when we refer to the interiority of our mental life and the interiority of organisms? Of course one might argue, as Thompson does, that the classical dualistic "zombie-argument" is just circular. It claims that we could imagine an organic body like ours but without a mind, while in fact for some embodiment theories we are just faced with two sides of the same coin (Thompson $2007,230 \mathrm{ff}$.). I am tempted to agree with this claim: if we could perfectly reconstruct a copy of my body, including my brain exactly as it is, this "body" would indeed (given the laws of this universe) probably have consciousness. But to merely claim this identity or strict correlation (even if only for this world) is just as much to beg the difficult question. Independent arguments are necessary to explain why and if such a connection between bodily interiority and awareness necessarily exists and if we are thereby allowed to identify these two concepts or not.

The fundamental problem remains that there is not yet (and may never be) an account that explains or fully re-describes "awareness" in non-awareness-related vocabulary. If we understand the inner ontological structure of organic bodies according to embodiment theories we are surely beyond a simple dualism of opposing "physical objects" and the "mental" realm, but it seems that there is more conceptual work to be done in order to "bridge the gap." 41 Thompson, in his newest book, agrees partly with this diagnosis. He also insists - rightly so - that consciousness can neither be reduced to nor re-described in non-mental vocabulary and stresses what he calls the epistemological "primacy of direct experience": "Consciousness itself has not been and cannot be observed through the scientific method, because the scientific method gives us no direct and independent access to consciousness itself. So the scientific method cannot have the final say on matters concerning consciousness... When we use the scientific method to investigate consciousness, we're always necessarily using and relying on consciousness itself" (Thompson 2015, 97-98). Rather than trying to re-describe mental events in non-mental terms, it might be much more advisable to reconsider our notions of nature and of matter, an option that Thompson also favors:

$[\mathrm{M}] \mathrm{y}$ viewpoint isn't a materialist one, for two reasons. First, consciousness has a cognitive primacy that materialism fails to see. There's no way to step outside consciousness and measure it against something else. Science always moves within the field of what consciousness reveals; it can enlarge this field and open up new vistas, but it can

${ }^{41}$ One of the most fascinating and comprehensive books on awareness from the school of enactivism so far is the brilliant new book of Thompson (2015), even if I still tend to think that a compulsory argument for either an "identification" of body and mind or for an ultimately satisfying view that balances the two without giving primacy to either of them $(2015,100 \mathrm{ff}$.) is missing. 
never get beyond the horizon set by consciousness. Second, since consciousness has this kind of primacy, it makes no sense to try to reductively explain consciousness in terms of something that's conceived to be essentially nonexperiential, like fundamental physical phenomena. Rather, understanding how consciousness is a natural phenomenon is going to require rethinking our scientific concepts of nature and physical being. (Thompson 2015, XXXV; see also $103 \mathrm{ff}$.)

It remains open how such a commitment to a "non-reductionism" and to emergentism can avoid varieties of dualism or be integrated into a naturalistic framework. In other words, how can we combine the necessary epistemological primacy of consciousness with its ontological aposteriority? ${ }^{42}$

2) Further, organic agency clearly requires "sense-making” as we have seen. Relevance, evaluations, and meaning - and in this sense normativity - begin with and are deeply connected to organic agency. If however one embraces traditional notions of normative concepts in ethics and epistemology then Tyler Burge is right to point out that it is "a root missmatch" to equate fitting cognition for survival with possible objective truth. ${ }^{43}$ One might add: it is also a mismatch to equate evaluations based on organic needs with the question of what is objectively good or ethically decent. There is an element in the traditional objectivist understanding of "the Truth" and "the Good" that either has to be given up when we embrace naturalistic versions of enactivism, ${ }^{44}$ or, if we stick to a more traditional notion of truth in ethics and epistemology, we are forced to move beyond identifying viability and organic well-being with objectivity or truth in epistemology and ethics. Thus, the gap between what is factual and causal, and what is "genuinely rational" and "normative" is, it seems, even more difficult to bridge than the one between organic interiority and awareness. ${ }^{45}$ Strong epis-

42 One is reminded of Schopenhauer's two basic (and I think contradictory) claims: if consciousness is a "product of the brain (or Will)" then every thought or fantasy of the mind seems just to be a construction or an illusion of "the Will” or "the brain": the brain is "real," the World (of the mind, as we see it) is a "Representation," or a "construction." But conversely, even the idea that the mind depends on the brain is part of how the world appears to us in our mind and thus can be understood only from within the perspective of the mind. I think that both views, which Thompson also wants to balance, do not easily form a balanced "convergent" circle or support each other in the way Thompson implies with his Buddhist poetic image of the relation of "name-and-form" as two sheaves of reeds leaning against each other (2015, 102; Thompson goes on to say that embodiment and consciousness are "not one, not two"). One might argue contrarily that epistemic priority of consciousness and ontological dependency or aposteriority are not views that mutually "prop each other up," but rather point to the opposing worldviews of materialism or idealism.

43 Burge (2010, $301 \mathrm{f}$.) criticizes Millikan's approach because he thinks that the validity constraints of higher cognitions cannot be reduced to the biological evaluation of the environment according to the goal of survival. What it means to represent the world correctly, Burge argues, is categorically different from representing the world merely in such a way that an organism might successfully survive.

44 See esp. Maturana's constructivism in (1970, 1980). Note the sketched version of Naturalistic Pessimism above.

${ }^{45}$ The idea that we need to find a place in naturalism for the fact that we could be motivated by "normative reasons" in ethics and epistemology without denying that reasons are "genuine," and thus different from "mere causations," lies at the center of McDowell's conception of a “second nature” (1996, 1998). 
temic realists like to define truth as being independent of, and not constituted by, the contingent existence or mental makeup of organic agents, and moral realists like to define values of objective validity independently of contingent acts of agreement. But with such questions we clearly leave the philosophy of biology behind and enter deep into epistemology and (meta-)ethics.

3) Both these questions concern those ideas that gave rise to a dualistic world view in the first place and that were mentioned in the "benevolent" interpretation of dualism above: dualism is based upon the intuition that normativity and awareness are fundamental features of reality that can't fully be re-described and explained (away) by non-normative or non-mental concepts (in more detail, see Spahn and Tewes 2011). If we want to answer these two questions then we obviously have to ask finally what method or viewpoint allows us to even discuss, let alone solve these problems? What is a good and fruitful methodological starting point to come to terms with these two questions? Are these empirical questions, questions about linguistic conventions, or genuine philosophical-conceptual questions? Thompson emphasizes the necessity of a dialogue between Eastern and Western traditions of thought and critically engages, in an inspiring way, in an exchange between neuroscience and Buddhism. ${ }^{46}$ Thompson's starting point here seems to be that both science and Buddhism are open to critical discussion and are both based upon taking seriously inner and outer experiences and observations (Thompson 2015, XXV). Nevertheless, he also points to the fact that certain fundamental questions remain "metaphysical" questions; they can't be solved by observation alone but rather concern the right interpretation of experiences. ${ }^{47}$ It seems that the modern Western preference for (scientific or methodical) "experiences" and "observations" over conceptual clarifications, even if we include inner experience and the rich tradition of Buddhist interpretations of awareness, might not be sufficient to make important steps forward in answering the first two questions. This preference for observations is surely justified as a necessary presupposition of all scientific investigations, but it can't itself be a necessary presupposition of all philosophical argumentation and discussions. The

46 The exchange between neuroscience and the Dalai Lama was initiated by Francisco Varela. For the idea of a "contemplative neuroscience," see Thompson (2015, 70-74).

47 Thompson rightly rejects the idea that neuroscience could "prove" the identity of the mind and the brain: "But neuroscience itself doesn't demonstrate this identity: rather the identity is a metaphysical interpretation of what neuroscience does show, namely, the contingency or dependence of certain kinds of mental events on certain kinds of neuronal events" (Thompson 2015, 101). Conversely, the Buddhist idea of the existence of a mind without a brain (or that the "clear mind state" is not contingent on the brain) is also a "metaphysical" thesis, and can't be solved by (internal) observations, see Thompson $(2015,90)$. Thompson further argues (I believe rightly so) that external scientific observational evidence for such an independence of mind-states from matter/brains has not yet been found (see for example his excellent chapter on near-death experiences, Thompson [2015, 299-314]). But the metaphysical question whether mind as such (not human states of cognition that might always rely on the body) is material or not, cannot, I think, by definition be solved by any inner or outer observations, since we can only observe bodies, or have inner experience as long as we are alive and have a body. Idealism, for example, can't be grounded upon, but also not be refuted by observations. It is a metaphysical view based upon metaphysical arguments. 
fact that methodological observations lead on average to reliable results can't, for example, be itself based on methodological observations, once we fully understand the philosophical importance of the problem of induction. Questions of how reliably science works (let's call it epistemology of the first order) are genuinely different from questions of how such a philosophical epistemology itself (epistemology of the second order) works. With regard to the first question we can be empiricists, but I am sure that this is impossible for the second question. It is a fact that methodological observations, given the right conceptual framework, lead on average to reliable results, but we can only argue for this and similar principles of science itself in a non-empirical, that is genuinely philosophical way. ${ }^{48}$

This last point is connected to the fact that many philosophical debates about embodiment still focus on a certain very traditional understanding of consciousness that belongs to the recent naturalistic-empirical tradition in philosophy, namely the claim that consciousness is mainly understood as the ability to be "subjectively aware" of something material or physical in the environment: the paradigm of cognition still seems to be the perception of external objects and their classification and interpretation etc. In keeping with the phenomenological tradition, Thompson adds to this the "inner sense" the awareness of subjective states of being aware of something internal or external. However, in many debates there is no focus on the ontological status of qualia themselves - that can't be put "into nature" like other objects, forces or energies. Furthermore, the cognition of philosophical, epistemological or ethical states of affairs or facts, if there are such facts or states of affairs, seems notoriously difficult to integrate into the limited framework of naturalistic philosophy or any epistemology that counts observation as the only path to verification and physical and cultural reality as the only possible conceptions of reality.

Again, given reductive naturalism, we can cast doubt on any philosophies and worldviews that postulate non-empirical logical facts or state of affairs in addition to the physical objects of science or the inner experiences of humans that sociology, psychology or descriptive phenomenology study. But it is also pos-

${ }^{48}$ See especially Wandschneider $(1986,1998)$. Further, the problem that there is something external to all observations itself (the problem of the "outer world") can clearly not be solved by observation without committing a circular fallacy, but that does not mean that it can't be solved at all. One might argue that there is a logical (but certainly not an empirical) self-contradiction in solipsism or radical constructivism that I can only allude to here: if we were to believe that all acts of consciousness are just "subjective" or have no other content independent of these acts themselves then the act by which this epistemological "insight" itself is grasped would be void of meaning. Different versions of radical skepticism lead to different versions of self-contradictions. Therefore, arguments from self-contradiction point to an interesting transcendental-conceptual method of philosophical investigations. In these cases we know that something is a logical fact not because of experience or because of mere verbal conventions or tautological definitions, but because our attempts to negate certain insights presuppose and rely upon the very same ideas or concepts that we want to deny. For the difference between different versions of arguments from self-contradiction, see Illies (2003, 30-49) and Hösle (1990, 163 f.). For the program of a transcendental-conceptual Logic ("ideale Logik" or "fundamentale Logik" in Braßel's and Wandschneider's terminology) that includes semantic investigations and thus goes beyond most analytical approaches to logic, see Wandschneider (1996) and esp. Braßel (2005). 
sible to turn the argument around: given a commitment to and arguments for the possibility of truth in epistemology and ethics and for the reality of qualia as facts in the world, we might reject such naturalistic ontologies and epistemologies as obviously incomplete. We can think of logical or mathematical proofs being sound, even if no one has discovered them yet, or of possible state of affairs being valuable even if factually nobody agrees. Can we, in a similar way, count as "real" the fact that there is something that empirical objects would feel like, would look like, would taste like, etc. for a given kind of mind, independent of the actual existence of a contingent given act of such a perception at a certain moment in the history of the universe?

We surely ascribe such a reality to the "outer world," and don't think that its existence depends upon our perception or recognition, but for certain reasons most contemporary philosophers hesitate to ascribe such objectivity to qualia as such or to their non-empirical content (especially, as we said, when it comes to ethical or logical facts). In this way we are, polemically speaking, reducing our theory of the mind to a theory of acts of being aware of spatio-temporal things or of our own feelings unfolding at a certain place and time. ${ }^{49}$ Against

49 It is certainly possible, against radical subjective idealism, to insist upon the fact that the world may exist without my act of recognizing it, while it seems tautological that "states of the mind" could surely not be independent of the mind, our mind or some mind. There can be, so to speak, no mind-independent reality of mind, only of "things." I think this argument is influential, but it is based upon a confusion of what the term 'mind-independent' should denote: if we would truly embrace it in all its glory we would necessarily end up in solipsism. I cannot discuss my objection here but only hint at it: according to the realist I do not really "create" the world by perceiving it (rather, I create contingent perceptions of a given object by opening my eyes and looking, etc., at it). In a similar way, one might argue that I do not really "create" the (inner content of the) sensation of coffee or colors for example; I only create perceptions of such "qualia" by certain acts: the world of the mind would then, similarly to the "external world," not be my or our creation, but a fact in the universe that can or cannot be perceived. When we refer to qualia, speak about them, etc., we try to re-describe experiencing their given objective structure. In this view this structure or this-ness is a fact in the universe. It can be actualized in my or your mind but it is not therefore a "dream," an "illusion," a creation, or a "fiction." If this were so, then also the fact that there is a world at all outside of my (or any) perception would have to be a dream, a fiction, etc. One might be tempted to say that we should only reify things of the outer sense, but never of the "inner sense" and one might rightly ask what could be an equivalent to empirical perception when it comes to "free floating thinking or even imagination." I think this preference for an "empirical conception of reality" is a widespread though not really convincing move: objectivity of thought cannot without self-contradiction reasonably be limited to possible intuitions in space and time and to empirical verification. Our "feeling" of "reality" might be linked to senso-motoric givenness and to our possibility of placing things in our intuition into space and time, but that is not the only reasonable conception of objectivity or reality. It is more plausible to think that the distinction between "real" and "not real" cuts through both the inner and the outer sense. We know of "external observations" that turn out to be hallucinations or illusions: not all things perceived in the outer sense are "real." But also not all things that can only be grasped by the inner sense are therefore "unreal." We can have dreams of unicorns or witchcraft, but we can also discover logical truths that are not empirical in nature, and whose validity is not dependent on my acts of cognitions or on mere conventions of symbols. Of course the arguments for and against such an objective idealistic ontology can't be discussed in this paper. For a recent similar and forceful view in connection with the bodymind problem, see Tewes (2015). 
Husserl, it seems, we are merging noeses and noemata in all those cases where we can't point to an empirical or psychological (or "cultural”) "object" or "state of affairs." Against this naturalistic view more complex ontologies - such as that of Frege, or even more so those philosophies in the tradition of objective Idealism (see recently Nagel 2012,17) - have always maintained that logical and ethical facts on the one hand and "awareness" and qualia of the mind on the other hand, are integral parts of reality as such that can neither be identified with or reduced to human mental acts nor with or to natural facts. They may therefore constitute a part of reality in its own right. Such a worldview might seem obsolete or even outrageous from the perspective of the modern Western naturalistic framework. A theory of mind or logical reality that goes beyond contingent acts of human cognition is, however, on the one hand still prominent in the Eastern traditions with which Thompson engages, but also formed the core of the idealistic tradition of Western philosophy before its turn to naturalism (see esp. the essays in Hösle 1996). Even if one rejects with Thompson $(2015,105)$ Strawson's recent attempts to defend a panpsychism, and also rejects an idealism that gives an ontological priority to the mind as alternatives to a naturalism or an emergentistic view, the search for a philosophically convincing ontology remains open.

\section{Bibliography}

Aristotle (1915) 1966. Ethica Nichomachea, in: The Works of Aristotle. Translated into English under the Editorship of Sir David Ross, Volume IX, Oxford: Oxford University Press 1966 [1915].

Axelrod, R. 1984. The Evolution of Cooperation. New York: Basic Books.

Axelrod, R. and W. D. Hamilton. 1981. The Evolution of Cooperation. Science 211: 13901396.

Ayer, A. J. (1936) 1952. Language, Truth, and Logic. New York: Dover Publications.

Bertalanffy, L.v. 1969. General System Theory; Foundations, Development, Applications. New York: G. Braziller.

Bertalanffy, L.v., W. Beier, and R. Laue. 1977. Biophysik des Fliessgleichgewichts. Braunschweig: Vieweg.

Blackmore, S. J. 1999. The Meme Machine. Oxford; New York: Oxford University Press.

Block, N.2005. Review of Alva Noë, Action in Perception. Journal of Philosophy 102: 259-279.

Blumenbach, J. F. 1781. Über den Bildungstrieb und das Zeugungsgeschäfte. Göttingen: Johann Christian Dieterich.

Bowler, P. J. 1988. The Non-Darwinian Revolution: Reinterpreting a Historical Myth. Baltimore: The John Hopkins University Press.

-. 2009. Revisiting the Eclipse of Darwinism. Journal of the History of Biology 38: 19-32.

Braßel, B. 2005. Das Programm der idealen Logik. Würzburg: Königshausen \& Neumann.

Burge, T. 2010. Origins of Objectivity. Oxford: Oxford University Press.

Clark, A. 2009. Spreading the Joy? Why the Machinery of Consciousness is (probably) still in the Head. Mind 118: 963-93.

Damasio, A. R. 1994. Descartes' Error: Emotion, Reason, and the Human Brain. New York: Putnam. 
Darwin, C. (1859) 1985. The Origin of Species: by Means of Natural Selection or the Preservation of Favored Races in the Struggle for Life. Harmondsworth, Middlesex: Penguin Books.

-. (1871) 1981. The Descent of Man, and Selection in Relation to Sex. Princeton, NJ: Princeton University Press.

Dawkins, R. (1976) 2006. The Selfish Gene, 30th Anniversary Edition. Oxford: Oxford University Press.

De Jaegher, H. and E. A. Di Paolo. 2008. Making Sense in Participation: An Enactive Approach to Social Cognition. In Enacting Intersubjectivity: A Cognitive and Social Perspective to the Study of Interactions, ed. F. Morganti, A. Carassa, and G. Riva, 33-47. Amsterdam: IOS Press.

Dennett, D. C. 1991. Consciousness Explained. Boston: Little, Brown and Co.

Di Paolo, E., M. Rohde, and H. De Jaegher. 2010. Horizons for the Enactive Mind: Values, Social Interaction, and Play. In Enaction: Towards a New Paradigm for Cognitive Science, ed. J. Stewart, O. Gapenne, and E. A. Di Paolo, 33-87. Cambridge, MA: MIT Press.

Frankl, V. E. (1940) 2006. Man's Search for Meaning. Boston: Beacon Press.

Freud, S. (1930) 1953-1974. Civilisation and its Discontent, in: The Standard Edition of the Complete Psychological Works of Siegmund Freud, edited and translated by James Strachey, Volume 21, 57-145, London: Hogarth Press 1953-1974.

Gehlen, A. (1940) 2009. Der Mensch. Seine Natur und seine Stellung in der Welt. Wiebelsheim: AULA.

Hösle, V. 1990. Die Krise der Gegenwart und die Verantwortung der Philosophie: Transzendentalpragmatik, Letztbegründung, Ethik. München: C. H. Beck.

-. 1996. Philosophiegeschichte und objektiver Idealismus. München: C. H. Beck.

-. 2001. Platonismus und Darwinismus. Freiburger Institut für Paläowissenschaftliche Studien (F.I.P.S.), Kleine Schriftenreihe 6. Freiburg: Verlag Wissenschaft und Öffentlichkeit.

Hösle, V. and C. Illies. 1998. Der Darwinismus als Metaphysik. In Jabrbuch für Philosophie des Forschungsinstituts für Philosophie Hannover 9: 97-127. Wien: Passagen.

Hume, D. (1740) 1978. A Treatise of Human Nature. Ed. Sir L. A. Selby-Bigge. Oxford, New York: Oxford University Press.

Illies, C. 2003. The Grounds of Ethical Judgement: New Transcendental Arguments in Moral Philosophy. Oxford: Clarendon Press.

-. 2006. Philosophische Anthropologie im biologischen Zeitalter: Zur Konvergenz von Moral und Natur. Frankfurt a. M.: Suhrkamp.

Jonas, H. 1966. The Phenomenon of Life: Toward a Philosophical Biology. New York: Harper \& Row.

-. 1973. Organismus und Freibeit: Ansätze zu einer philosophischen Biologie. Göttingen: Vandenhoeck \& Ruprecht.

Kant, I. 1781, 1787. Kritik der reinen Vernunft, in: Immanuel Kant: Werke in sechs Bänden, hrsg. v. W. Weischedel, Darmstadt: Wissenschaftliche Buchgesellschaft 1998, Band II.

-. 1790, 1793, 1799. Kritik der Urteilskraft, in: Immanuel Kant: Werke in sechs Bänden, hrsg. v. W. Weischedel, Darmstadt: Wissenschaftliche Buchgesellschaft 1998, Band V, 236-633.

Kitcher, P. 1993. Vier Arten, die Ethik zu "biologisieren”. In Evolution und Ethik, ed. K. Bayertz, 221-242. Stuttgart: Reclam.

Lorenz, K. 1963. Das sogenannte Böse: Zur Naturgeschichte der Aggression. Wien: G. Borotha-Schoeler.

-. 1973. Die Rückseite des Spiegels. München: Piper.

Marr, D. 1982. Vision: a Computational Investigation into the Human Representation and Processing of Visual Information. San Francisco: W. H. Freeman. 
Maturana, H. R. 1970. Biology of Cognition. Urbana, IL: University of Illinois, Biological Control Laboratory.

-. 1980. Autopoiesis and Cognition: The Realization of the Living. Dordrecht: D. Reidel Pub. Co.

-. 1992. The Tree of Knowledge: The Biological Roots of Human Understanding, rev. ed. Boston: Shambhala.

Maynard Smith, J. 1982. Evolution and the Theory of Games. Cambridge: Cambridge University Press.

Mayr, E. 1997. This Is Biology: The Science of the Living World. Cambridge, MA: Belknap Press of Harvard University Press.

McDowell, J. 1996. Mind and World: With a New Introduction. Cambridge, MA: Harvard University Press.

-. 1998. Two Sorts of Naturalism. In: Mind, Value, and Reality, 167-197. Cambridge, MA: Harvard University Press.

Menary, R. 2010. The Extended Mind. Cambridge, MA: MIT Press.

Merleau-Ponty, M. 1942. La structure du comportement. Paris: Presses universitaires de France.

Millikan, R. 1984. Language, Thought and Other Biological Categories: New Foundations for Realism. Cambridge, MA: MIT Press.

-. 2004. The Varieties of Meaning. Cambridge, MA: MIT Press.

Moore, G. E. 1903. Principia Ethica. Cambridge: Cambridge University Press.

Nagel, T. 2012. Mind and Cosmos: Why the Materialist Neo-Darwinian Conception of Nature Is Almost Certainly False. New York: Oxford University Press.

Noë, A. 2004. Action in Perception. Cambridge, MA: MIT Press.

Perler, D. and M. Wild, eds. 2005. Der Geist der Tiere: Philosophische Texte zu einer aktuellen Diskussion. Frankfurt a. M.: Suhrkamp.

Piaget, J. 1967. Biologie et connaissance: essai sur les relations entre les régulations organiques et les processus cognitifs. L'avenir de la science 42, Paris: Gallimard.

Popper, K. R. 1972. Objective Knowledge: An Evolutionary Approach. Oxford: Clarendon Press.

Proust, J. 1997. Comment l'esprit vient aux bêtes: essai sur la représentation. Paris: Gallimard.

-. 1999. Mind, Space and Objectivity in Non-Human Animals. Erkenntnis 51 (1): 41.

Rowlands, M. 2010. The New Science of the Mind: From Extended Mind to Embodied Phenomenology. Cambridge, MA: MIT Press.

Singer, W. 2005. Conditio humana aus neurobiologischer Perspektive. In Die Rolle der Seele in Kognitions- und Neurowissenschaft, ed. M. Peschl, 41-61. Würzburg: Königshausen \& Neumann.

Spahn, C. 2007. Lebendiger Begriff, begriffenes Leben: Zur Grundlegung der Philosophie des Organischen bei G. W. F. Hegel. Würzburg: Königshausen \& Neumann.

-. 2010. Nature and Nurture. In 21st Century Anthropology. A Reference Handbook, Vol. 2, ed. H. James Birx, 938-949. Los Angeles: SAGE.

-. 2011a. Prospects of Objective Knowledge. In Interdisciplinary Anthropology: The Continuing Evolution of Man, ed. W. Welsch, W. Singer, and A. Wunder, 55-77. Berlin: Springer.

-. 2011b. Qualia und Moralia: Auf der Suche nach dem Vernünftigen im Organischen und dem Natürlichen in der Vernunft. In Natur und Geist. Über ibre evolutionäre Verbältnisbestimmung, ed. C. Tewes and K. Vieweg, 31-44. Berlin: Akademie.

-. 2012. In What Way Can Considering Evolution Contribute to a Philosophical Epistemology? De Filosoof, Faculty Journal, Department of Philosophy 50 (February): 14-16.

Spahn, C. and C. Tewes. 2011. Naturalismus oder integrativer Monismus? Zur Verhältnisbestimmung von Natur und Geist. In Gott und Natur. Philosophische Positionen 
zum aktuellen Streit um die Evolutionstheorie, ed. K. Köchy and P. Kolmer, 141-185. Freiburg: Karl Alber Verlag.

Tewes, C. 2015. Was spricht für den Idealismus im Leib-Seele-Problem? In Idealismus heute. Aktuelle Perspektiven und neue Impulse, ed. V. Hösle and F. Suarez-Müller, 194-214. Darmstadt: Wissenschaftliche Buchgesellschaft.

Thompson, E. 2007. Mind in Life: Biology, Phenomenology, and the Sciences of Mind. Cambridge MA: Harvard University Press.

-. 2015. Waking, Dreaming, Being: Self and Consciousness in Neuroscience, Meditation, and Philosophy. New York: Columbia University Press.

Thompson, E. and D. Cosmelli. 2009. Spreading the Joy: Why the Machinery of Consciousness Is (probably) Still in the Head. Mind 118: 963-93.

-. 2013. Brain in a Vat or Body in a World? Brainbound versus Enactive Views of Experiences. Philosophical Topics 39 (1): 163-180.

Tinbergen, N. 1952. Instinktlebre: Vergleichende Erforschung angeborenen Verbaltens. Berlin: Parey.

Tooby, J. and L. Cosmides. 1992. The Psychological Foundations of Culture. In The Adapted Mind: Evolutionary Psychology and the Generation of Culture, ed. J. H. Barkow, L. Cosmides, and J. Tooby, 19-136. New York: Oxford University Press.

Trivers, R. L. 1971. Evolution of Reciprocal Altruism. Quarterly Review of Biology 46: $35-57$.

-. 1972. Parental Investment and Sexual Selection. In Sexual Selection and the Descent of Man 1871-1971, ed. B. Campbell, 136-179. Chicago: Aldine.

Varela, F.J., E. Thompson, and E. Rosch. 1991. The Embodied Mind: Cognitive Science and Human Experience. Cambridge, MA: MIT Press.

Vollmer, G. 1990. Evolutionäre Erkenntnistheorie : Angeborene Erkenntnisstrukturen im Kontext von Biologie, Psychologie, Linguistik, Philosophie und Wissenschaftstheorie. Stuttgart: Hirzel.

Wandschneider, D. 1986. Die Inkonsistenz empiristischer Argumentation im Zusammenhang mit dem Problem der Naturgesetzlichkeit. Zeitschrift für allgemeine Wissenschaftstheorie 17 (1): 131-42.

-. 1996. Letztbegründung und Dialektik. In Diskurs und Leidenschaft, ed. R. FornetBetancourt. 317-336, Aachen: Concordia, Reihe Monographien.

-. 1998. Die phänomenologische Auflösung des Induktionsproblems im "szientistischen Idealismus” der beobachtenden Vernunft. In Hegels Jenaer Naturphilosophie, ed. K. Vieweg. 369-382, München: Fink.

-. 2008. Naturphilosophie. Bamberg: C. C. Buchner.

Welsch, W. 2012. Homo Mundanus. Jenseits der anthropischen Denkform der Moderne. Weilerswist: Vellbrück.

Wilson, E. O. 1975. Sociobiology: The New Synthesis. Cambridge, MA: Belknap Press of Harvard University Press.

-. 1998. Consilience. The Unity of Knowledge. New York: Alfred A. Knopf. 
2. The Embodied Evolution

of Symbolic Competence 



\section{Introduction}

\section{Magnus Scblette}

An evolutionary account of the condition bumaine is inevitably confronted with a crucial issue, which has occupied philosophical thinking since antiquity. Any research on the essentials of human existence and the development of mankind draws on complex theoretical devices of research. Furthermore, the objects of research are defined by properties which are obviously significant to the very theories which bring those objects into the limelight. Anything known or knowable to humans about the origins and forms of their being therefore presupposes a well-established realm of meaning, which links the researcher with the objects he is examining. For that matter, a common designator of man has been the term 'animal rationale': Man is that particular animal that is rational to himself. He is subject as well as object of the complex investigative endeavors which aim at the clarification of the condition bumaine. And although he has been very successful at this enterprise so far, the required clarification comes with a blind spot. Since the realm of meaning constitutes the background of human self-investigation, it is very hard to see how this background may itself become the object of research that strives to understand its origins and the way it came into being.

How is a reasonable narrative about the lineage of symbolic competence possible? An evolutionary account of the condition humaine needs to find an answer to this question. It could easily enough rely on theories of meaning as they have been proposed in the Hobbes-Locke-tradition of language thought, reducing symbolic competence to instrumentally successful signification (Locke [1700] 2008, 257). According to this type of language theory symbolic competence consists of connecting words with ideas, which represent a previously perceived world. The words establish an order within the multitude of perceived objects, which allows cognition and orientation. Lines of filiation run from Locke's locus classicus to the stimulus-response connections of classical behaviorism on the one hand and to the representationalist theories of meaning in the philosophy of mind, on the other, encouraging a twofold-reduction of human symbolic competence (Taylor 2016, $3 \mathrm{ff} ., 103 \mathrm{ff}$.). They suggest reducing human symbolic competence either to a gradually more complex variant of a type of sign-use, which is attested among the higher mammals in animal kingdom, or to a form of information-processing disconnected from the lifeworldly entanglement of the particular individual organism actualizing this competence. The essays gathered in this section of this book reject both types of reductionism, the behaviorist and the representationalist, thereby defending the embodied evolu- 
tion of a specifically human symbolic competence. Let me roughly highlight this double rejection:

If we take human symbolic competence to be a faculty of direct reference mapping between signifiers and significates, we miss the point of specifically human language: its capacity of indirect reference (reference via an inferential network of signs), which presupposes the use of propositions with singular terms (Tugendhat 2003, $13 \mathrm{ff}$.). And if we take human symbolic competence to be instantiating an information-processing device, we will not become aware of the expressive dimension of language. Johann Gottfried Herder was one of the first prominent philosophers in modern thought to emphasize languages' acquirements of indirectly referring to perceived objects (whereby each word's and each sentence's reference presupposes a holistic web of meaning) and of expressing or articulating the speaker's attitude to those objects (Herder [1771] 1953). Charles Taylor has stressed the tradition of a "constitutive view" on language that bridges two hundred years from Herder and Humboldt to proponents of the main philosophical schools in the $20^{\text {th }}$ century - like pragmatist George Herbert Mead, analytic philosopher Ludwig Wittgenstein or phenomenologist Maurice Merleau-Ponty (Taylor 2016, 16). And the current discourse on embodied and enacted cognition is well aware of its philosophical antecedents in the aforesaid philosophies with their romantic predecessors such as Herder (Gallagher 2008). What Taylor has coined the "constitutive view" of language is a type of theory that considers language's formative impact on what counts as criteria of its rightness: "Being constitutive means that language makes possible its own content, in a sense, or opens us to the domain it encodes" (ibid., 40).

Following the constitutive view, language is enmeshed in man's volitional, emotional and cognitive states. It also mediates these states in the ongoing interaction with a recalcitrant world. Therefore, language is essential to the way humans enact the world in which they exist. The constitutive view fits into the conceptual framework of situated or embodied cognition according to which to use Hilary Putnam's illustrative phrase that he borrowed from Ecclesiastes 4, 12 - cognition consists in a "threefold cord of mind, body, and world" (Putnam 2001). The particular symbolic competence of humans, which we may refer to as their linguistic competence, has to be conceptualized within the triangular structure, which relates mind, body and world. It may then be understood in terms of enacting already established correlations of body, mind, and world in a novel way that reshapes the interrelated entities. In their introduction to this volume, Gregor Etzelmüller and Christian Tewes emphasize that "an evolutionary approach must explain how natural evolutionary processes could bring forth human beings who interact with and are responsive to the 'space of reasons' within the socio-cultural realm." An account of the evolution of symbolic competence has to deliver a reasonable theory about how natural evolutionary processes induced conditions of human co-existence under which established forms of presymbolic sign-use passed the threshold to the symbolic realm.

If the constitutive view of language is appropriate, only an account that builds on the paradigm of embodied cognition will be a promising candidate to under- 
stand the evolution of symbolic competence. The following contributions by Thomas Fuchs, Terrence Deacon, Jordan Zlatev, and Matthias Jung are substantial contributions to this enterprise.

Thomas Fuchs, Karl-Jaspers-Professor for the Philosophical Foundations of Psychiatry and Psychopathology at the University of Heidelberg, is entitled to the first contribution to this section because his essay mainly focuses on the ontogenetic transmission from presymbolic to symbolic sign-use in early childhood, whereas the following three essays explore the far distant regions of phylogenesis. Fuchs' argumentation starts by correlating the enactivist thesis, according to which cognition is considered to be a form of interaction between organism and environment, with the linguistic thesis of George Lakoff and Mark Johnson in their seminal book Metaphors We Live By (1980), that bodily experience has had an immense impact on the formation of body-related verbal metaphors. According to Fuchs, research suggests, that the semantic-syntactic structure of language imitates the operative intentionality of our body. Speech enacts meaning in a way that may be retranslated by the potential addressees in embodied understanding. According to Fuchs, the enactive account of language is backed up by neurobiological findings, which show that the understanding of words activates the same sensorimotor brain areas as the practical engagement those words refer to. The discovered correlation between language and bodily action serves Fuchs as a path to understanding the development of symbolic language. The second half of his essay deals with the embedding of language acquisition in the relationships of primary and secondary intersubjectivity, highlighting the dynamic between the successively verbalized (or rather 'linguistified') intersubjectivity on the one hand and its neurobiological effects due to the plasticity of the infant's brain on the other.

The newborn, Fuchs points out, perceives her mother not merely as a 'picture' or counterpart, but mimetically, by imitating her movements and expressions and feeling them from within. Fuchs follows the traits of the infant's socialization starting from the "protoconversations" between mother and child. The emergence of the first pointing gestures at the age of nine months transform the dyad of primary intersubjectivity between mother and child into the triadic situation of secondary intersubjectivity, where the actors reciprocally acknowledge each other's intentional orientation toward objects in the immediate environment. Subsequently, the child can take off to capture the world via bodily co-presence and cooperation. Fuchs sees the vocal gesture originally embedded in an intercorporeal mutual practice that is oriented towards a shared environment, then, during the further course of language development, separating the sign from the physical movement and transporting it into the invisible medium of sound. Via the communication of the mirror neuron system, the voice was able to call up the idea of the intended actions and objects in both speaker and listener. The brain functions become the matrix of language. On the other hand, correlates of semantic meaning are functionally and morphologically inscribed on the brain as neuronal patterns in the course of interaction. Language therefore is not just embodied in our bodily interaction with each other and the world but 
via brain functions incorporated into our bodies - the brain is a cultural organ, the affairs of our social life are organic events.

Fuchs' essay refutes the classical dichotomy between nature and nurture in favor of an irresolvable entanglement of both sides, each affecting and shaping the other. This idea is also picked up by Terrence Deacon in his essay entitled “On Human (Symbolic) Nature: How the Word Became Flesh”. Deacon, Professor for Biological Anthropology at the University of California at Berkeley, complements Fuchs' ontogenetic approach to the correlation of sociality, language and brain by the phylogenetic perspective. As beavers have bodies adapted to the aquatic niche that beavers create, symbolic communication, according to Deacon, became an artificial niche to which hominid brains had to adapt. The main focus of Deacon's essay is the specific cognitive efficiency of symbol use, which led to the mutual exertion of selection pressure from brain functions on language for special learning and production demands and from language structures on brain functions for learnability and ease of use.

Deacon offers an evolutionary theory of signs that is based in Peircean semiotics. According to his approach semiotic properties are not intrinsic. Anything can be taken as a sign for anything else in any respect (e.g. either icon, index or symbol) so long as an appropriate interpretant process is generated. Icons, characterized by similarity in form, constitute the basis of conceptually grasping indexical correlations, whose context-bound interrelatedness, again, feeds into the conceptualization of symbols as forms of inferential or indirect decontextualized reference. For Deacon, symbolic competence is therefore like cracking a code: One not only needs information about what refers to what, but also needs to understand the system of indices, which constitutes the matrix of symbols. Therefore "symbols are understood as higher-order semiotic relations embedded in a context of indexical and iconic modes of reference." It is obvious, that according to Deacon's semiotic approach, the development of pointing and joint attention becomes a key stage in the process of hominization. The sophistication with indexicality developed prior to speech and was transferred into the use of words via the vocal gesture, as Deacons argumentation complements the ontogenetic approach in Thomas Fuchs' essay.

One of the major innovations in the hominization process, Deacon emphasizes, was the evolution of procedural and episodic memory. Whereas the frontal-striatal-cerebellar circuit creates memory traces for skilled action by constant repetition and fine tuning, the sensory-hippocampal circuit creates memory traces for singular experiences by correlations between features. Both become essential for the emergence of narrative memory with its syntactical as well as semantic structure. Whereas articulatory and syntactical combinatorial skills are acquired procedurally, semantic relationships are acquired episodically. On the basis of narrative memory, icons can be juxtaposed to point to each other in a third iconism, which creates an index. Incongruous juxtapositions can convey abstract symbolic content behind the indexical 'surface' of the sign. Even more so, emotions linked to signs may by their correlation be combined in novel ways. Specifically, human emotions such as nostalgia, awe, humor, irony, derived from 
combinatory sign use, are evoked in the process of semiosis. Essential to Deacon's evolutionary theory of human sign use is the insight that symbolic reference emerges from and depends upon lower-order indexical and iconic forms of reference. Symbolic reference, which refers to the world only via indices, is thereby anchored in the sensual experiences of bodily creatures interacting with their environment.

Jordan Zlatev, Professor for Cognitive Semiotics at Lund University in Sweden, focuses in his essay "Preconditions in Human Embodiment for the Evolution of Symbolic Communication" on the cognitive-semiotic prerequisites for the emergence of symbolic communication in the process of hominization. Zlatev puts a strong emphasis on the development of human symbolic competence in a prolonged process of adaptation to the challenges of transformed social living conditions. His essay thus puts emphasis on bodily mimesis as a "missing link" to the further development of the 'symbolic species' (Terrence Deacon). Bodily mimesis, according to Zlatev, granted to human cognition and communication pre-linguistic features in five domains: Firstly, it allowed for systematic rehearsal of motor patterns necessary for the fine-tuning of complex skills; secondly, it founds highly demanding forms of imitation, in which a novel act is observed, modeled and eventually added to behavioral repertoire; it prepares, thirdly, the ground for episodic memory, bringing the re-enactment of an event through bodily motion under voluntary control; fourthly it provides the basis for the formation of rituals and ritual-mediated group mentality; and finally, through ritually-bound sequences of mime and gesture, bodily mimesis enables intentional communication. Zlatev's essay suggests answers to the questions of how bodily mimesis could evolve and which consequences it had for the development of full-fledged symbol use. The answer to the first question is "alloparenting", the answer to the second the "multimodal" character of communication.

As to the "alloparenting hypothesis" Zlatev argues in line with Sarah Blaffer-Hrdy (2009) that the nurturing attitudes of mothers were extended from their infants to other members of the group, allowing food and childcare to be more equally distributed. The tightening of social bonding, precipitated by the transition of social group organization to alloparenting, prepared the grounds for bodily mimesis. While bodily mimesis, according to Zlatev, opens the way to a representational relation between sign and object, the multimodality of communication allowed a gradual shift from predominantly bodily to predominantly vocal modes of expression. Since the vocal medium has less potential for iconic representation, it was prepared to overtake non-iconic representational tasks. Here the specific function of the vocal gesture comes to the fore: the vocal modality, while being grounded in the iconically based bodily gestures, could become increasingly instrumental for higher forms of representation.

For Matthias Jung, Professor of Philosophy at the University of Koblenz, the feature of symbolic - respectively indirect - reference, namely its dependence upon lower-order indexical and iconic forms of reference, is the main reason for conceptualizing the development of language as "Stages of Embodied Articulation". The depth of human experience, as he points out with explicit reference to 
Deacon's works, “is internally connected with our ability to handle linguistic and other symbols.” Supplementing Deacon's view on the organic basis of language in the development of narrative memory, Jung introduces the concept of articulation "as the embodied alternative to representationalist views of language". According to Jung, articulation is "the process of making explicit what the felt meaningfulness of action-associated qualities actually means, where its personal significance lies and to which entities in the world it refers." It is thereby embodied in two distinct aspects: On the one hand, the complex relation of semantic composition and syntactic ordering of meaning requires sensory-motor performances of the body; on the other hand, the enactment of meaning in speech depends upon bodily qualities and their felt change during the articulation process. Entangling hermeneutical accounts of experience with those of the pragmatist tradition, Jung argues for the creativity of language, which transforms the "underlying pervasive quality" of situations, in which man interacts with his environment, into meaning. Since subjective: emotional, sensual, qualitative aspects of the linguistic event of meaning-formation are enmeshed with the objective aspects of reference, truth-conditional theories of meaning fall short of the formative capacities of embodied language.

But Jung's argumentation points into yet another direction. Language, he says, is deeply embedded in our bodily practices and states of being, but it also has the potential to transcend its embedding. Referring to Deacon's semiotic account of the symbolic species, Jung locates the potential of languages to transcend the immediacy of context-bound interaction with the environment in their devices for symbolic reference. Symbolic reference, according to Jung, submits "second-order capabilities" of reflecting on qualitative states of being and action via a network of inferential semantics. Equipped with Deacon's semiotic differentiations, Jung finally ventures a synopsis of Robert Brandom's logical hierarchy of semantic explicitness in his seminal book Making it explicit (1994) with Merlin Donald's narrative about the co-development of human cognition in the cultural process (Donald 1991).

In a first step, adding insights from Brandom's neopragmatist entanglement of normative pragmatics with inferential semantics to his Deacon-based theory of signs, Jung conceptualizes "a ladder of articulation having three rungs - indexical, symbolical and symbolical-reflexive sign usage ... grounded in iconic reference" and thereby irresolvably embedded in the qualities of bodily interaction of the human organism with his environment. In a second step, he refers the aforesaid rungs in his ladder of articulation to Merlin Donald's phylogenetic stages in the development of linguistically impregnated human cultures. The point of this endeavor is to formulate a hypothesis about the successive emergence of linguistic characteristics which are specific to what Donald calls the episodic, mimetic, mythic and theoretic stages of human culture. According to this historical typology, full-fledged propositional language is thereby restricted to the evolvement of what Donald calls theoretical culture during the 1000-year period of the so-called Axial Age marked by the appearance of monotheistic religions and rationalist philosophies. 
The essays collected in this section formulate different strands of a research program which are interlinked but also develop into different directions. The challenge of conceptualizing the symbolic competence of the human species as essentially embodied in organic and social structures calls for the interdisciplinary coordination of perspectives, which encompass the child's first steps into her linguistic socialization as much as the formation of cultural frameworks, in which the conditions of decontextualized deliberation are institutionalized. It is the semiotic differentiation between iconic, indexical and symbolic means of conscious orientation, which formulates the pivot point of the four contentually diverse contributions. On the other side, the plausibility of the semiotic categories depends on the extent to which they help incorporating empirical fields of research into thinking about the constraints of symbolic competence in a non-dualist way.

\section{Bibliography}

Brandom, R. 1994. Making it Explicit. Reasoning, Representing and Discursive Commitment. Harvard: Harvard University Press.

Donald, M. 1991. Origins of the Modern Mind. Three Stages in the Evolution of Culture and Cognition. Harvard: Harvard University Press.

Gallagher, S. 2008. Philosophical Antecedents of Situated Cognition. In The Cambridge Handbook of Situated Cognition, ed. P. Robbins and M. Aydede, 35-52. Cambridge: Cambridge University Press.

Herder, J.G. (1771) 1953. Abhandlung über den Ursprung der Sprache. In Johann Gottfried Herder. Werke in zwei Bänden. Erster Band, 733-830. München: Hanser, engl. transl.: "Treatise on the Origin of Language”, in: Herder. Philosophical Writings, trans. Michael N. Forster, Cambridge UP 2004.

Hrdy, S. B. 2009. Mothers and Others. The Evolutionary Origin of Mutual Understanding. Harvard: Harvard University Press.

Lakoff, G. and M. Johnson. 1980. Metaphors We Live By. Chicago: University of Chicago Press.

Locke, J. (1700) 2008. An Essay concerning Human Understanding. Oxford: Oxford University Press.

Putnam, H. 2001. The Threefold Cord: Mind, Body and World. Columbia: Columbia University Press.

Taylor, C. 2016. The Language Animal. The Full Shape of the Human Linguistic Capacity. Cambridge, MA: Belknap Press.

Tugendhat, E. 2003. Egozentrizität und Mystik. Eine anthropologische Studie. München: Beck, engl. transl.: Egocentricity and Mysticism. An Anthropological Study, Columbia UP 2016 (forthcoming). 



\title{
The Embodied Development of Language
}

\author{
Thomas Fuchs
}

\begin{abstract}
The concepts of language prevalent in cultural and cognitive sciences regard it as a complex mental symbol system which is acquired mainly through maturation of suitable cognitive modules. In contrast, from an embodied and enactive point of view there is no fundamental separation between sensorimotor and symbolic interactions of an agent with its environment. The paper first presents arguments for an embodied basis of language production and comprehension, in particular results from cognitive neuroscience which link language processing to motor areas in the brain. The acquisition of language is then conceived as resulting from embodied interactions with others, starting from expressive or interbodily resonance, then proceeding to iconic gestures and finally leading to symbolic modes of communication. This development is essentially based on understanding others as intentional agents, which in turn is enabled by grasping their intentions as embodied in expressive, goal-directed, and pointing gestures in the context of shared practices.
\end{abstract}

\section{Introduction}

Since antiquity man has been primarily distinguished as the being that has language - the zoon logon echon, as Aristotle defines it, and later as the animal rationalis. According to this definition, on the one hand, humans are living beings like animals (animalia), and yet on the other hand are fundamentally different from them due to language and reason. Through these capacities alone, they achieve culture, art, science and technology. They are similar to their animal relations with regard to bodily needs, drives and affections; however, reasoned speech distinguishes them ahead of all other earthly creatures. Thus, Homo sapiens is an inherently ambivalent centaur being, a hybrid of animality and rationality, an animal rationale.

It may still be attributable to this traditional view of anthropology that for a long time both the cultural as well as the cognitive neurosciences only treated language as a disembodied mental symbol system. Starting with Fodor's "Language of Thought" (1975), words were conceived as producing images or symbols inside the head of the speaker or listener, whose brain would use them to construct a representation of the state of affairs "out there" (Fodor 1998; Pylyshyn 1984). The fact that language originates from speaking with one another, where this primarily represents a bodily movement of expression and a joint speech action, that is to say in brief - the bodily performance of speech was only acknowledged as an accidental attribute, which seemed to have no effects on its structure and the implied contents. 
Only recent decades of infant research and evolutionary anthropology have shown the wealth of communication and dialogue that already unfolds in the human individual before learning language (Trevarthen 1979, 2009; Stern 1985; Tomasello 2008). Bodily communication or body language, as we also call it, is mainly conveyed through facial expression and gestures, through the intonation of the voice and ultimately through the body's whole posture. As Darwin ([1872] 1998) already observed, this expressive communication in humans manifests a differentiation and diversity that is unique in the animal kingdom. However, it is also the foundation on which verbal-symbolic forms of communication may initially develop at all during early childhood. For as we shall see later, language acquisition crucially presupposes that children develop an understanding for the intentions of others; and at first these intentions are only accessible to them as embodied, namely as visible, expressive, goal-oriented and pointing movements, whose meaning is exposed in the context of practical bodily interaction.

In what follows, I will proceed from an embodied and enactive view on language and its development (Varela, Thompson, and Rosch 1991; Glenberg and Robertson 2000; Ziemke 2002; Zlatev 2007). I will argue for the following theses: (1) Language is not a representation of the world inside the head, but a form of embodied intersubjectivity: The meaning and function of words and sentences is derived from our bodily experience of interacting with the world, which we share in principle with others, and which is evoked both in ourselves and in others by our verbal utterances. This is reflected in recent research on the involvement of sensorimotor brain areas in language processing.

(2) The acquisition of language in infancy is not achieved through an abstract attribution of symbols to references, but through the infant's participation in shared intentional practices of interacting with the world. Only as embedded in an interactive "we-intentionality", can words be learnt and gain their meaning.

In both ways, language thus depends on intercorporeality (intercorporéité, Merleau-Ponty 1960), that means, on a sphere of reciprocal bodily understanding and interaction, from which words first draw their references and meanings. Following on from these practical interactions, the infant's brain is also influenced and structured by language: the brain only becomes an organ of the symbolic mind through social interactions (Fuchs 2010, 2011).

In the first part of my paper, I will argue for the embodied nature of language, including the anchoring of language in the brain. In the second part, I will give an account of the embodied development of language in early childhood. 


\section{Language, Embodiment, and the Brain}

\section{The Body as the Medium of Language}

In their seminal book "Metaphors we live by", Lakoff and Johnson (1980) have first emphasized the bodily basis of language. They described over 50 systematic schemes of body-related verbal metaphors: basic bodily experiences like those of in and out, up and down, front and back, warm and cold, fast and slow, near and far, etc., cover a wide range of applications in all dimensions of language. They become the basic schemes of conceptual development and imagery, and what we use to call metaphorical or figurative meanings are in fact derived from our bodily experience which is subliminally present and effective even in the seemingly most abstract discourse (see Johnson 1987).

The connection of language and the body has also been examined over the past two decades from the perspective of embodied and enactive cognition. This paradigm is based on the assumption that there is no strict separation of "lower" and "higher" cognitive functions, that is, between perception and movement on the one side and thought and language on the other. All forms of cognition are fundamentally considered as a form of interaction between an organism and its environment (Varela, Thompson, and Rosch 1991), which means that there is no abstract level of the mind as a computational symbol system. Instead, motor, sensory, and cognitive functions are always intermodally linked. This has also led to an embodied view of language as involving bodily systems of movement, posture, kinesthesia and proprioception, both in language production and comprehension (e. g. Glenberg and Robertson 2000; Ziemke 2002; Zwaan et al. 2004; Barsalou 2008; Cuffari, Di Paolo, and De Jaegher 2015).

Let us take an example: If we listen to a simple sentence such as "the book lies on the table", its meaning is constituted for us by a connection of several components:

(a) the evocation of two objects in our awareness, which does not only include their visual imagination, but also their affordances for our bodily action, for example, as something to grasp, to open and to read (the book), something solid to sit at or to lay things on (the table), etc.;

(b) our operative (motor, postural) bodily intentionality which lets us implicitly grasp the state of "lying", namely as being stretched out flat, wholly supported by the ground; ${ }^{1}$

1 This involvement of our body in the meaning becomes even more obvious if we think of the difference the German language makes between "lying" and "standing” objects: "Das Buch liegt auf dem Tisch" (the book "lies" on the table), but "die Tasse steht auf dem Tisch" (the cup "stands" on the table). This usage of the verbs mirrors the different postural imitations that are invoked in our body when looking at a flat versus an upright object. 
(c) a spatial relation which we know from your own bodily postures or actions (lying "on" something, being placed "next to", etc.);

(d) a temporal relation of simultaneity to our present experience ("lies”);

(e) a syntactical structure which generally combines a subject and a predicate in the same way as we experience ourselves as doing something ("the book lies", "the tree stands", "the bell rings", etc.). ${ }^{2}$

So what we implicitly understand when listening to the sentence above would have the unfolded meaning of "the thing-I-could-take-and-read is now lyinglike-I-would on the thing-I-could-sit-at", or similar. A sentence thus combines affordance-based terms into patterns of action and relation, or in other words, the syntax in a sense imitates the operative intentionality of our body. ${ }^{3}$ In its basic grammatical structure, a sentence expresses a subject acting on an object in a way that we could on principle perform ourselves; through this very structure, the sentence enacts its meaning and thus enables an embodied understanding, or to use an enactivist term, embodied sense-making (Weick 1995; De Jaegher \& Di Paolo 2007).

To this, we have to add the person speaking the sentence and her apparent intention in the interactive context, turning the utterance "the book lies on the table" either into an informative answer (there it is!), an implicit request (could you hand it over?), a philosophical example (let's take the following sentence ...), or whatsoever. Understanding another thus involves participating in her intentional attitude towards the situation. ${ }^{4}$ Moreover, listening to her also involves

2 The fundamental structure of a sentence (subject - predicate - object) implies an agent performing some kind of operation on an object, which is precisely the basic structure of our embodied relation to the world. Of course, there are many variations - the verb may be intransitive or signify a state rather than an action - but this does not change the fact that a sentence expresses what could on principle be our own experience.

${ }^{3}$ One might object that all these affordances and bodily conditions are far too complex to be present in the immediate understanding of the sentence. As we will see, however, there is now a lot of neurobiological evidence showing that this indeed the case (see below). But apart from that, the question is how one could ever come to understand the meaning of lying at all, if not by "what I know from my own lying", even if this embodied knowledge is only activated in the most remote way when hearing the word later on. For otherwise it would be very difficult and circuitous to explain what lying actually means, for example, "the spatial relation of an object being in close contact with another object underneath, touching it with its most extended side, whereas its smaller sides remain free and upright." And even then, we would run straightaway into the symbol grounding problem (Harnard 1990), for what the symbols "spatial", "contact", "touching", "cover", etc. in that definition mean could only be explained by even more complex definitions, and so on ad infinitum. Language cannot be a free-floating system of symbolic references - it must ultimately be grounded in embodied experience. This experience is primarily given as a knowing how based on bodily dispositions and habits, not as a knowing that represented in a propositional format (Fuchs 2016a).

${ }^{4}$ Usually, this does not require any explicit perspective-taking or mentalizing ("theory of mind"): we do not distinguish between an interlocutor's mental state and his utterances, as if the former would have to qualify the latter, but we understand his words as just what they mean in relation to the shared situation. The intention is inherent in the verbal expression itself. Only in cases of ambiguity or doubt, this unity of intention and utterance may be dissolved, and we apply explicit cognitive procedures of perspective-taking or inference ("what did he mean by that?", "what is he up to?", etc.). 
a tendency of subvocalizing her utterances. This becomes obvious for example when listening to a conversational partner who appears to hesitate or to be at loss for the right words, and without hesitation one supplies the missing words, completing the utterance of the speaker. For the speaker in turn, the attentive listener serves as a stimulus for his own speech, as Kleist ([1805] 1951, 43) has famously described in his essay On the gradual construction of thoughts during speech: "The other person's face is a curious source of inspiration for a person who speaks. A single glance which indicates that a half-expressed thought is already understood, bestows on us the other half of the formulation." ${ }^{5}$ Language production as well as comprehension may thus be described as a special kind of participatory sense-making (De Jaegher and Di Paolo 2007), namely as the co-enactment of a sense that is always in the making, through embodied protentions or co-anticipations of both speaker and listener.

If we take all this together, we can assume a prima facie evidence that

(a) language is not a free-floating, abstract symbol system, but a network of meanings evoking a certain way of embodied being-towards-the-world (êtreau-monde, Merleau-Ponty) or acting-towards-the-world;

(b) language production and comprehension are crucially based on embodied and enactive cognition, including the situated verbal interaction itself. That means, "words are patterns available for enacting certain forms of sense-making” (Cuffari, Di Paolo, and De Jaegher 2015), both in speaking and in understanding.

One could now argue that this bodily and operational basis of meaning and grammar does not apply to higher levels of abstraction: there seems to be no enactive account of abstract words like "conclusion", "peace" or "right", etc. However, a closer look reveals that even the meaning of abstract or metaphorical terms is ultimately based on bodily experience (see also Irwin 2015). Let us look at some examples:

- The noun "right" (or German Recht) is derived from the Indo-European roots reg- ("to move in a straight line, to straighten, to direct") and regtós ("straight, upright"). ${ }^{6}$ Thus, it is related to a bodily operation which implies

${ }^{5}$ It is worthwhile to follow Kleist's description in detail: “Often I sit at my desk, poring over documents and trying to discover the point of view from which some complicated controversy might be judged.... But, lo and behold, if I mention it to my sister, who is sitting behind me and working, I discover facts which whole hours of brooding, perhaps, would not have revealed. ... For since I always have some obscure preconception, distantly connected in some way with whatever I am looking for, I have only to begin boldly, and the mind, obliged to find an end for this beginning, transforms my confused concept as I speak into thoughts that are perfectly clear, so that, to my surprise, the end of the sentence coincides with the desired knowledge.... During this process nothing is more helpful to me than a sudden movement on my sister's part, as if she were about to interrupt me; for my mind, already tense, becomes even more excited by this attempt to deprive it of the speech of which it enjoys the possession and, like a great general in an awkward position, reaches an even higher tension and increases in capacity." (Kleist [1805] 1951, $42 \mathrm{ff}$.)

${ }^{6}$ Cf. also Greek orektos (stretched out, upright) or Latin rectus (straight, right). See Kluge (1989) and http://www.etymonline.com. 
an upright posture or gait and an experience of balance. This refers to the moral sphere as well: being a "righteous", honest or courageous person means an inner or moral attitude which is embodied in a corresponding posture of standing or walking upright. Similarly, the meaning of "justice" or "equity" (German Gerechtigkeit) is grounded on the experience of bodily equilibrium achieved in the upright position (as represented also in the balanced scales of Justitia).

- The words "concession" and "concede" are derived from the Latin cedere which means to withdraw, to give way. Thus, if I concede a right or a claim to someone, I withdraw, however slightly, from my primary bodily stance which may also be expressed by a conceding gesture of my arm.

- Apart from etymology, embodiment research may also support the bodily basis of metaphorical terms, as for example the connection between guilt and impurity, or cleansing, respectively. Pilate washed his hands and thus claimed to be innocent of Jesus' death, and Lady Macbeth develops a washing obsession after the murder of King Duncan. Recent research has now shown that cleansing can indeed wash away or alleviate feelings of guilt (Meier et al. 2012, Lee and Schwarz 2011, Zhong and Liljenquist 2006) and have a mildness influence on one's moral judgment (Schnall, Benton, and Harvey 2008).

- When we speak of a "warm welcome", we do so because we actually feel bodily warmth in this situation - the social atmosphere is felt as bodily sensation. Correspondingly, Zhong and Leonardelli (2008) found that test subjects, after having been exposed to a situation of social exclusion or ostracizing, estimated the room temperature to be colder than before. Moreover, Bargh and Shalev (2012) found that persons who experience social loneliness show an increased tendency to take warm baths or showers.

Generalizing such considerations and results, one can describe language as a system of interrelated terms which refer to all kinds of embodied operations and experiences, and which in their syntactical combination imitate our bodily interactions with the world. Even the most abstract terms are ultimately derived from some primary form of operation or interaction: Take "abstraction" as drawing away (from Latin abs-trabere), "detection” as pulling away a cover (de-tegere), "enlightenment" as sheding a light on something to become visible, or "negation" as an action or resistance against some kind of intrusion (for example, a rejecting gesture of one's hands or a shaking of the head to avoid intake). ${ }^{7}$

${ }^{7}$ Could this thesis even be extended to include abstract systems such as mathematical or logical structures and operations such as $\sqrt[3]{27}=3$, syllogisms or similar? It seems that from a certain degree of abstraction, such systems can still be comprehended or applied, but do no longer allow for any imagination based on sensorimotor experience. However, it soon becomes clear that even here, the abstract terms and operations are initially derived from experiences of bodily action in the way Piaget ([1936] 1952) has already described it (although he assumed that abstract thought disconnects from the level of primary sensorimotor or preconceptual thinking). Thus, addition, subtraction, multiplication, or division are mental operations which are only acquired initially by performing the concrete operations in an ostensive way (e. g. supported by 


\section{Neurobiological Findings}

In the last two decades, the embodiment of language has been increasingly confirmed by findings from neuroscience, which show that language processing in the brain is functionally connected to sensorimotor systems. Thus, if one listens to words, the same sensorimotor areas are activated as for the practical engagement with the objects that the words refer to, or in other words, language comprehension is crucially based on action-perception circuits in the brain (Gallese 2008; Pulvermüller and Fadiga 2010; Jirak et al. 2010). Let us look at some examples:

- Listening to the words "grasp", "go" or "shout" activates, alongside the receptive language areas, also the motor centers for the corresponding actions (Buccino et al. 2005; Jirak et al. 2010). There is even strong evidence for a somatotopy of language, that means a differential activation of motor centers according to the limb or action involved in the sentence one listens to: Pulvermüller (2005) identified specific fMRI-activity patterns in the pre-motor cortex for consonant verbs that refer to mouth, arm or leg movements, such as 'lick', 'pick' and 'kick'. In each case, the premotor cortex is differentially engaged in a topographical bodily pattern.

- When listening to verbs referring to hand movements (give, take, point, etc.) right-handed people show an activation of the left pre-motor cortex, lefthanded people an activation of the right (Willems, Hagoort, and Casasanto 2010). This shows that the verbs are processed according to the actual bodily movement that one could perform. Moreover, it strongly suggests that they have already been learnt in this embodied way: "to give" meant originally "handing something over to mom with my right hand" (or left hand, in the other case).

- Words related to odours (for example, "cinnamon") or to sounds (for example, "telephone") cause particular activation in olfactory and auditory brain areas, respectively (Pulvermüller and Fadiga 2010). Thus, listening to the sentence "the alarm sounded and John jumped out of bed" will activate areas both in the auditory and motor cortex related to sounds and movements (Kaschak et al. 2006; Winter and Bergen 2012).

- Moreover, Glenberg and collaborators (2008) and Boulenger, Hauk, and Pulvermüller (2009) found that the abstract usage of verbs such as "to give" or "to grasp" (to give a reason, to grasp a notion) activates the motor system no less than the concrete usage. Granted, these results are still open for debate,

one's finger or other countable objects). Of course, the habitualization of these operations leads to their formalization which does no longer need (nor afford) operative imagery. However, even though a number such as $1,455,578$ cannot be imagined in any sense, we still take it implicitly for granted that it is composed of as many steps of adding $1+1+1 \ldots$, and the same applies for all other kinds of mathematical operations - that is precisely why they are called "operations". The same could be shown for logical operations like conclusions (thus, the famous syllogism "All humans are mortal, Socrates is human, therefore Socrates is mortal" dips into a box in which all objects of a certain type have been put before and picks one out again). 
and it may also be possible that the context of words influences the degree to which the motor regions are involved in their comprehension (Jirak et al. 2010).

- Generally, merely listening to speech also activates motor brain regions that are involved in speech production (Wilson et al. 2004, Pulvermüller et al. 2006). This corresponds to the tendency of subvocalization during listening to an interlocutor mentioned above.

- Finally, it emerged that areas which were thought to have purely verbal functions like the Broca and Wernicke area actually combine language and bodily movement with one another, specifically via the mirror neuron system (Binkofski and Buccino 2004; Gallese 2008). "Mirror” or sensorimotor neurons, originally found in the premotor cortex of macaque monkeys, generally link one's own motor action to the same action as perceived in conspecifics, enabling a sensorimotor or embodied social perception (e. g., observing someone reaching for a cup activates one's own motor system for the same reaching action, even if only subliminally). In humans, Broca's area has been found to be the core region of the mirror neuron system, and there is increasing evidence showing that this system is at least participating in the connection of verbal sounds and possible action (Aziz-Zadeh et al. 2006; Aziz-Zadeh and Damasio 2008; Jirak et al. 2010).

All these strands of research are still in flux and a final evaluation is not possible yet. Nevertheless, there is at least strong evidence for an enactive concept of language as being crucially based on bodily perception and action. A consequent question is: Does the body also play a constitutive role for the acquisition of language, which also means for the establishment of neural action-perception circuits that are necessary to speak and understand language? In the introduction, I have already proposed that language developes as a form of embodied intersubjectivity. I now state some reasons in greater detail, looking at the development from pre-verbal to verbal stages of intersubjectivity in early childhood.

\section{The Embodied Development of Language}

\section{Primary Intersubjectivity}

Infants are attuned from birth to social interactions, in particular by showing a heightened attention to faces and their expressions (Valenza et al. 1996; Turati et al. 2002). Research studies conducted during the last two decades have mostly found that they are also able to imitate adults' gestures like sticking out their tongue, opening their mouth, frowning, and others (Meltzoff and Moore 1977, 1989). This capacity for spontaneous imitation of others' expressions has been considered a crucial basis of early social development (Meltzoff and Brooks 2001, Meltzoff and Prinz 2002). However, recent research with larger samples and a wider range of gestures presented to the infants challenges these results, finding 
no significant excess of matching over non-matching reactions (Oostenbroek et al. 2016). But even if it turns out that imitation is not an innate capacity, but develops in the course of mutual exchanges and matching reactions during the first months, it still functions as a major component of what Trevarthen (1979) has termed "primary intersubjectivity".

This stage is characterized by an increasing emotional resonance between infant and mother that develops via mutual bodily expressions and reactions. Usually, the mother intuitively answers the baby's signals and initiatives with suitable vocal and gestural reactions that stimulate further resonance. In the first months, mother and infant thus develop dynamic and synrhythmic "proto-conversations" (Trevarthen 2001, 2008), that is, fine-tuned sequences of alternating expressions with imitative utterances, smiles and gestures just like a conversation - the later verbal dialogue is already outlined here. Mothers and fathers intuitively use simplified, prototypical behavioral forms (welcome reaction, eye contact, musical utterances or "motherese", exaggerated facial expressions, among others) that correspond to the child's "musical repertoire" and preference for expressiveness (Papoušek and Papoušek 1987, 1995; Malloch 1999).

This early intensive dialogue is especially influenced by musical expressive qualities, by the rhythm and dynamics of facial, vocal, and gestural interaction that express changes of emotion and mood. They may best be described in qualities such as "crescendo", "decrescendo", flowing, frisking, smooth, explosive, etc., which Daniel Stern (1985) termed "vitality contours" or "vitality affects." For example, a sharply rising pitch contour in maternal vocalization alerts the infant, whereas the pitch is low and continuous in comforting or soothing (Fernald 1992, Papoušek 1994). Being the major bridge of emotional exchange, these expressive qualities lead to the mutual "affect attunement" of parent and infant that Stern highlighted. "Even in early weeks, infants learn little rituals of musicality, in vocal games, in simple rhyming songs, sharing with skill and affectionate good humour their recursive events ... babies are alert to the pulse and subtle harmonies of a mother's speech, turning to tones of sympathy, or withdrawing from their absence" (Trevarthen 2008, 18, 21). ${ }^{8}$ In the course of this preverbal communication, the child increasingly learns to connect the mother's or father's emotional expression with typical recurring situations and thus to distinguish its different meanings. The child also learns that his own reactions motivate the caregiver to specific behavior, and thereby develops interactive expectations. All this conveys to him the basic feeling of living with others in a shared world, of being perceived by them and being connected with them - a central precondition for the steps that now follow.

8 The baby's particular sensitivity to the lived synchrony of interaction was impressively demonstrated by Murray and Trevarthen (1985) who designed a Double Television set-up that enabled replay of the mother's affectionate and responsive talk with the baby. When a happy minute of the mother's live communication was later replayed to the baby (thus showing the same expressive qualities but lacking synchrony and responsiveness), the baby soon became distressed and turned away. 


\section{Secondary Intersubjectivity}

\section{(a) Joint Attention and the Pointing Gesture}

On the next level of secondary intersubjectivity, the phenomenon of "joint attention", which manifests itself from about the age of 9 months, signifies a key step towards symbolic communication (Trevarthen and Hubley 1978; Tomasello 2002; Bråten and Trevarthen 2007). At this age, babies begin along with adults to turn their attention to objects, in particular by following their pointing gestures. Soon the babies also proceed to steer the adults' attention to things through pointing themselves, and in doing so cast each other quick glances to reassure themselves of their attention. In an illuminating experiment by Tomasello and his group, infants aged about 12 months observed how one adult made a hole in a sheet of paper and filed it away in a clip folder. The adult now left the room and another adult entered, took the folder and placed it in a clearly visible cupboard, which he then locked. He left the room, the first adult re-entered and looked around, visibly searching for something, with a sheet of paper in his hand. In most cases, the infants looked attentively at the adults and then pointed to the cupboard (Liszkowski et al. 2006).

How can we interpret this experiment? Obviously, the infants recognized the adult's intention, only due to his previous action and now his questioning expression. Intentions are therefore not only something internal or mental, but they are also perceptible in the goal-oriented bodily actions of others and obtain their meaning from the context of the joint situation. There is no need first for a "Theory of Mind" (ToM) or some kind of inference or mind-reading in order to directly understand others' intentions in a practical context - after all, the usual time of acquiring a sophisticated knowledge of other minds (ToM) is not before the age of 4 years. Considered more closely, what does pointing imply?

Pointing first involves the mutual relation to a third entity that is seen by both partners, being aware that the other is also doing so. Hence, we are no longer concerned with the primary dyadic, but with a triadic situation comprising the infant, the adult and the mutually intended object or goal of an action (Tomasello 2002). The joint attention, which is visible in the parallel axes of the child's and adult's gazes, manifests a specifically human form of communication, namely conveying a message about a joint, external reference point. Here lies a fundamental limit to the mental capacities of other primates that cannot develop joint attention (Fuchs 2013). Even though great apes may become capable of so-called imperative pointing ("give me this!") when raised in human environments, there is no declarative or cooperative meaning attached to it (Gómez 2007). In contrast, as we saw in the above study, the infants also attempted to help the adult by pointing to the object being searched for. This communicative and cooperative attitude has been particularly highlighted by Tomasello and his group as a crucial difference from proto-pointing gestures shown by great apes (Tomasello et al. 2005; Tomasello and Carpenter 2007): only through this sharing of intentions, 
an actual "we-intentionality” is created ("look at this!", “now we are looking at this object together”).

Pointing is a gesture that only makes sense in an intersubjective context: it "indicates" the object with the index finger, instead of grasping it. The other person must understand this meaning, i. e. follow the direction of the finger into empty space until arriving at the object as its goal. The pointing gesture is the origin of mutually shared meanings and thus a precursor of the sign - the entity, which stands for something different, and represents it (Fuchs and De Jaegher 2009). Etymology also refers to the genetic link of pointing, sign and later speaking: in German, "zeigen" (to point) und "Zeichen" (sign) have the same Indo-Germanic root $<d e i k>$. This root also occurs in the Greek "deiknymi” (to point, to show) and "dáktylos" (finger), and also in the Latin "dicere" (to show, to speak) and "digitus" (finger). The same connection becomes manifest in German “deuten” (point) and "bedeuten” (signify) (see Kluge 1989, 807).

The pointing gesture is a grounding experience in still another way. Infants experience in this instance that other people also have a direction of attention that they can personally influence. Even though we should not be led to assume a mentalistic understanding of others at this level, infants at least begin to understand that the world looks different in their parent's eyes, yet that they can communicate with them about it. They show them an object because they notice that the adult has not seen it yet, but could soon see it, as shown in the experiment of the folder in the cupboard. In other words, infants develop an initial understanding of another perspective with which they identify by a kind of co-anticipation, assuming that an object has a meaning for the adult. Such a fundamental new stage of intersubjectivity is manifested here that Tomasello also refers to the "9-month revolution" (Tomasello 2002). ${ }^{9}$

\section{(b) Other Gestures}

Apart from the pointing gesture there are also other communicative gestures that develop in the second year of life. In almost all cultures, for example, shaking one's head means "no". The origin of this movement can be observed in babies who move their head to one side to avoid an unpleasant stimulation or to refuse further breast-feeding (Spitz 1957). Presumably this evolved into a ritualization during the course of phylogeny. As the signal must be clear, it was carried out more noticeably, i. e. by more markedly and repeatedly turning the head. On

\footnotetext{
${ }^{9}$ It should be mentioned here that Tomasello's account of infant pointing goes far into a mentalistic understanding of others even at this stage (see for example Tomasello et al. 2007). As Gómes (2007) has argued, there is also a more parsimonious explanation which emphasizes (as I did above) the embodied intentionality of gestures in the infant's experiential field: "behaviors are directly perceived as intentional, that is, as being directed to things other than themselves ... For example, understanding that gaze is directed to an object does not require attributing the mental experience of seeing the object - such directionality is directly attributed to gaze itself" (Gómez 2007, 730). Regarding intentional behaviour as field-related, one can even assume that an infant can "remember and predict the intentional availability of targets for others (e.g., whether they will or not be able to find an object hidden in their absence)" (l.c.).
} 
the other hand, nodding one's head represents "yes" in most cultures. Lowering the head probably meant a sort of gesture of humility signifying: I bow to what you say; I agree (Eibl-Eibesfeld 1972). These gestures are acquired in the course of the $2^{\text {nd }}$ year, with head shaking ("no") before nodding ("yes") (Kettner and Carpendale 2013).

Other gestures, which develop in the course of the second year of life, are of an iconic nature, i. e. they represent pantomime actions or recall something absent in the imagination: raising one's arms means "big", blowing means "too hot", panting represents a "dog”, flapping one's arms suggests a "bird” etc. (Tomasello 2009, $159 \mathrm{f}$.). Thus, the early development of non-verbal communication is characterized by deiktic and iconic gestures which supports an embodied view of language acquisition, although from the 14th month or so the gestures and vocalizations of this 'protolanguage' are already accompanied by the acquisition of verbal speech.

\section{(c) The Development of Language}

In the final months of the first year the words adults use to label people, objects or actions attract the infant's attention and invite imitation. Speech acquisition occurs not purely cognitively, however, as though language were just a sign system to be learned abstractly. According to the social pragmatic approach (Bruner 1983; Nelson 1996; Tomasello 2000), language acquisition is scaffolded by situations of intercorporeality, shared attention, joint practice, and ostensive cuing. The conditions for this are:

(1) the child's participation in an interactive framework that is already pre-verbally developed, in other words, verbal interaction presumes intercorporeal exchange;

(2) joint attention to a third entity, and specifically in the practical context that the speech refers to - that is, the triadic situation;

(3) understanding the communicative intentions of others as being based on their goal-directed movements, pointing or expressive gestures.

Hence, social practice represents the reference point and at the same time the scaffolding context within which a symbolic language can be learned. In concrete terms, this means that the first words are connected with already comprehensible gestures, in particular, the pointing gesture. For example, the parents ostensibly look at or point to objects and name them ("Look! A ball!"). The child now must understand that the parent intends for her (the child) to share attention with her to some outside entity, or in other words, the communicative intention (Tomasello 2000). Of course, grasping the word as meaningful does not yet imply higher conceptual capacities, but rather a typification of proto-concepts according to similarities of shape and behavior ("balls" means "such round, rolling things"). In the sequence, this leads to a reverse imitation: Now the child uses the first words ("there!", "ball”, etc.), often connected with a pointing gesture, to show the adult what she herself finds interesting and wants to share. The adult's 
understanding of the verbal gesture then acts as a reinforcement which stabilizes the new gestural meaning. ${ }^{10}$

A crucial question is how cognitively demanding this early communication should be conceived. Tomasello explains it already in terms of Grice's (1989) complex theory of language and meaning: "This is what a linguistic symbol is. It is a noise (or other behavior) that two or more individuals use with one another to direct one another's attention and thereby to share attention - and they both know this is what they are doing" (Tomasello 2000, 405). This is already a highlevel account of cognitive intentions, implying some kind of meta-perspective on the communication ("I know that you know what I mean"). It seems highly probable that this rather abstract level is only reached later on, whereas the early language use is based on situated and embodied interaction.

Thus, even if the verbal meanings can increasingly be detached from the concrete situation - at first, all of early speech acquisition is against the backdrop of interactive situations and short episodes: eating, washing, dressing, changing nappies, playing, building a tower out of blocks, feeding ducks, and so on. The child always first learns co-involvement with the relevant practical situation and to form mutual goals, and then he orders the speech, which he has heard, into this context (Bruner 1983). He learns the word "ball" when playing ball, the word "there" in association with the pointing gesture and the word "Ow!" in connection with an expression of pain etc. Children's perception of the environment is synchronized with the corresponding verbal expressions that denote it and with the adult's visible attention and intention. They only adopt a word for a new object when his or her attention is actually directed towards this object. If the adult is looking in another direction or the voice is coming from a tape, the child doesn't connect word and object (Tomasello 2000; Dittmann 2002, 43). The capacity for speech therefore only develops within social scaffolding through an intercorporeal practice that is oriented towards a shared environment.

In fact, the word is a vocal gesture and initially only complements the pointing gesture as a first sign. But the voice also separates the sign from the physical movement and transports it into the invisible, no longer localizable medium of sound (Fuchs 2010, 210). Thereby, the possibilities of referencing multiply, and ultimately the sound signs can even be detached from the concrete situation. They are capable of pointing to absent objects, for example to Mummy or Daddy when they are absent; they are even capable to pointing to "something like", that means to similar, general, or abstract objects. The gestural-iconic representation is then increasingly replaced by propositional speech, and the continued gestures accompanying verbal speech serve more visual aspects, for example, to illustrate forms, directions, and structures that are the topic of speech.

${ }^{10}$ Frequently, the interaction also selects wording from spontaneous sound production and the child's babbling, making them into meaningful signals: for example, when the child says "Mummy" or "Daddy", the parents presume her intention is to form these words and reinforce them accordingly. Recognizing the effect of her own sounds then leads the child to learn their "meaning". 


\section{Neurobiological Foundations}

As we can see from this brief outline of speech acquisition, the body as the medium of all action and interaction plays a fundamental role in the process. How is this reflected in the neuronal anchoring of language?

Neuroplasticity is a crucial presupposition for language development; in the course of meaningful interactions with others, the brain also becomes the matrix of language. Two aspects are significant here. Firstly, EEG studies show that up to the 2nd year of life the earlier developing right half of the brain which is the dominant hemisphere for processing music also manifests stronger activation while listening to language than the left half (Patel 2003; McMullen and Saffran 2004). This corresponds to the enhanced role of musical elements, namely, of speech melody, intonation, and rhythm for the perception of the toddler (Trevarthen 1998). The more advanced the development of symbolic speech, the more areas in the left brain take over verbally relevant functions, in particular, the Wernicke and Broca center and other premotor areas as well as the basal ganglia. However, even at a later stage in life, recent results suggest that the neuronal resources for processing speech and music still heavily overlap, in particular, in the Broca region and its counterpart in the right-half of the brain (Koelsch 2005, Koelsch et al. 2005). This suggests that at least in infancy the brain does not process music and speech as separate domains, but rather processes speech as a particular form of music, indeed that the musical capacities of bumans represent a decisive precondition for speech acquisition. ${ }^{11}$

Both music and language are organized temporally, with the relevant structures unfolding in time, as patterns and sequences of rhythm, emphasis, intonation, phrasing, and contour (McMullen and Saffran 2004). ${ }^{12}$ This is in correspondence with the central role of melodious-rhythmic interaction, vitality contours and affective resonance in the early mother-child dyad, which was mentioned above: The musicality of the interaction may be regarded as prefiguring the temporal dynamics in which language may then unfold. The theory of early "Communicative Musicality" is supported by acoustic analyses of the measures of rhythm, quality and dynamics in the vocal interplay between infants and adults (Malloch 1999). Here, an emotional aspect of speech development is involved that is especially manifest in prosody. Accordingly, recent neuroimaging results indicate that responses to human vocal sounds are strongest in the right superior temporal area (Belin, Zatorre, and Ahad 2002), near areas that have been implicated in processing of musical pitch (McMullen and Saffran 2004). This lends

11 The idea of singing being the ancestral origin of speech was first put forward by Giambattista Vico in his notion of "Parlare cantando" (cf. Trabant 1991).

12 This correspondence of temporal structure has already been noted by Adam Smith in his essay Of the imitative arts ([1777] 1982): "Time and measure are to instrumental music what order and method are to discourse; they break it into proper parts and divisions, by which we are enabled both to remember better what has gone before, and frequently to foresee somewhat of what is to come after ... the enjoyment of Music arises partly from memory and partly from foresight" (quoted after Trevarthen 2012, 259). 
plausibility to accounts of musical and linguistic co-evolution that emphasize emotional communication through prosody as a primary root of both systems.

The second aspect is related to the embedding of speech acquisition in interactive contexts. Specialized systems are required for the neuronal connection of action, perception, and meaning through speech, and there is now plenty of evidence to suggest a crucial role for the sensory-motor system of the mirror neurons. The localization of Broca's region in the inferior pre-motor cortex (responsible for speech production, but also for hand and mouth movement) and its coincidence with the main areas of the mirror neuron system suggests that language originally represented an interpersonal resonance system for action schemes: via the communication of the mirror neuron system, the voice was able to call up the idea of the intended actions and objects in both speaker and listener.

As mentioned above, the mirror neuron system (MNS) is activated both when observing a conspecific reach for or grasp an object and when imagining oneself reaching or grasping without actually moving one's hand. Thus, the system leads to matching an observed movement to the internally generated enactment of the same movement in the observer. ${ }^{13}$ Speculating on a connection to the evolution of language, Rizzolatti and Arbib (1998) have first assumed that the MNS also enables intentional meaning to be assigned to another's vocal gesture. The connection could be spelled out as follows (see in particular Gallese 2008; Jirak et al. 2010):

Mirror neurons also react to suggested goal-directed movements, i. e. they are activated when the hand of another individual reaches for an object that was already visible earlier, yet is now out of sight (Umiltá et al. 2001). This clearly corresponds to the pointing gesture which may be directed to a distant or even invisible object. Thus, the MNS would be suitable to support the connection of pointing and the object, by evoking one's own experience of movement and direction of gaze. The discovery of audiomotor mirror neurons in the Broca homologous area of monkeys also makes this plausible for vocal gestures (Kohler et al. 2002, Keysers et al. 2003). These neurons are activated (1) if the animal observes an action, which generates a sound - for example, knocking on a table or cracking a peanut: (2) if the animal performs the action itself, or also (3) if it only hears the knock or crack without seeing the movement. Transferring this to the voice, this would imply that the heard voice could potentially evoke the same action with an object that the listener could carry out himself.

Hence, in early speech acquisition when pointing and sound gestures are typically linked with each other, a neuronal coupling would be produced between

13 The question how this matching should be interpreted is still controversial, however. Gallese and Goldman (1998) have originally proposed a simulation theory of mind reading, and Gallese (2008) still defends an embodied simulation of others' expressions on a subpersonal level of the MNS. Such concept have been criticized by phenomenological authors, arguing against the complicated mechanism of an 'as-if'-simulation and backward projection of one's own bodily state onto others (Gallagher 2007, Fuchs and De Jaegher 2009). Instead, one's own bodily resonance may be simply inherent in one's perception of the other, namely as its'proximal' or tacit component (Fuchs 2016b). 
(1) the object being pointed to, (2) the related sound, and (3) one's own action with the object. As a result, the originally only accompanying sound becomes capable of evoking the intended object and the object-related action scheme in the listener. ${ }^{14}$ At the same time, the gesticulating pointing to objects recedes more and more into the background - as can also be observed in the development of infants.

In the acoustic medium, the word detaches itself from the speaker and is heard by him and the recipient together. The acoustic gesture is thus no longer subject-bound, but for both partners becomes a third entity, an intersubjective symbol. Mead (1973) already identified in this reciprocal aspect the decisive attribute of speech: the spoken word as a "significant gesture" becomes a symbol which basically causes the same reaction or idea in the speaker as in the listener. On a neurobiological level, this may be now understood as follows: communication in words is basically grounded in the fact that - in both speaker and listener via the medium of the MNS the word activates a congruence of neuronal patterns, and thus of ideas or action schemes. The concordant intention in both partners, which manifests itself in the word as an intersubjective symbol, would thus find its match in the resonance which forms between them on the neuronal level. Speech not only produces an intellectual connection among individuals, it additionally involves a biologically anchored interbodily resonance system. Thus, it is in virtue of our bodies acquiring, through social interaction, similar neurological structures that we can share the meaning of words and sentences. Although it must be added that the precise functional relevance of the MNS for the evolution and ontogeny of language remains far from being clarified, it already offers strong empirical support for an embodied and enactive view of language.

\section{Summary and Conclusion}

My intention in this paper was to show, based on theoretical considerations and empirical evidence, that language cannot be conceived as an abstract, disembodied system of symbols represented in the brains of separated individuals. Instead, language is both produced and understood as a form of embodied interaction, which in speaker and listener evokes the totality of possibilities for action that are mediated by the lived body. Thus, verbal communication is not a transfer of symbolic significances from one mind to another, but a "gesturing with words," co-enacting our actual and possible relations to the world, and scaffolded by our shared practical contexts. Particularly the pointing gesture, through uniting bodily movement and "we-intentionality," may be regarded as the lynchpin that leads from primary intercorporeality to the sharing of meanings through sym-

14 Apart from the studies on action-related word comprehension which were mentioned at the beginning, this connection is particularly supported by Aziz-Zadeh et al. (2006), who showed that the same cortical regions activated by action observation are also activated by the understanding of action-related sentences. 
bolic interaction. However, as Merleau-Ponty has argued, this transition never loses the gestural, enactive basis from which language first develops:

"The spoken word is a genuine gesture, and it contains its meaning in the same way as the gesture contains its. This is what makes communication possible. In order that I may understand the words of another person, it is clear that his vocabulary and syntax must be 'already known' to me. But that does not mean that words do their work by arousing in me 'representations' associated with them, and which in aggregate eventually reproduce in me the original 'representation' of the speaker. What I communicate with primarily is not 'representations' or thought, but a speaking subject, with a certain style of being and with the 'world' at which he directs his aim.” (Merleau-Ponty [1945] 1962, 213)

In other words, speech is primarily not a symbol system, but transformed gesture, enacted by the body, and evoking possible actions in it. Speaking and understanding are lived acts in which our experiences as embodied agents are always present, both in the content and in the syntactical structure that expresses it.

Speech capacity therefore does not develop merely from a biological Anlage or genetic disposition, but like no other human capacity it requires embedding in a sphere of shared meaning structures and communicative practice in order to evolve. Verbal meanings only exist between individuals just as pointing with one's finger only attains its meaning from the jointly oriented gaze. Words are carriers of intersubjective meanings, which have formed within a culture and increasingly differentiated into a complex referential system. To learn words, children must primarily be in intercorporeal, emotional and practical contact with others. They must further develop the capacity to focus on the same object and to share this intention with them. Scaffolded by these triadic practical situations the sound gestures may develop whereby we communicate with one another symbolically.

When in the embodied interaction with others the child learns their speech, then his brain functions as an organ of mediation that increasingly matches the heard words with neuronal patterns related to action, interaction and object experiences. This matching only occurs if the child experiences the others as intentional actors who intend to show him something through their speech and whose goal is the intended object. In short, the child must experience himself as the intended participant of communication. Only then - and not by means of a mechanical-associative connection - can the new words become sedimented as neuronal patterns that are associated with experiences of acting and interacting. The coupling of language perception and motor activity, which is now demonstrated by numerous imaging studies of the brain, shows that the meaning of words always remains connected to the interactive and embodied experiences in which they have been acquired.

The brain as such certainly does not become the location of meanings or the "symbol-processing organ", as it is sometimes referred to. The neuronal patterns, as correlates of speech, are only the necessary condition for the child understanding words as meaningful and thus participating in the joint world of the mind conveyed through symbols. Only such participation in the shared symbolic 
world is the sufficient condition for speech acquisition. Language is based on meanings, and meanings are ultimately based on embodied relationships. They are derived from the early childhood experience of joint attention, pointing, from the joint use of speech in practical contexts, and from the intersubjective symbolism of spoken words. Correlates of these meanings are functionally and morphologically inscribed on the brain as neuronal patterns in the course of interaction. In this way, language becomes enmeshed in our organic life: we incorporate into our bodies a linguistic style of being. This is also the reason why "linguistic events have a direct route to even our physiology, why the complex socio-cultural and interpersonal matrix disclosed by an insult or a compliment make our blood rush in quite different ways" (Cuffari, Di Paolo, and De Jaegher 2015, 1116). Language is nothing else than a manifestation of our embodied sociality.

\section{Bibliography}

Aziz-Zadeh, L. and A. Damasio. 2008. Embodied Semantics for Actions: Findings From Functional Brain Imaging. Journal de Physiologie - Paris 102: 35-39.

Aziz-Zadeh, L., S. M. Wilson, G. Rizzolatti, and M. Iacoboni. 2006. Congruent Embodied Representations for Visually Presented Actions and Linguistic Phrases Describing Actions. Current Biology 16: 1818-1823.

Bargh, J. A. and I. Shalev. 2012. The Substitutability of Physical and Social Warmth in Daily Life. Emotion 12(1): 154-62.

Barsalou, L. W. 2008. Grounded cognition. Annual Review of Psychology 59: 617-645.

Belin, P., R. J.Zatorre, and P. Ahad. 2002. Human Temporal-Lobe Response to Vocal Sounds. Cognitive Brain Research 13: 17-26.

Bråten, S. and C. Trevarthen. 2007. Prologue: From Infant Intersubjectivity and Participant Movements to Simulation and Conversation in Cultural Common Sense. In On Being Moved: From mirror neurons to empathy, ed. S. Bråten, 21-34. Amsterdam: John Benjamins Publishing.

Bruner, J. 1983. Child's Talk. New York: Norton.

Binkofski, F. and G. Buccino. 2004. Motor Functions of Broca's Region. Brain and Language 89: 362-369.

Boulenger, V., O. Hauk, and F. Pulvermüller. 2009. Grasping ideas with the motor system: semantic somatotopy in idiom comprehension. Cerebral cortex 19: 1905-1914.

Buccino, G., L. Riggio, G. Melli, F. Binkofski, V. Gallese, and G. Rizzolatti. 2005. Listening to Action-Related Sentences Modulates the Activity of the Motor System: A Combined TMS and Behavioral Study. Cognitive Brain Research 24: 355-363.

Cuffari, E. C., E. Di Paolo, and H. De Jaegher. 2015. From Participatory Sense-Making to Language: There and Back Again. Phenomenology and the Cognitive Sciences 14: 1089-1125.

De Jaegher, H. and E. Di Paolo. 2007. Participatory sense-making: an enactive approach to social cognition. Phenomenology and the Cognitive Sciences 6: 485-507.

Darwin, C. (1872) 1998. The Expression of the Emotions in Man and Animals. Introduction, Afterword and Commentaries by P. Ekman. London: Harper Collins Publishers.

Dittmann, J. 2002. Der Spracherwerb des Kindes. Verlauf und Störungen. München: Beck.

Eibl-Eibesfeldt, I. 1972. Similarities and Differences Between Cultures and Expressive Movements. In Non-verbal Communication, ed. R. A. Hinder, 37-48. Cambridge: Cambridge University Press. 
Fernald, A. 1992. Human Maternal Vocalizations to Infants as Biologically Relevant Signals. In Adapted Mind, ed. J.H. Barkow, L. Cosmides, and J. Toby, 391-426. New York: Oxford University Press.

Fodor, J. 1975. The Language of Thought. Cambridge: Harvard University Press

-. 1983. The Modularity of Mind. Cambridge, MA: MIT Press.

Fuchs, T. 2010. Das Gehirn - Ein Beziehungsorgan. Eine Phänomenologisch - Ökologische Konzeption. 3rd edition, Stuttgart: Kohlhammer.

-. 2011. The Brain - A Mediating Organ. Journal of Consciousness Studies 18: 196-221.

-. 2013. The Phenomenology and Development of Social Perspectives. Phenomenology and the Cognitive Sciences 12: 655-683.

-. 2016a. Embodied Knowledge - Embodied Memory. In: Analytic and Continental Philosophy. Methods and Perspectives. Proceedings of the 37th International Wittgenstein Symposium, ed. S. Rinofner-Kreidl, H. Wiltsche, 215-229. Berlin: De Gruyter.

-. $2016 \mathrm{~b}$ (in press). Intercorporeality and Interaffectivity. In: Intercorporeality: Emerging Socialities in Interaction, ed. C. Meyer, J. Streeck and S. Jordan. Oxford: Oxford University Press.

Fuchs, T. and H. De Jaegher. 2009. Enactive Intersubjectivity: Participatory Sense Making and Mutual Incorporation. Phenomenology and the Cognitive Sciences 8: 465-486.

Gallagher, S. 2007. Simulation trouble. Social Neuroscience 2: 353-365.

Gallese, V. and A. Goldman. 1998. Mirror Neurons and the Simulation Theory of Mind Reading. Trends in Cognitive Science 12: 493-501.

Gallese, V. 2008. Mirror Neurons and the Social Nature of Language: The Neural Exploitation Hypothesis. Social Neuroscience 3: 317-333.

Glenberg, A. M. and D. A. Robertson. 2000. Symbol Grounding and Meaning: A Comparison of High-Dimensional and Embodied Theories of Meaning. Journal of Memory and Language 43: 379-401.

Glenberg, A. M., M. Sato, L. Cattaneo, L. Riggio, D. Palumbo, and G. Buccino. 2008. Processing Abstract Language Modulates Motor System Activity. The Quarterly Journal of Experimental Psychology 61: 905-919.

Gómez, J.-C. 2007. Pointing Behaviors in Apes and Human Infants: A Balanced Interpretation. Child Development 78: 729-734

Grice, P. 1989. Studies in the Way of Words. Cambridge, MA: Harvard University Press.

Harnad, S. 1990. The Symbol Grounding Problem. Physica D 42: 335-346.

Kettner, V. A. and J. I. Carpendale. 2013. Developing Gestures For No and Yes: Head Shaking and Nodding in Infancy. Gesture 13: 193-209.

Irwin, B. A. 2015. An Enactivist Account of Abstract Words: Lessons From MerleauPonty. Phenomenology and the Cognitive Sciences (published online-first).

Jirak, D., M. M. Menz, G. Buccino, A. M. Borghi, and F. Binkofski. 2010. Grasping Language - A Short Story on Embodiment. Consciousness and Cognition 19: 711-720.

Johnson, M. 1987. The Body in the Mind. Chicago: University of Chicago Press.

Kaschak, M. P., R. A. Zwaan, M. Aveyard, and R. H. Yaxley. 2006. Perception of Auditory Motion Affects Language Processing. Cognitive Science 30(4): 733-744.

Keysers, C., E. Kohler, M. A. Umiltà, L. Nanetti, L. Fogassi and V. Gallese. 2003. Audiovisual Mirror Neurons and Action Recognition. Experimental Brain Research 153: 628-636.

Kleist, H.v. (1805) 1951. Über die allmähliche Verfertigung der Gedanken beim Reden. trans. M. Hamburger: On the Gradual Construction of Thoughts During Speech. German Life and Letters 5: 42-46.

Kluge, F. 1989. Etymologisches Wörterbuch der Deutschen Sprache. 22. Aufl. Berlin: De Gruyter. 
Koelsch, S. 2005. Ein neurokognitives Modell der Musikperzeption. Musiktherapeutische Umschan 26: 365-381.

Koelsch, S., T. Fritz, K. Schulze, D. Alsop, and G. Schlaug. 2005. Adults and Children Processing Music: An fMRI Study. Neuroimage 25: 1068-1076.

Kohler, E., C. Keysers, A. Umiltà, L. Fogassi, V. Gallese and G. Rizzolatti. 2002. Hearing Sounds, Understanding Actions: Action Representation in Mirror Neurons. Science 297: 846-848.

Lakoff, G. and M. Johnson. 1980. Metaphors We Live By. Chicago: University of Chicago Press.

Lee, S. W.S. and N. Schwarz. 2011. Clean Slate Effects: The Psychological Consequences of Physical Cleansing. Current Directions in Psychological Science 20: 307-311.

Liszkowski, U., M. Carpenter, T. Striano, and M. Tomasello. 2006. 12- and 18-Month-Olds Point to Provide Information for Others. Journal of Cognition and Development 7: 173-187.

Malloch, S. N. 1999. Mother and Infants and Communicative Musicality. Musice Scientice 3(1): 29-57.

McMullen, E. and J. R. Saffran. 2004. Music and Language: A Developmental Comparison. Music Perception: An Interdisciplinary Journal 21: 289-311.

Mead, G. H. 1973. Geist, Identität und Gesellschaft. Frankfurt: Suhrkamp.

Meier, B. P., S. Schnall, N. Schwarz and J. A. Bargh. 2012. Embodiment in Social Psychology. Topics in Cognitive Science 4(4): 705-716.

Meltzoff, A. N. and M. K. Moore. 1977. Imitation of Facial and Manual Gestures by Human Neonates. Science 198: 74-78.

-. 1989. Imitation in Newborn Infants: Exploring the Range of Gestures Imitated and the Underlying Mechanisms. Developmental Psychology 25: 954-962.

Meltzoff, A. N. and R. Brooks. 2001. 'Like me' as a Building Block for Understanding Other Minds: Bodily Acts, Attention, and Intention. In Intentions and Intentionality: Foundations of Social Cognition, ed. B. F. Malle, L. J. Moses, and D. A. Baldwin, 171-191. Cambridge, MA: MIT Press.

Meltzoff, A. N. and W. Prinz. 2002. The Imitative Mind. Development, Evolution and Brain Bases. Cambridge, MA: Cambridge University Press.

Merleau-Ponty, M. (1945) 1962. Phénomenologie de la Perception. Paris: Gallimard. trans. C. Smith: Phenomenology of Perception. London: Routledge and Kegan Paul.

-. 1960. Le Philosophe et Son Ombre. In Signes. Paris: Éditions Gallimard.

Murray, L. and C. Trevarthen. 1985. Emotional Regulation of Interactions Between Two-Montholds and Their Mothers. In Social perception in infants, ed. T. M. Field and N. A. Fox, 177-97. Norwood, N. J.: Ablex.

Nelson, K. 1996. Language in Cognitive Development. Cambridge University Press, Cambridge.

Oostenbroek, J., Suddendorf, T., Nielsen, M., Redshaw, J., Kennedy-Costantini, S., Davis, J., Clark, S., and Slaughter, V. 2016. Comprehensive Longitudinal Study Challenges the Existence of Neonatal Imitation in Humans. Current Biology 26: 13341338.

Papoušek, M. 1994. Melodies in Caregivers' Speech: A Species-Specific Guidance Towards Language. Early Development and Parenting 3: 5-17.

Papoušek, H. and M. Papoušek. 1987. Intuitive Parenting: A Dialectic Counterpart to the Infant's Integrative Competence. In Handbook of Infant Development, ed. H. R. Schaffer, 67-85. London: Academic Press.

-. 1995. Vorsprachliche Kommunikation: Anfänge, Formen, Störungen und psychotherapeutische Ansätze. In Die Kraft liebevoller Blicke. Psychotherapie und Babyforschung Bd. II., ed. H. G. Petzold, 123-142. Paderborn: Junfermann. 
Patel, A. 2003. Language, Music, Syntax and the Brain. Nature Neuroscience 6: 674-681.

Piaget, J. (1936) 1952. The Origins of Intelligence in Children. New York: International University Press.

Pulvermüller, F. 2005. Brain Mechanisms Linking Language and Action. Nature Rev. Neuroscience 6: $576-582$.

Pulvermüller, F., M. Huss, F. Kherif, F. M. del Prado Martin, O. Hauk, and Y. Shtyrov. 2006. Motor Cortex Maps Articulatory Features of Speech Sounds. Proceedings of the National Academy of Sciences 103: 7865-7870.

Pulvermüller, F. and L. Fadiga. 2010. Active Perception: Sensorimotor Circuits as a Cortical Basis for Language. Nature Rev. Neuroscience 6: 576-582.

Pylyshyn, Z. W. 1984. Computation and Cognition: Toward a Foundation for Cognitive Science. Cambridge, MA: MIT Press.

Rizzolatti, G. and M.A. Arbib. 1998. Language Within Our Grasp. Trends in Neurosciences 21: 188-194.

Schnall, S., J. Benton, and S. Harvey. 2008. With a Clean Conscience: Cleanliness Reduces the Severity of Moral Judgments. Psychological Science 19: 1219-1222.

Spitz, R. A. 1957. No and Yes: On the Genesis of Human Communication. New York: International Universities Press.

Stern, D. N. 1998. Die Lebenserfahrungen des Säuglings. Stuttgart: Klett-Cotta.

Tomasello, M. 2002. Die kulturelle Entwicklung des menschlichen Denkens. Zur Evolution der Kognition. Frankfurt: Suhrkamp.

-. 2008. The Origins of Human Communication. Cambridge, MA: MIT Press.

Tomasello, M., M. Carpenter, J. Call, T. Behne, and H. Moll. 2005. Understanding and Sharing Intentions: The Origins of Cultural Cognition. The Behavioral and Brain Sciences 28: 675-735.

Tomasello, M. and M. Carpenter. 2007. Shared Intentionality. Developmental Science 10: 121-125.

Tomasello, M., Carpenter, M., and Liszkowski, U. 2007. A New Look at Infant Pointing. Child Development 78: 705-722.

Trabant, J. 1991. Parlare Cantando: Language Singing in Vico and Herder. New Vico Studies 9: 1-16.

Trevarthen, C. 1979. Communication and Cooperation in Early Infancy: A Description of Primary Intersubjectivity. In Before Speech, ed. M. Bullowa, 321-347. Cambridge, MA: Cambridge University Press.

-. 1998. Language Development: Mechanisms in the Brain. In Encyclopedia of Neuroscience, ed. G. Adelman and B. Smith, 1018-1026. Amsterdam: Elsevier.

-. 2001. The Neurobiology of Early Communication: Intersubjective Regulations in Human Brain Development. In Handbook of Brain and Behaviour in Human Development, ed. A. F. Kalverboer, A. Gramsberg, 841-881. Dordrecht, Boston, London: Kluwer Academic Publishers.

-. 2008. The Musical Art of Infant Conversation: Narrating in the Time of Sympathetic Experience, Without Rational Interpretation, Before Words. Musicae Scientiae 12: $15-46$.

-. 2009. The Functions of Emotion in Infancy: The Regulation and Communication of Rhythm, Sympathy, and Meaning in Human Development. In The Healing Power of Emotion: Affective Neuroscience, Development, and Clinical Practice, ed. D. Fo-sha, D. J. Siegel, and M. F. Solomon, 55-85. New York: Norton.

-. 2012. Communicative Musicality: The Human Impulse to Create and Share Music. In Musical Imaginations: Multidisciplinary Perspectives on Creativity, Performance, and Perception, ed. D. Hargreaves, D. Miell, and R. MacDonald, 259-284. Oxford: Oxford University Press. 
Trevarthen, C. and P. Hubley. 1978. Secondary Intersubjectivity: Confidence, Confiding and Acts of Meaning in the First Year. In Action, Gesture and Symbol: The Emergence of Language, ed. A. E. Lock, 183-229. London, Oxford: Academic Press.

Turati, C., F. Simion, I. Milani, and C. Umiltà. 2002. Newborns' Preference for Faces: What is Crucial? Developmental Psychology 38: 875-882.

Umiltà, M. A., E. Kohler, V. Gallese, L. Fogassi, L. Fadiga, C. Keysers, and G. Rizzolatti. 2001. I Know What You Are Doing: A Neurophysiological Study. Neuron 31: 155-165.

Valenza, E., F. Simion, V. M. Cassia, and C. Umiltà. 1996. Face Preference at Birth. Journal of Experimental Psychology: Human Perception and Performance 22(4): 892-903.

Varela, F. J., E. Thompson, and E. Rosch. 1991. The Embodied Mind: Cognitive Science and Human Experience. Cambridge, MA: MIT Press.

Weick, K. E. 1995. Sensemaking in organizations. Thousand Oaks, CA: Sage.

Willems, R. M., P. Hagoort, and D. Casasanto. 2010. Body-Specific Representations of Action Verbs. Neural Evidence From Right- and Left-Handers. Psychological Science 21: 67-74.

Wilson, S. M., A. P. Saygin, M. I. Sereno, and M. Iacoboni. 2004. Listening to Speech Activates Motor Areas Involved in Speech Production. Nature Neuroscience 7: 701-702.

Winter, B. and B. Bergen. 2012. Language Comprehenders Represent Object Distance Both Visually and Auditorily. Language and Cognition 4: 1-16.

Ziemke, T. 2002. Special Issue on Situated and Embodied Cognition. Cognitive Systems Research 3(3): 271-274.

Zlatev, J. 2007. Embodiment, Language and Mimesis. In Body, Language and Mind vol.1, ed. T.Ziemke, J.Zlatev, and R. M. Frank, 297-337. De Gruyter Mouton.

Zhong, C. B. and G. J. Leonardelli. 2008. Cold and Lonely: Does Social Exclusion Feel Literally Cold? Psychological Science 19: 838-842.

Zhong, C. B. and K. Liljenquist. 2006. Washing Away Your Sins: Threatened Morality and Physical Cleansing. Science 313: 1451-1452.

Zwaan, R. A., C. J. Madden, R.H. Yaxley, and M. E. Aveyard. 2004. Moving Words: Dynamic Representations in Language Comprehension. Cognitive Science 28: 611-619. 


\title{
On Human (Symbolic) Nature: How the Word Became Flesh
}

\author{
Terrence W. Deacon
}

\begin{abstract}
The concept of human nature has been challenged by social scientists because of its inability to clearly delineate the distinction between the biologically inherited and experientially acquired attributes of being human. Yet the very fact of being susceptible to acquired cultural influences irrelevant to other species makes clear that this is an evolutionarily constrained susceptibility. Symbolic processes are the source of the most important and distinctively human acquired influences, and include both linguistically mediated and habitually reproduced social conventions. Susceptibility to these influences arose due to the evolution of neurological adaptations that support symbolic communication and cognition. Although human brains do not include any structures that lack ape homologues, the slight reorganization that made symbolic abilities ubiquitous has also created the possibility for socially transmitted information to radically reorganize mental functions. In this essay I re-analyze the concept of symbolic reference in order to overcome equivocal and ambiguous uses of the concept that obscure the special nature of these adaptations and thus blind research to the complex bio-cultural interactions that produce some of the most ubiquitous and unprecedented features of being human. These include modifications of memory functions, emotional experiences, the nature of identity, and the range of mental plasticity.
\end{abstract}

\section{Introduction}

Humans are a member of the family of African apes that also includes gorillas, chimpanzees, and bonobos. And yet this phylogenetic characterization of our species provides a very misleading characterization of what is shared with our ape cousins and what makes us distinct - in other words, what has traditionally been called human nature. The concept of human nature has a long and troubled history (Bock 1980, Hull 1986). Most often it has been used to designate some putative set of universally shared biologically inherited psychological traits constituting the so-called "psychic unity" of humankind (the term "psychic" is used here and below in its traditional non-mystical sense). As an essentialist enterprise, the effort to enumerate this set has failed (Ingold 2006). This turned out to be futile for the same reason that identifying the set of physiognomic traits that are shared by all and only members of the same species fails to unambiguously define a species.

Today, in the era of gene sequencing, taxonomists ground their determination of biological group membership on common genetic ancestry. Thus, as the open- 
ing statement of this essay indicates, we humans derive our genetic inheritance from the common ancestor of all African apes. Indeed, using genetic criteria it is possible to trace our ancestry all the way back to the so-called last universal common ancestor (LUCA) of all life on earth, grouping us with ever-larger more inclusive clades. The new rule of thumb for analyzing biological ancestry could thus be characterized as "follow the (genetic) information." Membership within a phylogenetic group - whether species or kingdom - is ultimately determined by all members sharing genetic continuity through a common ancestor.

But this cladistic approach, if pursued exhaustively, can lead to a problematic result even just in terms of phylogenetic genealogy. This is because organisms include large amounts of genetic material that gets shuffled and recombined during evolution, and in some case can involve highly distinct inheritance pathways. For example, the lateral transfer of genetic material between bacteria makes simple genealogical determination of the equivalent of a bacterial species essentially impossible. But even eukaryotic organisms such as ourselves, have dual ancestry. Though this is not the result of lateral gene transfer or because we have two parents. It is because the genealogies of our nuclear and mitochondrial genomes are quite radically separate. Our nuclear genes place us in a lineage that traces back to primitive single cell Archaea, whereas our mitochondria have a separate genome that shows them to be members of the Bacteria. These genetic lineages evolved in parallel and with respect to one another due to the endosymbiosis of a bacterial lineage within an archaean cell lineage well over a billion years ago. In the subsequent epoch of eukaryotic evolution, each genome has undergone radical reorganization in functional interaction with the other. As a result, there has been a sort of informational, though not molecular, lateral influence of the one on the other.

In many respects we humans also have dual psychic ancestry. One lineage is continuous with our African ape cousins, tracing back from there to all primates, mammals, terrestrial vertebrates, and so on. I will describe it as our primate mental genealogy, though many features antedate the origin of the primate clade. The other lineage is not traceable through molecular genetics, but through continuous social transmission. I will describe this as our symbolic genealogy.

This dual inheritance perspective is not new. And in many respects it remains quite controversial to describe it in terms of parallel evolutionary processes. But the relationship I am describing should not be confused with dual inheritance theories that dichotomize genetic and social transmission processes, as this is defined in so called bio-social evolution theories. In many respects my point is the opposite. It is their inextricable entanglement that I want to emphasize.

The interactions between biological and social information transmission is not what distinguishes these two cognitive-behavioral genealogies. The significant roles of epigenetic and niche-borne sources of inherited biases and constraints on the development of behavior and cognition are now well established. This undermines simple innate/learned, nature/nurture conceptions of behavioral evolution in social species in general, not just in humans. The transmission of cognitive, behavioral, and social predispositions down the primate-human lineage neces- 
sarily involved both social continuity and genetic continuity. So it is not the difference between molecular genetic and social transmission that I am interested in highlighting, but rather the entanglement of the symbolic component of social inheritance with non-symbolic social-cognitive inheritance, and how this interaction has influenced the evolution of their genetically and epigenetically mediated biological supports.

This brings up another parallel with the evolution of eukaryotic cells that should be mentioned before proceeding. The coupling of previously autonomous organisms which ultimately gave rise to mitochondrial and nuclear genomes did not merely constitute a cooperative relationship. It gave rise to an unprecedented new level of biological unity, and with it unprecedented new modes of evolution. The genetic information that characterized each of these once independent lineages has been fundamentally changed by their fusion and co-dependence. Each has been degraded and modified by the ubiquitous presence of the other over more than a billion years to the point that they are no longer even separable informational lineages.

Unlike the two lineages that came together to give rise to eukaryotic organisms, however, our primate and symbolic psychic lineages had unrelated origins and originated in epochs separated by tens of millions of years. The many overlapping cognitive, sensory-motor, and social-emotional predispositions that monkeys and apes inherited from the common ancestral anthropoid primate arose within the last 60 million years. In contrast the unbroken lineage of symbolic information almost certainly doesn't extend back more than about 2.5 million years, and may have a far more recent origin. The radical incommensurability of these yoked semiotic genealogies is what makes human nature so difficult to characterize in typical biological terms, and also dooms any effort to partition humanness into inherited and acquired (e. g., nature versus nurture) components. It is an unprecedented entanglement of genealogies that comprises a highly distinctive universally shared nature that sets us apart from all other species on earth.

Like the separate but interdependent genetics of mitochondrial and nuclear DNA, our symbolic genealogy has significantly influenced the evolution of our primate psychic genealogy, and vice versa. Like the fused genetic lineages that became eukaryotic information, these two psychic lineages that became entangled during our distinctive ancestry are no longer separable. Each has been fundamentally transformed by the other over this shared evolutionary history so that our biology can't be understood apart from the symbolic cultural context that it is informed by. This is why in my 1997 book (Deacon 1997) I described humans as a "symbolic species," analogous to the way we might characterize birds as aerial species and dolphins as aquatic species. But unlike these ecologically specialized lineages, the symbolic "ecology" that humans evolved to fit is not external to the human lineage. It is inextricably part of it. 


\section{Symbols Oversimplified}

In my view one of the most serious impediments to understanding what is distinct about human cognition is a fundamental misunderstanding about the nature of symbolic reference. As a result, our ability to understand the influence of our symbolic genealogy is severely compromised. This difficulty needs to be cleared up before we can hope to make sense of the common essence that defines humanness.

Ironically, the symbol concept has been the victim of oversimplification by linguistic theory itself. Language is the very phenomenon most in need of explanation and yet its unprecedented mode of referring is often treated as though it is self-evident. According to this common conception of linguistic symbolization it is merely an arbitrary (unconstrained) mapping relation between two classes of objects - signifiers and what they signify - linked only by convention.

From this perspective, linguistic reference appears as simple as possible, involving minimal defining criteria compared to other forms of reference. In comparison, iconic signs require some formal correspondence with what they signify and indexical signs require some factual connection with what they signify. Conceived in these terms, symbolic reference is often defined negatively; simply as reference that does not depend on either criterion; thus arbitrary. It would seem, then, that symbolic reference is primary and both iconic and indexical reference forms are the more complex and derived forms.

But this superficial characterization is deeply flawed. Bracketed from this analysis is any consideration of how these relations are interpreted and how they could have become established. Much of this is cryptically supplied by an unanalyzed concept of convention. Most uses of the term assume that it refers to formally or tacitly agreed-upon rules or habits of behavior or patterns of use in some domain of social interaction. Its basis has become a topic of considerable philosophical debate with such philosophical luminaries as Quine, Davidson, Lewis, and Millikan (to name just a few) presenting conflicting accounts. However, what is common to all is the recognition that the establishment of conventions occurs in a context of end-directed interaction among agents that each interpret the behaviors of the others as significant in some respect. In other words, conventions result from complex semiotic processes that are not at all arbitrary.

Though the problem doesn't begin with Ferdinand de Saussure's theory of language, the influence of this theory has perpetuated the problem. The concept of arbitrarity pertains to the relationship between sign vehicle attributes and the attributes of what it signifies. The problem arises from a synchronic (a.k.a. structuralist) account of how words refer. Only paying attention to the obvious fact that word sounds do not mimic or resemble what they refer to, their linkage to what they are about seems merely arbitrary. Moreover, signifiers (as Saussure termed the sign vehicles of language, e. g., words) are treated as the most basic types of signs by virtue of being defined by lacking properties shared with what they signify. 
Contrasting this with sign forms that share properties with what they signify, symbolic forms thereby appear arbitrary and simple. A bit of careful reflection, however, demonstrates that it is not sign vehicle attributes that determine whether reference is being mediated by iconic, indexical, or symbolic means.

Arbitrariness is a negative way of defining symbols. It basically tells us that neither formal likeness nor factual correlation are used as the basis for symbolic reference. But this is inadequate. It fails to specify exactly how the symbolic referential relation is established. So, even though this is a common shorthand way of characterizing symbolic reference it merely passes the buck, so to speak, to some assumed and undescribed means by which reference is established. In fact, all semiotic relationships include some degree of arbitrarity, because those attributes that are taken as the ground for the sign-object linkage can be chosen from many dimensions, and only some will be utilized (if any). What matters, then, is the interpretive process and how it makes use of sign vehicle attributes and the attributes of what is intended as a referent.

In this respect, we can say that anything can be chosen as a sign vehicle to represent anything else and by any mode, depending on the interpretive process involved. For example, depending on the interpreter and social context the same facial grimace can be interpreted as a sign of pain, a neurological disorder, or the communication of social censure. Or the sight of smoke can be seen to resemble a cloud, to indicate a fire, or to announce the election of a pope. The relevant property is selected by the interpreter and what is being referred to depends on the capacity of the interpreter to use that property in a particular way.

So, turning this initial logic of semiotic categorization on its head we can ask: What constitutes the interpretive capacity to comprehend a given referential relationship as either iconic, indexical, or symbolic? In the case of iconic and indexical relationships the sign vehicle itself includes properties that provide some evidence as to its relation to what it refers to. But where there is no interpretive support given by any sign vehicle property, all the interpretive clues must be supplied by the interpreter. This is, of course, made obvious when a traveler is confronted with an unfamiliar language or when an archaeologist comes upon a stone tablet inscribed with an unknown form of writing. Without some external source of information, some non-arbitrary semiotic basis, the meaning is effectively encrypted. In contrast, even though we know nothing of the beliefs and rituals of the stone-age people who painted on cave walls in Southern France, we immediately recognize the idealized forms of animals and humans painted by these stone-age "artists." Interpreting their iconic content is almost trivial, while interpreting their symbolic significance is forever beyond our reach. In order to be able to interpret the symbolic meaning of cave paintings or the words of an ancient lost language one would need some prior experience with the patterns and habits of use of these symbolic artifacts within a cultural network of other icons, indices, and symbols. In other words, this requires acquaintance with the semiotic conventions in which the symbols are embedded.

Similarly, children acquire their facility with language as its symbols are embedded in activities of pointing, soliciting shared attention, playful and instru- 
mental interactions, observing the complex correspondences between symbols, objects, and events in social activities, etc. All of these activities are constituted by non-symbolic forms of communication and interpretation. Only in this way can the apparent arbitrarity of symbolic reference be grounded in communications and actions that are not arbitrary.

So by simply equating symbolic reference with arbitrary correspondence its complex infrastructure and historical grounding is ignored. In addition, the simplified assumption that the nature of convention is just a correspondence relationship glosses over another critical distinction. This is the distinction between properties that distinguish types of sign vehicles and properties that determine how a sign vehicle refers to something that it is not. Both relations involve conventionality, but in different and partially independent ways. Symbols are in this respect doubly conventional. They are conventionally derived sign vehicles that refer to things via conventionally determined means.

As the father of semiotic theory Charles Peirce pointed out over a century ago, we must distinguish properties of a sign vehicle (e.g., a drawing or spoken word), from properties taken to link it to its object of reference. So a conventional sign vehicle can serve as the basis for iconic and indexical reference, as well as symbolic. There can be conventionalized typographical icons (e.g., the typographical smiley face).

Iconic reference depends on shared formal properties between the sign vehicle and what it refers to. For example, with a bit of imagination a face can be discerned on the full moon, or in a cloud formation, and it might even remind you of someone you know. But iconism can also be highly abstract. A mathematical equation refers iconically, once you know how to discern its symbol-mediated isometry (e. g., between the structure of the interpreted equation and a corresponding geometric or dynamical relationship). An equation can be interpreted to be iconic (e.g., of a parabolic trajectory) only, however, if you know how to discern the way that differences in the mathematical values or operations directly correspond to differences in the geometric object of reference.

Indices refer by contiguity in space, time, or deriving from a common substrate. A simple correlation can therefore be the ground for indexical reference. A lipstick smear on a man's shirt collar can be a troublesome indication to his wife, a urine scent on a branch can be a sexual index to a female lemur, and the mobbing call of a small bird can indicate the present of a raptor. What gets correlated and how (accidental, cultural, evolutionary) is arbitrary, but the fact of correlation is not.

A rat in a Skinner box pressing a bar in response to a bell in order to get a water reward has learned that the bell is an index of the state of the apparatus even though this pairing was the whim of an experimenter. The arbitrary pairing doesn't make the one a symbol of the other. Repeated pairing over the course of generations in the evolution of a species can similarly be the basis for an innate tendency to interpret something indexically. This is the case for the indexicality of a vervet monkey alarm call sound and its correlation with the presence of a type of predator and the urge to engage in an appropriate defense activity or 
escape behavior. Conventionalized indices are also quite common. For example, consider the white line painted down the middle of two lane roads. Though it is a conventionalized sign it refers indexically, not symbolically.

However, by virtue of an unfortunate shorthand it has become standard terminology to call most conventional sign vehicles symbols. Thus current vernacular habitually terms alphanumeric characters symbols. This usage reflects the fact that they have been explicitly developed over millennia to express symbolic relations. But when your computer begins randomly spewing alphanumeric characters onto your screen they are indices of a malfunction, not symbols of anything.

As Peirce recognized, symbolic reference involves a conventional type of sign vehicle that additionally represents its object of reference in a conventionally-mediated way (Peirce 1931). Something can be considered symbolic, then, only if the property determining its relationship to what it refers to is also a convention. Symbols are in this sense doubly conventional. So arbitrarity, by itself, is not diagnostic of symbolic reference. Nor can it be a critical defining feature of language.

Elsewhere (Deacon 1997, 2003) I have argued that although innate iconicity and indexicality can evolve by natural selection, symbolic reference cannot. This is because of their displacement from features shared with what they represent. Symbols lack the reliably repeated associations between properties that natural selection requires. This explains why there are no innate words, only innate calls (like laughter or sobbing). These are linked to reliable emotional states and characteristic external conditions, which reliably elicit these states.

This requirement has other important implications about language evolution, however, that are not generally recognized. Grammatical rules are even more abstract and less reliably correlated with anything in the brain or in the world than are words. In particular, the surface properties of utterances that provide the grammatical and syntactic information required to interpret a sentence are even less directly correlated with any physical property or relation in the world than are words. So they should be even less likely to arise as a product of biological evolution.

Yet the evolution of the capacity to communicate symbolically has clearly occurred during the course of human prehistory. We humans are the only species that regularly and reliably uses symbolic communication, and even with significant brain damage and mental impairment it is often still possible for people to have some linguistic facility. In contrast, even with extensive training, other quite intelligence species (e.g., apes, dolphins, and possibly parrots) can only acquire very minimal symbolic communicative abilities. This implies that there must have been significant atypical modifications to human brains in the course of their evolution to adapt them to the demands of symbol acquisition and use. So although there can be no innate symbols, there can be evolution to make the acquisition and use of symbolic communication comparatively easy. In turn, however, the regular use of symbolic communication and reasoning over our protracted evolutionary past as a symbolic species has almost certainly changed the ways we humans use even those cognitive abilities that long predate our symbolic awak- 
ening. These evolved modifications of human mental capacities have given rise to an unprecedented symbolic niche - culture - that forms the ubiquitous semiotic ecosystem in which we develop in response to. This atypically modified neurology and radically restructured social-ecological context together are responsible for the unprecedented deviation of human biology from the biology of effectively every other species on earth. So the virtual semiotic world of symbols in which we live and to which we have been innately predisposed and developmentally shaped has produced what I describe as our distinctive human symbolic nature.

\section{How the Disembodied Became Embodied}

To begin to understand the cognitive and neurological adaptations that aid symbolic thought and communication it is useful to first ask: Why is symbolic reference intrinsically difficult for non-human species?

One reason is that learning a symbolic relationship is a bit like cracking a code. One not only needs information about what refers to what, but also needs to understand the system of indexical relations linking symbols to one another and how this system relates to the physical world of properties, objects, and relations. This is because symbolic reference is intrinsically systemic and virtual. Learning correlations between symbols and objects is a start, but one must recognize that this is only a clue to the hidden logic of symbol-symbol (token-token) relationships (i. e., a network of indexical relations such as a linguistic lexicon). Recognizing the systemic correlations between symbol-symbol relations and relationships between properties and events in the real world requires the capacity to transfer relational information from one cognitive context to another quite different and abstract one. This demands a higher-order form of learning, something like an "insight," in which one learns how to re-use selected aspects of what has been learned in new ways.

Insight learning was first systematically studied by a Gestalt theorist: Wolfgang Kohler (1925). He was interested in how apes might problem-solve in a novel context in which the solution requires using previously acquired skills in novel combinations. For this he created "puzzles" in which trial and error problem solving would not have worked, such as piling boxes on top of one another and climbing on them to reach a suspended banana. To succeed it was necessary for the chimpanzee subject to be able to mentally experiment with new combinations of already understood relationships before enacting a plan of action. As a result, after a period of frustrated trial and error, one chimpanzee (Sultan) was seen to suddenly and deliberately arrange things to achieve the result, without trial and error approximations.

The role of insight learning with respect to language acquisition is strikingly exemplified by the account of Helen Keller's discovery of the symbolic nature of language (1905). Blind and deaf from a very early age, she had still not acquired language by early middle childhood. Her tutor, Anne Sullivan, eventually helped her acquire language abilities using a version of finger spelling that 
was impressed on her hand. Though Helen had acquired a modest set of signs that she could use to identify objects, the point at which she discovers their symbolic function, as opposed to just their indexical correlation with immediately touched objects, is described as a momentary insight. In an event that both Helen and Anne recount in their memoirs, it was an emotionally charged interaction taking place at a water pump that caused Helen to become aware that the signs she already knew constituted an interdependent system of relations that could be used to communicate about things and events not immediately present. In effect, she restructured knowledge that she had previously acquired into a system rather than merely a collection of correlations.

The transition from the one-to-one correlational relationships that is the ground of indexical reference to the system-to-system relationships that ground symbolic reference, as occurred in Helen Keller's epiphany, is not merely learning. It is rather a restructuring of existing knowledge due to the recognition of a previously unnoticed global relational iconism. So the ability to utilize higher-level relational thinking across different cognitive tasks and domains is essential to symbolic communication and reasoning because of the way symbolic reference is mediated by system-to-system correspondences. In other words, it is the recognition that the relational properties among symbol tokens abstractly mirror the abstract form of the relationships among properties, objects, and events in the world.

Recently this has become investigated in terms of a more general capacity, sometimes referred to as transfer learning. It is implicated in a variety of cognitive capacities. These include thinking in terms of analogies, understanding indirect inferential relations, dealing with complicated combinatorial relationships, and using information acquired in one domain to reason about problems in a very different domain. Importantly, all of these cognitive operations place a heavy burden on prefrontal cortex functions (e. g., see Deacon 1997; Vendetti and Bunge 2014; Yarkoni et al. 2011; among many others). Likewise studies attempting to directly test the cognitive-neural demands of making the shift from indexical to symbolic interpretations of the same relationships (e. g., Nieder 2009) have specifically shown the relevance of these prefrontal-dependent capacities for the origins of symbolic capacities.

In my 1997 book The Symbolic Species I review evidence from comparative neuroanatomy that suggests that major quantitative changes in the proportions of different human brain structures reflect adaptations for the unusual cognitive demands imposed by symbolic communication. In summary, I showed how embryological divergence of human brain/body proportions as compared to our close primate relatives altered axonal competition during development, favoring connections from relatively enlarged structures. The result was that reduced peripheral connections enabled structures less directly linked to the sensory-motor periphery to outcompete other connections. I predicted that this would have a number of structural-functional consequences. These should include relative enlargement of prefrontal and probably parietal cortex compared to peripherally specialized areas, motor cortex axons invading the nucleus ambiguous (which 
innervates laryngeal muscles), prefrontal connections displacing cortico-cortical and cortico-striatal projections from other systems, prefrontal-cerebellar connectivity increasing, and other less relevant changes.

In other words, human brains and language co-evolved so that languages were modified by selection favoring learnability and ease of use (via comparatively rapid historical change) and brain functions were modified by selection favoring the special learning and production demands of language (via comparatively slow evolutionary change). One important caveat to keep in mind, however, is this difference in the rate at which things change in culture and biology. For example, if we compare the amount of time that it takes for a language to split into mutually uninterpretable divergent forms to the amount of time it takes for large mammals like ourselves to split into distinct species unable to interbreed - i. e., thousand versus hundreds of thousands of years respectively - then we can assume at least two orders of magnitude of rate difference. Thus there will be perhaps hundreds of fundamental changes in the structures of human languages that only collectively affect selection with respect to common attributes. This means that rather than human brains reflecting specific structural features of language, only the most invariant sensory, motor, attentional, and mnemonic functions will have been selectively favored for their symbolic-linguistic contributions.

With respect to the symbolic cognition problem, then, one of these anatomical differences stands out as specifically relevant: prefrontal cortex expansion. The implication I draw from the coincidence of the distinctive prefrontal expansion in humans and the intense demand placed on transfer learning capacities is that this distinctively human neurological deviation is an adaptation evolved to ease the acquisition and use of symbolic communication and reasoning. But how could this have come about? Did brains become restructured for other reasons and just coincidentally provide the support for this unprecedented learning capacity?

I believe that this apparent "coincidence" has a simpler explanation. These anatomical changes reflect the demands of symbolic communication because they were favored in a context in which it was necessary to acquire and use symbolic communication in order to successfully survive and reproduce. What began as a minimal capacity for symbolic communication (which experiments have shown in apes) supported a very minimalistic form of symbolic social communication, but its value for group success and individual survival and reproduction became a force for both increased reliance on symbols and therefore increased demand on the neurological substrates that this required.

\section{Neural Adaptations to a Symbolic Niche}

Once symbolic communication became critical to hominid life it effectively became an artificial niche to which hominid cognition had to adapt. I argue that the changes in regional brain proportions and connections, briefly described above, exemplify ways that hominin biology was altered to succeed in this anom- 
alous niche. Analogous to the way that beavers bodies have become morphologically and physiologically adapted to the aquatic niche that beavers create, human brains have become adapted to the special demands of living in the symbolic niche they collectively create. And even the ecological niche that humans inhabit has been modified radically by the technological consequences of our symbolizing. So both human brains and the epigenetic context that shape how these unusual capacities are interdependently shaped into our mature mental predispositions are radically unlike what is found in any other species.

So how are we to characterize the distinctive nature of humanness if it is not possible to untangle the biological from the symbolic? One common response has been to give up the effort and to argue that any simple neo-Darwinian approach is made useless by this irreducible interdependence (e.g., Ingold 2007). But it would be misleading to further conclude that this semiotic-biological causal entanglement has produced a human mode of being that is entirely open-ended and lacks intrinsic constraints. Indeed, despite the unprecedented diversity of human social arrangements and languages, these distinctively human domains are nevertheless also statistically quite constrained. This is reflected in strong central tendencies - rather than some set of universals or list of common attributes - that independently have emerged again and again in human social arrangements, rituals, belief systems, and ways of communicating.

The approach I will take in the remainder of this essay is not, then, to attempt a survey of human social and psychological commonalities. The question I will instead address is: "What distinctive human predispositions can be inferred from our knowledge of this semiotic-biological foundation?” And specifically, I will focus on ways that the more or less virtual constraints imposed by our symbolic heritage have become physiologically embodied in ways that make them inseparable from what is considered biological.

\section{Neither Nature nor Nurture}

The first of these I will consider are those predispositions that are critical supports for symbolic-linguistic communication. These follow from the underlying semiotic support that is necessary to ground symbolic reference (as discussed above); specifically, the dependency of symbols on indexicality.

Because symbolic relations are indirect and "virtual" - constituted by symbol-symbol relations - an isolated symbol cannot determine reference to anything outside this symbolic domain. This has led to an extended history of arguments over the so-called symbol-grounding problem. I believe that this problem only appears to present a dilemma because symbolic reference is treated as primitive and simple. The dilemma disappears when symbols are understood as higher-order semiotic relations embedded in a context of indexical and iconic modes of reference.

Divorcing linguistic analysis from all forms of semiotic analysis except this simplified conception of symbolic reference, has also made it appear as though 
the highly systematic structure of language is also arbitrarily imposed. This has led to a half century of irresolvable nature/nurture debates concerning the possible locus of its cause. At one extreme, this structure has been attributed to innate sources of knowledge: nature (often described as universal grammar). Whereas at the other extreme, it is attributed to communicative habits and conventions that spontaneously develop in different societies: nurture. The innatist approach is criticized for being biologically implausible, while the social convention approach is criticized for being unable to account for the remarkable universality of many aspects of language structure. So is the resolution some combination of nature and nurture?

I have argued (Deacon 2003, 2011, 2012) that neither nature nor nurture nor even some combination of these provide an adequate solution to this debate. These options fail to notice an entire third realm of causal influences that results from the oversimplified conception of symbolization. This is the realm of semiotic constraints that arise due to the necessary semiotic infrastructure supporting symbolic reference.

Consider one of the most fundamental universals: the basic combinatorial nature of a sentence, or to be more technically accurate, the requirements for predication (which we might describe as communicating about something specific). Why is communicating linguistically intrinsically combinatorial? All languages produce structures that we recognize as sentences which either explicitly or implicitly combine at least two functionally different components. I believe that this necessarily follows from two important semiotic constraints. First, as noted above, symbol tokens mark positions within a systematic network of relations between symbols. As Saussure recognized, signifiers and signifieds (e. g., words and their meanings) are primarily markers of relative difference in relation to each other, independent of any individual real world binding to specific objects, events, or properties. Reference to individual facts of the world requires indexicality with its factual contiguity.

This dyadic sentential requirement derives from the inability of symbols to refer beyond the domain of other symbols without being additionally provided with indexical linkage. By itself a symbol token, like an isolated word, just pick out a position in the network of symbol-symbol associations. Only proper names have a specific link to a specific entity. So for a symbol to additionally have reference outside this virtual domain of symbolic associations it must be linked to an index or something that functions indexically. Consider someone uttering the word 'hard.' By itself it just brings to mind related associations, e. g., to words like soft, solid, difficult, and so on. But uttering the word and at the same time tapping on the table top with one's knuckles communicates that this particular surface is hard. The tapping is an index because of its immediate physical connection to the table. But this is not all. If the tapping were to take place minutes after the utterance there would be no such reference communicated. This demonstrates that there is a second indexical feature involved. The physical-temporal correlation between the tapping and the utterance also is interpreted indexically. In this way the combination provides a transitive indexical 
"bridge" linking the abstract symbol to the specific hardness of the real physical table. But notice that the tapping could have been replaced by the phrase "This [table] is ..." - perhaps also with the addition of a pointing gesture.

This constraint reflects fundamental features of both symbols and indices. Indexicality requires both the co-presence with a symbol token and embeddedness in a specific physical context. Contiguity of an index to a symbol (e.g., adjacency) provides both language-internal indexicality and language-external grounding. This dyadic linkage between semiotic functions in sentences and phrases within sentences, reflects the constraints on indexicality. This is what dictates phrase structure and its many syntactic correlates, such as the necessity of "merging" functionally different words into higher-order unit, limitations on between-phrase function-word movement, and long-distance dependency relations, to mention a few. Because of the factual contiguity constraint of indexicality, words functioning symbolically and indexically must be immediately linked, for the same reason that words and manual indices (such as the tapping described above) must be factually linked (though also employing iconic relations, such as gender agreement can provide some flexibility on what counts as adjacency). This imposes significant limitations on what can be "merged" with what in a phrase or clause, what parts of speech can be moved with respect to others, and what constitutes an embeddable phrasal unit.

This has an important bearing on questions concerning knowledge of language structure. Specifically, it suggests that grammatical roles and their associated syntactic constraints can be "discovered" both historically as new languages emerge and developmentally because there will be clear feedback concerning adequate or ambiguous specification of reference (Deacon 2011, 2012). The oftcited "poverty of the stimulus" argument, which suggests that there is nearly a complete lack of corrective feedback concerning grammatical and syntactic errors in early childhood, is simply looking in the wrong place for the appropriate source of feedback.

\section{Pointing and Joint Attention}

Young children have a bit of an innate head start when it comes to their ability to learn the constraints of indexicality. In the half year prior to acquiring their first words (another uniquely human predisposition discussed below) their communication with caregivers is aided by a predisposition to use their outstretched arm to point, often coupled with a non-linguistic vocalization such as whining to indicate an unfilled desire with respect to some object or activity. In addition, they quickly learn that a change in the direction of gaze of a caretaker is predictive of their future behavior with respect to something in that direction.

These distinctively human adaptations aiding cooperative indexicality are well known, and have become a major focus of comparative study. Whereas even human infants easily and spontaneously develop gaze following, pointing behaviors, and shared referential predispositions at an early age, well in advance of 
language acquisition, few other species come close to this level of performance, even with explicit training. This early indexical sophistication is an important precursor for the development of language. A sophisticated prior understanding of the constraints critical to unambiguous shared indexical reference provide a critical scaffold for the development of the core attributes of this universal grammatical constraint.

The constraints on successful indexicality are thus learned prior to speech and effectively transferred to use with words. Though the development of sophistication with indexicality will continue to be learned in the context of early language acquisition this mostly requires discovering how each particular language encodes these functions in words. As the first words are acquired (initially as vocal indices correlated with gestures) the functional forms of grammar and the structural constraints of syntax are in many respects already known. Applying this knowledge to language use requires the sophisticated transfer learning abilities that have also evolved, but not innate knowledge of grammar.

\section{Vocal Skill and Mimicry}

Another distinctive characteristic of humans that sets our species apart from essentially all other land mammals is our capacity to produce complex vocalizations that involve the articulation of the mouth and tongue with precisely synchronized control of the lungs and larynx. The innately produced calls of other land mammals are comparable to human laughter and sobbing, in being stereotypically structured and invariantly linked with specific arousal states. This mammalian limitation is a consequence of the fact that the descending neural control for the tongue, jaw, and facial muscles is distinct from that controlling the larynx and lungs. This is because the muscles of the mouth and face need to be under complex voluntary control in order to be able to adapt moment to moment to the variety of foods that need to be processed. In contrast, pharyngeal, laryngeal, and lung muscles need to function the same way time after time with each swallowing or breath, and need to do so infallibly and automatically. As a result, in the vast majority of land mammals these automatic systems are controlled locally in the brainstem, with little or no cortical input, as part of the visceral-motor system.

Species besides humans that have some skilled control of vocalization are only found among birds and cetaceans (the group including dolphins and whales). In these groups the production of sound uses structures other than a larynx. These include muscles constricting the pathway through the sinuses leading to the blow hole in cetaceans, and the muscular syrinx at the branch point of the bronchi in birds (see Deacon 1997 for details).

Along with these motor control adaptations, humans are predisposed to imitate the vocalizations of other humans. This is of course an important predisposition for early language acquisition, since each human language requires the ability to understand and produce many thousands of language-specific sound 
combinations (e. g., words). Although other species exhibit some mimicry capabilities, the human capacity is an order of magnitude more complex. Interestingly, almost all other species with significant imitative abilities exemplify this capacity in the aural-vocal domain, and have very limited behavioral mimicry capabilities. This phylogenetic bias probably reflects the fact that behavioral mimicry requires a complex visual-behavioral transformation to compensate for the mirror-image effect that reverses left and right, whereas sound mimicry requires no such transformation.

\section{Narrative Memory}

In all species of mammals learning depends on one or the other of two distinctively different mechanisms for establishing long-term stable memories, each depending on quite distinct neural substrates (e. g., see Baars and Gage 2007).

The acquisition of skills is accomplished by repetition of an activity, which progressively improves precision and efficiency of the activity and increasing its automaticity and reducing the need for consciously monitoring production. It is generally assumed that repetition progressively strengthens some complex synaptic pathways and weakens others. Thus retention and recall of this information is facilitated by the way that the signaling has become canalized by redundant synaptic strengthening. Since skill learning is particularly important for motor systems (even though also necessarily coupled with sensory feedback), the major brain systems involved mostly involve a frontal cortex to basal ganglia to thalamus to cortex loop, and a similar cortex to cerebellum to thalamus to cortex loop. Damage to structures or connections comprising these loops, significantly impairs this sort of memory formation. This mnemonic strategy is often called procedural memory for these reasons. It exemplifies the general principle that mnemonic strength and accessibility is a function of statistical redundancy.

In contrast, it is also critical to be able to store and retrieve experiential information in contexts where repetition is not possible. What has been called episodic or declarative memory is memory for events or episodes that occurred once, uniquely, are not repeated, and involve little in the way of repeatable actions. This requires the generation of redundancy of a different sort: redundancy of associations. This creates memory traces for singular experiences by correlations between features. Thus when we try to recall a specific experience from our past, it is generally necessary to triangulate to it using correlated associations, involving dates, places, typical social frames, and so forth. This form of mnemonic redundancy is formally orthogonal to the logic of procedural memory, and is thereby supported by quite distinct neural substrates. Thus, episodic memory is generated by neural circuits linking sensory cortices with the hippocampus and hippocampal damage significantly impairs the ability to consolidate new episodic memories.

Because of this functional segregation of these mnemonic systems, language can play an interesting mediating role. Indeed, it has become the foundation of 
an unprecedented new form of memory. Early in the process of language acquisition articulatory and syntactical combinatorial skills are acquired procedurally. In contrast, the symbolic reference that constitutes word meanings and their penumbra of semantic and experiential values are necessarily acquired. Because of this dualistic use of mnemonic systems language enables each mnemonic system to reciprocally cue the other. Narrative memory is the result. It forms the basis for promising, reasoning, theorizing, creating our identities, histories, politics, and art. Essentially, every form of socially maintained pragmatic knowledge, from religious belief to technology, is built from a growing matrix of narrative forms.

So although the neural substrates supporting these distinct mnemonic systems have not been fundamentally altered in human evolution from the ancestral primate condition, human cognition has been radically restructured by this novel mnemonic capability. The effects of this on the nature of human identity, agency, and social organization, as well as on the capacity of social groups to acquire and preserve complex knowledge over time, cannot be over-estimated.

\section{Emergent Emotions}

The consequences of these unprecedented adaptations for symbolic cognition weren't merely cognitive and linguistic, however. These capabilities have also incidentally produced uniquely human forms of emotional experience. Like the unique functional synergies that have re-organized the way that ancient mnemonic systems can be used, symbolic capacities have similarly re-organized the functions of the emotional systems of the brain. This has given rise to a whole class of human-unique emotional capacities (Deacon 2006). These might more accurately be described as symbolically modulated emotional relationships that also are realized by neuronal systems that we humans share with most other mammals.

Because symbol tokens represent their content indirectly and without sharing attributes or direct correlations with the thoughts they convey, the salience and intensity of their emotional correlates are also substantially reduced. This enables symbols to be combined and juxtaposed in many more diverse ways than other sorts of signs. This combinatorial freedom can lead to the expression of emotional interactions that could not otherwise occur. Because the correlated emotions aroused by symbol combinations are of low intensity they too are more easily manipulated and combined in novel ways. So it's not that we have evolved novel neurological systems for emotional expression, but that these processes can be set into novel synergistic and antagonistic and complementary combinations that would be very unlikely to occur in the absence of symbolic processes.

So what are some plausible candidates? Awe, nostalgia, righteous indignation, aesthetic appreciation, humor, irony, eureka ... All these involve unusual juxtapositions of more basic emotional dynamics, likely activated differently in the distributed structures responsible for emotion, including differences in homol- 
ogous structures in the two hemispheres. So not only can this involve the separate circuits that handle different arousal and hedonic states, but the bilaterality of these systems may also allow novel combinatorial interactions of otherwise mutually incompatible emotional dynamics in response to the flexibility of symbolic manipulation. This may help to explain the human fascination with activities that symbolically tweak our emotions in unusual and surprising ways.

For two prominent examples consider humor and artistic aesthetics. Humor involves a distinctive emotional replacement logic: a rapid shift of arousal from one state of expectation (often with tension) to another that completely dispels the tension. Curiously, this can be driven by purely linguistic twists of logical expectation, as in puns and jokes. This may involve a left to right hemispheric shift of activation because of the typical replacement of a typical logical expectation with a contextually parallel but absurdly unrelated consequence. In contrast, the aesthetic elation that can be elicited by artistic works often involves sustained emotional juxtapositions, rather than alternation. And other more complex emotional dynamics may be involved in the eureka experience of discovering new and surprising connections, the sense of irony elicited by juxtaposed opposites, or the sense of righteous indignation elicited by recognizing the failure to conform to expected norms.

\section{Religiosity}

Because of this suite of adaptations and the fact that linguistic communication and symbol-mediated cognition are integrated into every aspect of our lives we humans have essentially become symbolic savants (Deacon 1997; Deacon and Cashman 2009). We find ourselves intuitively and irresistibly "looking beyond the surface" for hidden meanings. The cognitive biases that evolved to make symbolic communication seem natural and effortless and quickly becomes the mental tool used to negotiate the vast majority of challenges we face, has likely also radically altered the way we tend to interpret even everyday non-symbolic experience. A linguistic description is an impoverished transcription of the thought that gave rise to it. The perceived sounds are clues pointing to an unseen thought not present to the senses and so must be reconstructed. The sounds and objects present to the senses are not meaningful in themselves, they are what we have to work with as intermediaries to discover the meaning making process that produced them.

The world's many spiritual traditions almost universally depict the existence of a world beyond the immediate physical world that is in some way more fundamental and ultimately determines the events and forms found in the world available to our senses (Deacon and Cashman 2009). Though what constitutes the constellation of beliefs and practices that define "religion" is a matter of contentious debate, in nearly every society we find traditions that assume that there exists a nonphysical world hidden from direct sensory experience. Deacon and Cashman (2009) describe this as the "bilayered world" assumption. Just as we 
intuitively assume that the words and actions of others are merely the superficial physical manifestations of an otherwise inaccessible agency that is the source of meaning and purpose expressed in these physical manifestations, so too have we come to look upon the happenings in the physical world to be the superficial expressions of some hidden teleology. It is as though we are unable to inhibit this compulsion to see everything in the image of symbols. Is it simply that since infancy we have needed to seek the meaning behind the curtain of the language and culture we are immersed in? Or did our long adaptation to a symbolic niche predispose us to reflexively think of the world we perceive as though it is text that needs to be interpreted, if only one knew how? Either way it has molded us into symbolic savants who incessantly and irresistibly see the world in bilayered terms.

In many respects, then, I consider the human discovery of the use of symbols to be the "original sin." Symbolic abilities have given us access to the knowledge contained in the forbidden fruit, and in this way we have been driven from the obliviousness of a pre-symbolic Eden. It provided access to unique emotions, indirect access to the thoughts and experiences of other minds, and a yearning for meaning in the narrative that is human identity. This transition has left us forever seeking a meaning hidden within a disembodied realm and thinking of the mundane physical world around us as deriving any of its value from this projected meaning source.

\section{Relaxation of Selection and Plasticity}

How could these many diverse cognitive and behavioral traits have become so functionally intertwined and interdependent as to provide such a novel means of thinking, feeling, and communicating? And how could the diverse brain systems supporting them have come to work in such a tight and unprecedented synergy? This is particularly challenging to explain because this symbolic capacity is in effect an emergent function, not some prior function just requiring fine-tuning. Our various inherited vocalizations, such as laughter, shrieks of fright, and cries of anguish, are comparatively localized in their neurological control (mostly subcortical) as are other modes of communication in animals. In comparison, language depends on a widely dispersed constellation of cortical systems, each of which can be found in other primate brains, but evolved for very different functions. These brain systems have become collectively recruited for language only because their previously evolved functions overlapped significantly with some processing demand necessitated by language, though evolved for quite different functions altogether.

A related mystery concerns the extent to which this dominant form of human communication depends extensively on information acquired by social transmission. Even for theories postulating an innate universal grammar, the vast quantity and high fidelity of the information constituting even a modest vocabulary stands out as exceedingly anomalous from a biological point of view. How did 
such a large fraction of our communicative capacity wind up offloaded onto social transmission? And what explains the remarkable reliability of this process?

These unprecedented emergent features of brain function and cognition suggest that a co-evolutionary logic alone is insufficient to explain them. Recent investigation of a parallel shift in both complexity and neural substrate in birdsong may, however, be able to shed some light on this.

In a comparative study of a long-domesticated bird, the Bengalese Finch, and its wild cousin, the White-Rump Munia, it was discovered that the domesticated lineage was a far more facile song-learner with a much more complex and flexible song than its wild cousin (e. g., see Okanoya 2004). This occurred despite the fact that the Bengalese Finch was bred in captivity for coloration, not singing. The domestic/wild difference of song complexity and song learning in these close finch breeds parallels what is found in comparisons between other bird species that are song-learners and non-learners. This difference also correlates with a much more extensive neural control of song in birds that learn a complex and variable song through social transmission.

The fact that this behavioral and neural complexity can arise spontaneously without specific breeding for singing is a surprising finding since it is generally assumed that song complexity evolves under the influence of intense sexual selection. In the case of the Bengalese Finch, his source of selection was eliminated by the intervention of human breeders.

In Deacon (2010), I argued that, paradoxically, it was the relaxation of natural and sexual selection on song structure that was responsible for its elaboration in this example. In brief, with song becoming irrelevant to species identification and mate choice in the domesticated lineage, territorial defense, mate attraction, predator avoidance, and so on, the innate mechanisms constraining song were allowed to degenerate. Mutations that resulted in degeneration of these systems and existing recessive alleles producing a less stereotypic song would not have been weeded out. The result would be the reduction of innate biases controlling song production. The domestic song could thus be described as both less constrained and more variable because it is subject to more kinds of perturbations. But with the specification of song structure no longer influenced by innate auditory biases and strictly controlled by the primary forebrain motor center of the songbird brain (called nucleus RA), other linked brain systems could begin to play a biasing role. With innate motor biases weakened, auditory experience, social context, learning biases, and attentional factors could all begin to influence singing. So the domestic song would be expected to become more variable, more complicated, and more influenced by social experience. These changes are what we observe in the case of the Bengalese Finch.

This is relevant to the human case, because a number of features of the human language adaptation also appear to involve a relaxation of innate constraints, allowing multiple brain systems and extrinsic influences to affect linguistic communication. The presence of infant babbling, increased variability and skilled modification of vocal behavior, decoupling of vocalization from arousal state, an extensive capacity for vocal imitation, etc., may all be consequences of a paral- 
lel relaxation of selection with respect to vocal communication. Freedom from constraint is also an important precondition for being able to correlate learned vocal behaviors with the wide diversity of objects, events, properties, and relationships that language is capable of referring to. It is also a plausible answer to the combinatorial synergy problem of brain functions (discussed above) because it provides an evolutionary route to multi-system coordination.

How might this analogue to the effects of domestication have arisen in human prehistory? Perhaps the most striking clues to an answer come from major changes in body structures and demographics occurring at various points in our hominin ancestry. The first and most obvious is a shift to bipedal locomotion somewhere in the period around 4 million years ago that relaxed selection on the upper limbs and hands enabling more flexible manipulation of objects. This was a precursor to the discovery of how to produce and use sharpened stones to gain access to the rich animal flesh resource that may date to over 3 million years into the past. But what may be the most radical change probably didn't occur until about 2 million years ago. This transition is characterized by a number of correlated anatomical and behavioral changes that indicate a major shift in all aspects of hominin life. Stone tool use becomes ubiquitous, bipedal locomotion becomes fine-tuned to modern forms, body size increases, brain size increases above typical ape levels, jaws and teeth are significantly reduced, and sexual dimorphism is decreased to modern levels. And perhaps most significantly, it is at this point that tool using hominins are, for the first time, found outside of Africa, in Central and Southeast Asia. There were likely other similar migration events occurring subsequently, but the last and most extensive migration out of Africa occurred as recently as 60,000 years ago, as anatomically modern-looking people swept over all of Eurasia to replace previous populations. Each of these events suggests that new levels of flexibility have become available giving rise to yet further phases of relaxation.

There is also increasing genetic evidence of relaxation of selection distinguishing humans from other primates. Although traditional assumptions about the role of genetic change in evolution have tended to focus on mutations that augment some function, evidence is growing that gene duplication and gene loss - including especially loss of non-coding regulatory sequences - has contributed to significant evolutionary change (e.g., Olsen 1999; Hunt et al. 2011). Human-specific loss of over 500 otherwise highly conserved non-coding regulatory sequences has recently been reported (McLean et al. 2011). This extensive loss of genetic regulation may be a signal of human-specific relaxation of selection and an increased sensitivity to epigenetic and environmental influences.

This argument inverts the claim that increased genetic specification of grammatical knowledge underlies our language capacity and is instead consistent with the explanation of language universals arising from extra-biological sources (such as suggested above). But perhaps the most important implications involve the incredible flexibility of human cognition and behaviors. We largely take this for granted, but in many respects it is one of the most robust and significant aspects of our distinctive nature. As a result, not only are human languages incredibly 
diverse, but all aspects of human culture show unprecedented diversity compared to anything found in the rest of the animal kingdom. Many of the distinctive symbolic adaptations discussed above reflect this increased openness to developmental and cultural influences that have led many to question the very concept of human nature.

\section{Conclusion}

In this essay I have reviewed extensive evidence that the unusual demands of symbolic communication and thought have restructured our brains, have provided an unprecedented degree of behavioral flexibility, and have embedded us in a symbolic ecosystem - culture - that is radically unlike the Umwelt of any other species. Symbolization has thereby modified nearly every aspect of what makes us human. The result is that our distinctive "nature" is as much symbolic as it is biological.

\section{Bibliography}

Baars, B. J. and N. M. Gage. 2007. Cognition, Brain, and Consciousness: Introduction to Cognitive Neuroscience. London: Elsevier Ltd.

Bock, K. E. 1980. Human Nature and History: A Response to Sociobiology. New York: Columbia University Press.

Deacon, T. 1997. The Symbolic Species: The Co-evolution of Language and the Brain. New York: W. W. Norton \& Co.

-. 2003. Universal Grammar and Semiotic Constraints. In Language Evolution, ed. M. Christiansen, and S. Kirby, 111-139. Oxford: Oxford University Press.

-. 2006. The Aesthetic Faculty. In The Artful Mind, ed. M. Turner, and S.Zeki, 21-53. Oxford: Oxford University Press.

-. 2009. Relaxed Selection and the Role of Epigenesis in the Evolution of Language. In Oxford Handbook of Development Behavioral Neuroscience, ed. M.S. Blumberg, J. H. Freeman, and S. R. Robinson, 730-752. New York: Oxford University Press.

-. 2010. A Role for Relaxed Selection in the Evolution of the Language Capacity. PNAS 107: 9000-9006.

-. 2011. The Symbol Concept. In The Oxford Handbook of Language Evolution, ed. M. Tallerman, and K. Gibson, 393-405. Oxford: Oxford University Press.

-. 2012. Beyond The Symbolic Species. In The Symbolic Species Evolved, ed. T. Schilhab, F. Stjernfeldt, and T. Deacon, 9-38. Dordrecht: Springer.

Deacon, T. and T. Cashman. 2009. The Role of Symbolic Capacity in the Origins of Religion. Journal of Religion, Nature \& Culture 3: 490-517.

-. 2012. Teleology versus Mechanism in Biology: Beyond Self-Organization. In Beyond Mechanism: Putting Life Back Into Biology, ed. B. Henning and A. Scarfe, 287-308. Lanham, MD: Lexington Books/Rowman \& Littlefield.

Hull, D. 1986. Human Nature. Proceedings of the Biennial Meeting of the Philosophy of Science Association 2: 3-13.

Hunt, B. G., L. Ometto, Y. Wurm, D. Shoemaker, S. V. Yi, L. Keller, and M. Goodisman. 2011. Relaxed Selection Is a Precursor to the Evolution of Phenotypic Plasticity. PNAS 108: 15936-15941. 
Ingold, T. 2006. Against Human Nature. In Evolutionary Epistemology, Language and Culture: A Non-Adaptationist, Systems Theoretical Approach, ed. N. Gontier, J.-P. van Bendegem, and D. Aerts, 259-281. Dordrecht: Springer.

-. The Trouble with 'Evolutionary Biology.' Anthropology Today 23 (2): 13-17.

Keller, H. 1905. The Story of My Life. In Parts I E II; Part III from the Letters and Reports of Anne Mansfield Sullivan (ca. 1867-1936), ed. J. A. Macy. New York: Doubleday, Page E Company.

Kohler, W. 1925. The Mentality of Apes. Trans. Ella Winter from the second revised edition. London: Kegan Paul, Trench, Trubner.

McLean, C. Y., P. L. Reno, A. A. Pollen, A. I. Bassan, T. D. Capellini, C. Guenther, V. B. Indjeian et al. 2011. Human-specific Loss of Regulatory DNA and the Evolution of $\mathrm{Hu}-$ man-specific Traits. Nature 471: 216-219.

Nieder, A. 2009. Prefrontal Cortex and the Evolution of Symbolic Reference. Current Opinion in Neurobiology 19: 99-108.

Okanoya, K. 2004. The Bengalese Finch: A Window on the Behavioral Neurobiology of Birdsong Syntax. Annals of the New York Academy of Sciences 1016: 724-735.

Olsen, M. 1999. When Less is More: Gene Loss as an Engine of Evolutionary Change. American Journal of Human Genetics 64: 18-23

Peirce, C. S. 1931. Collected Papers of Charles Sander Pierce. Vol. II Elements of Logic, ed. C. Hartshorn and P. Weiss. Cambridge, MA: Harvard University Press.

Vendetti, M. S. and S. A. Bunge. 2014. Evolutionary and Developmental Changes in the Lateral Frontoparietal Network: A Little Goes a Long Way for Higher-Level Cognition. Neuron 84: 906-917.

Yarkoni, T., R. A. Poldrack, T. E. Nichols, D. C. Van Essen, and T. D. Wager. 2011. Largescale Automated Synthesis of Human Functional Neuroimaging Data. Nature Methods 8: 665-670. 


\title{
Preconditions in Human Embodiment for the Evolution of Symbolic Communication
}

\author{
Jordan Zlatev
}

\begin{abstract}
The evolution of human language and thought is crucially based on the use of symbols, understood as socially-shared, conventional and systematic representations. In this chapter I argue that the evolution of human-specific symbolic communication took place over a prolonged period of time, relying on three key preconditions related to human embodiment. First was the evolution of a degree of intersubjectivity that is unique among great apes, most likely made possible by the adoption of cooperative breeding (alloparenting) as a key reproductive strategy. This in turn made possible the pro-sociality that is a further precondition of human communication, and which developed in pre-linguistic cultures via bodily mimesis (Donald 1991; Zlatev 2008). Finally, vocal-manual multimodality was essential for the transition from a predominantly bodily and iconic mode of representation to a predominantly (though not exclusively) vocal and symbolic mode. The suggested scenario implies extensive bio-cultural co-evolution, and draws upon extended Darwinian models such as multi-level selection theory, operating on both individuals and groups, with complex tradeoffs.
\end{abstract}

\section{Introduction: Whence the Symbolic Species?}

Human cognition and communication have unique properties when compared to those of non-human animals: this much is fairly uncontroversial. But as soon as we ask which properties these are, and how they have evolved, controversies loom large. It is often claimed that the representational and combinatorial powers of language are the key factors of human uniqueness (Christiansen and Kirby 2003), and one of the more successful definitions of us is as "the symbolic species” (Deacon 1997). To some extent, the term is apt, and the re-kindled interest in the topic of language evolution - as a means to address the classical questions of who we are and where we come from - is justified. But in effect, this is only a paraphrase of the initial "which and how" question, since there is little agreement on the fundamental features of language: are they "computational," cognitive, social, semiotic, or combinations of these (Chomsky 1986; Itkonen 2003; Zlatev 2007)? Likewise, the term "symbol" is one of the most ambiguous ones in the field, with many disagreements arising from misunderstandings (Heine and Kuteva 2007; Hurford 2007).

In the present chapter, consistent with discussions of the ontogenetic origin of symbolic communication (McCune 2008; Werner and Kaplan 1963; Zlatev 
and McCune 2014), I take a synthetic cognitive-semiotic perspective and define symbol use as the production and comprehension of signs which are (a) representational, i. e., they are understood by their users as denoting general or specific objects, properties and events (Sonesson 2007), (b) conventional, i. e., shared within a community (Itkonen 2003), though not necessarily "arbitrary" (see Section 3), (c) expressed along with communicative intent (Tomasello 1999; Zlatev 2013) and (d) systematically interrelated (Saussure [1916] 1983; Deacon 1997). The implications of this definition will become clear as we go along.

Symbol use - which is at the heart of language - is indeed at the essence of what makes human nature and culture unique. In this respect, I am in agreement with so called "discontinuity theories" of language origins (Tatterstall 2014). However, I part company when they assume that the transition to symbol use and language should therefore be regarded as abrupt, resulting either from sudden inventions or macro-mutations, sometimes said to coincide with "overt archeological evidence for symbolic activities" (ibid., 224) that are less than 100,000 years old. Rather, I argue that the evolution of symbolic communication was a prolonged process, and language would not have evolved, nor would it be learnable by children, or be able to function in general, if it were not for three key cognitive-semiotic properties, which all have to do with the nature of our embodiment. The first, on which I have placed considerable focus in the past, is that of bodily mimesis (Donald 1991, 1998, 2001, 2012; Zlatev 2007, 2008a, 2008b, 2014a). As summarized in Section 1, the Donald-Zlatev hypothesis is that an ancient, nearly two-million-old adaptation unleashed unprecedented capacities for representation, skill and imagination: an indispensable "missing link" on the way to language. However, with time I have realized that the bodily mimesis hypothesis is in itself not a sufficient evolutionary explanation of human symbolic origins.

On the one hand, bodily mimesis presupposes enhanced trust, empathy and cooperation within tightly knit social groups, or in one word: an advanced form of intersubjectivity that is sometimes called ultrasociality. While bodily mimesis and intersubjectivity may have to some degree co-evolved (Zlatev 2008a), it is very difficult to understand how the capacities described in Section 1 could have been established, without a prior evolution of uniquely human capacities and motivations for "sharing" (Zlatev et al. 2008). There have been numerous proposals in the literature for the contexts in which (human) ultrasociality evolved: from foraging (Tomasello et al. 2012) to agriculture (Gowdy and Krall 2016). However, considering a multitude of factors, including the coherence of the evolutionary sequence, nearly all such accounts leave something wanting. In Section 2, I summarize some theoretical proposals and argue that the combined evidence supports most strongly one particular theory for the evolution of uniquely human intersubjectivity: the alloparenting hypothesis (Hrdy 2009). As alloparenting has to do with reproduction, and is expressed in a multitude of bodily forms of interaction like emotional attunement, neonatal mirroring and joint attention, this is another aspect of human embodiment.

On the other hand, a body-based theory of the origins of symbolic communication is said to face a crucial challenge, regarded by some as "insuperable" (Ken- 
don 2009): why is the predominant channel of human language vocal, rather than gestural? In Section 3, I provide an answer, summarizing recent evidence from experimental semiotics (Galantucci and Garrod 2010), based on a third pre-condition that is also crucially tied to embodiment: the fundamentally multimodal, vocal-gestural nature of linguistic communication. The idea of "multimodal origins" has been gaining ground in the literature, as a middle ground of sorts between gesture-first and speech-first theories (Hurford 2007; Taglialatela et al. 2011). What I propose is a more specific version of the thesis, according to which the meanings expressed by the different channels of bodily expression - gesture and speech - need to be viewed as interacting but distinct, unlike some accounts by which they are inseparable (McNeill 2005, 2012).

Finally, I summarize the argument, and order the landmark evolutionary achievements in a hypothetical sequence stretching over the past four million years. Notably, the scenario presupposes modern developments in evolutionary theory that go beyond the gene-centered perspective of Neo-Darwinism.

\section{Bodily Mimesis: The "Missing Link" to Symbol Use}

In a nutshell, the bodily mimesis hypothesis states that "an adaptation for improved volitional control of the body gave our ancestors advantages in the domains of imitation, empathy and (gestural) intentional communication. This paved the way for the evolution of language, with no other biological adaptation ... apart from improved vocal control" (Zlatev 2014a, 166). Etymologically stemming from the Greek verb mimeisthai (to imitate), the concept of mimesis and its cognitive role was noted by Aristotle, who commented on "man's natural propensity, from childhood onwards, to engage in mimetic activity (and this distinguishes man from other creatures, that he is thoroughly mimetic, and through mimesis takes his first steps in understanding" (Aristotle 1987, 34). In effect, Donald naturalized the concept, in framing (bodily) mimesis as "an embodied, analogue, and primordial mode of representation" based on "a unified neuro-cognitive adaptation that formed the early foundation of a distinctly human mind-sharing culture" (Donald 2012, 180-181). Consistent with the bodily mimesis hypothesis, the archeological record for Homo erectus (1.8-0.5 MYA) provides evidence for complex Acheulean (so-called "Mode 2") tool-manufacture, campfires and long-distance migration over most of Eurasia. The paleontological evidence shows basically modern human anatomy, and a double increase in brain size compared to predecessors. Adding evidence from neuroscience, psychology, anthropology and primatology (Donald 2001; Zlatev 2014a) supports the thesis that an adaptation for enhanced voluntary control of the body served as the key to a "cultural style that can still be recognized as typically human" (Donald 2001, 261), and yet was not dependent on language.

An attractive feature of the hypothesis is its parsimony: while the original adaptive function of bodily mimesis could have been tool production, it would have naturally been extended to much else: "pantomime, imitation, gesturing, 
shared attention, ritualized behaviors, and many games. It is also the basis of skill rehearsal, in which a previous act is mimed, over and over, to improve it" (Donald 2001, 240). In sum, bodily mimesis granted to human cognition and communication pre-linguistic, and yet uniquely developed features in at least the following five domains.

Complex skills: Many motor skills do not rely on a mimetic controller. However, the kinds of motor patterns necessary for complex skills like bipolar axe production and precision throwing require systematic rehearsal and the ability to "compare, in imagination, the performed act with the intended one" (Donald 2012, 182). Mimesis brings the ability to "shift attention from the external world, and redirect it to [our] own bodies and actions" (Donald 1998, 45), and thus to align the performed and observed movements.

Social learning: Again, simpler forms of social learning like goal emulation, response facilitation and stimulus enhancement, are available to many primate species. However, true imitation - in which a novel act is observed, modeled and eventually added to the behavioral repertoire - is much more restricted (Tomasello 1999). Only human children reproduce an observed action with high fidelity even when some of the steps are clearly not functional to achieving the goal, i. e., what is now known as "over-imitation" (Horner and Whiten 2005). From the other side (that of the teacher), helping by overtly demonstrating, guiding and when necessary correcting is also a universal, human-specific trait (Gergely and Csibra 2006).

Memory and planning: The ability to (consciously) remember some event experienced in the past is characteristic of episodic memory, allowing mental access to "a particular experience (witnessed, or felt, or thought something) in a particular place at a particular time” (Tulving 2005, 15). It is also important for planning and guiding of future actions. There has been accumulating evidence that at least some episodic memory is not specifically human (Hurford 2007) and Donald (1991) has referred to the minds and cultures of chimpanzees as "episodic," acknowledging that they are not strictly limited to the here and now, allowing "flashbacks," even if these are not under voluntary control. The mimetic controller adds to this not only volitional control of the imagination, but the ability to explicitly re-enact a past or future event through bodily motion.

Rites and rituals: Moving further into the social domain and combining the functions discussed above - skill rehearsal, re-enactment and (over-)imitation provides the bases for another universal of human cultures: rituals. These involve more or less formalized, invariant and stylized bodily performances, loaded with affective meaning, and serving social bonding (Bell 1997). Donald writes of "reciprocal mimesis" (Donald 1991, 6) as the means for establishing such forms of "group mentality." The highly normative and (in part) symbolic character of many rituals transcends the borders of the "mimetic stage," intermixing with the subsequent "mythic stage" in Donald's evolutionary model, characterized by narrative and language.

Mime and gesture: A re-enacted hunting dance is clearly representational, in the sense that expression and content are clearly differentiated for both the per- 
former and audience. In a general sense, it is also communicative. But rituals are generally performative rather than informative, and lack the full sense of (Gricean) intentional communication, in which there is both an intention to inform the audience of something new, and a higher-order intention for the audience to understand this (Sperber and Wilson 1995). Hence, it is doubtful if "mime and non-linguistic gesture come for free with skill” (Donald 2012, 182). The cooperative motivations and cognitive capacities for the use of communicative intentions need to be seen as an extension of the motoric aspects of mimesis (Zlatev et al. 2013). And arguably, "Gricean intentions" are still needed to bridge the gap between animal expressive communication and language (pace Bar-on 2013).

In sum, the concept of bodily mimesis is both specific and rich in relations and extensions: from the motoric skill to social cognition and human-specific culture. Hence, it has been necessary both to constrain it and to provide it with a hierarchical structure, distinguishing simpler from more elaborated forms (e.g., Zlatev 2008b). Adapting a definition used in previous publications, an actual or imagined act of cognition or communication is an act of bodily mimesis if: (a) it involves a cross-modal mapping between exteroception (e. g., vision) and proprioception (e. g., kinesthesia); (b) it is under conscious control and is perceived by the subject to be similar to some other action, object or event; (c) the subject intends the act to stand for some action, object or event for an addressee, and for the addressee to recognize this intention; (d) it is not fully conventional and normative; and (e) it does not divide (semi)compositionally into meaningful sub-acts that systematically relate to other similar acts, as in grammar. A conceptual-empirical model known as the Mimesis Hierarchy (Zlatev 2013) follows from this definition by assuming that these features build incrementally upon one another, so that possessing only (a) yields proto-mimesis, whereas (a) and (b) together give dyadic mimesis, while adding (c) leads to full triadic mimesis. With the last two (negative) criteria in the definition follow the two "post-mimetic" stages: (d) protolanguage, with signs following criteria for correctness, but lacking systematic relations among them; and (e) language, with sufficient systematicity to allow the construction of discourse and narratives.

Table 1 shows the five stages of the Mimesis Hierarchy, alongside corresponding social-communicative skills. Reviews of comparative psychological and social neuroscience research (Zlatev 2008a, 2008b) show abundant evidence for proto-mimesis in non-human primates, and some for dyadic mimesis in non-human apes, and especially chimpanzees. But without extensive human enculturation, triadic mimesis skills are inaccessible, and even the most successful "language apes" like the bonobo Kanzi do not appear to master them fully (which can explain their inability to acquire anything more than proto-linguistic skills). The conclusion is thus that it is the lack of bodily mimesis, rather than any "language acquisition device" or such that prevents non-human primates from evolving both cumulative culture and language. Another review of the comparative evidence reaches similar conclusions (Vaesen 2012): seven pre-linguistic domains show clear differences between human and great ape capacities: (1) motoric: hand-eye coordination; (2) social-cognitive: imitation, teaching, and social rea- 
soning; and (3) general cognitive capacities: causal reasoning, function-based categorization (e. g., related to tools) and executive control (e.g., related to planning). Considering the functions of bodily mimesis outlined earlier (i. e., skill, planning, imitation, rites, gesture), there is considerable overlap between these lists: from the most specific (1), to the social consequences of bodily mimesis in (2) and the most general ones in (3). In other words, the poly-functional nature of bodily mimesis may help explain the internal coherence among the features that distinguish human and animal cognition, apart from language.

Further, by comparing the features and skills in Table 1 with the definition of symbol use presented in the introduction, it becomes clear how bodily mimesis can function as the bridge from primate episodic cognition to language. Iconic gestures ("pantomime") are clearly representational, and declarative pointing and joint attention bring with them the markers of communicative intent. Fully conventional (normative) signs like emblems and single words take on an additional property of symbols, but only once their systematic and combinatorial potential is realized (in grammar and story-telling) is there "fully symbolic communication."

Table 1. The five stages of the Mimesis Hierarchy, in rising order, with incremental features and corresponding cognitive-semiotic structures.

\begin{tabular}{|c|c|c|c|}
\hline \multicolumn{2}{|c|}{ Stage } & \multirow{2}{*}{$\begin{array}{l}\text { Novel feature } \\
\text { Semiotic } \\
\text { systematicity }\end{array}$} & \multirow{2}{*}{$\begin{array}{l}\text { Cognitive-semiotic structures } \\
\text { - Fully symbolic communication } \\
\text { - Grammar } \\
\text { - Narrative }\end{array}$} \\
\hline$\# 5$ & Language & & \\
\hline$\# 4$ & Protolanguage & $\begin{array}{l}\text { Conventionality/ } \\
\text { normativity }\end{array}$ & $\begin{array}{l}\text { - Emblems } \\
\text { - Words } \\
\text { - Multimodal utterances }\end{array}$ \\
\hline \#3 & Triadic mimesis & $\begin{array}{l}\text { Communicative } \\
\text { intent }\end{array}$ & $\begin{array}{l}\text { - Declarative pointing } \\
\text { - Iconic gestures } \\
\text { - Joint attention }\end{array}$ \\
\hline$\# 2$ & Dyadic mimesis & $\begin{array}{l}\text { Volitional } \\
\text { re-enactment }\end{array}$ & $\begin{array}{l}\text { - Full imitation } \\
\text { - Imperative pointing } \\
\text { - Shared attention }\end{array}$ \\
\hline$\# 1$ & Proto-mimesis & $\begin{array}{l}\text { Mapping extero- } \\
\text { ception and } \\
\text { proprioception }\end{array}$ & $\begin{array}{l}\text { - Emotional contagion } \\
\text { - Attention contagion } \\
\text { - Neonatal mirroring } \\
\text { - Mutual gaze }\end{array}$ \\
\hline
\end{tabular}


This model may be somewhat schematic, but it helps to appreciate both the potential and the limits of bodily mimesis. Cognitive-semiotic enhancements in the five domains highlighted earlier provide the essential ingredients of a type of culture that is not based on language, but on shared skills, (simple) rites, (public) representations, and non-linguistic communicative signs. The kind of imitation that comes with bodily mimesis makes cumulative cultures possible (Richerson and Boyd 2005) since "only imitation gives rise to cumulative cultural evolution of complex behaviors and artifacts" (ibid., 108). Yet, the rate of cultural evolution was very slow until the appearance of the "symbolic artifacts" emphasized by many archeologists (Tatterstall 2014), and it is possible that fully modern-like languages are contemporaneous and brought about through historical, rather than biological processes (Heine and Kuteva 2007), during the past 100,000 years.

Let us postpone the discussion of the transition from bodily mimesis to language as a "conventional-normative semiotic system for communication and thought” (Zlatev 2008b, 137) for Section 3, where we explore the nature of the transitional "protolanguage" and its (gradual) evolution to modern-like languages, in part thanks to the multimodal character of bodily mimesis. But first, we need to consider the evolution of a pre-requisite for bodily mimesis itself: the type of social context that would generate a "society of intimates" (Givón and Young 2002).

\section{The Intersubjective Preconditions for Mimesis}

Human cultures and minds are special with respect to the extent of their intersubjective sharing. Our species is sometimes characterized as being not simply social, but "ultrasocial" (Campbell 1982; Tomasello 1999). As far as the sharing of cognitive phenomena such as linguistic rules, moral norms and religious beliefs is concerned, human uniqueness is indisputable. But these are relative latecomers in evolution, as well as in ontogenetic development, and cannot help us get at the roots of intersubjectivity. As stated in a recent anthology on the social origins of language: "the major transition, all of us agree, must have been the establishment within social groups of unprecedentedly co-operative, trusting relationships. Only then could language ... begin to evolve” (Dor, Knight, and Lewis 2014,14 ). More basic than the sharing of norms and beliefs is that of food and care for children and elderly, as is customary for hunter-gatherers (Wiessner 2002). But what factors brought this about? In a recent target article, Gowdy and Krall $(2016,1)$ discuss the "lack of consensus in the biological sciences in classifying social behavior" and opt for the following definition: "Ultrasociality refers to the most social of animal organizations, with full time division of labor, specialists who gather no food but are fed by others, effective sharing of information about sources of food and danger, self-sacrificial effort in collective defense" (Campbell 1982, 160). Curiously, however, this ends up including "ants, termites and humans in several scattered archaic city-states," but not hunter-gatherer societies. Indeed, the social organization that our ancestors lived in 
since the dawn of Homo until some 10,000 years ago lacked the kind of ant-like subjugation of the individual to the interests of the collective that Gowdy and Krall (2016) claim became dominant with the rise of organized agriculture (and become global with market capitalism). Thus we need to look elsewhere for the preconditions of mimetic cultures.

One reason why strong forms of pro-social behavior such as altruism are always both relevant and controversial in evolutionary discussions is that, at least on the face of it, they contradict a basic principle of the "modern synthesis" in evolutionary theory: "costly group-beneficial behavior cannot evolve unless the benefits of group-beneficial behavior flow non-randomly to the genes that give rise to the behavior" (Richerson and Boyd 2005, 198). A number of well-known approaches have turned out not to generalize to all cases, and to human contexts specifically. Kin-selection (Hamilton 1964) can explain cooperation only in species that are very closely related, such as social insects and bees. Reciprocity can evolve and become stable in small groups, but becomes easily vulnerable to "free-riders." The two mechanisms to resolve this have their own problems: tracking reputations can influence willingness to cooperate, but this requires fairly advanced memory systems and "gossip," which of course presupposes language, and hence cannot be evoked as precondition for it. Finally, retribution for non-collaborators requires group-wide moral norms, and some system to police these into action, or else become vulnerable to "second-order free-riders" (who like most of us prefer to look the other way, rather than risk harm).

The debates go on in evolutionary theory, but as more and more are willing to move beyond the "Geno-centrism" of Neo-Darwinism into various extensions such as epigenetics (Jablonka and Lamb 2007) and developmental systems (Oyama et al. 2000), an old Darwinian idea - that selection may operate on whole groups, and not just on individual and their genes - has been revived and elaborated as multi-level selection (MLS) theory (Richerson and Boyd 2005; Sober and Wilson 1999). In a recent publication (Zlatev 2014b), I considered four specific theories for the evolution of human-specific sociality in the light of such developments, and attempted to evaluate them with respect to the following factors: (a) Is there an explanation why a higher level of sociality evolved in the Homo genus, rather than in other animals? (b) What kind of evolutionary mechanism is provided, and is it plausible? (c) Is the timing proposed consistent with relevant anatomical changes (e.g., bipedalism, reduced canines, and reduced sexual dimorphism)? (d) Is the theory consistent with anthropological evidence from extant hunter-gatherer societies, as well as technologically advanced ones? (e) Is the theory consistent with evidence on how the features claimed to be unique for human sociality develop in children? In this section, I will briefly review the claims of these theories and how they relate to these five criteria, and in addition, consider their compatibility with the bodily mimesis hypothesis from Section 1.

The four theories can be summarized as follows. Dunbar (1996) proposes that life on the savannah necessitated larger groups, which required a novel mechanism for social bonding, realized first as vocal grooming. Deacon (1997) hypothesizes that a sex contract in a multi-male/multi-female group paved the way to 
both morality and symbolic reference. Tomasello (2008) advocates a cognitive adaptation for joint intentionality and Hrdy (2009) argues that a transition to alloparenting as dominant reproductive and rearing strategy radically altered our ancestors' interpersonal relations. The first two of these theories focus on the origin of language, with sociality as something of a "byproduct," while the latter two are explicitly formulated as precursor theories. Let us consider the cases for each below in terms of the first four criteria, leaving the final developmental factor (e) for special consideration at the end.

\subsection{Vocal Grooming and Gossip}

Dunbar shows that physical grooming is an important mechanism of social bonding and coalition building in primates. However, it is time-consuming and therefore sets limits on group sizes. With life in open territory, larger groups are beneficial for minimizing the risk of predation, foraging and territory defense. The solution found by our ancestors was first "vocal grooming," on top of which "language evolved to service social bonds in a more generic sense by providing a substitute for social grooming" (Dunbar 2009, 14). But why was this evolutionary path not taken by other primates? The ecological conditions of gelada baboons are apparently similar. In partial support for Dunbar's theory gelada also live in large terrestrial groups/herds of 200-300 individuals, and have evolved enhanced vocal signaling to keep contact with members of the same "harem" (a one-male reproductive unit), but nothing resembling a symbolic communication system allowing them to "gossip."

Dunbar famously bases his theory on fairly robust correlations between group and neo-cortex sizes in primates from monkeys to humans, interpolating the likely group sizes of extinct hominins. The central proposal is that neo-cortex increased in response to larger groups: "Since maintaining coherent groups is cognitively demanding, brain size (or more specifically neo-cortex volume) will evolve to match the cognitive demands of the species' optimal group size" (Sutcliffe et al. 2012, 51). References to "mating opportunities" suggest standard individual-level selection: individuals with larger brains, a larger number of friends and better vocal grooming reproduced more successfully. But one may argue that group size optimality is not a property determined by individual brains, and that some form of multi-level selection may be needed to converge on "optimal" groups, both in terms of size, and the adequate means to bond their members. Present-day human social groups fall into three categories - small, medium, and large, corresponding to "bands," "cultural lineage groups," and "tribes" - with respective size ranges of $30-50,100-200$, and $500-2500$ members each. The number that best matches Dunbar's estimates for "optimal group size" given human neo-cortex is approximately 150 . This so-called "Dunbar's number" has been criticized: in Western societies "the range in network size is vast, with $90 \%$ of the adult population knowing anywhere between 250 and 1,710 other people, and half knowing between 400 and 800" (Wellman 2012, 174). On the other 
hand, most anthropologists emphasize the band of 30-50 people as the most significant group for hunter-gatherers, and it is likely that in such "societies of intimates" the initial adaptations for human-specific intersubjectivity took place.

On the issue of timing, Dunbar (2009) points to the relatively scarce fossil evidence of a larger thoracic vertebral canal (MacLarnon and Hewitt 1999), which has been (controversially) interpreted as an index of improved vocal control in Homo heidelbergensis and concludes that "the 0.5 mya [R]ubicon may mark the appearance of some form of intensely music-like exchanges, with full grammatical language (i. e. language as we know it today) emerging only later - perhaps with the appearance of anatomically modern humans around $200 \mathrm{kya}$ " (Dunbar $2009,29)$. But 0.5 MYA is a very late onset for the process leading to human-specific sociality, given the many earlier changes, from Ardipithecus ramidus at 4.4 MYA (involving reduced canines, reduced sexual dimorphism and partial bipedalism) to the mimetic culture of Homo erectus, from 1.8 MYA (see Section 1).

\subsection{Sex Contract}

Deacon's explanation for the evolution of human-specific sociality is likewise ecological: our ancestors needed to survive in large male-female groups, with slowly maturing infants (due to bipedalism). This called for extensive maternal care and paternal provisioning, which could only be stabilized through a contract that required symbolic marking of the social rights and obligations of sex-partners. As summarized by Deacon (1997): “The need to mark these reciprocally altruistic relationships arose as an evolutionary adaptation to the extreme instability of the combination of group hunting/scavenging and male provisioning of mates and offspring" (ibid., 401). In other words, Deacon proposes that social norms and symbol use spearheaded the process that made human societies more cooperative rather than vice-versa. Analogous to Dunbar, Deacon correlates the evolution of "symbolic reference" with increase in brain size, but emphasizes the prefrontal cortex, responsible for much of "higher" social cognition and executive functions, as especially important.

Since at the root of the adaptation proposed by Deacon is not pair-bonding per se (which could perhaps be accounted for by direct reciprocity, and expected to result in an adaptation for emotional attachment: love), but symbolically mediated social norms, beneficial for the social group as whole, group selection is even more strongly implied than in the case of Dunbar - groups that found a way to ensure sex-based division of labor and paternal provisioning out-competed those that did not. When did this happen? Deacon also appeals to novel ecological conditions of "life on the savannah" for what started the process, but places the beginning of the transition at more than 3 MYA, with australopithecines. Since for Deacon this should mark the onset of symbol use (rather than "music-like exchanges"), this is a remarkably early date, without any support in the archaeological record. Also, since for hunter-gatherers the distribution 
of food concerns not "nuclear families" but the whole group (Wiessner 2002), Deacon's scenario seems rather unrealistic. Paternal provisioning is far from universal: "Across cultures and between individuals, more variation exists in the form and extent of paternal investment in humans than in all other primates combined" (Hrdy 2009, 162).

\subsection{Joint Intentionality}

Departing from child and language development, Tomasello has over the past two decades presented different versions of the thesis that the evolution of language must be grounded in prior adaptations for social-cultural life. Tomasello (2008) argues for two such adaptations: a capacity for shared intentionality, needed for conducting planned actions jointly, and a pro-social motivation to share (information). More recently, these are referred to as joint intentionality, and a two-step process is ascribed to its evolution. The first step is the following: "a change of ecology ... lead humans to an interdependent lifestyle, especially collaborative foraging, which resulted in the evolution of new skills and motivation for collaborating with others (joint intentionality), and gave individuals special incentives for helping their partners altruistically as well" (Tomasello et al. 2012, 685). Evidence of active collaborative hunting and gathering is said to coincide with the emergence of Homo heidelbergensis ca. 800,000 years ago.

The second major step involves extending such "small-scale collaboration" to whole communities on the basis of cultural conventions, norms and institutions, setting the stage for cultural group selection and cumulative cultural evolution. Timewise, this is said to coincide with the appearance of Homo sapiens. The original way of dividing shared intentionality and pro-social motivation had the troublesome implication that motivational/emotional aspects would have evolved only at a secondary stage. With the extended scenario, altruistic motives are expected to evolve during the first step: "obligate collaborative foraging produced interdependence among members of a group, and this makes it in my direct interest to help others who might be my future partners" (Tomasello et al. 2012, 679). In sum, while Tomasello does not explicitly endorse multi-level selection, the overall account, ranging from reproductive benefits for individuals, through "social selection," to cultural group selection, is fully consistent with it. Further, the authors state that "in this context humans became cooperative breeders, regularly providing childcare to offspring who were not their own" (Tomasello et al. 2012, 680), explicitly acknowledging the final theory under discussion.

\subsection{Alloparenting}

Hrdy (2009) proposes that what started the cascade of processes which led to increased brain size, human-specific cognitive abilities and ultimately language was a switch in reproductive and rearing strategy: "Without doubt, highly com- 
plex coevolutionary processes were involved in the evolution of expanded lifespans, prolonged childhoods, and bigger brains. What I want to stress here, however, is that cooperative breeding was the pre-existing condition that permitted the evolution of these traits in the hominin line" (Hrdy 2009, 277; italics added). Such a switch is plausible, since it is only in our species among the Great Apes that childcare is extensively shared among group members. While in orangutans, gorillas, chimpanzees, and even bonobos, mothers are the only ones to hold and nurse infants due to fear of kidnapping or infanticide, other alloparenting primates like marmosets and tamarins are "unusually altruistic, displaying a curiously human impulse to give” (Hrdy 2009, 96).

Unlike the other authors discussed here, Hrdy does not appeal to changes in ecological conditions for what started the process: our ancestors did not bave to make the transition to alloparenting (characterizing only $3 \%$ of mammal species), but the groups who did so were evolutionary winners, as alloparenting allows for unusually fast rates of reproduction, despite large-brained, and slowly maturing babies: "Mothers can overshoot their capacities to provide, and fathers can vary, because both sexes evolved in a highly fluid system where alloparents often provided the compensatory assistance" (Hrdy 2009, 167). The hypothesis is that the nurturing tendencies of mothers were extended to other members of the group, including fathers but not limited to them, allowing food and childcare to be more equally distributed within the group than, for example, in Deacon's scenario. The proposal is also distinct from the so-called grandmother bypothesis, according to which women live longer than female apes after ceasing to ovulate, due to their positive role in the survival of grandchildren (Hawkes 2004). Hrdy observes that human longevity increased for both men and women, and while (maternal) grandmothers typically function as alloparents, other group members do as well: "Efe babies average 14 different caretakers in the first days of life” (Hrdy 2009, 9).

Hrdy argues that this process can be accounted for by an evolutionary model that generalizes Hamilton's Rule $r B>C$ where $r$ does not refer only to genetic relatedness (and hence standard kin selection) since "once the neural and physiological underpinnings for helping behavior were in place, helpers did not need to be close kin" (Hrdy 2009, 88). Since kin selection can be seen as a special form of group selection (Sober and Wilson 1999) this "generalized kin selection" can be seen as a form of multi-level selection, with selection between groups favoring those which have adapted the "winning strategy." The transition is proposed to have occurred with Homo ergaster/erectus around 1.8 MYA, based on evidence for changes in diet (including meat), sexual division of labor, larger brains and longer life-spans. However, this was hardly an abrupt transition, since the traces of a process of "self-domestication" can be found in Ardipithecus ramidus, with reduced sexual dimorphism and partial bipedalism. This suggests a co-evolutionary scenario of more immature infants, prolonged childhood, more need for shared care and provisioning, in which alloparenting was not the single initial factor, as suggested by Hrdy, but was itself facilitated by increased altriciality (i. e., very immature babies). In birds, cooperative breeding has been found to be 
more likely to evolve in taxa where chicks are helpless rather than in those where they are soon able to survive on their own (Cockburn 2006).

Hrdy bases her theory extensively on the child-care practices of extant hunter-gatherer groups, reminding us that these should not be viewed as "living fossils," but rather as the closest models for what the lives of our pre-agricultural ancestors could have been like. It is significant that in culturally, geographically and environmentally highly distinct hunter-gathering societies such as Aka, Efé, !Kung San (Central Africa), Himba (Western Africa), Yanomamo (Venezuela), and Agta (Philippines), care is shared between mothers and alloparents, and in some cases fathers.

\subsection{Ontogenetic Development and Summary}

I single out here ontogeny as a special criterion, in line with the emphasis in current evolutionary thinking on the need to integrate evolution and development in a united evo-devo approach, as "evidence from genetics, embryology, and developmental biology has converged to offer a more epigenetic, contingent, and dynamic view of how organisms develop" (Lickliter and Honeycutt 2003, 819). Or as summarized by Thompson (2007): "The core idea of this new synthesis that all important changes in evolution are alternations in development" (ibid., 195). As shown below, of the four theories, only those of Tomasello and Hrdy explicitly address development, and that of Hrdy is arguably the one that is most consistent with an integrated evo-devo approach.

The key adaptations of Dunbar's theory are expanded neo-cortex and improved vocalization. Concerning the latter, human-specific vocalizations indeed start early in the first year of life, but the neo-cortex undergoes extensive expansion first in late childhood (6-11 years), after much of language and symbolic competence are already in place. Deacon (1997) repeatedly points out that symbols (in his particular definition) are easily acquired only by young brains. But why this should be so is not fully clear, as the supposedly key pre-frontal cortex matures slowly in development, and not fully until adulthood. Tomasello and colleagues focus on early-developing social skills such as sharing impulses, joint attention and declarative pointing, which are claimed to be human-specific, universal and pre-linguistic (Liszkowski et al. 2012; Tomasello et al. 2005). While some of these claims have been contested (Leavens and Racine 2009; Leavens, Hopkins, and Bard 2005), evidence of prolonged childhood compared to apes, and apes consistently failing tasks that require cooperative intentions where even preverbal children succeed, strongly suggest an evolutionary adaptation for intersubjectivity realized in early childhood. What is less clear is how it could have been selected for in the kinds of contexts envisioned by Tomasello and colleagues, such as (cooperative) foraging.

Hrdy explicitly evokes the infant intersubjectivity approach in developmental psychology (Bråten 2006; Trevarthen 1979), which can be summarized in the words of its key exponent: "We are born to generate shifting states of self-aware- 
ness, to show them to other persons, and to provoke interest and affectionate responses from them" (Trevarthen 2011,119). This approach has been criticized for being too "adaptationist" and inconsistent with current evo-devo thinking (Racine et al. 2014), but placed in the perspective of Hrdy's theory, this is clearly not so. According to the latter, growing up in the context of alloparenting, the human child is both selected for and develops enhanced understanding of perspective and self-awareness:

A baby thus had far more incentive to monitor his mother's whereabouts and to maintain visual and vocal contact with her, as well as far more motivation to pay attention to her state of mind, and to the willingness of others who might be available to care for him when his mother was disinclined. (Hrdy 2009, 114)

Furthermore, the adaptation is assumed to have concerned not only children, but the whole interactional niche, involving a "self-reinforcing evolutionary process of parents and alloparents who are more sensitive to infantile signals and babies who are better at emitting them" (Hrdy 2009, 220). As pointed out earlier, this can be conceived in terms of post-Neo-Darwinist ideas like niche-construction and multi-level selection, and is not predicated on simplistic kinds of evolutionary psychology.

We can now sum up the comparison of the four theories of the evolution of human-specific sociality in terms of the five factors given at the beginning of this section. Concerning (a), Dunbar, Deacon and Tomasello appeal to specific (though somewhat different) ecological conditions for the transition, but in all cases other primates could be seen to share these conditions and yet do not evolve the human intersubjective skills. In comparison, Hrdy's argument that alloparenting was the "pre-existing condition" that started the process is the most persuasive one. Concerning (b), I have suggested that all four theories imply, explicitly or implicitly, multi-level selection, providing more arguments elsewhere (Zlatev 2014b).

With respect to $(c)$ the alloparenting hypothesis is most consistent with the evidence for early origins of human intersubjectvity, with or even prior to Homo erectus of 1.8 MYA. Dunbar's claimed transition to vocal grooming around 0.5 MYA is much too late to explain the earlier biological and cultural changes to our species, while Deacon's presumed onset of symbolism at 3 MYA is much too early. However, as will be suggested in the conclusions, their ideas may be applicable to specific aspects, in a composite evolutionary account. Concerning (d), the kind and size of the relevant social groups, the "band" of 30-50 members seems to be key, corresponding most closely to the social niche in which human-specific intersubjectivity first arose, prior to language. On the other hand, identifying and cooperating with the "clan" of 150 or so people, and the "tribe" of 500 , not to mention still larger circles like "nations," clearly require moral rules and symbolically mediated shared values, which presuppose language. Finally, we saw above how both alloparenting and shared intentionality can be aligned with evidence from child development, with some degree of correspondence to developmental stages. Bråten (together with Trevarthen) distinguishes, schematically, between (1) primary intersubjectivity, from the first months of life 
onwards, based on "direct sympathy with actual others' expressions of feelings in intimate reciprocal subject-subject contact”; (2) secondary intersubjectivity, from 9 months, involving "objects of joint attention and emotional referencing are brought into play within trusting relations of companionship ... sometimes inviting imitative learning"; and (3) tertiary intersubjectivity, based on "symbolic conversation with actual or virtual companions” (Bråten 2007, 3). We may link these developmental stages to evolutionary stages, and the Mimesis Hierarchy (see Table 1, Section 1) as follows: (1') human-specific traits of intersubjectivity evolved first on the level of "direct sympathy" between child and alloparents; (2') these were subsequently extended to the cognitive skills of shared intentionality and mimesis (dyadic and triadic); (3') only (much) later, with the evolution of proto-language and language, were "symbolic conversations" and the kind of moral norms envisioned by Deacon made possible.

Thus, of the four theories reviewed in this section, Hrdy's theory fares best with respect to all the comparative criteria - as an explanation of why and how the unique type of human intersubjectivity, combining features of Great Ape intelligence and tamarin-like altruism, could evolve. It is consistent with Tomasello's theory of joint intentionality, after reversing the originally proposed order in which the more cognitive aspects preceded the motivational ones, placing the onset on "direct sympathy with actual others' expressions of feelings" in the context of communal reproduction, child care and practical teaching. The bodily and emotion-laden nature of these basic capacities, practices and skills motivates seeing them as expressions of human embodiment, anticipating and perhaps further co-evolving with bodily mimesis.

\section{From Bodily Mimesis to Language: The Role of Multimodality}

As described in Section 1, and schematically shown in the Mimesis Hierarchy, bodily mimesis provides two of the four features of symbol use given in the introduction. First: a representational relation between sign and object, above all on the basis of an iconic (resemblance-based) ground. Second: the understanding of communicative intent, a second-order intention for the addressee to grasp the communicator's first-order informative intention. I have argued that the combination of these two features is a pre-linguistic, human-specific characteristic. While Tomasello's shared intentionality hypothesis, and in particular joint attention, with its higher-order intentionality ("I wish for you to see that I see X"), highlights the second feature, bodily mimesis accommodates both (Zlatev 2008a; Zlatev et al. 2013).

But what about the highest two layers of the Mimesis Hierarchy which, as reflected in Table 1, imply the remaining two features of symbolic communication: the use of conventional (i. e., socially shared) and systematic signs? This question was addressed in a recent doctoral thesis with the characteristic title The Evolution of Symbolic Communication: An Embodied Perspective, posing the problem as follows: 
A major step in the evolutionary process by which human communication could have emerged has been proposed in the bodily mimesis hypothesis... This ability provides a foundation from which symbolic communication can arise, but how such a transition would have taken place has not been fully examined. This thesis examines the gap between bodily mimesis and symbolic communication. (Brown 2012,1)

It should be noted that Brown (2012) assumes a different definition of "symbol" than that used here: a customary (negative) definition stating that the symbolic sign is not to stand in a motivated relation to its referent, but rather to be "arbitrary." This until recently generally accepted take on the "linguistic sign" in the steps of Saussure ([1916] 1983), has been seriously questioned (Alhner and Zlatev 2010; Perniss, Thompson, and Vigliocco 2010), to the extent of claiming that non-arbitrariness, i. e., degrees of similarity and contiguity between sign and object, may constitute an essential prerequisite for language acquisition (Imai and Kita 2014). Nevertheless, Brown's approach to the evolution of symbols, grounded in experimental semiotics and computational simulations, is highly useful for the present account due to the following considerations. First, it helps explain the gradual shift from a predominantly bodily to a predominantly vocal mode of expression (Zlatev, Donald, and Sonesson 2010). Second, it explains why the process was accompanied by a partial loss of iconicity, and thus greater reliance on knowing linguistic conventions (Zlatev 2014a). Third, the combination of the previous two transitions may have provided a key contribution for the evolution of the final feature of symbols: elaborate within-system relations and combinatorial potential. Let us motivate these three factors in turn.

As pointed out in Section 1, the "switch to speech" is currently considered the key problem of gesture-first theories of language origins (Fitch 2010). Multimodal origin theories avoid this, but at a price. Two different kinds of multimodal theories of language origins can be distinguished. First, single system theories claim that "hand and mouth" were tightly interlinked from the start, with a distribution of semiotic labor - the gestural modality carrying the iconic/imagistic representation, and the vocal the symbolic/arbitrary ones (McNeill, 2005, 2012). The problem with such theories is that they both predict a stronger link between the two modalities than what is attested, e. g., in ontogenetic development (Bates et al. 1979; Zlatev 2015), and that they underestimate the degree of non-arbitrariness in speech, (e.g. Dingemanse 2012), an insight that is being increasingly supported, as pointed out earlier.

The kind of multimodality predicted by bodily mimesis is of a weaker kind, with the bodily-visual and vocal-auditory channels in coordination, but still operating as two supplementary modes of expression, according to the second type of multimodal theories: interactive ones (Kita and Özyurek 2003). Due to a number of factors, such as its proximity to fully-fledged actions, the bodily channel would have dominated communication initially. Then, over a prolonged period of time, a gradual shift of the communicative load toward the vocal channel can be expected to have taken place: "a transitional period of over half a million years ... from the appearance of Homo erectus to that of archaic Homo sapiens ... during all this time, humans regularly communicated bi-mod- 
ally, only gradually shifting from a code that foregrounded gesture to one that foregrounded voice" (Collins 2013, 136). The question is why this happened, as simple ecological explanations (e. g., "speech could take place in the dark") are unconvincing (Fitch 2010). The answer given by Brown (2012) and others using the tools of experimental semiotics (Galantucci and Garrod 2010) lies in the semiotic properties of the modalities: the vocal medium has less potential for representing meaning on the basis of similarity than the manual-bodily medium (Fay, Arbib and Garrod 2013). Furthermore, there is a trade-off: when relatively few messages need to be communicated, an "iconic code" is advantageous, but with larger vocabularies, iconic coding leads to ambiguity, and hence a more arbitrary mapping is more stable (Monaghan, Mattock and Walker 2012). As summarized by Brown (2012): "the vocal modality would have become predominantly symbolic because its lower non-arbitrary capacity increases the likelihood that vocalizations are perceived as arbitrary" (ibid., 134).

However, as the iconicity of the vocal channel decreases, it is even more important that the non-arbitrariness of bodily mimesis functions as a support structure: "the conventionalization process requires a rich and supportive communicative infrastructure in which novel arbitrary signs can be used ... so that the intended form-meaning relationships could be correctly interpreted" (ibid., 81). Thus, parallel use of both channels while bodily mimesis maintained its iconic-indexical ground was needed for the stabilization of a code based on fully conventional (i. e., commonly known) and iconically bleached ("arbitrary") signs. In contrast, this process would not have worked if mimesis (or "pantomime") had first been reduced to "protosign," as per the definition given by Arbib (2005): "a manual-based communication system ... elements for the formation of compounds which can be paired with meanings in a more or less arbitrary fashion" (ibid., 195). If gestural communication had become such, it could not function as the "rich and supportive communicative infrastructure" needed to support the learning of vocal signs that have lost their transparency, and it would be much harder to explain why the gestural modality does not continue to dominate in language. In sum, according to this argument (which is admittedly tentative since it is based on indirect evidence from experiments and simulations), the "weaker" form of multimodality of bodily mimesis was necessary both for the relative shift in communicative load from body to mouth, as language remains multimodal (Zlatev et al. 2010), and for the establishment of a protolanguage with a large number of fully conventional spoken signs (Monaghan, Mattock, and Walker 2012).

At this stage, the organization of signs into systems of paradigmatic oppositions and syntagmatic/syntactic relations would have been facilitated: as the "arbitrary" signifiers are more detached from their referents, they become to a larger extent objects of attention in their own right. This would have stimulated learning their interrelations: the linguistic system of oppositions, as envisaged by structuralism, and of the combination of words into propositions. This, I believe, is at the core of Deacon's conception of "symbolic reference" (Deacon 1997), according to which symbols are essentially interconnected in systems, and not 
primarily related to the world of experience, in the manner of iconic and indexical signs. At the same time, such symbols would not be able to function without the latter, an example of what Deacon (2003) elsewhere refers to as "semiotic constraints." Note that the present proposal, which to my knowledge has not been previously elaborated, is not that "the transition to speech" was a necessary precondition for the evolution of grammar. First of all, such a statement would be falsified by the elaborate grammars of signed languages. Rather, the suggestion is that for hearing people, there was a natural drift toward the vocal modality with larger vocabularies (which came along with more complex cultures), and the combination of large vocabularies and relatively “arbitrary" signs, was a key push toward the organization of linguistic systems. In fact, there is evidence for a certain degree of complementarity between iconicity and system character in signed languages as well, where the process from bodily mimesis to full-scale symbol use can be observed to take place over a number of generations (Sandler 2012).

\section{Summary and Conclusions}

We began by proposing that the question of the evolution of language is largely synonymous with that of the evolution of symbols, after defining these as conventional, intentional and systematic representations. Communication, and eventually thought, on the basis of such signs is qualitatively different from that of non-human animals (Zlatev 2009). Yet there was no cataclysmic event which brought about this in a leap: evolution is a miser, and proceeds in relatively small steps. The resolution of this apparent contradiction requires a scenario of the evolution of symbol use that takes place over a prolonged period of time, and a number of more or less distinct stages. We may conclude by bringing together the three key preconditions of symbolic communication related to human embodiment focused on in this chapter - alloparenting, bodily mimesis and multimodality - in a composite account, accommodating certain points from other theories that were mentioned along the way.

Hrdy's thesis that a transition to alloparenting, unique among the Great Apes and very rare among mammals, characterized the evolutionary niche in which human intersubjectivity evolved, offers the best explanation so far of what set human evolution on a different track from that of the other Great Apes. This transition can be hypothesized to have begun even before the evolution of Homo ergaster-erectus of ca. 1.8 MYA, and perhaps even to have served as one of the crucial factors that led to this, including the stabilization of bipedalism.

The second major transition was that of Donald's "mimetic culture" based on tool production, pedagogy, division of labor, cooperative hunting and foraging. All these presuppose mimetic, multimodal but predominantly gestural, communication with communicative intentions: "triadic mimesis." Importantly, this was not yet language, and arguably not even a protolanguage (see Table 1). Tomasello's theories and empirical support for pre-linguistic shared/joint intentionality is fully compatible with this account. 
The next step in the scenario is the hypothesized gradual transition to a vocal-gestural protolanguage, leading to Homo heidelbergensis ca. 0.5 MYA, the ancestor species to both us and the Neanderthals. As argued in Section 3, the multimodal nature of bodily mimesis, and the different intrinsic capacities of gesture and speech for iconicity, would have played a key role for the transition. In addition, Dunbar's hypothesis of "vocal-grooming" suggests a second reason why vocal communication (and improved volitional control of the vocal apparatus) could have been enhanced. Initially, gesture and vocalization could mostly have served different functions - representation and affiliation, respectively - but over time, vocalizations would have been "reinterpreted" as communicative signs, given the tight synchronization of multimodal, gestural-vocal communication.

With the larger, more organized and technologically advanced social groups of anatomically modern Homo sapiens from ca. 200,000 years ago, a larger vocabulary of conventional expressions, increasingly relying on the vocal channel, would have provided the impetus for systematization. Thus the capacity for learning and using intricate sign-sign relationships would have come about: the final feature from the definition of symbol use used in this chapter. To some extent, Deacon's "sex contract," linking linguistic and moral norms, may have played a role in this process, but much later than originally proposed. Thus the human potential for system building may, in a sense, be "innate," and yet not codified in the genes, or a "language instinct," in a way that is consistent with current evo-devo thinking. Importantly, this potential is in the words of Arbib (2005) "post-biological," i. e., emerging through processes of cultural evolution, such as grammaticalization.

Equally importantly, such a composite scenario of the evolution of human symbolic communication, which I believe is both supported by the evidence and theoretically coherent, goes beyond "mainstream" models of evolution based only on individual-level and gene-level selection. In the footsteps of Donald's (1991) original stage-model of human cognitive semiotic evolution, it presupposes extensive bio-cultural co-evolution, and extended Darwinian models such as multi-level selection theory, operating on both individuals and groups, with complex tradeoffs between them. Less complex models could hardly be expected to be able to account for the evolution of the one and only "symbolic species" on our planet.

\section{Acknowledgements}

I wish to thank Johan Blomberg, Slawomir Wacewicz and Christian Tewes for helpful comments on previous versions of this text. 


\section{Bibliography}

Alhner, F. and J.Zlatev. 2010. Cross-modal Iconicity: A Cognitive Semiotic Approach to Sound Symbolism. Sign System Studies 38: 298-348.

Arbib, M. 2005. From Monkey-like Action Recognition to Human Language: An Evolutionary Framework for Neurolinguistics. Behavioral and Brain Sciences 28: 105-168.

Aristotle. 1987. The Poetics of Aristotle. Trans. S. Halliwell. Chapel Hill: The University of North Caroline Press.

Bar-On, D. 2013. Origins of Meaning: Must We 'Go Gricean'? Mind and Language 28, 3: 342-375.

Bates, E., L. Benigni, I. Bretherton, L. Camioni, and V. Volterra. 1979. The Emergence of Symbols: Cognition and Communication in Infancy. New York: Academic Press.

Bell, C. 1997. Ritual: Perspectives and Dimensions. New York: Oxford University Press.

Brăten, S. 2006. Intersubjective Communication and Emotion in Early Ontogeny. New York: Cambridge University Press.

-. 2007. On Being Moved: From Mirror Neurons to Empathy. Amsterdam: John Benjamins.

Brown, J. E. 2012. "The Evolution of Symbolic Communication: An Embodied Perspective." PhD Thesis, University of Edinburgh.

Campbell, D. 1982. Legal and Primary-Group Social Controls. In Law, Biology and Culture: The Evolution of Law, ed. M. Gruter and P. Bohannan, 59-171. Berkeley, CA: Bepress.

Chomsky, N.1986. Knowledge of Language: Its Nature, Origin, and Use. New York: Praeger.

Christiansen, M.H. and S. Kirby. 2003. Language Evolution: The Hardest Problem in Science? In Language Evolution, ed. M. H. Christiansen and S. Kirby, 1-15. Oxford: Oxford University Press.

Cockburn, A. 2006. Prevalence of Different Modes of Parental Care in Birds. Proceedings of the Royal Society of London B: Biological Sciences 273 (1592): 1375-1383.

Collins, C. 2013. Paleopoetics: The Evolution of the Literary Imagination. New York: Columbia University Press.

Deacon, T. 1997. The Symbolic Species. New York: Norton.

-. 2003. Universal Grammar and Semiotic Constraints. In Language Evolution, ed. M. H. Christiansen and S. Kirby, 111-139. Oxford: Oxford University Press.

Dingemanse, M. 2012. Advances in the Cross-Linguistic Study of Ideophones. Language and Linguistics Compass 6: 654-672.

Donald, M. 1991. Origins of the Moden Mind: Three Stages in the Evolution of Human Culture. Cambridge, MA: Harvard University Press.

-. 1998. Mimesis and the Executive Suite: Missing Links in Language Evolution. In Appoaches to the Evolution of Language: Social and Cognitive Biases, ed. J. R. Hurford, M. Studdert-Kennedy, and C. Knight, 44-67. Cambridge: Cambridge University Press.

-. 2001. A Mind so Rare: The Evolution of Human Consciousness. New York: Norton.

-. 2012. The Mimetic Origins of Language. In The Oxford Handbook of Language Evolution, 180-183. Oxford: Oxford University Press.

Dor, D., C. Knight, and J. Lewis. 2014. The Social Origins of Language, Studies in the Evolution of Language, Vol. 19. Oxford: Oxford University Press.

Dunbar, R. 1996. Grooming, Gossip, and the Evolution of Language. Cambridge, MA: Harvard University Press.

-. 2009. Why only Humans have Language. In The Prehistory of Language, ed. R. Botha and C. Knight, 12-35. Oxford: Oxford University Press.

Fay, N., M. Arbib, and D. Garrod. 2013. How to Bootstrap a Human Communication System. Cognitive Science 37 (7): 1356-1367. 
Fitch, W. T. 2010. The Evolution of Language. Cambridge: Cambridge University Press.

Galantucci, B. and S. Garrod. 2010. Experimental Semiotics: A New Approach for Studying the Emergence and the Evolution of Human Communication. Interaction Studies 11: $1-13$

Gergely, G. and G. Csibra. 2006. Sylvia's Recipe: The Role of Imitation and Pedagogy in the Transmission of Human Culture. In Roots of Human Sociality: Culture, Cognition, and Human Interaction, ed. N. Enfield and S. Levinson, 229-255. Oxford: Berg Publishers.

Givón, T. and P. Young. 2002. Cooperation and Interpersonal Manipulation in the Society of Intimates. Typological Studies in Language 48: 23-56.

Gowdy, J. and L. Krall. 2016. The Economic Origins of Ultrasociality. Behavioral and Brain Sciences 39: 160.

Hamilton, W. D. 1964. The Genetical Evolution of Social Behaviour. Journal of Theoretical Biology 7: 1-16.

Hawkes, K. 2004. Human Longevity: the Grandmother Effect. Nature 428 (6979): $128-129$.

Heine, B. and T. Kuteva. 2007. The Genesis of Grammar: A Reconstruction. Oxford: Oxford University Press.

Horner, V.K. and A. Whiten. 2005. Causal Knowledge and Imitation/Emulation Switching in Chimpanzees (Pan Troglodytes) and Children. Animal Cognition 8: 164-181.

Hrdy, S. B. 2009. Mothers and Others: The Evolutionary Origins of Mutual Understanding. Cambridge, MA: Harvard University Press.

Hurford, J. R. 2007. The Origins of Meaning; Language in the Light of Evolution. Oxford: Oxford University Press.

Imai, M. and S. Kita. 2014. The Sound Symbolism Bootstrapping Hypothesis for Language Acquisition and Language Evolution. Philosophical Transactions of the Royal Society B 369, 2013098.

Itkonen, E. 2003. What is Language? A Study in the Philosophy of Linguistics. Turku: Turku University Press.

Jablonka, E. and M. J. Lamb. 2007. Précis of Evolution in Four Dimensions. Behavioral and Brain Sciences 30 (4): 353-365.

Kita, S. and A. Özyurek. 2003. What Does Cross-Linguistic Variation in Semantic Coordination of Speech and Gesture Reveal? Evidence for an Interface Representation of Spatial Thinking and Speaking. Journal of Memory and Language 48: 16-32.

Leavens, D. A., W.D. Hopkins, and K. A. Bard. 2005. Understanding the Point of Chimpanzee Pointing. Current Directions in Psychological Science 14: 185-189.

Leavens, D. and T. P. Racine. 2009. Joint Attention in Apes and Humans: Are Humans Unique? Journal of Consciousness Studies 16 (6-8): 240-267.

Lickliter, R. and H. Honeycutt. 2003. Developmental Dynamics: Toward a Biologically Plausible Evolutionary Psychology. Psychological Bulletin 129 (6): 819.

Liszkowski, U., P. Brown, T. Callaghan, A. Takida, and C. de Vos. 2012. A Prelinguistic Gestural Universal of Human Communication. Cognitive Science 36 (4): 698-713.

MacLarnon, A. M. and G. P. Hewitt. 1999. The Evolution of Human Speech: The Role of Enhanced Breathing Control. American Journal of Physical Antbropology 109 (3): 341-363.

McCune, L. 2008. How Children Learn How to Learn Language. Oxford: Oxford University Press.

McNeill, D. 2005. Gesture and Thought. Chicago: University of Chicago Press.

-. 2012. How Language Began: Gesture and Speech in Human Evolution. Cambridge: Cambridge University Press. 
Monaghan, P., K. Mattock, and P. Walker. 2012. The Role of Sound Symbolism in Word Learning. Journal of Experimental Psychology: Leaning, Memory and Cognition 38: $1152-1164$.

Oyama, S., P. Taylor, A. Fogel, R. Lickliter, K. Sterelny, K. C. Smith, and C. van der Weele. 2000. The Ontogeny of Information: Developmental Systems and Evolution. Durham, NC: Duke University Press.

Perniss, P., T. Thompson, and G. Vigliocco. 2010. Iconicity as a General Property of Language: Evidence from Spoken and Signed Languages. Frontiers in Psychology 1: $1-15$.

Racine, T. P., T.J. Wereha, O. Vasileva, D. Tafreshi, and J.J. Thompson. 2014. The Evolution of Joint Attention: a Review and Critique. In The Evolution of Social Communication in Primates, ed. M. Pina and N. Gontier, 127-145. Berlin: Springer.

Richerson, P.J. and R. Boyd. 2005. Not by Genes Alone: How Culture Transformed Human Evolution. Chicago: University of Chicago Press.

Sandler, W. 2012. Dedicated Gestures, and the Emergence of Sign Language. Gesture 12: 265-307.

Saussure, F.d. (1916) 1983. Course in General Linguistics. Trans. Roy Harris. London: Duckworth.

Sober, E. and D.S. Wilson. 1999. Unto Others: The Evolution and Psychology of Unselfish Behavior. Cambridge, MA: Harvard University Press.

Sonesson, G. 2007. From the Meaning of Embodiment to the Embodiment of Meaning: A Study in Phenomenological Semiotics. Body, Language and Mind. Vol. 1: Embodiment: 85-128. Berlin: Mouton de Gruyter.

Sperber, D. and D. Wilson. 1995. Relevance: Communication and Cognition. Malden, MA: Blackwell Publishing.

Sutcliffe, A., R. Dunbar, J. Binder, and H. Arrow. 2012. Relationships and the Social Brain: Integrating Psychological and Evolutionary Perspectives. British Journal of Psychology 103 (2): 149-168.

Taglialatela, J. P., J. L. Russell, J. A. Schaeffer, and W. D. Hopkins. 2011. Chimpanzee Vocal Signaling Points to a Multimodal Origin of Human Language. PloS One 6 (4): e18852.

Tatterstall, I. 2014. Communication and Human Uniqueness. In The Evolution of Social Communication in Primates, ed. M. Pina and N. Gontier, 219-228. Berlin: Springer.

Thompson, E. 2007. Mind in Life: Biology, Phenomenology, and the Sciences of Mind. Cambridge, MA: Harvard University Press.

Tomasello, M. 1999. The Cultural Origins of Human Cognition. Cambridge, MA: Harvard University Press.

-. 2008. The Origins of Human Communication. Cambridge, MA: MIT Press.

Tomasello, M., M. Carpenter, J. Call, T. Behne, and H. Moll. 2005. Understanding and Sharing Intentions: The Origins of Cultural Cognition. Behavioral and Brain Sciences 28 (5): 675-691.

Tomasello, M., A. P. Melis, C. Tennie, E. Wyman, and E. Herrmann 2012. Two Key Steps in the Evolution of Human Cooperation. Current Antbropology 53 (6): 673-692.

Trevarthen, C. 1979. Communication and Cooperation in Early Infancy: A Description of Primary Intersubjectivity. In Before Speech: The Beginning of Interpersonal Communication, ed. M. Bullowa, 321-347. New York: Cambridge University Press.

-. 2011. What Is It Like To Be A Person Who Knows Nothing? Defining the Active Intersubjective Mind of a Newborn Human Being. Infant and Child Development 20 (1): 119-135.

Tulving, E. 2005. Episodic Memory and Autonoesis: Uniquely Human? In The Missing Link in Cognition: Origins of Self-reflective Consciousness, ed. H. Terrace and J. Metcalfe, 3-56. Oxford: Oxford University Press. 
Vaesen, K. 2012. The Cognitive Bases of Human Tool Use. Behavioral and Brain Sciences 35: $203-262$.

Wellman, B. 2012. Is Dunbar's Number Up? British Journal of Psychology 103 (2): 174176.

Werner, H. and B. Kaplan. 1963. Symbol Formation: An Organismic-Developmental Approach to Language and the Expression of Thought. New York: Wiley.

Wiessner, P. 2002. Hunting, Healing, and Hxaro Exchange: A Long-Term Perspective on !Kung (Ju/'Hoansi) Large-Game Hunting. Evolution and Human Behavior 23 (6): 407-436.

Zlatev, J. 2007. Language, Embodiment and Mimesis. In Body, Language, Mind. Vol.1: Embodiment, ed. T.Ziemke, J.Zlatev, and R. Frank, 297-337. Berlin: de Gruyter.

-. 2008a. The Coevolution of Intersubjectivity and Bodily Mimesis. In The Shared Mind: Perspectives on Intersubjectivity, ed. J.Zlatev, T. Racine, C.Sinha, and E. Itkonen, 215-244. Amsterdam: Benjamins.

-. 2008b. From Proto-Mimesis to Language: Evidence from Primatology and Social Neuroscience. Journal of Physiology - Paris 102: 137-152.

-. 2009. Levels of Meaning, Embodiment, and Communication. Cybernetics and Human Knowing 16: 149-174.

-. 2013. The Mimesis Hierarchy of Semiotic Development: Five Stages of Intersubjectivity in Children. Public Journal of Semiotics 4: 47-70.

-. 2014a. Bodily Mimesis and the Transition to Speech. In The Evolution of Social Communication in Primates, ed. P. Marco and N. Gontier, 165-178. Berlin: Springer.

-. 2014b. The Co-Evolution of Human Intersubjectivity, Morality and Language. In The Social Origins of Language, ed. D. Dor, C. Knight, and J. Lewis, 249-266. Oxford: Oxford University Press.

-. 2015. The Emergence of Gestures. In The Handbook of Language Emergence, ed. B. MacWhinney and W. O'Grady, 458-477. New York: Wiley.

Zlatev, J., M. Donald, and G. Sonesson. 2010. From Body to Mouth (and Body). In The Evolution of Language, ed. A. D. M. Smith, M. Schouwstra, B. de Boer, and K. Smith, 527-528. London: World Scientific.

Zlatev, J., E. Madsen, S. Lenninger, T. Persson, S. Sayehli, G. Sonesson, and J. v. d. Weijer. 2013. Understanding Communicative Intentions and Semiotic Vehicles by Children and Chimpanzees. Cognitive Development 28: 312-329.

Zlatev, J. and L. McCune. 2014. Towards an Integrated Model of Semiotic Development. In Cognitive Development: Theories, Stages, Processes and Challenges, ed. R. Chen, 59-76. New York: Nova Publishers.

Zlatev, J., T. Racine, C.Sinha, and E. Itkonen. 2008. The Shared Mind: Perspectives on Intersubjectivity. Amsterdam: Benjamins. 



\title{
Stages of Embodied Articulation
}

\author{
Matthias Jung
}

Abstract: Human beings are embodied symbol-users. By performing bodily movements humans are capable of creating symbolic meanings which are intimately connected with the corporeal experience of living-in-the-world, but at the same time transcend the boundaries of the organism within its environment. The concept of articulation enables us to do justice to these two complementary aspects of the human life-form. Having introduced the notion of articulation, Peirce's semiotic theory is then employed to show how the functional integration of different types of signs shapes our semiotic capabilities and with them our consciousness. Finally, the distinctions which have been elaborated are put to the test by connecting them with the history of cultural evolution.

\section{Introduction}

In the last two decades, the general claim that human cognition is not only embrained but also embodied has developed from an outsider position to something only a little short of a mainstream truism. As long as the details are left unspecified, most (second-generation) $)^{1}$ cognitive scientists and philosophers would concede that functioning brains (even if channels for sensory input and motor output are provided) are necessary but in no way sufficient conditions for cognition. In the meantime, the surge of enthusiasm about embodiment has reached an impressive height and produced rather extreme positions like radical enactivism (see Hutto and Myin 2013). The contested question now seems to be not whether cognition is embodied but how deep the embodiment goes.

Conceptualizing cognition and mind in terms of embodiment undoubtedly has many advantages. It helps us to avoid unconvincing dualist anthropologies and to keep both feet on the ground of what can either be phenomenologically described or scientifically analyzed. It situates cognition in the real world and thus immunizes against the alienating effects of neuro-constructivism in the fashion of Thomas Metzinger (2009). ${ }^{2}$ In moral philosophy, to mention a hitherto

${ }^{1}$ Notoriously, the first generation was dominated by the disembodied computer-model of the brain and a concomitant functionalism which implied the multi-realizabilty of cognitive processes.

${ }^{2}$ Alva Noë (2010) lucidly exposes and criticizes the widespread "idea that the world is a figment conjured for us by our brains" (ibid., 131) in its fatal consequences for our ability to feel at home in the world. 
mostly neglected but important point, it allows us to situate overly abstract universalisms within the enactive primary intersubjectivity of corporeal persons. ${ }^{3}$

It is, however, possible to overemphasize embodiment. This happens when, attempting to describe cognitive processes in terms of skillful coping of the organism within its specific environment, ${ }^{4}$ we lose sight of something essential for human cognition: our capacity to transcend the here and now of our embodiment. Embodied cognition is not necessarily driven by instrumental goals, as the term "coping” might be taken to imply. In this regard, the manner in which the American pragmatists are often highlighted as pioneers of the coping-conception of embodiment is characteristic. Teed Rockwell, to give one example, refers to Dewey's philosophy as paradigmatic for the pragmatist alternative to dualism and opines that for Dewey "an experience is always constituted by a goal-directed activity” (Rockwell 2005, 164). It is true that Dewey often underlines goal-directedness, but it would be a real mistake to overlook the degree to which he, for example in Art As Experience (Dewey [1934] 2008b), also acknowledges the importance of intrinsically satisfying activities with no instrumental or external purpose. Hence the importance of play in his account of embodied action. ${ }^{5}$ Such embodied activities do not lend themselves to an understanding in terms of coping. Ultimately, "every experience is the result of interaction between a live creature and some aspect of the world in which he lives" (Dewey [1934] 2008b, 50). But this leaves ample room for the aesthetic dimension and for the transcending force of higher-order goals. ${ }^{6}$ It is therefore important to avoid a one-sided reception of pragmatism as a philosophical source of embodied cognition. Only by acknowledging the degree to which the classic pragmatists arguing from within the interactional unity of organism and environment - leave room for the depth and uniqueness of human experience, can we wholeheartedly appreciate their contribution to embodied cognition.

The aforementioned depth of human experience is internally connected with our ability to handle linguistic and other symbols. In the course of our evolutionary development, the structure of our minds, as Terrence Deacon has argued convincingly, co-varied with the evolution of semiotic capabilities (Deacon 1997, 449-450). We have embodied minds, shaped by our usage of symbols as well as by our corporeality. Our symbolically (and intersubjectively ${ }^{7}$ ) enlarged consciousness enables us to escape the realm of direct experience. We live in normatively structured cultures, in which possible worlds play an integral part of our orientation in the real world. We are capable of purposeless, entirely non-instrumental thought, of "musement" in the meaning Peirce gave to that

\footnotetext{
3 For an important move in this direction, see Meuter 2006, chap. 8.

4 Hubert Dreyfus, deeply influenced by Heidegger, is the most prominent promotor of this conception (e.g., Dreyfus 2014).

5 See the section "Action and Play" in Jung (2010, 157-158).

6 The importance of - innerworldly - transcendence in Dewey's thought is carefully elaborated in Kestenbaum (2002).

7 As Michael Tomasello's “shared intentionality hypothesis" reminds us, see Tomasello (2014). For a concise summarizing diagram, see ibid. (140).
} 
term. ${ }^{8}$ Man's cognitive feats include the construction of cosmological models, the development of comprehensive worldviews and - last but not least - the writing of books about embodiment. These capabilities are difficult to account for in terms of any narrow conception of situated interaction with the environment. And they cannot be dismissed as mere epiphenomenal side-effects of the selective advantages produced by flexible coping-strategies within the environment. This point was forcefully made in Thomas Nagel's recent Mind in Cosmos (Nagel 2012). On the other hand, fortifying the distinction between body and mind into a dualism is clearly no alternative, since the latter is distinguished from the former by rendering the respective components "in terms that make ... their characteristic relations to one another ultimately unintelligible. (Descartes's dualism is, as always, the paradigm.)" (Brandom 1998, 615). Dualism avoids reductive naturalism only at the cost of denying essential embodiment. The challenge, in my eyes, thus lies in articulating our condition as situated but at the same time situation-transcending organisms in terms of embodied performances. As Teed Rockwell succinctly puts it: we are neither brain nor ghost and need to look for a nondualist alternative to the mind-brain identity theory (Rockwell 2005, 164).

The term "embodiment" itself already suggests the integration of organic functions with something different that is usually described as mind or "Geist" in the German tradition (admittedly, "Geist" has a more dualistic ring to it than "mind"). There's a different danger lurking here, though: embodiment may dualistically - be misunderstood as the contingent coupling of organic features and independently describable cognitive properties. This temptation has ancient Platonic roots but can, prima facie, also be detected in modern functionalist talk, when multiple realizability is conceived of in a manner which separates functional description and physical instantiation.

So how can we adequately think about our human condition as embodied symbol users? In this paper, I will develop a suggestion centered on the concept of articulation. Many animals communicate via highly sophisticated systems of signs, and the meaning of facial expressions, gestures, posture, gait, etc., can often be understood across species-borders, as Darwin famously showed (Darwin 1998). But there is no evidence available that any other species except Homo sapiens uses symbolic language in which meaning is conveyed by articulated strings of signs which combine direct and indirect reference to the world. Articulation is a genuinely corporeal activity, but it creates meanings which are detachable from the here and now of their articulation. By articulating what is meaningful for us in the course of our interactions with our environment, human culture brings forth a realm of meanings which are relatively independent from their origin and may in turn influence the embodied experiences of future generations, which will produce further creative fusions of actual and sedimented experience, and so on. These repeated feedback-loops between articulated meanings and novel,

\footnotetext{
${ }^{8}$ Peirce has even based his famous and controversial "Neglected Argument" (for the existence of God) on this state of mind, of which only symbol users are capable, see Peirce (1998, 434-450).
} 
unanticipated experience - hermeneutic cycles, as Wilhelm Dilthey would have called them - introduce second-order reflexivity into first-order bodily coping, without ever severing the connection to the lived body. Insofar as the idea of coping implies that the purpose of intelligent behavior is predetermined by the affordances and obstacles present in the immediate environment of the organism, a decisive transition occurs and the fixed distinction of means and ends is dissolved: real-time coping is supplemented with genuine reflection upon the reasonableness of purposes, instrumental reasoning becomes embedded in second-order thinking, mere preferences become evaluated by values and norms. In the course of all this, the living-organism-within-its-environment remains the anchor point.

All expressive systems are ultimately owned in the same way as any other motor system: that is, they are self-rooted.... The conscious mind may have reinvented itself and greatly extended its reach in language, but it has never lost its vestigial roots in embodiment. (Donald 2001, 137)

In what follows, I will try to elaborate this conception in three steps. First, I will sketch the concept of articulation as the embodied alternative to representationalist views of language, second I will introduce some basic insights from Peirce's theory of signs as a key to understanding the deep integration of symbolic transcendence and embodied sign-usage, and finally I will try to connect these insights with actual developments of cultural history.

\section{Making Us Explicit - Articulation as the Practice of Embodied Sign-Usage}

As pragmatists, phenomenologists and exponents of the hermeneutic tradition have pointed out time and again, we live in a reality of qualitative meanings. "The world in which we immediately live, that in which we strive, succeed, and are defeated is preeminently a qualitative world. What we act for, suffer, and enjoy are things in their qualitative determinations" (Dewey [1930] 1998, 195). These meanings come to us in the first instance not as representational content, but as something which is implicit in our practices and may be felt more or less intensely in the form of what John Dewey calls a "single pervasive quality" (ibid., 198). Distinct qualities accompany the situations we live through and individuate them. These qualities, however, are prima facie unarticulated. They contain no countable parts and no distinction of reference and meaning. Articulation is the process of making explicit what the felt meaningfulness of action-associated qualities actually means: where its personal or social significance lies and to which entities in the world it refers. Symbol-users sometimes accomplish this without actually articulating themselves, but even mute, fully-fledged thinking presupposes linguistic abilities and is best thought of as internalized conversation. ${ }^{9}$

9 The pragmatist philosopher who most forcefully developed the internal relation between thinking and intersubjective symbol-use is G. H. Mead: "We sometimes speak as if a person 
The etymology is helpful here: the Latin articulus literally means the bodily joint whose inflections allow us to structure movements in general. The same applies to expressive movements: they are articulated, that is, their meaning is brought about by the position, direction and order of the performed gesture. Today, not only Michael Tomasello ${ }^{10}$ is convinced that gesturing was at the beginning of symbolic language, and this means that the syntactical character of language is derivative of the process of bodily articulation in the literal sense. At the beginning of the $19^{\text {th }}$ century, Wilhelm von Humboldt ${ }^{11}$ discovered the phenomenon later called "double articulation," the fact that human languages are structured twice: words are composed out of smaller discrete phonemes, meanings are composed out of morphemes. Thus articulation is essentially embodied: it consists in the functional coupling of semiotic meaning to bodily movements characterized by discrete parts and multiple, but limited, degrees of freedom in connecting them. In his book The Symbolic Species (1997), Terrence Deacon offers a convincing case for the sensory-motor character even of phonetic articulation: empirical research shows that the gestalt-like comprehension of "articulatory gestures" 12 lies behind our astonishing ability to detect words in the fast and blurred soundstream of speech. If we had to rely on acoustic impressions alone, we would never be able to discriminate the meaning of spoken language - it is the activation of patterns which match oral-vocal movements that does the job.

Semiotic articulation (in Humboldt's terminology "Reflektion") is thus based upon somatic articulation, which is not only dependent upon our body scheme and the coordinated movements it enables but is also directly connected with the felt meanings the Deweyan unifying pervasive qualities convey. The phenomenal gestalt of a pervasive quality and the expressive meaning conveyed by the person's countenance, posture, gesture, gait, etc., are two sides of the same coin. If our embeddedness within our environment were characterized by fluent transactions, in which no hitches occurred, that would be the whole story and my paper would have to end here. But as a matter of fact, we live in a resistive world in which the interactional loops between organism and surrounding are constantly disintegrated and have to be restored again and again. As Peirce,

could build up an entire argument in his mind, and then put it into words to convey it to someone else. Actually, our thinking always takes place by means of some sort of symbols. It is possible that one could have the meaning of the word 'chair' in his experience without there being a symbol, but we would not be thinking about it in that case.... In a thought process there has to be some sort of a symbol that can refer to this meaning, that is, tend to call out this response, and also serve this purpose for other persons as well" (Mead 1967, 146).

${ }^{10}$ See the section "Symbolizing in Pantomime" in Tomasello (2014, 59-66).

11 "Es vereinigen sich also im Menschen zwei Gebiete, welche der Theilung bis auf eine übersehbare Zahl fester Elemente, der Verbindung dieser aber bis ins Unendliche fähig sind, und in welchem jeder Theil seine eigentümliche Natur immer zugleich als Verhältnis zu den zu ihm gehörenden darstellt. Der Mensch besitzt die Kraft, diese Gebiete zu teilen, geistig durch Reflexion, körperlich durch Articulation, und ihre Theile wieder zu verbinden" (von Humboldt 1994, 13).

12 "We tend to perceive speech sounds in terms of 'articulatory gestures,' whose boundaries and distinctions correspond to articulatory (i. e., somatomotor) features, not just sound features"(Deacon 1997, 359). 
Dewey, and Dilthey pointed out in unison, the need for articulation is the need for reflective transformation of problematic situations into less problematic ones (Since the pragmatist use of the term problem is likely to cause misunderstandings, a clarification is in order: for pragmatists, problems are not be conceived of as exceptional occurrences with negative value. They are as much part and parcel of our interactions as uninhibited exchange; problems in the ordinary meaning of the term form only a more troublesome subspecies of them.). In the light of this conception, making matters explicit is always driven by a felt dissonance, be it ever so slight, within the interactional cycle, which in order to be solved has to be articulated. During this process, the holistic gestalt qualities which accompany action are transformed into semiotic performances containing discrete parts in an articulated sequence which determines its meaning.

If we conceive of language, as I suggest, as the problem-driven explication ${ }^{13}$ of felt qualities by both bodily and symbolic means, the veridical representation of matters of fact features as only one subspecies of the genus and not, as truth-conditional theories of meaning would have it, as what language is all about. "Truths are but one class of meanings, namely, those in which a claim to verifiability by their consequences is an intrinsic part of their meaning" (Dewey [1927-1928] 2008a, 4-5). The gestalt qualities of the lived body in its environment can never be represented in language anyway, since they have no parts at all that might be mapped in an isomorphic manner onto linguistic content. Their function in the process of articulation is to deliver a sense of directedness, not to be captured in their entirety. As Wittgenstein reminded us, the signs we use in our speech acts are the only means we have to determine their meanings (see Wittgenstein [1953] 1971, 220). But conversely it is also true that only the felt meaning which accompanies the process of articulation guides us in choosing the right words in a given situation. In this manner, articulation is always embodied in two distinct aspects: first, both the semantic composition of words out of smaller units and the syntactic ordering of meaning within a sentence depend upon sensory-motor performances of the body; and second, the successive determination of meaning within speech depends upon corporeal qualities and their felt change during the articulation process. Depending on the necessities of the context, this process may develop in a variety of ways, focusing either on subjective, intersubjective or objective features of the problematic situation. Representational theories of linguistic meaning misconceive their topic by taking the referential aspect, which allows us to separate and make explicit intentional correlates of consciousness, for the axis around which language revolves.

${ }^{13}$ It is very easy to misconceive the phrase "problem-driven." For the classical pragmatists, it precisely doesn't mean that language and reason are mere instruments for the solving of pregiven problems. Whereas intelligence - the term used across the border between human beings and other animals - is always in the service of coping, mankind's problems stem mostly from second-order ends not already fixed by survival-problems. Of course it is possible to still call this kind of problem-solving "coping," but then what is coped with is the conditio bumana - not as something eternally fixed, but as the result of contingent historical developments. 
Charles Taylor, in a widely read paper on theories of meaning, has therefore proposed substituting representationalist accounts with expressivist ones (see Taylor 1985, 284-291). Taylor conceives of language as articulatory in principle: by articulating the implicit meaning of our interactions with the environment, we achieve three crucial things: we generate explicit (ibid., 256-258) and public (ibid., 259-260) consciousness and shape the distinctions we need to handle our human affairs (ibid., 260-263). My only critique of Taylor's conception - labeled by him variously as the "Herder-Humboldt-Haman," "romantic" or "expressivist" (ibid., 255-256) theory - would be that it unnecessarily plays down the limited, but still very important role of veridical, propositional speech. From a pragmatist point of view, the gestalt qualities with which articulation begins emerge from the entire interactional cycle and not from the subject alone. There is, strictly speaking, no such entity as an individual subject there: the latter emerges, as George Herbert Mead has shown, only during the process in which the problematic situation is reconstructed. ${ }^{14}$ Thus, if we talk about expressivist theories of meaning, we should carefully avoid all subjectivist implications: what is expressed in articulation is not inwardness - "Innerlichkeit" in the romantic sense - but the meaning of the fully-fledged mind-brain-body-world-cycle. Facts about the world, explicable in propositional language, are integral parts of this relational structure. The need to separate subjective and objective components and thus to distinguish expressive (in the narrower sense) and referential utterances emerges as part of the problematic situation. Consequently for pragmatists, the term expressivism should be taken to signify the generic condition of which expressions of subjectivity and of facts about the world are only subspecies, differentiated from each other not as natural kinds, but as pragmatic requirements of problematic situations.

To sum up, articulation is the embodied explication of problematic ${ }^{15}$ situations. It is aptly called expressive, because it creates explicit consciousness, starting from meaningful yet underdetermined situations. Articulation is not a pictorial relationship between some already predetermined content and semiotic meaning, but is concerned instead with reconstructing problematic situations in a manner which enables interaction to be resumed. Sometimes, however, this implies separating the objective, referential aspects of a given situation from the ones pertaining to the interaction-unity between organism and environment: propositional language emerges. The instruments for this and other remarkable feats of explication are signs: signs in their mutual interdependence and division of labor. An anthropological theory of articulation needs a semiotic foundation, and it is Charles Sanders Peirce who delivered it.

14 "It is in this phase of subjectivity, with its activities of attention in the solution of the problem, i. e., in the construction of the hypothesis of the new world, that the individual qua individual has his functional expression or rather is that function" (Mead 1964, 52). For a detailed account, see Jung (2009, 217-252).

${ }^{15}$ But see 13 for a non-reductionist understanding of the term "problematic." 


\section{Peirce's Triadic Semiotics and the Mutual Dependence of Embodiment and Symbolic Transcendence}

The fruitfulness of Peirce's semiotics for the study of embodied cognition is convincingly exemplified in Terrence Deacon's The Symbolic Species (Deacon 1997, 69-101). Deacon begins by pointing out that dyadic concepts of reference, those which work with some sort of mapping or one-to-one correspondence between words and entities or events in the world - as exemplified by the semiology of de Saussure - fail to explain the indirect character of human language. "The correspondence between words and objects is a secondary relationship, subordinate to a web of associative relationships of a quite different sort which even allows us reference to impossible things" (ibid., 70). Symbolic reference is special not in being referential but in being symbolical, and that is: indirect. That's the reason only a triadic semiotics like Peirce's can do justice to the distinctive features of language. Monkey alarm calls refer to the world as well as human symbols, but human symbols refer primarily to other symbols and only indirectly to the world. This indirectness of human languages enables us to think and talk about virtually everything: the existence or nonexistence of God, normative and evaluative claims dealing with nonexistent but desirable states of affairs, modal and temporal differences, abstract entities like the ones of particle physics, etc. It comes at a price, of course: symbols allow us to transcend embodied direct experience and thus to develop reflexivity and be guided by counterfactual ideals, but symbolic communication still depends on direct experience for its connection to reality. "No combination of words," Peirce says bluntly, "(excluding proper nouns, and in the absence of gestures or other indicative concomitants of speech), can ever convey the slightest information" (Peirce 1998, 7). A symbol denotes a "kind of thing" (ibid., 9), not anything particular, and it establishes its meaning indirectly, by the manner in which it is woven into a holistic network of other signs, not via direct acquaintance. Thus symbols are dependent upon non-symbolic, direct reference, which is accomplished by the united workings of iconic and indexical signs.

This threefold distinction of symbols, indices, and icons is the most important among Peirce's many subtle classifications of signs, because it enables us to understand the functional integration upon which human sign-usage is based. Even though human languages are entirely shaped by their symbolic character, symbols are crucially dependent upon icons and indices, which convey direct experience. ${ }^{16}$ Icons are imitative signs, picking out certain likenesses among things which are in the direct presence of the sign-user. Indices presuppose icons.

16 Note that in Peirce's rendering only indexical signs connect language with experience. Likenesses, according to Peirce, are always ambiguous, as long as they are not interpreted by an index (see Peirce 1998, 7, for an example). My own, slightly different, account here is influenced by Dewey's concept of unifying qualities as explained at the beginning of section 1 of this paper. These qualities I regard as iconic signs for identifying specific situations as resembling other situations in the history of the symbol user's experience. Insofar as they actually function in this manner, they presuppose direct acquaintance. 
To quote Peirce, they "show something about things, on account of their being physically connected with them" (ibid., 5). In order to do so, an indexical sign must iconically be identified as the token of a certain type, and it must establish some corporeal relationship between the sign-user and what is designated. Symbolic meaning transcends the here and now of the sign-user by virtue of establishing meaning and reference independently from direct experience. But the chain connecting the symbolic web to the immediate reality of qualitatively experienced gestalts and what Peirce calls the indexical experience of acting and being acted upon can only be prolonged, but never broken, if our thoughts are to be distinguished from the mere flight of fancy.

Every given instance of reasoning, Peirce insists, must involve a triad of signs, namely icons, indices and symbols (ibid., 9). It is only their functional integration which endows our language with its symbolic character, not the symbolic sign in isolation. This Peircean insight is absolutely crucial. Symbols are embodied in icons and indices, and we are embodied symbol-users, whose bodies and social/physical environments provide the means for this anchoring-process. Without the lived body, its phenomenal presence in the world and its articulated sensory-motor behavior, symbol-usage would not encounter the "outward clash" (Peirce 1992, 233) - Peirce's congenial term for the experience of unanticipated, resistive reality which only acting brings forth. Without this resistance, language would become entirely self-referential. On the other hand, without symbolic signs, we would not be able to transcend our immediate reality and become the reflective beings we at least sometimes are. It is the outstanding achievement of Peirce to have developed a fully integrated picture of symbolic sign-usage, and thus to have shown how embodiment and transcendence are internally connected.

Peirce himself never reflected systematically on the evolutionary path to symbolic sign-usage - from the time when there were no signs at all over the time when iconic and iconic-indexical signs evolved to the present stage of fully-fledged symbolic language. But Terrence W. Deacon has developed an elaborate, albeit necessarily somewhat speculative account of this process in Peircean terms (see Deacon 1997). From the standpoint of anthropology, the decisive fact is that the emergence of symbolic communication does indeed lead to a fundamental change - Deacon calls it crossing the "symbolic threshold"17 - of a categorical, not quantitative nature. Symbolic communication released the human mind by enabling a mode of intentionality no longer restricted to the organism's immediate surrounding. This change, though, was not accomplished by leaving behind immediate experience but rather by means of a functional reintegration of the evolutionarily older forms of sign-usage: "iconic and indexical referential relationships are implicit and essential components of symbolic reference” (Deacon 1997, 450).

Thus, no account of human embodied cognition will be satisfying which excludes our symbolic (that is, reflective) second-order-capabilities. On the other hand, no adequate account of our symbolically structured minds will ever be

17 See the "The Symbolic Threshold" in Deacon (1997, 79-92). 
able to get rid of the fact that even the most abstract reasoning is embodied. Climbing the stages of articulation is not comparable to Wittgenstein's famous ladder that we must cast away once we have mounted it (see Wittgenstein ([1921] $1963,6.54)$; it is more like acquiring new capabilities by using the ladder to climb continually up and down. Jacob's ladder from the book of Genesis might offer a more appropriate metaphor. ${ }^{18}$ In this manner, we can conceive of distinct, but internally connected rungs or stages of embodied articulation. Each rung has semiotic and anthropological properties which may, with appropriate care and restraint, be used heuristically to better understand cultural developments. In the last part of my paper, I will try to sketch the rungs of this ladder.

\section{The Ladder of Articulation}

The most fascinating developments in recent evolutionary anthropology are those which allow for the deep integration of nature and culture in a non-reductionist manner. Michael Tomasello has shown us from an evolutionary perspective how it is natural for us to develop our cognitive equipment by cultural transmission (Tomasello 1999), and Merlin Donald has distinguished stages of consciousness that he takes in their entirety to be part of our current cultures and at the same time to describe actual phases of human evolution (see Donald 1991, 2001). In what follows, I will make use of Donald's work and rely on the triadic distinction of signs together with insights drawn from Robert Brandom in order to bring out its full anthropological significance. Brandom has written a famous book titled Making it Explicit (1998), in which he conceptualizes language in terms of making explicit the meaning of our actions and the norms embodied in them. This is a pragmatic stance I endorse, despite the fact that Brandom almost entirely leaves out embodiment and focuses narrowly on propositional language. His key insight is nevertheless very helpful: pragmatically, language can be seen as the explication of something already implicit in practice, but capable of distinct modes of explication. These modes can be ordered in an ascending sequence, leading to logically (not only quantitatively) higher degrees of explicitness.

Brandom's ladder of explication begins with intersubjective practices that go beyond causally explainable "matter-of-factual-regularities" - as regulism ${ }^{19}$ would have it - insofar as they bear the normative character of signs endowed with conditions of correctness, but have not yet reached the stage of articulated normativity. Unfortunately, Brandom makes no attempt to connect his reflections with empirical research about evolutionary stages of sign-usage. But the context suggests that, to give just one example, the “joint-intentionality"-phase

18 In Gen 28:12, Jacob has a dream in which angels are constantly climbing up and down a ladder connecting heaven and earth.

19 For the distinction between regulism (the conviction that [linguistic] normativity presupposes explicit rules) und regularism (the opposite claim, that normativity can be reduced to factual regularities), see Tomasello (1999, 29). 
of human development, preceding the later collective intentionality, in Michael Tomasello's account of cognitive evolution (Tomasello 2014, 32-79), ${ }^{20}$ can be seen as an instantiation of Brandom's first rung. The "natural gestures of pointing and pantomiming" (Tomasello 2014, 33) which characterize this phase exhibit both deep embodiedness and implicit normativity. In the terminology of Peirce, we are in the realm of indexical (which necessarily include iconic) signs. But from the vantage point of embodied cognition it is quite obvious that Brandom starts too late. In order for gestural, normative signification to get off the ground, we need qualitative experience. The ladder has to be fixed on the ground, so to speak, and this is accomplished by bodily expressions and the unified qualities that accompany them. They are endowed with a unified gestalt that allows them to function as iconic (proto-)signs. Articulation then starts with the conscious stylization which gestures enable - presumably beginning with limb and wholebody movements and later becoming vocalized. These gestures establish indexical relations between a community of sign-users and their habits of pointing to certain aspects of the world and of the community itself (as in rituals) and are crucial in creating and solidifying the common ground of shared intentionality. But indexical explication, albeit the starting point of articulation, still presupposes the physical co-presence of sign and signified.

This changes radically once the symbolic threshold is crossed. On the symbolic level, meaning and reference are established and stabilized non-indexically by complex, inferential, so to say "horizontal" relationships between symbols in holistic networks. The connection to embodied experience thus becomes indirect, which amounts to loosening the iconic and indexical bond with embodied experience without cutting it off. In the course of the child's language-acquisition, for example, the concepts 'mother' and 'father' are always introduced indexically, namely by direct reference to concrete mothers and fathers. But after the transformation to symbolicity has taken place, the meaning of these concepts becomes detachable from personal experience and is fixed in the linguistic and logical relations between the concepts 'mother,' 'father,' 'children,' 'parent,' 'family,' 'relatives,' 'male,' 'female,' etc. To be sure, individuals and communities still have to anchor indirect reference in their embodied experiences, but the "naked" meaning is then established and stabilized irrespective of direct acquaintance. Thus, even people who never saw a single snowflake fall are enabled to understand what the word 'snow' means. Once natural languages have reached this decisive stage of indirect reference, according to Brandom, they are always characterized by rational expressivity, that is, by the mastery of a language which uses logical operators that allow us to make explicit which inferential relationships and validity claims are implied in speaking. ${ }^{21}$ Language allows us to make

${ }^{20}$ It is perhaps no mere coincidence that the chapter's motto is taken from Brandom's Making it Explicit.

21 For the concept of rational expressivity, see Brandom (1998, 105-117). “The fundamental characteristic role of logic vocabulary is to make it possible to talk and think explicitly about the inferentially articulated semantic contents implicitly conferred on expressions (among other things) by their role in rational practice” (Brandom 1998, 117). 
explicit not only the intentional correlates of our actions, but also the way in which we are related to these correlates.

After symbolic communication is established in cultural evolution and ontogeny, the highest possible level of sign-usage is attained. But Brandom's ladder is not finished yet. Its last rung consists of what he calls logical expressivity. Language can be used not only to articulate, roughly spoken, the relation of meaning and reference, as in rational expressivity. ${ }^{22}$ Symbol-usage generates the possibility of second-order thinking, of taking a reflective stance towards language itself and the kind of embodied consciousness it brings forth. Here are the concluding sentences of his book: "We are more than rational, expressive beings. We are also logical, self-expressive beings. We not only make it explicit, we make ourselves explicit as making it explicit” (Brandom 1998, 650). For Brandom, self-expressiveness is essentially about the way in which the ability of human beings to make explicit the structures of our linguistically shaped relation to the world effects our understanding of what and who we are. From the perspective of an anthropology of embodiment, this position leaves out the entire embodiment-part and the whole range of non- and pre-linguistic experiences implied in it. But if we keep in mind - pace Brandom - that even logical expressivity is strictly dependent upon our corporeal entanglement with the world for the meaningfulness and the verification of second-order-concepts, his hierarchy of explication proofs is an essential tool for conceptualizing the tense relation between embodiment and symbolic transcendence. In this way, we are left with a ladder of articulation having three rungs - indexical, symbolical and symbolical-reflexive sign-usage and which is grounded in iconic reference. The human life-form results from the practice, so to speak, of moving up and down this ladder time and again, not from the use of symbolic signs in isolation.

The final step of my argument will now be to connect these insights with Merlin Donald's reflections on the evolution of human cognition and culture. Donald distinguishes four successive layers, namely the episodic, mimetic, mythic, and theoretic stages of culture, whose inner structures and connectedness can be elucidated by using the Peircian and Brandomian conceptions I sketched above. In the course of each stage (except the first), embodied cognitive capacities are tethered more strongly to intersubjective modes of consciousness and intentionality and to culture, ${ }^{23}$ thereby enlarging our ability to think about the world in a context-independent manner. Whether, in the long run, Donald's ideas will stand up against the background of our ever-changing and growing empirical knowledge about human evolution is of course a matter that cannot be decided in advance nor out of the philosopher's armchair. But for the time being, the matching of his evolutionary perspective with semiotic and anthropologic categories may be

22 To avoid confusion: Even rational expressivity already uses logical vocabulary. Logical expressivity bends back the logical vocabulary to the community of symbol-users in order to make explicit what it means to be such a community.

23 "Collectivity has thus become the essence of human reality" (Donald 2001, 298). More recently, Michael Tomasello's "shared intentionality hypothesis" (see Tomasello 2014) has given this intuition a much more detailed account. 
taken as evidence for the ladder of embodied articulation. The following sketch will be a very rough and oversimplified one, but it should suffice to bring out the general structure.

Donald's first stage, the episodic one, is shared by all primates, whom he describes as members of the "consciousness club" (Donald 2001). Its characteristic novel forms are episodic event perceptions, bringing forth self-awareness and event sensitivity. The episodic consciousness of primates can pragmatically be interpreted in terms of Deweyean gestalt-like qualitative unities, which allow us to identify and re-identify situations and thus enable iconic sign-usage. Brandom's first rung of the explication-ladder may then be seen to parallel Donald's second stage, the mimetic one of the early hominids, in the course of which physical interactions are stylized and often ritualized. Donald conceives of it as "an extension of conscious control into the domain of action" that "enabled playacting, body language, precise imitation and gesture” (261). These new capabilities exhibit an indexical structure, in which certain activities stand for collectively shareable meanings. As far as I can see, Donald's account is fully compatible with Tomasello's recent emphasis on cooperation and shared common ground. Donald also highlights the importance of mimetic skills for the refinement of toolmaking. On this level, hominids can develop shared intentionality and articulate the emotional and practical meaning of shared situations by the mutual attunement of mimics and gestures. Nevertheless, the decisive transition in the evolution of modern humans is the next one, the crossing of the symbolic threshold, characterized by Donald as "the spiraling coevolution of thought and symbol" (274). It enables indirect reference and what Brandom calls "rational expressivity": the ability to explicate the logical relations between the "assertional commitments" (Brandom 1998, 167) involved in discourse, thereby pondering the reasons backing up the interlocutor's claims. Symbolic language detaches the content of speech from immediate experience while still depending on the latter and thus allows us to articulate context-independent meanings by context-dependent means. In short: "language transcends embodiment at the same time that it depends on it" (Gallagher 2005, 127). Donald associates the development of symbolic language with the emergence of Homo sapiens, finding its culmination in the evolution of Homo sapiens sapiens. His key-word for the - as he calls it mythic culture thus enabled is narrativity. Human affairs and the experience of nature are fused in orally-transmitted narrations which enable what Tomasello calls the "ratchet effect" of culture (Tomasello 1999, 37-40), the cumulative building of increasingly complex artefacts.

With symbolic communication, the triadic structure of sign-usage has unfolded. But it is only when the theoretical and last stage of Donald's explanation is reached, that the power of symbols is fully realized. The last stage of embodied articulation does not correspond to an evolutionarily unprecedented form of sign-usage, but to the qualitative step within symbolicity. This happens when the reflective distance built into indirect reference is extended from objects to the practice of symbol-usage itself - what Brandom calls logical expressivity, which enables second-order thinking, allows for the explication of the difference 
between sign and signified and therefore for the emergence of concepts of transcendence. The ability to make explicit, over and above the explication of referential claims, the relation between the signs we use, the thoughts we can think and the world, is inherent already in the mastery of symbols. But only when this ability pervades social structures does it gain world-changing significance. Donald links this last transition, starting about 40,000 years ago, to the development of external storage systems such as writing (see Donald 2001, 260, table 7.1), and in some recent publications he has explicitly connected it to the Axial-Age debate in sociology and history (Donald 2012, 47-76). The concept of an Axial Age emerged around the turn of the $20^{\text {th }}$ century ${ }^{24}$ and has recently become a focal point for ongoing debates about the possibility of multiple modernities, the relationship between science and religion, about universalist moral systems, etc. Basically, the term refers to the time period in the middle of the first millennium B.C., when the incipient stages of science together with novel forms of cultural expression (e.g., theater), universalistic ethics and religions, rational organizations of the state - sometimes accompanied by surges in radical political criticism - emerged in China, India, Persia and the near East/South-eastern Europe (Israel and Greece). ${ }^{25}$

And it is here that we've come full circle. I started with the idea that embodied cognition must, in a non-dualist manner, include our symbolic capability of transcending. And I end by expressing my conviction that, in the evolution of human culture, it is indeed possible to identify, in many different cultures and partly independent from each other, a developmental stage where the tension between the inseparable dimensions of symbolic transcendence and particular, localized experience becomes the driving force of culture: the Axial Age. ${ }^{26}$ The breakthroughs which shaped human culture in its present form are historically, anthropologically, and semiotically connected with reaching the last stage of embodied articulation: semiotic transcendence.

${ }^{24}$ Hans Joas' "The Axial Age Debate as Religious Discourse" (2012, 9-29) offers a concise outline of the developing debate and its current relevance. For a more extensive version of this text, see Joas (2014).

${ }_{25}$ The locus classicus is Jaspers ([1949] 1983). In the discussion which followed, two of Jaspers's original claims proved to be untenable: his overly rigid time-frame and his contention that the Axial civilizations developed essentially independently from one another. Furthermore, historians and sociologists have criticized the concept for its inherent over-generalizations. Consequently, it would perhaps be useful to shift focus from a too strongly unifying concept called Axial Age (implying the existence of parallel developmental stages forming one distinct historical period) to the analysis of diverse Axial developments, conceptually unified by breakthroughs in second-order thinking and concepts of transcendence. Such a move would place the emphasis on the systematic, not the empirical concept, but need not deny the special importance of the half millennium between roughly 800 and 300 B. C.

${ }^{26}$ In A Secular Age (2007), Charles Taylor depicts the impact of the "Axial revolution" as “The Great Disembedding” (Taylor 2007, 146). Embeddedness and Embodiedness are not identical, but they overlap substantially and the former implies the latter. Thus, from the perspective of embodied cognition and Peircean semiotics, desembedding can never be complete: it signifies a shift in the inextricable dialectic relation between immediate experience and symbolic transcendence, not the dissolution of the tense unity they form. 


\section{Bibliography}

Brandom, R. B. 1998. Making it Explicit. Reasoning, Representing E Discursive Commitment. Cambridge, MA: Harvard University Press.

Darwin, C. 1998. The Expression of the Emotions in Man and Animals. New York: HarperCollins.

Deacon, T. W. 1997. The Symbolic Species. The Co-evolution of Language and the Brain. New York: W. W. Norton \& Company.

Dewey, J. (1930) 1998. Qualitative Thought. In The Essential Dewey, vol. 1, Pragmatism, Education, Democracy, ed. L. A. Hickman and T. M. Alexander, 195-205. Bloomington: Indiana University Press.

-. (1927-1928) 2008a. Philosophy and Civilisation. In The Later Works, 1925-1953, vol. 3, ed. J. A. Boydston, 3-24. Carbondale: Southern Illinois University Press.

-. (1934) 2008b. Art as Experience. In The Later Works, 1925-1953, vol. 10, ed. J. A. Boydston. Carbondale: Southern Illinois University Press.

Dreyfus, H. 2014. Skillful Coping. Essays on the Phenomenology of Everyday Perception and Action. Ed. M. Wrathall. Oxford: Oxford University Press.

Donald, M. 1991. Origins of the Modern Mind. Three Stages in the Evolution of Culture and Cognition. Cambridge, MA: Harvard University Press.

-. 2001. A Mind So Rare: The Evolution of Human Consciousness. New York: W. W. Norton \& Company.

-. 2012. An Evolutionary Approach to Culture: Implications for the Study of the Axial Age. In The Axial Age and its Consequences, ed. R. Bellah and H. Joas, 47-76. Cambridge, MA: Harvard University Press.

Gallagher, S. 2005. How the Body Shapes the Mind. Oxford: Oxford University Press.

von Humboldt, W. 1994. Ueber das vergleichende Sprachstudium. In Über die Sprache. Reden vor der Akademie, ed. J. Trabant, 11-32. Tübingen: Francke.

Hutto, D. D. and E. Myin. 2013. Radicalizing Enactivism: Basic Minds Without Content. Cambridge, MA: MIT Press.

Jaspers, K. (1949) 1983. Vom Ursprung und Ziel der Geschichte. München: Piper.

Joas, H. 2012. The Axial Age Debate as Religious Discourse. In The Axial Age and its Consequences, ed. R. Bellah and H. Joas, 9-29. Cambridge, MA: Harvard University Press.

-. 2014. Was ist die Achsenzeit? Eine wissenschaftliche Debatte als Diskurs über Transzendenz. Basel: Schwabe.

Jung, M. 2009. Handlung, Erleben, Mitteilung - Bewusstsein als Interaktionsphase. In Funktionen des Erlebens, ed. M. Jung and J. C. Heilinger, 217-252. Berlin: de Gruyter.

-. 2010. John Dewey and Action. In The Cambridge Companion to Dewey, ed. M. Cochran. Cambridge: Cambridge University Press.

Kestenbaum, V. 2002. The Grace and the Severity of the Ideal. John Dewey and the Transcendent. Chicago: The University of Chicago Press.

Mead, G.H. 1964. The Definition of the Psychical. In Selected Writings, ed. A. J. Reck, 25-59. Chicago: Chicago University Press.

-. (1967) 2009. Mind, Self and Society. From the Standpoint of a Social Behaviorist. Ed. C. W. Morris. Chicago: Chicago University Press.

Metzinger, T. 2009. The Ego-Tunnel. The Science of the Mind and the Myth of the Self. New York: Basic Books.

Meuter, N. 2006. Anthropologie des Ausdrucks. Die Expressivität des Menschen zwischen Natur und Kultur. München: Fink.

Nagel, T. 2012. Mind and Cosmos. Why the Materialist Neo-Darwinian Conception of Nature is Almost Certainly False. Oxford: Oxford University Press. 
Noë, A. 2010. Out of Our Heads, Why You Are Not Your Brain and Other Lessons from the Biology of Consciousness. New York: Hill and Wang.

Peirce, C.S. 1992. An American Plato. In The Essential Peirce, vol.1, 1867-1893, ed. N. Houser and C. Kloesl, 229-241. Bloomington: Indiana University Press.

-. 1998. A Neglected Argument for the Reality of God. In The Essential Peirce. Selected Philosophical Writings, vol.2, 1893-1913, ed. N. Houser and C. Kloesl, 434-450. Bloomington: Indiana University Press.

Rockwell, W. T. 2005. Neither Brain nor Ghost. A Nondualist Alternative to the MindBrain Identity Theory. Cambridge, MA: MIT Press.

Taylor, C. 1985. Theories of Meaning. In Human Agency and Language. Philosophical Papers 1, 284-291. Cambridge: Cambridge University Press.

-. 2007. A Secular Age. Cambridge, MA: Harvard University Press.

Tomasello, M. 1999. The Cultural Origins of Human Cognition, Cambridge, MA: Harvard University Press.

-. 2014. A Natural History of Human Thinking. Cambridge, MA: Harvard University Press.

Wittgenstein, L. (1921) 1963. Tractatus logico-philosophicus. Frankfurt a. M.: Suhrkamp.

-. (1953) 1971. Philosophische Untersuchungen, Teil 1. Frankfurt a. M.: Suhrkamp. 
3. Embodiment as a Bridging Concept for Evolutionary and Historical Anthropology 



\section{Introduction}

\section{Alexander Massmann}

"In the future I see open fields for far more important researches. ... Much light will be thrown on the origin of man and his history" (Darwin [1859] 2009, 428). With these well-known words from the last pages of On the Origin of Species, Charles Darwin (1809-1882) marked the starting point of evolutionary anthropology. The closing remarks include the evolution of human beings into a panoramic perspective on how the earliest forms of life fanned out into life's current diversity. In keeping with the logic that characterizes evolution throughout, Darwin also sees human beings acquire "each mental power and capacity by gradation" (428) only.

A rigorous inclusion of humanity in the general evolutionary dynamic of life implies a dual heritage in anthropology. To begin with, attention to evolutionary dynamics in anthropology will insist on the fundamentally embodied nature of all dimensions of human life. To speak about human experience includes speaking about particular bodies, which have a history, albeit on a time scale differing from cultural history as commonly conceived. One would expect the path dependence of the genesis of bodies to have a bearing on how human life is lived and experienced.

How processes of variation and selection have contributed significantly to what it is to be human can be illustrated with several examples. Darwin's second anthropological book, The Expression of the Emotions in Man and Animals ([1872] 1999), points to physiological phenomena, shared by humans with other animals, such as blushing, an increased pulse, and sweating or raised hair associated with particular emotional states. Cultural anthropologists may object that different cultures vary in the wider meaning such emotional expressions take on. But the relevance of evolutionary inheritance can at least be illustrated with the fact that an artist's choice of material will neither determine the overall meaning and expression of the resulting piece of art, nor will it be irrelevant.

Further, infanticide is not particularly rare in several mammal species. The common evolutionary explanation is that a new resident male increases its own genetic representation in the future gene pool by reducing the offspring of rival males and rendering the mother sexually receptive sooner (Hausfater and Hrdy 2008). It turns out even that among humans, it is more often the stepparent than any other person who is responsible for serious neglect or even the death of a child (Voland and Stephan 2000).

The other aspect of a prominent role of evolution in anthropology, a counterbalance to the aspect just illustrated, consists in the suspicion that evolution- 
ary anthropology may represent a temptation to an illegitimate reductionism. Darwin's closing argument in the Origin includes a note that is both distinctly 'hawkish' and progressivist in suggesting that, "as natural selection works solely by and for the good of each being, all corporeal and mental endowments will tend to progress towards perfection." The final pages of Origin see human psychology "securely based on the foundation" of evolutionary biology. The evolution of "higher animals" is inconceivable unless resulting gradually "from the war of nature” (Darwin [1859] 2009, 428f.). Such a harsh, progressivist view implicitly plays off unambiguous adaptive optimality against an abiding diversity of particular traits within a species. But abiding diversity, in contrast to variation evolving into adaptedness, is the hallmark of human culture. It is described and interpreted by anthropologists who probe the unexpected varieties of human modes of being in the world. Yet the marginalization of human diversity by a hierarchy of higher and lower forms of civilization, quite in keeping with the colonial politics of the time, can also be found in Darwin's work (e.g., Darwin [1871] 2004, 134-139).

In this context it should also be noted, however, that Darwin's case for a singular, natural origin of all humanity was inspired by his passion for emancipatory politics, which he articulated in discussions about slavery in North America (Moore and Desmond 2004). On a less explosive level, moreover, it is remarkable how Darwin already hinted at diversity within an animal species in discussing, for example, the production and use of tools among animals (Darwin [1871] 2004, 102-104). More recently, this has become a powerful research program that has led some primatologists to speak wholeheartedly of cultures among chimpanzees (Wrangham et al. 1996; de Waal 2001).

If Darwin's emphasis on natural selection is to serve as a criterion even in anthropology, however, it may seem that human morality, reason, meaning making, religion, and the arts are but strange guises of a 'Darwinian' opportunism. This critique is voiced by many who are at home in the humanities, notably social and cultural anthropologists. In this section, this tradition is represented also by historical anthropology, a discipline working less with ethnographic rather than diachronic, historical methods. Devoted to the phenomena of contingency and change, cultural anthropology approaches the human person from a different angle than the supposedly universal truths of science. Certainly the importance of the evolutionary time scale does not render the cultural time scale insignificant.

The current section takes up this discussion with the question of the evolution of morality. Eve-Marie Engels, professor of ethics in the life sciences at the University of Tübingen, presents a nuanced image of how Darwin's first anthropological book, The Descent of Man (1871), laid out a concept for the evolution of morality. Darwin proposes a naturalism that is not reductionist (Engels in this volume). With the evolution of morality, Darwinism does not simply present us with "nature, red in tooth and claw" (Tennyson [1849] 2008, 153). Certainly the basic Darwinian principle remains intact: a current trait can be accounted for by a variation of similar traits in previous generations. At that stage, the partic- 
ular quality of the trait in one lineage proved to be more adaptive, contributing to more numerous offspring. The trait in question can be sensory perception, speed, timidity, or camouflage, but in this case we are concerned with a more pronounced social instinct and a feeling of sympathy.

It is notably David Hume's and Adam Smith's moral sense theory that construes morality in terms of feeling. Any human being in trouble, not just a close relative, evokes a feeling of sympathy. But according to Darwin ([1871] 1999), in our early human ancestors, the feeling of sympathy must have been more limited in scope. Nonetheless - may I add, perhaps as if by the working of natural moral law? - a helpful intervention fueled by sympathy, a unilateral expense to begin with, must have been rewarded by natural selection in the long run. Now a "coevolution" of nature and culture" (Engels in this volume) sets in that hones the beginnings of morality. This concerns not only the acuteness and the radius of sympathy, but also the uniquely human moral sense of right and wrong, human conscience. Darwin even affirms Kant's notion of duty, also if it implies a costly sacrifice that reduces one's Darwinian fitness.

$\mathrm{He}$ also suggests that self-sacrificial morality is unlikely to have arisen without the religious exhortation to "do good in return for evil, to love your enemy" (Darwin; Engels in this volume). Indeed, while "unashamedly naturalistic," Darwin's central concept of natural selection "did not have to be atheistic, but could also be associated with theism, deism, or agnosticism” (Brooke 2013, 38).

Biologists have worked on the evolution of morality beyond Darwin's groundwork discussed by Engels. A crucial point is that even a morality inspired by religion may die a Darwinian death, with highly religious populations being outreproduced and replaced by less religious ones, or 'altruistic' ones by more 'selfish' ones. Darwin's own answer is that natural selection acts on a group level (Wilson 2003). As soon as morality is in place - whether established in religious or other ways - another dynamic is required for its preservation, i.e., fitness:

A tribe including many members who, from possessing in a high degree the spirit of patriotism, fidelity, obedience, courage, and sympathy, were always ready to aid one another and to sacrifice themselves for the common good, would be victorious over most other tribes; and this would be natural selection. (Darwin [1871] 2004, 157f.)

We might also envisage the 'victory' of the 'altruists' over 'egoists' as a non-conscious process, as an unnoticed spread of a culture of helpfulness. In addition, David S. Wilson has shown that 'altruistic' populations are more likely to populate new locations than 'selfish' ones (Wilson 2015, 25f.).

In contrast with between-group competition, 'selfish' organisms have an advantage in within-group competition, however. An arsenal of three to five other theories addresses this problem in Darwinian terms (Nowak 2013, Clutton-Brock 2009, Zahavi and Zahavi 1999). Kin selection can account for behavior that is costly in direct proportion to genetic relatedness. Direct reciprocity ("tit for tat") widens the circle of beneficiaries. This tendency is even more pronounced in indirect reciprocity, in which my reputation of helpfulness is instrumental to my recompense by a third party. Only group selection can account for 
the evolutionary stability of a behavior incurring a net fitness loss for the individual, but in one way or another, all of these models rely on an ultimate fitness gain through cooperation.

So does Darwin's concept of the evolution of morality account for all crucial elements of human morality? Darwin assumes that in moral conflict, the feeling of sympathy will take over after considering the facts. Here as in moral sense theory, however, there is little point in reasoning critically if a moral obligation exists, if my conscience is too dull, or if I may or should disregard a misguided conscience. Can Darwin, then, truly account for the critical sense of Kant's 'ought,' as he claims? According to Kant, ethics may have an emotional component, but this is the feeling of respect for law engendered by reason (Kant [1797] 2012, 64). Fundamentally, it is not feeling that compels a Kantian to act, but reason working independently of the heteronomous promptings of feeling. From a religious rather than a philosophical point of view, can we explain the Good Samaritan, or a culture that argues for costly commitment regardless of returns (Rolston 2004)? To ask these questions may be to engage reductionism further than Darwin intended. But does this leave Darwin's thesis untouched that "the difference in mind between man and the higher animals, great as it is, certainly is one of degree and not of kind" (Darwin [1871] 2004, 151)?

Does human culture have to be accounted for, at least in one crucial respect, in non-Darwinian terms, or is a reconciliation between evolutionary and cultural anthropology possible? Gregor Etzelmüller, professor of systematic theology and religious studies in Osnabrück (Germany), suggests that the concept of the (lived) body (Leib-Körper) provides a bridge between nature and culture, between evolutionary and historical anthropology. The classic distinction between body (Körper) and lived body (Leib) goes back to Edmund Husserl (1859-1938). While I can turn my body into an object, my lived body evades objectification. In both its subjective and objective aspect, the (lived) body is both nature and a being that transcends nature in culture. Etzelmüller (this volume) expresses this ambiguity with the concept of the (lived) body as the "tipping point" between nature and culture, which he adopts from the philosopher Bernhard Waldenfels (*1934).

Hence there is no such thing as the mere biology of human life. Nonetheless, the human person as a protagonist is always also a particular biological constellation, in an essential rather than an ancillary sense. Moreover, even the behavior of comparatively simple organisms does not follow the mere dictates of natural law. Niche-construction, rather than an adaptation in which the organism assimilates to a given, static environment, is a typical case. Further, even a flagellate displays a certain kind of autonomy in predictably swimming away from a particular substance, as the philosopher Evan Thompson (*1962, Thompson 2010,163) argues. Thus if we continue to speak about "nature" in biology rather than "culture" as well, this involves a significant abstraction or extrapolation.

This is especially true, however, concerning human life with its characteristic reflexive behavior. With the unique practices of morality and meaning-making, humanity constitutes a category of its own in the history of life. In turn, life 
forms lacking these characteristics, even if displaying a certain kind of autonomy, appear "natural” by comparison. Etzelmüller (in this volume) notes that according to Darwin, wide-spread social instincts in animals only require an increase in intellectual power to attain a fully-fledged moral sense. Yet once this moral sense is attained, we are speaking no longer about an animal, but the human person. A change by degree that can be traced in the body amounts to a qualitative shift, constituting a "tipping point." Nature takes on a cultural dynamic. While different kinds of animals stretch the boundaries of nature in different ways, nature is nonetheless the category to assign them.

But does this not render the relation between humanity and biology all too tenuous, bordering on the metaphysical? Etzelmüller draws upon the concept of the plasticity of human development as described by the Swiss zoologist Adolf Portmann (1897-1982, Portmann 1990) in answering no. Portmann calls humans secondarily altricial. Born with sense organs no longer blocked by a protective membrane and with mobile limbs, humans might be called precocial. But the retarded development of the brain, the sensory systems, and the wider anatomy constitutes an overlay of altriciality, which humans have 'reinvented' on a new level. Due to their slow and drawn-out development, humans depend strongly on environmental factors. Biological facts are crucially involved in that the "low speed' of human somatic development allows for social and psychic shaping, thus bringing forth the human being as an entity characterized by an irreducible mutual interdependence of nature and culture, body and psyche" (Etzelmüller in this volume). Due to the plasticity of human development, there is a feedback of the cultural dynamic into human biology. "Humans are cultural beings by nature" (Etzelmüller in this volume).

While chimpanzees, our closest zoological relatives, display a remarkable sense of social connection, for humans sociality is the very fabric of the universe, and humans take cooperation to another level. Cooperation among apes, although remarkable, is limited due to their effective physical adaptedness, which is more pronounced than in humans. By contrast, the extreme degree of human social and cooperative adaptedness comes to the fore in the phenomenon of "over-imitation." Human children make a "leap of faith" (Etzelmüller in this volume) in hypothesizing about meaning in imitation, as in taking a moment of silence before a worship service. Conspicuously, they even imitate random minutiae. An extreme degree of "shared intentionality" (Michael Tomasello, $* 1950$, Tomasello 2010, 154, Searle 1997) is the fertile ground that allows human symbolic communication to thrive. It is this new cultural dynamic that shapes the body structure in the slow human physical development. Moreover, in dialogue with the philosopher Judith Butler (*1956), the notion of plasticity allows Etzelmüller to explore critically the question whether biology is fate.

The antithesis between the lived body as a "tipping point" and a Cartesian ontology then opens up new perspectives for a dialogue between biology and historical anthropology. Etzelmüller illustrates this with anthropological traditions from ancient Egypt, Mesopotamia, ancient Greece, and notably the Old Testament and the Apostle Paul. For example, the Hebrew word commonly mis- 
translated as soul denotes the entire person in all her needfulness. Paul, far from a somatophobic spiritualizer, saw the organismic constellation of the congregation as the matrix in which the relationship with the divine is embodied.

In sum, due to the plasticity of human development, the possibility of a non-linear genesis of genuinely novel phenomena in life due to culture can be captured by the metaphor of the tipping point. But does the metaphor suggest a realm of culture that is homogeneous in having emancipated itself, as it were, from nature? Or are there natural factors that modulate expressions of human culture in a non-trivial sense? And if the lived body is the tipping point between nature and culture, what species do we need to talk about? Ethological studies indicate that among apes, there is shared attention, even to the point of vocal symbolic communication (Boesch 2002, Seyfarth and Cheney 2012, Crockford et al. 2012, Crockford, Wittig, and Zuberbühler 2014). Should we speak of several tipping points and distinguish different kinds of culture - although this would threaten to relativize the remarkable characteristics of human culture? Concerning humanity, it is perhaps helpful also to draw on another metaphor by Waldenfels, which conceives of humanity as living on the "threshold," or in the no-man's-land between nature and culture, which is crossed again and again but cannot be left behind (Waldenfels 2001; 2016, $104 \mathrm{f} ., 114 \mathrm{f}$.)

The imitation of seemingly pointless details, so-called “over-imitation," may point to a key ingredient in the unique constellation of human culture. Imitation is involved in shared attention, which betrays a fundamentally social mode of being in the world and lays the foundation for symbolic communication. At the same time, over-imitation may just be the tip of the iceberg. Christoph Wulf who is professor of anthropology and philosophy of education at Freie Universität Berlin as well as director of Berlin's Interdisciplinary Center for Historical Anthropology - points to the dramatic cultural importance of mimesis. With Walter Benjamin's depiction of his butterfly chase, Wulf (in this volume) points out how Benjamin's grasping the phenomenon of the butterfly is fundamentally embodied, mimetic, and enactive. Among other things, mimesis also subverts our anthropological distinction of inner and outer, since children even learn feelings in mimesis, something we often take for granted as one's own private domain. Moving on to more encompassing social forms, Wulf highlights especially that in mimesis, humans create a dense texture of social customs and expectations. In creating similarities rather than exact replicas, mimesis differs from mimicry. Social customs evolve and diversify. At some point, a birthday came to be associated with gift giving, which reinforces an existing personal bond. "This ritual knowledge, which enables us to act competently in rituals, evolves from ... participation in ritual activities" (Wulf in this volume). Mimesis inscribes institutions into the body. Rituals then serve as templates in meaning making.

Wulf's presentation of mimesis confronts us again with the abiding riddle why humans can even trade in meaning in the first place. "Receptivity and activity overlap," according to Wulf (in this volume). In imitation, children perform a "leap of faith" (Etzelmüller in this volume) in assuming that there is a point to the current activity. Bernhard Waldenfels analyzes this phenomenon as an 
ineluctable interlacing of "pathos" and "response" (Waldenfels 2016, 43, 45). In his terminology, pathos denotes both a sensitivity for what we encounter and its interpretation, but the interpretation is neither a simple causal process nor a free-wheeling creation without 'friction' with reality. The response is an articulation, vocal or physical in other ways, that does not, although one's own, take its orientation from oneself but from without, a factor that eludes us. Thus, in participating in rituals, we both discover and create forms that allow for shared meaning $(30,43-45)$.

Such receptive spontaneity may perhaps be seen as a cultural analogy to niche-construction in Darwinian evolution. Indeed "ritual knowledge ... evolves" (Wulf, in this volume), and we might see a resemblance to Darwinian 'descent with modification' in mimesis. Darwinian natural selection constrains variation. In culture, however, the constraints in the evolution of rituals are rather loose. The supposition of meaning in participating in a ritual can be called a "leap of faith" also because it creates with little constraint, allowing for wide variation. Humans cooperating in a hunt may understand the process in widely differing terms, as 'bread winning,' as a check to chaos in nature, or as camaraderie, as long as the endeavor is sufficiently plausible and successful. According to Wulf, the "practical knowledge and social activity," which is constituted in the participation in rituals, "are shaped largely by historical and cultural factors" (Wulf in this volume). Yet there is no clean separation between history and culture on the one hand and practical knowledge and social activity on the other, and mimesis plays a significant part in the genesis of the entire complex. Is there perhaps a "natural" factor involved in the beginnings of this complex?

In differentiating between culture and nature, a comparison between humans and primates can often be instructive. Primatologists have argued for chimpanzee cultures based on differing practices of tool and symbol use (Boesch and Tomasello 1998). Is this diversity a cultural outgrowth of an overall natural primate life? Regarding the rituals with which a primate population maintains fundamental social cohesion, local distinctions have not been found so far. However, when primatologists kept rhesus macaques and stump tail macaques together, they found that the species with the more "despotic" temperament adopted the manners - one might almost want to say instincts - of the more "egalitarian" one, although the behavioral repertoire is not entirely plastic, with only some parts having changed (de Waal and Johanowicz 1993; see also Sapolsky 2006).

We may also boil down the mimetic constellation to even simpler constellations. For example, when a polecat is confronted with a rat, hunting behavior kicks in practically without learning, but still young polecat must learn from its mother how to kill the catch by imitating her bite in the neck (Wuketits 2002). How does this mammal "learn" and "know" that biting is the thing to do? How does an animal discern the affordances involved in "open instincts"? It seems to be a case of pathos and response as well that accounts for the intentional salience of the biting. The philosopher Hans Jonas (1903-93) speculates that intentionality arises when animal emotion provides coordination, the missing connection that allows for foraging and hunting to result from sense perception and motility 
(Jonas 1966). Is this kind of animal emotion the first rudimentary intertwining of pathos and response in evolution?

The irreducibility of pathos and response may seem to create a Cartesian or a vitalist temptation to attribute it to a factor impinging from outside. But while Cartesianism conceives of spirit in ontological opposition to matter, it is instructive to look at alternative ontologies from a historical point of view. Ancient stoicism, for example, understands spirit as an exceedingly fine substance, presupposing a spectrum from coarser to finer materiality (Sedley 1999). Annette Weissenrieder's contribution to this volume illustrates yet another way ancient thinkers conceived of the human mind in relation to the human body. She is professor of New Testament at San Francisco Theological Seminary, and her article on the explanation of epileptic seizures in medical texts from the ancient Mediterranean world takes us into material issues in historical anthropology.

Two texts from the so-called Hippocratic Corpus, "De morbo sacro" and "De flatibus" ("On the sacred disease," "On winds"), describe epilepsy in a way that has struck interpreters as remarkably rational and naturalist. The Hippocratic Corpus is a large, heterogeneous assemblage of about 60 ancient Greek writings of unknown origin on medical issues. It is attributed to the elusive $5^{\text {th }}$ century figure Hippocrates and was discussed by prominent thinkers such as Galen and Celsus (Craik 2014).

Weissenrieder (in this volume) describes how in the antique world, epilepsy, or "the sacred disease," was often understood in religious terms as an effect of demons or a lunar deity. But in interpreting the symptoms of the disease, "De morbo sacro" credits pneuma, which can be translated as air or breath, but also as spirit, with 'bearing' human consciousness, while in "De flatibus," this is a property of blood. Once the circulation of air in the body stands still, blocked by phlegm - or, depending on the text, if the flow of blood is blocked by air regular consciousness breaks down, seizures occur. In part, such diagnoses are informed by animal dissection. While other treatises of the Hippocratic Corpus see the heart crucially involved in human cognition and even "De morbo sacro" attributes a certain role to other organs than the brain as well, these treatises see the human intellect depending on the brain in particular. But "these medical texts refuse to envisage a separation of body and mind ... none of these texts proposes a concept of 'soul' or 'mental activity,"” highlighting the role of "sensory and motor skills" (Weissenrieder in this volume) for the constitution of the mind.

Prominent depictions of epilepsy in the New Testament gospels employ a terminology very similar to the 'Hippocratic' medical tradition. In keeping with the 'natural' bent of the medical explanations, the Gospel of Luke does not see demons involved in epilepsy. The Gospel of Mark, by contrast, does see evil spirits at work. Their malice seems to speak against the disease as divine punishment, which relieves the patient of a significant 'spiritual' burden. At the same time, given the concepts the Gospels share with the hippocratic traditions, a more overtly 'religious' view is no less medical, according to Weissenrieder (in this volume). On the other hand, certain translations adopt the agential dimension of 
the demonological interpretation in portraying the disease as an active force, but stop short of attributing the disease to a demon.

On the whole, neither the Hippocratic Corpus nor the New Testament texts in question, apart from the demonological traditions, see consciousness as an entity of a second kind in addition to the fundamental physical dimension. This is also why the importance of material traditions in historical anthropology certainly goes beyond chronicling and book keeping - even though we can no longer affirm the idea that, for example, epilepsy is due to phlegm blocking the flow of air. Rather, historical anthropology confronts our contemporary discourses with an alternative choice of categories and issues. Today's decisions might otherwise remain hidden in plain sight.

The philosopher John McDowell (cf. McDowell 1996) analyzes the epistemological issues involved. This reminds us of the close interrelationship between the scientific, historical, and philosophical dimensions involved in this section. Using Kant as an important touchstone, he argues that human understanding is both receptive and spontaneous. Sense data do not simply impinge causally on our minds, but we are ourselves responsibly involved in human thought achieving "friction" with reality. McDowell appreciates Kant's notion of concepts as an interface between my intuition and the world. Concepts are created transcendentally by human reason rather than constituted empirically, but they allow for a consistent account of nature.

As part of his critique of Cartesianism, however, McDowell fears that the transcendental nature of concepts gives rise to "rampant platonism" (McDowell 1996, 83). By contrast, he wishes to "reinstate friction between thought and the world" by taking "capacities of spontaneity to be in play all the way out to the ultimate grounds of empirical judgments" (ibid., $66 \mathrm{f}$.). He compares the genesis of a cogent use of spontaneity with the formation of ethical character according to Aristotle, which he calls "second nature" (ibid., 84). McDowell assumes that in "practical wisdom," "our eyes are opened" to the objective "requirements of reason” (ibid., 79). Yet even this argument does not fully overcome a conspicuous platonism if it assumes a discovery of reality without ambiguities and discrepancies. What is required is a plausible account of how the requirements of reason come to correspond to the realities of the world.

I have suggested that this connection is made in an embodied intertwining of pathos and response, with an imitator making a "leap of faith," or when a young polecat grasps the anatomical affordances of the prey animal in its paws. Not that this explains much, if for such an explanation a reduction to more basic principles is required. But it does show that there is no inherent reason why we should expect such a "leap of faith" to pick out unerringly the one true objective reality. Affordances can indeed be multivalent. This is not to argue for relativism. On the contrary, the exploration of alternative conceptual schemes can be enriching and eye-opening. By contrast, it would be ironic if we dismissed the Hippocratic corpus as unscientific in the name of modern empirical science, while in fact falling into "rampant platonism" in failing to account for the historical genesis of the guiding concepts of our own science. 


\section{Bibliography}

Boesch, C. 2002. Cooperative Hunting Roles among Taï Chimpanzees. Human Nature 13 (1): 27-46.

Boesch, C., and M. Tomasello. 1998. Chimpanzee and Human Cultures. Current Anthropology 39 (5): 591-614.

Brooke, J. H. 2013. "Ready to Aid One Another": Darwin on Nature, God, and Cooperation. In Evolution, Games, and God: The Principle of Cooperation, ed. M. A. Nowak and S. Coakley, 37-59. Cambridge, MA, London: Harvard University Press.

Clutton-Brock, T. H. 2009. Cooperation between Non-Kin in Animal Societies. Nature 462 (7269): 51-57.

Craik, E. M. 2014. The "Hippocratic" Corpus: Content and Context. Abingdon, New York: Routledge.

Crockford, C., R. M. Wittig, R. Mundry, and K.Zuberbühler. 2012. Wild Chimpanzees Inform Ignorant Group Members of Danger. Current Biology 22 (2): 142-46.

Crockford, C., R. M. Wittig, and K. Zuberbühler. 2014. An Intentional Vocalization Draws Others' Attention: A Playback Experiment with Wild Chimpanzees. Animal Cognition 18 (3): 581-91.

Darwin, C. (1871) 2004. The Descent of Man, and Selection in Relation to Sex. Ed. J. Moore and A. Desmond. London: Penguin Classics.

-. (1872) 1999. The Expression of the Emotions in Man and Animals. Edited by Paul Ekman. 3rd ed. London: Harper Collins: Fontana.

-. (1859) 2009. The Origin of Species: By Means of Natural Selection: Or the Preservation of Favoured Races in the Struggle for Life. Cambridge Library Collection. Cambridge, UK, New York: Cambridge University Press.

de Waal, F. 2001. The Ape and The Sushi Master: Cultural Reflections of a Primatologist. New York: Basic Books.

de Waal, F. and D. L. Johanowicz. 1993. Modification of Reconciliation Behavior through Social Experience: An Experiment with Two Macaque Species. Child Development 64 (3): 897-908.

Engels, E.-M. 2016. The Roots of Human Morals and Culture in Pre-Human Sympathy: Charles Darwin's Natural and Cultural History of Morals. In this volume.

Etzelmüller, G. 2016. The Lived Body as the Tipping Point Between an Evolutionary and a Historical Anthropology. In this volume.

Hausfater, G. and S. B. Hrdy. 2008. Infanticide: Comparative and Evolutionary Perspectives. New Brunswick, N. J.: Aldine.

Jonas, H. 1966. The Phenomenon of Life: Toward a Philosophy of Biology. Evanston: Northwestern University Press.

Kant, I. (1797) 2012. Groundwork of the Metaphysics of Morals. Ed. M. Gregor and J. Timmermann.2nd ed. Cambridge Texts in the History of Philosophy. Cambridge: Cambridge University Press.

McDowell, J. 1996. Mind and World. Cambridge, MA: Harvard University Press.

Moore, J. and A. Desmond. 2004. Introduction. In Charles Darwin. The Descent of Man, and Selection in Relation to Sex, ed. J. Moore and A. Desmond, xi-lxvi. Penguin Classics. London: Penguin.

Nowak, M. A. 2013. Five Rules for the Evolution of Cooperation. In Evolution, Games and God: The Principle of Cooperation, ed. idem and S. Coakley, 99-114. Cambridge, MA, London, UK: Harvard University Press.

Portmann, A. 1990. A Zoologist Looks at Humankind. Trans. J. Schaefer. New York: Columbia University Press, 1990. 
Rolston, H. III. 2004. The Good Samaritan and His Genes. In Evolution and Ethics: Human Morality in Biological and Religious Perspective, ed. P. Clayton and J. Schloss, 238-53. Grand Rapids: Eerdmans.

Sapolsky, R. M. 2006. Culture in Animals: The Case of a Non-Human Primate Culture of Low Aggression and High Affiliation. Social Forces 85 (1): 217-33.

Searle, J. R. 1997. The Construction of Social Reality. Free Press.

Sedley, D. 1999. Hellenistic Physics and Metaphysics. In The Cambridge History of Hellenistic Philosophy, ed. K. Algra, J. Barnes, et al., 355-411. Cambridge, UK, New York: Cambridge University Press.

Seyfarth, R. M., and D. L. Cheney. 2012. Animal Cognition: Chimpanzee Alarm Calls Depend On What Others Know. Current Biology 22 (2): R51-R52.

Tennyson, A. (1849) 2008. In Memoriam A. H. H. In Selected Poems. 2nd edition. Penguin Classics. London, New York: Penguin. 96-198.

Thompson, E. 2010. Mind in Life: Biology, Phenomenology, and the Sciences of Mind. Cambridge, MA, London: Belknap Press.

Tomasello, M. 2010. Origins of Human Communication. The Jean Nicod Lectures. A Bradford Book. Cambridge, MA, London: MIT Press.

Voland, E., and A. Stephan. 2000. "The Hate That Love Generated" - Sexually Selected Neglect of One's Own Offspring in Humans. In Infanticide by Males and Its Implications, ed. C. P. van Schaik and C. H. Janson, 447-65. Cambridge, UK: Cambridge University Press.

Waldenfels, B. 2001. Verfremdung der Moderne: Phänomenologische Grenzgänge. Essener kulturwissenschaftliche Vorträge 10. Göttingen: Wallstein.

-. 2016. Grundmotive einer Phänomenologie des Fremden. 5th ed. Frankfurt a. M.: Suhrkamp.

Weissenrieder, A. 2016. "It Proceeded from the Entrance of a Demon into the Man": Epileptic Seizures in Ancient Medical Texts and the New Testament. In this volume.

Wilson, D. S.2003. Darwin's Cathedral: Evolution, Religion, and the Nature of Society. University of Chicago Press.

-. 2015. Does Altruism Exist?: Culture, Genes, and the Welfare of Others. New Haven and London: Yale University Press and Templeton Press.

Wrangham, R.W., W. C. McGrew, F.B.de Waal, P. G. Heltne, L. A. Marquardt. 1996. Chimpanzee Cultures. Cambridge, MA: Harvard University Press.

Wuketits, F. M. 2002. Was ist Soziobiologie? Beck Wissen 2199. München: C. H. Beck.

Wulf, C. 2016. The Creation of Body Knowledge in Mimetic Processes. In this volume.

Zahavi, A. and A.Zahavi. 1999. The Handicap Principle: A Missing Piece of Darwin's Puzzle. Oxford, New York: Oxford University Press. 



\title{
The Lived Body as the Tipping Point Between an Evolutionary and a Historical Anthropology*
}

\author{
Gregor Etzelmüller
}

\begin{abstract}
The article raises the question how evolution brings forth a (lived) body that is able to transcend itself in shaping itself culturally. Taking up an idea of Charles Darwin, it will be argued that it is human vulnerability, a specific dimension of the human bodily condition, that makes the human person a social, cooperative creature. In a second step the article focuses on the shift from natural evolution to cultural development. An embodied leap of faith seems to be at the beginnings of typically human communication. Evolution has brought forth a body that tends to imitate others by nature - and which is thus shaped culturally from the outset. Insofar as evolutionary anthropology explores the human as a being that is, by nature, open to the cultural shaping of its nature, it opens up bridges for a sustained and robust dialogue with historical anthropology. In pointing out alternatives to practices and understandings that seem a matter of course, historical anthropology, too, relativizes the modern understanding of humanity that is characterized by the dualism of spirit and body.
\end{abstract}

\section{Introduction}

In spite of all interdisciplinary efforts in the two fields, it seems that evolutionary anthropology and historical anthropology continue to drift apart. What appears to be the result of natural processes to thinkers in evolutionary anthropology is understood as a cultural construct by representatives of historical anthropology. An approach in anthropology that assigns fundamental importance to the human body, however, might counteract the centrifugal trend in anthropological studies. For the body is the place where "nature and culture" reach a "tipping point." "Both the process of evolution (Darwin 2004; Pinker 2011) and social expectations and practices (Bourdieu 1984; 2001, Schroer 2005; Karle 2014) are inscribed into one's own (lived) body (Leib-Körper). At this point, the paradigm of embodiment thus raises the crucial question how evolution brings forth a (lived) body that is able to transcend itself in shaping itself culturally (see also Jung's contribution in this volume).

* Translation by Alexander Massmann. 


\section{The Human Person as the Tipping point of Natural Selection and a Culture of Mercy}

Charles Darwin already pointed out that with the human person, various evolutionary processes - in his view primarily the reduction of instincts and the development of cognitive capabilities - brought forth a living being who can follow the guidance of reasons, or more precisely, of "preferences ... that are constitutive for a person” (Pauen 2007, 270).

$[\mathrm{A}] \mathrm{s}$ the power of reasoning becomes clearer so that man can value justly the judgments of his fellows, he will feel himself impelled, apart from any transitory pleasure or pain, to certain lines of conduct. He may then declare ... I am the supreme judge of my own conduct, and in the words of Kant, I will not in my own person violate the dignity of humanity. (Darwin 2004, 133)

The synthesis of Darwin and Kant that Jürgen Habermas pursues (Habermas 2005, $156 \mathrm{f} ., 175,188$ ) is thus already visible in Darwin's own work (see Engels this volume).

The meaning of the "tipping point" can be illustrated with the phenomenon of natural selection. Natural selection brings forth a living being that can disregard the principle of selection to which it owes its own existence (Engels 2010). The human person can act in ways contrary to selection. In The Descent of Man, Darwin writes: "we build asylums for the imbecile, the maimed, and the sick; we institute poor-laws; and our medical men exert their utmost skill to save the life of every one to the last moment." While Darwin admits that no animal breeder would act in this way, he notes that we cannot "check our sympathy, even at the urging of hard reason, without deterioration in the noblest part of our nature" (Darwin 2004, 159). ${ }^{2}$

Since communities characterized by mutual support and self-restraint for the benefit of others have better chances of survival, natural selection rewards such caring communities. Steven Pinker puts it in more general terms: "today no biologist doubts that evolutionary dynamics like mutualism, kinship, and various forms of reciprocity can select for psychological faculties that, under the right circumstances, can lead people to coexist peacefully” (Pinker 2011, 573). There can in fact be selection pressure that positively supports greater cooperation (Tomasello 2014, 31; de Waal 2009; Wilson 2012).

According to Darwin, there is a notable correlation between the specifically human bodily condition, especially in its vulnerability, and the particular human cooperation. Darwin concedes "that man is one of the most helpless and defence-

1 "Umschlagstelle von Natur und Kultur": I am here adopting a concept from Bernhard Waldenfels who gave the keynote address at the Heidelberg symposium, entitled "The Lived Body as the Tipping Point of Nature and Culture” (Dec. 4, 2014, in German). As the lecture was delivered freely without a fixed manuscript, it is not documented in this volume.

2 Darwin's position is diametrically opposed to that of the British philosopher Herbert Spencer, who wrote with regard to the poor, "the whole effort of nature is to get rid of such, to clear the world of them, and make room for better" (cited according to de Waal 2009, 28). 
less creatures in the world." But it seems precisely this fact that contributed to human success in evolution. It stands to reason, according to Darwin,

that an animal possessing great size, strength, and ferocity, and which, like the gorilla, could defend itself from all enemies, would not perhaps have become social: and this would most effectually have checked the acquirement of the higher mental qualities, such as sympathy and the love of his fellows. Hence it might have been an immense advantage to man to have sprung from some comparatively weak creature." (Darwin 2004, 83 f., see also Engels's contribution to this volume)

What makes the human person a social, cooperative creature is thus a dimension of the human bodily condition, that is, human vulnerability.

\section{The Shift from Natural Evolution to Cultural Development}

In the history of humanity, natural evolution reaches a tipping point at which it shifts to the mode of a cultural process. As a consequence, the dynamics of change accelerate dramatically (Jung 2009). Not long ago - in the Mesolithic age - humans had to defend themselves against wild animals (De Waal 2009), but soon they began to dominate their environment, in such a way that late modernity often does not even know nature anymore except through the media.

A simple consideration illustrates that the rise of modern humanity cannot be described in biological terms only ${ }^{3}$ :

The fact is, there simply has not been enough time for processes of biological evolution involving genetic variation and natural selection to have created, one by one, each of the cognitive skills necessary for modern humans to invent and maintain complex tool-use industries and technologies, complex forms of symbolic communication and representation, and complex social organizations and institutions. (Tomasello 2001, 2)

As a result, we need to ask when and how natural evolution shifts to cultural development in such a way that the dynamics of change rapidly accelerate. In this endeavor, we will need to keep in mind that, on the one hand, the origins of human culture are already prefigured in the evolution of living organisms. This is a point made, quite rightly, in enactivism: Every living organism not only adapts to the environment, but also contributes to the shape of the environment - thus, in a sense, creating it. On the other hand, however, the extraordinary character of human culture and its genesis must not be ignored either. ${ }^{4}$ Human culture must be described in continuity with and in discontinuity to the behavior of living organisms in general.

Enactivism presents human culture within the framework of the behavior of living organisms. "Organisms shape the physicochemical environment

${ }^{3}$ See also the emphatic warning issued already by Adolf Portmann not to understand "the procedures of human history [merely as] the natural continuation of the evolution of organic forms" (Portmann 1944, 21; translator's note: all text with a German bibliography translated by A.M.).

${ }^{4}$ Spahn draws attention to this dual perspective in his contribution to this volume. 
into a milieu (an Umwelt)" (Thompson 2007, 74). In recognizing organisms as “"niche-constructing' beings" (Thompson 2007, 95), we see our ability to give rise to a world structured by meaning prepared in the history of life. Other organisms contribute actively to the shape of their environment, thus influencing the course of evolution.

In observing other creatures struggling to continue their existence - starting with bacteria that actively swim away from a chemical repellent - we can, through the evidence of our own experience and the Darwinian evidence of the continuity of life, view inwardness and purposiveness as proper to living being. (Thompson 2007, 163)

That even lower organisms are endowed with subjectivity is not a mere postulate of thought, but seems likely in the face of the experiments of the biologist Martin Heisenberg from Würzburg. He was able to demonstrate that in a flight simulator even the behavior of fruit flies engages in a certain degree of freedom (Heisenberg 1983; 1985; 1997; 2002). We can already observe in nature that animal behavior weighs options off against each other:

For example, consider a squirrel on a tree branch gearing up to jump to another. One can see the muscles preparing, but in some cases the squirrel decides the leap is too far and so, after feigning some jumps, climbs down the trunk and then back up the other branch. The most straightforward description of this event is that the squirrel is observing and evaluating a simulation of what it would experience if it leaped. (Tomasello 2014, 14)

Based on that evaluation, the squirrel decides against jumping.

The embodied character of mental life refers not only to the fact "that mind even on its highest reaches remains part of the organic," but also challenges us to see that "the organic even in its lowest forms prefigures mind" (Jonas 2001, 1).

At the same time we need to be aware of the difference that distinguishes human culture from the niche-construction of a bacterium. Certainly, potential differences are always prepared in evolution. From the perspective of evolutionary anthropology, a specifically human sociality cannot be conceived of without the rise of social instincts among animals (Tomasello 2009). The evolutionary benefit of social instincts can help explain why "helping others with simple physical problems ... is a naturally emerging behavior" (Tomasello 2009, 7; Tomasello 2014, 51 f.). Children as young as two years old, regardless of their culture, show such behavior, which can also be observed in elephants and chimpanzees (Tomasello 2009, 8-12; see also the impressive account in de Waal). This raises the question of when exactly social instincts arise that allow for a kind of behavior that is of a different quality than that of a bacterium actively swimming away from a chemical repellent.

According to Frans de Waal, the evolution of social instincts

probably started with the birth of parental care. During 200 million years of mammalian evolution, females sensitive to their offspring outreproduced those who were cold and distant. When pups, cubs, calves, or babies are cold, hungry, or in danger, their mother needs to react instantaneously. There must have been incredible selection pressure on this sensitivity: Females who failed to respond never propagated their genes. (De Waal 2009, 67) 
Once the social instincts are in place, however, they can also refer to other objects, no longer being directed necessarily towards one's own offspring alone (de Waal 2009, $181 \mathrm{f}$.).

In this context, another one of Darwin's observation is apposite. It seemed to him highly "probable - namely, that any animal whatever, endowed with well marked social instincts ... would inevitably acquire a moral sense or conscience, as soon as its intellectual powers has become as well, or nearly as well developed, as in man" (Darwin 2004, 102). Accordingly, the decisive, if only gradual, difference is located on the level of the cognitive. To be sure, human cognitive capabilities are the result of a long evolutionary process, but what distinguishes humanity from other living organisms, primates especially, seems to be the capability of cultural learning. Humans "can learn not just from the other but through the other" (Tomasello 2001, 6). For this reason, it is not necessary for every individual to invent the wheel anew, but humans can build on what was learned in previous generations. This unique procedure of "cultural transmission" creates a "ratchet effect" (Tomasello 2001, 4) that speeds up cultural developments immensely. In this way, cultural development is decoupled from natural processes (see Jung 2009). To learn "through the other" means, in an elementary sense, to learn by imitating the other's physical behavior. "Imitation requires identification with a body of flesh and blood. We're beginning to realize how much human and animal cognition runs via the body" (de Waal 2009, 59). In imitating the other, synchronizing with her, we are experiencing the world from her perspective - through her body, as it were.

In humans, this form of imitation appears especially pervasive, as the phenomenon of so-called over-imitation demonstrates. ${ }^{5}$ It has only been observed in human children, but occurs in all cultures. ${ }^{6}$ Starting at about age three, toddlers tend to imitate another's action "overly precisely" (Hoehl et al. 2014). In contrast to chimpanzees, for example, toddlers imitate even those aspects that are obviously causally irrelevant for reaching the goal. An experiment by Horner and Whiten often referred to in this context demonstrates this with great force.

Young wild-born chimpanzees from an African sanctuary and 3- to 4-year-old children observed a human demonstrator use a tool to retrieve a reward from a puzzle-box. The demonstration involved both causally relevant and irrelevant actions, and the box was presented in each of two conditions: opaque and clear. In the opaque condition, causal information about the effect of the tool inside the box was not available, and hence it was impossible to differentiate between the relevant and irrelevant parts of the demonstration. However, in the clear condition causal information was available, and subjects could potentially determine which actions were necessary. When chimpanzees were pre-

5 In technical terms, developmental psychologists speak of “over-imitation," which can be confusing as children imitate with high precision, while from a logical and analytical point of view over-imitation seems doubtful. The term is intended to clarify that children imitate even those elements in an action that are obviously irrelevant for reaching the goal. I am grateful to Stefanie Höhl (Heidelberg) for the literature referenced in the following.

6 See Froese and Leavens 2014, 2: “Over-Imitation has been consistently documented for children, but not for young and older chimpanzees ... and it has been demonstrated in a cross-cultural context." 
sented with the opaque box, they reproduced both the relevant and irrelevant actions, thus imitating the overall structure of the task. When the box was presented in the clear condition they instead ignored the irrelevant actions in favour of a more efficient, emulative technique. These results suggest that emulation is the favoured strategy of chimpanzees when sufficient causal information is available. However, if such information is not available, chimpanzees are prone to employ a more comprehensive copy of an observed action. In contrast to the chimpanzees, children employed imitation to solve the task in both conditions, at the expense of efficiency. (Horner and Whiten 2005, 164)

Interestingly, children imitate the action that is obviously causally irrelevant even if they assume they are not observed or if they are encouraged to reach the goal in such a way as makes sense to them. Lyons and colleagues demonstrated that in many cases, children imitate an action with high precision even if this constitutes a disadvantage in a competitive situation: "children will continue to overimitate even when doing so imposes motivationally salient costs" (Lyons et al. 2011, 1163).

In addition, toddlers imitate actions even if they were not demonstrated intentionally, i. e., in a pedagogic context or by trusted care givers:

Interestingly, and in contrast to our prediction, children initially reenacted the irrelevant actions no matter whether these actions were demonstrated by a pedagogical experimenter or by an unfamiliar and non-communicative experimenter. This was true even though the no-contact experimenter never interacted with children and avoided any contact before or during the experiment. The no-contact experimenter never expressed the intention to teach or show anyone how to operate the container and instead made it clear that he or she removed tokens from the container in order to exchange them for stickers.... Our results suggest that preschoolers imitate irrelevant actions even when performed by a complete stranger in the absence of communication and instruction. The incidental observation of actions whose purpose is opaque in relation to the goal of the action, thus, seems to be sufficient to trigger overimitation in 5-year-olds. (Hoehl et al. 2014, 131)

Human children seem to assume that an action performed by a parent or a care giver, even by any person in general, is meaningful, even if they do not see or understand its point. The child imitates these actions as exactly as possible precisely because it does not see the point.

On this view, and in direct contrast to the traditional view of imitation, understanding the other's goals and intentions does not facilitate imitation, but actually hinders it because such direct insight obscures the precise means. Imitation requires individuals to change attention from what the other's goals are to bow the other's actions are precisely realized, while emulation is possible without this extra effort." (Froese and Leavens 2014, 5)

Accordingly, it is not the recognition of the other as an intentional actor ${ }^{7}$ that is foundational for the specifically human mode of learning by imitation, but

7 Tomasello has revised his previous thesis according to which only humans can understand conspecifics as intentional agents. Recent research has shown that "nonhuman great apes not only are intentional agents themselves but also understand others as intentional agents" (2014, 20 , see also ix-x). 
the assumption of there being a point to imitation, in the sense of a leap of faith. Humans assume that the other's actions are meaningful even if her action does not seem so at first blush. This supposition of meaning allows for a cultural dynamic that will turn out to be of particular importance even for the human biology.

That this assumption of meaning amounts to a leap of faith is demonstrated by experiments that confront toddlers with a conflict. They are presented with two different courses of action, and then they need to decide themselves what strategy to pursue.

After being shown two strategies, they chose to maintain the strategy or switch to the strategy employed by the pedagogical experimenter (pedagogical-then-no-contact and no-contact-then-pedagogical conditions), with whom they presumably shared a stronger bond (i. e., social affiliation) and whose normative behavior they may have been more motivated to copy. In the pedagogical-then-pedagogical condition, both experimenters were equally familiar and pedagogical. Here, it seems that children's behavior was flexible and they performed the strategy they had seen last. (Hoehl et al. 2014, 131)

The capability of toddlers to imitate the actions of others precisely shapes even their neuronal system. The difference between human children and apes is even reflected in their mirror system. While in apes mirror neurons seem to react only to goal-directed action, "the human mirror system ... codes both transitive and intransitive motor acts, it is able to code both the goal of the motor act and the movement of which the act is composed" (Rizzolatti and Sinigaglia 2008, 124).

The significance of so-called over-imitation in a typically human mode of life is revealed only once the difference between the contexts of one's lifeworld and the experimental setup in developmental psychology is taken into account. The experiments just mentioned induce children into imitating a pointless action and the reason they are set up this way is that researchers know about the significance of over-imitation for children. Thus, in the lab, over-imitation seems pointless, requiring a time commitment and incurring a competitive disadvantage. But what seems odd in the lab does indeed serve a purpose in one's life world. In this latter context, children imitate the actions of their adult care givers as well, yet these actions are typically goal directed. On the one hand, evolution and history have optimized a multitude of types of action. On the other hand, non-instrumental actions such as greeting rituals often fulfill a precise purpose in the lifeworld.

In learning by imitation, human children not only explore the world that is already opened up for them with respect to cognition, but an entire world of pragmatic options, even if their meaning is not plain to them. We can observe this every day: For example, toddlers learn to close the fridge long before their parents have explained, linguistically, the causality of an open fridge, melting ice, spoiling groceries, and a higher electricity bill. The fridge must be closed, and if an adult leaves it open too long, a child may well close it spontaneously. In mimetic learning, children grasp the difference between the necessity of closing the fridge and the possibility of leaving other doors, or windows, open. The same holds for non-instrumental actions. When attending a church service, chil- 
dren imitate their parents in taking a moment standing for meditation or a silent prayer before taking a seat in the pew. This way they learn to enact, in an embodied way, the difference that distinguishes liturgical communication from communication in the everyday world - long before they are able to grasp this difference intellectually, and this will then enable them to adopt an autonomous stance toward these different modes of communication.

The specific way humans learn culturally, their ability not only to learn from, but also through others, is manifest on an elementary level in the child's imitation of another. Thus, there is no point at which this kind of learning has taken place in a non-embodied form. Humans begin to learn culturally with one person imitating the other, even over-imitating that person, in the terminology of developmental psychology. Embodied imitation is the tipping point at which natural evolution shifts to cultural development. Evolution has brought forth a life form that tends to imitate others by nature - and which is thus shaped culturally from the outset.

That the specific mode of human cultural learning is fundamentally embodied, rather than dependent on language, can be seen both in human phylogenesis and ontogenesis. Joint attention is the original social context of the supposition that there is in fact a point to what the other person is doing (Tomasello 2001, 84-94): by use of a bodily action, such as pointing gestures or the adoption of a particular direction of view, persons draw the other's attention to a third entity, at the same time ascertaining that the other grasps the communicated intention and acts accordingly.

With respect to phylogenesis, there is ample evidence that the early hominin environment required cooperation. Early humans were a far cry from dominating the savannah (de Waal 2009, 18-19). Instead, their survival depended fundamentally on cooperation. Unable to survive individually, humans have always depended on communication. To be successful, however, communication requires the assumption that a movement or an utterance does indeed transport meaning, and to respond appropriately to such a gesture is to make a leap of faith. Since both communicating parties are interested in communicating successfully, however, they are both interested in trust not being exploited for illicit purposes. Any pointing gesture thus carries the message, you should - and you can - trust me! The pointing gesture not only carries informational content, but also communicates the intention to inform, as Tomasello shows. What is more, any pointing gesture at once even communicates the call to accept it, in trust, at face value. Since successful communication is selected for, however, over time the pointing gesture takes on the meaning “you can trust me!” In phylogenesis, the assumption of another's action carrying meaning, to which one responds with a leap of faith, is at first embodied in "pointing and pantomiming" (Tomasello 2014, 49).

In ontogenesis, children are introduced into human communication by way of "pointing and pantomiming" (Tomasello 2014, 49; see also Fuchs's contribution to this volume) as well. Starting at the age of nine to 12 months, children trust "that the adult is pointing out to them something relevant to their current 
search" (Tomasello 2014, 52). We might say that in trust they adapt to a physical gesture. If this trust turns out to be justified again and again, toddlers aged 14 to 18 months themselves start to "use the pointing gesture to coordinate their joint activity" (Tomasello 2014, 51; see the literature used there).

Iconic gestures go beyond the simple pointing gesture "by actually symbolizing an entity, action, or situation in an external icon" (Tomasello 2014, 60). A necessary prerequisite of iconic gestures consists in the physical ability to perform an action resembling the real action itself (Tomasello 2014, 61). Based simply on their physical abilities, primates would be capable of "pantomiming," but the function of their hands is much more strongly constrained: from the get-go the use of an ape's hand is to hold on tight to its mother. By contrast, the human toddler is able to make free use of her hands in various ways (Portmann 1944, 30). She thus discovers the possibilities her hands are given. But even if the motor requirements for gestures are fulfilled, pantomiming may not actually occur. Gestures make sense only once I assume that my partner in communication knows that I want to communicate something (Tomasello 2014, 61).

Both in phylogenesis and ontogenesis, the first forms that practice perspective taking are embodied in elementary forms of behavior. Perspective taking does not require the capacity to speak (Tomasello 2014, 127; see also Fuchs's contribution to this volume). It is embodied articulation, not language, that is at the beginnings of communication (Jung 2009; Breyer 2015, 35-39). Embodied articulation is the tipping point at which natural evolution shifts into cultural development.

\section{Evolutionary Anthropology Advocating for a Plurality of Culture}

Humans are cultural beings by nature. To a large extent they shape their own behavior by learning and imitating other humans physically, rather than following natural instincts. That the development of the human person is shaped by her cultural environment is clear from this, and at different times and in different contexts, the human person develops differently. Based on this mutual interdependence of natural and cultural processes, we can conclude that humans do not live in a particular culture by nature. This has also been pointed out by the Jewish philosopher Michael Landmann (1913-84): "what is prefigured already in nature is the mere fact of culture, but not its particular shape" (Landmann 1961, 60). There is no type of culture that derives from human nature, from the human essence, by necessity.

For that reason it is not correct either to conceive of culture in the singular as a human creation. Humans do not create culture in the singular, but particular people create their own culture. Humanity creates cultures. That humans may create culture in the first place already implies that they create them again and again in different ways, that they may create the most diverse cultures. (Landmann 1961, 26) 
Yet, since humans not only create diverse cultures but are themselves shaped by their respective cultures, there is no definite human essence. As both creators and creatures of culture, we encounter the human essence always in the plural.

The human person is just as diverse ... as the cultures that shape the person. It is not that the human person brings forth different cultures while remaining the same, passing through them untouched as a constant entity. In creating cultures, humans finish creating themselves, and in each culture they provide themselves with a different form and direction (Landmann 1961, 61).

Due to the shape of a culture created by humans, human nature itself changes in history. "Historical variability is the radical human fate" (Landmann 1961, 26). The notion of an unchanging human core thus turns out to be a fiction. "If we wish to speak of a core, this would be nothing but open plasticity" (Landmann 1961, 62, see 27).

This philosophical realization is correlated with the biological fact of the enormous human plasticity in ontogenesis. The elementary formation of the human baby during the first year is characterized by the exchange with others, which amounts to cultural formation.

Thus already during the first year, the life of the human child takes place in the realm of the 'historic,' in a time during which the human neonate would - if it were a mammal in all respects - still have to gain shape under the most pure conditions of natural law, i. e., in the darkness of the womb. (Portmann 1944, 70, see 81)

But even processes seemingly purely somatic "such as gaining an upright gait, the formation of the spine and the pelvis" (Portmann 1944, 125) take place in touch with the social environment, in one's "own activity of striving, learning, and imitation" (Portmann 1944, 70). This is even clearer for the "development of the truly human possibilities in practicing and maturing” (Portmann 1944, 101), as in the varied uses of the hand, which can learn to write in an 'elegant hand,' to play the piano brilliantly, to touch gently. The 'low speed' of human somatic development allows for social and psychic shaping, thus bringing forth the human being as an entity characterized by an irreducible mutual interdependence of nature and culture, body and psyche (Portmann 1944).

What Portmann described in developmental and behavioral respect corresponds to current thinking about the plasticity of the human brain. "The human brain is not only the most complex, but also the most adaptable organ that we know of. As the neurosciences show, all our experiences, perceptions, and interactions with the environment modify the neuronal structures throughout our lives” (Fuchs 2013, 156). Notably, the long maturation period of the human brain is of great significance, since at birth it amounts "only to a little more than $25 \%$ of an adult's brain volume," appearing not to be fully matured even at age ten (Grupe et. al., 67). Evolution has thus brought forth an organ that for its development depends on an environment conducive to life, only reaching its detailed structure in interaction with the environment. The influence of the environment extends even into the precise neuronal structures. 
Thus, brain research confirms an insight that Portmann, as a biologist, reached in the 1940s: "We see the biological characteristics of the human precisely in how inherited factors irreducibly grow together with the various social effects - in ways that are final and unique from the point of view of the individual" (Portmann 1944, 127).

The extent to which the human person is open to social influences can also be seen in human sexuality: "even that part of human behavior that is most instinctual, sexuality, is open for a far-reaching freedom of personal decision” (Portmann 1944, 59) - and thus for cultural shaping.

Insofar as evolutionary anthropology explores the human as a being that is, by nature, open to the cultural shaping of its nature, it opens up bridges for a sustained and robust dialogue with historical anthropology, which draws on methods from cultural studies. Evolutionary anthropology explores the human as a living being that is shaped culturally even in its very nature. It also raises awareness of the fact that by nature the human person is destined for culture, although not for any specific culture. For that reason, human nature varies historically. The human being is historical and cultural by nature, calling for historical description. Taking the tipping point of nature and culture seriously, we see that the (lived) body is a bridge from evolutionary to historical anthropology.

\section{Evolutionary and Cultural Anthropologies}

Historical anthropology sees its task in pointing out historical alternatives to what seems a matter of course $e^{8}$ and in working against "unwitting retrojections of contemporary conceptions onto the past" (Tanner 2009, 147, see 154). The human body represents the "starting point of historical anthropology" as well, but here the body is seen less in its evolutionary genesis, but "rather in its historical and cultural character" (Wulf 2004, 134). The cultural and historical formation of the body is in the foreground - and not only the lived body (Leib), but even the seemingly natural body as it is described in medicine and physiology. "Even the scientific anthropologies thus appear with a temporal index, becoming accessible for analysis in their historically contingent shape" (Tanner 2009, 151).

To put it succinctly in terms of discourse analysis: discourses as societal practices that must be explored historically are inscribed into the body, thus bringing forth bodies as their material effects. ${ }^{9}$ This thesis contradicts modern dualism,

\footnotetext{
${ }^{8}$ See Süssmuth 1984, 8: “There are several tasks of historical anthropology: to detect forms of human culture covered by other historical layers, to uncover manifold expressions of life, to contribute to a critique of ideologies in making clear distinctions between stereotypical conceptions of imposed and constant characteristics of human motivations, attitudes, and forms of behavior."

9 I am grateful for Michael Bergunder's challenge to reflect more deeply about the relation between embodiment and discourse theories. The phrasing used here follows Bergunder's understanding of discourses as "social practices having material effects" (Bergunder 2014, 263), which he develops in discussing Foucault, Butler, and Laclau.
} 
as does evolutionary anthropology - only from the other shore, to continue the image of the bridge. Michael Bergunder points out that the notion of the material effects of discourse overcomes the "usual, but unsatisfactory, dichotomising into discursive and non-discursive, into thought and reality” (Bergunder 2014, 263). The notion of discursive practices having material effects is thus directed against a dualism that distinguishes thought as mere representation of reality and reality itself to begin with. In a second step, the question would be how the two aspects can be brought together. According to Bergunder, by contrast, there is no reality that is not always already shaped by discourse. We should take the intertwining of culture and nature as a starting point, rather than the supposed dichotomy. The idea that at first there was nature without culture, which would then be reshaped by culture, is ill-suited to how things are. In this respect, the cultural approach of discourse analysis runs parallel to a central insight of the philosophy of embodiment.

In another respect, both discourse theory and the embodiment paradigm choose intersubjectivity as their starting point in describing human reality. The individual subject exists only in intersubjective relationships. The subjective Spirit - even if taken in the sense of a material reality of the brain - gains shape through interaction with the objective Spirit, in being formed by discourses in society. The Spirit of a time objectifies itself in law, religion, education, the arts, and the sciences. There is no point at which an individual may become aware of himself in which he has not already been shaped historically.

Neither the cultural studies approach nor the embodiment paradigm takes the individual Spirit as its point of departure, after which one might go on to ask how this individual Spirit relates to others and to material reality. Instead, both approaches begin by looking at the interrelationship of sociality, individuality, and material reality. Human persons "create and modify their being in engaging the things that they move and by which they are moved" (Tanner 2009, 155). Yet precisely in this respect, human persons resemble all other organisms, as emphasized both by Foucault and enactivist thinkers (Foucault 1978; Thompson 2007, 74, see Malafouris this volume). Thus, we can identify two points of agreement between the cultural studies approach and the embodiment paradigm. To begin with, they share the concern to overcome the modern dichotomy of thought and reality, but they also agree in their fundamental focus on intersubjectivity. These two points of agreement, however, raise two important questions.

1. What conditions do bodies have to fulfill in order to be shaped by discursive practices?

2. What properties must a reality have so that individuals do not only constitute the discourse, but are always already shaped by it? To put it differently: What is required for top-down effects that is a feedback between the objective spirit and the subjectively lived bodies?

According to Judith Butler, the task of cultural anthropology is to discover the body as a "site of a number of possibilities that are expanding in cultural ways" (Butler 1997a, 11). As the body is not determined by nature, it can become a site of unexplored possibilities. This view is not far from Portmann's notion of 
the human as born "premature" physiologically. The human being is that which is non-determined by nature - see already Nietzsche's argument to this effect (Nietzsche 2003, 56: section 62). Thus, there is a biological basis, as it were, for the cultural studies approach (see Foucault 1978, xix). This also implies at the same time, however, that the biological description does not necessarily lead to essentialism. For biology itself indicates that, based on nature, we cannot say what a lived body is and what possibilities it holds. "Even to the biologist the human person must appear as that very particular being with a history, as the living being with its particular second nature, i. e., culture" (Portmann 1944, 20). It is precisely the embodiment approach that discovers the human body as less than completely determined, as a "site of a number of possibilities that are expanding due to culture” (Portmann 1944, 20).

If the human person, or humanity, materializes realities in discourse, as the cultural studies approach maintains, this implies not only that the human person is open to the world, but also that the world is open for the human person. If the world were completely determined physically, discursive practices would be unable to change the world. By contrast, the cultural studies approach asserts that discursive practices gain shape in the world and thereby shape the world themselves. The environment of the human person does not simply determine human behavior, but in exchange with the environment, the human person shapes herself and her world. That is exactly the thesis which the approach called enactivism proposes within the framework of the embodiment paradigm: in acting, the human person participates in bringing forth her world.

That the cultural studies approach is partly in agreement with the embodiment approach can also be observed in Judith Butler's texts, notably in her ambivalent relation to Maurice Merleau-Ponty's phenomenology. According to Merleau-Ponty, the lived body is the tipping point of nature and culture, that is - in Butler's words - "the site where the materiality of language and that of the world ... are perpetually negotiated" (Butler 1993, 69). For precisely this reason Butler is fascinated with Merleau-Ponty's approach: "It seemed to me that he offered a promising denaturalization of the gendered body" (Butler 1997b, 185). He appears to understand the lived body as a "'historical idea' rather than 'a natural species”" (Butler 1989, 85). The body is conceived of "as a modality of existence, the 'place' in which possibilities are realized” (Butler 1989, 86). Butler concedes that in principle, Merleau-Ponty does not think of the body in essentialist terms (Butler 1989, 94), but she sees his argument in The Phenomenology of Perception amounting implicitly to a "universalization of the male subject" (Butler 1989, 98), which misses the point that gender is always already constituted in discourse. Merleau-Ponty describes the individual sexual situation without taking into consideration that individuals always face "a sedimented sexuality" (Butler 1989, 90). It is only for this reason, Butler argues, that Merleau-Ponty can understand the universal dialectics of slave and master as an implication of lived experience (Butler 1989, 96). He cites current practice, thus again reinforcing it. Doing so, he normatively asserts and strengthens a supposed essence of women: 
"The slave must be the Other, the exact opposite of the Subject, but nevertheless remain his possession” (Butler 1989, 96). By contrast, Butler points out, what seems natural to Merleau-Ponty must be deconstructed as the result of a historical genesis. ${ }^{10}$

If we take seriously that by nature the human person requires description in historical terms, then there is no longer any living being 'behind' history that is merely natural. Instead, in history there is nothing but the (lived) body as the tipping point of nature and culture. This (lived) body, however, can also be described precisely with respect to biology. Without the plasticity of the human person and her brain, which can be described from a biological point of view, without the human ability to interact socially, which can be described by an evolutionary anthropology (Tomasello 2014, ch. 1), and without the elementary intercorporeity, in which human persons are always already oriented towards each other, which can be highlighted in developmental psychology (Fuchs 2013, 188-208, Hoehl et al. 2014), we would not be able to conceive of a natural being that can be specified historically. At the same time, biology and psychology describe processes that illustrate how comprehensive the influence of social practices on the human person is. Developmental psychology shows "that learning begins already long before birth" (Pauen 2012, 10). Further insights into how toddlers learn a particular language demonstrate the far-reaching consequences of discursive practices as well. According to studies with infants, "new-born babies are sensitive to all sounds of all languages at first, but they lose this sensitivity already around the end of their first year, from then on being able only to distinguish the sounds of those languages they hear particularly often" (Pauen 2012, 12). Our social environment not only opens up possibilities for us, it also stunts certain possibilities of early infancy. Since our regular social communication involves the significant capacity to distinguish between different human faces, but not between the faces of apes, infants lose their initial capability of identifying chimpanzee faces individually (Pauen 2012,13). Neurology shows "that such influences are correlated with changes in brain structure" (Pauen 2012, 13) - that indeed the social aspect shapes the natural dimension. Notably the development of the prefrontal cortex is "open to influences by the social environment in an especially high degree. The predisposition toward neuronal and synaptic circuit patterns of the prefrontal cortex is actualized not via genetic guidance, but via one's own experience” (Hüther 2012,16). Exactly which neuronal circuits will develop depends on "the stimuli for particular kinds of use which [children and youths] experience during their education and socialization. In consequence, at least this area of the human brain must be considered a social product" (Hüther 2012, 17; see Tanner 2009, 152).

${ }^{10}$ According to Butler, in his "incomplete and posthumously published The Visible and the Invisible," Merleau-Ponty "achieved a philosophical distance from the sexual Cartesianism of his phenomenological colleagues," suggesting instead an "ontology of the tactile, a description of sensual life" (Butler 1989, $97 \mathrm{f}$.). 
Moreover, the study of epigenetics shows that an organism's experiences and its behavior can have an influence on its sex cells. In 2014, a research group in Zurich headed by Isabelle M. Mansuy, a researcher in neuroepigenetics, succeeded in demonstrating the inheritance of early childhood traumata by offspring in mice (Gapp et al. 2014). Presumably microRNA - short copies of genetic material influencing gene expression - is involved in transmitting inherited information in this case. The research group discovered

that traumatic stress in early life altered mouse microRNA (miRNA) expression, and behavioral and metabolic responses in the progeny. Injection of sperm RNAs from traumatized males into fertilized wild-type oocytes reproduced the behavioral and metabolic alterations in the resulting offspring. (Gapp et al. 2014, 667)

If these results can be replicated and shown to apply to humans as well, we will be dealing with a scientific account of how social experience shapes embodied beings across generations. Neuroepigenetics demonstrates that even the reality of each of us is historically sedimented.

The fact that discursive practices have real effects and that social relationships shape what is supposedly natural hearkens back to the challenges that psychosomatics has always presented for our understanding. The difficulty is how psychic realities as well as social ones generate physical realities - that is, diseases, as in psychosomatic medicine, or potentially gender, as Judith Butler argues. A sophisticated psychosomatics presupposes an interdependence between psychic and physical factors that is non-linear, however. In this respect, the physical dimension, the body, also plays a role in the genesis of a reality that is always shaped by this interdependence. Since social interaction typically involves vision, for example, the ability to interact communally is also due to the particularities in the morphology of the human eye, the extraordinary human sociality to our particular vulnerability, as Darwin noted (see Darwin 2004, $83 \mathrm{f}$.$) .$

The (lived) body is indeed the "site of a number of possibilities that are expanding in cultural ways" (Butler 1997a, 11), but it has a say in fulfilling these possibilities - and thus there are limits to the ways it might be shaped.

\section{Historical Anthropology and the Anthropology of Embodiment}

The bridge between evolutionary anthropology and historical anthropology not only opens a passage from the former to the latter, however. In pointing out alternatives to practices and understandings that seem a matter of course, historical anthropology relativizes the modern understanding of humanity that is characterized by the dualism of spirit and body. It is just one perception among several options that have been instantiated historically. The typically modern anthropological distinction between inside and outside is culturally conditioned as well. 
In our languages of self-understanding, the opposition 'inside-outside' plays an important role. We think of our thoughts, ideas, or feelings as being 'within' us, while the objects in the world which these mental states bear on are 'without'. Or else we think of our capacities or potentialities as 'inner', awaiting the development which will manifest them or realize them in the public world. The unconscious is for us within, and we think of the depths of the unsaid, the unsayable, the powerful inchoate feelings and affinities and fears which dispute with us the control of our lives, as inner. (Taylor 2001, 111)

Yet, this feeling of interiority is not atemporal, but is part of a "historically limited mode of self-interpretation, one which has become dominant in the modern West” (Taylor 2001, 111).

The anthology Der ganze Mensch: Zur Anthropologie der Antike und ibrer europäischen Nachgeschichte ("The Entire Human Person: Anthropology in the Antique World and its European Post-History”), edited by Bernd Janowski (Janowski 2012), shows in an exemplary manner that the antique world, in all its variety, thought in quite different ways. The introduction states explicitly that when modern scholars, "with an integrative intention, critical of academic theory," asked for the whole person in the sense of an "alternative idea," there have "again and again been surprising points of contact with the anthropological discourses of the antique world" (Janowski 2012, 9f.). In ancient Egypt, "the body is conceived of as an entirety composed of individual parts," and human life is thought of as "integration into social relationships" (Janowski 2012, 10). Mesopotamian traditions are not aware of the "modern separation between the physical, psychic-mental, and the social aspects” of one's life (Janowski 2012, 10; see Steinert 2012). Even ancient Greece cannot be simply considered a precursor of modern dualism. Even for Homer there is "quite obviously ... no center of our consciousness" (Bremmer 2012, 176). Neither does the medical-philosophical tradition of the antique world understand the inner human dimension as the true person, but rather as that which is internal to the body (Weissenrieder 2016).

Pursuing a consistently historical interpretation, the exegesis of the Old and the New Testament has also contributed to the relativization of modern dualism. The Old Testament scholar Hans Walter Wolff from Heidelberg pointed out in his groundbreaking Anthropology of the Old Testament (1973; see Wolff 1975; see Janowski 2013) that from the Old Testament perspective, the human person appears as embodied throughout. The Hebrew term that is commonly translated as soul, næpæ̌́, denotes "needy man, who aspires to life and is therefore living." The term grasps the human person "primarily in his need and desire," but also in his "emotional excitability and vulnerability," as a pathic being, as a psychosomatic unity. The concept is characterized by the dimension of "vitality", which also applies to the animal” (Wolff 1975, 25). The human person is flesh, and so the Hebrew term for flesh $b \bar{a} \bar{s} \bar{a} r$ can stand in for the personal pronoun in rhetorical parallelism (Ps 119:120; see Wolff 1975, 28).

For the most part, a dualist anthropology is foreign to the New Testament as well. Paul's letters do not portray, for example, the soul as the site at which the relationship with the divine takes place. Instead, they call the body the temple of 
the Holy Spirit (1 Cor. 6:19). Paul understands the present body as a soma psychikon, as a psychosomatic unity (1 Cor. 15:44; see Theißen 2016; Weissenrieder 2016; Etzelmüller 2016). He is impressed by the (lived) body

as an organismic interaction of a limited plurality of organs. Paul thus chooses the body as an ideal image for the congregation and the church: a polyphonic interplay of organs resulting in mutual strengthening and edification, not by way of mono-hierarchical forms of power, but structured only through changing constellations of functional prioritization and subordination. (Welker 2013, 72; see Wilckens 1980, 67)

Moreover, Paul is sensitive to the fact that conflicts in the congregation can result in physical disease (1 Cor. 11:30; see Weissenrieder, Etzelmüller 2010, 25f.), but he also sees how the supposed deficiency of his own body contributes to the creation of the kind of community ${ }^{11}$ that says "those members of the body that we think less honorable we clothe with greater honor, and our less respectable members are treated with greater respect” (1 Cor. 12:23; see Martin 1995, 102).

Over against an anthropology that is construed in dualistic or mentalistic ways, to biblical scholars it seems only appropriate - and only possible to highlight the alien character of the Biblical perception of the human person. But surprisingly, the historical reconstruction of the anthropologies of the Old and the New Testament shows an understanding of the human person that is much closer to current research and discourse about embodiment in the neurosciences, in biology, medicine, and philosophy than those understandings of the human that we usually consider modern. Anthropologies of the Old and the New Testament thus do not merely open up perspectives on texts that are historically distanced, but do uncover alternatives to classical modern anthropology. In this sense, historical anthropology turns out to hold great potential for future research on anthropology.

In conclusion, the human person turns out to be the tipping point between an evolutionary and a historical anthropology. On the one hand, evolutionary anthropology reveals that by nature, the human person is destined for culture, hence being "mere plasticity." On the other hand, historical anthropology shows that foreign, historical perceptions of humans knew about this plasticity, i. e., the ineluctable, non-linear interdependence between physical and psychic, individual and social, as well as between natural and cultural processes.

It was in view of the Old Testament, which sees the human person as fundamentally characterized by the dialogue with God, that Wolff asserted: "In his dialogue with God above all, man sees himself as called into question, searched

11 Presumably, Paul suffered from "a physical deficiency that adversely affected his rhetorical performance” (Martin 1995, 54). This may have been the physical result of the abuse he suffered as a missionary. In that case, his body would have manifested, in the eyes of his antique environment, the lowly, dishonorable state of the apostle. For in Roman culture, "a beaten body was a dishonoured body" (see Glancy 2010, 41). Yet, Paul's wish for this weakness to be cured was not granted. Instead, he came to embody the word of the risen Christ, "My grace is sufficient for you" (2 Cor. 12:9). The supposed deficit of the apostle's body thus comes to embody the word of the cross and contributes to the transvaluation of all values which it brings about. 
out and thus much less established for what he is than called to new things" (Wolff 1975, 3, translation revised; see also Weissenrieder's contribution to this volume). In accordance with this, the New Testament also sees the human being as characterized by an open future. The First Epistle of John puts it paradigmatically: "what we will be has not yet been revealed" (1 John 3:2). This biblical insight goes well together with an evolutionary anthropology, which does not aim at a description of the human essence, but rather at a better understanding of those natural processes that allow the human person to create and establish something new again and again. The results of a historical genesis should not be misunderstood as the description of the human essence, but as enabling the very process of transcending.

As the searchlights of evolutionary and historical anthropology converge on the lived body, the human person appears as a living being that cannot be described appropriately in naturalistic terms, but as a complex unity resulting from the interdependence of natural and cultural processes. This complex constellation is condensed precisely in human embodiment. For this reason, "every substantial presentation of the human being must renounce the artificial separation between approaches in the sciences and in the humanities, which makes sense only for more narrow purposes" (Portmann 1944, 125). An embodied being that is mere plasticity and that is consequently open in its future demands an interdisciplinary anthropology that includes the diverse disciplines and provides new perspectives on the human (lived) body as the site of possibilities not yet realized.

\section{Bibliography}

Bergunder, M. 2014. What is Religion? The Unexplained Subject Matter of Religious Studies. Method E Theory in the Study of Religion 26 (3): 246-286.

Bourdieu, P. 1984. Distinction: A Social Critique of the Judgement of Taste. Trans. Richard Nice. Cambridge, MA: Harvard University Press, 1984.

-. 2001. Masculine Domination. Trans. R. Nice. Stanford: Stanford University Press, 2001.

Bremmer, J. N. 2012. Die Karriere der Seele: Vom antiken Griechenland ins Moderne Europa. In Der ganze Mensch: Zur Anthropologie der Antike und ibrer europäischen Nachgeschichte, ed. B. Janowski, 173-198. Berlin: Akademie-Verlag.

Breyer, T. 2015. Verkörperte Intersubjektivität und Empathie: Philosophisch-anthropologische Untersuchungen. Philosophische Abhandlungen 110. Frankfurt: Klostermann.

Butler, J. 1989. Sexual Ideology and Phenomenological Description: A Feminist Critique of Merleau-Ponty's Phenomenology of Perception. In The Thinking Muse: Feminism and Modern French Philosophy, ed. A. Jeffner and I. M. Young, 85-100. Bloomington and Indianapolis: Indiana University Press.

-. 1993. Bodies that Matter: On the Discursive Limits of 'Sex'. New York: Routledge.

-. 1997a. Vorwort zur deutschen Ausgabe. In Körper von Gewicht: Die Diskursiven Grenzen des Geschlechts. Trans. K. Wördemann, 9-12. Frankfurt a. M.: Suhrkamp, 1997.

-. 1997b. Postskriptum, April 1997. In Geschlechtsideologie und Phänomenologische Beschreibung: Eine feministische Kritik an Merleau-Pontys Pbänomenologie der 
Wabrnehmung. In Phänomenologie und Geschlechterdifferenz, ed. S. Stoller and H. Vetter, 166-86. Wien: Universitätsverlag.

Darwin, C. 2004. The Descent of Man, and Selection in Relation to Sex, ed. J. Moore and A. Desmond. Penguin Classics. London: Penguin.

de Waal, F. 2009. The Age of Empathy: Nature's Lessons for a Kinder Society. New York: Harmony Books.

Engels, E. M. 2010. Charles Darwins Kritik an der Lehre vom 'Intelligent Design.' In Schöpfungsglaube vor der Herausforderung des Kreationismus, ed. B. Janowski, F. Schweitzer, and C. Schwöbel, 69-106. Theologie Interdisziplinär 6. NeukirchenVluyn: Neukirchener Verlagsgesellschaft.

-. 2016. The Roots of Human Morals and Culture in Pre-Human Sympathy Charles Darwin's Natural and Cultural History of Morals. In this volume.

Etzelmüller, G. 2016. Verkörperung als Paradigma theologischer Anthropologie. In Verkörperung als Paradigma theologischer Anthropologie, ed. G. Etzelmüller and A. Weissenrieder, 219-242. Theologische Bibliothek Töpelmann 172. Berlin and New York: de Gruyter.

Foucault, M. 1978. Introduction. In Georges Canguilhem. On the Normal and the Pathological. Trans. Carolyn R. Fawcett, ix-xx. Studies in the History of Modern Science 3, Dordrecht, Boston, and London: Reidel.

Froese, T. and D. A. Leavens. 2014. The Direct Perception Hypothesis: Perceiving the Intention of Another's Action Hinders its Precise Imitation. Frontiers in Psychology 5:65. $1-15$.

Fuchs, T. 2013. Das Gehirn - ein Beziehungsorgan: Eine phänomenologisch-ökologische Konzeption. $4^{\text {th }}$ ed. Stuttgart: Kohlhammer.

Gapp, K., A. Jawaid, P. Sarkies, J. Bohacek, P. Pelczar, J. Prados, L. Farinelli, E. Miska, and I. M. Mansuy. 2014. Implication of Sperm RNAs in Transgenerational Inheritance of the Effects of Early Trauma in Mice. Nature Neuroscience 17: 667-671.

Glancy, J. 2010. Corporal Knowledge: Early Christian Bodies. Oxford: Oxford University Press.

Grupe, G., K. Christiansen, I. Schröder, and U. Wittwer-Backofen. 2012. Anthropologie: Ein einfübrendes Lesebuch. $2^{\text {nd }}$ ed. Berlin, Heidelberg: Springer.

Habermas, J. 2005. Zwischen Naturalismus und Religion: Philosophische Aufsätze. Frankfurt a. M.: Suhrkamp.

Heisenberg, M. 1983. Initiale Aktivität und Willkürverhalten bei Tieren. Naturwissenschaften 70: 70-78.

-. 1985. Freiheit aus der Sicht der Verhaltensforschung. In Ordnung und Unordnung, ed. G. Becker et al., 74-82. Weinheim: Beltz.

-. 1997. Das Gehirn des Menschen aus biologischer Sicht. In Der Mensch und sein Gehirn: Die Folgen der Evolution, ed. H. Meier and D. Ploog, 157-186. Munich, Zurich: Piper.

-. 2002. Freiheit als Eigenschaft des Verhaltens. Nova Acta Leopoldina 86: 79-95.

Hoehl, S., M.Zettersten, H. Scheihauf, S. Grätz, and S. Pauen. 2014. The Role of Social Interaction and Pedagogical Cues for Eliciting and Reducing Overimitation in Preschoolers. Journal of Experimental Child Psychology 122: 122-133.

Horner, V. and A. Whiten. 2005. Causal Knowledge and Imitation/Emulation Switching in Chimpanzees (Pan Troglodytes) and Children (Homo Sapiens). Animal Cognition 8 (3): 164-181.

Hüther, G. 2012. Verschaltungen im Gestrüpp: Kindliche Hirnentwicklung. Aus Politik und Zeitgeschichte 22-24: 15-19.

Janowski, B. 2012. Der ganze Mensch: Zur Anthropologie der Antike und ibrer europäischen Nachgeschichte. Berlin: Akademie-Verlag. 
-. 2012. 'Der ganze Mensch': Zur Geschichte und Absicht einer integrativen Formel. In Der ganze Mensch: Zur Anthropologie der Antike und ibrer europäischen Nachgeschichte, ed. B. Janowski, 9-21. Berlin: Akademie-Verlag.

-. 2013. Hans Walter Wolff und die alttestamentliche Anthropologie. In Neu aufbrechen, den Menschen zu suchen und zu erkennen: Symposium anlässlich des 100. Geburtstages von Hans Walter Wolff, ed. J. C. Gertz and M. Oeming, 77-112. Biblisch-Theologische Studien 139. Neukirchen-Vluyn: Neukirchener.

Jonas, H. 2001. The Phenomenon of Life: Towards a Philosophical Biology. Northwestern University Studies in Phenomenology and Existential Philosophy. Evanston: Northwestern University Press.

Jung, M. 2009. Der bewusste Ausdruck: Anthropologie der Artikulation. Humanprojekt 4. Berlin, New York: de Gruyter.

Karle, I. 2014. Beyond Distinct Gender Identities: The Social Construction of the Human Body. In The Depth of the Human Person: A Multidisciplinary Approach, ed. M. Welker, 333-350. Grand Rapids, Cambridge: Eerdmans.

Landmann, M. 1961. Der Mensch als Schöpfer und Geschöpf der Kultur: Geschichts- und Sozialanthropologie. München, Basel: Reinhardt.

Lyons, D. E., D. H. Damrosch, J. K. Lin, D. M. Macris, and F. C. Keil. 2011. The Scope and Limits of Overimitation in the Transmission of Artefact Culture. Philosophical Transactions of the Royal Society London B: Biological Sciences 366: 1158-1167.

Malafouris, L. 2016. On Human Becoming and Incompleteness: A Material Engagement Approach to the Study of Embodiment in Evolution and Culture. In this volume.

Martin, D. B. 1995. The Corinthian Body. New Haven and London: Yale University Press.

Nietzsche, F. 2003. Beyond Good and Evil: Prelude to a Philosophy of the Future, ed. R. P. Horstmann and J. Norman. Trans. J. Norman. Cambridge Texts in the History of Philosophy. Reprint. Cambridge, UK: Cambridge University Press, 2003.

Pauen, M. 2007. Ursachen und Gründe: Zwei zentrale Begriffe in der Debatte um Naturalismus und Willensfreiheit. In Naturgeschichte der Freibeit, Humanprojekt. Interdisziplinäre Anthropologie I, ed. J. C. Heilinger, 247-272. Berlin and New York: de Gruyter.

Pauen, S. 2012. Wie lernen Kleinkinder? Entwicklungspsychologische Erkenntnisse und ihre Bedeutung für Politik und Gesellschaft. Aus Politik und Zeitgeschichte 22-24: 8-14.

Pinker, S. 2011. The Better Angels of our Nature: The Decline of Violence in History and its Causes. London: Allen Lane/Penguin.

Portmann, A. 1944. Biologische Fragmente: Zu einer Lehre vom Menschen. Basel: Schwabe. Rizzolatti, G. and C. Sinigaglia. 2008. Mirrors in the Brain: How our Minds Share Actions and Emotions. Oxford: Oxford University Press.

Schroer, M. 2005. Zur Soziologie des Körpers. In Soziologie des Körpers, 7-47. stw 1740. Frankfurt a. M.: Suhrkamp.

Spahn, C. 2016. Beyond Dualism? The Implications of Evolutionary Theory for an Anthropological Determination of Human Being. In this volume.

Steinert, U. 2012. Aspekte des Menschseins im alten Mesopotamien: Eine Studie zu Person und Identität im 2. und 1. Jt. v. Chr., Cuneiform Monographs 44, Leiden, Boston: Brill.

Süssmuth, H. 1984. Historische Anthropologie: Der Mensch in der Geschichte. Kleine Vandenhoeck-Reihe 1499. Göttingen: Vandenhoeck \& Ruprecht.

Tanner, J. 2009. Historische Anthropologie. In Handbuch Anthropologie: Der Mensch zwischen Natur, Kultur und Technik, ed. E. Bohlken and C. Thies, 147-156. Stuttgart, Weimar: Metzler.

Taylor, C. 2001. Sources of the Self: The Making of the Modern Identity. $10^{\text {th }}$ printing. Cambridge, MA: Harvard University Press. 
Theißen, G. 2016. Verkörperung als Botschaft: Transformative Religion und Theologie im Urchristentum. In Verkörperung als Paradigma Theologischer Anthropologie, ed. G. Etzelmüller and A. Weissenrieder, 159-181. Theologische Bibliothek Töpelmann 172. Berlin and New York: de Gruyter.

Thompson, E. 2007. Mind in Life: Biology, Phenomenology, and the Sciences of Mind. Cambridge, MA, London: Belknap/Harvard University Press.

Tomasello, M.2001. The Cultural Origins of Human Cognition. Reprint. Cambridge, MA, London: Harvard University Press.

-. 2009. Why We Cooperate. With Carol Dweck, Joan Silk, et al. A Boston Review Book. Cambridge, MA and London: MIT Press.

-. 2014. A Natural History of Human Thinking. Cambridge, MA, London: Harvard University Press.

Waldenfels, B. 2014. “Der Leib als Umschlagstelle von Natur und Kultur.” (Unpublished Lecture, Given at the University of Heidelberg, Dec. 4, 2014.)

Weissenrieder, A. 2016. "It Proceeded from the Entrance of a Demon into the Man". Epileptic Seizures in Ancient Medical Texts and the New Testament. In this volume.

-. 2016. Verkörperung des Inneren Menschen? 2. Korinther 4,16 im Lichte Antiker Medizinischer und Philosophischer Traditionen. In Verkörperung als Paradigma Theologischer Anthropologie, ed. G. Etzelmüller and A. Weissenrieder, 183-218. Theologische Bibliothek Töpelmann 172. Berlin, New York: de Gruyter.

Weissenrieder, A. and G. Etzelmüller. 2010. Christentum und Medizin: Welche Kopplungen sind lebensförderlich? In Religion und Krankheit, ed. G. Etzelmüller and A. Weissenrieder, 11-34. Darmstadt: Wissenschaftliche Buchgesellschaft.

Welker, M. 2013. Was ist ein 'Geistiger Leib'? In Interdisziplinäre Anthropologie: Leib, Geist, Kultur. ed. T. Breyer, G. Etzelmüller, et al., 65-83. Schriften des Marsilius-Kollegs 10. Heidelberg: Winter.

Wilckens, U. 1980. Der Brief an die Römer. Vol. 2: Röm 6-11. Evangelisch-Katholischer Kommentar. Zurich, Einsiedeln, and Cologne: Benziger.

Wilson, E. O. 2012. The Social Conquest of Earth. London, New York: Liveright/Norton. Wolff, H. W. 1975. Anthropology of the Old Testament. Trans. M. Kohl. Reprint. London: SCM and Minneapolis: Augsburg Fortress, 1975.

Wulf, C. 2004. Anthropologie: Geschichte, Kultur, Philosophie. Hamburg: Rowohlt. 



\title{
The Roots of Human Morals and Culture in Pre-Human Sympathy
}

\section{Charles Darwin's Natural and Cultural History of Morals}

\author{
Eve-Marie Engels
}

\begin{abstract}
The human being was a topic for Darwin's revolutionary theory of descent from the very beginning (1838). Darwin's evolutionary anthropology sought to explain not only our bodily structure but also the origin of our emotions, of conscience and of the moral sense. For Darwin "sympathy" is a fundamental element of the social instincts in animals as well as a basic element of our moral sense, one which links us to our nonhuman predecessors. At the same time Darwin adheres to the idea that morality is a uniquely human trait, not reducible to animal instincts; without traces of such sympathetic instincts a human being would be an "unnatural monster". In accordance with his general theory of descent Darwin emphasizes the continuity between humans and other animals as well as new traits unique to humans: self-consciousness, verbal language and morality. This distinguishes him from some of his current adherents.
\end{abstract}

\section{Introduction}

The aim of my contribution is to introduce Charles Darwin as the founder of an evolutionary anthropology, who has a particular interest in emphasizing man as a being capable of morals. In his natural history of the human being, as outlined in his work Descent of Man and further supported in The Expression of the Emotions in Man and Animals, Darwin links human evolution to that of other animals. In doing so he draws on the old principle of continuity, which has a long tradition. In the context of Darwin's theory, however, the static principle of continuity is turned into a dynamic one. Different species are linked together by their evolutionary history, and humans are an animal species that has descended from prehuman animals and embodies their phylogenetic experience by inheritance. Humans share certain traits with other extinct and living animals through descent. On the other hand each new species has particular features which allow it to be distinguished from other species. Darwin characterizes the human being as the only one which possesses moral faculties, a moral sense. This moral sense is a complex human trait with different elements, some of which have their roots in our animal history. However Darwin is not a reductionist; he does not claim that humans' moral sense can be reduced to animals' social instincts. 


\section{Darwin's Scientific and Philosophical Revolution}

With his theory of descent Darwin created a paradigmatic framework for understanding the evolution of organisms. ${ }^{1} \mathrm{He}$ offered an explanation of the origin of new species and of the functional character of organisms' traits, their adaptations, by invoking a unitary scientific principle or mechanism. The revolutionary character of his solution consists in having achieved this without drawing on religious or other metaphysical assumptions, such as a Creator God, an intelligent designer, or Aristotelian final causes and goal-directed forces immanent in nature. Darwin's goal was to "overthrow the dogma of separate creations" (Darwin [1877] 1989, 65) ${ }^{2}$, the idea that each single species has been created by God and the idea of the constancy of species. Species have neither been created separately nor are they immutable. Darwin wanted to provide evidence for the view that species come into being via the transformation of other species, and that this can be explained by natural laws. From the first edition of his Origin of Species in 1859 onwards Darwin declined to explain the origin of life as such and that of mental faculties: "I must premise, that I have nothing to do with the origin of the primary mental powers, any more than I have with that of life itself" (Darwin [1859] 1964, 207; see similarly Darwin's last edition [1876] 1988, 214). In his letter of April 18, 1863 to the Athenaum on some reviewers' reactions to the Origin's use of "Pentateuchal" terminology (he had written of "some one primordial form, into which life was first breathed", Darwin [1859] 1964, 484) Darwin admits that "in a purely scientific work I ought perhaps not to have used such terms; but they well serve to confess that our ignorance is as profound on the origin of life as on the origin of force or matter" (Darwin 1999, 324).

Darwin was not the first to conceive of the mutability of species. From the 4th American printing (1860) and more extensively from the 3rd English edition (1861) onwards he mentions in his introduction authors who anticipated the idea, among them Buffon, Darwin's grandfather Erasmus Darwin, and Lamarck. Although Darwin particularly emphasizes Lamarck, he also mentions some Lamarckian assumptions which he does not find convincing, such as his concept of progressive development and his idea of an ongoing spontaneous generation. However, with respect to inheritance he draws on Lamarck's theory of the inheritance of acquired characters, which was widespread at that time.

The starting point of Darwin's "theory of descent with modification, through variation and Natural Selection"” is the assumption that there is an analogy

${ }^{1}$ In my book Charles Darwin (Engels 2007), published in the philosophical series "Denker," I have explored in more detail the philosophical aspects of Darwin's theory of descent, his evolutionary anthropology and his moral philosophy. For Darwin's philosophical revolution see Engels 2008.

2 The first edition of Descent of Man was published in 1871, a second augmented and corrected edition in 1874. A revised and augmented edition of 1874 was published in 1877 . I am using the edition of 1877 (Darwin 1989), published in two volumes. When I refer to the second one, I will quote as "Darwin 1989, II."

3 This is the complete term of Darwin's theory which he uses in the 6th edition of Origin of Species (1876). 
between the origin of new breeds of plants and animals by domestication ("methodical selection," "artificial selection") and that of new species in free nature. Methodical or artificial selection is a main topic in Darwin's The Variation of Animals and Plants under Domestication ([1875] 1988), first published in 1868. Breeders select those individuals of a race which have certain traits or characters useful for the breeders' purpose, and let them propagate. In the course of many generations these traits (insofar as they can be inherited) gradually prevail or take the form intended by the breeder. “The key is man's power of accumulative selection: nature gives successive variations; man adds them up in certain directions useful to him. In this sense he may be said to make for himself useful breeds" (Darwin [1859] 1964, 30).

In free nature there is an analogous mechanism of selection. Here, however, the selected traits are useful for an organism itself in its environment. Darwin proceeds from the observation that two organisms of the same species are never completely identical. There are always differences between them, variations, however small they may be, and thus also differences in the organisms' adaptation to an environment. Those organisms of a species whose traits are better adapted to their environment have a higher chance of survival and thus on average bear more offspring than the others. This means that a natural selection of the better adapted takes place. Due to inheritance, those traits which are advantageous for survival can accumulate over generations and so increasingly change compared to the traits of the aboriginal stock. Over long periods of time, from individual variants hereditary varieties, subspecies and finally new species thereby evolve. Darwin advocates a gradualism and repeatedly draws on the principle of continuity of natural philosophy: "Natura non facit saltum" (Darwin [1859] 1964, 194, 206, 210, 243, 460, 471). However, this principle is no longer static; it becomes dynamic and it stands for a real relationship between species. Natural selection thus leads not only to the dying out of species but also the generation of new species. Species descend from other species, and from one species several others can evolve by adapting to different places in the "economy of nature” (Darwin [1859] 1964, 331, 470). The finches of the Galápagos Islands are a striking example of this "principle of divergence" (Darwin [1859] 1964, 112, 118). Today we call these places in the economy of nature ecologic niches.

In contrast to artificial selection there is no breeder in nature who purposively chooses organisms for propagation. How can selection be applied to organisms living in a state of nature? Since Darwin no longer drew on the Creator as the intelligent designer of species he had to discover some kind of a non-personal natural mechanism fulfilling the function of selection. Here Darwin leaves the analogy between artificial and natural selection. He names this natural mechanism the "struggle for life" or "struggle for existence" and draws upon Malthus' law of population. The political economist and clergyman Thomas Robert Malthus points to a disproportion between the arithmetical progression of the means of subsistence $(1,2,3,4,5,6)$ and the geometrical progression (when unchecked) of the human species $(1,2,4,8,16,32)$ (Malthus 1989, 1:15). Since human populations by and large exhibit stability, there must be a mechanism which limits this increase. 
Malthus assumes the constant effect of preventive checks (late marriage or restraint from marriage due to sexual abstinence or celibacy) and positive checks (common diseases and epidemics, wars, pestilence, plague, convulsions of nature, famine) (Malthus 1989, 1:16-18). He terms the preventive checks "moral restraint," meaning "a restraint from marriage from prudential motives, with a conduct strictly moral during the period of this restraint” (Malthus 1989, 1:18n4).

Reading Malthus' Essay on 28 September 1838, the idea struck Darwin of how to apply the concept of natural selection to free nature.

Hence, as more individuals are produced than can possibly survive, there must in every case be a struggle for existence, either one individual with another of the same species, or with the individuals of distinct species, or with the physical conditions of life. It is the doctrine of Malthus applied with manifold force to the whole animal and vegetable kingdoms; for in this case there can be no artificial increase of food, and no prudential restraint from marriage. (Darwin [1859] 1964, 63)

But a scarcity in resources is for Darwin only one of many causes of this often misunderstood "struggle for life." Even in situations of abundance of food there can be a struggle for life with differential outcomes for reproduction. Life may depend on skills such as better hiding, faster climbing and smarter behaviour. Cognitive, social, and moral faculties can be as important as bodily vigour in this struggle for life in which only the fittest survive. From the 5th edition of Origin of Species onwards Darwin adds to "natural selection" the term "survival of the fittest," coined by Herbert Spencer (1864), "fit" meaning adapted to fulfil the demands of a given situation (Spencer 1864, 1:444f.).

For Darwin there is no exception to Malthus' law and he already includes the human being in his calculation: "Even slow-breeding man has doubled in twenty-five years, and at this rate, in a few thousand years, there would literally not be standing room for his progeny" (Darwin [1859] 1964, 64). Particularly the moral or preventive checks described by Malthus play an important role in Darwin's later arguments in his Descent of Man in the context of his concerns for the future of mankind. Darwin rejected artificial birth control, a growing topic in the 19th century, fearing it would threaten chastity and thereby the basis of the family bond. The weakening of the family bond would be the "greatest of all possible evils to mankind," as he wrote in a letter to Charles Bradlaugh (Darwin 1877). ${ }^{4} \mathrm{He}$ was concerned about the loss of morality and of the social and institutional security the family provided (see Engels 2012).

From the outset, the metaphors of "natural selection" and "struggle for existence" were subject to much misunderstanding. They can have quite different

\footnotetext{
${ }^{4}$ I thank the editors of the Darwin Correspondence Project, University of Cambridge, for access to unpublished material from the correspondence between Charles Bradlaugh and Darwin. The quoted material is at a pre-publication stage, and the Project cannot be held responsible for any errors of transcription remaining. I also thank the copyright holder of this letter, Mr. William Huxley Darwin (London), for his permission to quote the letter. Finally I thank Dr. Paul White from the Darwin Correspondence Project for our helpful correspondence on the interpretation of this letter.
} 
meanings: intraspecific as well as interspecific competition, and the struggle of individuals or groups against environmental dangers (drought, coldness, wetness, etc.). Darwin moreover uses "the term Struggle for Existence in a large and metaphorical sense, including dependence of one being on another, and including (which is more important) not only the life of the individual, but success in leaving progeny" (Darwin [1859] 1964, 62). The phrase "struggle for life" has often been interpreted as a bloody or deadly fight between individuals, races or species. In a letter to William Preyer Darwin expresses apprehensions about the equivocality of the phrase "struggle for existence," suspecting "that the German term Kampf \&c. does not give quite the same idea.” (Darwin 2009, 161).

Depending on the situation, the struggle for existence can be coped with by competition or cooperation. The necessity of cooperation for survival and for the spread of social virtues is outlined extensively in Darwin's Descent of Man. The emphasis on mutual aid is a line of Darwin-reception which was pursued particularly in Russia (see Todes 1989). A prominent example is Petr Kropotkin's book Mutual Aid. A Factor of Evolution (1902). ${ }^{5}$

Already at the end of the first edition of Origin of Species Darwin alludes to the importance of his theory of descent for our understanding of the human being (Darwin [1859] 1964, 488): “In the distant future I see open fields for far more important researches. Psychology will be based on a new foundation, that of the necessary acquirement of each mental power and capacity by gradation. Light will be thrown on the origin of man and his history" (Darwin [1859] 1964, 488).

\section{Darwin's Descent of Man}

\subsection{Darwin's Early Notebooks}

Darwin's conviction, that humans and other animals are relatives can be traced back to his early Notebooks of 1837 (Barrett et al. 1987). Already in July 1837, twenty-two years before the publication of his Origin of Species, Darwin began writing a series of notebooks in which he treated the revolutionary subject of the "transmutation of species." A second series, which he started in the middle of 1838, was focused on philosophical subjects: "metaphysical enquiries." 6 These notebooks show that Darwin was already convinced of the idea of evolution as

${ }^{5}$ For an overview of the reception of Charles Darwin in European countries, see Engels and Glick (2008).

6 There are further notebooks on geological, ornithological and more subjects, but I will concentrate on the above mentioned two. The different notebooks have been transcribed and edited by Barrett et al. (1987), and I use their edition here. There are other, earlier transcriptions and editions of Darwin's notebooks by Gruber and Barrett (1974) and by Sir Gavin de Beer, M. J. Rowlands, and B. M. Skramowsky on the transmutation of species in Bulletin of the British Museum (Natural History) Historical Series Vol. 2, London 1969 und Historical Series Vol. 3 London 1971. 
opposed to the doctrine of separate creation and constancy of species. In 1842 he wrote a 35-page sketch of his theory and enlarged it into a 230-page essay in 1844. These manuscripts were published posthumously by Darwin's son Francis (F. Darwin [1909] 1986).

The scientific and metaphysical notebooks, which were not intended for publication, manifest in an impressive way the encyclopedic interests Darwin already had as a young man. When he set out on the voyage of the Beagle (1831-1836) he was only twenty-two years old, and when he began to write his notebooks, which reveal the germ of his revolutionary theory, he was twenty-eight. The Notebooks as well as Darwin's Marginalia (Di Gregorio 1990) present his comments on a wide range of literature. He reads, extracts, and comments on authors of geology, botany, zoology, other natural sciences, medicine, psychology, philosophy, theology, political economics, history and other fields. Darwin's Notebooks can be characterized as a workshop for his out-of-the-ordinary ideas, his thought experiments in exploring radical theoretical possibilities. In his Notebooks "we can, if we look carefully, almost catch his thought on the wing" (Gruber and Barrett 1974, xv).

In the following I will present a very short selection of Darwin's notes which concern his awareness of the radical nature of his ideas (e.g., C 76), his criticism of traditional philosophy (e.g., M 84e, M 128), his early views on the origin of species (e.g., B 101), his conception of the descent of humans from other animals (e.g., C $196 \mathrm{f}$.), his view of the difference between animal instincts and human reasoning powers (C 77-78), and other subjects: ${ }^{7}$

It is absurd to talk of one animal being higher than another.- We consider those, where the $\left\{\begin{array}{c}\text { cerebral structure } \\ \text { intellectual faculties }\end{array}\right\}$ most developed, as highest.-

A bee doubtless would when the instincts were.-

(Barrett et al. 1987, B 74, 189)

Astronomers might formerly have said that God ordered, each planet to move in its particular destiny.- In same manner God orders each animal created with certain form in certain country, but how much more simple, \& sublime power let attraction act according to certain laws such are inevitable consequen let animal be created, then by the fixed laws of generation, such will be their successors.-

(Barrett et al. 1987, B 101, 195)

If all men were dead then monkeys make men.- Men makes angels-

(Barrett et al. 1987, B 169, 213)

Animals- whom we have made our slaves we do not like to consider our equals.- «Do not slave holders wish to make the black man other kind? ...» Animals with affections, imitation, fear .... pain. sorrow for the dead.- respect

(Barrett et al. 1987, B 231, 228)

Once grant that «species» one genus may pass into each other.- grant that one instinct to be acquired (if the medullary point in ovum. has such organization as to ... force

${ }^{7}$ Darwin's orthographical and grammatical peculiarities and mistakes have been maintained. The brackets « » are Darwin’s insertions, « means Darwin’s deletion. 
in one man the developement of a brain capable of producing more glowing imagining or more profound reasoning than other- if this be granted!!) \& whole fabric totters \& falls.- look abroad, study gradation. study unity of type- Study geographical distribution [C 76] study relation of fossil with recent. the fabric falls! But Man ... is Mammalian.- his ... origin has not been indefinite- he is not a deity, his end «under present form» will come, (or how dredfully we are deceived) then he is no exception.he possesses some of the same general instincts, ... \& < moral > feelings as animals.- they on other hand can reason- but Man has reasoning powers in excess. instead of [C 77] definite instincts.- this is a replacements in mental machinery- so analogous to what we see in bodily. that ... it does not stagger me.- What circumstances may have been necessary to have made man! [C 78]

(Barrett et al. 1987, C 76-78, 263)

Reflect much over my view of particular instinct being memory transmitted without consciousness «a most possible thing. see men walking in sleep».- an action becomes habitual is probably first stage, $\&$ an habitual action implies want of consciousness $\&$ will \& therefore may be called instinctive.- But why do some actions become heredetary \& instinctive \& not others.-

(Barrett et al. 1987, C 171, 292)

Man in his arrogance thinks himself a great work. worthy the interposition of a deity, more humble \& I believe true to consider him created from animals.-

(Barrett et al. 1987, C 196f., 300)

If I be asked by what power the creator has added thought to ... so many animals of different types. I will confess my profound ignorance.- but seeing such passions acquired \& heredetary \& such definite thoughts, I will never allow that because there is a chasm between Man ... and animals that man has different origin.

(Barrett et al. 1987, C 222e-223, 310)

Origin of man now proved.- Metaphysic must flourish.- He who understands baboon ... would do more towards metaphysics than Locke.

(Barrett et al. 1987, M 84e, 539)

Plato ... says in Phaedo that our "necessary ideas" arise from the preexistence of the soul, are not derivable from experience.- ... read monkeys for preexistence.

(Barrett et al. 1987, M 128, 551)

The distinction «as often said» of language in man is very great from all animals- but do not overrate- animals communicate to each other. ... they likewise must understand each other expressions, sounds, \& signal movements.- some say dogs understand expression of man's face.- ... How far they communicate not easy to know,- but this capability of understanding language is considerable, thus carthorse \& dog.- birds many cries. monkeys communicate to each other.

(Barrett et al. 1987, M 96 f., 542f.)

I am tempted to say that those actions which have been found necessary for long generation, (as friendship to fellow animals in social animals) are those which are good \& consequently give pleasure ... Descent of Man Moral Sense (bold type in the original). $\left(\right.$ Barrett et al. 1987, M 132e, 552) ${ }^{8}$

8 The words in bold type (italic in the text) are Darwin's later annotation and refer to “Comparison of the Mental Powers of Man and the Lower Animals” ([1871] 1981, chap.3, 70-106). In the second edition (Darwin [1877] 1989), which I use in my article, this is the 4th chapter. 


\subsection{Darwin's Moral Science and Ethics}

Darwin waited twelve years, however, before publishing his Descent of Man (1871), as he thought that his book would only add to the prejudices against his views. But now "the case wears a wholly different aspect" (Darwin 1981, 3). What made the difference? Years before Darwin published his Descent of Man, other scientists and philosophers had applied his ideas to explain the origin of man. He mentions Huxley ([1863] 2009), C. Vogt (1863), Wallace (1864), Rolle (1866), ${ }^{9}$ Büchner (1868), Haeckel (1868) and others. So the public was prepared for the idea that the human beings had descended from animals. Nevertheless the idea contained much explosive force. Frances Power Cobbe wrote in her review of Darwin's Descent of Man: "Let me say it at once. These doctrines appear to me simply the most dangerous which have ever been set forth since the days of Mandeville" (Cobbe 1871, 175). I will come back to these doctrines later.

In his Descent of Man Darwin treats four issues. He considers "firstly, whether man, like every other species, is descended from some pre-existing form; secondly, the manner of his development; and thirdly, the value of the differences between the so-called races of man" (Darwin [1877] 1989, 4).

The fourth question is the most important for our context. It is the question of the origin of the "moral sense or conscience," because "as far as I know, no one has approached it exclusively from the side of Natural History. The investigation possesses, also, some independent interest, as an attempt to see how far the study of the lower animals throws light on one of the highest psychical faculties of man" (Darwin [1877] 1989, 102).

Darwin wants to investigate the scope of his theory of descent for understanding humans' moral sense. Is it possible to clarify man's capacity for morality, which we consider as specifically human, by drawing on the theory of descent? Can man's special position as a moral being be clarified within an evolutionary naturalism? As we shall see, Darwin does not reject traditional concepts of ethics and religion, but combines them with his naturalistic framework and thus brings evolutionary naturalism and ethical traditions together. As living beings humans are part of organic nature, but as self-conscious, reflecting beings they can also go beyond it.

Talking about ethics in the context of Darwin's work we thus have to consider two frameworks of reference. One framework is the evolutionary and cultural context of the description and explanation of humans as a species. How did our moral ability and attitudes evolve, and what were the conditions for their evolution? The other context is that of a normative ethics, of evaluation. What makes up human morals? Which moral values and norms should we follow? Which actions are virtuous? In the light of the implicit or explicit answers to these questions human action is judged.

\footnotetext{
${ }^{9}$ Rolle's preface is from Christmas 1865, the book was published in 1866 (see bibliography).
} 


\subsubsection{The Evolution of the Human Being, "The Most Dominant Animal"}

Before treating the evolutionary origin of the moral sense Darwin first has to provide evidence for his assumption that man has descended from animals. In the first chapter he makes use of three traditional criteria for showing the similarities between animals of different species of the same class or of different classes of the same phylum. These criteria had already been established by experts of various disciplines (embryology, morphology, brain anatomy, etc.) independently of a theory of descent. The three criteria are homologies, which is the resemblance of the "Bauplan" of different species of the same class or of different classes of the same phylum, the resemblance of embryos of different species founded in embryology, and rudiments, organs which have lost the function they previously had in phylogenetically earlier organisms. Darwin argues that the best explanation for these phenomena is a common descent of the organisms and their adaptation to different conditions of life. He then reconstructs the evolution of man.

An important step in the evolution of the human being is the evolution of upright posture (bipedy), with its consequences for other bodily changes, particularly the evolution of the brain with its specific human functions. The front limbs were released from their function of locomotion and could evolve into free usable human arms and hands with their specific relation between thumb and forefinger. The sense of touch was refined and allowed a more targeted use of objects. Darwin mentions the important function of the opposable thumb, typical of the great apes. It allows objects to be seized and worked on. Darwin also assumes a mutual influence between the evolution of articulate language, which for him has its origin in imitation, and the evolution of the brain. Here he particularly relies on Chauncey Wright (Wright 1870). But he is careful to avoid definite statements about the role of natural selection and other motors of change: "It is very difficult to decide how far these correlated modifications are the result of Natural Selection, and how far of the inherited effects of the increased use of certain parts, or of the action of one part on another" (Darwin [1877] 1989, 57).

Like all other animals, the early progenitors of humans must have tended to increase beyond their means of subsistence and thus must have been exposed to the struggle for life and to natural selection. "Beneficial variations of all kinds will thus, either occasionally or habitually, have been preserved, and injurious ones eliminated" (Darwin [1877] 1989, 52). Darwin refers to Alfred Russel Wallace and his "admirable Paper" of 1864, in which Wallace writes of a certain stage of human evolution when, along with changes of the brain (the "organ of the mind") and other organs, the "truly human faculties" such as human speech, social, moral and intellectual faculties had evolved and become permanent. At this point man "would be kept in harmony with the slowly changing universe around him, by an advance in mind, rather than by a change in body" (Wallace 1864, clxvi). Man could develop technologies to construct objects the way he needed them for getting along with the world, instead of having to adapt himself to the world. "Man in the rudest state in which he now exists is the most dominant animal that has ever appeared on this earth. He has spread more widely 
than any other highly organized form: and all others have yielded before him" (Darwin [1877] 1989, 52). Mankind owes this dominant position, his "immense superiority," to his corporeal and intellectual qualities, his self-consciousness, a verbal language as well as his social qualities and habits. The invention and use of various technologies such as weapons, tools, traps, the discovery of fire, the construction of rafts and boats gave him an enormous advantage over other animals.

Darwin then responds to the Duke of Argyll's objection, that humans' divergence "from the structure of brutes, in the direction of greater physical helplessness and weakness" cannot be explained by natural selection. How could these human deficiencies have a selective advantage (Darwin [1877] 1989, 67)? How could a "Mängelwesen," as humans are later described in Arnold Gehlen's (2004) philosophical anthropology, evolve at all by natural selection? Darwin replies that we do not know from which species, strong or weak, man is descended, but - and he turns the table - "it might have been an immense advantage to man to have sprung from some comparatively weak creature." Precisely because of our vulnerability we might have become social and might have acquired our higher mental and social qualities (Darwin [1877] 1989, $67 \mathrm{f}$.).

Above all, humans had a selective advantage due to their cognitive flexibility in connection with language. In contrast to other animals, humans do not have to change their body in order to be able to adapt to their environment, but they can change their environment and submit it to their needs. Whereas animals may slowly adapt, bodily and behaviorally, to the challenges of a changing world, human culture (including technology) allows us to make far more rapid adaptations of nature to our wants and needs.

The lower animals, on the other hand, must have their bodily structure modified in order to survive under greatly changed conditions. They must be rendered stronger, or acquire more effective teeth or claws, for defence against new enemies; or they must be reduced in size, so as to escape detection and danger. When they migrate into a colder climate, they must become clothed with thicker fur, or have their constitutions altered. If they fail to be thus modified, they will cease to exist. (Darwin [1877] 1989, 132)

There is a dark side to human advantage and our ability to adapt nature, however. Today's climate change and environmental catastrophes manifest in a dramatic way our species' limits. Humans may become victims of their own exploitation of nature.

\subsubsection{Mental Faculties in Animals and Their Instinct of Sympathy}

Darwin's work was provocative for several reasons. Most controversially, man's appearance on earth can be explained by blind mechanisms of evolution without having to assume God's creative power, wisdom and goodness. ${ }^{10}$ Man's existence is contingent, "for he owes his birth to a long line of progenitors. If any single link in this chain had never existed, man would not have been exactly

10 This was the research program of Natural Theology (Gundry 1946); see also Richards 1981 and 1987. 
what he now is" (Darwin [1877] 1989, 171). A second source of provocation which seemed no less threatening to many of Darwin's contemporaries was the assumption that mankind had apelike progenitors. For Darwin, there was even no "fundamental difference" between man and the other animals with respect to their “mental faculties" or "mental powers" (Darwin [1877] 1989, 69f.). "We must also admit that there is a much wider interval in mental power between one of the lowest fishes, as a lamprey or lancelet, and one of the higher apes, than between an ape and man; yet this interval is filled up with numberless gradations" (Darwin [1877] 1989, 70).

But no matter how large the difference between humans and other animals may be, it is certainly "one of degree and not of kind" (Darwin [1877] 1989, 130). Darwin firmly assigns mankind a place in the animal kingdom (Darwin [1877] 1989, 152). At the end of Descent of Man he repeats the observations of the first chapters, that - irrespective of all noble qualities - "Man still bears in his bodily frame the indelible stamp of his lowly origin” (Darwin [1877] 1989, II, 644).

Humans and other animals share not only bodily features but also certain instincts as well as mental faculties, i. e., emotional and intellectual faculties. Darwin mentions the instincts of "self-preservation, sexual love, the love of the mother for her new-born offspring, the desire possessed by the latter to suck, and so forth. But man, perhaps, has somewhat fewer instincts than those possessed by the animals which come next to him in the series" (Darwin [1877] 1989, 70).

His representation of the mental faculties of animals provides an excellent up-to-date overview of contemporary comparative ethology in the 19th century. His knowledge is based on his observations and experience with his own animals and children, to a greater extent on the international literature of renowned scientists and experts of a wide range of different fields as well as on the personal experience of his correspondents. He makes full reference to Alfred Brehm's well known Thierleben (Brehm 1864) as well as to Johann Rudolph Rengger's Naturgeschichte der Sängethiere von Paraguay (Rengger 1830). Even today, Darwin's descriptions of the mental powers of animals and humans have not lost their relevance. He gives a long list of feelings: pleasure and pain, happiness and misery, fear, suspicion, revenge, jealousy, shame, love of approbation or praise, magnanimity, rage, sense of humor, love of a dog for his master, maternal affection (Darwin [1877] 1989, 73). In animals there are also "more intellectual emotions and faculties, which are very important, as forming the basis for the development of the higher mental powers." These are wonder, curiosity, imitation, attention, memory, imagination, deliberation, choice, association of ideas, "reason, though in very different degrees," sagacity, caution, cunning, tool use (Darwin [1877] 1989, 75-84).

"That animals sometimes are far from feeling any sympathy is too certain; for they will expel a wounded animal from the herd, or gore or worry it to death" (Darwin [1877] 1989, 106). Yet Darwin's picture of animals is basically positive in comparison with that of Wallace and Huxley (see Engels 2015). Many animals feel sympathy for animals of the same species as well as for those of other spe- 
cies. Examples are adoption and helpful behavior among animals, which occur even across species: dogs are defended by Rhesus monkeys, for instance, when attacked by other dogs; each warns and helps the other.

Many animals, however, certainly sympathize with each other's distress or danger. This is the case even with birds. Captain Stansbury ... found on a salt lake in Utah an old and completely blind pelican, which was very fat, and must have been well fed for a long time by his companions. Mr. Blyth, as he informs me, saw Indian crows feeding two or three of their companions which were blind; and I have heard of an analogous case with the domestic cock. We may, if we choose, call these actions instinctive; but such cases are much too rare for the development of any special instinct. ... I have myself seen a dog, who never passed a cat who lay sick in a basket, and was a great friend of his, without giving her a few licks with his tongue, the surest sign of kind feeling in a dog. (Darwin [1877] 1989, 106f.)

Because man has descended from non-human beings who were already equipped with social instincts, we do not come into this world as tabula rasa, but rather with an evolutionary heritage of social dispositions. We know today that even infants aged only 14 to 18 months try in surprisingly manifold ways to help an adult who needs support in solving a problem, even if he or she is not their relative. It seems that such behavior is a natural disposition (see Tomasello 2009). The great importance attached by Darwin to emotions in humans and animals manifests itself in the fact that he dedicated a whole book to the subject, The Expression of the Emotions in Man and Animals (Darwin [1872] 1998). With this book Darwin pursues two goals. Firstly, he wants to provide evidence for the unity of mankind by showing human universals across different cultures and races. Secondly, he wants to support his view that humans have descended from other animals. The primatologist Frans de Waal has endorsed Darwin's ideas about the roots of human empathy in prehuman animals by showing that also some species of our primate relatives, like capuchin monkeys and the great apes, have a sense of fairness. Some can have an emotional and even a cognitive empathy for others beyond their own species (de Waal 2006).

We humans have mental faculties because we have inherited them from our predecessors, from other animals. We have inherited not only instinctive impulses from animals but also intellectual faculties. In the quotation Darwin attributes intelligence to birds, which are flexible enough to perform actions which are appropriate for specific situations, so that this cannot merely be fixed, inherited instinctive behavior (see Notebook C $77 \mathrm{f}$., above). Nevertheless humans are the only living being capable of morals. This peculiarity expresses itself in another, specifically human trait, in blushing. No animal has this capacity, a human universal that occurs in the "various races of man." Darwin dedicates a whole chapter, chapter 13 of his Expression of the Emotions in Man and Animals to this human peculiarity. The cause of blushing in the context of morals is the thought that others think that we are guilty or know that we are guilty (Darwin [1872] $1998,331)$. So the ability to blush presupposes the capacity for reflection. Other reasons for blushing are violations of etiquette and modesty. Today's ethology and studies of animal intelligence show more and more the broad range of cogni- 
tive, emotional and social competence in nonhuman animals, from which serious questions of animal ethics arise, calling our attitude towards animals into question (Benz-Schwarzburg and Knight 2011; Benz-Schwarzburg 2012).

\subsubsection{Sympathy as "Foundation-Stone" of Social Instincts}

The point of departure for Darwin's reflections on human morals is his assumption that primitive man, the early, human progenitors of civilized man, possessed well-developed social instincts like those already to be found in many animals, including the "ape-like progenitors" of primitive and modern man (Darwin [1877] 1989, 113 f.). Because our progenitors descended from non-human beings who were already invested with social instincts, they did not come into this world as tabula rasa, but rather with an evolutionary heritage of social instincts. An important element of such social instincts is sympathy for members of the same community or tribe. Darwin explains the emergence of these instincts in terms of his theory of natural selection, ascribing to them a function necessary for preserving the community. Social instincts include parental and filial affections, sociability, pleasure in one's fellows, faithfulness, willingness to help, etc. (Darwin [1877] 1989, 102f.).

For Darwin, sympathy "forms an essential part of the social instinct, and is indeed its foundation-stone" (Darwin [1877] 1989, 103). The "instinct of sympathy" is the root of our "moral sense or conscience" because our moral sense, like the instinct of sympathy, is directed towards the good of the community, not egoistically towards our own happiness (Darwin [1877] 1989, 137). The radius of social instincts originally only extended to the members of the same community or tribe, not to all members of the species. Initially, man was not interested in preserving the species but in preserving his own community and tribe. Cooperation among members of the same community and tribe ensured survival in confrontation with nature and foreign groups, thus becoming a strategy in the struggle for existence.

However, compared to our early apelike and human progenitors, our instincts are reduced in several ways, in their number, their specialization and their strength. The condition for the development of genuine morals is this reduction of instincts along with the evolution of reason, judgment and language, with "free intelligence" and "free will" (Darwin [1877] 1989, 71f.). Nevertheless the social instincts still give the impulse to our social and moral actions. However, they have to be oriented by reason.

Thus although the "first foundation or origin of the moral sense lies in the social instincts, including sympathy" (Darwin [1877] 1989, II, 637) and these constitute the roots of our "moral sense," they do not alone suffice to explain the phenomenon of morality. According to Darwin, genuine morality consists in the "moral sense or conscience," in a "sense of right and wrong," this being something only man possesses. Sometimes Darwin uses "conscience” as equivalent to "moral sense"; sometimes he makes a finer distinction between them. The moral sense "tells us what we ought to do" while the conscience "reproves us if we 
disobey it" (Darwin [1877] 1989, 121). The moral sense is our interior lawgiver, conscience our interior sanctioning authority.

\subsubsection{Man, the Animal Capable of Morals}

The evolution of the human intellectual capacities for self-consciousness and verbal language is for Darwin not only important for humans' spreading over the earth, but is also a necessary condition for the possibility of a moral sense. Thus only the conduct of humans can be called "moral."

A moral being is one who is capable of comparing his past and future actions or motives, and of approving or disapproving of them. We have no reason to suppose that any of the lower animals have this capacity; therefore, when a Newfoundland dog drags a child out of water, or a monkey faces danger to rescue its comrade, or takes charge of an orphan monkey, we do not call its conduct moral. But in the case of man, who alone can with certainty be ranked as a moral being, actions of a certain class are called moral, whether performed deliberately, after a struggle with opposing motives, or impulsively through instinct, or from the effects of slowly-gained habit. (Darwin [1877] 1989, 115f.).

Even if animals manifest behavior which in us we would call moral, it cannot count as moral according to this criterion. On the other hand, humans' actions do not cease to be moral when done without deliberation or hesitation. The power of moral judgment makes the all-important difference (Darwin [1877] $1989,115 \mathrm{f}$.). It presupposes the power of reasoning, self-consciousness, a verbal language and the understanding and formulation of moral imperatives, like Kant's (Darwin [1877] 1989, 113 f.).

At the same time, according to Darwin we do not value moral conduct only because of the actor's self-consciousness, but also for the sympathy involved in such action. "A man who possessed no trace of such instincts would be an unnatural monster” (Darwin [1877] 1989, 116). Thus Darwin describes human morality as a complex phenomenon, involving quite different capabilities, reason as well as emotions, particularly sympathy, and virtues.

Darwin follows up on the English-Scottish tradition of the moral sense (see Hume [1777] 1992; Smith 2000; Bain 1868) but goes beyond it in his biological understanding of the human being as well as in his understanding of the moral sense and morals. Hume wrote in his Enquiry Concerning the Principles of Morals:

It is needless to push our researches so far as to ask, why we have humanity or a fellow-feeling with others. It is sufficient, that this is experienced to be a principle in human nature. We must stop somewhere in our examination of causes; and there are, in every science, some general principles, beyond which we cannot hope to find any principle more general. No man is absolutely indifferent to the happiness and misery of others. The first has a natural tendency to give pleasure; the second pain. This every one may find in himself. It is not probable, that these principles can be resolved into principles more simple and universal, whatever attempts may have been made to that purpose. But if it were possible, it belongs not to the present subject; and we may here safely consider these principles as original: ... (Hume [1777] 1992, 219f.n1; italics added) 
Hume's remark reflects the scientific knowledge of his time, before Darwin's theory of descent was formulated, but it also shows that Hume avoids a theological-metaphysical explanation. As was revealed in Hume's posthumously published Dialogues concerning Natural Religion ([1779] 1993), one of the repudiations of Natural Theology's argument from design is found in the notion of self-organizational powers in nature. In entertaining such an idea, Hume was ahead of his time. Darwin's grandfather Erasmus Darwin, who already held evolutionary ideas, quotes Hume in his Zoonomia; or the Laws of Organic Life (E. Darwin 1794, 1:509).

Charles Darwin extends their view with an evolutionary perspective which includes the experience of the evolving human species from prehuman animals and semi-human progenitors. Humans embody the experience of these progenitors through inheritance over evolutionary timescales. Darwin claims that his own approach has greater explanatory power than that of Smith and Bain, because he can account for phenomena which otherwise would remain puzzling. Smith writes in the first chapter of his Theory of Moral Sentiments (1759), entitled "Of Sympathy," that "it is by changing places in fancy with the sufferer, that we come either to conceive or to be affected by what he feels" (Smith 2000, 4). Similarly, for Alexander Bain "SyмPATHy is to enter into the feelings of another, and to act them out, as if they were our own" (Bain 1868, 276). Bain believes that the basis of our sympathy for the suffering of others lies in our strong proclivity to retain our own former states of pain and pleasure. "The sight of another person enduring hunger, cold, fatigue, revives in us some recollection of these states, which are painful even in idea" (Bain 1868, 279). The same holds for pleasurable experiences. Thus, "Sympathy is, indirectly, a source of pleasure to the sympathizer” (Bain 1868, 281). Darwin, however,

cannot see how this view explains the fact that sympathy is excited, in an immeasurably stronger degree, by a beloved, than by an indifferent person. The mere sight of suffering, independently of love, would suffice to call up in us vivid recollections and associations. The explanation may lie in the fact that, with all animals, sympathy is directed solely towards the members of the same community, and therefore towards known, and more or less beloved members, but not to all the individuals of the same species. (Darwin [1877] 1989, 110)

This is not meant as a plea for graduated sympathy and helpfulness but as a description of animals' and humans' natural disposition. Darwin's idea of moral progress in the individual as well as in human history is that of an expanding circle. He thinks that sympathy has been increased through natural selection, "for those communities, which included the greatest number of the most sympathetic members, would flourish best, and rear the greatest number of offspring” (Darwin [1877] 1989, 111).

The close link between humans and animals does not only manifest itself in Darwin's idea that humans have inherited the faculty of sympathy from their non-human animal ancestors, but also in Darwin's taking the line of argumentation in the other direction by imagining what would happen to animals' faculties if their intellectual powers progressed. It seems to him "in a high degree 
probable - namely, that any animal whatever, endowed with well-marked social instincts ... the parental and filial affections being here included, would inevitably acquire a moral sense or conscience, as soon as its intellectual powers had become as well, or nearly as well developed, as in man” (Darwin [1877] 1989, 102; italics added).

Already now "besides love and sympathy, animals, exhibit other qualities connected with the social instincts, which in us would be called moral" (Darwin [1877] 1989, 107; italics added). Darwin refers to the power of self-command in dogs, faithfulness among animals, obedience, etc.

For Darwin the "moral sense," in the strict sense of humans' power of moral judgments, presupposes self-consciousness, a verbal language, by which not only public opinion but also general moral and ethical rules can be articulated, and the capacity to base one's judgment on moral imperatives like Kant's:

I fully subscribe to the judgement of those writers ${ }^{1}$ who maintain that of all the differences between man and the lower animals, the moral sense or conscience is by far the most important. This sense, as Mackintosh ${ }^{2}$ remarks, "has a rightful supremacy over every other principle of human action"; it is summed up in that short but imperious word ought, so full of high significance. It is the most noble of all the attributes of man, leading him without a moment's hesitation to risk his life for that of a fellow-creature; or after due deliberation, impelled simply by the deep feeling of right or duty, to sacrifice it in some great cause. Immanuel Kant exclaims, "Duty! Wondrous thought, that workest neither by fond insinuation, flattery, nor by any threat, but merely by holding up thy naked law in the soul, and so extorting for thyself always reverence, if not always obedience; before whom all appetites are dumb, however secretly they rebel; whence thy original?"3 (Darwin [1877] 1989, $101 \mathrm{f}$.) ${ }^{11}$

Darwin tries to mediate different ethical traditions which emphasize specific aspects of the human being and of morality. These are elements of the theory of the moral sense (Hume, Smith), of virtue ethics and of an ethics of duty (Kant). Our social virtues are an expression of our moral sense and, following Darwin's line of thought, I propose to describe them as the refined and enlarged "offspring” of animals' and early humans' social instincts. Humans are not made from one piece. Our complex brain with its different parts has evolved over millions of years and embodies the phylogenetic past of other animals. Since humans are "created from animals," or less metaphorically, since we are descendants of other animals, but are special animals, we embody instinctive, emotional, and rational elements. This has to be mirrored in ethics. And we have to look at other animals in order to learn something about ourselves, because children resemble their parents. Darwin doubts that the moral sense is a faculty which develops only during individual ontogeny and rejects John Stuart Mill's and Bain's position:

Mr. J. S. Mill ... remarks, "if, as is my own belief, the moral feelings are not innate, but acquired, they are not for that reason less natural.” It is with hesitation that I venture to differ at all from so profound a thinker, but it can hardly be disputed that the social

${ }^{11}$ In these three footnotes he refers to Quatrefages, Mackintosh, and Kant. 
feelings are instinctive or innate in the lower animals; and why should they not be so in man? Mr. Bain (see, for instance, The Emotions and the Will, 1865, p. 481) and others believe that the moral sense is acquired by each individual during his lifetime. On the general theory of evolution this is at least extremely improbable. The ignoring of all transmitted mental qualities will, as it seems to me, be hereafter judged as a most serious blemish in the works of Mr. Mill. (Darwin [1877] 1989, 102n3)

Darwin's statements about the moral sense give rise to questions and misunderstanding. In the quotation he talks about "moral feelings," "social feelings" and the "moral sense." On the one hand he uses the term "moral sense" in the strict meaning of humans' competence in moral judgments, which presuppose self-consciousness, verbal language and the capacity to base one's judgment on moral imperatives like Kant's. The moral sense is a specific capacity of humans that other animals do not have. On the other hand, his footnote on Mill and Bain indicates that he also uses the term in the sense of "social feelings" which are "instinctive or innate in the lower animals." And Darwin wonders why these feelings should not be so in man. Although he assumes that human instincts are reduced with respect to their number, degree of specialization and strength (Darwin [1877] 1989, 113, [1877] 1989, II, 636), he sometimes talks of instincts where he can only mean instinctive impulses. This is linguistic carelessness, even stronger in the first edition, which is only partially corrected in the second edition and by which Darwin has invited the objection that he has no uniform notion of sympathy but uses it sometimes in the tradition of Hume and Smith, sometimes in the sense of "instinct." With this double notion of sympathy, so the objection further runs, Darwin is presupposing measures of ethical value which are not deducible from evolution as such (M. Vogt 1997, $130 \mathrm{f}$.).

However, Darwin judges certain natural phenomena such as sympatby in the light of ethical measures of value, which he can neither deduce from evolution nor claims to do so. Thus we can defend Darwin's project as coherent: he does not operate with two notions of sympathy but traces one element of our moral sense - sympathy - back to its evolutionary past, to trace its roots in the social instincts of animals. This is in fact compatible with his concept of moral sense as a human capacity, which is more differentiated than animal instincts but connected with them through natural history. Moreover, when reading Darwin we have to keep in mind the stage of human evolution to which Darwin refers. In our early human predecessors sympathy was more strongly instinctive than later and today. And finally it would be worthwhile investigating more closely Hume's and Smith's meaning of sympathy. Hume himself uses the notion of instinct in connection with "sympathy": "The social virtues of humanity and benevolence exert their influence immediately by a direct tendency or instinct ... A parent flies to the relief of his child; transported by that natural sympathy which actuates him, and which affords no leisure to reflect on the sentiments or conduct of the rest of mankind in like circumstances" (Hume [1777] 1992, 303). 


\section{Morals, a Phenomenon of Cultural History with Roots in Natural History}

Darwin's fourth question is the question of the origin of the "moral sense or conscience," because "no one has approached it exclusively from the side of natural history." Darwin's “investigation possesses, also, some independent interest, as an attempt to see how far the study of the lower animals throws light on one of the highest psychical faculties of man" (Darwin [1877] 1989, 102).

This is no rhetorical question, but a truly scientific and philosophical program. Darwin's answer is as complex as the way leading to it (Engels 2011).

Humans' moral faculties are rooted in the natural history of man, in the social instincts as well as in the mental abilities of prehuman animals. But these roots are not sufficient to explain and describe our moral sense or conscience. In the course of human evolution, starting from animal predecessors through semi-human progenitors to modern humans, we have inherited instincts and, later, instinctive impulses, directed towards the wellbeing of others, which are the necessary condition for the formation of our moral sense. "A man who possessed no trace of such instincts would be an unnatural monster" (Darwin [1877] 1989, 116). Instincts, mental abilities and verbal language as conditions for human morals evolved via the general mechanisms of evolution but were already from the early evolution of mankind onwards intertwined with habits and cultural factors, a process which today is called the "coevolution" of nature and culture. Since Darwin, like many of his contemporaries, advocated the Lamarckian theory of the inheritance of acquired characters, habits, practiced over a long period of time, could become traits of organisms. In the course of time, morals became increasingly a cultural phenomenon, albeit with roots in natural history. Our "sympathies" became "more tender and more widely diffused" (Darwin [1877] 1989, 127). Darwin describes moral progress as an expanding circle of sympathy, of humanity. A tribe, whose members supported each other, "would succeed better and conquer the other ... Selfish and contentious people will not cohere, and without coherence nothing can be affected. A tribe rich in the above qualities would spread and be victorious over other tribes" (Darwin [1877] 1989, 135). This kind of group selection, happening repeatedly in the course of history, goes hand in hand with moral progress: "thus the social and moral qualities would tend slowly to advance and be diffused throughout the world" (Darwin [1877] 1989, 135). Not only the number of virtuous individuals thereby increased, but also the quality of sympathy, the standard of morality and the circle of beings who become beneficiaries of our moral and social virtues. Moral progress manifests itself in caring for the "imbecile," "maimed," and finally for animals (Darwin [1877] 1989, 129). For Darwin, "disinterested love for all living creatures" is "the most noble attitude of man" (Darwin [1877] 1989, 130).

Although Darwin revolutionized our view of life by offering a scientific explanation of the origin of species, and although he was an agnostic, religion played an important role in the cultural progress of humanity. 
To do good in return for evil, to love your enemy, is a height of morality to which it may be doubted whether the social instincts would, by themselves, have ever led us. It is necessary that these instincts, together with sympathy, should have been highly cultivated and extended by the aid of reason, instruction, and the love or fear of God, before any such golden rule would ever be thought of and obeyed. (Darwin [1877] 1989, 117)

Darwin's outlook on "future generations" is optimistic, because “we may expect that virtuous habits will grow stronger, becoming perhaps fixed by inheritance. In this case the struggle between our higher and lower impulses will be less severe, and virtue will be triumphant." (Darwin [1877] 1989, 129f.)

Important as the struggle for existence has been and even still is, yet as far as the highest part of man's nature is concerned there are other agencies more important. For the moral qualities are advanced, either directly or indirectly, much more through the effects of habit, the reasoning powers, instruction, religion, etc., than through Natural Selection; though to this latter agency may be safely attributed the social instincts, which afforded the basis for the development of the moral sense. (Darwin [1877] 1989, II, 643)

So the answer to Darwin's question, whether our moral sense can be adequately approached exclusively from the side of natural history, is negative. Morals are a phenomenon of cultural history with roots in natural history.

\section{Bibliography}

Bain, A. 1868. Mental and Moral Science. A Compendium of Psychology and Ethics. London: Longmans, Green, and Co.

Barrett, P. H., P. J. Gautrey, S. Herbert, D. Kohn, and S. Smith, eds. 1987. Charles Darwin's Notebooks, 1836-1844. Cambridge: Cambridge University Press.

Benz-Schwarzburg, J. 2012. Verwandte im Geiste - Fremde im Recht. Sozio-kognitive Fähigkeiten bei Tieren und ibre Relevanz für Tierethik und Tierschutz. Erlangen: Harald Fischer Verlag.

Benz-Schwarzburg, J. and A. Knight. 2011. Cognitive Relatives yet Moral Strangers? Journal of Animal Ethics 1 (1): 9-36.

Brehm, A. E. 1864-1867. Illustriertes Thierleben. 4 Vols. Hilburghausen: Verlag des Bibliographischen Instituts.

Büchner, L. 1868. Sechs Vorlesungen über die Darwin'sche Theorie von der Verwandlung der Arten. Leipzig: Theodor Thomas.

Cobbe, F. P. 1871. Darwinism in Morals. The Theological Review 33: 167-192.

Darwin, C. (1859) 1964. On the Origin of Species. Cambridge, MA: Harvard University Press.

-. (1876) 1988. The Origin of Species. In The Works of Charles Darwin, vol.16, ed. P. H. Barrett and R. B. Freeman. London: William Pickering.

-. (1871) 1981. The Descent of Man, and Selection to Sex. Facsimile of the $1^{\text {st }}$ ed. 1871. Princeton, NJ: Princeton University Press.

-. (1877) 1989. The Descent of Man, and Selection in Relation to Sex. Two Parts. In The Works of Charles Darwin, vols. 21 and 22 (Part one and two), $2^{\text {nd }}$ ed. rev. and aug., ed. P. H. Barrett and R. B. Freeman. London: William Pickering.

-. (1872) 1998. The Expression of the Emotions in Man and Animals. Introduction, Afterword and Commentaries by P. Ekman. London: HarperCollins Publishers.

-. (1875) 1988. The Variation of Animals and Plants under Domestication. $2^{\text {nd }}$ ed. rev. Vols. 19, 20, ed. P. H. Barrett and R. B. Freeman. London: William Pickering. 
-. 1877. To Charles Bradlaugh, 6 June 1877. In The Correspondence of Charles Darwin, ed. Darwin Correspondence Project, University of Cambridge (not yet published).

-. (1999). To Athenæum 18 April [1863]. In The Correspondence of Charles Darwin, vol.11, ed. F. Burkhardt, D. Porter, S. A. Dean, J. R. Topham, and S. Wilmot, 324-326. Cambridge: Cambridge University Press.

-. (2009). To William Preyer 29 March. [1869]. In The Correspondence of Charles Darwin, vol. 17, ed. F. Burkhardt, J. A. Secord, S. A. Dean, S. Evans, S. Innes, A. M. Pearn, and P. White, 161-162. Cambridge: Cambridge University Press.

Darwin, E. 1794-1796. Zoonomia: Or, The Lawes of Organic Life. 2 vols. London: Printed for J. Johnson in St. Paul's Church-Yard.

Darwin, F. (1909) 1986. The Foundations of the Origin of Species. Two Essays Written in 1842 and 1844. In The Works of Charles Darwin, vol.10, ed. P. H. Barrett and R. B. Freeman. London: William Pickering.

De Waal, F. 2006. Primates and Philosophers. How Morality Evolved. Princeton, NJ: Princeton University Press.

Di Gregorio, M. A. 1990. Charles Darwin's Marginalia. Vol. I. With the assistance of N. W. Gill. New York: Garland Publishing.

Engels, E.-M. 2007. Charles Darwin. München: Beck.

-. 2008. Darwin's Philosophical Revolution: Evolutionary Naturalism and First Reactions to his Theory. In The Reception of Charles Darwin in Europe. Vol. 1, ed. E.-M. Engels and T. F. Glick, 23-53. London: Continuum.

-. 2011. Der Mensch, das moralfähige Tier - Zur Anthropologie und Ethik von Charles Darwin. In Charles Darwin und seine Bedeutung für die Wissenschaften, ed. E.-M. Engels, O. Betz, H.-R. Köhler, and T. Potthast, 145-179. Tübingen: Attempto.

-. 2012. Darwin's Care for Humanity. In Sex, Reproduction and Darwinism, ed. F. de Souza, and G. Munévar, 173-194; 246-251. London: Pickering \& Chatto.

-. 2015. Darwin/Darwinismus. In Lexikon der Mensch-Tier-Beziebungen, ed. A. Ferrari and K. Petrus, 69-73. Bielefeld: Transcript.

Engels, E.-M. and T.F. Glick, eds. 2008. The Reception of Charles Darwin in Europe. 2 Vols. London: Continuum.

Gehlen, A. 2004. Der Mensch. Seine Natur und seine Stellung in der Welt. 14th ed. Wiebelsheim: Aula.

Gruber, H. E. and P. H. Barrett. 1974. Darwin on Man. London: Wildwood House.

Gundry, D. W. 1946. The Bridgewater Treatises and Their Authors. History. The Journal of the Historical Association 31: 140-152.

Haeckel, E. 1868. Natürliche Schöpfungsgeschichte. Berlin: Georg Reimer.

Hume, D. (1777) 1992. Enquiries Concerning Human Understanding and Concerning the Principles of Morals. Ed. L. A. Selby-Bigge. $3^{\text {rd }}$ ed. Oxford: Clarendon Press.

-. (1779) 1993. Dialogues Concerning Natural Religion and The Natural History of Religion. Ed. J. C. A. Gaskin. Oxford: Oxford University Press.

Huxley, T. H. (1863) 2009. Evidence as to Man's Place in Nature. Cambridge: Cambridge University Press.

Kropotkin, P. 1902. Mutual Aid. A Factor of Evolution. London: W. Heinemann.

Malthus, T. R. 1989. An Essay on the Principle of Population. 2 vols. The version published in 1803, with the variora of 1806, 1807, 1817 and 1826. Ed. P. James. Cambridge: Cambridge University Press.

Rengger, J. R. 1830. Naturgeschichte der Sängetiere von Paraguay. Basel: Schweighauser.

Richards, R. 1981. Instinct and Intelligence in British Natural Theology. Some Contributions to Darwin's Theory of the Evolution of Behavior. Journal of the History of Biology 14: 193-230.

-. 1987. Darwin and the Emergence of Evolutionary Theories of Mind and Behavior. Chicago: The University of Chicago Press. 
Rolle, F. 1866. Der Mensch, seine Abstammung und Gesittung im Lichte der Darwin'schen Lebre von der Art-Entstehung und auf Grundlage der newern geologischen Entdeckungen dargestellt. Frankfurt/Main: Joh. Christ. Hermann'sche Verlagsbuchhandlung.

Smith, A. 2000. The Theory of Moral Sentiments. New York: Prometheus Books.

Spencer, H. 1864. The Principles of Biology. 2 vols. London: Williams and Norgate.

Todes, D. 1989. Darwin without Malthus. The Struggle for Existence in Darwin's Evolutionary Thought. Oxford: Oxford University Press.

Tomasello, M. 2009. Why We Cooperate. Cambridge, MA: The MIT Press.

Vogt, C. 1863. Vorlesungen über den Menschen seine Stellung in der Schöpfung und in der Geschichte der Erde. Gießen: Ricker'sche Buchhandlung.

Vogt, M. 1997. Sozialdarwinismus. Wissenschaftstheorie, politische und theologisch-ethische Aspekte der Evolutionstheorie. Freiburg: Herder.

Wallace, A. R. 1864. The Origin of Human Races and the Antiquity of Man Deduced from the Theory of "Natural Selection." Journal of the Anthropological Society of London 2: clviii-clxx.

Wright, C. 1870. Review of "Contributions to the Theory of Natural Selection. A Series of Essays.” By Alfred Russel Wallace. The North American Review 111: 282-311. 



\title{
The Creation of Body Knowledge in Mimetic Processes
}

\author{
Christoph Wulf
}

Abstract: To a great extent, cultural learning is mimetic learning, which is at the center of many processes of education, self-education, and human development. It is directed towards other people, social communities and cultural heritages and ensures that they are kept alive. Mimetic learning is a sensory, body-based form of learning in which images, schemas and movements needed to perform actions are learned. This embodiment is responsible for lasting effects that play an important role in all social and cultural fields. Mimetic processes create both similarities to, and differences from, the situations or persons to which or whom they relate. By participating in the living practices of other people, humans expand their own life-worlds and create for themselves new ways of experiencing and acting. Receptivity and activity overlap. In all areas of human existence rituals and gestures are important for the mimetic development of body knowledge. Embodied knowledge is indispensable to religion, politics, economy, science, families, and education. It helps us deal with difference and alterity and create a sense of community and social relationships. It also enables us to assign meaning and structure to human relations. Ritual knowledge facilitates both continuity and change, as well as experiences of transition and transcendence.

\section{Introduction: \\ Mimetic Processes: Cultural Learning, Aesthetic Experience and the Emergence of Violence}

Infants and small children relate to the people with whom they live: parents, elder siblings, other relatives and acquaintances. They try to be like them by, for example, answering a smile with a smile. However, they also initiate responses in adults by using skills they have already acquired (Dornes 1993; Stern 2003). These exchanges also enable small children to learn feelings. They learn to evoke their own feelings towards other people and to elicit them in others. The brain develops in the course of its exchanges with the environment, i.e., certain capacities are trained, others fade (Rizzolatti and Sinigaglia 2008; Fuchs 2008). The cultural conditions of early life are imprinted in the brains and bodies of small children. Anyone who has not learned to see, hear or speak at an early age has tremendous difficulties acquiring these skills at a later age. Initially, the mimetic actions of infants and children do not allow for a separation of subject and object; this occurs only at a later stage of development. At first, the world is perceived as magical, i. e., not only humans but also objects are experienced as being alive. As 
rationality becomes more developed the capacity to experience the world in this way gradually becomes less central. However it is this capacity upon which children draw to transform the external world into images in mimetic processes and to incorporate them into their internal image worlds (Gebauer and Wulf 1995).

In his autobiography Berlin Childhood around 1900, Walter Benjamin (2006) illustrated how children incorporate their cultural environments in processes of assimilation. In the course of these processes, children assimilate aspects of the parental home such as the rooms, particular corners, objects and atmospheres. They are incorporated as "imprints" of the images and stored in the child's imaginary world, where they are subsequently transformed into new images and memories which help the child gain access to other cultural worlds. Benjamin reports experiences from his childhood in which real space and imaginary space so interpenetrate each other to such an extent that they become one space. The child becomes similar to a thing, so that the thing becomes a part of them. Things look back and seem to be making themselves similar to the child. Thus, for example the passage describing a butterfly hunt: "The old rules of hunting took over between us: the more my being, down to its very fibers, adapted to my prey (the more I got butterflies in my stomach), the more the butterfly took on in all that it did (and didn't do) the color of human resolution, until finally it was as if capturing it was the price, was the only way I would regain my humanity" (Benjamin 1980c, 244). The mimetic character of the child's experience, the way incidents are expanded by the mimetic faculty, is clear. The child gets butterflies in his stomach, and at the same time the butterfly takes on human traits. The capture of the butterfly makes it possible to erect boundaries again and to secure the child's form of human being. On the level of appearances, only in overcoming the object can the child preserve the constitution of its self-consciousness. Berliner Kindheit relates many similar experiences. Some are charged with anxiety and fear, such as the encounter with the "Little Hunchback," who can be regarded as a symbol of preservative forgetting, because he takes from the child his unconscious experiences and preserves them, thus preparing the way for memory. Things shrivel up; they enter memory in distorted form. The fright that the encounter occasions is a moment of self-awareness and at the same time an obstacle to and repression of more far reaching self-experience. The child, on the other hand, approximates himself completely to the environment, which is comparable to mimicry, and on the other, experiences his power over spaces and objects through the dedication of his magical interaction with them. Spaces and objects "look back." Culture is handed on by means of these processes of incorporating and making sense of cultural products. The mimetic ability to transform the external material world into images, transferring them into our internal worlds of images and making them accessible to others enables individuals to actively shape cultural realities (Gebauer and Wulf 1995, 1998).

These processes encompass not only our modes of dealing with the material products of culture but also social relationships and forms of activity and the way social life is staged and performed. In particular, it involves forms of practical knowledge that are learned mimetically in body-oriented, sensory processes 
and which enable us to act competently in institutions and organizations (Wulf 2006b). Ritual knowledge is an important aspect of this practical social knowledge, and this is the means by which institutions become rooted in the human body, enabling us to orient ourselves in social situations. Images, schemas and movements are learned in mimetic processes, and these render the individual capable of action. Since mimetic processes involve products of history and culture, scenes, arrangements and performances, these processes are among the most important ways of handing down culture from one generation to the next (Wulf 2014). Without our mimetic abilities, cultural learning and "double inheritance," i. e., the handing down of cultural products along with biological inheritance, which enables culture to change and develop, would not be possible (Tomasello 1999).

Writing - an assemblage of non-sensory similarities - elicits mimetic processes that help to bring to life what is read (Benjamin 1980a, 1980b). It is the same with other products of culture that also require a mimetic relationship for them to come alive. Without such a relationship, they represent simply a cultural possibility that can only realize its full potential through processes of education and self-education. Such processes are particularly important in the transfer of culture from one generation to the next, since these processes require a metamorphosis to keep forms of living, knowledge, art or technology alive. As mimetic processes are not simply methods of copying worlds that have already been symbolically interpreted but also consist in our taking and then incorporating "impressions" of such worlds, these mimetic relationships always contain creative aspects which alter the original. This creates a cultural dynamism between generations and cultures which constantly gives rise to new things.

To a great extent, cultural learning is mimetic learning, which is at the center of many processes of education and self-education. It is directed towards other people, social communities and cultural heritages and ensures that they are kept alive. Mimetic learning is a sensory, body-based form of learning in which images, schemas and movements needed to perform actions are learned. This embodiment is responsible for the lasting effects that play an important role in all areas of cultural development. "Becoming similar" to the world in mimetic actions becomes an opportunity to leave egocentrism, logocentrism and ethnocentrism behind and to open oneself to experiences of otherness (Wulf 2006a, 2016). However, mimetic processes are also linked to aspirations to forms and experiences of higher levels of life, in which vital experiences can be sought and found. In the experience of love, mimetic movements invokes "the power to see similarity in the dissimilar" (Adorno 1978, 191). No knowledge is possible without the production of similarities, without mimesis. It is certainly taken as true for scientific knowledge that mimesis is indispensable to the process of knowing. "Cognition itself cannot be conceived without the supplement of mimesis, however that may be sublimated. Without mimesis the break between subject and object would be absolute and cognition impossible" (Adorno 1982, 143). If a mimetic element is indispensable to scientific knowledge, it is at the heart of aesthetic experience. Mimesis makes it possible to comprehend the self-equiv- 
alency of the artwork and occasions a knowledge from within, which exists independently of theories and concepts. Aesthetic experience arouses "a sense of being overwhelmed in the presence of a phenomenon that is nonconceptual while at the same time being determinate" (Adorno 1984, 236), and this sense is largely beyond the reach of planning and resists precise localization. In the sudden density of a moment there occurs an aesthetic shock, which can rock the foundation of the I. The mimetic impulse leads to momentary contact with what is nondeterminate in the similar-to-itself artwork. The works' similarity is not referred to something outside itself, which is why mimesis in this context cannot denote the imitation of something that preexisted the work; rather, mimesis is similar to the self-referential creative force of the natura naturans, the nonobjective aspect of nature. Aesthetic experience arises in the "fine distance" between recipient and artwork and represents a nonscientific form of knowledge. "The continued existence of mimesis, understood as the non-conceptual affinity of subjective creation with its objective and unposited other, defines art as a form of cognition and to that extent as'rational.' ... Art expands cognition into an area where it was said to be non-existent” (Adorno 1984, 80). Art is a refuge for "mimetic behavior" (ibid. 79), "the organ of mimesis" (ibid. 162). Aesthetic experience refers mimetic movement beyond works of art and beyond the subject to possibilities of historical development. It can thereby become the carrier of hopes, expectations, and promises. Its central concern is a non-functionalized, improved relation between rationality and sensuousness.

In contrast to their role in aesthetic experience mimetic processes can also be connected with the processes whereby we are infected by experiences in which our subjectivity dissolves into chaos and uncontrolled violence. These processes also involve confrontations with power, domination, violence and oppression, which are part of every culture and into which mimetic processes are repeatedly immersed. The vicious circle of violence is an example of the mimetic structure of many forms of violence (Girard 1977, 1987). The starting point of this theory of violence is the insight that in mimesis there lies a necessity inextricably connected with being human, a reason for the emergence of violence among humans. Mimetic appropriation of attitudes and behaviors creates competition and rivalry, which then become the start of acts of violence. Violent behavior is especially imitated. In most societies every act of violence is followed by a retaliatory act of violence, an occurrence which threatens the cohesion of the society. Two strategies present themselves as methods of mastering the potential for violence emerging from mimesis: prohibition and ritual. By means of prohibitions everything which threatens the sense of community is supposed to be excluded. This includes conflicts of competition, rivalry and violence, to all of which mimesis gives rise. Mimetic behavior which aims at eliminating differences which are essential to the structural maintenance of the internal order of a society, such as those behaviors necessitated by hierarchies and the division of functions, is forbidden. These essential types of behavior must be preserved because they fulfill an integrative function and the society would be threatened if unlimited mimesis were allowed. It is necessary to restrain mimesis with pro- 
hibitions in order to strike a balance between its powers of social cohesion and social dissolution. While prohibitions strive to suppress violence that threatens the cohesion of a society by excluding the mimetic rivalry which contains the potential for such violence, rituals represent the attempt to channel manifest mimetic crises in such a manner that integration within the society is not endangered. When prohibitions are violated, a mimetic crisis arises that jeopardizes the social consensus with a vicious circle of reciprocal violence. It is the task of rituals to master the threat to the cohesion of a society in mimetic conflict by involving its members in a cooperative act. While prohibitions aim to prevent mimetic crises from arising in the first place, rituals pursue the goal of overcoming such crises by the repetition of certain acts intended to foster integration and the maintenance of the society (Dieckmann, Wulf, and Wimmer 1996; Paragrana 2011; Capeloa Gil and Wulf 2015).

\section{Social Action and Mimetically-Acquired Practical Knowledge}

The capacity for social action is acquired mimetically in cultural learning processes. This has been shown in numerous studies in recent years. In mimetic processes, for example games, gift exchange and ritual behavior, people develop skills that differ from one culture to another. For people to be able to act "correctly" in each situation, practical knowledge is necessary. This is acquired in sensory, body-orientated mimetic learning processes in each different field of activity. However, the corresponding cultural characteristics of social behavior can only be learned using mimetic approximations. Practical knowledge and social activity are shaped largely by historical and cultural factors.

In a first approximation social acts can be regarded as mimetic,

- if as movements they relate to other movements;

- if they can be understood as performances or enactments of the body;

- if they are independent actions that can be understood in their own terms and which relate to other actions or worlds (cf. Gebauer and Wulf 1998).

Thus non-mimetic actions would be, for instance, mental calculation, decisions, reflex actions or routine behavior as well as one-off acts or rule-breaking.

In contrast to attempts to differentiate forms of mimesis and to place these in hierarchical order, my research begins from the assumption that, while mimetic elements play a key role in numerous socio-cultural, cognitive and language-based phenomena, the attempt to build a hierarchy of different types of mimesis is misguided, due to the multimodal bodily- and imagination-based character of mimesis itself (cf. Zlatev, this volume).

The relationship between social action, practical knowledge and mimetic acquisition of knowledge is demonstrated by the following example taken from everyday contemporary culture:

On the morning of her birthday he prepares a glass dish decorated with ivy leaves and fills it with water, he makes little boats from walnut shells and places 
a candle in each one. Next to the dish there is a birthday cake, a large bunch of roses, a bottle of champagne and the ring, which is packed in a large box, to make it more of a surprise. Breakfast is waiting on the table that has been elaborately set; his wife waits outside the room until he has lit all of the candles and opened the champagne. He takes her in his arms, they exchange a few words of affection. A woman is about to celebrate her birthday and her partner wants to give her a present. He wonders what she might like. Initially he does not have many ideas. It should not be something useful that she would buy herself. He rejects the idea of the fondue set she has shown him in a catalogue. This would be more a present for them both than a birthday present and he finds this a little too impersonal for his partner. His thoughts focus on what she would like and what would really give her pleasure. He looks through the art books in a bookshop and then through the latest novels that have just come out, when he remembers that last year she gave him an album of photographs from the early days of photography, so he decides that a book would not be the correct choice. In an antique shop he looks for a candlestick or an old lamp. He likes what he finds but is still not satisfied. Then he sees a garnet ring. He remembers that she once told him that her grandmother had such a ring which she had loved to try on when she was a little girl. Now he is certain that he has found the right present; and she is delighted with the preparations and the present he selected so lovingly. They both sit down; they eat and have breakfast - taking slightly longer than usual. The day begins.

This scene shows us how a man looks for a birthday present and, after going through a good deal of trouble, finds it, and how he stages and performs the giving of the present and the small, early morning birthday party. His efforts are successful and bring great joy. Even when searching for the present, the man avoids decisions that would make the present less meaningful for his wife. He selects neither a useful nor a "joint" present; he also avoids giving her a similar present to the one she had given him recently. After a long search he finds something that is particularly suitable for his partner and which will appeal to her individually. His sensitive selection of a present is complemented by the loving preparations of the breakfast table with the candles floating amidst the leaves, the roses, the champagne, a birthday cake, the wrapped present, the elaborately set table, the tender words and the embrace.

How does the man celebrating his wife's birthday know what he has to do to show her his affection and to turn his efforts into a confirmation of the emotional quality of their shared life? Nobody has given him a set of rules to follow when celebrating birthdays or giving presents. Nevertheless, the man has acquired from action- and language-games a knowledge of how to behave appropriately, what criteria are important when selecting the gift and setting the stage to give it. How does the recipient know what the chosen present and the early morning celebration arranged in such a manner mean and how she is supposed to react for the breakfast to become a celebration of their togetherness? No one has ever told her what the rules are either. However, they both know their roles, what they need to do and how they should respond to each other so that the morning becomes a celebration of their life together. 
Such situations are only successful because all the participants have a practical knowledge of what they need to do, how they should respond to each other and how they should present themselves. Their actions are derived from practical knowledge of how, when and which situations are to be performed and how their performance and staging can meet or contradict the expectations of others. They have learned this in the many opportunities provided by everyday life, where they perceived through their own senses the way their parents prepared birthday celebrations for them, their siblings or for each other. In these earlier situations there may well not have been candles floating amidst ivy leaves or thoughts that resulted in the purchase of a garnet ring. However, there will have been other scenes involving the search for presents to delight the recipients, the loving thoughtful attitude towards the birthday celebrant and the joy of a shared life. There will have been other birthday scenes where, for example, siblings expressed their affection in a teasingly aggressive manner, where "Happy Birthday" was sung and presents were given that had been expressly asked for. In spite of such differences, birthday parties resemble each other in a number of aspects. In mimetic processes inner pictures, feelings and performative sequences arise in the participants, which serve as material for them to fashion the scene of the giving or receiving of a present or of celebrating or being celebrated in similar situations.

Anywhere where someone acts in reference to an existing social practice and thereby creates a social practice, there is a mimetic relationship between the two. This occurs, for instance, in the example of the birthday present, or when one acts according to a social model or uses the body to express a social concept. As we have seen, this does not simply involve actions of imitation. Mimetic actions are no mere reproductions that follow a pre-existing image precisely. Social practices performed in a mimetic manner lead to the creation of something individual.

Unlike the process of mimicry, which merely requires an adaptation to given conditions, a mimetic process - as can be seen in the example of the birthday present - creates both similarities to and differences from the situations or persons to which or whom they relate. In adapting and becoming similar to situations experienced earlier and to worlds that bear the mark of the culture of which they are part, subjects acquire the skills required to behave appropriately in a certain social situation. By participating in the living practices of other people, they expand their own life-worlds and create for themselves new ways of experiencing and acting. Receptivity and activity overlap. In this process, the given world becomes interwoven with the individual experience of those who form a mimetic relationship with it. We recreate the situations and external worlds experienced earlier, and by duplicating them, turn them into our own. It is only by confronting earlier situations or external worlds that these gain their individuality. It is not until this happens that our excess drive loses its indeterminate nature and is directed into individual wishes and needs. The confronting of the external world and the creation of the self occur as part of one and the same system. The external and internal worlds become increasingly similar and can only be experienced in their mutual dependency. Thus the internal and the external take on similarities 
and begin to correspond to each other. People make themselves similar to the outside world and change as they do so; this transformation involves the changing of their perception of the external world and of themselves.

Mimetic processes lead us to perceive similarities and create links to our social environment and it is through experiencing this that people make sense of the world. One of the earliest human skills was to create similarities, and these can be seen clearly in phenomena that correspond in a sensory way. Similarities can occur between two faces or in processes where one person imitates the actions of another. Forms of similarity can also be found between the living and the inanimate. One of the purposes of the human body is to create and express similarities. Dance and language illustrate this clearly (Brandstetter and Wulf 2007), as here there is no difference between representation and expression on the one hand, and performance and behavior on the other. They form two aspects that are not separate in the act of mimesis, but inextricably linked.

The acquisition of practical knowledge in mimetic processes does not necessarily involve similarities. If mimetic knowledge is acquired by relating to social actions or performative behavior from the world of the past, then it is only possible to identify the perspective of the mimetic relationship by comparing the two worlds. Similarity is nevertheless the most frequent trigger for the mimetic impulse. However, creating a magical contact can also become the initial point of mimetic action. Previous social actions are carried out for a second time in mimetic learning processes. The relationship is created not by theoretical thinking but aisthetically, through the senses (Michaels and Wulf 2014). The second action differs from the first not by challenging it or altering it but by re-performing it; thus the mimetic action has both a revelatory and a performative character and its performance creates its own aesthetic qualities. Mimetic processes relate to social worlds already created by humankind that are either real or imaginary.

The dynamic character of social activities is connected with the practical nature of the knowledge required for the enacting of such situations. As practical knowledge it is less subject to rational controls than is analytical knowledge. This is also because practical, ritual knowledge is not a reflexive, self-aware knowledge. It only becomes this in the context of conflicts and crises where the actions that result from it require justification. If social practice is not questioned, practical knowledge remains "semi-conscious." Like habitus knowledge it embraces images, schemas and forms of activity which are used for the staging and bodily performance of social acts without requiring any reflection on their appropriateness. They are simply known and called upon for the staging of social practices.

Human beings' residual instinct, the hiatus between stimulus and response and also their "eccentricity" (Plessner 1982) are prerequisites for the extraordinary plasticity of humankind and the opportunities this provides for acquiring practical knowledge in mimetic processes, thereby allowing social action to be conceptualized, staged and performed. This practical knowledge also includes the body movements that are used to stage scenes of social action. Discipline and control of body movements result in a disciplined and controlled practi- 
cal knowledge which is stored in the body memory and enables human beings to enact the corresponding forms of symbolic and scenic actions. This practical knowledge is based on the social forms of action and performance established in a particular culture, and is therefore a pronounced but specific knowledge, limited in terms of its historical and cultural horizons.

Imitative change and adaptation of previous worlds take place in mimetic processes (Hüppauf, and Wulf 2009). This is the innovative factor of mimetic actions. Social practices are mimetic if they relate to other actions and can themselves be seen as social arrangements that constitute independent social practices and also relate to other practices. Social actions are made possible by the acquisition of practical knowledge in the course of mimetic processes. The practical knowledge necessary for social actions is not only historical and cultural but also bodily and ludic knowledge; it is formed in face-to-face situations and is not semantically unequivocal; it has aesthetic components and elements of the imaginary and therefore cannot be reduced to intentionality; it incorporates an excess of meaning and can be seen in the social staging and performance of religion, politics and everyday life (Kraus et al. 2016).

\section{Mimetic Embodiment in Rituals}

In all areas of human existence rituals and gestures are important for the mimetic development of body knowledge. Embodied ritual knowledge is indispensable in religion, politics, economy, science, families, and education. It helps us to deal with difference and alterity and to create a sense of community and social relationships. It also enables us to assign meaning and structure to human relations. Ritual knowledge facilitates both continuity and change, as well as experiences of transition and transcendence. Given the significance of rituals in so many areas of social life, it is no surprise that there is no generally accepted theory of rituals, since the positions of the individual academic disciplines differ too widely. Scholars now generally agree that it makes little sense to reduce the wealth and diversity of studies on rituals to individual theories and lines of research. What is needed is rather to be aware of a wide variety of aspects and to render the complexity of the field explicit (Wulf et al. 2001, 2004, 2007, 2010, 2011).

All approaches to classifying rituals are faced with the fact that rituals are always the product of multidimensional processes of symbolization and construction. The phenomena studied are also more complex than the concepts and theories used to describe them. This also applies to the attempt to organize the field of ritual studies by types of occasion and to distinguish, for instance, the following kinds of rituals:

- Rituals of transition (birth and childhood, initiation and adolescence, marriage, death)

- Rituals of institution or taking up office (taking on new tasks and positions)

- Seasonal rituals (birthdays, days of remembrance, national holidays)

- Rituals of intensification (eating, celebrating, love, sexuality) 
- Rituals of rebellion (peace and ecological movements, rituals of youth)

- Rituals of interaction (greetings, taking leave, conflicts) (Gebauer and Wulf 1998, 130).

Other attempts at classification are also conceivable and can provide orientation in the complex field of ritual research. It is possible to differentiate between the following types of ritual activity: ritualization, convention, ceremony, liturgy, celebration (Grimes 1985).

In all rituals mimetic processes contribute to the development of practical knowledge. When we look at the staging and performance of rituals, the bodies of the participants are implicitly involved. How do these appear in a ritual? How are they enacted? What does their arrangement in the ritual tell us about the community, the individuals, and their culture? The movements and practices of bodies need to be considered. How are they used to exploit the ritual space, and what rhythm do they follow? The distance between bodies and the manner in which they approach each other or distance themselves are significant. What positions do they take up? Do they stand or sit? What movements do they make when they dance? The configurations of the body are symbolically encoded and convey messages. In terms of gestures, which we can consider to be language without words, it is possible to distinguish between iconic and symbolic gestures. Iconic gestures are simple "pictorial” gestures with meanings that are largely independent of the knowledge of a historical time or a particular culture. Examples of such gestures are giving indications of dimensions with simple hand movements or expressing tiredness and the need for sleep by inclining the head and placing the hands together beside it. Symbolic gestures, on the other hand, have different meanings depending on the historical era or culture, and more precise historical and cultural knowledge is required to understand them (Wulf and Fischer-Lichte 2010). In each case, the "logic" of the body, that is, its presentation and expression, plays an important role in the performance of gestures and rituals. This is especially true of the preconscious perception of bodily expressions, which forms the basis upon which the atmosphere of ritual arrangements is felt. The bodies of other people look at us before we become consciously aware of them, and in this way they determine our perception of them. In order for the performance of rituals to result in embodiment-processes, people need to experience the flow of energies and forces between them as a physical and psychological process that takes place at the outer reaches of consciousness.

During the staging and performance of rituals, a new social reality is created and incorporated by the past (Wulf and Zirfas 2007). This reality is not completely new, since previous versions of it have existed before; however, it has not existed in this particular form at this particular location before this particular time. Drawing on earlier rituals, every staging and performance creates a new ritual reality and a new ritual community. This ritual community can develop for the first time among the people who carry out the ritual actions, but it can also be experienced as a repetition through which the community confirms its status as such. The performance of rituals is decisive for the forming of the members of 
communities. The community presents itself in the staging and style of the performance. In the ritual presentation it expresses something that cannot be portrayed in any other way. The ritual staging can therefore be seen as a "window" that provides a glimpse into the deep structure of the community and the culture that creates it. The staging and performing of rituals render something visible that was previously invisible and embody it in the participants of the ritual. The staging of rituals always includes a reference to previous ritual performances. However, this can vary greatly. In some cases the connection between old and new ritual performances is very close; in others it is very loose. However, in both cases the performance and incorporation of the ritual establishes a form of continuity that is important for the effectiveness of the ritual. Often, the historical continuity is incorporated by the participants of the ritual and stabilizes the social order of the community, legitimizing it. This frequently serves to uphold the current distribution of power and maintain bodily-based social hierarchies. It requires a critical analysis of power relations.

Rituals are tied to time and space, and their cultural and historical conditions are embodied in these terms. Different spaces have differing effects on the structure, quality, and style of the rituals that take place within them. Ritual spaces differ from physical spaces. On the one hand, they create ritual stagings and performances; on the other hand, rituals create ritual spaces using body movements, settings, and symbolic and indexical frames. Rituals and space are not related in terms of subject/object or cause and effect, but interactively. Both rituals and spaces are performative (Wulf, Göhlich, and Zirfas 2001). On the one hand, a decorated gymnasium provides the space for a school dance, just as a church provides the space for a confirmation ceremony. On the other hand, the school dance transforms the gymnasium into a ballroom, and the confirmation ceremony transforms the church into a living, sacred space. The intermeshing of real, virtual, symbolic, and imaginary spaces with the bodily movements of those taking part plays an important role in the development of ritual activities.

This intermeshing of real, virtual, symbolic, and imaginary spaces with bodily movements takes place in an environment shaped by historical and cultural factors (Wulf 2013a, 2013b). This results in an atmosphere that affects the mood of all the participants in the ritual. Actions that have already been carried out here before and for which the space is suitable are repeated as part of an attempt to adjust to the atmosphere, structure, and function of the space in which the ritual is being carried out. The participants change by mimetically recreating the conditions of the space around them. The performative effects and embodiment of ritual spaces such as the church, the family living room, and the virtual space of electronic media are very different from one another and have different socializing effects.

The other constituent condition of ritual activity apart from space is time. Two complementary views are important for the manner in which humans deal with time. First, rituals play a major role in introducing children to the time structure of society. Parents attempt to adjust their children's rhythms to the time rhythms of society and thus to accustom even infants to the manner of 
structuring time that is the social norm. Childhood rituals are used to ensure that time becomes the main structuring influence in children's lives. Second, in our ritualized handling of time, we acquire practical knowledge that is indispensable for the staging and performance of rituals. Insofar as the management of time results from cultural learning processes, rituals play a very important part in this. Their repetitive character helps to inscribe the order of time into our bodies, which then become structured by time. Many rituals are repeated cyclically. Their purpose is to assure us of the presence of the community and to reaffirm its order and potential for transformation. The aim of rituals is to stage continuity, timelessness, and constancy. They are oriented toward processuality and the projectivity of communities and individuals. As we structure our time in a ritualized manner, we learn to manage it as a social skill. In today's societies the ritual organization of time lends a structure to every aspect of individual and communal life.

Between the beginning and the end of a ritual, different sequences of ritual activity occur in which different kinds of actions are expected and carried out. The rule-bound nature of ritual activity is closely linked to its sequentiality. The ritual actions follow an order that is also chronological and embodied. Periods of time are created in and by means of rituals which differ from the uniformity of everyday life and become moments of heightened intensity. This kind of intensification is due to the exceptional character of the events and is also achieved by highly condensing them and speeding them up. In many rituals, time becomes sacred time. Memory and reconnection with the past are therefore constitutive elements of religions, which, with the aid of rituals, transfer sacred content from communicative memory to cultural memory. They thereby render it accessible, so that it can be used to shape the future. In the experience of sacred time, it is not so much the length of time that counts as its intensity. On the one hand, rites of passage make it possible to experience different stages of life as phases with their own temporal dynamics; on the other, they create continuity and meaning in the process of life. In the time structures of rituals, different times often overlap, resulting in highly complex temporal experiences.

Rituals are essential for worship and the embodiment of religion, regardless of whether one sees their importance in the creation and practicing of religious feelings in cult ceremonies or focuses on their capacity to create sacrality, in which society makes an image of itself. The magical character of ceremonies of promotion to a higher office also has many aspects in common with sacred ceremonies. Even a candlelit dinner for two, where the candles on the table emphasize the special atmosphere of sharing a meal, raises the question as to whether the scene has elements borrowed from the sphere of the sacred and transferred to everyday life. The upgrading of such customs by adding sacred symbols may be connected to the far-reaching changes in attitudes toward religion and sacred matters that we are currently experiencing.

During the performance of rituals, the participants refer simultaneously and directly to the actions of other participants. They do so largely by means of mimesis, using the senses, the movements of the body, and a joint orientation 
toward words, sounds, language, and music. A ritual can only take place as a structured whole if all actions are successfully coordinated, precisely orchestrated and adequately embodied. Here the staging is indispensable; however, the performance itself is the decisive factor, as the ritual actions need to be exactly coordinated. Otherwise the results are farcical, and the ritual breaks down. If the interaction is to be harmonious, the ritual activities must be mimetically coordinated with each other. If this is achieved, energies can "flow" between the ritual participants, and this is experienced as intense, pleasant, and bonding (Csikszentmihalyi 1990). As in dance or courtship behavior, the rational control of actions also has limits in rituals. We only have the feeling that a ritual is successful if a mimetically created harmony that is beyond rational control develops between one body and another, one movement and another, and one gesture and another. These mimetic processes form the basis of the feeling of belonging to a community as well as the experience of the sacred.

Whereas the synchronous dimension of mimetic processes relates to the importance of mimetic processes in the actual conduct of rituals, the diachronous dimension relates to the historical aspects of rituals. Rituals always relate to others that have taken place before - either ones in which one has participated or ones of which one has heard. Thus the historical dimension is essential for the creation and the incorporation of rituals. Ritual actions include mimetic references to earlier rituals. As these references are made by mimesis, they create an "impression" of earlier performances of the ritual, which is then adapted to suit the current context. Depending on the requirements, some aspects of the ritual may be transformed in this process. Creating a mimetic link between the current world and a previous world ensures historical continuity, which legitimizes the current ritual activity, even if it differs from its predecessor. This use of mimesis to refer to or reconnect with previous performances of a ritual does not mean that it is recreated in exactly the same way every time. To make a reference by mimesis is to "adjust oneself to become similar," that is, to repeat a similar action that would not be possible if the previous ritual activity had not taken place. In some cases the result of this mimetic referencing also leads to a critical distancing from the reference point of the ritual, although this point of reference does not become superfluous. In mimetic referencing processes, the configurations and arrangements of the ritual action are updated and modified to match the context of our own activities. Mimetic constellations, staging styles, and types of movement are acquired and modified according to necessity or what the person thinks fit. The "repetition" of earlier rituals does not result in a copy in a photographic sense. Rather, through the inclusion of mimetically transferred and assimilated elements, something new is created in the repetition for everyone involved. The older version is merged into the new in a dialectical fashion. The ritual that has been updated by a mimetic process contains the old ritual, which has been given a new face and new clothing (Wulf 2005; Michaels and Wulf 2013).

Mimetic processes play an important role in the staging, performing and incorporation of ritual events: they produce the practical knowledge necessary for the ritual actions in question. This ritual knowledge, which enables us to 
act competently in rituals, evolves from real or imaginary participation in ritual activities. In mimetic processes people take part in ritual actions that are corporeal and are both independent and related to other ritual acts or arrangements. In so doing, they undergo an expansion in order to accommodate the ritual practice. Thus, through mimetic referencing, they undergo a process of adjusting to the ritual activities in which corporeality and performativity play an important role. These processes incorporate ritual configurations, scenes, sequences of events, images, and behavior patterns, all of which, in other contexts, contribute to the competent execution of a ritual practice.

\section{Outlook}

Mimetic processes are principally but not exclusively orientated towards other people. In mimetic processes people also incorporate their cultural environments. In the course of these processes, children assimilate aspects of their home, such as rooms, particular corners, objects and atmospheres. People and objects of the environment are incorporated as "imprints" and stored in the body and in the imaginary world, where they are subsequently transformed into new images and memories that help gain access to people and culture. Culture is handed on by means of these processes of incorporating and making sense of people and cultural products. The mimetic ability to transform people and the external material world into images, transferring them into the internal worlds of images and making them accessible to others enables individuals to actively handle the relationship with other people and shape cultural realities. These processes encompass our modes of dealing with the material products of culture and with the social relationships and forms of activity and the way social life is staged and performed. In particular this involves forms of practical knowledge that are learned mimetically in body-oriented, sensory processes and enable us to act competently in institutions and organizations. Ritual knowledge, for example, is an important area of this practical social knowledge, and this is the means by which "imprints" of people and institutions become rooted in the human body, enabling us to orient ourselves and act in social situations. Images, schemas and movements are learned and embodied in mimetic processes, and these render the individual capable of action. Since mimetic processes involve other human beings and products of history and culture, scenes, arrangements and performances, these processes are among the most important ways of handing down culture from one generation to the next. In mimetic processes, physical and historical-cultural elements are inextricably interwoven, so that it is of equal significance what humans have in common and what distinguishes them in historical and cultural respects. In anthropology today several paradigms can be distinguished in which these two perspectives play a role (Wulf 2013a). While research in the fields of hominization, evolutionary anthropology and philosophical anthropology investigate primarily what we as Homo sapiens have in common, research on historical anthropology and cultural anthropology investi- 
gates, respectively, the diachronic and synchronic differences between us. Given this situation, new perspectives might arise where the research on hominization, evolutionary anthropology and philosophical anthropology to turn to the particular or individual and research in historical or cultural anthropology turns to the general or universal. How difficult these turns are in epistemological respects has become clear in many of the large-scale joint-research projects of recent years.

\section{Bibliography}

Adorno, T. W. 1978. Minima Moralia: Reflections on a Damaged Life. Trans. E. F. N. Jepcott. London: Verso.

-. 1982. Against Epistemology. A Metacritique. Studies in Husserl and the Phenomenological Antinomies. Trans. Willis Domingo. Oxford: Basil Blackwell.

-. 1984. Aesthetic Theory. Trans. C. Lenhardt. London: Routledge \& Kegan Paul.

Benjamin, W. 1980a. Über das mimetische Vermögen. In Gesammelte Schriften. Vol. 2, 1, ed. R. Tiedemann and H. Schweppenhäuser, 210-212. Frankfurt a. M.: Suhrkamp.

-. 1980b. Lehre vom Ähnlichen. In Gesammelte Schriften. Vol. 2, 1, ed. R. Tiedemann and H. Schweppenhäuser, 204-210. Frankfurt a. M.: Suhrkamp.

-. 1980c. Berliner Kindheit um Neunzehnhundert. In Gesammelte Schriften. Vol. 4, 1, ed. R. Tiedemann and H. Schweppenhäuser, 235-304. Frankfurt a. M.: Suhrkamp.

-. 2006. Berlin Childhood around 1900. Cambridge, MA: Belknap Press of Harvard University Press.

Brandstetter, G. and C. Wulf, eds. 2007. Tanz als Anthropologie. München: Wilhelm Fink.

Capeloa Gil, I. and C. Wulf. 2015. Hazardous Future. Disaster, Representation and the Assessment of Risk. Berlin: de Gruyter.

Csikszentmihalyi, M. 1990. Flow. The Psychology of Optimal Experience. New York: Harper \& Row.

Dornes, M. 1993. Der kompetente Säugling. Frankfurt a. M.: Fischer.

Dieckmann, B., C. Wulf, and M. Wimmer. 1996. Violence. Nationalism, Racism, Xenophobia. Muenster: Waxmann.

Fuchs, T. 2008. Das Gehirn - ein Beziehungsorgan. Eine phänomenologisch-ökologische Konzeption. Stuttgart: Kohlhammer.

Gebauer, G. and C. Wulf. 1995. Mimesis. Culture, Art, Society. Berkeley: California University Press.

-. 1998. Spiel, Ritual, Geste. Mimetisches Handeln in der sozialen Welt. Reinbek: Rowohlt.

Girard, R. 1977. Violence and the Sacred. Baltimore, MD: Johns Hopkins University.

-. 1987. Things Hidden since the Foundation of the World. Stanford, CA: Stanford University Press.

Grimes, R. L. 1985. Research in Ritual Studies. Methuen, MA: Scarecrow Press.

Hüppauf, B. and C. Wulf, eds. 2009. Dynamics and Performativity of Imagination. The Image between the Visible and the Invisible. New York: Routledge.

Kraus, A., J. Budde, M. Hietzges, and C. Wulf, eds. 2016. Handbuch Schreigendes Wissen. Weinheim: Beltz Junventa.

Michaels, A. and C. Wulf, eds. 2013. Emotions in Rituals and Performances. New Delhi: Routledge.

-. 2014. Exploring the Senses. New Delhi: Routledge.

Plessner, H. 1982. Ausdruck der menschlichen Natur. In Gesammelte Schriften, vol.7, 391-398. Frankfurt a. M.: Suhrkamp. 
Rizzolatti, G. and C. Sinigaglia. 2008. Empathie und Spiegelneurone. Die biologische Basis des Mitgefühls. Frankfurt a. M.: Suhrkamp.

Stern, D. 2003. Die Lebenserfahrung des Sänglings. Stuttgart: Klett-Cotta.

Tomasello, M. 1999. The Cultural Origins of Human Cognition. Cambridge, MA: Harvard University Press.

Wulf, C. 2005. Zur Genese des Sozialen: Mimesis, Performativität, Ritual. Bielefeld: Transcript.

-. 2006a. Anthropologie kultureller Vielfalt. Interkulturelle Bildung in Zeiten der Globalisierung. Bielefeld: Transcript.

-. 2006b. Praxis. In Theorizing Rituals: Issues, Topics, Approaches, Concepts. ed. J. Kreinath, J. Snoek, M. Stausberg, and M. Leiden, 395-411. Amsterdam: Brill.

-, ed. 2010. Vom Menschen. Handbuch Historische Anthropologie. Weinheim/: Beltz; 2nd ed.: Der Mensch und seine Kultur. Cologne: Anaconda.

-. 2013a. Anthropology. A Continental Perspective. Chicago: The University of Chicago Press.

-. 2013b. Das Rätsel des Humanen. München: Wilhelm Fink.

-. 2014. Bilder des Menschen. Imaginäre und performative Grundlagen der Kultur. Bielefeld: Transcript.

-, ed. 2016. Exploring Alterity in a Globalized World. London: Routledge.

Wulf, C., B. Althans, K. Audehm, C. Bausch, M. Göhlich, S. Sting, A. Tervooren, M. Wagner-Willi, and J.Zirfas, eds. 2001. Das Soziale als Ritual. Zur performativen Bildung von Gemeinschaften. Opladen: Leske und Budrich.

-, eds. 2010. Ritual and Identity. London: Tufnell Press.

Wulf, C., B. Althans, K. Audehm, C. Bausch, B. Jörissen, M. Göhlich, R. Mattig et al., eds. 2004. Bildung im Ritual. Schule, Familie, Jugend, Medien. Wiesbaden: VS Verlag für Sozialwissenschaften.

Wulf, C., B. Althans, K. Audehm, G. Blaschke, N. Ferrin, I. Kellermann, R. Mattig, and S. Schinkel, eds. 2011. Die Geste in Erziehung, Bildung und Sozialisation. Wiesbaden: VS Verlag für Sozialwissenschaften.

Wulf, C., B. Althans, G. Blaschke, N. Ferrin, M. Göhlich, B. Jörissen, R. Mattig et al., eds. 2007. Lernkulturen im Umbruch. Wiesbaden: VS Verlag für Sozialwissenschaften.

Wulf, C. and E. Fischer-Lichte, eds. 2010. Gesten. Inszenierung, Aufführung, Praxis. München: Wilhelm Fink.

Wulf, C., M. Göhlich, and J.Zirfas. 2001. Grundlagen des Performativen. Eine Einführung in die Zusammenhänge von Sprache, Macht und Handeln. Weinheim: Juventa.

Wulf, C. and J.Zirfas, eds. 2007. Pädagogik des Performativen. Theorien, Methoden, Perspektiven. Weinheim: Beltz.

-, eds. 2011. “Töten.” Special Issue, Paragrana. Internationale Zeitschrift für Historische Anthropologie 20 (1).

Zlatev, J. 2016. Preconditions in Human Embodiment for the Evolution of Symbolic Communication. In this volume. 


\title{
"It Proceeded from the Entrance of a Demon into the Man"1
}

\section{Epileptic Seizures in Ancient Medical Texts and the New Testament}

\author{
Annette Weissenrieder
}

And as he is who falls and knows not how, By force of demons who to earth down drag him, Or other oppilation that binds man, When he arises and around him looks, Wholly bewildered by the mighty anguish

(Dante Alighieri)

Abstract: This paper aims to show how epilepsy was investigated in "rational" medical and theological discourses in antiquity: cognitive and somatic manifestations of epilepsy are intertwined and an epileptic attack is interpreted as a complete loss of consciousness in these sources. In Mark 9:14-29 and Luke 9:37-43, the Synoptic Gospels narrate Jesus' healing of an epileptic boy which describe the attack using the standard term from ancient medicine. This view is further reinforced by Matthew's version of the story where the sickness of the boy is described with the verb seleniazomai, "to be moonstruck" (17:15). It is in fact remarkable that the Gospels, but especially Luke, interpret "epilepsy” linguistically in the context of ancient medicine, still mention a demon. The texts distinguish between the sick person and the disease that invades the patient as an independent force from the outside. If a religious community assumes that it can explain the patient's etiology and thus any deviations from the healthy state through religion, and if it therefore ascribes illnesses to unclean spirits or demons and describes overcoming as a process of conquering these forces, that does not make the system any less medical. This insight is particularly apt when the physical symptoms are expressly understood in a medical sense, but the etiology is described demonologically.

\section{Introduction}

In the Inferno, Dante Alighieri makes a connection between "the falling evil," that is, the "force of demons" in a human being, and a medical interpretation, namely "congesting" or blocking the ventricles of the brain, which is signified

${ }^{1}$ Aretaeus SC 1.4; for further informations see pp. 274-75. 
by the Latin term oppilatio, a well-known term in medieval medicine. This term oppilatio is often used by medieval physicians describing epileptic phenomena ("falls and knows not how," "oppilation"). At the same time, Dante is referring to a demonological explanation of a human being suffering from the falling sickness ("force of demons") as well as to a medical phenomenon in a person's brain. The oldest source referring both to the demonological (-magical) explanation and to medical knowledge comes from first-century C.E. physician Aretaeus of Cappadocia, who refers to epilepsy as the result of demonic possession, or the punishment of the goddess of the moon. ${ }^{2}$ The context in which this explanation is found, however, also reflects medical knowledge: the brain as the cause of epileptic seizures. Aretaeus' interpretation may have influenced Greek and Latin Church fathers. ${ }^{3}$ Origen of Alexandria $(\dagger 254)$ writes:

Physicians may offer natural theories since according to their view it is not an unclean spirit but a bodily affection and physiology which presents itself in bodily humors in the head which move according to the movement of the moon which has a humid structure as well. We, however, also believe the enaggelion in the point that this disease [the lunacy] in those affected with it, is obviously brought about by an unclean and dumb spirit. (Origen, In Matth. Comment. 13.3-6)

Origen refers to the illness construct of "epilepsy," which he assumes physicians (iatroi) refer to as a "bodily affection" (sōmatikon sumptōma) caused by "humors in the head" (ta bugra ... ta en tē kefalè) and that he - reminiscent of Galen - associates with the course of the moon. ${ }^{4}$ Both the "humors in the head" and "the course of the moon" are grammatically paralleled with Origen's own theological perspective, "the unclean and dumb spirit." Therefore it is clear that Origen largely knows about the physicians' medical understanding. However, is also indisputable that he rejects these. Similar ideas are also expressed in a satirical dialogue by Lucian, who writes, "[e]veryone knows about the Syrian from Palestine, the adept in it, how many he takes in hand who fall down in the light of the moon and roll their eyes and fill their mouths with foam; nevertheless, he restores them to health and sends them away normal in mind, delivering them from their straits for a large fee" (Lucian Philopseudes 16, LCL 1921, 344-345). All four of these sources have one thing in common: They seem to be aware of the etiological cause of an epileptic phenomenon in the head and brain, while they connect its consequences to a form of demonic obsession that is expressed physically. Therefore, the sources allow the supposition that the brain is interpreted as "a relational organ" (Fuchs 2011, 347-358), which is embodied by different interpretations.

2 Aretaeus SC 1.4. Temkin $(1994,40)$ defines demonic possession as "the intrusion of a god, demon or ghost of a hitherto normal individual who now behaves like a willing or reluctant instrument of the intrudent."

${ }^{3}$ See Aretaeus SC 1.4.

${ }^{4}$ Cf. Galen De diebus decretoriis 3.2 (9.902f. Kühn); De locis affectis 3.9 (8.175-177; 233 Kühn). 
In this paper, I aim to show how epilepsy was investigated in "rational" medical and theological discourses in antiquity. In the following, I will describe the terms, some grammatical issues, theoretical foundations, and explanatory strategies of the medical and theological discourses of epileptic phenomena more precisely. In addition to the Greek text of the New Testament, I will also make use of the Old Latin version of the New Testament, the Vetus Latina, though briefly, in order to evaluate the reception of medicine in theological discourse. For one thing is worth noting: cognitive and somatic manifestations of epilepsy are intertwined in these sources.

\section{Phlegm, pneuma, Blood and Bile and the Question of the Cause of Epilepsy}

"Rational medicine" is widespread, above all, in the Corpus Hippocraticum (abbreviated thereafter $\mathrm{CH}$ ) and in later works by medical theorists such as Herophilus and Galen (cf. Tieleman 1996), who brought physical and scientific legality to the fore (cf. Weissenrieder 2003; Weissenrieder and Etzelmüller 2007). "Rational" is understood here as logikos in the sense of theoretically substantiated, indicating a closeness to the natural philosophy of antiquity. The beginning of rational medicine refers therefore most clearly "in the discovery of disease as a natural process: a particular type of cause produces as a rule a particular type of effect" (see Tieleman 2010, 83-95, here 90; 2013, 101-115). Many texts of the Hippocratic Corpus the treatise refer to epileptic phenomena (cf. Weissenrieder 2003, chap. 7): $:^{5}$ The adjective epileptos means that a person suffers from an attack of a disease and the adjective epileptikos refers to any kind of attack. ${ }^{6}$ Within the Hippocratic Corpus De morbo sacro and De flatibus take similar positions, in so far as both texts offer an etiological interpretation of the illness of epilepsy; however, they take different paths in their theoretical foundation, especially with regard to the question of the seat of cognition. Both texts agree in the fact that they accept a "consciousness-bearing" substance which spreads in the body. And both texts see a connection between the cognitive abilities and physical reactions and a blockage of these "consciousness-bearing" substances in the body. Whereas De morbo sacro regards pneuma as the reason of epilepsy, De flatibus refers to the blood. We come first to De morbo sacro:

The setting of the fourth chapter is the portrayal of the "epileptic phenomena."7

\footnotetext{
${ }^{5}$ See e. g. CH Coac. 157, 339, 445, 450, 511, 587; Epid. 7.1; Aphor. 2.45; 3.16, 20; Prorr. 1.131; Mul. 2.151.

${ }^{6}$ Temkin writes: "The distinction between systematic epilepsy, i. e., a syndrome which might be associated with various diseases, and the possible existence of an 'essential' or 'genuine' disease, epilepsy, is of relatively modern origin and was of little importance in antiquity" (Temkin 1994, 28; cf. Weissenrieder 2003, chap. 7; Wohlers 1997).

7 If not mentioned otherwise, the translations are mine.
} 
We also take in the majority of spirit (pneuma) along these vessels, for they are vents for our body. By drawing in air (aêr), they conduct it into the rest of the body along the smaller vessels and they refresh the spirit (pneuma) in the rest of the body and once again they discharge the air. For the spirit is not able to stand still but moves up and down. Should it stand still somewhere and be cut off, that part where the spirit stands still becomes powerless. [CH De morbo sacro 4.1-9 (VI 368.1-9 Littré)]

The fourth chapter is thus to be classified in an etiological sense. Here the author describes the function of the veins in breathing. The chapter provides the basis for his further discussion of air and its role in the formation of consciousness. The theory developed here forms the basis for numerous Hippocratic treatises (cf. De aere aquis locis 10$).{ }^{8}$ The ancient medical texts do not provide information as to how deeply the pneuma infiltrates the body. We read in the Hippocratic treatise De victu 2.38: "As there is breath in living things, it is also present in all the remainders." ${ }^{9}$ It is the whole body that inhales and exhales. After the spirit (pneuma) finds its way to the brain, it takes effect in the stomach, the lungs and the "veins". It is significant that the text shifts between outside air, a $\bar{e}$, and inside air, breath/spirit (pneuma). The movement of the pneuma in the body is also further specified in chapter 7 by the verb $x \bar{u} m b a l l e t a i$, which has a fundamental meaning of "bringing together" or "uniting" (LSJ 1996). It is the interior pneuma that, according to the treatise, links the various body parts. Notable is the verb reviving/refreshing, anapsüchousi, which lacks a clearly stated object. Hence, it is preferable to understand the object of the verb as pneuma, which is as breath/spirit the air inside the body. ${ }^{10}$ The consciousness-bearing substance is thus the breath, pneuma, in contrast to De flatibus, where blood is considered the central substance. This pneuma must always remain in motion. Otherwise the body part in which the pneuma pauses will become powerless.

The seventh chapter provides the heart of the work De morbo sacro. In essence, the author explains in chapter 7 that the majority of pneuma is inhaled by the respiratory system and the veins "by dragging the air into the body and carrying it to the bodily members" (van der Eijk 2005, 131). An “epileptic" seizure is portrayed as the result of phlegm flowing into the air passages, rendering the blood vessels unable to distribute the vital pneuma. This accumulation is based on the prenatal or postnatal katharsis of phlegm in the brain. The illness is caused by a blockage of passages that, spreading out from the brain to the rest of the body, is responsible for transporting pneuma. The pneuma can be understood here as a "consciousness-bearing" material (van der Eijk 2005, $131 \mathrm{ff}$; see Temkin 1936, 141-44, Temkin 1994). This accumulation results in different

${ }^{8}$ One finds examples of other systems in $\mathrm{CH}$ De carnibus 5 , according to which all veins come from the heart.

${ }^{9}$ Galen refers several times to this aphorism; see De sanitate tuenda I 10.22 f. (CMG V 4.2) and III 4.7 (179.10); De opt.const. 4 (IV 746 Kühn); see also Gal De sanitate tuenda CMG V 10 $2.2126 \mathrm{ff}$.

10 This view would then be similar to that of Philistion and Diocles of Carystus and the single purpose of breathing attributed to Hippocrates by Galen (De Usu Respirationis 1.2-3; VII 769 K). For air in De morbo sacro see Hippocrate 2003, 255-258; see also Debru 1996, $180 \mathrm{ff}$. 
symptoms, depending on the part of the body in question: close to heart it leads to palpitation and asthma, in the abdomen it leads to diarrhea, and in the vessels it leads to a spasm of the hands, crossed eyes, foaming at the mouth, symptoms of choking or suffocation. With this enumeration, the author takes up the symptoms already mentioned in chapter one, but explains them here scientifically, adding aphonia to the list. In contrast to the magician's understanding of this illness, mentioned in chapter 1 of this treatise, the mental affection is of primary importance for the physician. The mental phenomena of illness are here associated with the brain. It is noteworthy that the author grants the heart a reaction to the bodily processes.

If the phlegm should be shut off from these passages but make its descent into the channels that I have mentioned, the patient becomes speechless and chokes and froth flows out of the patient's mouth. His teeth are clenched and his hands are drawn up. His eyes are distorted and perceive nothing. ... A patient becomes speechless whenever the phlegm shuts off the air because it has come down into the channels, and the patient receives air neither in his brain nor in his hollow channels nor in his ventricles but he stops breathing. Whenever a human receives breath through the mouth or nostrils, it goes first to the brain. The greatest part of it, however, goes into the internal cavity, some of it to the lung but some of it to the channels. From these body parts, it is dispersed to the remaining parts along the channels. As much breath as enters into the internal cavity cools the internal cavity and nothing flows to another part. The air in the lung and the channels, however, flows together into the ventricles as it enters also into the brain. Thus, the air furnishes intelligence and movement to the bodily members. Whenever therefore the channels of the air are clogged by the phlegm and do not receive the air, the result is that they render the buman speechless and senseless. The hands become powerless and drawn after the blood becomes calm and not dispersed as usual. The eyes are distorted as the small channels are shut off from the air and they throb. The foam from the mouth is furnished from the lung, for whenever the breath does not enter it, it foams and sputters as though dying.... This symptom occurs whenever the breath does not enter the mouth as usual. The patient kicks with his feet whenever the air is shut up in the members since it cannot pass through to the outside because of the phlegm. As the air rushes upward and downward through the blood, it engenders spasm and pain. Therefore, the patient kicks. The patient suffers all these symptoms whenever the cold phlegm flows in the blood while it is warm, for it chills the blood and makes it stand still. If the flow of phlegm should be copious and thick, it kills the patient straightaway, for it masters the blood by its cold and congeals it. [CH De morbo sacro 7.1-39 (VI 372.4-374.13 Littré)]

The author is specifically interested in demonstrating that the brain is the cause of attacks of the "sacred disease" (cf. Jouanna 2003, 93 ff.; Grensemann 1968, $200 \mathrm{ff}$.). In chapter 16, this author addresses a wide-ranging discussion in the ancient world about the bodily organ responsible for thinking and intelligence. The earliest position seems to be that the diaphragm is the seat of intelligence. Authors such as Diogenes of Apollonia (VS 64 A 20) and the Hippocratic author of De carnibus argue very forcefully that the heart is the central organ of reason, decision-making, and intelligence, and this view was widespread in the ancient world. Against this popular position, the author of De morbo sacro weighs in on the side of those who assert the brain to be the intellectual center of the human 
body. Notable in chapter 7 is the verb "perceiving, have understanding" - phroneousin (see line 13 underlined), which does not have a clear subject: the subject could be either patients or eyes (accusativus limitationes). In order to decide one way or the other, it is significant to note that the other references to the patient in the treatise all use singular verbs. The plural verb form thus indicates the changes in the eyes during a serious attack. In consequence, the eyes and not the patient are the subject of perceiving and understanding.

Finally, chapter 16 represents the theoretical explanation of the attacks of the "sacred disease" described in chapters 13-15. Chapter 14 describes attacks of this disease that occur most often at night, while chapter 15 is devoted to those attacks that occur during the day. The nocturnal attacks are caused by bile and lead to shrieking and destructive behaviors directed both inward and outward, and the body is "like a corpse" (see also Mark 9:26). The attacks during the day are caused by phlegm and cause an altered consciousness and convulsions. These various diurnal and nocturnal symptoms arise from a single source, namely the brain. The brain is the chief organ of the body in general. Nevertheless, it is air that enables intelligence, comprehension, and discernment. Sense organs, speech, and motor skills are mediated through the commands of the brain. The commands are communicated through the air to the individual organs. These organs also have the ability of discernment. The air arrives first in the brain, making the brain the comprehensive center of intelligence and discernment. Now the brain perceives not only the consciousness or intelligence (phronesis) from the air but also climatic changes that alter the air, and the "sacred disease" is therefore affected by these environmental factors. The brain is described as interpreting (bermeneus) what came from the air outside. With this thought, the author returns to ideas expressed at the beginning of his treatise.

The author differentiates the diaphragm and the heart from the brain. Both the heart and the diaphragm certainly have aisthesis in the sense of reflex and sensation, but not phronessis (consciousness; intelligence) in the sense of mental abilities. The brain already plays an important role in the explanation of epilepsy, which he describes in chapter 11 [see CH De morbo sacro 11.3-5 (VI 382 Littré)] as an illness caused by an accumulation of phlegm in and around the brain. $\mathrm{He}$ employs an empirical argument: If one opened the skull of a goat that died of an epileptic fit, one would find a large amount of phlegm around the brain.

For this reason I believe that the brain possesses the greatest power in the human being, for it is the interpreter (bermeneus) for us of the phenomena of what comes to the body through the air if it is healthy. Further, the air supplies the intelligence (phronesis) and the eyes, the ears, the tongue, the hands, and the feet carry out such things as much as the brain knows, for the phenomenon of intelligence is produced in the entire body so long as the body partakes of air. But the brain is the interpreter for comprehension. For when a human draws in breath (pneuma), it arrives first in the brain, and thus the air is distributed into the remaining body after it has deposited its greatest strength in the brain, namely, the thing that has both consciousness and understanding (phronessis and gnome $\bar{e}$. For if the air were to arrive in the body first and afterwards in the brain, the power of discernment (diagnosis) would be left in the fleshly parts and in the vessels, it would come into the brain while it is warm and not pure but mixed with the moist 
secretions both from the fleshly parts and the blood so as to be no longer accurate. Therefore, I say that the brain is the interpreter of comprehension (sünesis).... Some say that we owe our consciousness to the heart and that this is the member which grieves and feels anxiety. This is not so; rather it is torn just like the diaphragm and even more than that, for the same causes, for blood vessels stretch into it [the heart] from all over the body, and the heart has been connected so as to perceive if any pain or strain occurs in the human being. Now, it is necessary for the body to shudder and strain for the extent of time that a human experiences distress and to contract when it feels pain, and when it is overwhelmed by joy it experiences the same. Therefore, in the heart as well as the diaphragm, feeling is the most acute. Certainly, in neither of the two share in consciousness at all, but of all these things, the brain perceives the intelligence of the air before the other members that are in the body. Thus, also the brain first perceives if any more violent change occurs in the air by the seasons of the year and if the air itself becomes at variance with itself. Therefore also, I say that the most acute and most severe and deadliest illnesses as well as the illnesses hardest to discern by the inexperienced fall within the province of the brain. [Corpus Hippocraticum De morbo sacro 16.1-17.26 (VI 390.10-394.8 Littré)]

The author attempts to describe the abilities of the brain by using various terms such as phronèsis (consciousness, intelligence), phronimos (consciousness), sünesis (comprehension, understanding), diagnōsis (discerning, thinking), and gnomē (understanding). It is clear that phronēsis is presented as the most comprehensive of these mental abilities. In English, phronesis is rendered as intelligence of thinking. According to Hüffmeier and Miller, it should be understood more as "having one's senses together" (Hüffmeier 1961, 58; cf. Miller 1948). The term implies perception and movement, but it also refers to a force by which a living being can deal with its surroundings. It is also remarkable that phronesis can be found throughout the body (through pneuma). Or to say it differently: Pneuma operates in the body, but sunesis (understanding) is restricted to the brain (see van der Eijk 2005, 127). The brain is not only a source of understanding, but also of feeling, although the heart and the diaphragm take part in that as well. All the other terms are dependent on and derivative of phronessis. Sunesis is rendered as comprebension which is mediated to all other organs through the brain. Comprehension is linked to sense and understanding, which according De morbo sacro is transmitted to all organs. Sense, discernment, and understanding are all distinguished as brain activities and are also broadly provided to the other organs by the air, though not to the degree that the air enables the brain to perform these functions.

In antiquity, the work De flatibus, part of the Corpus Hippocraticum, was already recognized as one of the writings ${ }^{11}$ that represent the doctrine of the "Hypothetics," whose basic premise is that sicknesses have a single cause (see CH Flat., ed. Heiberg; CMG I, 1, 91-101; Ducatillon 1983, Jouanna 2003, Segal 1970). The author of De flatibus does not himself use the term bypothesis, but

11 The treatise De flatibus has an epideictic character, and we can suppose that it was conceived as a lecture for an audience. I refer primarily to (Krug 1993, 43 ff.; Langholf 1989). This treatise shows that the Hippocratics not only had to distinguish themselves from magicians and Templar healers, but also had to establish themselves in opposition to intellectual healers who offered healing informed by their philosophy. 
in essence he treats a principle source, pneuma, as a bypothesis. He understands

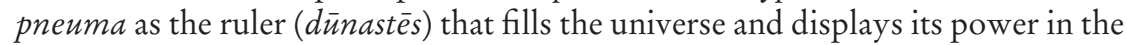
human body. ${ }^{12}$

I propose that none of the contributory causes in the body is more for intelligence than blood. Whenever blood remains in its usual state, intelligence also remains. When the blood is altered, intelligence also undergoes a change. There are many examples testifying that this is so. First sleep, which is common to all animals, testifies to what was just said. When sleep comes upon the body, the blood is cooled, for sleep produces a chilling by its nature. The passages become more sluggish by the blood's being chilled. Now this chilling is clear because bodies fall and become heavy, for all heavy things produce a falling downward. The eyes close, and intelligence is altered, and some other visions that are called dreams spend time there. Second, when the blood suddenly increases by drunkenness, souls and the thoughts in souls undergo a change. Souls become forgetful of present evils and hopeful of future goods. I could state many such instances in which changes of blood change the intelligence. If therefore all the blood should be completely disordered, the intelligence must be utterly destroyed, for the things learnt and recognized are habits. [CH De flatibus 14.1-15.8 (VI 110.14-114.20 Littré)]

Thus it is clear that this author is not merely talking about the possible effects of an epileptic attack, but also says that all physiological and sensory/motor skills are lost. In other words, an epileptic attack is interpreted as a complete loss of consciousness. The medical authors do not tend to hold theoretical discussions about the seat of reason, as we would expect from Plato or Aristotle. However, ancient writers are continually confronted with the theoretical issue when they are faced with illnesses involving epileptic phenomena, mania, melancholy and strokes. The Hippocratic author grants breath/air a central role in the life of an organism, and air also plays an important role in causing illness. In addition to causes outside the body, nourishment and digestion are also important since food always contains air. Thus, air itself is now seen as a potential blockage or hindrance, while in the text De morbo sacro it was more likely to be hindered in its flow throughout the body: the blood ${ }^{13}$ is cooled by the air, making it flow more slowly and providing the body with a reduced supply of consciousness. However, the author of the text also understands this to mean that any change in the blood changes the intelligence, phronesis; in other words, as long as the blood is moving normally though the body, intelligence remains constant. This movement is said to stop during sleep, when the blood cools and slows down.

In his treatise De somno et vigilia, Aristotle thus builds on De flatibus in a certain sense, since both suspect that there is a connection between sleep and epi-

12 Although the author's primary interest continues to be focused on pneuma, he mentions individual phenomena under the catchword nourishment, trophe $\bar{e}$. In the text, he characterizes pneuma as a genre for which two species are basic, which in turn are differentiated according to their topos: breath is pneuma in the body and air is pneuma outside of bodies $p h \bar{u} s \bar{e}$ (Ionic for Attic $p \bar{h} \bar{u} s$ ) refers to a pair of bellows, and the analogy to the chest cavity of animals is appropriate. The dynamic movement produced by blasts of air from a bellows is also analogous to the "blasts of air" in bodies that produce movement, as the rest of the text demonstrates.

13 Van der Eijk $(2005,132)$ analyzes the text as a "haematocentric approach to epilepsy." 
lepsy, though with one difference: blood, which was still central to the argument in De flatibus, now fades into the background as a cause.

Sleep arises from evaporation due to food [...] Young children sleep deeply because all the food is borne upwards. An indication of this is that in early youth the upper parts of the body are larger in comparison with the lower parts which is due to the fact that growth takes place in the upward direction. Hence too they are liable to epilepsy; for sleep is like epilepsy; indeed sleep is a type of epileptic fit. This is why in many people epilepsy begins in sleep [...]. (Aristotle, De somno et vigilia 3.457a4ff.) $)^{14}$

Aristotle thus interprets epilepsy as a form of sleep without ascribing a pathological significance to it. Sleep, he says, results from the process of digesting food, which is heated by the warmth of sleep and not only reaches the brain in the form of pneuma-breath, a warm steam, but also makes the head heavier, which makes the person sleepy. As in De morbo sacro, he says that children are more susceptible to this phenomenon.

One final text is ascribed to Diocles and his pupil Praxagoras, which is handed down to us through Anonymous Parisinus. As is his custom, Anonymous deals with the positions of Praxagoras, Diocles of Carystus, and Hippocrates. The reference to Praxagoras in this fragment is uncertain. In his well-known 1895 study, "Anecdota medica Graeca," R. Fuchs already pointed out that the description is more like Erasistratos' etiology of illness preserved in Codex Paris. 636, folio $132 \mathrm{r}^{15}$

The text begins by naming epilepsias, ${ }^{16}$ which initially means simply an occasional seizure that, however, does not need to coincide with the symptoms of an illness. Here the name can simply refer to any seizure. ${ }^{17}$

Praxagoras says that it [epilepsy] originates around the thick artery when phlegmatic humours come to exist in it. He says that by forming bubbles, these humors shut off the passageway of the psychic pneuma from the heart. Thus, this spirit agitates and wrenches the body. He says that the affliction ceases when the bubbles settle down again. Diocles himself also thinks that there is a stoppage around the same place. $\mathrm{He}$ also agrees that the other things happen according to the same causes, but it is Praxagoras who says these things happen. Diocles has neglected to state the nature of the cause of the stoppage but says that patients recover with more difficulty at the termination of the affliction if there are impediments to the spirit. Hippocrates states the cause of the blockage to be moist phlegm. He said that all the brain is filled with moistures, and the psychic spirit is shut off, but not everywhere, since the sinews through which the bodily members were set in motion are filled by it. He said that the onset of an epileptic seizure and the convulsion and the spasm occur by the charging of the spirit through these sinews. He said that the affliction becomes acute either because of the degree of

14 The translation (van der Eijk 2005, 133) also refers to the connection between sleep and physiological explanations like the ones found in Aristotle's De somno et vigilia.

15 Cf. also Garofalo 1988: Erasistrati fragmenta, fr. 293.

16 See CH De morbo sacro; De aere, aquis, locis 3 (II 18 Littré); see (Temkin 1936, 137 ff.)

17 The basic texts for the illnesses described with the terms cited here are Corpus Hippocraticum De morbo sacro; De aere, aquis, locis $3 \mathrm{f}$; De flatibus 14 and Arist. De omno et vigiliae 457 a 7-10. For the ancient understanding of epilepsy see Grensemann 1968, $5 \mathrm{f}$. In particular on the following section see Temkin 1936; 1994; on the Aristotle text cf. Debru 1996, 25-41. 
the problematic matter is so great as to suffocate the heat or because of the degree of the body's lack of tone so that the patient is not able to resist the spasms but gives in to them beforehand. [Anonymus Parisinus, De morbis acutis et chroniis $3(18,10-20,6$ Garofalo)]

The basic view is that a psychic phenomenon comes from the heart and causes convulsions in the body. The opposing view is offered by the author of the work De morbo sacro (chap. 9-11), part of the Corpus Hippocraticum. The external causes of the flow of phlegm are basically named here. The treatise interprets the modalities of the course of an illness under changing bodily conditions on the basis of which prognoses are formed, since children and the aged are in particular danger because of their veins.

To summarize this point: Ancient medical texts use the same terms - nosos, nosema and pathos - to describe illnesses located both in the body and in the brain. So ancient definitions of epileptic phenomena are described as a "disorder of the body" (CH Epid. VI 8.31.), an "interruption of the leading function" of the body (Gal. De sympt.diff. 3, VII 58-59 Kühn), and in his Definitiones medicae, Pseudo-Galen interprets the illness as a seizure of the thinking faculty "when the evil is abating and past its height" (Ps.-Gal. DM 240, XIX 414 Kühn). Therefore, these medical texts refuse to envisage a separation of body and mind, as remarked by Putnam and Cellucci (see Putnam 1995, 3; Cellucci 2005, 390 ff.); instead, the texts identify this illness as responsible for influencing a person's thinking, sensory and motor faculties, and behavior. It should also be noted that none of these texts proposes a concept of "soul" or "mental activity." At this point, it should be clear that theories of embodiment and this article presented here share an important insight (see Thompson 2010, Gallagher 2013, Fuchs 2013): "Mind, therefore, is not incidentally but intimately embodied and intimately embedded in its world" (Haugeland 1998, 237). Ancient interpretations of epilepsy show that the brain (the heart and the blood) is not a neural network that remain hidden in a supposed interior space, largely separate from the world; instead, it is to be seen as a dynamic form of physical being-in-the-world. The texts also show that sensory and motor skills should not be interpreted separately from "consciousness," but as a way of defining what the mind is.

\section{The Synoptic Gospels}

In Matt 17:14-21, Mark 9:14-29 and Luke 9:37-43, the Synoptic Gospels narrate Jesus' healing of an epileptic boy. The narratives portray the illness as an embodiment or "disembodiment" demons, and spirit/breath into or out of a human body. The terms used in Greek are "to cast" (ekballein) out and departing (exerchomai). Both terms allude to notions of action and movement of a demon which often leads to an evaluation

18 This term was first used in context of demonology by Stuckenbruck $(2014,174)$. 
of the terminology as "exorcistic rather than therapeutic" (Kelley 2011, 210). Do the Synoptic Gospels therefore show an emphasis of terms referring to an exorcism instead of a illness and healing?

Of course, the previous observations raise doubts about this analysis. First of all, it is noteworthy that Mark 9:14-29 as well as Luke 9:37 describe the attack using the standard term from ancient medicine. Not only do medical texts as well as Mark and Luke refer to a young child (paida in the Greek text; infans and pueris in Latin) who suffered from epilepsy from "childhood" (ek paidiothen Mark 9:21), ${ }^{19}$ the medical texts also refer to the boy's grinding his teeth (Mark 9:18 NTG ${ }^{29}$; strindet dentibus Vetus Latina), ${ }^{20}$ foaming (Mark 9:18, 20 and Luke 9:39), ${ }^{21}$ and convulsing (Mark 9:20; Luke 9:39). ${ }^{22}$ Linguistically, these descriptions are completely consistent with the manner of speaking found in medical texts. The precise naming of the symptoms not only shows that the authors must have been familiar with the symptoms of the illness; the list, which is mentioned repeatedly by Mark in particular, also indicates that the text's readers were able to classify the symptoms and assign them to an illness. This view is further reinforced by Matthew's version of the story. The tale of the boy with a falling sickness is given its special narrative profile through a dual interpretation of the sickness of the boy, who falls into the fire and into the water; the parallelism of the repetition emphasizes its frequency (pollakis, "often"). However, the story is noteworthy because of the use of the verb seleniazomai, "to be moonstruck" (17:15). In medical literature, this term is first used by Aretaeus of Cappadocia, who also takes a commonly held view in the context of a medical etiology, writing:

But also it is reckoned a disgraceful form of disease; for it is supposed that it is an infliction on persons who have sinned against the Moon (Selene): and hence some have called it the Sacred Disease, and that for more reasons than one, as from the greatness of the evil, for the Greek word hieros also signifies great; or because the cure of it is not human, but divine; or from the opinion that it proceeded from the entrance of a demon into the man: from one, or all these causes together, it has been called Sacred. [Aretaeus SC I 4.2 (38.28ff. Hude; translation: Adams 1856, 297)].

According to Aretaeus, Selene is said to punish crimes against the moon goddess with "epileptic phemonena." The type of crime can be better understood through a brief notice by Callimachus ( $3^{\text {rd }}$ century BCE), which he took from the chronicler Chenomedes; the text mentions that the goddess Artemis had afflicted Cydippe, a woman of Naxos, with quartan fever, then a bad cold and finally epileptic attacks (see Call. Aet. 75,121 ff.). Artemis sends these attacks because Cydippe had promised Artemis, against her will, to marry a man named

\footnotetext{
19 Cael. Aur. Morb. Chron. I 4,60: puerilis passio.

20 Cael. Aur. Morb. Chron. I 65: illisio dentium atque stridor et languae prolaptio.

21 Cael. Aur. Morb. Chron. I 65; Cel. III,23,1: per os atque nares spumarum.

22 Luke: occupaverit Vetus Latina; Cael. Aur. Morb. Chron. I $65 \mathrm{ff}$. facilis sensus obtinente passione accupato corpore, sensum privation accessione or apprehendere mentem partier appre-
} bendat. 
Acontius and then tried to back out of her oath. In this sense, "epileptic phenomena" are beyond human intervention, but certainly are the result of human guilt (Keydell 1969, 390). Aretaeus' comments could thus indicate that the boy's falling sickness was caused by a divine power as a punishment. However, there are several reasons that the text does not necessarily follow this interpretation: ${ }^{23}$ Galen is investigating the influence of the environment, the macrocosm, on the human body. ${ }^{24}$ In this sense, the illness is caused by phlegma, which comes from environmental influences that cannot be affected by people. This is also made clear by a short passage in De morbo sacro:

This disease, which is called sacred, arises from the same causes as the others, from the things that come and go away and from cold and sun and winds that change and never rest. These things are divine, so that one ought not to separate this disease and regard it as being more divine than the others; it is rather that all are divine and all are human (alla panta theia kai anthropina panta), and each of them has a nature and a power of its own. [CH De morbo sacro 18.1-2 6.394 Littré (translation: van der Eijk 2005, 51)]

One way of understanding this grammatical construction is that theios, the divine, can be included as an attribute or prophasis along with the climatic factors. Accordingly, it would be the climatic factors that are beyond human influence. An illness is only divine if it is caused by factors that are beyond human influence, such as heat or cold, the sun or the moon. If we follow Galen's medical interpretation, it is possible that the term seleniazomai, "to be moonstruck," (Matt 17:15) refers to the supposedly unchanging position of the moon. This clearly cannot be influenced by human power, as the triple reference to the disciples' inability makes clear in the Matthaean version of the Gospel (v. 16, 19, 20). This is all the more serious because in Matt 10:1, 8, the disciples are charged with healing the sick. Thus, an inability to provide healing indicates a lack of faith (v. 19). In Matthew 21:22, the request is syntactically subordinate to the prayer, which also creates a dependency at the content level. The past tense form, "which you already received," implies that the prayer has been heard. In the prayer, the petitioner subordinates himself to the will of God (Matt 21:22) and asks that God's will be done through his request. Thus, the "correct" request is through the mode of prayer, but the prayer itself shows the relationship to God. This perspective is further emphasized when we include a redactional change to the Matthaean version, interpreting Jesus' question "When shall I be with you?" as a reference to the Emmanuel Christology in the first chapter, God's being with you. Secondly, the Matthaean version emphasizes the boy's physical suffering (paschein) or feeling badly (kakōs echein; see the manuscripts $\mathrm{C} \mathrm{D} \mathrm{W} \mathrm{f} \mathrm{f}^{13} \mathrm{M}$ lat syr) in verse 15 . In any case, the question of guilt is not mentioned. We can therefore see that despite the variations in the terminology employed in the Synoptic Gospels, in each case the boy's condition can be identified as epilepsy; the terms refer to the boy's illness and suffering.

${ }^{23}$ Gal. De diebus decretoriiis 3.2 (IX $902 \mathrm{f}$. Kühn).

${ }^{24}$ Gal. In Hipp. Aphor. comment. 3.22 (XVII B 624 Kühn). 
Ancient medicine is familiar with two models of epilepsy as described above (see Weissenrieder 2003, chap.7). Both of these start from the assumption of damage to the brain due to bodily fluids brought on by the congestion of breath in the arteries. One form manifests itself in conspicuous seizures; the other results in paralysis. Whereas both phenomena are mentioned together in the Gospel of Mark (Mark 9) - an impossible scenario in the context of ancient medicine - the author of the Gospel of Luke omits the symptoms describing paralysis in the boy and limits himself to the depiction of a seizure brought on by phlegm, which includes a fit of dramatic physical movement.

It is in fact remarkable that Luke, who interprets epilepsy in the context of ancient medicine, still mentions a demon (Luke 9:42). Any interpretation of this text always has to take the ambiguity of the term pneuma into consideration: On the one hand, the unclean spirit refers to a demonic interpretation of the illness, epilepsy (also mentioned in De morbo sacro); on the other hand, pneuma also refers to unclean pneuma inside the human body, which is the cause of the epilepsy. If one follows the interpretation of the unclean spirit as a demon, the question regarding the function of this demon arises (For further details on epilepsy see Wohlers 1999; Temkin 1994; Weissenrieder, 2003).

A hint is found in the story of the healing of Peter's mother, who was ill with a high fever (Luke 4:39). In addressing the fever, Jesus treats the fever as a demon and thus as a responsive person. Luke distinguishes between the sick person and the disease that invades the patient as an independent force from the outside. A comparable situation can also be found in Mark 9:20, where the pneuma recognizes Jesus and the boy then loses consciousness and experiences an attack; or in Mark 9:25, where Jesus merely addresses the unclean spirit, not the boy: "you mute and deaf spirit, I command you ..." Precisely for this reason, the patient is freed of the religious responsibility for his illness. This results in the paradoxical situation that the introduction of a demon is to be interpreted as antidemonic. Accordingly, the mention of the demon in Luke's story of the healing of the epileptic boy demonstrates that the cause of the disease is not to be found in his alleged sins, but is to understand as being beyond human control. Or, in a medical and sociological sense: if a religious community assumes that it can explain the patient's etiology and thus any deviations from the healthy state through religion, if it ascribes illnesses to unclean spirits or demons and describes overcoming them not as a physiological process, but as a process of conquering these forces, that does not make the system any less medical. This insight is particularly apt when the physical symptoms are expressly understood in a medical sense, but the etiology is described demonologically. It is also worth noting that in Greek manuscripts of Luke 9:42 ( $\mathrm{k}, \mathrm{A}, \mathrm{C}, \mathrm{D}, \mathrm{L}$ ) exaiphnēs belongs to krazein: The boy cries out suddenly. In the Old Latin manuscript tradition (D and e), however, exaiphnès belongs to pneuma which reflects the medical tradition: the pneuma seizes him suddenly. The demonological system of interpretation (the unclean, silent spirit) is thus seen as an expression of the medical understanding that the illness is caused by an unclean pneuma. Potential therapeutic and exorcistic meanings can overlap here. This is particularly clear if 
we look once again at the Latin manuscripts. In the so-called Vetus Latina, the Old Latin Bible manuscripts, which consists of manuscripts from the $2^{\text {nd }}$ century C.E. on, terms refer to the action of eliminating the demons: "scolding," (see Luke 9:42) "throwing out" or "eliminating by force" increpare, eicere, exigere, excludere, expellere, or depellere. Some texts seem to take on the perspective of the evil spirit when they speak of "quitting" and "decamping" - exire, recedere, abscedere, absistere. In Roman medicine, one faces a medical phenomenon: symptoms and diseases that may not have a corporeal existence are presented as if they were physical objects, and they are indeed often associated with predicates that imply they are animate, even human agents of the action of the verb. Actions and states are also ascribed to body parts and remedies, which include a notion of movement, aggressive attack, force, capacity, and occupation. The medical historian D. R. Langslow calls this an "element of corporeality, animacy, and even anthropomorphism used for physiological processes" (Langslow 2000,194). The Gospel narratives do not reflect on the different causes of epileptic seizures described above, nor do they raise the question of convulsion coming from the heart or the brain. However, the symptoms seem to reflect the ancient medical discussion in different ways. There are first of all metaphors for an action of the human body, ranging from involuntary to voluntary: Almost all manuscripts of the Vetus Latina of Mark and Matthew translate the polysemous Greek verbs ballo and ekballō with eicere in the European text. Celsus has eicere for "vomiting" of bodily substances, Scribonius has reiectio and reicientes for "vomiting, bringing up" of poison, of blood and other substances ${ }^{25}$ and Cassius Felix uses reiactatio "vomiting, bringing up" of blood or phlegm, from the verb used figuratively from its basic sense "to throw back violently."

Luke often refers to mittere and dimittere. In the narrative on Peter's mother-in-law, the fevers do bestow supernatural abilities and may be ascribed to the evil powers that cause pain as mentioned in 4:36. These evil powers would then be opposed by Jesus. This conclusion is contradicted, however, by examples in ancient Roman medicine relating to the actions of diseases and remedies. The medical historian Langslow thus refers to a disease "approaching or more aggressively attacking the patient, seizing the patient, letting him go, and departing." 26 Celsus uses decedere for the "abatement of an illness" and remittere (remissio) for the "remission or abatement of an illness," and he speaks mainly of fever as a disease leaving the patient. In addition, he speaks of the even more aggressive notion of occupare and occupatio, as we can also see in Luke's version of the epileptic boy (114.9). Finally, Cassius Felix uses dimittere as well as remittere and apprehesio, or "seizure" of the senses, (see André 1963, 60-62, 65) and he also speaks of an illness being born, nativitas, which refers to an illness's initial growth. ${ }^{27}$ The

25 Scribonius Largus 47.22-23; 48.19; 49.1 with reference to sanguinis; 62.20 stercus per os eicientem.

26 For the following see André 1963, 47-67 and Langslow 1992, 196.

27 Cf. André 1963, 65, with reference to Cass 38.1. (Vietmeier 1937, 53-54). 
results suggest, carefully expressed, that the terms eicere, dimittere and remittere used in the Vetus Latina translation do not refer to exorcism, but are much more like a medical description of an illness that was experienced by ancient medical scientists as "approaching” or even "aggressively attacking” people (Langslow 1992, 196ff.). The fact that fever was evaluated as one of these illnesses that seize or occupy a person is obvious from these sources (Vietmeier 1937, 53-54). The results suggest, carefully expressed, that the terms "to cast out" and "departing of a demon" used in the Synoptic Gospels and its translations into Latin translation do not refer to exorcism, but much more resemble a medical description of an illness, which was experienced by ancient medical scientists as "approaching” or even "aggressively attacking" people. The terminology is rather therapeutic than exorcistic.

\section{Conclusion}

Mental life is not just embedded in the world, but also evokes this world. Cognition can be understood as the shaping of a meaningfully structured environment. This creates access to a "deep continuity of life and mind" (Thompson 2010, 128). If we follow the ancient texts, this mental life is disrupted by the illness of epilepsy - in the sense of a systematic disruption whose phenomenology is based on a physiological disruption of human consciousness. All of the ancient texts (including those of the New Testament) associate epilepsy with the loss of the patient's cognitive activity, the brain and the (unclean) air (pneuma), the blood, the heart, or a demon. And all of the texts refer to the loss of sensory stimuli - blindness, deafness, or the ability to feel pain. Thus, all of these texts also describe a loss of communication with the outside world. If we read the ancient sources from the perspective of embodying the mind, the non-linear causality of the interactions between mental and physical processes thus becomes clear.

\section{Acknowledgements}

I am grateful to the organizers of the conference for inviting me, especially Prof. Dr. Gregor Etzelmüller. My interpretation of the ancient medical sources was also discussed in a workshop organized by Prof. Dr. Christoph Markschies and Dr. Eva Elm and the Sonderforschungsbereich "Dämonen und Heil" at the Humboldt Universität zu Berlin, in June 2015.

\section{Bibliography}

André, J. 1963. Remarques sur la Traduction des Mots Grecs dans les Textes Médicaux du Ve. Siècle (Cassius Felix et Caelius Aurelianus). RPh 59: 47-67.

Aretaei Cappadocis. 1828. Opera Omnia, ed. C. G. Kühn. Lipsius: Teubner. 
Aretaeus, the Cappadocian 1856. The extant works of Aretaeus, the Cappadocian, edited and translated F. Adams, London: Sydenham Society.

Cellucci, C. 2005. Mente Incarnate e Conscenza. In Per Una Storia del Concetto di Mente, ed. E. Canone, 383-410. Florence: Olschki.

Debru, A. 1996. Le Corps Respirant. La Pensée Physiologique chez Galien. New York: Brill.

Ducatillon, J. 1983. Le Traité des Vents et la Question Hippocratique. In Formes de Pensée dans la Collection Hippocratique. Actes du IVe Colloque International Hippocratique, Lausanne 1981, ed. F. Lasserre and P. Mudry, 263-276. Geneva: Libraire Droz.

Duminil, M. P. 1983. Hippocrate. Le Sang, les Vaisseaux, le Coeur dans la Collection Hippocratique. Anatomie et Physiologie. Paris: Les Belles Lettres.

Fuchs, T. 2011. Hirnwelt oder Lebenswelt? Zur Kritik des Neurokonstruktivismus. Dentsche Zeitschrift für Philosophie 59: 347-358.

-. 2013. Das Gehirn - ein Beziehungsorgan: Eine phänomenologisch-ökologische Konzeption. Stuttgart: Kohlhammer.

Galen. Opera Omnia, inklusive des Werks von Pseudo-Galen, 22 vol., 1821-1833, Hildesheim 1964-1965, ed. C. G. Kühn. Leipzig: C. Cnobloch.

Gallagher, S. 2013. How the Body Shapes the Mind. Oxford: Clarendon Press.

Garofalo, I. 1998. Erasistrati Fragmenta. Biblioteca di Studi Antichi 62. Pisa: Giardini.

Grensemann, H. 1968. Hippokrates. Die Hippokratische Schrift 'Über die Heilige Krankbeit'. Ars medica II, 1. Berlin: de Gruyter.

Haugeland, J. 1998. Having Thought. Essays in the Metaphysics of Mind. Cambridge, MA: Harvard University Press.

Hüffmeier, F. 1961. Phronesis in den Schriften des Corpus Hippocraticum. Hermes 89: 51-84.

Hippocrate. 2003. La Maladie Sacrée, ed. and transl. J. Jouanna; Budé II, 3. Paris: Les Belles Lettres.

-. 2003. Des Vents - De l'Art, ed. and transl. J. Jouanna; Budé V, 1. Paris: Les Belles Lettres.

Kelley, N. 2011. The Punishment of the Devil was Apparent in the Torment of the Human Body: Epilepsy in Ancient Christianity. In Disability Studies and Biblical Literature, ed. C. Moss and J. Schippers, 205-223. New York: Palgrave MacMillan.

Keydell, R. 1969. Art. Kydippe, RP 3: 390.

Krug, A. 1993. Heilkult und Heilkunst. Medizin in der Antike. München: Beck.

Langholf, V. 1989. Beobachtungen zur Struktur einiger Traktate des 'Corpus Hippocraticum.' Sudhoffs Archiv 73: 64-77.

Langslow, D. R. 1992. The Development of Latin Medical Terminology: Some Working Hypotheses. Proceeding of the Cambridge Philological Society 37: 106-130.

-. 2000. Medical Latin in the Roman Empire. Oxford: Oxford University Press.

Liddell, H. G. and R. Scott. 1996. A Greek English Lexicon. A New Edition Revised and Augmented throughout by Sir Henry St. Jones. Oxford: Clarendon Press.

Littré, É. M. P. 1850. Oeuvres complètes d'Hippocrate: Traduction nouvelle avec Grec. Paris: Baillieres.

Lucian. 1921. Text and Translation, ed. A. M. Harmon; LCL3; Cambridge: Harvard University Press.

Miller, H. W. 1948. A Medical Theory of Cognition. Transactions and Proceedings of the American Philological Association 79: 168-183.

Segal, C. 1970. Lucratius, Epilepsy, and the Hippocratic On Breaths. Classical Philology 65 (3): 180-182.

Stuckenbruck, L. T. 2014. The Myth of Rebellious Angels. Tübingen: Mohr Siebeck. 
Temkin, O. 1936. Epilepsy in an Anonymous Greek Work on Acute and Chronic Diseases. Bulletin of the History of Medicine 5: 137-144.

-. 1994. The Falling Sickness. A History of Epilepsy from the Greeks to the Beginnings of Modern Neurology. Baltimore/London: John Hopkins Press.

Thompson, E. 2010. Mind in Life: Biology, Phenomenology, and the Sciences of Mind. Cambridge, MA/London: Harvard University Press.

Putnam, H. 1995. Words and Life. Cambridge, MA: Harvard University Press.

Tieleman, T. 1996. Galen and Chrysippus on the Soul: Argument and Refutation in the "De placitis," Books II-III. Philosophia Antiqua 58. Leiden: Brill.

-. 2010. Religion und Therapie in Galen. In Religion und Krankheit, ed. G. Etzelmüller and A. Weissenrieder, 83-95. Darmstadt: Wissenschaftliche Buchgesellschaft.

-. 2013. Miracle and Natural Cause in Galen. In Miracle Stories Revisited. Studies on the Bible and its Reception, vol. 1, ed. S. Alkier and A. Weissenrieder, 101-115. Berlin: de Gruyter.

van der Eijk, P. 2005. The Heart, the Brain, the Blood and the Pneuma: Hippocrates, Diocles and Aristotle on the Location of Cognitive Processes. In Medicine and Philosophy in in Classical Antiquity: Doctors and Philosophers on Nature, Soul, Health and Disease, 119-136. Cambridge: University Press.

Vietmeier, K. 1937. Beobachtungen über Caelius Aurelianus als Übersetzer medizinischer Fachausdrücke verlorener griechischer Schriften des methodischen Arztes Soranos von Ephesos. Gütersloh: Thiele.

Weissenrieder, A. 2003. Images of Illness in the Gospel of Luke. Insights of Ancient Medical Texts. Tübingen: Mohr Siebeck.

Weissenrieder A. and G. Etzelmüller. 2007. Christlicher Glaube und Medizin. Stationen einer Beziehung. Christian Beliefs and Medicine. Stations of Relationship. Deutsche Medizinische Wochenschrift 132: 2747-2753.

Wohlers, M. 1999. Heilige Krankheit. Epilepsie in antiker Medizin, Astrologie und Religion. Marburger Theologische Studien 57. Marburg: Elwert. 

4. The Mutual Intertwinement of Nature and Culture 



\section{Introduction}

\section{Miriam Noël Haidle}

Numerous characteristics have been identified that distinguish contemporary humans from other living animals. Among the physical differences are upright locomotion, hands with opposable thumbs, a vastly larger relative brain size, a markedly higher gene expression rate in the brain, and a course of life-history with extended childhood and post-reproductive phase. Contemporary humans possess complex tools, language, art, music, religion, and explicit normative rules. Their social life is marked by joint attention, cumulative culture, a theory of mind, extended co-operation (even a division of labor), and the teaching of naïve individuals by experts. Humans reflect upon experiences in their deep past, plan for years ahead, think about what happens after death, examine causalities in structured ways, debate ethical issues, and create new problems and solutions. They settle around the whole world, reach out into space, exploit a wide range of natural resources, transform a multitude of environmental factors, create new materials and build highly artificial settings. Contemporary humans differ in many aspects of body, mind, and behavior (i. e., tackling problems they encounter) from other living animals. The differences lie in the nature and in the culture of Homo sapiens.

But how are human nature and culture entangled? How far do physical, cognitive, and behavioral features distinguish us from other species? Are the distinctions absolute or a matter of degree? How independent are they and in what ways do they interrelate? What were the prime movers and developmental factors that created Homo sapiens? Which of these prime movers and developmental factors have been necessary, which ones sufficient to develop humans as they are and as they perform today? Since Darwin published his ground-breaking volumes $O n$ the origin of species by means of natural selection (1859) and The descent of man, and selection in relation to sex (1871), supernatural explanations of human origins have been excluded from scientific discussion. Yet, while evolutionary-biological processes such as genetic mutation and selection are widely seen as necessary in explaining human cultural behavior to some extent, it is questionable whether they provide a sufficient explanation, particularly of the diversity of that behavior. The articles in this section follow context-based approaches to get closer to the central question of how the specific combination of natural and cultural characteristics exhibited by contemporary humans could have developed in physical and cognitive interaction with the material world. The contributions present different lines of arguments while sharing a common emphasis on evolutionary 
processes shaping human capacities rather than singular developmental leaps that miraculously altered the human being. The focus here is on the mutual development of body, mind, behavior and environment, and the reciprocal effects each has on the other. Only in this way, the contributors agree, can one explain aspects and processes of continuity and change in human nature/culture.

Basing itself on Material Engagement Theory (Malafouris 2013), Lambros Malafouris' paper “On human becoming and incompleteness: a material engagement approach to the study of embodiment in evolution and culture" explores openness to creative evolution as a special feature of human evolvability. Following a radical embodied and enactive approach, thinking can be understood as "thinging." Things become vital agents in human thinking through acts of engagement and enactive signification: though lacking intentionality, things nevertheless offer possibilities, set limits, open paths, merge with other things to create new contexts, providing humans with changing spheres for interaction. The mind is always thinking about, with, and through the material world. And it is embodied not only in having a neural and neurological basis or in being expressed by bodily actions or perceiving information through physical organs, but also by being formed through the body while engaging with the world. Cognition, perception, mediated bodily action, and material culture form a continuum that not only appears in a situated setting, but also shapes human ontogeny and evolution. The human mind is intertwined with a plastic culture which brings about metaplasticity, a form of human biosocial becoming. The minds of Homo sapiens are not complete or fixed, but open and incomplete. Malafouris does not deny genetically evolved structures, but presents material engagement as the main constituent of cognitive evolution. He identifies "creative thinging" as part of our human nature.

In his contribution "Metaplasticit-ies. Material engagement meets mutational enhancement," Duilio Garofoli seeks to combine Material Engagement Theory with certain features of human cognition acquired through evolutionary-biological processes. He addresses the question of the deeper history of the genus Homo: according to Malafouris (2013), humans have been materially engaged since they started to produce and use their own tools around 3.3 million years ago. Thus, metaplasticity, the integration of cultural and neural levels of plasticity, began quite early in the course of human evolution. At the same time, Garofoli recognizes the problem that "not every human biological system, provided with the appropriate conditions of cultural engagement with artefacts, can bring forth any potential cognitive function.” To overcome this problem, Garofoli introduces a neuroconstructivist approach, bringing together genetic enhancement of the neurological system and the more context-based MET. Material engagement is seen as a necessary, but not sufficient condition for the emergence of cognitive functions in contemporary humans. Neural plasticity cannot be extended without limit; there are constraints on the brain/body system. Garofoli rejects models of innate psychological entities, representations of how to cope with the world, such as those proposed by evolutionary psychology. Instead, he points to an acquired modularity of the neurological system: there, the innate component lies 
in physical properties of the bodily system that allow the acquisition of partial representations. For Garofoli there is no strict relationship between selection of biological enhancements and specific behavioral innovations; there is no gene for art or religion or football. Instead, mutational enhancement over the course of evolution allowed different ranges of metaplasticity. The capacity for a metaplastic development of cognition in humans did not evolve in a single biological step after which only material engagement created new forms of cognitive and cultural expressions. Rather, the basic neural system itself underwent mutational enhancement. And with these different neural systems different Homo species engaged with the material, natural and cultural world in different ways, developing not just metaplasticity, but possibly several different metaplasticit-ies. Cultural products such as body ornaments are thus not the result of a specific mutation for personal adornment, nor are they brought forth only by material engagement. They rest on distinct neural structures in combination with multiple forms of material engagement; these might involve different "cognitions", even if their material products, for example shell ornaments, might look the same.

In "Artefacting minds: material engagement theory and joint action," Shaun Gallagher and Tailer G. Ransom expand Material Engagement Theory from the individual level to the development of joint actions and their cognitive background. Usually, joint actions are associated with cognitively strong preconditions such as shared beliefs, normative commitments, or interlocking intentions. Recent studies, however, have shown that joint actions are possible at a lower level of cognitive sophistication, on the basis of simple embodied dyadic interactions with joint attention directed to relevant factors. Gallagher and Ransom point here to the role played by intersections between agents, material things, and social structures in the emergence, construction and constitution of mental processes in joint actions. Taking examples of spontaneous joint actions (dancing), distributed joint actions (terrorist cells), and joint actions guided by joint attention (moving a bookcase) the authors define these as dynamical embodied intersubjective interactions and distinguish them from joint intention and joint attention which may (but don't have to be) associated with joint action. In joint actions there is generally no simulation, imitation, or motoric matching. The movements are coordinated but distinct, and there is often no theoretical inference. Rather, things and environments shape the movements' dynamics. The intentions-in-action are dynamical processes; in joint actions the actors share the overall goal, but use different movements and means. On the basis of MET and Bruno Latour's (2005) Actor-Network Theory, Gallagher and Ransom argue that intentions are formed via material engagement. Action and agency are embodied and situated in a particular physical and artefactual environment connected to values, skills and know-how that constrain the affordances of that environment. The affordance of a certain artefact can shape human intention, but changes in the use of an artefact may introduce alterations in affordance: joint actions may expand or shrink the affordance space - the range of possibilities for action depending on the body and the environment - relative to what individuals may accomplish on their own. 
In his contribution, Wolfgang Welsch takes a broader perspective on the evolution of human cultural capabilities and draws the attention to "bodily changes during the proto-cultural period and their ongoing impact." His central concern is to resolve the nature versus culture dichotomy, and to this end he views cultural evolution not in opposition to human nature, but as lying at its foundation. Welsch defines three periods of human evolution: a phase displaying merely biological adaptation; a proto-cultural phase which started around two million years ago and ended around 40,000 years ago; and finally a cultural phase in which biological and genetic changes largely cease. Key physical, behavioral, and social changes originating in the proto-cultural phase include the increase in body size, changes in sexuality, growth of the brain and changes in its structure, the development of an apparatus of self-reference, an increase in reflectivity, and the capacity to learn from others. The perception of objects as tools and their deliberate manufacture show feedback processes occurring between brain development and behavior. The proto-cultural phase, according to Welsch, displayed a remarkable acceleration of cultural innovation processes in comparison with biological adaptions; indeed, as Welsch argues, biological evolution essentially stopped around 40,000 years ago with the Upper Paleolithic Revolution. Since that time, cultural evolution has held sway and a cumulative culture can be observed. Welsch concludes that our whole cultural evolution is based on the human constitution achieved in the proto-cultural period. Human universals such as the phenomenal experience of color, facial expressions of basic emotions, the understanding of elementary gestures, and certain aesthetic preferences originate in that period: "our biological and proto-cultural preconditions are not something dubious that we would better leave behind. On the contrary, they are productive potentials that enable everything to come."

\section{Bibliography}

Darwin, C. 1859. On the Origin of Species by Means of Natural Selection, or the Preservation of Favoured Races in the Struggle for Life. London: John Murray.

-. 1871. The Descent of Man, and Selection in Relation to Sex. London: John Murray.

Latour, B. 2005. Reassembling the Social: An Introduction to Actor-Network-Theory. Oxford: Oxford University Press.

Malafouris, L. 2013. How Things Shape the Mind. A Theory of Material Engagement. Cambridge, MA: MIT Press. 


\title{
On Human Becoming and Incompleteness: A Material Engagement Approach to the Study of Embodiment in Evolution and Culture
}

\author{
Lambros Malafouris
}

Abstract: What is the meaning of embodiment in evolution and culture? My aim in this essay is to explore that question from the perspective of material engagement theory (MET) (Malafouris 2013). In particular, I want to describe and explain how the argument for the constitutive intertwining of mind with the material world challenges the traditional (neo-evolutionary) ways of understanding the importance of embodiment, plasticity, and material culture in human evolution. Based on the relational enactive foundation of material engagement theory and building on notions of metaplasticity and creative thinging this essay will propose a different view of human becoming. This new understanding challenges neo-Darwinism and its foundation in evolutionary psychology and recognizes the impact of material culture on the making and evolution of human intelligence.

\section{Introduction}

I begin with an image and a question that can help us to focus and visualize the main underlying problem that I will try to tackle in this paper. The image depicts a process of making, specifically, the forming of a clay vessel by the hands of the potter on the wheel (Figure 1). The question concerns the meaning of embodiment relevant to the human abilities of making and growing: What is the mean-

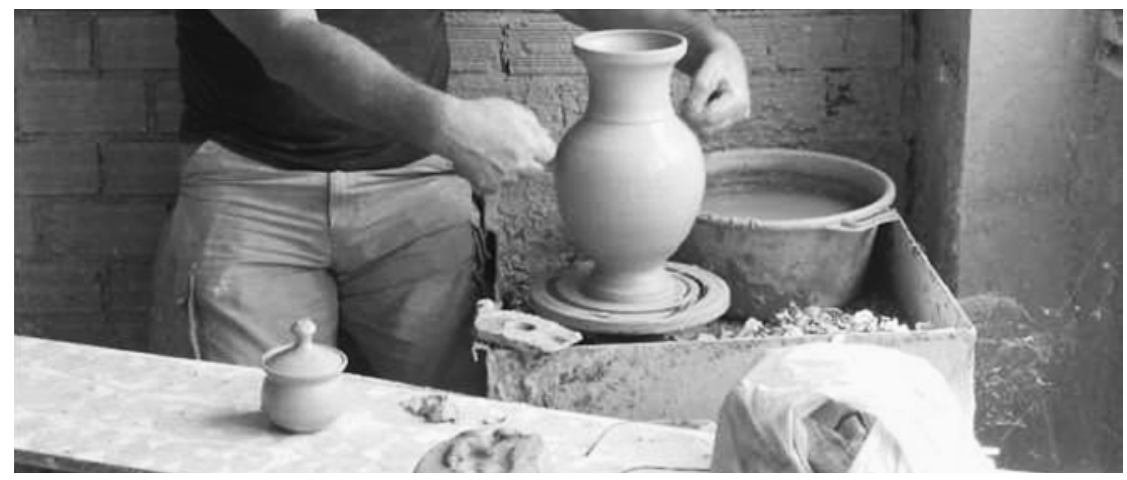

Figure 1: What is the meaning of embodiment in the context of making? (photo by author). 
ing of embodiment in the context of making? The short answer I would like to propose is that we humans are embodying and self-bounding creatures able to influence our developmental paths by changing our means of material engagement and by altering, mediating and regulating the flows of energy and matter. Human beings evolve by creating material things and assemblages which scaffold the ecology of our minds and shape the boundaries of our thinking. This openness of the human mind to Bergsonian creative evolution - not just Darwinian natural selection - is one of the distinctive features of our species' ability to evolve. I call that special feature of human evolvability ${ }^{1}$ metaplasticity; it operates by means of creative material engagement (Malafouris 2015). This special feature also indicates that the nature of human intelligence is to remain amenable to deep reorganization and reconstitution, thus, to remain incomplete. In this paper, I will try to explore, the major implications of this ongoing dialectic of co-constitution of people and things for the traditional ways we understand the process human becoming, in particular, for the ways we understand the importance of embodiment and culture in human evolution. In the first part of the paper I make some basic points on the use and abuse of the notions of embodiment, evolution, and culture. I cannot discuss the complex histories and debates that surround those concepts in different disciplines. I just want to clarify my understanding of those terms in order to avoid confusion about the claims I am going to make in the second part of this paper. I begin with embodiment. Specifically, I am interested, in what we call embodied cognition.

\section{From Embodiment to the Act of Embodying}

I admit there is something disconcerting about the term embodied mind. It can be argued that from the perspective point of phenomenology, ecological psychology, process, or pragmatist philosophy a term like 'embodiment' looks, rather, unnecessary. Indeed, for those committed to a genuinely dynamical grounding of human mind in bodily movement and to the tight connections between perception and action the skin was never a boundary. In order to appreciate the value and contribution of the embodied cognition approach it is necessary to understand the history of this theoretical paradigm as a critique of cognitivism ${ }^{2}$. Seen in this context the need for embodied cognition becomes clearer: the notion of embodied mind is needed because a big part of cognitive science operates still on the implicit assumption that the mind is essentially a disembodied representational engine inside the head of the individual. In recent decades the embodied cognition approach has been challenging that assumption trying to illustrate the

1 For a good discussion on the concept of evolvability, the question whether the ability to evolve itself evolves, its role within an extended evolutionary synthesis see Pigliucci (2008): Is evolvability evolvable?

2 I will use the term cognitivism in this paper to refer broadly to classical computational, representational, internalist and nativist modular theories of cognition. 
important ways that the body (and by extension the world) matters for thinking. Unfortunately, despite the growing contemporary interest in embodied cognition there is little agreement about just what it means (Gallagher 2005; Kiverstein 2012). Not surprisingly the label embodied cognition is used to refer to a variety of theories, some of which are more 'radical' than others (Shapiro 2014; Gallagher 2005; Kiverstein 2012).

Viewed from the perspective of material engagement theory those hard or 'radical' meanings and uses of the term embodied mind (e. g., Chemero 2009; Fuchs and De Jaegher 2009; Hutto and Myin 2013) according to which cognition is grounded in situated action, and is interdependently constituted by the specific kind of body we possess, are certainly more helpful than weak or 'simple' forms of embodiment ${ }^{3}$.

One important question that can help us understand those differences between radical and weak uses of embodied cognition concerns what is meant by 'body' here. I argue that if notions of embodiment are to have any real explanatory value in the study of culture and human evolution the 'body' ought to mean something different than the brain. This is not because the brain should be kept separate from the body, rather it is because if in our discussion of embodiment we allow the brain to qualify as part of the body we run the risk to trivialize the claim that the body is crucial to mental life ending up with some version of brain identity theory (Goldman and de Vignemont 2009, 154). What I call embodied cognitivism, namely, the limited representational view of embodied mind as something contained, localized in, caused by, or identifiable with the brain must be overcome. The embodied mind must be more than a collection of neural representations or it is an empty concept. This last point is worth stressing.

Another basic question in this context, of course, concerns the nature of the exact contribution of the body to cognition and about whether this contribution can be accounted for by way of classical computational models or whether something more radical is needed. It is necessary when we speak of embodiment as the condition of cognitive extension to clarify some important, often implicit, distinctions, such as those between derived and non-derived content, and between causal influence and constituency (Wheeler 2014, 378). More important for my purpose in this chapter, is the question of where does material culture fit into this emerging picture of the embodied mind? I should clarify then, that the meaning of embodied cognition in the context of MET is not signifying a simple shift from the disembodied computational image of mind to one that is now grounded in neural structures and brain networks. It is also not associated with a view of the mind as existing within the body's interior. Rather, it signifies that the details of embodiment and thus of action, situation, and mediation matter. Seen in this way embodiment is less of a property and more of a process, specifically, an act of embodying. This act of embodying forms the precondition for material engagement and vice versa. Taken together the two processes, embodying and mate-

\footnotetext{
${ }^{3}$ Here I draw on a useful distinction, proposed by Andy Clark (1999), between two varieties of embodiment: 'simple' versus 'radical' embodiment.
} 
rial engagement, explain the dynamical nature and variety of forms that human mental processes can take, and how they connect to the world they are about. Thus, it no longer makes any sense to separate an 'inner' domain of mentality from an 'outer' domain of materiality. Rather, embodied cognition involves and emerges from situated dynamic interactions between different types of materials and activities. The fact that a variety of material extra-neural resources (bodily, artefactual, or semiotic) contribute to human cognition also implies that given the unique properties of each material resource they will make a separate and distinct contribution. Embodiment is what brings those diverge resources and their properties together to form what we define as the human mind.

On that note I turn now to the concepts of evolution and culture.

\section{Evolution and Culture}

I will keep it simple. So far as basic biological constitution is concerned I take we all agree that human beings, like any other creature, can be described as the products of Darwinian evolution operating through dynamics of variation (mutation and recombination), inheritance, competition and selection. Things, however, are different, and remain heavily contested, when it comes to the neo-Darwinian theory or the so-called Modern Synthesis and the way that this has been applied in the study of culture and human cognitive evolution (see for review Mesoudi 2011; Mesoudi, Whiten, and Laland 2006). Indeed, there are limits to what can be explained by way of natural selection acting on genetic variation. Take for instance the traditional culture-gene coevolution theory by Boyd and Richerson (1985). It is based on the fundamental premise that there are two distinguishable, yet, complementary and interacting evolutionary processes, i. e., genetic evolution and cultural evolution. In summary, the main claim is that cultural evolution exhibits the Darwinian properties of variation, inheritance, and the accumulation of successive cultural modifications over time. In other words, the major claim is that 'culture' evolves like an organism does. Beliefs like genes, spread, persist or disappear. The question for a theory of cultural evolution is to understand why and how? Seeing culture as an evolutionary system in its own right implies that cultural change, as a form of non-genetic inheritance, could then be studied using similar methods and concepts to those already in use to study of biological evolution (e.g., population-dynamic concepts and evolutionary models). Such a unified approach to the study of cultural change might look appealing from a methodological or empirical perspective. However, it embodies various shortcomings especially from a material engagement perspective. I will just mention one important shortcoming that is more relevant to my purpose here: Neo-Darwinian theory is inherently 'cognitivist', it adopts fully the basic essentialist premises of evolutionary psychology which views the human mind as a collection of functionally specialized (i. e., domain-specific) universal computational modules fixed (via natural selection) inside the head (Cosmides and Tooby 1987; Barkow, Cosmides, and Tooby 1992; Pinker 2003). An immediate implication of adopting, 
implicitly or explicitly, such an internalist computational view of mind is that for the majority of the proponents of the Darwinian models of cultural evolution culture is essentially seen as acquired information (knowledge, beliefs, and values) that is inherited through social learning, stored in human brains, and expressed in behavior and artifacts (e. g., Mesoudi, Whiten, and Laland 2006; Richerson and Boyd 2005). For instance, Richerson and Boyd define culture as "information capable of affecting individual's behavior that the acquire from other members of their species through teaching, imitation, and other forms of social transmission" $(2005,5)$. As they explain, their use of the term information refers to any kind of mental state (idea, knowledge, belief, value, attitude or skill), conscious or not, "that is acquired or modified by social learning and affects behavior" (ibid).

I want to argue that this view of culture as a collection of internal representations inside the head and the epiphenomenal conception of material culture that it implies is deeply flawed. For one thing it misrepresents materiality as a passive means of adaptation and offers an erroneous view the relationship between cognition and material culture as one based on representation and information processing. For another it reiterates the nature/culture dichotomy. I should clarify here that my quarrel with the neo-Darwinian (Modern and Extended Synthesis) does not lie in the existence of non-genetic inheritance ${ }^{4}$ systems, rather, it concerns the ways those systems become conceptualized as disembodied information processes and the possible role they have been argued to play in human evolution. In principle I agree with the general epistemic contention that the most fundamental questions of human becoming "can only be answered by a theory in which culture has its proper role and in which it is intimately intertwined with other aspects of human biology" (Richerson and Boyd 2005, 4). But I cannot see how such an objective can be accomplished by reiterating conventional ideas of what count as culture, biology, mind or life. I take that very few people will seriously deny that culture is rooted in human biology or that we have an evolved psychology that shapes what we learn and the ways we think. But the important question, I want to suggest, is not one of influence or mere interaction, rather it is a question about constitution. Put it simply it is a question about what is the domain that really matters for explaining the phenomenon we are seeking to understand. What does it mean to say that "culture is part of biology" (Richerson and Boyd 2005, 4)? What is exactly the nature of the relationship or connection between biology and culture implied when we argue that "we are largely what our genes and our culture make us" (ibid, 7)? I will argue that as long the cognitive system and its development remains confined to the organism alone, environmental and cultural resources might influence the process of development and evolution but they remain fundamentally distinct and secondary if compared with genetic influences.

To be fair one could argue that, to some extent, the 'population thinking' approach to cultural evolution manages to avoid the fallacy of classical socio-bi-

${ }^{4}$ By non-genetic inheritance I refer broadly to changes (e.g., epigenetic, ecological or cultural) that occur independently of, or do not involve direct changes in the DNA sequence. 
ology (and memetics) by recognizing that culture change is not a mere extension of biological evolution. As Richerson and Boyd write: “[c]ulture is interesting and important because its evolutionary behavior is distinctly different from that of genes ... [c]ulture would never have evolved unless it could do things that genes can't!" $(2005,7)$.

Nonetheless, without denying that for gene-culture coevolution theory 'culture' is indeed recognized as an important influence the kind of 'interaction' that one can find at the heart of the gene-culture coevolution approach is far too soft to provide an adequate description of the relation between cognition and material culture.

Evolutionary psychology and neo-Darwinianism misrepresent human becoming because they misconstrue the relational ontology of embodiment, materiality and action. Their notion of culture divorced from the embodied, participatory and situated character of human ways of perceiving, acting, and thinking, losses its explanatory power and value.

How can we understand better those processes? I propose that we should view the human mind as the emergent dynamical product of this developmental conflation of embodiment, evolution and culture. What this implies is that when we speak about interaction between embodiment, evolution and culture we should not be thinking of three independently realized processes that somehow interact but, instead, of an ongoing ontological conflation, a constitutive intertwining of mind and matter. The later means that the nature of interaction is dynamic, transactional and participatory. Such a radical relational conception of human becoming able to accommodate the plasticity and situated character of material engagement can help us overcome the classical distinction between proximate (non-genetic) and ultimate (genetic) causes in biology (see Laland et al. 2013) offering new analytical bridges between the developmental and the evolutionary aspects of human cognitive becoming (see also Malafouris 2010; Malafouris 2015; Gosden and Malafouris 2015).

\section{Thinking as Thinging}

I now return to the image of the potter working with the clay (Figure 1). I want you to notice that what we see here is a single moment in the life history of this material object - if we can call it an object at this stage. If we want we could look at a different moment from an earlier stage in the process of making. We could look for instance at the very beginning where the act of centering the clay takes place, or instead, we can look at the final stages of the process, or maybe at the final product itself. However, there is a reason I chose to focus on that specific moment, rather than at some other stage of the process of making. There is something about that specific moment that is tying together the totality of the form-making processes brought about in the making of this particular thing (see also Gosden and Malafouris 2015). This moment binds together past, present and future in a way that is less visible at earlier stages of the process and is prob- 
ably completely invisible at the end (when the object is finished). Mainly this is because it is during that moment that the underlying tension between mind and matter is best revealed. The phenomenal consciousness of this object spreads in-between the states of matter and form. It is stable enough to be identified and named as a vase. But still, it remains plastic, open to change and transformation: an experiential mixture of materiality, affectivity, and creativity. In a way then this image speaks of two kinds of 'uncertainty' in the potter's way of thinking and feeling with and through the soft clay: one about origins and the other about endings. Uncertainty about origins refers to the processes responsible for the potter's form-making abilities. What kind of cognitive recourses are involved? There is also uncertainty about endings because as long as the object remains plastic it remains unfinished. I want to emphasize that moment of ongoing plasticity and incompleteness because I want to use it as the grounding metaphor for my perspective on human becoming and the evolution of mind. I will return to exemplify that in the last section. First I want to dwell a bit further on the transactional and participatory character of form-making. I said that this image helps us to visualize the ongoing dynamical tension between matter and form. What brings this tension about is, of course, the potter's body. But what is it exactly that moves the hand of the potter enabling the creation of this specific form out of clay? Let's take one step at a time starting with something more basic: Exactly how are things related to thinking? Where does the mind of the potter stop and the form of the object begin?

According to cognitivism the answer is simple: The potter's head offers a 'natural' demarcation line for separating pure mind-stuff from the clay. What about the cultural transmission and evolution of the embodied skills and ways of thinking involved in the art of potting? As we discussed the neo-Darwinian approaches to cultural evolution are firmly grounded in evolutionary psychology and its computational 'internalist' foundation. Whatever the culturally situated information and skills that enable the potter's fingers to know or discover the precise force, place and time of the appropriate grip they become acquired, learned and transmitted, like any other cultural recourse involved in pottery making, by means of internal representations. I imagine that this substitution of bodily skills with neural representations makes good sense if your approach to culture follows Richerson and Boyd's conviction "that most cultural variation is caused by information stored in human brains - information that got into those brains by learning from others" $(2008,5)$. But the neurocentric ontology of this representational logic is problematic: First, it precludes any causal role for material culture in human thought and its products often resemble a lifeless abstraction; second, it misleads us to think that all that really matters for the study of mind in culture, or of culture in mind, is to understand the nature of the internal mental representations and the input/output mechanisms that enable their formation, acquisition, and cultural transmission. In other words, on this construal, embodiment does not matter in culture or human evolution.

Here is the problem or paradox then: If you are committed to cognitivism you don't need embodiment or culture; all you need is system able to process 'inter- 
nal representations,' that is, disembodied information learned and transmitted across generations.

Many people working in the sciences of the mind from a variety of embodied, extended, distributed and enactive perspectives think that there are alternative ways to conceptualize the relations between brains, bodies and the world avoiding the representationalist treatment of cognition as a computational 'black box' (Varela, Thompson, and Rosch et al. 1993; Clark 1997, 2008; Hutchins 1995, 2010; Hutto and Myin 2013; Chemero 2009; Wheeler 2005; Noë 2004; Sutton 2008; Menary 2010). Similarly, the material engagement approach that I advocate (Malafouris 2013) insists on the one hand, that the brain can only be understood as one element of a larger intelligent bodily system that incorporates material culture, and on the other hand, that the physical location and ontology of human intelligence remains an open question. As we discussed earlier embodiment, at least in the radical sense that matters for human cognitive evolution and is consistent with theoretical principles of MET, comes with the price of continuity between cognition and material culture, or else between cognition, perception and mediated bodily action. The implications of this continuity are not well understood yet. MET insists that only a thorough reconfiguration of the relation between cognition and material culture can provide a feasible alternative framework to battle the cognitivism of classical evolutionary psychology. The organism's worldly engagements, and acts of making, become the new analytical unit for the study of mind. Obviously, I do not mean to question the neural bases of cognition. I want simply to underline that from a material culture-perspective one can hardly find any convincing reason - besides convention - why the study of the mind should be restricted to neural processes occurring inside an individual's brain. Rather, what we see is both equivalence and complementarity of what is 'inside' with what is 'outside'. Brains, bodies and things participate equally, albeit in different ways, in human cognitive life.

At that point I would like to introduce the notion of thinging, and more specifically what I call creative thinging (Malafouris 2014; cf. what Gallagher and Ransom call artifacting (this volume). I use those notions to articulate and draw attention into the varieties or kinds of cognitive life instantiated specifically in acts of thinking and feeling with, through, and about material things. The philosopher Martin Heidegger, in his famous essay "Das Ding” (The Thing) (1975, 166), uses the same term, i. e., "thinging" to express how things "gather" space and time tying together their material constituents. My own use of the term thinging tries to retain something of this original sense of 'gathering' at its heart. But I also diverge from the Heideggerian phenomenological path, especially relevant to one aspect of 'thingness' that is typically cast in the shadow: the vitality and agency of things in human thinking, or else, the cognitive life of things (Malafouris 2008a; Malafouris and Renfrew 2010; Bennett 2010; Sutton 2002). More specifically, I use this term to differentiate the active participatory process by which things are presented to us through acts of engagement and enactive signification, from the passive process by which things are often construed as re-presented in us by way of internalization and mental substitution. Thinging 
is inherently dynamical and non-representational, although in certain contexts it can become the vehicle for external representation.

Thinging incorporates time-varying and culture specific artefacts, material assemblages and bodily techniques; it also extends to sensory and cognitive prostheses and interfaces of any kind. This description of thinging is probably too general to serve taxonomic considerations. But in its lack of analytic closure it opens up new possibilities for transgressing some of our common-sense assumptions about what minds and things are, and about how they relate and connect to each other. The analytical value of the notion thinging, then, lies in helping us to disentangle, on the one hand, from a cognitivist view of what mind consists of, and on the other from a narrowly modernist definition of what matter consists of. In any case, the basic premise that I want to communicate through the notion of thinging is quite simple: We humans are thingers. What is more important, we have been altering our own developmental paths by making new things and by changing our means of material engagement. Not only most of our thinking is thinking with, through and about things, it is also, creative thinging (Malafouris 2014). Humans are creators of new things which constantly change the ecology of our minds and re-shape the boundaries of our thinking. This brings us back to the question I raised at the beginning: How can we make sense of the embodied form-making activities that made us what we are?

No other animal has been or can be defined as a species on the basis of its relationship with the variety of things and material forms that it makes. We humans are precisely a species of this rather strange sort. Let me explain, I say we are strange, not because, I deny that other animals are capable of thinging - the rapidly expanding literature on animal tool use proves that to a certain extent they are $^{5}$. Nor do I say we are strange because I believe that by making things, and by thinking through things we overcome the limits of our 'nature' and enter into a separate 'cultural' realm. No other controversy in the study of human becoming has been more unhelpful, misleading and sterile than that between nature and culture. There is no moment in the history of human evolution where biology gave way to culture. The following quote from Griffiths and Stotz (2000, 44-5) nicely summarize the crux of the problem:

Human development is, not uniquely but certainly outstandingly, reliant on external scaffolding. This scaffolding is commonly referred to as culture. Part of the rationale of the traditional idea of human nature was to isolate features that do not depend on culture. These 'biological' features represent our true nature - the naked ape stripped of its cultural clothes. It seems to us that this traditional project is as misguided as seeking to investigate the true nature of an ant by removing the distorting influence of the nest! Human beings and their cultures have co-evolved as surely as ants and hives or dogs and packs. Human nature must inevitably be a product of a developmental matrix which includes a great deal of cultural scaffolding. (Griffiths and Stotz 2000, 44-5).

\footnotetext{
5 The ability of using elementary tools it is well demonstrated, in both nature and captivity, most famously by chimpanzees (Pan troglodytes) and New Caledonian crows (Corvus moneduloides) (for a good review see Biro, Haslam, and Rutz 2013.
} 
By the same token, the kind of thinking in anthropology, rooted in A. L. Kroeber's tradition of 'superorganicism' (Kroeber 1917) which dismisses any need to incorporate biology in the study of human culture is obviously false and must be rejected. None of the empirical phenomena people often describe under the headings of human biology and human culture - not even the most basic human actions and movements - makes sense, or even exists, in isolation. Human becoming has always been inseparably linked with the developmental contingencies of action. No bodily activity - not even our basic capacity for bipedal locomotion is immune to the situated dynamics of real life ontogenetic development.

As the anthropologist Marcel Maus neatly points out in his famous essay on bodily techniques, there is no "natural" way to move our bodies (Mauss [1935] $1973,74)$. But I suggest it would be equally wrong to think of bodily movement as an isolated 'culturally' induced phenomenon. There is no "natural" or "cultural" way to move our bodies. Instead, we should think of the acting body as a relational developmental achievement of situated material engagement. For instance, the potter's body, entering into the craft of pottery making, working with clay and developing the skills of the practice, it becomes more than a body; it becomes situated. That is, it becomes specific and different (it embodies a unique developmental life history). It becomes affected by the physical and psychoactive properties of clay, by the patterns and rhythms of coordination, and by the kinesthetic experiences, muscular memories and skills attached to it. As we discussed from a material engagement-perspective the meaning of the term body, far from reductive and fixed, refers to the details of bodily implementation (neural and extra-neural) and action-taking potentials as those can be determined by the nature of local interactions, cultural practices and prostheses. In a way then the potter's body and mind are one.

This last point brings us right at the heart of the phenomenon I am trying to capture when I talk about human strangeness: we humans have made thinging in general, and creative thinging in particular, part of our nature. Put it simply, with thinging biological heredity becomes creative evolution. Instead of simply reproducing ourselves, we rather extend ourselves and we construct new cognitive and material ecologies for growing and instituting our minds. We create things which in turn create us (for different perspectives on that see Malafouris 2013; Malafouris and Renfrew 2010; Hodder 2012; Gosden 2008, 2005; Olsen 2010; Webmoor and Witmore 2008; Knappett and Malafouris 2008b; Bennett 2010; Knappett 2005, 2011; Renfrew 2004, 2007; Schiffer and Miller 1999; Latour 2005). This is what I call the priority of material engagement.

\section{Metaplasticity and Incompleteness}

What does it mean? To answer that I return to the comment about 'human incompleteness' I've made before. We are used to think of our 'sapient' minds, with all our unique capacities, as the apex of evolution. This vision of humanity as 'complete' and 'fixed', and the concomitant neo-Darwinian ideals of cognitive 
and behavioral 'modernity,' is almost certainly wrong (Nagel 2012; Tallis 2011; Ingold 2013; Ingold and Pálsson 2013; Barrett 2011, 2014; Dupré 2008). From the perspective of the material engagement approach the human mind emerge as an 'open' unfinished project, potentially, in a permanent state of on-going evolution. I said before that we humans are different from other animals in that we have made thinging our nature. I would also insist that we humans are also a species extensive and, thus, 'incomplete.' What I am proposing is that we humans, unlike any other species, seem to have more than just a plastic mind: we have a mind which is also inextricably intertwined with a plastic culture. I am not saying that other species are not plastic or intelligent in their own special ways. And, obviously, I am not just talking about brain or neural plasticity here. I am trying to gesture towards a different kind of plasticity: the plasticity of a mind not limited by the skin. I call that metaplasticity (Malafouris 2015; 2013, 2010).

The notion of metaplasticity seen as an expression for human biosocial becoming can be linked with other non-genetic forms of biological organization but it should not be confused or conflated with notions of individual phenotypic plasticity and variation. It represents an alternative to the Neo-Darwinian forms of non-genetic inheritance (like epigenetic inheritance or cultural transmission) that is based on the logic of enactivism and operates by means of creative material engagement.

The focus here is on understanding the nature of plastic changes, not at the level of the individual, but in the broader systemic context (cultural or social) where thinging takes place. At this higher level of engagement with the material world where neural and cultural plasticity interact and exchange properties, material culture competes, equally with any other brain region, for a place in the human cognitive system. The active mind has no a priori location. Human beings, understood as real living creatures that move and act in this world, are the developmental products of this conflation or 'meshwork' (Varela, Thompson, and Rosch 1993; Knappett 2011) of brains, bodies and things. If you separate the three - if you separate brain, body and culture - you end up with a number of overly simplified abstractions about lifeless categories. I am not denying the analytical value of those abstractions for many disciplines; but I do think they are misleading when it comes to understanding human becoming.

\section{Conclusion}

Our understanding of how evolution works is rapidly changing (see Laland et al. 2014). Many important shortcoming of mainstream neo-Darwinian evolutionary thought have been exposed. For instance, since the introduction of niche-construction theory to evolutionary biology by Lewontin in the 1980 s (Lewontin 1983, 2000), there has been surmounting evidence on how organisms often "act as co-directors of their own and other species' evolution" by modifying natural selection in their environment (Laland, Odling-Smee, and Myles 2010, 139; Laland, Odling-Smee, and Feldman 2000; Laland et al. 2009; Lewens 2003; Odling-Smee, 
Laland, and Feldman 2003; Oyama, Griffiths, and Gray 2001; Sterelny 2003; Donohue 2005). Moreover, the recent calls for an Extended Evolutionary Synthesis has been very influential in understanding better how non-genetic processes by which organisms grow and develop can be drivers of evolution, and thus must be seen as part of evolutionary theory (see Laland et al. 2014; Pigliucci and Muller 2010; Jablonka, Lamb, and Zeligowski 2014; Odling-Smee et al. 2003).

I find the general idea of an Extended Evolutionary Synthesis appealing - for the same reasons that I find the broad idea of extended cognition appealing. Still many of the conceptual barriers confronting neo-Darwinian theories remain. To give one example, a further problem neo-Darwinian models inherit from evolutionary psychology, beyond the mentioned computational character of psychological adaptations and the related arguments about their modular internal structure, is the false dichotomy between evolved (innate) domain-specific structures and learned behaviors. One reason for the persistence of this false opposition, of a universal evolved human nature versus our culture-specific ways of learning, can be found in the influence that computational thinking still has on the way psychology traditionally understands the basic structure of human mental architecture. Another reason, as Barrett et al. point out, is probably because the main argument about human evolution "is framed in terms of adaptation, when the real issue ... [is] the degree of plasticity or flexibility shown by our learning mechanisms" (Barrett, Pollet, and Stulp 2014, 5).

From the perspective of MET, I suggest that we need to resist any tendency at reducing the nature of change in human beings to a series of underlying domain-specific adaptations and shift the focus, instead, on processes of deep enculturation, dynamical enskilment, and 'profound embodiment' (Clark 2008; Wheeler and Clark 2008; Chiel and Beer 1997). What this means essentially is that there is no pre-determined central driver (genetic or cultural) but instead a temporally emergent coalition of situated developmental forces. Moving away from the previously described dichotomous account of development and evolution toward an account incorporating elements from developmental systems theory (DST) (Oyama, Griffiths, and Gray 2001; Griffiths and Gray 1994, 2001; Griffiths and Stotz 2000), 'niche-construction theory' (Odling-Smee et al. 2003; Sterenly 2003; Stotz 2014), and neuroconstructivism (Mareschal et al. 2007; Quartz and Sejnowski 1997) and probabilistic epigenesis (Gottlieb 2003, 2007) will put us in a better position to recognize the multiplicity and dynamical character of resources contributing to the developmental process. Although some more recent 'neo-evolutionary' approaches, adopting a more interactive outlook have been successful in pointing out the importance of what Karola Stotz calls "the human-being-in-its-developmental-niche" (Stotz 2010, 498-99) and dispense with the need of genetically specified abstractions they retain still much of the basic neo-Darwinian logic of the adaptationist program ${ }^{6}$ (Sterelny 2003;

\footnotetext{
${ }^{6}$ For example, Richerson and Boyd argue "that the human cultural system arose as an adaptation, because it can evolve fancy adaptations to changing environments rather more swiftly than is possible by genes alone" $(2005,7)$.
} 
Jablonka and Lamb 2005; Richerson and Boyd 2008). What the study of culture and human evolution needs is not a more extended version of the neo-Darwinian representational logic but instead a more radical, process-based, post-Darwinian enactive conceptualization. Of course, that is more easily said than done. As pointed out by Varela, Thompson, and Rosch at their seminal work The Embodied Mind: Cognitive Science and Human Experience:

Part of the difficulty in moving beyond the adaptationist framework is to determine what to do after we abandon the idea of natural selection as the main explanation, so that every structure, mechanism, trait, or disposition cannot be explained away by its contribution to survival value. The temptation is to say, But then are things there for no reason at all? The task in evolutionary biology is to change the logical geography of the debate by studying the tangled, circular relations of congruence among the items to be explained. (Varela, Thompson, and Rosch [1991] 1993, 195; italics added)

The perspective of material engagement, I propose here, brings with it a new emphasis on materiality and on the long-term developmental mechanisms by which the bidirectional, mutual constitution of brain and culture occurs. From the perspective of material engagement theory the recognition of the material world as a constitutive and efficacious part of the human cognitive system means that interaction elicited by our surroundings (human or nonhuman) not only influences our cognitive abilities and affective responses from the very beginning but also shapes the form and the constitutive mechanisms of interaction. Different forms of material culture demand and dictate different kinds of engagement and forms of relatedness. New materialities (objects, materials, or assemblages) bring about new modes of acting and thinking. Those changes are not simply epiphenomenal added layers of complexity and variation on a stable evolved core of natural cognitive capacities. Instead, they penetrate and alter the historical constitution of that core.

This recognition, that the relationship between an organism and its relative niche can be modified and that those alterations (conscious in the case of humans) matter to the evolutionary process, also demonstrates the evolutionary significance of the huge variability we see archaeologically in material culture and the built environment. If there is anything truly distinctive and universal about human mind it must be its openness to cultural influence and variation. This is not to deny that any evolved genetically guided structures exist, but instead to recognize that genetic and neurobiological underpinnings do not determine anything before and outside the specific developmental pathways and situational affordances of material engagement.

\section{Bibliography}

Barkow, J. H., L. Cosmides, and J. Tooby. 1992. The Adapted Mind: Evolutionary Psychology and the Generation of Culture. New York: Oxford University Press.

Barrett, L. 2011. Beyond the Brain: How Body and Environment Shape Animal and Human Minds. Princeton, NJ: Princeton University Press. 
Barrett, L., T. V. Pollet, and G. Stulp. 2014. From Computers to Cultivation: Reconceptualizing Evolutionary Psychology. Frontiers in Psychology 5: 1-14.

Bennett, J. 2010. Vibrant Matter: A Political Ecology of Things. Durham, NC: Duke University Press.

Boyd, R. and P.J.Richerson. 1985 Culture and the Evolutionary Process. Chicago: University of Chicago Press.

Biro, D., M. Haslam, and C. Rutz 2013. Tool use as adaptation. Philosophical Transactions of the Royal Society B: Biological Sciences 368 (1630): 20120408.

Chemero, A. 2009. Radical Embodied Cognitive Science. Cambridge, MA: MIT Press.

Chiel, H.J. and R. D. Beer. 1997. The brain has a body: Adaptive behavior emerges from interactions of nervous system, body and environment. Trends in Neuroscience, 20, $553-557$.

Clark, A. 1997. Being There: Putting Brain, Body and World Together Again. Cambridge, MA: MIT Press.

-. 1999. An embodied cognitive science? Trends in cognitive sciences 3 (9): 345-351.

-. 2008. Supersizing the Mind: Embodiment, Action, and Cognitive Extension. Oxford: Oxford University Press.

Cosmides, L. and J. Tooby. 1987. From Evolution to Behavior: Evolutionary Psychology as the Missing Link. In The Latest on the Best: Essays on Evolution and Optimality, ed. J. Dupré, 277-306. Cambridge, MA: MIT Press

Dupré, J. 2008. Against Maladaptationism: Or What's Wrong with Evolutionary Psychology? In Knowledge as Social Order: Rethinking the Sociology of Barry Barnes, ed. M. Mazzotti, 165-80. Aldershot, England: Ashgate.

Fuchs, T. and H. De Jaegher 2009. Enactive intersubjectivity: Participatory sense-making and mutual incorporation. Phenomenology and the Cognitive Sciences 8 (4): 465-486. DOI: $10.1007 /$ s11097-009-9136-4

Gallagher, S. 2005. How the body shapes the mind. New York: Cambridge University Press.

Gallagher, S. and T. G. Ransom. 2016. Artifacting Minds: Material Engagement Theory and Joint Action. In this volume.

Gosden, C. 2005. What Do Objects Want? Journal of Archaeological Method and Theory 12: $193-211$.

-. 2008. Social ontologies. Philosophical Transactions of the Royal Society of London Series $B, 363,2003-10$.

Gosden C. and L. Malafouris, 2015 Process archaeology (P-Arch). World Archaeology, 47 (5): 1-17.

Gottlieb, G. 2003. On Making Behavioral Genetics Truly Developmental. Human Development 46: 337-55.

-. 2007. Probabilistic Epigenesis. Developmental Science 10: 1-11.

Goldman, A. and de Vignemont, F. 2009. Is social cognition embodied? Trends in cognitive sciences 13 (4): 154-159.

Griffiths, P.E. and R. D. Gray. 1994. Developmental Systems and Evolutionary Explanation. Journal of Philosopby XCI: 277-304.

-. 2001. Darwinism and Developmental Systems. In Cycles of Contingency: Developmental Systems and Evolution, ed. S. Oyama, P. E. Griffiths, and R. D. Gray, 195-217. Cambridge, MA: MIT Press.

Griffiths, P. E. and K. Stotz. 2000. How the Mind Grows: A Developmental Perspective on the Biology of Cognition. Synthese 122: 29-51.

Heidegger, M. 1975. “The thing”. In Poetry, Language, Thought. Hofstadter, A. (trans.). New York: Harper \& Row, 161-184.

Hodder, I. 2012. Entangled: An Archaeology of the Relationships between Humans and Things. John Wiley \& Sons. 
Hutchins, E. 1995. Cognition in the Wild. Cambridge, MA: MIT Press.

-. 2010. Cognitive Ecology. Topics in Cognitive Science 2: 705-15.

Hutto, D. and E. Myin. 2013. Radicalizing Enactivism. Cambridge, MA: MIT Press.

Ingold, T. 2013. Prospect. In Biosocial Becomings: Integrating Social and Biological Anthropology, ed. T. Ingold and G. Pálsson, 1-21. Cambridge: Cambridge University Press.

Ingold, T. and G. Pálsson, eds. 2013. Integrating Social and Biological Anthropology. Cambridge: Cambridge University Press.

Jablonka, E. and Lamb, M. J. 2005. Evolution in four dimensions. Cambridge, MA: MIT Press.

Jablonka, E., M. J. Lamb, and A. Zeligowski 2014. Evolution in Four Dimensions: Genetic, Epigenetic, Behavioral, and Symbolic Variation in the History of Life. Cambridge, MA: MIT Press.

Kiverstein, J. 2012. The meaning of embodiment. Topics in Cognitive Science, 4(4), 740758.

Knappett, C. 2011. An Archaeology of Interaction: Network Perspectives on Material Culture and Society. Oxford: Oxford University Press.

-. 2005. Thinking through Material Culture: An Interdisciplinary Perspective. Philadelphia: University of Pennsylvania Press,.

Knappett, C., and L. Malafouris, eds. 2008. Material Agency: Towards a Non-anthropocentric Approach. New York: Springer.

Kroeber, A. L. 1917. The superorganic. American anthropologist 19 (2): 163-213.

Laland, K. N., J. Odling-Smee, and M. W. Feldman. 2000. Niche Construction, and Cultural Change. Behavioral and Brain Sciences 23: 131-46

Laland, K. N., J. Odling-Smee, and S. Myles. 2010. How culture shaped the human genome: bringing genetics and the human sciences together. Nature Reviews Genetics 11: (2): 137-148.

Laland, K. N., J. Odling-Smee, W. Hoppitt, and T. Uller. 2013. More on how and why: cause and effect in biology revisited. Biology E Philosophy 28 (5): 719-745.

Laland, K., T. Uller, M. Feldman, K. Sterelny, G. B. Müller, A. Moczek, E. Jablonka, et al. 2014. Does evolutionary theory need a rethink? Nature 514 (7521): 161.

Latour, B. 2005. Reassembling the Social: An Introduction to Actor-Network-Theory. Oxford: Oxford University Press,.

Lewens, T. 2003. Prospects for Evolutionary Policy. Philosophy 78 (2003): 495-514.

Lewontin, R. C. 1983. Gene, Organism and Environment. In Evolution from Molecules to Men, ed. D. S. Bendall, 273-85. Cambridge: Cambridge University Press.

-. 2000. The Triple Helix: Gene, Organism, and Environment. Cambridge, MA: Harvard University Press.

Malafouris, L. 2008a. At the Potter's Wheel: An Argument for Material Agency. In Material Agency: Towards a Non-anthropocentric Perspective, ed. C. Knappett and L. Malafouris, 9-36. New York: Springer,.

-. 2008b. Beads for a Plastic Mind: The 'Blind Man's Stick' (BMS) Hypothesis and the Active Nature of Material Culture. Cambridge Archaeological Journal 18: 401-14.

-. 2010. Metaplasticity and the Human Becoming: Principles of Neuroarchaeology. Journal of Anthropological Sciences 88: 49-72.

-. 2013. How Things Shape the Mind: A Theory of Material Engagement. Cambridge, MA: MIT Press.

-. 2014. "Creative thinging: The feeling of and for clay." Pragmatics and Cognition 22 (1): $140-158$.

-. 2015. Metaplasticity and the Primacy of Material Engagement. Time and Mind, 8(4): $351-371$ 
Malafouris, L. and C. Renfrew. 2010. The Cognitive Life of Things: Archaeology, Material Engagement and the Extended Mind. In The Cognitive Life of Things: Recasting the Boundaries of the Mind, ed. L. Malafouris and C. Renfrew, 1-12. Cambridge: McDonald Institute Monographs.

Mauss, Marcel. (1935) 1973. Techniques of the Body. Economy and Society 2 (1973): 70-88.

Mareschal, D., M. Johnson, S. Sirois, M. Spratling, M. Thomas, and G. Westermann, G., eds. 2007. Neuroconstructivism, Vol. I: How the brain constructs cognition. Oxford, UK: Oxford University Press.

Menary, R. 2010. The Extended Mind. Cambridge MA: MIT Press.

Mesoudi, A. 2011. Cultural Evolution: How Darwinian Theory Can Explain Human Culture and Synthesize the Social Sciences. Chicago: University of Chicago Press.

Mesoudi, A., A. Whiten, and K. N. Laland. 2006. Towards a Unified Science of Cultural Evolution. Behavioral and Brain Sciences 29: 329-83.

Noë, A. 2004. Action in Perception. Cambridge, MA: MIT Press.

Odling-Smee, F.J., K. N.Laland, and M. W. Feldman 2003. Niche Construction: The Neglected Process in Evolution. Princeton, NJ: Princeton University Press.

Olsen, B. 2010. In Defense of Things: Archaeology and the Ontology of Objects. Plymouth, UK: Altamira.

Oyama, S., P. E. Griffiths, and R. D. Gray 2001. Cycles of Contingency: Developmental Systems and Evolution. Cambridge, MA: MIT Press.

Pigliucci, M. and G. B. Muller 2010. Evolution: the extended synthesis. Cambridge, MA: MIT Press.

Pigliucci, M. 2008. Is evolvability evolvable? Nature Reviews Genetics 9 (1): 75-82.

Pinker, S. 2003. The Blank Slate: The Modern Denial of Human Nature. London: Penguin.

Quartz, S. R. and T.J. Sejnowski. 1997. The Neural Basis of Cognitive Development: A Constructivist Manifesto. Behavioral and Brain Sciences 20: 537-96.

Renfrew, C. 2004. Towards a Theory of Material Engagement. In Rethinking Materiality: The Engagement of Mind with the Material World, ed. E. DeMarrais, C. Gosden, and C. Renfrew, 23-32. Cambridge: The McDonald Institute for Archaeological Research.

-. 2007. Prehistory: The Making of the Human Mind. London: Weidenfeld \& Nicolson,

Richerson, P.J. and R. Boyd. 2005. Not by Genes Alone: How Culture Transformed Human Evolution. Chicago: University of Chicago Press.

Shapiro, L. ed. 2014. The Routledge Handbook of Embodied Cognition. Routledge.

Schiffer, M. B. and A. R. Miller. 1999. The Material Life of Human Beings: Artifacts, Behaviour and Communication. London: Routledge.

Sterelny, K. 2003. Thought in a hostile world: The evolution of buman cognition. New York: Wiley-Blackwell.

Stotz, K. 2010. Human Nature and Cognitive-Developmental Niche Construction. Phenomenology and the Cognitive Sciences 9: 483-501.

-. 2014. Extended Evolutionary Psychology: The Importance of Transgenerational Developmental Plasticity. Evolutionary Psychology and Neuroscience 5: 1-14.

Sutton, J. 2002. Porous memory and the cognitive life of things, in Prefiguring Cyberculture: An Intellectual History, eds. D. Tofts, A. Jonson \& A. Cavallaro. Cambridge (MA): MIT Press, 130-41.

-. 2008. Material Agency, Skills and History: Distributed Cognition and the Archaeology of Memory. In Material Agency: Towards a Non-anthropocentric Perspective, ed. C. Knappett and L. Malafouris, 37-56. New York: Springer.

Tallis, R. 2011. Aping Mankind: Neuromania, Darwinitis and the Misrepresentation of Humanity. Durham: Acumen.

Varela F. J., E. Thompson, and E. Rosch. (1991) 1993. The Embodied Mind: Cognitive Science and Human Experience. Cambridge, MA: MIT Press. 
Webmoor, T. and C. L. Witmore. 2008. Things Are Us! A Commentary on Human/Things Relations under the Banner of a 'Social' Archaeology. Norwegian Archaeological Review 41: 53-70.

Wheeler, M. 2005. Reconstructing the cognitive world: The next step. MIT press.

-. 2014. The future prospects for embodied cognition. In L. Shapiro (Ed.), The Routledge handbook of embodied cognition. Routledge.

Wheeler, M. and A. Clark. 2008. Culture, Embodiment and Genes: Unravelling the Triple Helix. Philosophical Transactions of the Royal Society B: Biological Sciences 363: $3563-75$. 



\title{
Metaplasticit-ies: Material Engagement Meets Mutational Enhancement
}

\author{
Duilio Garofoli
}

\begin{abstract}
Material engagement theory (Malafouris 2013) rejects the idea that human cognitive evolution is the result of a passive process of natural selection. Cognitive capabilities and behavioral outcomes do not stem from hard-wired neurocognitive modules as the most appropriate solutions to adaptive problems. In contrast to these neurocentric tenets, cognitive evolution is explained as an enactive process, according to which humans bring forth new meanings and cognitive abilities by thinking with artifacts and through artifacts. Human minds thus emerge from a bio-cultural transformative process driven by the plasticity of culture in relation to the plasticity of the brain (i. e., metaplasticity). However, material engagement theory is threatened by a serious risk of misuse in cognitive evolution theory. Neuroplasticity, in fact, does not warrant that every human biological system, provided with the appropriate conditions of cultural engagement with artifacts, can bring forth any potential cognitive function. Drawing upon the principles of neuroconstructivism, I will argue that the enactive/transformative process of material engagement is compatible with a concept of "acquired" modularity, and at the same time also limited by innate constraints within human biological systems. Thus, new cognitive properties could still require mutational enhancement as a necessary condition of their being acquired. In this way, I will explore the possibility that different hominins, situated within different environments/cultures and subject to specific biological alterations, could have evolved different metaplasticit-ies.
\end{abstract}

\section{Introduction}

Cognitive archaeology is a developing discipline that tackles the problem of human cognitive evolution by integrating knowledge from several fields of enquiry, including paleoanthropology (Benazzi et al. 2011; Bruner 2010), empirical archaeology (Conard and Bolus 2003; d'Errico 2003; Zilhão 2011a), cognitive neuroscience (Coolidge and Wynn 2005; Stout and Chaminade 2007), genetics (Krause et al. 2007), archaeological theory (Hodder 2012; Knappett 2005), embodied cognition (Malafouris 2013), semiotics (Preucel 2007), linguistics (Botha 2010; Noble and Davidson 1996; Johansson 2015) and philosophy of mind (Sterelny 2011). These different approaches are brought together by the logic of reconstructing the properties of ancient minds from the behavioral traces left in the archaeological record of extinct human populations (Renfrew 1994; Garofoli and Haidle 2014; Wynn and Coolidge 2009; Dubreuil 2011). 
Since the first era of this discipline, the most critical problem has focused on what Colin Renfrew (1996) has called "the sapient paradox," namely the apparent mismatch between the rise of a modern anatomy and the emergence of a set of behaviors that overlap with those shown by historical/ethnographic human populations. Current evidence in paleoanthropology shows indeed that modern human anatomical forms could be identified in the African Middle Stone Age as early as $200 \mathrm{kya}$ (McDougal, Brown, and Fleagle 2005; White et al. 2003). By contrast, behaviors that resemble those found in ethnographic populations, which define the controversial concept of "behavioral modernity" (Wadley 2001; Henshilwood and Marean 2003; Conard 2010; Garofoli 2016; Shea 2011) took much longer to manifest in the record. Great interest was therefore focused on defining the mechanisms that explained this disconnection. Three alternative models were defined over time as a result of this research. These models differentiate for the role that culture and biology respectively undertake in explaining the emergence of behavioral modernity and, in particular, the cognitive capabilities and limits of archaic human populations (e.g., Neanderthals), as opposed to those of modern humans (d'Errico and Stringer 2011; Nowell 2010).

The first perspective, commonly referred to as the "human revolution" model (Mellars and Stringer 1989; Bar-Yosef 1998; Klein and Edgar 2002), argues that the European Upper Paleolithic archaeological record shows discontinuity in both the quality and quantity of new artifact categories, compared to the African Middle Stone Age (Klein 2008; Mellars 2005). Besides some limited and controversial cases (d'Errico and Nowell 2000), ivory figurines (Wynn, Coolidge, and Bright 2009) and cave art (Fritz and Tosello 2007), for example, have no precedents in the Middle Stone Age. At the same time, artifacts such as body ornaments or laminar technologies, which are poorly represented in Middle Stone Age sites, become abundant during the Upper Paleolithic (Klein 2000). In this sense, revolutionary models argue that the Upper Paleolithic marks a discrete leap in modern human technological sophistication (cf. Welsch, this volume). This approach is usually combined with the idea that mutational enhancement, possibly incurring in some human populations in Africa at ca. $60 \mathrm{kya}$, represents the most plausible explanation for the cultural enhancement that characterized the florescence of innovations typical of the Upper Paleolithic (Klein 2000; Mellars 2006). At the same time, these models defend the idea that cognitive enhancements exerted a remarkable role in the replacement of Neanderthals by modern human populations (Lewis-Williams 2002; Mithen 1996; Stringer and Gamble 1993; Wynn and Coolidge 2004).

Although the "human revolution" has long received great attention in cognitive archaeology, new archaeological data from several South African Middle Stone Age sites has led to a reconsideration of these initial assumptions. Evidence has come to light of artifact kinds, previously associated with the Upper Paleolithic only, dating back prior to the $60 \mathrm{kya}$ threshold postulated by the partisans of the "Upper Paleolithic Revolution" (Nowell 2010). In particular, engraved ochre fragments (see Anderson 2012, for review), perforated shells used as body ornaments (d'Errico et al. 2005; Bouzouggar et al. 2007; d'Errico et al. 
2009), burials (Schwarcz et al. 1988), laminar tools (Barham 2001; Henshilwood 2009) and bone tools (Yellen et al. 1995; d'Errico and Henshilwood 2007) have been reported in several locations within the African continent. In this light, McBrearty and Brooks (2000) argued that the apparently discrete explosion of innovations characterizing the Upper Paleolithic was in fact produced by an underlying gradualist dynamic. A slow incremental process of cultural evolution deeply rooted in the African Middle Stone Age proceeded for millennia until it reached a threshold. After this threshold, social and technological factors began to mutually reinforce each other, causing cascade events of population growth, technological diffusion, and leaps in innovation, leading modern humans to migrate out of Africa and invade Europe. The Upper Paleolithic revolution is thus only an illusion provided by a threshold effect, while cultural, demographic and environmental factors, rather than mutations within the brain, are sufficient to explain the burst of innovation typical of the Upper Paleolithic. However, this approach still maintains that the emergence of Upper Paleolithic innovations is a prerogative of modern humans alone (d'Errico and Stringer 2011, 1061).

The opposite extreme to the biological position is offered by the "cultural school" (d'Errico 2003; Speth 2004; Villa and Roebroeks 2014; Zilhão 2007). Partisans of this model attempted to demonstrate that Upper Paleolithic innovations are neither constrained by biology, nor necessarily connected with modern anatomy. Instead, variations in the environmental, demographic and social conditions (i. e., the context) were to be considered as the unique factors leading different human populations in different locations and times to develop more advanced behavioral practices as a response to ecological pressures. Recent studies that ascribe to Neanderthal populations in Europe the independent production of body ornaments (e. g., Finlayson et al. 2012; Zilhão et al. 2010), pigments (d'Errico and Soressi 2002), hafted weapons (Mazza et al. 2006), burials (Grün and Stringer 2000), laminar blades (d'Errico et al. 1998), bone tools (Soressi et al. 2013) were used to give credit to this idea (for review, see d'Errico 2003; Zilhão 2007, 2011a, 2011b, 2012; Villa and Roebroeks 2014). In consequence, advocates of the cultural school argue that the same cognitive/biological bases were present in humans since the Middle Pleistocene (ca. 500 kya; see Nowell 2010 and references therein; d'Errico and Stringer 2011; Soffer 2009) and hence that modern and archaic populations were cognitively equivalent (Burdukiewicz 2014; Zilhão 2007).

The debate about the emergence of modern behavior is dominated by a series of intuitions that have roots in a secular opposition between contrasting philosophical traditions. While the biological models find a parallel with the rationalist conceptions of innate abilities, cultural/context-based approaches rest instead on empiricist grounds. At the same time, modern-centric intuitions are now viewed as distorting any theories which compare Homo sapiens and Neanderthal cognition. Mutational enhancement approaches are perceived as integral to the Victorian idea that modern humans evolved a complete/perfect brain, which allowed them to prevail over the primitives (Zilhão 2011a). On the other hand, the guilt for having downplayed Neanderthals for decades (Papagianni and Morse 2013) 
has fostered new dogmatic positions which reject the existence of any biological differences between human species (Klein 2008, 272).

These intuitions have exerted dramatic influence on cognitive archaeological debates, generating opposition between theoretical agendas which often lack solid justification. Many of the current approaches have neglected to explain the very mechanisms of interaction between cultural changes in the archaeological record and the brain. The context-based models introduced above lack a detailed analysis of how context-related factors interact with the brain. Biological models, in turn, do not clearly explain how neural mutations can affect culture. The absence of such explanations has led scholars from different traditions to create some emblematic "proxies" (in the sense both of substitutes and approximations) for the various existing models, where summary connections between brains, minds and culture have been postulated and then criticized.

Only recently, some proposals (d'Errico and Banks 2013) have focused on elucidating how technological and cultural evolution could be related to variations in the ecological niche (Banks, d'Errico, and Zilhão 2013), addressing in particular the relationship between artifacts and adaptive environments (e.g., Lahr and Foley 1998). An additional focus has regarded the relationship between the transmission of cultural innovations and demographic dynamics, attempting to show that the appearance of new technologies in the record is constrained by minimum population size (Shennan 2001; Powell, Shennan, and Thomas 2009; but see Read 2012 and French 2016 for limitations). However, models of cultural and cognitive evolution which integrate historical-social aspects with biological factors are still rare (Haidle et al. 2015).

\section{Aims}

In this chapter I will provide a more coherent analysis of the mechanisms underlying the proxy models adopted in cognitive archaeology. My aim will be, first, to illuminate the potential relationship between genes, cognitive functions and behavior in both biology and context-based models. I will show that the proxy for biological models corresponds to a hard-core version of evolutionary psychology, where genes exert strong influence on hard-wired cognitive modules, which in turn generate inflexible adaptive behaviors. On the other hand, I will argue that the context-based models best fit with a mechanism of cognitive transformation that combines elements of cognitive/cultural niche construction, embodied cognition and neuroplasticity. I will refer in particular to Lambros Malafouris' (2013) material engagement theory as a thorough instance of these family of mechanisms in cognitive evolution.

After making evident the main differences between these approaches, I will show that material engagement theory and, more generally, plasticity-based mechanisms are compatible with mutational enhancement models. The tension between these approaches is resolved when we abandon the idea, intrinsic to biological proxies, that neural mutations acting on hard-wired modules are suffi- 
cient conditions for the emergence of new technologies in the record. In line with Wheeler and Clark's (2008) neuroconstructivist lean, I will argue that modules are not innately specified, but can be acquired along an embodied and culturally situated developmental trajectory. Furthermore, modules do not run genetically determined programs that lead to the production of adaptive behaviors and tools. On the contrary, modules can integrate aspects of the material world within their functional outcome (Wilson 2008). Thus, plasticity of the brain, in relation to the plasticity offered by material culture (what Malafouris [2010] calls "metaplasticity") can lead to the production of a massive series of different extended mental architectures. However, although the gamut of possible architectures is great, metaplasticity is limited by constraints distributed within the whole brainbody-world system. Therefore, mutational enhancement could still represent a necessary condition for the acquisition of extended cognitive modules. I will conclude that the co-evolution of cognitive niches, bodies and brains can lead different human species to evolve along alternative metaplastic trajectories, since their plasticity could be subject to different constraints and degrees of freedom.

\section{The "Magic Mutation": Explaining the Trick}

In cognitive archaeology, biological models are often criticized by their detractors for being based on “magic mutations" (e. g., Malafouris 2007, 295). This expression is often used to argue that the mechanisms connecting neural enhancement with the emergence of innovations in the Upper Paleolithic record are poorly defined by partisans of the biological approach.

This blurry picture about the actual nature of biological models can be clarified by drawing upon Steven Mithen's (1994, 1996, 2014) work. In accord with the multidisciplinary goal and methods of a conditional cognitive archaeology (Abramiuk 2012, 30-33; Garofoli and Haidle 2014; Haidle 2014; Wynn and Coolidge 2009), this author has integrated the archaeological record with theories of mental architecture, embracing the idea that the human mind is modular (Mithen 1996). In cognitive science, several authors have elaborated different definitions of the concept of module. According to Jerry Fodor (1983, 1985), a module is a specialized, encapsulated mental organ that has evolved to handle specific information types of particular relevance to the species. Encapsulation means that it is impossible to interfere with the inner workings of a module. Modules also act unconsciously and fast; they provide determined output in relation to specific input, regardless of the content of the input itself, while they do not provide information about the intermediate steps of this process. Modules develop along a universal ontogenetic trajectory, are localized within the brain and break as a result of pathological conditions leaving the rest of modular architecture unaltered (see Elman et al. 1996, for review).

While moderate proposals argue that not all cognition is modular (Fodor 1983), more extreme approaches claim that the human mind is constituted by a massive set of modules (Carruthers 2006; Sperber 1996). Other radical versions 
defend the existence of Darwinian algorithms within the mind, namely functions that are selected to cope with specific adaptive problems such as kin recognition, foraging, or even the identification of potential dangerous animals (Cosmides and Tooby 1987, 2013; New, Cosmides, and Tooby 2007). These stances are united by the idea that modules or Darwinian algorithms are hard-wired by natural selection in order to provide certain adaptive behaviors.

Such a view resonates with a conception of culture close to that advocated by supporters of the New Archaeology (see Abramiuk 2012, sec. 1.2 and 2.1.1). According to this school of thought, culture represents an extra-somatic means of adaptation to environmental problems (White 1959), which is subject to evolutionary mechanisms similar to those related to the selection of adaptive anatomical structures (Binford 2001). Cultural practices are thus selected in relation to their ability to maximize human reproductive fitness (see Wylie 2002, 67-70, for review). The idea that artifacts are the mere byproduct of intrinsic neuro-cognitive modules suggested that culture is thoroughly the product of Darwinian selection acting on genes. Most importantly, modules are domain-specific processors, namely computational entities that deal with a specific kind of informational content. In other words, modules are hard-wired with innate bodies of knowledge that they exploit to solve adaptive problems, a conception known as representational nativism (Elman et al. 1996, 367-371; Spelke 1994; cf. Zahidi and Myin, this volume).

Drawing upon Howard Gardner's (1983) approach, Mithen (1994, 1996) argued that the evolution of the human mind was characterized by the selection of specific intelligences, each related to a particular domain of human life (e.g., technical, social, linguistic). These intelligences evolved as encapsulated domains, each hard-wired with specific algorithms and causally related to specific behavioral outcomes. The evolution of archaic human lineages was characterized by an increasing specialization (and separation) of these different intelligences (Mithen 2014). The archaeological record of Neanderthals, for example, shows that great achievements were reached in technological intelligence, such as the production of highly sophisticated hafted spears (e. g., Mazza et al. 2006), and social intelligence, as supported by the ambush strategies likely involved in big game hunting (Villa et al. 2005). However, the absence of manifestations of art comparable to those of the modern human Upper Paleolithic (Wynn and Coolidge 2004) proves that Neanderthals lacked integration between such modules (Mithen 2014). This integration requires the evolution of a metacognitive module in modern humans, mostly scaffolded by language, which is capable of establishing connections between the various intelligences. The appearance of such a new mental architecture could be explained by both cladogenetic mechanisms similar to those described by Klein (2000), where bottlenecks lead to a dramatic radiation of neurally modern Homo sapiens, as opposed to the nearly modern Homo belmei. Alternatively, anagenesis could be also considered for a gradual transformation of an archaic Neanderthal-like architecture into a fully modern one. Both mechanisms are compatible with strong instructionism (Wheeler 2006; Wheeler and Clark 1999), namely the idea that genes fully specify their outcome. In this 
way, genetic mutations can produce neurobiological changes, which can directly modify innate representations, change Darwinian algorithms, alter domains of intelligence and ultimately lead to the production of adaptive behaviors. The combination of strong instructionism, hard-wired modularity and culture as extra-somatic adaptation represents well the "Neo-Darwinian synthesis" of cognitive evolution (cf. Malafouris, this volume; Iliopoulos and Garofoli 2016). This represents the extended version of the proxy model commonly associated with revolutionary and biologically oriented approaches in cognitive archaeology. ${ }^{1}$

\section{Context-Based Approaches}

In the current cognitive archaeological debate, two growing bodies of theory, namely the incremental (McBrearty and Brooks 2000) and the cognitive equivalence model (d'Errico 2003; Zilhão 2007), favor cultural mechanisms over biological enhancements as drivers of cognitive evolution. However, the detailed mechanisms concerning how context can profoundly alter cognition have barely been explored and currently remain underspecified in archaeological theory. A more thorough explanation for such mechanisms requires multidisciplinary integration with knowledge drawn from different domains (see Laland and O'Brien 2010, for an example).

It is broadly recognized in evolutionary biology that many organisms can act upon their living environments, creating selective environments to which they (and their offspring) adapt (Laland, Odling-Smee, and Feldman 2000, 2001; Odling-Smee, Laland, and Feldman 2003). Humans have adopted culture to create and maintain such selective environments, a process currently known as cultural niche construction (Kendal et al. 2011; Laland and O’Brien 2011; see Watkins 2016, for review).

Cultural niches are not simply an outstanding example of active ecological engineering, they also show the uniquely human ability to modify the informational structures and opportunities within the environment. Rather than simply selecting among alternative sources of information, humans can alter the very way information can be drawn from the environment, acting in this way as "epistemic engineers” (Sterelny 2003, 148; Clark 2008, 66-68). Consider, for example, the creation of learning environments for the transmission of lithic technologies,

\footnotetext{
${ }^{1}$ Nevertheless, one must acknowledge that not all the existing models in evolutionary psychology actually subscribe to this whole set of requirements at one and the same time. Some moderate versions, for example, concede that the content processed by algorithms which evolved in a "Stone Age world" can be partly attuned to the challenges of contemporary settings (e. g., an "avoid a stampeding elephant" algorithm is modulated to "detect an approaching truck"; see Sperber 2005). In addition, evolutionary psychologists have attempted many times to reject the charge of "genetic determinism," by emphasizing the importance of the environment in the emergence of domain-specific functions (e. g., Kurzban 2010). However, the psychologist Alison Nash (2014) has recently countered that the environment, within this line of defense, pays only "lip service" to the developmental process, by passively "triggering" a genetically determined program which nevertheless rests inscribed in the brain by natural selection.
} 
such as the Acheulean bifacial hand-axes (Wynn 2002). Being situated within dedicated "tool working" areas of activity, youngsters at first become familiar with lithic technologies by coming into contact with the byproducts of the experts' flaking activity (i.e., the debris). Then, they can directly interact with experts and rely on the technical solutions already figured out in the past. In this way, the embedment within an epistemically altered environment eliminates the need for reinventing the same technology many times (Sterelny 2011, chap.2). Furthermore, apprenticeship systems facilitate the production of artifacts by superseding costly individual cognitive strategies with more economic distributed ones. The cognitive load that is required to support the invention of an artifact, such as the construction of mental plans and networks of inferences, is reduced by simply becoming attuned to the actions of a teacher. In sum, cultural niches are represented by collections of practices, values, norms and relationships, built on through the engagement with social agents and artifacts, which emerge within an epistemically engineered environment.

Cultural niches act also as cognitive niches, since they represent new developmental contexts for children. Embedded in such engineered environments, children are exposed to new meanings and relationships since their early age, which deeply alter their cognitive development (Kendall 2011). This allows transformation of the human mind throughout ontogenesis. At the same time, the emergence of new socially relevant situations also influences the adult mind. Interaction with young learners can indeed lead expert adults to acquire new values, like the importance for group survival of transmitting a technique to the new generations. This long-term goal can lead experts to develop a higher degree of tolerance toward younger individuals, which in turn can alter social relationships (and cognition) within a group.

Most crucially, the cultural/cognitive niche is also vertically transmitted, since the epistemically modified environments are bequeathed from one generation to the next, allowing the accumulation and transfer of a huge cultural and cognitive “capital” along a Lamarckian dimension (Jablonka and Lamb 2005). In addition, the process of downstream epistemic engineering could be incremental, for the inherited environment could be further modified by the following generations (Sterelny 2011). Importantly, the vertical transmission of a cultural/cognitive niche relies on the idea that the human mind is massively plastic, rather than being represented by a set of fixed modular adaptations to a Paleolithic world (Sterelny 2003, chap. 10). Thus, the mind is specifically adapted to keep pace with continuously changing cultural/cognitive niches. Adaptations of this kind involve enhancements in neuroplasticity mechanisms combined with an extension of childhood/adolescence periods (Bock and Sellen 2002), which are critical for learning. The downstream transmission of developmental contexts, coupled with the plasticity of the human mind, represent a plausible account of the mechanisms sought by cultural models in cognitive archaeology. ${ }^{2}$

${ }^{2}$ However, Iliopoulos and Garofoli (2016) have recently argued that even cultural models, like biological ones, are compatible with a hard-core evolutionary psychology argument (and 


\section{Embodied/Extended Variants}

An additional issue is how the plastic human mind gets dynamically attuned to an inherited cultural niche. Various explanations have been proposed to address this problem. According to Sterelny (2003), the human mind evolved as a domain-general device, provided with basic abilities, such as processing statistical regularities, focusing the attention on some targets, or inhibiting irrelevant stimuli. Such domain-general abilities are connected to critical stimuli that are made salient by the cultural niche itself. In this way, there is no need to develop hard-wired modules able to identify and process specific contents, if these contents are already evident within the world. A domain-general cognitive architecture requires simply attunement to such situated information. The process of cognitive transformation introduced earlier is the result of different adaptations to different cultural niches.

Another body of theories, known as the extended cognition hypothesis (Clark 2008; Rowlands 2010; Menary 2007), argues that the engagement with the cultural niche is not simply the product of an interaction between internal cognitive properties and salient external aspects. Artifacts and social agents could in fact be constitutive of human cognition, resulting in a massive series of hybrid mental architectures (Clark 2008). The plasticity of the brain allows humans to dynamically assemble such hybrid systems in relation to the situation in which they are enculturated, rather than having a fixed internal architecture inflexibly operating on the world.

The most radical approach to such an active externalist view is likely represented by Lambros Malafouris' (2013) material engagement theory. Malafouris (2013, chap. 4) argues that a proper theory of extended mind requires embracing the idea that humans not only use artifacts to assist their cognitive processes, but that they think through artifacts themselves (see also Knappett 2005). At the base of this theory is the idea that the enactive engagement with artifacts leads to the emergence of new cognitive and behavioral possibilities for human agents. For example, the action of tracing a curved line on a cave wall with pigments allows an Upper Paleolithic human to perceive the back of an animal (Malafouris 2007). The affordances for action provided by the material properties of pigments, coupled with explorative actions on a cave wall, bring forth a new series of meanings, represented by pictorial images. The pictorial action gradually leads humans to reflexively think about the creative process itself, scaffolding the emergence of a concept of image. Once images are socially shared, they can ground new meanings and social relationships that can enact the construction of appropriate socio-cognitive abilities.

they often adopt evolutionary psychology rhetoric). Indeed, provided that both these models accept the existence of innate domain-specific modules, the difference between the two could simply boil down to whether genes are the main cause of development (i. e., genetic determinism) or whether the environment "triggers" innately specified functions (see note 1). 
Thus material culture is plastic in that it affords many possibilities for cognitive transformation, which in turn is made possible by the plasticity of the human brain. Integration between the cultural and the neural levels of plasticity defines the concept of metaplasticity (Malafouris 2010), which stands at the crux of material engagement theory.

\section{Acquired Modularity}

The concept of metaplasticity is introduced as a diametric alternative to the modular approach defended by evolutionary psychologists (Malafouris 2013, 46). Where the latter seeks the existence of a universal human nature grounded into innate psychological entities (Buss 1994; Pinker 1994), the former argues that human minds are incomplete by nature (Malafouris, this volume), since they emerge as the product of material and social engagement. Such a rejection of modularity is based on two main reasons. The idea that the mind and the cultural/cognitive niche co-construct each other seems to better explain the existing empirical evidence than does the modular approach (Dekker and Karmiloff-Smith 2011). Williams syndrome, in particular, has been used as a paradigm to show that developmental disorders are not caused by "broken" modules (Karmiloff-Smith 2012b; Karmiloff-Smith and Farran 2012), which leave the rest of the architecture intact (for this approach, see Baron-Cohen 1998; Carruthers 2002; Pinker 1999, 262; van der Lely 2005). On the contrary, small innate biases caused by genetic mutations can influence a child's activity, leading to dysfunctional outcomes within a developmental trajectory. As a result, the whole mental architecture is affected by this initial variation, even if the long-term effects of such an early impairment are more evident in some specific cognitive functions than in others (Karmiloff-Smith 2009).

On the other hand, metaplasticity and material engagement theory also emerged as a rejection of some deep metaphysical problems that afflict the innate modularity approach. Without going into details, it is worth mentioning the notorious problem of homuncularism that is inextricably associated with representational nativism (Gibson 1979; cf. Zahidi and Myin, this volume). There are issues with the assumption that modules are agents that interpret the content of some stimuli by means of innate knowledge. In fact, modules are clusters of neurons, which can either respond to stimuli or not, and thus it is unclear how they can "know," "simulate" or "talk about" aspects of the world (Gallagher 2008). Another problem with innate modularity lies in the idea that human behavior and cognitive life are a priori shaped by algorithms which evolved during the Paleolithic (Pinker 1994), thereby reducing the richness and variability of human life and culture to a mere by-product of natural selection (Tallis 2011).

However, these problematic aspects with modularity have led proponents of metaplasticity (and similar massive plasticity approaches) to assume overdefensive positions. In fact, the opposition between modularity and metaplasticity exists only insofar as we consider the innate aspect of modularity, namely that 
modules are hard-wired in the neural system and that they harbor native representations (see above). Wheeler and Clark (2008) have applied a neuroconstructivist approach (Mareschal et al. 2007; Farran and Karmiloff-Smith 2012; Westermann et al. 2007) to show that the existence of encapsulated, domain-specific modules is not necessarily innate. Drawing in particular upon Karmiloff-Smith (1992), they have argued that given the early plasticity of the human brain, modules can be progressively acquired during development. The modularization process combines native biases in, for example, the ability to plan saccades (Brown et al. 2003), with the proactivity of the subject to engage with the world. Partial representations, namely neural activations that correlate with aspects of the world, are built in throughout development and used as bricks to construct more complex representations (Mareschal et al. 2007, 101-102). ${ }^{3}$ The acquisition of partial representations restricts the plasticity of the system, reducing the gamut of possible outcomes, so that development itself becomes a critical constraint in influencing the developmental outcome (Karmiloff-Smith 1998). Regions of the brain acquire partial representations through processes of competition for information and bring about new functional outcomes and more complex representations by cooperative processes with other regions (Mareschal et al. 2007, 97). This view constitutes what in general is defined as the interactive specialization model of development as opposed to the maturational one, according to which modules develop along deterministic trajectories and abilities present at birth are unlocked according to a pre-specified time program (Johnson 2001; Johnson and de Haan 2011, 13-14).

\section{Body Ornaments as Transformative Technologies}

In this section I will adopt early body ornaments as a case study to argue that cognitive modules can be acquired through a process of material engagement. Body ornaments present some concrete advantages in comparison to the Oldowan tool-making example sketchily introduced by Malafouris $(2013,175)$ as a paradigm for cognitive transformation. Like the production of Oldowan handaxes (Wynn 2002), early beads can act as material scaffolds for the emergence of new meaningful relationships and cognitive processes at the individual level. At the same time, ornaments specifically bring forth social relationships and socio-cognitive abilities, altering the human cultural/cognitive niche. This case study also makes evident the bridging between niche construction and material engagement theory.

\footnotetext{
${ }^{3}$ For neuroconstructivists, a representation is "an information state within the brain of an organism that contributes to adaptive behavior within a given environment" (Mareschal et al. $2007,4)$. Although these authors accept the notion of computation over mental representations, their approach appears open to an anti-representational amendment (see Zahidi and Myin, this volume).
} 
As previously suggested, perforated shells have been found at several African Middle Stone Age sites (e.g., d'Errico et al. 2005) and supposedly in some late Neanderthal sites in Europe (Zilhão 2012). Pierced shells are considered to be body ornaments by an abductive argument that focuses on the following factors: the shells show signs of active manipulation, which consist in the intentional perforation of the item, although the exploitation of natural openings is also possible (Bouzouggar et al. 2007). Furthermore, evidence of use-wear recorded on the perforation edge is consistent with the use of a string to form pendants (d'Errico et al. 2005; Henshilwood et al. 2004). In addition, evidence for the transportation of shell beads from coastal shores to distant regions, alongside the limited nourishment value of these mollusks, strengthens the idea that these objects could be socially relevant (Bouzouggar et al. 2007; d'Errico et al. 2009). The presence of such body ornaments within the archaeological record of ancient human populations has been associated with symbolism and said to prove behavioral and cognitive modernity (e.g., Henshilwood and Marean 2003; Kuhn and Stiner 2007). Furthermore, at a deeper level of cognitive realization, Henshilwood and Dubreuil $(2009,2011)$ have argued that such ornaments prove the acquisition by their users of shared conceptual abstractions and meta-representational capabilities necessary to implement full-blown mindreading. Against this paradigm, a growing number of authors have contended that shell beads cannot be considered as symbols at a semiotic level (Coolidge and Wynn 2011; Rossano 2010; Iliopoulos 2016). In addition, an enactive approach to social perception (Hutto 2011; Gallagher and Hutto 2008; De Jaegher and Di Paolo 2007; Fuchs and De Jaegher 2009) suffices to explain the emergence of these ornaments in the record without the need for the costly cognitive abilities that are considered necessary to produce full symbolism. The enactive argument implies that the meaning of a shell bead is directly perceived as an embodied emotion toward the shell itself (Gallagher 2008). Indeed, the properties of the nacre are special in themselves and foster an aesthetic reaction from the observer. The person displaying the shell can directly perceive the embodied emotional reaction of the observer. In this way, the embodied reaction grounds the meaning of the shell, without the need to infer it through mindreading. This meaning motivates individuals to wear shells as ornaments and to maintain this practice in the long term. This in turn alters the cognitive niche in which children develop, shaping the perception of adorned individuals for subsequent generations (see Garofoli 2015, for the full version of this argument).

Placed in the context of Malafouris' (2013) approach, the engagement with non-symbolic body ornaments represents a necessary condition for the acquisition of more advanced cognitive abilities. The meaning of an ornament is first maintained as a concept grounded in the perception of embodied emotions (see Barsalou 1999; Prinz 2002, for a similar approach). Once body adornment becomes embedded within the culture of a group, embodied concepts are gradually targeted by vocalizations and turned into words (Barsalou et al. 2008; see the contributions to this volume by Fuchs, and Zahidi and Myin, for an embodied approach to language acquisition). Concrete word concepts scaffold the emer- 
gence of abstractions and lead to the production of a language composed of propositional chunks. In turn, propositional language could be used to build a contextual/behavioral narrative, namely a set of stories that describes the actions and reasons of some agents within a concrete social context, often involving social norms (Fiebich 2016). Let us consider the following example:

"Everybody cheers the man with many beads," said the mother to the child, "for he has hunted many animals and he is the strongest man of the tribe."

A narrative of this kind leads children to understand the ornament's meaning without necessarily reinventing it from an initial phase of embodied perception. This phase becomes part of the cognitive niche by being embedded in narrative practice, so that children can rely on such narratives to directly understand what being the chieftain means (Gallagher and Hutto 2008). Thus, narratives can augment the direct perception of embodied aspects of the world. Furthermore, the concepts brought forth by body ornaments can be recycled and plastically adapted to other situations, thus further transforming the mind and the cognitive niche. An increase in the use of language as a cognitive tool in social contexts in turn scaffolds the production of linguistic meta-representations of the kind "I know that you know." This leads to the formation of narratives that do not simply contain a description of concrete actions and reasons of the protagonists, but which also focus onto mental contents (Fiebich 2016; Milligan, Astington, and Dack 2007). For example:

"The augur looked at the twirling birds and waited in silence. The warriors believed he wished to say not to start the fight."

The education of children in such mentalistic narratives allows the acquisition of full-blown mindreading, namely the ability to understand others in terms of mental states (Apperly 2011).

The crucial point in relation to material engagement theory is that body ornaments and narratives, by altering the cognitive niche and the plastic human brain, can mediate the construction of specific functional modules. Indeed, these artifacts can lead to the emergence of modules which process embodied concepts, linguistic abstractions, propositional meanings and meta-representations (see Barnard 2010, for a cognitive architecture compatible with this argument).

\section{Innateness Rethought}

Neuroconstructivism does not only solve the apparent tension between material engagement theory and the modularity of mind. It also shows that context-based approaches are compatible with mutational enhancement models. Acquired modularity implies that modules are constructed along a trajectory of multilevel interactions, which range from the cellular to the cultural level.

At the crux of acquired modularity lies the concept of "domain-relevance" of brain regions as opposed to that of domain-specificity typical of hard-wired 
modularity (Karmiloff-Smith 2009, 2012a; Dekker and Karmiloff-Smith 2011). Domain relevance rejects the main idea at the heart of representational nativism, namely that neural systems harbor innate knowledge. On the contrary, the innate component lies here in the physical properties of neural cells, layers, regions and bodily systems which constrain "the kinds of information that can be received, and hence the kinds of problems that can be solved and hence the kinds of representations that can subsequently be stored" (Elman et al. 1996, 30). Thus, this form of nativism focuses on the structural limits to the acquisition of cognitive functions. In particular, the functional potential of a set of elements within a neural network is constrained by the relationship of this element with the rest of the network. While at the local level cortical regions could show larger degrees of freedom, at the level of regional connectivity (embrainment) the neural architecture is partly determined by its mutual relationship with other regions (Elman et al. 1996, 27-30; Johnson and de Haan 2011, 14). Furthermore, functional acquisition is constrained by the structure of the body, as well as by how the neural system is physically connected to the body itself (Mareschal et al. 2007, chap. 4; Gallagher 2005). A five finger hand or a standing posture influence the type of affordances for action that can be brought forth by engaging with the world and thus shape the kind of information reaching the brain. Development itself then provides new constraints by reducing the functional flexibility of brain regions as a result of progressive specialization (Elman et al. 1996, 33; see above). In sum, the physical constraints of some domain-relevant regions alter the probabilities that interaction with the environment will lead to the emergence of a specific cognitive function (Gottlieb 2007).

This way of rethinking innateness, however, does not eliminate the fact that innate components are critical for development and evolution. Giving up neurocentric/deterministic ideas of hard-wired modularity does not leave room to an extreme form of classic empiricism. The emergence of a cognitive function merely becomes distributed among many levels and is subject to a similarly distributed set of constraints. Although a concept of strong neurocentric causality disappears when abandoning representational nativism, this does not imply that all hominid brains are unstructured monads that can give rise to every potential outcome in relation to their specific engagement with the world. Modularization still implies the existence of a structural flexibility that allows the acquisition of a particular function. Roughly put, whatever the origins of a cognitive function are, either brought forth by material engagement or innately specified, its very existence implies that such a function has some minimal neurobiological requirements to be implemented.

\section{Impossible Modularization}

Let us consider the emergence of body ornaments in late Neanderthal cultures (e. g., Zilhão 2012). In relation to the discussion above, these artifacts could have been used by Neanderthals to transform their mind, as well as their cognitive 
niche. Such a transformation could require the emergence of new modules to process linguistic propositions that are built upon an initial stage of enactive perception of embodied emotions. However, this shift is only a logical possibility and does not represent a necessary outcome. Likewise, even if Neanderthals had basic linguistic forms that allowed them to create propositional meaning, the shift from this level to that of linguistic meta-representations is not a priori warranted.

Focusing on this second case, constraints upon the emergence of meta-representations could lie in two different places. First, the physical properties of the Neanderthal neural architecture could have insufficient flexibility to allow the production of meta-representations. In this way, Neanderthals would not be provided with domain-relevant regions that allow them to acquire a specific module for a theory of mind. For example, the degree of connectivity of the Neanderthal temporal lobe could be different from that of modern humans, preventing them from acquiring some language-based cognitive functions. The architecture of the brain is constrained in a way that no neuronal recycling or plastic rearrangements (e. g., Dehaene and Cohen 2007) allow such meta-representations to be acquired. Second, constraints could exist in the embodiment of a cognitive function, so that even if Neanderthals did possess brain regions flexible enough to acquire meta-representations, the relationship between these relevant regions and other aspects of the brain-body system nevertheless impedes the acquisition of this function. For example, higher levels of expression of hormone receptors for the control of stress could have disrupted the acquisition of a "language of diplomacy" in Neanderthals, even if their brains per se were sufficiently plastic to acquire this function (Ambrose 2010). Higher levels of stress could have exerted repercussions at the social level, impeding the consolidation of some necessary conditions for the emergence of full-blown mindreading. A potential inflexibility of these receptors to culturally-mediated modulations could have made the acquisition of such cognitive abilities impossible in the long term.

In this way, material engagement represents only a necessary condition for the emergence of cognitive functions. On the other hand, it is logically possible that biological constraints need to be released in order to allow a function to modularize.

\section{Limitless extension}

In the previous section I have argued that the brain-body system of archaic humans such as Neanderthals could have had an inferior level of intrinsic plasticity. However, it could be objected that since development is a situated process, the acquisition of a cognitive function cannot simply be evaluated by looking at the internal constraints of the brain-body system. In fact, acquired modules can be extended (Clark 2008) and based on the manipulation of material vehicles (Menary 2007). Such an extended approach raises important new considerations about the neurobiological limits to cognitive transformation in human evolu- 
tion. Indeed, limited neuroplasticity does not preclude a cognitive function being acquired by off-loading the burden onto the material world.

It is worth considering, for instance, the notorious case of bartenders discussed in extended cognition literature (Beach 1988; Clark 2001). Bartenders have to face the problem of preparing specific cocktails at certain hours of the evening. One strategy to realize this task would be to create an internal model of the detailed relationships between cocktail types and time-frames. This strategy, although possible, appears to be very costly in cognitive terms, since many relationships need be memorized and retrieved at the right time. Bartenders, however, can structure their environment in order to facilitate such a problem-solving activity. They usually adopt the material features of glasses (such as shape and size) to define associations with particular kind of cocktails. In addition, they organize their working environments, so that different glasses are spatially arranged in a way that corresponds to the chronological order in which cocktails will be prepared. Bartenders thus use variation in the glass shapes (which is allowed by the plastic properties of glass) to solve a mnemonic problem of association. At the same time, they use spatial disposition to visualize time relationships. We could now return to the problem of cognitive evolution, considering this example as a paradigmatic case of mentalistic vs. situated strategies. We could then take the case of a primitive agent who has insufficient neural flexibility to implement the mentalistic cognitive approaches to spatial problem solving, and who could realize only situated strategies. Would this mean that the primitive agent has inferior visuospatial abilities compared to the modern bartender who can do both? Recently, Bruner and Lozano (2014) seem to have reached a similar conclusion in relation to the Neanderthal use of teeth as a third-hand interface to assist praxis, which has limited counterpart in ancient Homo sapiens populations. These authors have argued that the use of an additional interface in Neanderthals, instead of stone tools, is due to the absence of efficient visuospatial integration in this species compared to Homo sapiens. It could be argued, however, that mentalistic operations are not intrinsically more efficient than the embodied version Bruner and Lozano (2014) ascribe to Neanderthals, if judged in terms of results. Therefore, one can conclude that cognitive extensions can in principle be as efficient as mentalistic processes, at least in the short term, and thus that intrinsic limits to plasticity can be fully compensated by cognitive extensions without great adaptive loss.

However, an extreme application of this argument raises a series of important problems. Even though cognitive extensions can reduce the cognitive load and allow a less plastic system to acquire a function, this process is not limitless. Extensions cannot create an infinite range of cognitive capabilities potentially realizable by a brain-body system. If we take this idea as valid, it could be argued that any brain-body system, independently of its intrinsic limits, could acquire whatever final hybrid configuration. In other words, this would mean that while brains and bodies are constrained, their engagement with the world it is not. The problem of limitless neural plasticity discussed above is thus transferred to the material dimension. Such an assumption implies that the material engagement 
with the world can result in any possible cognitive outcome, since the world is infinitely plastic. These conditions force us to face the situation depicted in the 1992 movie The Lawnmower Man, where the engagement with a set of mysterious virtual reality machines, coupled with the administration of neurotrophic drugs, leads a simple mind to acquire super-human powers such as telepathy or psychokinesis.

\section{Relational and Situated Constraints}

In contrast with limitless plasticity, when artifacts are integrated within the developmental process, they become part of a wholly constrained system. Constraints emerge in the very relationship between the brain-body system and material culture, so that the kind of possible hybridizations are also limited. Given the intrinsic plasticity of a system, it is therefore possible that some hybrid architectures cannot be assembled. For example, monkeys are generally considered to have limited capacity to solve analogies, specifically the ability to understand relationships between relationships (e.g., AA analogous to $\mathrm{BB}$ and different from CD, see Holyoak and Thagard 1994). Evidence in comparative psychology shows that apes can use tokens for "same" or "different" relationships between pairs of elements, thus facilitating analogical reasoning. Conversely, monkeys seem to fail to use such material anchors to solve analogies (see Thompson and Oden 2000 , for review). In this sense, their limited neural plasticity does not simply impede them from performing analogies in their minds; it also hampers the application of extended strategies.

On the other hand, constraints upon cognitive extension could also be fully located within the world. That is, there could be intrinsic properties of the world which impose mandatory solutions upon cognitive agents. Let us consider the case of mentalistic mindreading discussed in the previous sections and in particular the construction of a linguistic meta-representation "I know that you wish $\mathrm{X}$." This linguistic representation allows an individual to represent the propositional attitude of another subject towards a mental state X "as such” (you wish $\mathrm{X}$ ) and to represent his own propositional attitudes toward this proposition (I know that). Such a form of mentalistic mindreading has been argued to be dependent on language acquisition in developing children (Milligan, Astington, and Dack 2007; Ruffman et al. 2003). Although there is no space to support the following assumption in this chapter, let us suppose that there is simply no way to represent the content of someone else's mind which is not language-based. In this way, the adoption of writing or Braille code alters the physical vehicle through which language is constructed, but it does not provide a substantially different cognitive strategy than orally produced language. Considering Malafouris' (2010) concept of metaplasticity, in this case the material side, rather than the neural one, is tightly constrained. Thus, only agents who have an embrained and embodied system that allows the acquisition of meta-representational language can acquire full-blown mindreading, since alternative cultural strategies are impeded. 


\section{Metaplasticit-ies}

According to Wheeler and Clark (2008), the universal character of human nature is actually a meta-nature, represented by the possibility of dynamically assembling a vast gamut of extended cognitive architectures. The structure of these architectures depends on the trajectory of embodied and situated modularization that takes place during development. The element of constancy lies here in the possibility for radical variation in the cognitive outcome, rather than in a series of innate, fixed and universally shared cognitive modules, a conception that is close to Malafouris' (2010) metaplasticity.

In this chapter, I hope to have sufficiently argued that such a meta-nature has limits, represented by the constraints that are distributed within the whole brainbody-world system which characterizes extended cognitive architectures. In particular, I have identified constraints: (a) intrinsic to neuroplasticity, (b) located within the brain-body-artifact relationship, and (c) fully situated in the structure of the world. Crucially to the current argument, such limits could be altered by mutational enhancement, allowing the modularization of cognitive functions which could not be constructed with different levels of plasticity (Garofoli 2013; Roberts 2016).

The critical point is that the context/culture-based mechanisms currently adopted to explain the rise of innovations in the archaeological record are complementary with and not opposed to mutational enhancement. Cognitive niche shaping (Watkins 2016), downward transmission of epistemically engineered environments (Sterelny 2011), cultural transmission augmented by demographic effects (French 2016), coupled with neuroplasticity can transform the mind only within certain limits. Mutational enhancement could thus still represent a necessary condition for the emergence of some specific cognitive abilities (cf. Coolidge and Wynn 2005; Russell 1996), although no longer a sufficient condition, as in the innate modularity approach.

The view of cognitive evolution emerging from these arguments is close to that embraced by Cochran and Harpending (2009). The shaping of a cognitive/cultural niche creates conditions of selection for neural substrates that allow the acquisition of adaptive cognitive functions (Lewontin 2000; Laland, OdlingSmee, and Feldman 2000; Iriki and Taoka 2012). The Lamarckian transmission of the cognitive/cultural niche to further generations makes possible the durable selection of these substrates over time (Jablonka and Lamb 2005).

This position has remarkable implications within the current debate about the mechanisms of cognitive evolution. The human meta-nature could have been triggered by small alterations on an ancestral brain architecture (Wheeler and Clark 2008, 3572), which correlated with drastic changes in the innovations characterizing the Middle to Upper Paleolithic transition (Bar-Yosef 2002). In this way, metaplasticity appears, in principle, to even be compatible with those revolutionary models in cognitive archaeology which ascribe the Upper Paleolithic revolution to discrete mutations in brain architecture (e. g., Klein 2000). However, models like the incremental approach (McBrearty and Brooks 2000), 
according to which innovations slowly emerged until a threshold was reached that generated the illusion of a technological revolution, could also be compatible with the idea that some forms of mutational enhancement were running beneath these incremental dynamics.

However, we do not necessarily have to imagine metaplasticity, or meta-nature, as a modern human feature, that abruptly or gradually emerged during Homo sapiens' evolutionary history. An analysis of material culture in human evolution shows that humans were social beings interacting with tools much earlier than the appearance of Upper Paleolithic cultures. The flaked cobbles of the Oldowan and early Acheulean cultures (Wynn 2002), the emergence of simple spears (Thieme 2005) and shelters (Gamble 1999) in some Homo beidelbergensis camps, the hafted weapons of Neanderthals (Mazza et al. 2006) and early moderns (Wadley, Hodgskiss, and Grant 2009) and the emergence of figurative art in the Homo sapiens Upper Paleolithic record (Fritz and Tosello 2007) suggest that these different hominins were materially engaged. This does not mean that these species shared the same meta-nature, since they could have developed along alternative trajectories, constituted by different domain-relevant neural architectures, body structures and cognitive/cultural niches. Hence, these hominins could have realized their own metaplasticit-ies, characterized by a series of intrinsic constraints and idiosyncratic neural augmentations.

\section{Conclusions: Epistemological Implications for Cognitive Archaeology}

In the light of the considerations advanced above, the traditional approach to a conditional cognitive archaeology (e. g., Abramiuk 2012, 30-33) needs to be rethought. Viewing cultural innovations as the product of new innate modules allowed scholars to infer the timing for such biological changes by directly looking at variations within the archaeological record (e.g., Mithen 1996). The dynamic and emergent aspects of metaplasticity tell against this approach. Mutations affecting domain-relevant properties of the brain could show effects on domain-specific cognitive functions only after millennia of material engagement and plastic rearrangements. Thus, no strict relationship exists between innovation and selection of biological enhancements.

Furthermore, conditional cognitive archaeology has to shift focus from abilities that are de-contextualized and static to metaplastic trajectories, highlighting differences and similarities between the trajectories undertaken by different human populations. This approach implies identifying the artifacts which scaffolded processes of cognitive transformation in the record, as well as the cognitive abilities that are minimally constrained by this transformation. The approach becomes much more complex when we consider the possibility that metaplastic trajectories could have been augmented by mutational enhancement (see Hutchins 2008, 2018). Conditions for the incurrence of such mutations need to be identified as integrated within a trajectory of change. 
In this sense, it is worth asking whether the metaplastic trajectories taken by Neanderthal populations prove the existence of an equivalent level of cognitive flexibility in this species compared with modern humans, or whether the modern human Upper Paleolithic trajectory strongly departs from those of the Neanderthals (Garofoli 2013). Considering the case study discussed earlier in this chapter, it is possible that Neanderthals were provided only with embodied strategies of social cognition, or at most with a level of flexibility that allowed the construction of behavioral-contextual narratives. In this way, Neanderthals could have invented their body ornaments and used them to transform their social cognition along a trajectory that is different from that of modern humans. Perhaps, their metaplasticity was limited in its construction of meta-representations and full blown theory of mind. The minimal conditions for the presence of such abilities ought to be identified in Neanderthal material culture in a process of transformation similar to the one introduced here (see Mithen 2014, on similar grounds). At the same time, Neanderthal metaplasticity could have been equally (if not even more) efficient in the realm of embodied cognition, imagistic reasoning or basic language constructions. Thus, the potential absence of meta-representations in Neanderthals could have led this species to develop cognitive abilities that have no counterpart in modern humans. Langbroek's (2014) idea that humans could have evolved different "cognitions," is illustrated well by the existence of different metaplasticit-ies.

\section{Acknowledgements}

I would like to thank the organizers of the Heidelberg conference "Embodiment in evolution and culture," from which this volume has been derived, in particular Gregor Etzelmüller, Thomas Fuchs, Grit Schwarzkopf and Christian Tewes. Special thanks to Christian Tewes for assistance during the preparation of this chapter and to Adrian Wilding for language revision. Thanks also to Miriam Haidle for having involved me in this project. I borrowed the expression "unstructured monad" used in this chapter from Ezequiel Di Paolo (personal communication).

\section{Bibliography}

Abramiuk, M. 2012. The Foundations of Cognitive Archaeology. Cambridge, MA: MIT Press.

Ambrose, S. 2010. Evolution of Composite-Tool Technology, Constructive Memory, and Language: Implications for the Evolution of Modern Human Behavior. Current Anthropology 51: S135-147.

Anderson, H. 2012. Crossing the Line: The Early Expression of Pattern in Middle Stone Age Africa. Journal of World Prehistory 25 (3-4): 183-204.

Apperly, I. A. 2011. Mindreaders. New York: Psychology. 
Banks, W.E., F. d'Errico, and J.Zilhão. 2013. Human-Climate Interaction During the Early Upper Paleolithic: Testing the Hypothesis of an Adaptive Shift Between the Proto-Aurignacian and the Early Aurignacian. Journal of Human Evolution 64: $39-55$.

Barham, L. S. 2001. Central Africa and the Emergence of Regional Identity in the Middle Pleistocene. In Human roots: Africa and Asia in the Middle Pleistocene, ed. L. S. Barham and K. Robson-Brown, 65-80. Bristol: Western Academic and Specialist Press.

Barnard, P. J. 2010. From Executive Mechanisms Underlying Perception and Action to the Parallel Processing of Meaning. Current Antbropology 51: S39-53.

Baron-Cohen, S. 1998. Does the Study of Autism Justify Minimalist Innate Modularity? Learning and Individual Differences 10 (3): 179-191.

Barsalou, L. W. 1999. Perceptual Symbol Systems. Behavioral and Brain Sciences 22: $577-660$.

Barsalou, L. W., A. Santos, W. K. Simmons, and C. D. Wilson. 2008. Language and Simulation in Conceptual Processing. In Symbols, Embodiment, and Meaning, ed. M. de Vega, A. Glenberg, and A. Graesser, 245-284. Oxford: Oxford University Press.

Bar-Yosef, O. 1998. On the Nature of Transitions: The Middle to Upper Palaeolithic and Neolithic revolution. Cambridge Archaeological Journal 8 (2): 141-163.

-. 2002. The Upper Paleolithic Revolution. Annual Review of Anthropology 31: 363-393.

Beach, K. 1988. The Role of External Mnemonic Symbols in Acquiring an Occupation. In Practical Aspects of Memory, ed. M. M. Gruneberg, P. E. Morris, and R. N. Sykes, 342-346. New York: Wiley.

Benazzi, S., K. Douka, C. Fornai, C. C. Bauer, O. Kullmer, J. Svoboda, I. Pap et al. 2011. Early Dispersal of Modern Humans in Europe and Implications for Neanderthal Behaviour. Nature 479: 525-528.

Binford, L. R. 2001. Constructing Frames of Reference. An Analytical Method for Archaeological Theory Building Using Ethnographic and Environmental Data Sets. Berkeley: University of California Press.

Bock, J. and D. W. Sellen. 2002. Childhood and the Evolution of the Human Life Course. Human Nature 13 (2): 153-159.

Botha, R. 2010. On the Soundness of Inferring Modern Language from Symbolic Behaviour. Cambridge Archaeological Journal 20: 345-356.

Bouzouggar, A., N. Barton, M. Vanhaeren, F. d'Errico, S. Collcutt, T. Higham, E. Hodge, et al. 2007. 82,000-year-old Shell Beads from North Africa and Implications for the Origins of Modern Human Behavior. Proceedings of the National Academy of Science U.S.A. 104: 9964-9969.

Brown, J.H., M. H. Johnson, S. J. Paterson, R. Gilmore, E. Longhi, and A. KarmiloffSmith. 2003. Spatial Representation and Attention in Toddlers with Williams Syndrome and Down Syndrome. Neuropsychologia 41: 1037-1046.

Bruner, E. 2010. Morphological Differences in the Parietal Lobes within the Human Genus: A Neurofunctional Perspective. Current Anthropology 51: S77-78.

Bruner, E. and L. Marina. 2014. Extended Mind and Visuospatial Integration: Three Hands for the Neandertal Lineage. Journal of Anthropological Sciences 92: 273-280.

Burdukiewicz, J. M. 2014. The Origin of Symbolic Behavior of Middle Palaeolithic Humans: Recent Controversies. Quaternary International 326-327: 398-405.

Buss, D. M. 1994. The Evolution of Desire: Strategies of Human Mating. New York: Basic Books.

Carruthers, P. 2002. The Cognitive Functions of Language. Behavioral and Brain Sciences 25: 657-674.

-. 2006. The Architecture of the Mind. Massive Modularity and the Flexibility of Thought. New York: Oxford University Press. 
Clark, A. 2001. Mindware: An Introduction to the Philosophy of Cognitive Science. New York: Oxford University Press.

-. 2008. Supersizing the Mind: Embodiment, Action and Cognitive Extension. Oxford: Oxford University Press.

Cochran, G. and H. Harpending. 2009. The 10,000 Year Explosion: How Civilization Accelerated Human Evolution. New York: Basic Books.

Conard, N. J. 2010. Cultural Modernity: Consensus or Conundrum? Proceedings of the National Academy of Science U.S.A. 107: 7621-7622.

Conard, N. J. and M. Bolus. 2003. Radiocarbon Dating the Appearance of Modern Humans and the Timing of Cultural Innovations in Europe: New Results and New Challenges. Journal of Human Evolution 44: 331-371.

Coolidge, F. L. and T. Wynn. 2005. Working Memory, its Executive Functions, and the Emergence of Modern Thinking. Cambridge Archaeological Journal 15 (1): 5-26.

-. 2011. Comment to C.S. Henshilwood and B. Dubreuil "The Still Bay and Howiesons Poort, 77-59 ka: Symbolic Material Culture and the Evolution of the Mind during the African Middle Stone Age." Current Anthropology 52 (3): 361-400.

Cosmides, L. and J. Tooby. 1987. From Evolution to Behaviour: Evolutionary Psychology as the Missing Link. In The Latest on the Best: Essays on Evolution and Optimality, ed. J. Dupre, 277-306. Cambridge, MA: MIT Press.

-. 2013. Evolutionary Psychology: New Perspectives on Cognition and Motivation. Annual Reviews of Psychology 64: 201-229.

Dehaene, S. and L. Cohen. 2007. Cultural Recycling of Cortical Maps. Neuron 56 (2): 384-398.

De Jaegher, H. and E. Di Paolo. 2007. Participatory Sense-Making: An Enactive Approach to Social Cognition. Phenomenology and the Cognitive Sciences 6: 485-507.

Dekker, T. M. and A. Karmiloff-Smith. 2011. The Dynamics of Ontogeny: A Neuroconstructivist Perspective on Genes, Brains, Cognition and Behavior. In Gene Expression to Neurobiology and Behavior: Human Brain Development and Developmental Disorders, ed. O. Braddick, J. Atkinson, and G. M. Innocenti, 23-33. Burlington: Elsevier/ Academic Press.

d'Errico, F. 2003. The Invisible Frontier. A Multiple Species Model for the Origin of Behavioral Modernity. Evolutionary Anthropology 12: 188-202.

d'Errico, F. and W. E. Banks. 2013. Identifying Mechanisms behind Middle Paleolithic and Middle Stone Age Cultural Trajectories. Current Anthropology 54: S371-387.

d'Errico, F. and C. S. Henshilwood. 2007. Additional Evidence for Bone Technology in the Southern African Middle Stone Age. Journal of Human Evolution 52: 142-163.

d'Errico, F., C. Henshilwood, M. Vanhaeren, and K. van Niekerk. 2005. Nassarius Kraussianus Shell Beads from Blombos Cave: Evidence for Symbolic Behaviour in the Middle Stone Age. Journal of Human Evolution 48 (1): 3-24.

d'Errico, F. and A. Nowell. 2000. A New Look at the Berekhat Ram Figurine: Implications for the Origins of Symbolism. Cambridge Archaeological Journal 10: 123-167.

d'Errico, F. and M. Soressi. 2002. Systematic Use of Manganese Pigment by the Pech-del'Azé Neandertals: Implications for the Origin of Behavioral Modernity. Journal of Human Evolution 42: A13.

d'Errico, F. and C. B. Stringer. 2011. Evolution, Revolution or Saltation Scenario for the Emergence of Modern Cultures? Philosophical Transactions of the Royal Society of London B 366: 1060-1069.

d'Errico, F., M. Vanhaeren, N. Barton, A. Bouzouggar, H. Mienis, D. Richter, J.-J. Hublin, S. P. McPherron, and P. Lozuet. 2009. Additional Evidence on the Use of Personal Ornaments in the Middle Paleolithic of North Africa. Proceedings of the National Academy of Sciences U.S.A. 106: 16051-16056. 
d'Errico, F., J.Zilhão, M. Julien, D. Baffier, and J. Pelegrin. 1998. Neanderthal Acculturation in Western Europe? A Critical Review of the Evidence and its Interpretation. Current Anthropology 39: S1-44.

Dubreuil, B. 2011. The Other Middle-Range Theories: Mapping Behaviour and the Evolution of the Mind. In Homo Symbolicus: The Dawn of Language, Imagination and Spirituality, ed. C. S. Henshilwood and F. d'Errico, 185-204. Amsterdam: John Benjamins.

Elman, J. E., K. Plunkett, D. Parisi, A. Karmiloff-Smith, E. Bates, and M. H. Johnson. 1996. Rethinking Innateness: A Connectionist Perspective on Development. Cambridge, MA: MIT Press.

Farran, E. K. and A. Karmiloff-Smith. 2012. Neurodevelopmental Disorders Across the Lifespan: A Neuroconstructivist Approach. Oxford: Oxford University Press.

Fiebich, A. 2016. Narratives, Culture, and Folk Psychology. Phenomenology and the Cognitive Sciences 15 (1): 135-149.

Finlayson, C., K. Brown, R. Blasco, J. Rosell, J. J. Negro, G. R. Bortolotti, G. Finlayson et al. 2012. Birds of a Feather: Neanderthal Exploitation of Raptors and Corvids. PLoS ONE 7 (9): e45927.

Fodor, J. A. 1983. The Modularity of Mind: An Essay in Faculty Psychology. Cambridge, MA: MIT Press.

-. 1985. Multiple Book Review of the Modularity of Mind. Behavioral and Brain Sciences 8: 1-42.

French, J. C. 2016. Demography and the Palaeolithic Archaeological Record. Journal of Archaeological Method and Theory 23 (1): 150-199.

Fritz, C. and Tosello, G. 2007. The Hidden Meaning of Forms: Methods of Recording Paleolithic Parietal Art. Journal of Archaeological Method and Theory 14 (1): 48-80.

Fuchs, T. and H. De Jaegher. 2009. Enactive Intersubjectivity: Participatory Sense-Making and Mutual Incorporation. Phenomenology and the Cognitive Sciences 8: 465-486.

Gallagher, S. 2005. How the Body Shapes the Mind. New York: Oxford University Press.

-. 2008. Direct Perception in the Intersubjective Context. Consciousness and Cognition 17: 535-543.

Gallagher, S. and D. Hutto. 2008. Primary Interaction and Narrative Practice. In The Shared Mind: Perspectives On Intersubjectivity, ed. J.Zlatev, T. Racine, C. Sinha, and E. Itkonen, 17-38. Amsterdam: John Benjamins.

Gamble, C. 1999. Paleolithic Societies of Europe. Cambridge: Cambridge University Press.

Gardner, H. 1983. Frames of Mind: The Theory of Multiple Intelligences. New York: Basic Books.

Garofoli, D. 2013. Critique of "How Things Shape the Mind. A Theory of Material Engagement," by Lambros Malafouris. Journal of Mind and Behavior 34 (3-4): 299-310.

-. 2015. Do Early Body Ornaments Prove Cognitive Modernity? A Critical Analysis From Situated Cognition. Phenomenology and the Cognitive Sciences 14: 803-825.

-. 2016. Cognitive Archaeology Without Behavioral Modernity: An Eliminativist Attempt. Quaternary International 405, Part A (The material dimensions of cognition): $125-135$.

Garofoli, D. and M. N. Haidle. 2014. Epistemological Problems in Cognitive Archaeology: An Anti-Relativistic Agenda Towards Methodological Uniformity. Journal of Anthropological Sciences 92: 7-41.

Gibson, J. J. 1979. The Ecological Approach to Visual Perception. Boston: Houghton Mifflin.

Gottlieb, G. 2007. Probabilistic Epigenesis. Developmental Science 10: 1-11.

Grün, R. and C. B. Stringer. 2000. Tabun Revisited: Revised ESR Chronology and New ESR and Useries Analyses of Dental Material From Tabun C1. Journal of Human Evolution 39: 601-612. 
Haidle, M. N. 2014. Building a Bridge - An Archeologist's Perspective on the Evolution of Causal Cognition. Frontiers in Psychology 5: 1472.

Haidle, M. N., M. Bolus, M. Collard, N. J. Conard, D. Garofoli, M. Lombard, A. Newell, C. Tennie, and A. Whiten. 2015. The Nature of Culture: An Eight-Grade Model for the Evolution and Expansion of Cultural Capacities in Hominins and Other Animals. Journal of Anthropological Sciences 93: 43-70.

Henshilwood, C.S. 2009. The "Upper Palaeolithic" of Southern Africa: The Still Bay and Howiesons Poort Techno-Traditions. In African Genesis: Perspectives on Hominid Evolution, ed. S. Reynolds and A. Gallagher, 38-50. Johannesburg: Witwatersrand University Press.

Henshilwood, C.S., F. d'Errico, M. Vanhaeren, K. van Niekerk, and Z.Jacobs. 2004. Middle Stone Age Shell Beads from South Africa. Science 304: 404.

Henshilwood, C.S. and B. Dubreuil. 2009. Reading the Artefacts: Gleaning Language Skills from the Middle Stone Age in Southern Africa. In The Cradle of Language, ed. R. Botha and C. Knight, 41-60. Oxford: Oxford University Press.

-. 2011. The Still Bay and Howiesons Poort, 77-59 ka: Symbolic Material Culture and the Evolution of the Mind during the African Middle Stone Age. Current Anthropology 52: 361-400.

Henshilwood, C.S. and C. W. Marean. 2003. The Origin of Modern Human Behavior. Current Anthropology 44: 627-651.

Hodder, I. 2012. Entangled: An Archaeology of the Relationships Between Humans and Things. Oxford: Wiley-Blackwell.

Holyoak, K. and P. Thagard. 1994. Mental Leaps: Analogy in Creative Thought. Cambridge, MA: MIT Press.

Hutchins, E. 2008. The Role of Cultural Practices in the Emergence of Modern Human Intelligence. Philosophical Transactions of the Royal Society Biological Sciences 363: 2011-2019.

Hutto, D. D. 2011. Elementary Mind Minding, Enactivist-Style. In Joint Attention: New Developments in Philosophy, Psychology, and Neuroscience, ed. A. Seemann, 307-341. Cambridge, MA: MIT Press.

Iliopoulos, A. 2016. The Material Dimensions of Signification: Rethinking the Nature and Emergence of Semiosis in the Debate on Human Origins. Quaternary International 405, Part A (The material dimensions of cognition): 111-124.

Iliopoulos, A. and D. Garofoli. 2016. The Material Dimensions of Cognition: Reexamining the Nature and Emergence of the Human Mind. Quaternary International 405, Part A (The material dimensions of cognition): 1-7.

Iriki, A. and M. Taoka. 2012. Triadic (Ecological, Neural, Cognitive) Niche Construction: A Scenario of Human Brain Evolution Extrapolating Tool Use and Language from the Control of Reaching Actions. Philosophical Transactions of the Royal Society of London B Biological Sciences 367: 10-23.

Jablonka, E. and M.Lamb. 2005. Evolution in Four Dimensions: Genetic, Epigenetic, Behavioral, and Symbolic Variation in the History of Life. Cambridge, MA: MIT Press.

Johansson, S. 2015. Language Abilities in Neanderthals. Annual Review of Linguistics 1: $311-332$.

Johnson, M. H. 2001. Functional Brain Development in Humans. Nature Revieres Neuroscience 2: 475-483.

Johnson, M.H. and M. de Haan. 2011. Developmental Cognitive Neuroscience. Malden: Wiley-Blackwell.

Karmiloff-Smith, A. 1992. Beyond Modularity: A Developmental Approach to Cognitive Science. Cambridge, MA: MIT Press. 
-. 1998. Development Itself is the Key to Understanding Developmental Disorders. Trends in Cognitive Sciences 2. (10): 389-398.

-. 2009. Nativism versus Neuroconstructivism: Rethinking the Study of Developmental Disorders. Developmental Psychology 45 (1): 56-63.

-. 2012a. Is Development Domain Specific or Domain General? A Third Alternative. In The Journey from Child to Scientist: Integrating Cognitive Development and the Education Sciences, ed. S. M. Carver and J. Shrager, 127-140. Washington, D. C.: American Psychological Association.

-. 2012b. Perspectives on the Dynamic Development of Cognitive Capacities: Insights from Williams Syndrome. Current Opinion in Neurology 25: 106-111.

Karmiloff-Smith, A., and E. K. Farran. 2012. Williams Syndrome: A Model for the Neuroconstructivist Approach. In Neurodevelopmental Disorders Across the Lifespan: A Neuroconstructivist Approach, ed. E. K. Farran and A. Karmiloff-Smith, 1-12. Oxford: Oxford University Press.

Kendall, J. R. 2011. Cultural Niche Construction and Human Learning Environments: Investigating Sociocultural Perspectives. Biological Theory 6 (3): 241-250.

Klein, R. G. 2000. Archaeology and the Evolution of Human Behavior. Evolutionary Anthropology 9: 17-36.

-. 2008. Out of Africa and the Evolution of Human Behavior. Evolutionary Anthropology 17: 267-281.

Klein, R. G., and B. Edgar. 2002. The Dawn of Human Culture. New York: John Wiley and Sons.

Knappett, C. 2005. Thinking through Material Culture: An Interdisciplinary Perspective. Philadelphia: University of Pennsylvania Press.

Krause, J., C. Lalueza-Fox, L. Orlando, W. Enard, R. E. Green, H. A. Burbano, J.-J. Hublin et al. 2007. The Derived FOXP2 Variant of Modern Humans Was Shared with Neandertals. Current Biology 17: 1908-1912.

Kuhn, S. and M. Stiner. 2007. Paleolithic Ornaments: Implications for Cognition, Demography and Identity. Diogenes 54: 40-48.

Kurzban, R. 2010. Grand Challenges of Evolutionary psychology. Frontiers in Psychology 1 (3): doi: $10.3389 /$ fpsyg. 2010.00003

Lahr, M. M. and R. A. Foley. 1998. Towards a Theory of Modern Human Origins: Geography, Demography, and Diversity in Recent Human Evolution. Supplement American Journal of Physical Antbropology 41(27): 137-176.

Laland, K. N. and M.J. O’Brien. 2010. Niche Construction Theory and Archaeology. Journal of Archaeological Method and Theory 17 (4): 303-322.

-. 2011. Cultural Niche Construction: An Introduction. Biological Theory 6 (3): 191202.

Laland, K. N., J. Odling-Smee, and M. W. Feldman. 2000. Niche Construction, Biological Evolution, and Cultural Change. Behavioral and Brain Sciences 23 (1): 131-175.

-. 2001: Cultural Niche Construction and Human Evolution. Journal of Evolutionary Biology 14 (1): 22-33.

Langbroek, M. 2014. Ice Age Mentalists: Debating Neurological and Behavioural Perspectives on the Neandertal and Modern Mind. Journal of Anthropological Sciences 92: 285-289.

Lewis-Williams, D. 2002. The Mind in the Cave: Consciousness and the Origins of Art. New York: Thames and Hudson.

Lewontin, R. C. 2000. The Triple Helix: Gene, Organism and Environment. Cambridge, MA: Harvard University Press.

Malafouris, L. 2007. Before and Beyond Representation: Towards an Enactive Conception of the Palaeolithic Image. In Image and Imagination: A Global History of Figurative 
Representation, ed. C. Renfrew and I. Morley, 289-302. Cambridge: McDonald Institute Monographs.

-. 2010. Metaplasticity and the Human Becoming. Principles of Neuroarchaeology. Journal of Anthropological Sciences 88: 49-72.

-. 2013. How Things Shape the Mind: A Theory of Material Engagement. Cambridge, MA: MIT Press.

Mareschal, D., M. H.Johnson, S. Sirois, M. Spratling, M.S. C. Thomas, and G. Westermann. 2007. Neuroconstructivism: How the Brain Constructs Cognition. New York: Oxford University Press.

Mazza, P. P. A., F. Martini, B. Sala, M. Magi, M. P. Colombini, G. Giachi, F. Landucci et al. 2006. A New Palaeolithic Discovery: Tar-hafted Stone Tools in a European Mid-Pleistocene Bone-Bearing Bed. Journal of Archaeological Sciences 33: 1310-1318.

McBrearty, S. and A. Brooks. 2000. The Revolution That Wasn't: A New Interpretation of the Origin of Modern Behavior. Journal of Human Evolution 39: 453-563.

McDougal, I., F. H. Brown, and J. G. Fleagle. 2005. Stratigraphic Placement and Age of Modern Humans from Kibish, Ethiopia. Nature 433: 733-736.

Mellars, P. 2005. The Impossible Coincidence: A Single Species Model for the Origins of Modern Human Behavior in Europe. Evolutionary Anthropology 14: 12-27.

-. 2006. Why Did Modern Human Populations Disperse From Africa ca 60,000 Years Ago? A New Model. Proceedings of the National Academy of Science U.S.A. 103: 9381-9386.

Mellars, P. and C. Stringer. 1989. The Human Revolution: Behavioural and Biological Perspectives on the Origins of Modern Humans. Edinburgh: Edinburgh University Press.

Menary, R. 2007. Cognitive Integration: Mind and Cognition Unbounded. Basingstoke: Palgrave Macmillan.

Milligan, K., J. W. Astington, and L. A. Dack. 2007. Language and Theory of Mind: Meta-Analysis of the Relation between Language Ability and False-Belief Understanding. Child Development 78: 622-646.

Mithen, S. 1994. From Domain Specific to Generalized Intelligence: A Cognitive Interpretation of the Middle/Upper Palaeolihtic Transition. In The Ancient Mind: Elements of Cognitive Archaeology, ed. C. Renfrew and E. B. W.Zubrow, 29-39. Cambridge: Cambridge University Press.

-. 1996. The Prehistory of the Mind: A Search for the Origins of Art, Religion, and Science. London: Thames and Hudson.

-. 2014. The Cognition of Homo Neanderthalensis and Homo Sapiens: Does the Use of Pigment Necessarily Imply Symbolic Thought? In Dynamics of Learning in Neanderthals and Modern Humans. Vol.2, ed. T. Akazawa, N. Ogihara, H. C. Tanabe, and H. Terashima, 7-16. Tokyo: Springer.

Nash, A. 2014. Are Stone-Age Genes Created out of Whole Cloth? Evaluating Claims about the Evolution of Behavior. Dialectical Anthropology 38: 305-332.

New, J., L. Cosmides, and J. Tooby. 2007. Category-Specific Attention for Animals Reflects Ancestral Priorities, Not Expertise. Proceedings of the National Academy of Sciences U.S.A. 104: 16598-16603.

Noble, W. and I. Davidson. 1996. Human Evolution, Language and Mind. Cambridge: Cambridge University Press.

Nowell, A. 2010. Defining Behavioral Modernity in the Context of Neandertal and Anatomically Modern Human Populations. Annual Reviews Anthropology 39: 437-452.

Odling-Smee, J. F., K. N. Laland, and M. W. Feldman. 2003. Niche Construction: The Neglected Process in Evolution. Princeton: Princeton University Press.

Papagianni, D. and M. A. Morse. 2013. The Neanderthals Rediscovered. How Modern Science Is Rewriting Their Story. London: Thames and Hudson. 
Pinker, S. 1994. The Language Instinct. New York: William Morrow.

-. 1999. Words and Rules. London: Weidenfeld and Nicolson.

Powell, A., S. Shennan, and M. G. Thomas. 2009. Late Pleistocene Demography and the Appearance of Modern Human Behavior. Science 324: 1298-1301.

Preucel, R. 2007. Archaeological Semiotics. Malden: Blackwell Publishing.

Prinz, J. J. 2002. Furnishing the Mind: Concepts and Their Perceptual Basis. Cambridge, MA: MIT Press.

Read, D. 2012. Population Size Does Not Predict Artifact Complexity: Analysis of Data from Tasmania, Arctic Hunter Gatherers, and Oceania Fishing Groups. eScholarship University of California: http://www.escholarship.org/uc/item/61n4303q.

Renfrew, C. 1994. Towards a Cognitive Archaeology. In The Ancient Mind: Elements of Cognitive Archaeology, ed. C. Renfrew and E. B. W.Zubrow, 3-12. Cambridge: Cambridge University Press.

-. 1996. The Sapient Behaviour Paradox: How to Test for Potential? In Modelling the Early Human Mind, ed. P. Mellars and K. Gibson, 11-15. Cambridge: McDonald Institute.

Roberts, P. 2016. 'We Have Never Been Behaviourally Modern': The Implications of Material Engagement Theory and Metaplasticity for Understanding the Late Pleistocene Record of Human Behavior. Quaternary International 405, Part A (The material dimensions of cognition): 8-20.

Rossano, M. 2010. Making Friends, Making Tools and Making Symbols. Current Antbropology 51: S89-S98.

Rowlands, M. 2010. The New Science of the Mind: From Extended Mind to Embodied Phenomenology. Cambridge, MA: MIT Press.

Ruffman, T., L. Slade, K. Rowlandson, C. Rumsey, and A. Garnham. 2003. How Language Relates to Belief, Desire, and Emotion Understanding. Cognitive Development 18 (2): 139-158.

Russell, J. 1996. Development and Evolution of the Symbolic Function: The Role of Working Memory. In Modelling the Early Human Mind, ed. P. Mellars and K. Gibson, 159-177. Cambridge: McDonald Institute.

Schwarcz, H. P., R. Grün, B. Vandermeersch, O. Bar-Yosef, H. Valladas, and E. Tchernov. 1988. ESR Dates for the Hominid Burial Site of Qafzeh in Israel. Journal of Human Evolution 17: 733-737.

Shea, J. J. 2011. Homo Sapiens Is as Homo Sapiens Was. Current Anthropology 52: 1-35.

Shennan, S. 2001. Demography and Cultural Innovation: A Model and its Implications for the Emergence of Modern Human Culture. Cambridge Archaeological Journal 11: 5-16.

Soffer, O. 2009. Defining Modernity, Establishing Rubicons, Imagining the Other and the Neanderthal Enigma. In Sourcebook of Paleolithic Transitions, ed. M. Camps and P. Chauhan, 43-64. New York: Springer.

Soressi, M., S. P. McPherron, M. Lenoir, T. Dogandzic, P. Goldberg, Z. Jacobs, and Y. Maigrot. 2013. Neandertals Made the First Specialized Bone Tools in Europe. Proceedings of the National Academy of Sciences U.S.A. 110: 14186-14190.

Spelke, E. S. 1994. Initial Knowledge, Six Suggestions. Cognition 50: 431-445.

Speth, J. 2004. News Flash: Negative Evidence Convicts Neanderthals of Gross Mental Incompetence. World Archaeology 36 (4): 519-526.

Sperber, D. 1996. Explaining Culture: A Naturalistic Approach. Oxford: Blackwell.

-. 2005. Modularity and Relevance: How Can a Massively Modular Mind Be Flexible and Context-Sensitive? In The Innate Mind: Structure and Content, ed. P. Carruthers, S. Laurence, and S. Stich, 53-68. Oxford: Oxford University Press.

Sterelny, K. 2003. Thought in a Hostile World. New York: Blackwell.

-. 2011. The Evolved Apprentice: How Evolution Made Humans Unique. Cambridge, MA: MIT Press. 
Stout, D. and T. Chaminade. 2007. The Evolutionary Neuroscience of Tool Making. Neuropsychologia 45: 1091-1100.

Stringer, C. B. and C. Gamble. 1993. In Search of the Neandertals. New York: Thames and Hudson.

Tallis, R. 2011. Aping Mankind: Neuromania, Darwinitis and the Misrepresentation of Humanity. Durham: Acumen Editions.

Thieme, H. 2005. The Lower Palaeolithic Art of Hunting: The Case of Schöningen 13 II-4, Lower Saxony, Germany. In The Hominid Individual in Context: Archaeological Investigations of Lower and Middle Paleolithic Landscapes, Locales and Artefacts, ed. C. Gamble and M. Porr, 115-132. New York: Routledge.

Thompson, R. K. R. and D. L. Oden. 2000. Categorical Perception and Conceptual Judgments by Nonhuman Primates: The Paleological Monkey and the Analogical Ape. Cognitive Science 24 (3): 363-396.

van der Lely, H. K. J. 2005. Domain-Specific Cognitive Systems: Insight from Grammatical Specific Language Impairment. Trends in Cognitive Sciences 9: 53-59.

Villa, P. and W. Roebroeks. 2014. Neandertal Demise: An Archaeological Analysis of the Modern Human Superiority Complex. PLoS ONE 9 (4): e96424.

Villa, P., E. Soto, M.Santonja Gómez, A. Pérez-González, R. Mora, J. Parcerisas, and C. Sesé. 2005. New Data From Ambrona: Closing The Hunting Versus Scavenging Debate. Quaternary International 126-128: 223-250.

Yellen, J. E., A. S. Brooks, E. Cornelissen, M. J. Mehlman, and K. Stewart. 1995. A Middle Stone Age Worked Bone Industry from Katanda, Upper Semliki Valley, Zaire. Science 268: 553-556.

Wadley, L. 2001. What is Cultural Modernity? A General View and a South African Perspective from Rose Cottage Cave. Cambridge Archaeological Journal 11 (2): 201221.

Wadley, L., T. Hodgskiss, and M. Grant. 2009. Implications for Complex Cognition from the Hafting of Tools with Compound Adhesives in the Middle Stone Age, South Africa. Proceedings of the National Academy of Sciences U.S.A. 106: 9590-9594.

Watkins, T. 2016. The Cultural Dimension of Cognition. Quaternary International 405, Part A (The material dimensions of cognition): 91-97.

Welsch, W. 2016. Bodily Changes During the Proto-Cultural Period and Their Ongoing Impact on Culture. In this volume.

Westermann, G., D. Mareschal, M. H. Johnson, S. Sirois, M. W. Spratling, and M. S. Thomas. 2007. Neuroconstructivism. Developmental Science 10 (1): 75-83.

Wheeler, M. 2006. Traits, Genes and Coding. In Handbook of the Philosophy of Biology, ed. M. Ruse, 381-411. Amsterdam: Elsevier.

Wheeler, M. and A.Clark. 1999. Genic Representation: Reconciling Content and Causal Complexity. British Journal for the Philosopby of Science 50 (1): 103-135.

-. 2008. Culture, Embodiment, and Genes: Unraveling the Triple Helix. Philosophical Transactions of the Royal Society Series Biological Sciences 363: 3563-3575.

White, L. 1959. The Evolution of Culture: The Development of Civilization to the Fall of Rome. New York: McGraw-Hill.

White, T. D., B. Asfaw, D. DeGusta, H. Gilbert, G. D. Richards, G. Suwa, and F. C. Howell. 2003. Pleistocene Homo sapiens from Middle Awash, Ethiopia. Nature 423: 742-747.

Wilson, R. A. 2008. The Drink You Have When You're Not Having a Drink. Mind and Language 23(3): 273-283.

Wylie, A. 2002. Thinking Through Things. Essays in the Philosophy of Archaeology. Berkeley: University of California Press.

Wynn, T. 2002. Archaeology and Cognitive Evolution. Behavioral and Brain Sciences 25: $389-438$. 
Wynn, T. and F. L. Coolidge. 2004. The Expert Neandertal Mind. Journal of Human Evolution 46: 467-487.

-. 2009. Implications of a Strict Standard for Recognizing Modern Cognition in Prehistory. In Cognitive Archaeology and Human Evolution, ed. S. de Beaune, F. L. Coolidge, and T. Wynn, 117-128. New York: Cambridge University Press.

Wynn, T., F. L. Coolidge, and M. Bright. 2009. Hohlenstein-Stadel and the Evolution of Human Conceptual Thought. Cambridge Archaeological Journal 19 (1): 73-83.

Zilhão, J. 2007. The Emergence of Ornaments and Art: An Archaeological Perspective on the Origins of Behavioral Modernity. Journal of Archaeological Research 15 (1): 1-54.

-. 2011a. The Emergence of Language, Art and Symbolic Thinking. A Neanderthal Test of Competing Hypotheses. In Homo Symbolicus: The Dawn of Language, Imagination and Spirituality, ed. C. S. Henshilwood and F. d'Errico, 111-132. Amsterdam: John Benjamins.

-. 2011b. Aliens From Outer Time? Why The "Human Revolution" Is Wrong, and Where Do We Go from Here? In Continuity and Discontinuity in the Peopling of Europe, ed. S. Condemi and G. C. Weniger, 331-366. Dordrecht: Springer Editions.

-. 2012. Personal Ornaments and Symbolism among the Neanderthals. In Origins of Human Innovation and Creativity. Developments in Quaternary Science. Vol. 16., ed. S. Elias, 35-49. London: Elsevier.

Zahidi, K. and E. Myin. 2016. Radically Enactive Numerical Cognition. In this volume.

Zilhão, J., D. E. Angelucci, E. Badal-García, F. d'Errico, F. Daniel, L. Dayet, K. Douka et al. 2010. Symbolic Use of Marine Shells and Mineral Pigments by Iberian Neandertals. Proceedings of the National Academy of Science U.S.A. 107: 1023-1028. 



\title{
Artifacting Minds: Material Engagement Theory and Joint Action
}

\author{
Shaun Gallagher/Tailer G. Ransom
}

\begin{abstract}
In this chapter we argue that an account of joint or collective action should make explicit the dynamical relations, not just among intersubjective forces, including normative and institutional practices, but also among the material conditions imposed by artifacts, tools, media, etc. Not only do joint actions emerge at these intersections, but also various aspects of the relevant cognitive processes (intentions, beliefs, commitments, common knowledge, etc.), rather than pre-existing in any complete form, emerge in the dynamical mix of agents, material things, and social structures. We employ Material Engagement Theory (MET) to explicate an account of these factors, and we show how this approach to joint action is consistent with an enactivist theory of cognition.
\end{abstract}

\section{Introduction}

There has been a lot of ink spilled on the topic of joint and collective action. Established accounts tend to ground joint actions in terms of individual agents with separate intentions, plus shared beliefs about the activity that is jointly accomplished (Tuomela 2005); or individuals with separate intentions, who form plural subjects together through their normative commitments to act jointly (Gilbert 2009); or individuals with interlocking intentions, meshing sub-plans and common knowledge of each other's intention to act together (Bratman 2009). These are just a sample of contemporary accounts of joint or collective action. Recently, however, efforts have been made to show how joint actions are possible without these strong, cognitively sophisticated preconditions which complicate or rule out some forms of activity such as team reasoning (Pacherie 2012), interacting with children or other less "cognitively complicated" critters (Tollefsen 2005) or some simple embodied interactions with others, which do not need to involve anything more complicated or conceptually/representationally loaded than a basic dyadic interaction with joint attention directed at the relevant state-of-affairs (Fiebich and Gallagher 2013). In this chapter we argue that any account of joint or collective action should make explicit the dynamical relations, not just among intersubjective forces, including normative and institutional practices, but also among the material conditions imposed by artifacts, tools, media, etc. The idea is that not only do joint actions emerge at these intersections, but also various aspects of the relevant cognitive processes (intentions, beliefs, com- 
mitments, common knowledge, etc.), rather than pre-existing in any complete form, are constituted in the dynamical mix of agents, material things, and social structures.

The approach we take in this chapter fits well with a project that Lambros Malafouris (2013) develops under the title Material Engagement Theory (MET). Although Malafouris applies MET to questions about agency and intentionality, he does not discuss joint agency or shared intentions. We think his analysis can be extended to include considerations about joint actions. Our aim is to provide a MET analysis of joint action, and to show how this approach is consistent with enactivist accounts of cognition. At the very least this type of analysis will highlight some traditionally ignored features of joint action, and we think it provides some innovative insights.

\section{MET and the Concept of Agency}

From the perspective of the philosophy of mind, MET is a non-representationalist version of distributed cognition, or the extended mind hypothesis (Clark and Chalmers 1998), and has close kinship with enactivist approaches to cognition. MET does not rule out representations altogether; it leaves room for external representations that may be the products of our practices and engagements with the material world, but it does not endorse an account of the mind constituted by internal representations of the external world. Malafouris, working within the perspective of archeology and anthropology, characterizes MET in terms of post-processual thinking, niche-construction theory, developmental systems theory, and Actor-Network Theory (2013, 33, 40, 123). MET is situated primarily as an approach to material culture. Its purview involves, accordingly, an evolutionary framework with the aim of providing a deep historical account of how the contemporary human mind emerged out of a dynamic interplay of anatomical, neurological, cultural and material forces. It also has clear application to philosophy of mind and cognitive science, especially in relation to embodied cognition approaches. It focuses on what Edwin Hutchins $(2010,705)$ has called "cognitive ecology," "the web of mutual dependence among the elements of a cognitive ecosystem” (see Malafouris 2013, 36). The elements are not just neural, but extend to include elements of the body and the environment. In this regard, however, the aim of MET isn't just a mapping of how brains, bodies, and environments have co-evolved in terms of causal correlations. It also seeks "to discern the possible ways in which the actual nature of the relationship between them might have changed in the course of human evolution" $(2013,39)$. This involves what Malafouris calls "metaplasticity," where evolutionary change is not explained simply in biological terms of brain plasticity, but includes the effects of our engagement with culture and the material environment ( $45 \mathrm{ff}$.). "MET is particularly concerned with the mechanisms that mediate those plastic changes, not at the level of the individual, but at the systemic level of enculturation and social practice" (50). 
According to MET, the embodied mind stretches into the surrounding material reality and consists in the interaction between brain, body, and things or artifacts. Malafouris provides a good example of this in his discussion of the Mycenaean Linear B tablets. Linear B is a script, predating the Greek alphabet, used to write the earliest form of Greek on clay tablets. For users of the Linear B tablets, cognitive processes incorporated certain practices made possible by the tablets, employing not simply the representational mnemonic function they served, but also the physical manipulation of the medium allowed by the material tablets. Such physical manipulation not only facilitated problem solving by a spatial (re)organizing of complex cognitive tasks but also transformed the physical boundaries of the problem space and thereby restructured the problem-solving process (Malafouris 2013, 72). The spatial arrangement of information afforded by the use of clay tablets made a certain kind of cognition, which previously did not happen, possible. Furthermore, the detailed cultural practices that grew up around the production and use of such tablets contributed to a reorganization of knowledge. They introduced a different set of skills and affordances that "radically reconfigure the cognitive ecology and the dynamics of the Mycenaean memory field" (81), shifting cognition into a new operation: reading. “The Linear B tablets, by 'being there' in the 'outside' world, enable the Mycenaean scribe to substitute visual recognition for recall, thereby transforming the difficult 'internal' memory problem into an easier 'external' perceptual one" (82). The tablet format did this, moreover, in a way that allowed others to see the process and to comment and improve on it. In this respect it introduced more than a form of epistemic environmental scaffolding, in support of intelligent action (Sterelny 2010); it rather introduced a kind of entanglement, which describes the dynamical network of co-dependent cognitive and practical relations between humans and material things (Hodder 2011).

We might think of these tablets as external to the cognitive system. But 'internal' and 'external' are relative terms; a material artifact, may be physically external to the human organism, but at the same time, to the extent that it involves a practice, and therefore a coupling to the organism, it can be internal to the cognitive system (Malafouris 2013, 84; also see Aydin 2013). Importantly, in contrast to the emphasis on parity, isomorphism or cognitive equivalents found in the extended mind paradigm (Clark and Chalmers 1998; Clark 2008), MET emphasizes the differences introduced into cognition, generated by differences among artifacts, brains and bodies, much like the productive effects one gets when one combines the different media of spatial hand gestures with sequential vocal speech (see McNeill 1992). The engagement with artifacts, such as the Linear B tablets, contributes something to the cognitive process that changes it. In this sense, what happens in this engagement is not a mere offloading of some cognitive capacity, which finds its origin first in the brain, and is then exteriorized onto a stable, material scaffolding. Instead we find the generation of new cognitive capabilities and practical affordances that emerge out of the interaction between the scribe and the tablet, where the dynamically intertwined features of, and contributions to, the system are not reducible to its individual parts. 
Moreover, things, with which we engage, from the Linear B tablets to the contemporary iPhone, don't simply sit there in their materiality, but also act as "emotional anchors" that elicit affective and aesthetic responses. Material things are not simply seen; they are manipulated, exchanged, desired, "fetishized, revered, ridiculed, and so on" (Malafouris 2013, 87), and can have transformative, metaplastic effects on cognition. Accordingly, they take on additional cultural significance and become part of a broader "web of cultural meaning" that enables a range of nuanced and normative action-perception cycles.

To bring us closer to the issue of joint action, let's consider the application of MET to the question of agency. On the analysis given by Malafouris, agency should not be considered a property of humans, or of things, but a property of the material engagement that involves both humans and things. He employs Bruno Latour's Actor-Network Theory (ANT) to clarify this idea. On this theory, actions occur in networks that include people and things without giving any sort of a priori privilege to the role played by one type of element as the supposed "prime mover" of the process (128). People don't act outside of some context or simply from some de novo intention formed abstractly inside their mind. It is certainly true that people sometimes engage in acts of deliberations that one cannot simply deduce or explain from the contextual priors alone. They may consider different options for actions (reasons or means to realize an action) and search for new problem-solving strategies for specific challenges that they encounter in their surroundings. But this intention formation is itself shaped by the affordances offered by the environment - it's a mentality shaped by the environs, which may also be guided or informed by others, and may in fact take place through intersubjective discussions. Accordingly, prior intentions have contextual priors that explain why someone may have formed them the way they did. That means intention formation is embedded, not only in an individual's life history, but in that individual's relations with others, in her cultural milieu, and, in effect, in the world, as a phenomenologist might say. Intentions emerge in a world populated with artifacts, or things that do things: a speed bump that modifies any intention you may have to speed down a neighborhood street; or a cumbersome weight attached to a hotel room key that forces you to organize your intentions and actions so that you leave the key at the desk rather than carry it away (Malafouris 2013, 124; Latour 1991, 104). Intention formation is not something that simply happens in your head; intentions are being formed via engagement with the materiality of things in the surrounding environment. Malafouris quotes Gell in this regard: agency is "a global characteristic of the world of people and things in which we live, rather than an attribute of the human psyche, exclusively" (1998, 20; quoted in Malafouris 2013, 136).

To flesh out this idea Malafouris turns to Searle's concept of 'intention-in-action'. Here we'll adopt Pacherie's $(2006 ; 2007)$ terminology that distinguishes between a prior or distal (D-) intention, an intention-in-action or present (P-) intention, and a motor (M-) intention. At least in some cases a P-intention is unconnected to any $\mathrm{D}$-intention. And in every case it is in the action, which is occurring in the world, constrained or enabled by particular artefacts that make 
way or get in the way of the action. The agent is constantly adjusting her action to the physical contours and demands of the environment. The notion of the M-intention highlights the fact that an intention is also fully embodied insofar as every P-intention has to be cashed out in detailed, dynamic, mostly non-conscious motoric terms. Action and agency are material in the very real sense that they are ineliminably embodied and situated in a particular physical and artifactual environment. The context of action is even wider than the immediate physicality involved, however, since it is always situated in a "Background" (to use Searle's term) or habitus (to use Bourdieu's). The background/habitus should be considered part of the extended mind - a part that includes cultural values, skills, know-how, and so forth - all of which constrain the affordances presented in any environment.

Here we want to link up Malafouris's account of action to the notion of affordance space. This concept derives from Gibson's notion of affordance: it defines a range of possibilities for action that depend on both body and environment. Such affordance spaces can be physical, but also social and cultural (see Brincker 2014; Gallagher 2015).

An affordance space is defined as the (abstract) range of possibilities provided by any active movement in body or change in environment. An individual's occurrent affordance space is defined by evolution (the fact that she has hands, for instance), development (her life-stage - infant, adult, aged), and by social and cultural practices (normative constraints) - all of which enable and constrain the individual's action possibilities. The human affordance space, for example, differs from a non-human animal's due to differences in evolution. Humans have hands and capacities for certain kinds of movement, and for that reason, a variety of cognitive possibilities are afforded and constrained by these anatomical differences. A child's affordance space differs from an adult's due to differences in developmental factors. Humans learn to move or think in specific ways across developmental parameters. One individual's affordance space differs from another's due to differences in experience, skill level, education and normative constraints, etc. Humans are enabled or constrained to move or to think in particular ways due to their prior experiences and plastic changes in both brain and body, but also within the constraints of their environment.

The concept of metaplasticity is also relevant here. In this respect, it's not just, as Malafouris suggests, that the affordance offered by a certain artifact can shape or reshape a human intention $(2013,143)$. Beyond that possibility, changes in practice, whether intentional or accidental, whether due to an agentive decision, or to the material particularities of the environment, may introduce modulations into the affordance space that, in the long term, loop back into the background, and change the habitus in potentially radical ways. This is still consistent with Malafouris's own aim in his analysis of intention and agency, namely, that intention formation "should be understood as a distributed, emergent and interactive phenomenon rather than as a subjective mental state. The artifact should not be construed as the passive content or object of human intentionality, but as the concrete substantiating instance that brings forth the intentional state" (144). 
Bringing together these different concepts of distributed agency, embodied and situated intentions, affordance spaces, and metaplasticity, MET is consistent with much of the extended and also enactive conceptions of cognition. To put this in somewhat dramatic terms, we might say that the mind itself is an artifact something made or formed in the complex mélange of bodies, material things, physical, social, and cultural environments, practices, backgrounds, and so forth. It's not the person simpliciter who makes, or makes up, his mind; rather, the mind is "artifacted" at the intersection of all of these forces. ${ }^{1}$ This, however, would be too one-sided, since we also want to emphasize the enactive nature of cognition by saying that the mind is artifacting as it is engaged in the realization of actions. Artifacts or material items do not simply enforce, even if they constrain, specific actions. There is a dynamical coupling between the organism (as a self-sustaining system) and its environment that may sometimes assume an asymmetric structure in which one can differentiate varying degrees of active engagement and passive experiences (e.g., Di Paolo 2005). As such, the organism achieves a degree of autonomy that actively modifies the environment for the sake of self-maintenance and adaptation.

\section{METing out Joint Action}

Despite claiming that agency and cognition are not the accomplishments of an individual agent per se, the analyses and examples offered by Malafouris are mostly about individuals engaged with things and environments. Intentions are distributed across the individual and the surrounding environment; an individual is driving his car over a speed bump; an individual guest is returning a key to the hotel desk; an individual scribe is engaged in holding a clay tablet (but not interacting with an official standing next to him or the small boy in the foreground [this is Chadwick's description as rehearsed by Malafouris 2013, 78]), an individual potter is sitting at the potter's wheel, and an individual knapper is working with the stone. To be sure, these examples lead to important and insightful analyses; and furthermore there is no question of MET denying the importance of social interaction, as Malafouris makes clear numerous times in his book. Given all of this we think it may be productive to look at the case of joint action through the lens of MET - something that may offer insights into joint action, and at the same time, extend MET into more intersubjective interactive settings.

1 It's curious that the term 'artifacting' is in use as a noun, but as yet does not have a dictionary definition (see, e.g., https://www.wordnik.com/words/artifacting). It is used as a technical term in media-technology to mean something like an accidental trace of the technological apparatus or a compression that interferes with or detracts from the quality or aesthetics of the product. In this respect, however, it means precisely the same thing as (and may be slang for) the term 'artifact,' as when one says, for example, that we can see in the photo an artifact due to optical refraction or a lens effect. In this paper we are using the term 'artifacting' as a verb, along with its odd derivative adjective 'artifacted.' 
Joint action has been defined as "any form of social interaction whereby two or more individuals coordinate their actions in space and time to bring about a change in the environment" (Sebanz, Bekkering, and Knoblich 2006, 70). Consider three examples of joint action.

(1) Spontaneous joint action. I'm standing next to you at a party. Music starts and I am so moved by the beat that I grab you and we start dancing. In this case there is no formation of a D-intention (for either of us); a shared P-intention only starts to emerge as we continue to dance and perhaps adopt tactics to avoid bumping into others. In thinking about how this joint action is initiated we could say that the music elicited the initial step. Maybe both of us were motivated by the music. The action that resulted started by shaping my bodily movements into a specific dance step, thereby generating an M-intention, assuming that my action was not just automatic or unintentional. In this joint action of dancing, we are both hearing the music, but it's not clear that we are jointly attending to the music in any unambiguous way. Indeed, it is not clear that joint attention is involved at all.

(2) Distributed joint action. You and I are currently involved in a large complex action that is spatially distributed in such a way that we are not in the same location. Indeed, we may not even know each other. For example, we may be engaged in a terrorist action to blow up a significant national landmark. To accomplish this action we are located in two different places and engaged in two different sub-actions. We have the same goal and we know that we are acting jointly to accomplish this goal. But it's possible that we belong to two different terrorist cells and are following instructions given to us by a third party who explains the project to us in great detail. In this case our shared D-intention governs our joint action, but our P- and M- intentions are entirely different and distinct. In this case, there is no joint attention involved; indeed, since we are in different places, following different instructions and are doing different things, joint attention is not involved. Following a set of instructions in some sense takes the place of guidance by joint attention. In this particular example, the joint action doesn't even involve embodied intersubjective interaction.

(3) Joint action guided by joint attention. I call your attention to the bookcase and convince you to help me move it to the next room. Moving the bookcase is a joint action guided by a relatively abstract $\mathrm{D}$-intention (to move the bookcase) that begins as my individual $\mathrm{D}$-intention but is then shared by you. The most significant contribution comes from our interactive P-intentions (deciding how to turn the bookcase so that it fits through the door, etc.) that involve an ongoing shaping of our coordinated $\mathrm{M}$-intentions as we move the bookcase through the door, etc. In this case there is clear joint attention, shifting, perhaps, from bookcase, to doorway, and back.

These three examples are not meant to be exhaustive; there are many other examples of joint action that involve different intentional structures and degrees of joint attention. 
What can MET tell us about such joint actions? Clearly each of these joint actions involves material engagement and rich contexts (that can also be explicated in terms of background or habitus). With respect to the various aspects of cognition involved, they are best characterized as extended across brains-bodies-agents-environments, in ways that incorporate relevant background components. With respect to intentions, for example, the relevant shared intentions may be primarily in our bodies or emerging in our actions (as in 1). Alternatively, a shared D-intention may be primarily a matter of communicating or instructing (as in 2), and is thus instantiated in other joint actions of communication, or even written down (or encoded) in more than one set of instructions. This type of arrangement may in fact characterize many of the actions, practices or rituals of institutions that document their goals and establish formal statements of their strategies. Even in the last example (3), the shared D-intention is not so much a similarity or identity of the mental states of two individuals; it gets shared through communicative means. Oftentimes a D-intention is actually formed through communicative means, coming into my thoughts through something I overheard, for example, and taking shape only when I express an incomplete or obscure idea that you make more precise in your communicative response. It's not always clear whose idea it was to do X, because the intention was literally distributed across several individuals and shared with all of the imprecisions involved in communicative acts.

Both (1) and (3) involve dynamical embodied intersubjective interactions without which the joint action itself would not be accomplished. Dancing together obviously involves some degree of intersubjectively coordinated movement (the degree of which will depend on the dance, from something close to $0 \%$ in free form dance to something close to $100 \%$ while dancing the tango). Indeed, the joint action is nothing other than this movement. Moving the bookcase also requires some degree of coordination of our individually embodied movements.

We note three things about these kinds of intersubjectively coordinated movements. First, unless there is some kind of imitation involved, the dynamics of the interaction are not based on mirroring. One might imagine a form of dance where one partner simply imitates the other partner, but this would be some idealized process (and clearly not the basis for either free form or tango). In most cases we cannot appeal to a purely brain-based simulation doctrine of motoric matching or imitation to explain how we accomplish the joint action. Our movements are coordinated but also necessarily different. In moving the bookcase, imitation would likely defeat the purpose. We mention this because there is obviously some form of social cognition involved in these examples (that is, one agent understands what the other is doing, and vice versa, at least to some degree), but at least in (1) and (3) the social cognition is neither a form of simulation nor a theoretical inference. Specifically, in these cases, intentions are not hidden away inside the heads of the agents; they are communicated and in some sense take shape in the bodily movements themselves.

Second, things and environments shape the movement dynamics. This is an important insight that comes from MET. Joint action involves material engage- 
ment. This seems obvious in (3). The bookcase is of a certain shape, size, weight and material, and these properties constrain our movements. Getting the bookcase through the door may involve standing it upright and twisting it just so but this entirely depends on how the bookcase is shaped, its weight relative to our strength, etc. Similar things can be said about the doorway - its height and width, where it is positioned in the room, the proximity of other walls and corners, etc. I move my arm to this grip and then move the bookcase this way precisely because I need to move it at a certain angle through a certain angle of the door - and this is something that has to be coordinated with you, either by some short speech act, or gesture, or movement itself. In fact, when moving the bookcase, some of the dynamic joint motor intentions can be coordinated without any direct communication between participants, but through joint attention to the artifact in the specific context of our project. If, for instance, I feel the bookcase start to tip slightly to the right (your left), this change is something I will automatically react to by changing the position of my hands or the amount of force I apply to one part of the bookcase or another. In this case, I counter-balance your movements (and vice versa), not because I directly attend to the motor dynamics of your body and the intentions that they are supposed to express, but rather because we attend to the bookcase and couple our movements through this material artifact. This project of moving and balancing immanently couples our movements often without the need for explicit communicative acts, and can be achieved simply because we jointly attend and react to the situation from unique, but coordinated perspectives on the same thing. In this respect, things and environments shape our M- and P-intentions, and the very dynamics of our movements. It's also quite possible that the jointness of our action (with all the cognitive aspects of intention and attention) fails or dissipates temporarily and then quickly gets reestablished because it is supported by the structure that is reflected in just the way that our bodies are dynamically coupled to each other, to the things and to the specifics of the environment. Intentions are thus constitutively formed and sustained by the whole network of social and material possibilities for a particular action in the course of its being enacted.

In this regard, one could give a much more detailed explication for these different kinds of coordination by means of the conceptual tools and the corresponding empirical results in dynamical systems theory. These would include the analysis of movement patterns that reflect a combination of stability and variability over time, and an understanding of coordinated actions as self-organizing under constraints, such that in some cases, "individuals spontaneously are pulled into the orbit of another's incidental movements," and in some cases environmental constraints push coordinated joint action into being (Marsh et al. 2009, 322). "The dynamical perspective makes the prediction that there are some 'dances we cannot do' - whether those dances be alone or with another individual” (Marsh et al. 2009, 322, citing Schmidt and Richardson 2008). In this context there has been a significant amount of research on social synchrony that allows for precise predictions about what conditions will suit the coordination of movement in tandem with others. Studies using magnetic sensor systems have 
shown that rhythmic movements as well as aperiodic, highly stochastic movements enter into the dynamics of interpersonal coordination (Fowler et al. 2008; Schmidt and Richardson 2008; van Ulzen et al. 2008). Using dynamical equations, other studies have shown that individuals engaged in interactive contexts move in and out of coordination with significantly more time in in-phase or antiphase behavior than in non-coordinated states (Richardson, Marsh, and Schmidt 2005). As Marsh et al. (2009) make clear, these processes are not driven by centralized neural or cognitive processes like simulation or those typically explained in game theoretic approaches; rather neural activation is entrained by the dynamical processes that extend over body and environment. The MET hypothesis in this context is that the introduction of a significant artifact or instrument in the immediate environment of the joint action would alter the (intersubjective) dynamics and redefine that joint action.

Third, as Malafouris indicates, all of this plays out most directly in the P-intentionality involved in the action. For each individual, we should consider the P-intention (the intention-in-action) as a dynamical process. And, precisely for reasons given in the previous two points, there is no coincidence of $\mathrm{P}$-intentions between you and me as we move the bookcase, or as we dance. Accordingly, as Searle (1980) indicates, shared intentions-in-action may have the same goal in common, but they don't necessarily have the means in common. We can make this an even stronger claim: they necessarily don't have the means in common. For example, to move the bookcase through a certain angle, I will have to place my hands differently from yours. Even in dancing, my response to you will differentiate my P-intention from yours, since you are likely responding to me in a different way than I am responding to you, whether in a formal tango or a libertarian free form. Even in the case where I try to imitate you, the dynamics of my movement (the instantiation of the P-intention in the $\mathrm{M}$-intention) are necessarily different given that my body has a different starting position, size, shape, weight, energy level, motor habit, etc. from yours. How I am going to respond, in micro-detail, is just as constrained by my body-schematic and affective parameters as our joint action is constrained by the specific material properties of the bookcase.

One might be tempted to say that the P-intention is constituted by the integration of bottom-up material constraints that relate to the specifics of body-environment, and top-down elements that derive from the $\mathrm{D}$-intention. Two things may be inaccurate in this characterization, however. First, there may not be a $\mathrm{D}$-intention in the mix of things (as in 1). Second, the $\mathrm{D}$-intention has no privilege in regard to material engagement. That is, a $\mathrm{D}$-intention is just as constrained by the specifics of body-environment as the P-intention. It's unlikely, for example, that I will form a D-intention to invite you to join me in eating my car (to use an example from Malafouris). "That is so because such an intention is not afforded. A car might afford a variety of human intentions, some of them quite odd indeed, but at the same time it constrains and limits them” $(2013,143)$. The very materiality of a car limits the possibilities; moreover, such limitations and impossibilities are reflected in (background) cultural practices (or the absence of 
such practices). In that case, if one is tempted to call a $\mathrm{D}$-intention a top-down element, one has to acknowledge that the $\mathrm{D}$-intention is also determined by bottom-up material constraints, and the affordance space offered by the material aspects of action and culture. ${ }^{2}$

The notion of affordance space also helps us to understand some important aspects of joint actions. Joint actions may expand or shrink the affordance space relative to what individuals may accomplish on their own. On the one hand, the expansion of the affordance space should be intuitive. Although I cannot move this bookcase on my own, I can move it if I can get you to help me. Our joint action makes possible something that was impossible via individual action. And although I may be able to dance alone, I can't do the tango alone. In some regard, when I add my skills (strengths) to your different set of skills (strengths), we both may gain by expanding the affordance space to offer more possibilities. On the other hand, some joint actions may limit my individual affordances. If we decide to go for a walk together, your slower gate may prevent me from achieving the right level of exercise. Or my commitment to the joint action may prevent me from doing something else. In joint actions one often has to compromise for the sake of accomplishing the goal, and such compromises can lead to a contraction of either or both of the individuals' affordance spaces.

The affordance spaces defined by joint-action possibilities are not only dependent on the individuals (their skill levels, strengths, weaknesses, etc.) involved, but on the material aspects of things, environments, and cultures. It's precisely the weight and shape of the bookcase that prevents me from moving it on my own; and it may even take three people to move (although in most cases we suggest that removing the books can solve this problem). It's precisely the fact that there is a wide doorway that creates an affordance for this move. And it's precisely the effects of the cultural background and my background reading skills that motivate me to keep the bookcase rather than use it as firewood. Using it as firewood is a possibility in our affordance space, but the fact that the bookcase is an original Memphis-Milano design makes it a more remote possibility. You would likely object to my suggestion that we burn a Memphis-Milano bookcase, even if it were not aesthetically pleasing to you.

${ }^{2}$ A good example of this can be found in the house-building practices of the Eipomek in Papua New Guinea. The process starts with a general D-intention to build a house, which requires a joint action by a number of participants. The size of the house will be determined by the actual number of men that show up to participate in the construction on the day the house is built. The available material and specific social practices determine other features of the house. With the material at hand, the Eipo men build perfectly round houses using implicit geometrical knowledge and concepts for which they do not possess a term, e. g., circle or metrical distance (Thiering 2015, $218 \mathrm{ff}$.). Thiering argues that the building process is shaped by the affordances of the construction material, and the processes in joint actions that reflect an embodied (situated/distributed) shared knowledge. 


\section{Conclusion}

Although researchers are quick to admit that, for example, "studies on joint action challenge the assumption traditionally held in cognitive psychology that perception, action, and higher-level cognitive processes can be understood by investigating individual minds in isolation" when it comes to explaining joint action the focus remains on identifying the mechanisms within individuals that allow for its possibility, for example, the mechanisms that would "allow individuals to share representations" (Sebanz, Harold, and Knoblich 2006, 70). It will pay to focus briefly on Sebanz, Harold, and Knoblich's (2006) excellent review article on joint action because it nicely summarizes standard interpretations, but also points in a direction indicated by MET.

There are plenty of accounts of joint action that place the majority of emphasis on shared beliefs, representations, predictions, etc., that is, accounts that seemingly start and finish inside the subject's head. Shared internal representations, for example, are said to enable the initiation of coordinated action or to keep the action on track once engaged (e. g., Sebanz, Harold, and Knoblich 2006, 70). On such views, establishing joint attention, in the service of joint action, is considered to be the establishment of shared mental representations rather than being itself a basic joint action that involves movement and related bodily and environmental constraints (Fiebich and Gallagher 2013). Thus, standard explanations of joint action suggest that to be able to engage in complementary movements that contribute to the joint action one requires a "joint goal representation" that would suppress what is considered to be the default process of imitating or simulating or mirroring what one sees. In turn, both imitation and joint action rely upon the ability to predict or "infer" action goals, and to "represent the other's task in a functionally equivalent way to one's own" (Sebanz, Harold, and Knoblich 2006, 70).

When experimental subjects become participants or agents in joint action, however, rather than mere observers or predictors of the other's action, researchers discover that the material aspects of bodies and things have an effect on joint action. Thus, in one experiment co-actors modify their actions depending on the length of the wooden board they have to grasp (acting as either an individual or as co-actor), and on the physical length of the other agent's arms (Marsh et al. 2006; Knoblich and Jordan 2003). Thus, "pairs with large mean arm spans made the transition from taking turns to lifting jointly at a longer plank length than pairs with small mean arm spans" (Sebanz, Harold, and Knoblich 2006, 73). One might think, and MET certainly would propose, that the important elements modulating joint action in this case are the material lengths of the arms and boards involved in the task. A more standard interpretation, however, retreats from this view and takes the important elements to be the individual agent's "beliefs" - so that actions are adjusted "not only by what individuals believe they can do, but also by what they believe they can do with others" (Sebanz, Harold, and Knoblich 2006, 73).

This seems a clear example of the different direction of analysis proposed by MET. According to MET, the material aspects of things (their shape and weight and substance) and environments (their physical and social and cultural affor- 
dances) as well as other people (their postures, movements and expressions) matter and enter into the constitution of joint action. Joint action is not simply action in close proximity accompanied by shared mental representations of goals and tasks. It's materially constrained and materially enabled interaction that redefines the affordance space and allows us to do things that we could not do on our own.

We conclude by noting again that there are many different kinds of joint and collective actions. We suggest that the embodied-enactive-extended approach offered by MET can provide critical analyses of the more complex forms of joint actions that go beyond things like dancing or moving bookcases. For example, material artifacts afford the possibility for coordinating social forces in ways that might not otherwise be possible, and in this regard there are cases where specific kinds of material engagement can disclose new social affordances. Consider, for example, something like the Arab Spring or the Occupy movements, where governments were preventing or discouraging people from gathering together in the same space to organize and resist. Despite this prohibition, coordinated collective activity was not only made possible through the use of social media; the form that activity could take was in large part enabled and constrained by the detailed affordances of material culture - the technology, the buildings, the places directly tied to cultural practices - allowing for particular kinds of social-political action that would have been impossible in the absence of such materially constituted affordances. The same kinds of material and social affordances defined the types of actions and sanctions (including bodily threats) utilized by government agencies in response. A larger scale analysis of the overall dynamics that emerge from such confrontations is beyond the scope of this chapter, but we mention such examples to indicate at least one direction of future research.

\section{Acknowledgements}

The authors thank Deborah Tollefsen, Christian Tewes, and an anonymous reviewer for helpful comments on an earlier draft. SG also acknowledges support from the Humboldt Foundation's Anneliese Maier Research Award.

\section{Bibliography}

Aydin, C. 2013. The Artifactual Mind: Overcoming the 'Inside - Outside' Dualism in the Extended Mind Thesis and Recognizing the Technological Dimension of Cognition. Phenomenology and the Cognitive Sciences, 14 (1): 73-94.

Bratman, M. E. 2009. Shared Agency. In Philosophy of the Social Sciences: Philosophical Theory and Scientific Practice, ed. C. Mantzavinos, 41-59. Cambridge: Cambridge University Press.

Brincker, M. 2014. Navigating Beyond 'Here and Now' Affordances - On Sensorimotor Maturation and 'False Belief' Performance. Frontiers in Psychology 5: 1433. doi: 10.3389/fpsyg.2014.01433. 
Clark, A. 2008. Supersizing the Mind: Embodiment, Action and Cognitive Extension. Oxford: Oxford University Press.

Clark, A. and D. Chalmers. 1998. The Extended Mind. Analysis 58: 7-19.

Di Paolo, E. A. 2005. Autopoiesis, Adaptivity, Teleology, Agency. Phenomenology and the Cognitive Sciences 4 (4): 429-452.

Fiebich, A. and S. Gallagher. 2013. Joint Attention in Joint Action. Philosophical Psychology 26 (4): 571-87.

Fowler, C. A., M.J. Richardson, K. L. Marsh, and K. Shockley. 2008. Language Use, Coordination, and the Emergence of Cooperative Action. In Coordination: Neural, Behavioral and Social Dynamics, ed. A. Fuchs and V. K. Jirsa, 261-279. Berlin: SpringerVerlag.

Gallagher, S. 2015. Doing the Math: Calculating the Role of Evolution and Enculturation in the Origins of Mathematical Reasoning. Progress in Biophysics and Molecular Biology 119: 341-346.

Gell, A. 1998. Art and Agency: An Anthropological Theory. Oxford: Oxford University Press.

Gilbert, M. 2009. Shared Intention and Personal Intention. Philosophical Studies, 144: 167-187.

Hodder, I. (2011). Human-thing entanglement: Towards an integrated archeological perspective. Journal of the Royal Anthropological Institute 17 (1): 154-177.

Hutchins, E. 2010. Cognitive Ecology. Topics in Cognitive Science 2: 705-715.

Knoblich, G., and J.S. Jordan. 2003. Action Coordination in Groups and Individuals: Learning Anticipatory Control. Journal of Experimental Psychology: Learning, Memory, and Cognition, 29 (5): 1006.

Latour, B. 1991. Technology is Society Made Durable. In A Sociology of Monsters: Essays on Power, Technology and Domination, ed. J. Law (pp. 103-131), 103-132. London: Routledge

Malafouris, L. 2013. How Things Shape the Mind. Cambridge, MA: MIT Press.

Marsh, K. L., M. J. Richardson, R. M. Baron, and R. C. Schmidt. 2006. Contrasting Approaches to Perceiving and Acting with Others. Ecological Psychology, 18 (1): 1-38.

Marsh, K. L., M. J. Richardson, and R. C. Schmidt. 2009. Social Connection through Joint Action and Interpersonal Coordination. Topics in Cognitive Science 1:320-339.

McNeill, D. 1992. Hand and Mind: What Gestures Reveal about Thought. Chicago: University of Chicago Press.

Pacherie, E. 2006. Towards a Dynamic Theory of Intentions. In Does Consciousness Cause Behavior? An Investigation of the Nature of Volition, ed. S. Pockett, W. P. Banks, and S. Gallagher, 145-167. Cambridge, MA: MIT Press.

-. 2007. The Sense of Control and the Sense of Agency. Psyche 13 (1): 1-30. http://psyche. cs.monash.edu.au/.

-. (2012). The Phenomenology of Joint Action: Self-Agency vs. Joint-Agency. In Joint Attention: New Developments, ed. A. Seeman, 343-389. Cambridge, MA: MIT Press.

Richardson, M. J., K. L. Marsh, and R. C. Schmidt. 2005. Effects of Visual and Verbal Couplings on Unintentional Interpersonal Coordination. Journal of Experimental Psychology: Human Perception and Performance 31: 62-79.

Schmidt, R. C. and M.J. Richardson. 2008. Dynamics of Interpersonal Coordination. In Coordination: Neural, Behavioral and Social Dynamics, ed. A. Fuchs and V. K. Jirsa, 281-307. Berlin: Springer-Verlag.

Searle, J.R. 1980. The Intentionality of Intention and Action. Cognitive Science 4 (1): 47-70.

Sebanz, N., H. Bekkering, and G. Knoblich. 2006. Joint Action: Bodies and Minds Moving Together. Trends in Cognitive Sciences 10 (2): 70-76. 
Sterelny, K. 2010. Minds: Extended or Scaffolded? Phenomenology and the Cognitive Sciences 9 (4): 465-481.

Thiering, M. 2015. Spatial Semiotics and Spatial Mental Models: Figure-Ground Asymmetries in Language. Applied Cognitive Linguistics 27. Berlin: Mouton de Gruyter.

Tollefsen, D. 2005. Let's Pretend: Children and Joint Action. Philosophy and the Social Sciences 35: 75-97.

Tuomela, R. 2005. We-Intentions Revisted. Philosophical Studies 53: 367-389.

van Ulzen, N. R., C. J. C. Lamoth, A. Daffertshofer, G. R. Semin, and P. J. Beek. 2008. Characteristics of Instructed and Uninstructed Interpersonal Coordination While Walking Side-by-Side. Neuroscience Letters 432 (2): 88-93. 



\title{
Bodily Changes During the Proto-Cultural Period and Their Ongoing Impact on Culture
}

\author{
Wolfgang Welsch
}

Abstract: The paper focuses on the importance of the protocultural period of humankind. During this period - extending from roughly 2.5 million years ago to 40,000 years ago - homo sapiens attained the characteristic constitution it still has today. In our present basic bodily, emotional, affective and even cognitive setup we are still much the same as our ancestors were at the end of the protocultural period and at the beginning of the subsequent take-off of cultural evolution 40,000 years ago. The protocultural period has brought about "the innate, generic constitution of modern man" (Clifford Geertz). This will be explained with respect to bodily, practical, and social changes during that period. Our protocultural setup sustains, on a general level, all our subsequent cultural evolution and, in specific cases, directly inspires cultural creations.

\section{Introduction}

I want to draw attention to the importance of the proto-cultural period of humankind. This period extended from roughly 2.5 million years ago to 40,000 years ago. It was during this period that Homo sapiens attained his characteristic constitution as it still persists today. We are, I suggest, in our basic bodily, emotional, affective and even cognitive setup still much the same as our ancestors were at the end of the proto-cultural period and at the beginning of the subsequent take-off of cultural evolution 40,000 years ago (see Welsch [2012] 2015, 715-735). ${ }^{1}$

The text that was most inspirational to me when developing this view was Clifford Geertz' article “'The Impact of the Concept of Culture on the Concept of Man," published almost 50 years ago. In this article Geertz characterized the proto-cultural stage as an "overlap period of cultural and biological change" (Geertz [1966] 1973, 48) resulting in the human constitution as we know it today. During this period, Geertz stated, man "quite literally created himself” (ibid.) by producing "the innate, generic constitution of modern man" (Geertz [1962] 1973, 67).

${ }^{1}$ The following is largely based on the more detailed account provided in Welsch ([2012] 2015, 715-735). 


\section{Classification of the Proto-Cultural Period}

In the development towards the modern human, three stages are to be distinguished. It goes without saying that this division is rather schematic; future research may move one or the other date a bit but the overall picture that I draw is likely to hold water. And don't let us ignore that in addition to individual data one must develop an overall picture - an account of where continuity prevails and where big changes take place.

The first stage began about 7 million years ago when the hominid line split off from the chimpanzee line, a period which lasted until about 2.5 million years ago when the first members of the genus Homo appeared. During this first and comparatively longest stage (it lasted four and a half million years, after all) surprisingly little happened. The australopithecines (the various precursor forms of Homo) differed little from their closest relatives, the chimpanzees. Their body size was similar, their brain volume was barely larger, and many among them still spent part of their lives in trees. All this did not change for a long time.

The second stage began about 2.5 million years ago and lasted until about 40,000 years ago. This stage I call the proto-cultural period. It was of paramount importance for the human constitution. At the beginning of this phase our ancestors were, as I said, hardly any different from the first australopithecines about 7 million years ago. But at the end of the proto-cultural period (40,000 years ago), humans not only had a brain three times the size of the beginning, but were producing highly sophisticated weapons, figurative representations and musical instruments. During this period (between 2.5 million years and 40,000 years ago) the crucial development must have occurred that transformed the human animal into a fully-fledged human being.

Finally, the third stage - that of cultural evolution - began about 40,000 years ago and extends to the present day. During the proto-cultural period there was an interplay of biological evolution and cultural innovation. While increasing their cultural activities, people also underwent biological and genetic modifications. Crucially, this no longer occurs during cultural evolution. Now the development is primarily cultural with barely any biological or genetic alterations. ${ }^{2}$

How did the proto-cultural changes come about, and what did they consist in?

\section{Proto-Cultural Changes}

\section{a. Physical changes}

Firstly, physical changes occurred. Upright walking was perfected, the hand was refined, body size increased considerably (while Homo babilis, about 2.1 million years ago, was little taller than 1 meter, Homo erectus, only 300,000 years later,

${ }^{2}$ Genetic changes include, in particular, skin and eye color. Other alterations are a consequence of agriculture. Currently immunological adjustments are taking center stage. 
measured up to 1.80 meter). And by reducing their body hair (whilst reinforcing their head hair), humans have increasingly disassociated themselves from their closest relatives, the great apes. ${ }^{3}$ Changes occurred in the area of sexuality too: males lost their penis bones, female breasts became sexualized, a-fronte practices began to replace the conventional a-tergo practices, and in this context not only the sense preferences changed dramatically but, as Freud suspected, human sexuality altogether was forever "heavily damaged" (Freud [1930] 1974, 234n4)

Humans' external appearance and essential practices changed significantly during the proto-cultural period. As products of this period we are clearly distinguished from our animal relatives as well as from our hominid ancestors.

\section{b. A Brain Beyond Comparison}

The human brain also changed considerably during the proto-cultural period. First, there was a rapid increase in volume: from about 400 cubic centimeters in the early australopithecines to more than $600 \mathrm{~cm}^{3}$ in Homo rudolfensis (the first species of Homo, about 2.5 million years ago), then notably to Homo erectus (before about 1.8 million years) with almost $1000 \mathrm{~cm}^{3}$, and to Homo sapiens (200-150,000 years ago) with more than $1200 \mathrm{~cm}^{3}-$ an increase by a factor of 3 over 2.5 million years.

But the increase in size (caused mainly by the growth of the neocortex ${ }^{4}$ ) is only one thing; at least as important are the structural changes which occurred over that time. It was during the proto-cultural period that the basic configuration of the human brain developed that is still characteristic of our brains. The human brain turned increasingly into an apparatus of self-reference. The relationship between external and internal functions of the brain shifted more and more in favor of the latter. At the end of this period, only 10 percent of cortex volume served outer reference (perception and motor skills), while 90 percent served inner reference (internal coordination processes) (cf. Storch, Welsch, and Wink 2001, 375). ${ }^{5}$

${ }^{3}$ Hairlessness is not, as previously supposed, an adaption to climate change (in this case our ancestors would, in the first place, have been obliged to reduce their head hair instead of reinforcing it) but a result of sexual selection. Hair growth reduction became attractive as a means to visibly distinguish ourselves from our closest apish relatives. In mating, those candidates were preferred who matched this new human fashion - which entailed a spread of hairlessness in the genome of our ancestors. In this way, an aesthetic inclination generated a new distinctive human feature. One might add: it is an aesthetic phenomenon insofar as our hairlessness is only a visual appearance. In fact, human bodies possess as many or even more hairs than many primate species, it's just that our hairs have in most cases become so unremarkable that the optical illusion of hairlessness or nakedness arises (see Menninghaus 2003, 88). Gender-specific variations (stronger hair and especially beard growth in men) are to be seen as strategies of gender distinction (dimorphism).

${ }^{4}$ In humans the neocortex accounts for about three quarters of the brain weight.

5 A comparison with rats (with whom, after all, we share 90 percent of our genome) makes the amount of change evident. In rats, the ratio of external to internal communication is (conversely to our case) 90:10 (cf. Storch, Welsch, and Wink 2001, 375). The quantitative reversal of 
The extent of this imbalance becomes fully clear when one looks at the number of neural connections instead of the ratios of volume. Of the roughly $10^{14}$ connections in our brain only every ten millionth serves purposes of external communication, whereas all others serve purposes of internal communication (Spitzer 2002, 52). Thus the internal communication paths outweigh those of external communications by the enormous ratio of $10^{7}: 1$. Each external connection is counterbalanced by 10 million internal connections! Our brain is predominantly an apparatus for self-reference. We humans are world champions of internal communication, of reflection in the broadest sense. Herein lies the unique feature of the human brain. ${ }^{6}$

\section{Tool Development and New Social Requirements as the Driving Factors}

How did this brain configuration come about? Basically through feedback processes between brain development on the one hand and the human activities made possible by it on the other. The brain did not just grow on its own, like a tumor. Neither the increase in its size nor the growth in its self-referentiality were results of an autonomous process, so to say a greenhouse of the skull. Instead the human brain has evolved via feedback from the activities it afforded. The increased performance of the enlarged brain enabled new activities; to master these amounted to a selective advantage, which in turn reinforced the increase. Thus, brain optimization and activity development have continually provoked each other. ${ }^{7}$

Two types of cultural activity were particularly important for this: the development of tools and new requirements of social life.

Clearly indicative of the connection between brain development and tool development is their synchrony. The oldest tools date back to around 2.6 million

the ratio in humans amounts to a qualitative leap. In human evolution it was probably above all the prefrontal lobe and the rear associative cortex that grew disproportionally compared to the primary sensorimotor structures (cf. Preuss 2001, $154 \mathrm{f} ., 2000,1223-25)$. It is also noteworthy that the overall genetic similarity between humans and chimpanzees (more than 98 percent) applies much less to the brain. In the course of human evolution, gene expression patterns concerning the brain have changed significantly more than those in chimpanzees: about 5.5 times as much (By contrast, there exist no such changes in other cells e. g., liver or blood cells [Pääbo et al. 2002, 341]). This is a further indication of how important brain modifications were in the course of hominization.

6 To be sure, this growth of reflectivity already began with the great apes, but in humans it has reached an unparalleled extent.

7 “His [man's] large and efficient brain is a consequence of culture as much as its cause. He does not have a culture because he has a large brain; he has a large brain because several million years ago his little-brained ancestors tried the cultural way to survival. Of course, the correct way to view this is a feedback process ... The cultural things themselves propelled him into getting a larger brain" (Fox 1991, 283 f.). Already in 1966 Clifford Geertz had stated: "Between the cultural pattern, the body, and the brain, a positive feedback system was created in which each shaped the progress of the other" (Geertz [1966] 1973, 48). 
years ago, ${ }^{8}$ just when a significant enlargement of the brain occurred. The steps in brain development which followed were likewise associated with advances in tool technology, both in Homo habilis, who was the first to chip sharp-edged pieces from stones (thus the suffix "the skillful" - babilis), and in Homo erectus, who developed an intensified culture of tools.

It is perfectly understandable that tool techniques require reflective skills. The perception of an encountered object as a tool already requires the re-interpretation of that object from the perspective of an internal purpose. Likewise its transformation into a better tool (such as in the production of bifaces) requires anticipatory imagination and an assessment of one's operations in comparison with one's goal. And the production of completely novel tools (such as spears, bows and arrows) again requires a high degree of internal reflection: first the draft of a new possibility, then the equally internal calculation of the means to achieve this goal. All these operations are essentially based on internal, reflective processes.

In addition to new tool technologies, new social demands promoted the increase in reflectivity. Already in the animal kingdom we notice a correlation between brain size and social life: animals living in large groups generally have more brain mass than those who interact with only a few conspecifics. ${ }^{9}$ During the proto-cultural period human social life became more and more complex and so required an increase in reflection. Conventionally, humans had already learned to master the understanding of intentions and states of consciousness of conspecifics. Now, however, they developed new social rules (division of labor, group privileges, initiation rites, etc.) and new practices (building huts, strategies for hunting, tanning of animal skins, etc.) for which they did not possess genetic programs, but which needed a brain capable of learning. Thus a pressure of social selection emerged in favor of learning and reflectivity. In this way social life, too, has contributed to the optimization of the brain. ${ }^{10}$

In short: the proto-cultural progress of both tool technology and social complexity fostered reflective capacities. Through a continuous feedback between

8 The Oldowan-tools were found in Ethiopia.

9 Sometimes there is even a directly proportional correlation between the size of the group and the size of the cortex (the part of the brain that is responsible for the intelligent processing of information). While the typical group size is $\mathbf{5 0}$ members in chimpanzees, it is 150 members in hunter-gatherer populations - which corresponds perfectly to the fact that the human brain has about three times the size of a chimpanzee brain.

${ }^{10}$ It is in this context that the emergence of human language or proto-language should be considered. It certainly helped increase the associative-reflective abilities to a remarkable extent. However, we know to date very little about the evolution of language - almost nothing. Already the Australopithecines and Homo habilis showed an increase in Broca's area (which, in humans, is responsible for syntactic language), but this does not entail that they used language (presumably their vocal apparatus was not yet sufficiently developed for that). Moreover, even in chimpanzees and orang-outangs an increase occurred in those parts of the brain where our language centers reside: Broca's area (for syntax) and Wernicke's area (for semantics). Damage to these parts of the brains in apes results in disturbance of their vocal communication. It appears that the human ability to speak rests on a prehuman prototype. The most common hypothesis concerning the origin of verbal language is that it was first developed (as a proto-language) by Homo erectus (about 1.8 million years ago). The formation of syntactic language may have occurred only $50-40,000$ years ago. 
activity innovation and brain optimization, the extremely reflective and efficient brain that is typical of humans emerged. The specificity of the human brain (the overwhelming dominance of internal, reflective functions over external, stimulative functions) is a result of the proto-cultural development. And, as we shall soon see, an extremely momentous result at that.

\section{The Dynamics of Proto-Cultural Development before the Take-Off of Cultural Evolution}

Let us take a brief look at the dynamics of proto-cultural development. In the beginning (2.5 million years ago) the number and importance of proto-cultural inventions and achievements was still low. But slowly these grew and became increasingly important for survival in nature and against competitors. Thus a selection pressure developed which managed such capacities and encouraged further ones.

In this way, over time, the balance between natural and cultural virtues shifted towards the latter. Previously, only physical assets had been decisive for the success of individuals and groups. When hunting, for example, the faster was the more successful. But now the slower could compensate for his physical handicap with a more sophisticated strategy. The physical virtues were increasingly confronted by cultural and intellectual advantages. These represented a new kind of power and created chances for physical underdogs to gain the upper hand.

In the course of this development, the efforts of early humans shifted more and more to proto-cultural achievements. These became the decisive factors of success in the competition between different groups and between different individuals within the same group. Proto-cultural advancement was augmented, and investment in proto-cultural achievements (inventions, learning processes) became key - both for the group and the individuals. Proto-cultural stocks grew faster and faster, shifting into an acceleration phase.

It is foreseeable that eventually a point will be reached, in evolutionary terms, from which only cultural innovation can lead any further (Biological optimization, which takes a long time, would now be simply too slow). This point was actually achieved about 40,000 years ago. ${ }^{11}$ At that time the proto-cultural acceleration passed over into the take-off of a new type of evolution, of cultural evolution. The arrow of culture was released from the bow of proto-culture. ${ }^{12}$

${ }^{11}$ Before that date Homo sapiens had already spread beyond Africa, about 100,000 years ago to Israel, about 60,000 years ago to East Asia and about 45 to 40,000 years ago to Europe (Cro-Magnon) (see Mithen 1996, 172f.). But in each of these lines, and independently of each other, a transition to cultural evolution occurred around 40,000 years ago.

${ }^{12}$ It is similar to Uranium: If one accumulates Uranium 233, up to about $12 \mathrm{~kg}$ not much happens, but once one reaches $15 \mathrm{~kg}$, an unstoppable chain reaction breaks out. One might imagine the explosion of cultural evolution in a similar manner. The initially slow increase in proto-cultural goods and activities gained momentum, accelerating to the point where it reached a critical mass: the take-off of cultural evolution. 
Humanity entered a new evolutionary mode. Long before, in the time of the australopithecines, everything had moved along the paths of natural evolution; subsequently, during the proto-cultural period, there was an interplay of biological and cultural development; now, however, humanity crossed the Rubicon towards an exclusively cultural evolution. A new mode of transmission became the order of the day: learning and tradition-building instead of genetic transfer; and the previously occasional invention of cultural achievements shifted into the cumulative mode of cultural evolution (see Tomasello 1999, 37). From now on, the cultural path alone was open. ${ }^{13}$

To briefly indicate its main stages: in Europe, the take-off of cultural evolution led to the Upper Paleolithic Revolution (45,000 to 30,000 years ago) with its gigantic explosion of creativity, where the first musical instruments ${ }^{14}$ and the earliest statues (human and animal figures) emerged, ${ }^{15}$ but also new tools (narrow blade technology) and highly refined weapons (balanced spears and harpoons) were developed, ${ }^{16}$ soon followed by the first cave paintings (Chauvet, Lascaux). ${ }^{17}$ The next big step was taken with the Neolithic Revolution in the Middle East more than 10,000 years ago, when agriculture began, cities were founded and a sedentary life form replaced that of hunter-gathering. ${ }^{18}$ Finally, about 6000 years ago, the stage of advanced civilizations which extends to the present day began.

\section{The Emergence of Culture from Nature and Proto-Culture}

In short, proto-culture first emerged from nature, and then culture from proto-culture. For this to occur, nothing had to be added from the "outside." Proto-culture developed from natural beginnings, and culture emerged through the increasing dynamics of proto-culture.

This explanation dispenses with the crux of older theories - the recourse to an external factor supposedly responsible for the foundation of culture. The old dichotomous thinking - nature versus culture - had necessitated such an assumption. Today, however, we can understand how culture de facto emerged from nature via the mediating pivot of proto-culture. Culture did not originate through some disruption or through the influence of higher forces; it has emerged step by step from nature and proto-culture.

${ }^{13}$ By analogy with Kant's claim that "the critical path alone is still open" (Kant [1781] 1965, 668 f., A 856).

${ }_{14}$ These are bone flutes - the oldest specimen (to date) was found in 1995 in Slovenia.

${ }^{15}$ Currently the oldest find is a human figure made from a mammoth tusk (discovered near Kostenski, about $400 \mathrm{~km}$ south of Moscow).

16 They date back to around 37,000 years ago.

17 The paintings in Chauvet could be 30,000 years old, those at Lascaux may have originated 17,000 years ago.

18 The Neolithic Revolution occurred (like the earlier turn to cultural evolution) independently in the Middle East, in Africa, in China, in America and in Europe (there relatively late, in Central Europe only in the 6th century BC). 


\section{The Great Importance of the Proto-Cultural Development}

Let me once again underscore the great importance of the proto-cultural development. It has created our - physical as well as intellectual - nature, the nature which everyone still bears within himself. It has made us the special beings we are - from physical characteristics such as baldness and upright walking via our behavior in sexual and social life through to the unique brain configuration that has become vital for our existence as learning and cultural beings. And lastly, the proto-cultural development even fired the starting pistol for cultural evolution. One can hardly overestimate its importance. The proto-cultural development accomplished the transition from the human animal to a fully-fledged human being. ${ }^{19}$

\section{a. Cultural Evolution Still Rests on Proto-Culturally Formed Human Nature}

The whole of cultural evolution is based on the human constitution as it was achieved at the end of the proto-cultural period. Since then our genetic setup has hardly changed. We are still born as stone-age babies.

At the same time, this makes clear how great and eminently powerful our proto-culturally accumulated capital was: it was good enough to carry all future cultural evolution. From the first works of art to Picasso, from the invention of writing to the internet, from the pyramids to moon landing, people have been able to produce their cultural achievements with the help of their proto-cultural set-up. Einstein invented the theory of relativity on the basis of a Stone Age brain. ${ }^{20}$

In particular it was the brain-configuration reached at the end of the proto-cultural development which has made possible not only the leap into cultural

19 To be sure, proto-culture already exists in the animal kingdom. Some higher animals have developed practices that one could hardly not describe as proto-cultural. Examples are the use and production of tools, forms of social organization, efficient ways of communication, aesthetic practices, and the emergence of cultural diversity. This occurrence of proto-culture in the animal kingdom is not surprising at all. It is generally the case that we find nothing completely new in the human sphere. Everything exists in weaker form already in other animals - and the closer they are to us, the more the traits crucial to us have already undergone an increase in their evolution. We make the most of the prehuman talents bequeathed to us. It is just that our relatives stopped in their proto-cultural activities at a relatively modest level. As far as one can see, nowhere in the animal kingdom did an acceleration get under way that would have lead to a take-off of culture in the emphatic sense. This has only happened in human evolution.

20 Certainly the environment - especially the cultural environment - plays an important role during epigenetic development. A Stone Age brain was not capable of inventing the theory of relativity during the Stone Age, it could do so only in the social cognitope of highly sophisticated modern physics. The brain is not just a biological but also a social entity. But the basic configuration of the apparatus which is capable of all the subsequent cultural achievements was developed in the Stone Age and is no different today than it was then. Wolf Singer has expressed this relationship between ancestral hardware and cultural software as follows: if a Stone Age baby were to grow up in our civilization, it would look the same and achieve the same learning results as our children; conversely, were a baby from the 21st century to grow up in a Stone Age culture, it would be indistinguishable from genuine Stone Age children (cf. Singer 2002, 44). 
evolution but also all the extraordinary achievements during its course. Through proto-culture the treasure of our nature blossomed, and culture is acting it out. ${ }^{21}$

\section{b. Universals: Continuing Witnesses to the Proto-Cultural Level of Development}

We can easily verify our proto-cultural imprint by looking at details of our emotional life, our aesthetic assessments and our cognitive activities. The keyword here is "universals." These universals are firmly connected with human nature as it was attained at the end of the proto-cultural period - and they are still effective across otherwise existing cultural differences, precisely because that nature is common to all humans (see Welsch [2012] 2015, 731-734).

During the heyday of cultural relativism, the existence of universals was systematically denied. Since then, hard scientific evidence has emerged to refute such relativism. Brent Berlin's and Paul Kay's study on Basic Color Terms from 1969 was groundbreaking (Berlin and Kay 1969). The authors showed that the members of different cultures, even when dividing the color spectrum according to the number of color words their language possesses (the number ranges from two to eleven), give amazingly matching answers when asked which color sample (from about 300) best represents a color type for which they have a word. Thus the phenomenal experience of color is not culturally determined, but universal. Berlin and Kay found, furthermore, that the sequence of the appearance of color words is by no means arbitrary. If a language has only two color words, these are always white and black; the third one is always red; the fourth is either green or yellow, and the fifth yellow or green; followed by blue and brown; and only at the end come purple, pink, orange and gray. There are hard universals for phenomenal judgments and linguistic (incidentally, also phonological) sequences. ${ }^{22}$

In addition, universals exist in the relation of facial expression to basic emotions, the understanding of elementary gestures, and to some aesthetic preferences. ${ }^{23}$ All these universal features are anchored in the still persistent proto-cultural nature of all humans. ${ }^{24}$ To express it in a take home message: what is universal, is proto-cultural; what is not, is cultural.

21 This once again throws light on the aforementioned congruence between the end of the genetic modification of humans and the beginning of cultural evolution. This simultaneity is no coincidence but a congruence on both sides. Through proto-cultural development, humans' biological setup had become good enough to support the entire future cultural evolution. And conversely, it belongs to the nature of cultural evolution to have only very little genetic retroaction and to rely instead on cultural continuity and progress.

${ }^{22}$ In the meantime, similar sequences have been demonstrated for the classification of plants and animals (cf. Brown 1991, 14).

${ }_{23}$ On aesthetic universals, see especially Welsch (2012, 292-330).

24 Darwin already emphasized the "similarity, or rather identity" of humans in their elementary skills and behavior: One could "hardly fail to be deeply impressed with the close similarity between the men of all races in tastes, dispositions and habits" (Darwin [1871] 1981, 232). 


\section{c. The Ongoing Impact of our Proto-cultural Nature on our Cultural Existence}

Our basic proto-cultural nature will certainly be refined (sometimes also twisted) in epigenetic and cultural processes. But it supports and determines us from the ground up. It provides our possibilities - and sets certain limits. To take a practical example, just try to produce, whenever you feel joy, the expression of sadness, and vice versa. With some effort you may succeed for a short time, but you will then begin to feel a deep inner confusion, and the experiment is best stopped before you end up in a mental hospital. It is wise to trust these old routines rather than delve too deep into them - otherwise our cultural activity breaks down.

Proto-cultural achievements not only tacitly support all our cultural activity, but sometimes even explicitly come to the fore. For example, when Renaissance art introduced perspective, it did not invent perspectival seeing (this had been successfully practiced from time immemorial) but only raised this age-old habit to its representational principle; it switched from the hitherto culturally predominant pattern of the self-being of things to the phylogenetically much older perspectival perception of things in space - and was thus able to create wonderful paintings which we feel closer to than previous iconic representations. Here an age-old proto-cultural invention was culturally brought to bear; the recourse to ancient stock gave culture a new direction. And this holds true for other innovations in the arts, such as impressionism or op-art: each draws on old practices of everyday perception, making them explicit.

Contemporary mass media is another example: they widely rely on the proto-culturally bred ability to decipher facial expressions. Hence the many closeups of faces in film and television, which convey important information, and do so independently of specific languages and cultures, by making recourse to universally shared age-old patterns of understanding. ${ }^{25}$

These are just a few examples of the impact of proto-cultural achievements on our cultural existence. We often understand the latter better when we take their proto-cultural grounding into account.

I am not saying that all cultural phenomena could be sufficiently explained by pointing out their proto-cultural contents. That would be an erroneous claim. But some traits of cultural phenomena do have proto-cultural explanations.

With respect to our evolutionary heritage, I suggest, a fundamental change of thinking, a reorientation in contrast to deep-seated habits is needed. Our biological and proto-cultural preconditions are not something dubious that we would

25 "It is the emotional displays on the generic side of the ledger that the mass arts gravitate toward - such as fear, elation, sadness, anger, surprise, lust, and so on. This is the stuff upon which mass art thrives, as a quick review of the most popular motion pictures attests. Moving-image mass art is able to convey, to a significant degree, this emotional information so effectively to large and diverse audiences of heterogeneous backgrounds because of its reliance on close-ups of faces, something that within a certain range of emotional expressions, ones particularly germane to the territory mass art cultivates, audiences can comprehend in large part by dint of their innate biological equipment" (Carroll 2004, 104). 
better leave behind. On the contrary, they are productive potentials which enabled everything which was to come. Their vector points forward as well as backwards. As our proto-cultural nature once allowed the emergence of culture, so it still provides us with the energy to continue the path of culture. Cultural evolution has not occurred against the grain of our biological nature, but grew out of it, and its subsequent course unfolds that nature's potentials rather than choking them off. We should cherish this potential in its proto-cultural as well as cultural form.

\section{Bibliography}

Berlin, B. and P. Kay. 1969. Basic Color Terms: Their Universality and Evolution. Berkeley: University of California Press.

Brown, D. E. 1991. Rethinking Universality: Six Cases. In Human Universals, 9-38. New York: McGraw-Hill.

Carroll, N. 2004. Art and Human Nature. The Journal of Aesthetics and Art Criticism 62 (2): 95-107.

Darwin, C. (1871) 1981. The Descent of Man, and Selection in Relation to Sex, Vol.1. Princeton: Princeton University Press.

Fox, R. 1991. Encounter with Anthropology. New Brunswick, NJ: Transaction.

Freud, S. (1930) 1974. Das Unbehagen in der Kultur. In Studienausgabe, Bd. 9, ed. A. Mitscherlich, J. Strachey, and A. Richards, 197-270. Frankfurt/Main: S. Fischer.

Geertz, C. (1962) 1973. The Growth of Culture and the Evolution of Mind. In The Interpretation of Cultures, 55-83. New York: Basic Books.

-. (1966) 1973. The Impact of the Concept of Culture on the Concept of Man. In The Interpretation of Cultures, 33-54. New York: Basic Books.

Kant, I. (1781) 1965. Critique of Pure Reason. Trans. N. K. Smith. New York: St. Martin's. Menninghaus, W. 2003. Das Versprechen der Schönheit. Frankfurt/Main: Suhrkamp.

Mithen, S. 1996. The Prehistory of the Mind. London: Thames and Hudson.

Pääbo, S., W. Enard, P. Khaitovich, J. Klose, S. Zöllner, F. Heissig, P. Giavalisco et al. 2002. Intra- and Interspecific Variation in Primate Gene Expression Patterns. Science (296): $340-342$.

Preuss, T. M. 2000. What's Human about the Human Brain? In The Nerw Cognitive Neurosciences, ed. M. S. Gazzaniga, 1219-1234. Cambridge, MA: MIT Press.

-. 2001. The Discovery of Cerebral Diversity: An Unwelcome Scientific Revolution. In Evolutionary Anatomy of the Primate Cerebral Cortex, ed. D. Falk and K. R. Gibson, 138-164. Cambridge, MA: Cambridge University Press.

Singer, W. 2002. Der Beobachter im Gehirn. Essays zur Hirnforschung. Frankfurt/Main: Suhrkamp.

Spitzer, M. 2002. Lernen. Gebirnforschung und die Schule des Lebens. Heidelberg: Spektrum Akademischer Verlag.

Storch, V., U. Welsch, and M. Wink. 2001. Evolutionsbiologie. Berlin: Springer.

Tomasello, M. 1999. The Cultural Origins of Human Cognition. Cambridge, MA: Harvard University Press.

Welsch, W. (2012) 2015. Homo Mundanus: Jenseits der anthropischen Denkform der Moderne. 2nd ed. Weilerswist: Velbrück Wissenschaft.

-. 2012. Zur universalen Schätzung des Schönen. In Blickwechsel. Neue Wege der Ästhetik, 292-230. Stuttgart: Reclam. 



\section{Contributors}

Prof. Dr. Terrence W. Deacon

Anthropology Department

University of California, Berkeley

deacon@berkeley.ed

Prof. Dr. Eve-Marie Engels

Professor for Ethics in the Life Sciences

Department of Biology

Eberhard Karls University of Tübingen

eve-marie.engels@uni-tuebingen.de

Prof. Dr. Gregor Etzelmüller

Institute for Protestant Theology

Osnabrück University

gregor.etzelmueller@uni-osnabrueck.de

Prof. Dr. Dr. Thomas Fuchs

Karl Jaspers Professor for Philosophical Foundations of Psychiatry and

Psychotherapy/Head of the section "Phänomenologische Psychopathologie

und Psychotherapie" (Phenomenological Psychopathology and Psychotherapy)

Clinic for General Psychiatry

University Clinic Heidelberg

thomas.fuchs@urz.uni-heidelberg.de

Prof. Dr. Shaun Gallagher

(Humboldt Foundation Anneliese Maier Research Fellow 2012-17)

Lillian and Morrie Moss Chair of Excellence in Philosophy

University of Memphis

gallaghr33@gmail.com

Dr. Duilio Garofoli

Cognitive Archaeology Unit

Institute for Archaeological Sciences

Eberhard Karls University of Tübingen/Research Center "The Role of Culture in Early Expansions of Humans" of the Heidelberg Academy of Sciences and Humanities

dui.garo@gmail.com 
PD Dr. Miriam Noël Haidle

Research Center "The Role of Culture in Early Expansions of Humans" (ROCEEH) of the Heidelberg Academy of Sciences and Humanities/ Senckenberg Research Institute/Institute for Archaeological Sciences Eberhard Karls University of Tübingen miriam.haidle@uni-tuebingen.de

Prof. Dr. Matthias Jung

Professor for Philosophy

Faculty for Arts and Humanities

University of Koblenz-Landau

mjung@uni-koblenz.de

Dr. Lambros Malafouris

Johnson Research Fellow in Creativity, Cognition and Material Culture Keble College, Oxford

Institute of Archaeology

University of Oxford

lambros.malafouris@keble.ox.ac.uk

Dr. Alexander Maßmann

The Faraday Institute for Science and Religion

St Edmund's College

University of Cambridge

am2251@cam.ac.uk

Prof. Dr. Erik Myin

Centre for Philosophical Psychology

Department of Philosophy

University of Antwerp

erik.myin@uantwerpen.be

Tailer G. Ransom

Department of Philosophy

University of Memphis

tgransom@memphis.edu

PD Dr. Magnus Schlette

Head of the Section "Theology and Science"

Forschungsstätte der Evangelischen Studiengemeinschaft Heidelberg/

Faculty of Philosophy

University of Erfurt

magnus.schlette@fest-heidelberg.de 
Prof. Dr. Christian Spahn

Department of Philosophy

College of Humanities

Keimyung University

cspahn@kmu.ac.kr

Dr. Mog Stapleton

Postdoctoral Fellow in the Research Group "Philosophy of Neuroscience"

Werner Reichardt Centre for Integrative Neuroscience

Eberhard Karls University of Tübingen

mog.stapleton.philosophy@gmail.com

PD Dr. Christian Tewes

Section "Phänomenologische Psychopathologie und Psychotherapie"

(Phenomenological Psychopathology and Psychotherapy)

Ruprecht Karls University of Heidelberg/Faculty of Philosophy

Friedrich-Schiller-University Jena

tewes@mk.uni-heidelberg.de

Prof. Dr. Annette Weissenrieder

Professor of New Testament

San Francisco Theological Seminary/Core Doctoral Faculty Member

Graduate Theological Union Berkeley

aweissenrieder@sfts.edu

Prof. Dr. Wolfgang Welsch

Faculty of Philosophy

Friedrich-Schiller-University Jena

Wolfgang.Welsch@uni-jena.de

Prof. Dr. Christoph Wulf

Anthropology and Education Section

Department of Education and Psychology/Freie Universität Berlin chrwulf@zedat.fu-berlin.de

Prof. Dr. Karim Zahidi

Department of Philosophy

University of Antwerp

karim.zahidi@uantwerpen.be

Prof. Dr. Jordan Zlatev

Professor for Cognitive Semiotics

Centre for Languages and Literature

Lund University

jordan.zlatev@ling.lu.se 



\section{Index of Persons}

Abramiuk, M. $311 \mathrm{f}$., 325

Adams, F. 1f., 18, 34

Adorno, T. W. $251 \mathrm{f}$.

Ahad, P. 120

Aitken, K.J. 49

Aizawa, K. 1 f., 18, 34

Alhner, F. 166

Alighieri, D. 265

Ambrose, S. 321

Anderson, H. 308

Anderson, M. L. 17

André, J. 278 (FN 26, 27)

Anjum, R. L. 33 (FN 2)

Apperly, I. A. 319

Arbib, M. A. 121, 167, 169

Aretaeus of Cappadocia 265f. (FN 1-3), $275 \mathrm{f}$.

Argyll, Duke of 236

Aristotle 44, 75 (FN 6), 82, 107, 153, 201, $273 \mathrm{f}$. (FN 14, 17)

Astington, J. W. 319, 323

Axelrod, R. 74

Aydin, C. 339

Ayer, A.J. 76 (FN 10)

Aziz-Zadeh, L. 114, 122 (FN 14)

Baars, J. 51, 143

Bain, A. 240-243

Banks, W.E. 310

Bar, M. $22 \mathrm{f}$.

Bar-On, D. 155

Bar-Yosef, O. 308, 324

Barandiaran, X.E. 44

Bard, K. A. 163

Bargh, J. A. 112

Barham, L. S. 309

Barkow, J. H. 292

Barnard, P.J. 319

Baron-Cohen, S. 316

Barrett, L. F. 22 f., $299 \mathrm{f}$.

Barrett, P. H. 231-233 (FN 6)
Barsalou, L. W. 109, 318

Bates, E. 166

Beach, K. 322

Beer, R. D. 300

Behnke, E. A. 45, 49

Beier, W. 83

Bekkering, H. 343

Belin, P. 120

Bell, C. 154

Benazzi, S. 307

Benjamin, W. 198, $250 \mathrm{f}$.

Bennett, J. 296, 298

Benton, J. 112

Benz-Schwarzburg, J. 239

Bergen, B. 113

Bergunder, M. 215 f. (FN 9)

Berlin, B. 361

Bertalanffy, L.v. 82 (FN 29)

Bickhard, M. H. 34

Binford, L. R. 312

Binkofski, F. 114

Biro, D. 297 (FN 5)

Blackmore, S. J. 74

Block, N. 85

Blumenbach, J. F. 81 (FN 27)

Bock, J. 314

Bock, K.E. 129

Bolus, M. 307

Bongard, J. 18

Botha, R. 307

Boulenger, V. 113

Bourdieu, P. 49, 52, 205, 341

Bouzouggar, A. 308, 318

Bowler, P.J. 74 (FN 2)

Boyd, R. 157f., 292-295, 300f. (FN 6)

Bradlaugh, C. 230 (FN 4)

Brandom, R. B. 104, 177, 184-187

(FN 20, 21)

Brandstetter, G. 256

Brannon, E. M. 64

Braßel, B. 91 (FN 48) 
Bråten, S. 116, 163-165

Bratman, M.E. 337

Brehm, A.E. 237

Bremmer, J. N. 220

Breyer, T. 213

Bright, M. 308

Brincker, M. 341

Brooks, A. 309, 313, 324

Brooks, R. 114

Brown, D. E. 361 (FN 22)

Brown, F. H. 308

Brown, J.E. $166 \mathrm{f}$.

Brown, J. H. 317

Bruner, E. 307, 322

Bruner, J. $118 \mathrm{f}$.

Buccino, G. $113 \mathrm{f}$.

Büchner, L. 234

Buffon, Comte de 228

Burdukiewicz, J. M. 309

Burge, T. 79f. (FN 18, 20, 22), 89 (FN 43)

Buss, D. M. 316

Butler, J. 3, 197, 215-219 (FN 9, 10)

Butterworth, B. 62

Campbell, D. 157

Campbell, R. 33 f. (FN 2), 42

Capeloa Gil, I. 253

Carpendale, J. I. 118

Carpenter, M. 116

Carroll, N. 362 (FN 25)

Carruthers, P. 311, 316

Casasanto, D. 113

Casey, E.S. $47 \mathrm{f}$.

Cellucci, C. 274

Chalmers, D. J. 14, 18, 31 (FN 1), 33, 36, 79 (FN 16), $338 \mathrm{f}$.

Chaminade, T. 307

Chemero, A. 59, 291, 296

Chiel, H.J. 300

Chomsky, N. 151

Christiansen, M.H. 151

Clark, A. 1, 14, 17-21 (FN 2), 24 f., 28 f., 31 (FN 1), 33-37, 41, 43, 79 (FN 16), 86 (FN 40), 291 (FN 3), 296, 300, 311-313, 315, 317, $321 \mathrm{f} ., 324,338 \mathrm{f}$.

Cobbe, F. P. 234

Cochran, G. 324

Cockburn, A. 163

Cohen, L. 321

Collins, C. 167

Conard, N. J. $307 \mathrm{f}$.
Coolidge, F. L. 307 f., 311 f., 318, 324

Cosmelli, D. 13, 16, 20 f., 24 (FN 5), 85-87 (FN 40)

Cosmides, L. 57 (FN 2), 74 (FN 2), 292, 312

Craig, A. D. 22

Csibra, G. 154

Csikszentmihalyi, M. 261

Csordas, T.J. 53

Cuffari, E. C. 109, 111, 124

Dack, L. A. 319, 323

Damasio, A. R. 79 (FN 17), 114

Darwin, C. 8, 13, 73-75 (FN 1), 108, 177, 193-197, 205-207 (FN 2), 209, 219, 227-245 (FN 1-8), 285, 361 (FN 24)

Darwin, E. 228, 241

Darwin, F. 232

Davidson, D. 7, 132

Davidson, I. 307

Dawkins, R. 3, $74 \mathrm{f}$. (FN 2)

Deacon, T. W. 1, 48, 101-104, 131, 135, 137, 140-142, 144 f., 147, 151 f., 158, 160-165, 167-169, 176, 179 (FN 12), $182 \mathrm{f}$. (FN 17)

De Beer, Sir G. 231 (FN 6)

Debru, A. 268 (FN 10), 273 (FN 17)

De Cruz, H. 62

De Haan, M. 317, 320

Dehaene, S. 62, 64-69, 321

De Jaegher, H. 7, 44, 50, 85, 109-111, 117, 121 (FN 13), 124, 291, 318

D’Errico, F. 307-310, 313, 318

Dekker, T. M. 316, 320

Dennett, D. C. 3, 76 (FN 11)

De Saussure, F. 132, 140, 152, 166, 182

Descartes, R. 177

De Smedt, J. 62

De Vignemont, F. 291

Devlin, K. 62

De Waal, F. 3, 194, 199, 206-209 (FN 2), 212, 238

Dewey, J. 176 (FN 6), 178-180, 182 (FN 16), 187

Dieckmann, B. 253

Di Gregorio, M. A. 232

Dilthey, W. 178, 180

Dingemanse, M. 166

Diocles of Carystus 268 (FN 10), 273

Diogenes of Apollonia 269 
Di Paolo, E. A. 4, 7, 20, 25 (FN 6), 32, 42-45, 50, 85, 109-111, 124, 318, 342

Dittmann, J. 119

Donald, M. 1, 4, 6, 33, 40, 47 f., 53, 104, 151-155, 166, 168 f., 178, 184, 186-188 (FN 23)

Donohue, K. 300

Dor, D. 157

Dornes, M. 249

Dreyfus, H. 176 (FN 4)

Dubreuil, B. 307, 318

Ducatillon, J. 271

Dunbar, R. 158-160, 163 f., 169

Dupré, J. 299

Edgar, B. 308

Eibl-Eibesfeldt, I. 118

Elman, J. E. 311 f., 320

Engels, E. M. 194f., 206 f., 228 (FN 1), $230 \mathrm{f}$. (FN 5), 237, 244

Etzelmüller, G. 100, 196-198, 221, 267

Fadiga, L. 113

Farran, E. K. $316 f$.

Fay, N. 167

Feldman, M. W. 46, 299f., 313, 324

Fernald, A. 115

Fiebich, A. 319, 337, 348

Finlayson, C. 309

Fischer-Lichte, E. 258

Fitch, W.T. $166 \mathrm{f}$.

Fleagle, J. G. 308

Flegg, G. 60-62

Fodor, J. A. 107, 311

Foley, R. A. 310

Foucault, M. 215-217 (FN 9)

Fowler, C. A. 346

Fox, R. 356 (FN 7)

Frankl, V.E. 75 (FN 7)

Frege, G. 93

French, J. C. 310, 324

Freud, S. 77, 355

Fritz, C. 308,325

Froese, T. 6, 49, 209f. (FN 6)

Fuchs, R., 273

Fuchs, T. 6, 49, $101 \mathrm{f} ., 108,110$ (FN 3), 116f., 119, 121 (FN 13), 212-214, 218, 249, 266, 274, 291, 318

Gage, N.M. 51, 143

Galantucci, B. 153, 167
Galen/Pseudo-Galen 200, 266-268 (FN 4, 9, 10), 274, 276

Gallagher, S. 1, 6, 13, 100, 121 (FN 13), 187, 274, 287, 291, 296, 316, 318-320, $337,341,348$

Gallese, V. 113 f., 121 (FN 13)

Gallistel, C. R. 57, 62, 69

Gamble, C. 308, 325

Gapp, K. A. 219

Gärdenfors, P. 48

Gardner, H. 312

Garofoli, D. 66 (FN 9), 286f., 307 f., 311, $313 \mathrm{f}$. (FN 2), 318, 324, 326

Garrod, D. 153, 167

Gebauer, G. 250, 253, 258

Geertz, C. 353, 356 (FN 7)

Gehlen, A. 1, 75 (FN 6), 236

Gell, A. 340

Gergely, G. 154

Gibson, J. J. 68, 316, 341

Gilbert, M. 337

Girard, R. 252

Giuliano, T. $51 \mathrm{f}$.

Givón, T. 152

Glancy, J. 221 (FN 11)

Glasersfeld, E. v. 3

Glenberg, A. M. 108 f., 113

Glick, T.F. 231 (FN 5)

Göhlich, M. 259

Goldman, A. 121 (FN 13), 291

Gómez, J. C. 116f. (FN 9)

Goschke, T. 47

Gosden, C. 294, 298

Gottlieb, G. 300, 320

Gowdy, J. 152, 157 f.

Grant, M. 325

Gray, R. D. 300

Grensemann, H. 269, 273 (FN 17)

Grice, P. 119, 155

Griffiths, P.E. 297, 300

Grimes, R. L. 258

Gruber, H.E. $231 \mathrm{f}$. (FN 6)

Gruber, O. 47

Grün, R. 309

Grupe, G. 214

Gundry, D. W. 236 (FN 10)

Habermas, J. 206

Haeckel, E. 74 (FN 2), 234

Hagoort, P. 113

Haidle, M. N. 307, $310 \mathrm{f}$. 
Halbwachs, M. 52

Hamilton, W. D. 74, 158, 162

Harnard, S. 110 (FN 3)

Harpending, H. 324

Harvey, S. 112

Haslam, M. 297 (FN 5)

Haugeland, J. 274

Hauk, O. 113

Hawkes, K. 162

Hegel, G. W. F. 82 (FN 29)

Heidegger, M. 83 (FN 33), 176 (FN 4), 296

Heine, B. 151, 157

Heisenberg, M. 208

Henshilwood, C. S. 308 f., 318

Herder, J. G. 100, 181

Herophilus 267

Hertel, P. $51 \mathrm{f}$.

Hewitt, G. P. 160

Hippocrates 200, 268 (FN 10), 273

Hodder, I. 298, 307, 339

Hodgskiss, T. 325

Hoehl, S. 209-211, 218

Holyoak, K. 323

Honeycutt, H. 163

Hopkins, W. D. 163

Horgan, T. 35

Horner, V. K. 154, $209 \mathrm{f}$.

Hösle, V. 74, 91 (FN 48), 93

Hrdy, S. B. 103, 152, 159, 161-165, 168, 193

Hubley, P. 116

Hüffmeier, F. 271

Humboldt, W.v. 100, 179 (FN 11), 181

Hume, D. 76, 195, 240-243

Hüppauf, B. 257

Hurford, J. R. 151, 153 f.

Hurley, S. 17, 33

Husserl, E. 81, 93, 196

Hutchins, E. 296, 325, 338

Hüther, G. 218

Hutto, D. D. 15, 57-59 (FN 1, 3), 175, 291, 296, 318f.

Huxley, T. H. 234, 237

Iliopoulos, A. $313 \mathrm{f}$. (FN 2), 318

Illies, C. 74 (FN 2), 76 (FN 12), 91 (FN 48)

Imai, M. 166

Ingold, T. 129, 139, 299

Iriki, A. 324
Irwin, B. A. 111

Itkonen, E. $151 \mathrm{f}$.

Jablonka, E. 158, 300 f., 314, 324

James, W. 23

Janowski, B. 220

Jaspers, K. 188 (FN 25)

Jirak, D. 113 f., 121

Joas, H. 188 (FN 24)

Johansson, S. 307

Johnson, M. H. 317, 320

Johnson, M. L. 101, 109

Jonas, H. 4, 42, 76 (FN 8), 81-84 (FN 25, 29, 33-35), 199 f., 208

Jordan, J. S. 348

Jordan, K. E. 62-65

Jouanna, J. 269, 271

Jung, M. 1, 101, 103 f., 176 (FN 5), 181 (FN 14), 205, 207, 209, 213

Kant, I. 76, 81 f. (FN 31), 195 f., 201, 206, 240, 242 f. (FN 11), 359 (FN 13)

Kaplan, B. 151

Karle, I. 205

Karmiloff-Smith, A. 316f., 320

Kaschak, M. P. 113

Kay, P. 361

Kelley, N. 275

Kelso, J. A.S. 34, 41

Kendall, J.R. 314

Kestenbaum, V. 176 (FN 6)

Kettner, V. A. 118

Keydell, R. 276

Keysers, C. 121

Kirby, S. 151

Kita, S. 166

Kitcher, P. 76 (FN 12)

Kiverstein, J. 291

Klein, R. G. 308, 310, 312, 324

Kleist, H.v. 111 (FN 5)

Kluge, F. 111 (FN 6), 117

Knappett, C. 298 f., 307, 315

Knight, A. 239

Knight, C. 157

Knoblich, G. 343, 348

Kobayashi, H. 5

Koelsch, S. 120

Kohler, E. 121

Kohler, W. 136

Kohshima, S. 5

Krall, L. 152, $157 \mathrm{f}$. 
Kraus, A. 257

Krause, J. 307

Kroeber, A. L. 298

Kropotkin, P. 231

Krug, A. 271 (FN 11)

Kuhn, S. 318

Kühn, C. G. 266 (FN 4), 268 (FN 9), 274, 276 (FN 23, 24)

Kurzban, R. 313 (FN 1)

Kuteva, T. 151, 157

Laclau, E. 215 (FN 9)

Lahr, M. M. 310

Lakoff, G. 62, 69 f., 101, 109

Laland, K. N. 46, 292-294, 299f., 313, 324

Lamarck, Chevalier de 228

Lamb, M.J. 158, 300f., 314, 324

Landmann, M. $213 \mathrm{f}$.

Langbroek, M. 326

Langholf, V. 271 (FN 11)

Langslow, D. R. 278f. (FN 26)

Latour, B. 287, 298, 340

Laue, R. 83

Leavens, D. A. 163 , 209f. (FN 6)

Lee, S. W.S. 112

Legrand, D. 45

Leonardelli, G.J. 112

Lewens, T. 299

Lewis, J. 157

Lewis-Williams, D. 308

Lewontin, R. C. 299,324

Lickliter, R. 163

Liljenquist, K. 112

Liszkowski, U. 116, 163

Locke, J. 99, 233

Lorenz, K. 74 (FN 2), 77, 80

Lucian 266

Lyons, D. E. 210

\section{Mackintosh, J. 242 (FN 11)}

MacLarnon, A. M. 160

Malafouris, L. 6, 60 (FN 5), 216, 286, 289f., 294, 296-299, 307, 310f., 313, 315-318, 323 f., 338-342, 346

Malloch, S. N. 115,120

Malthus, T.R. $229 \mathrm{f}$.

Mansuy, I. M. 219

Marean, C. W. 308, 318

Mareschal, D. 300, 317 (FN 3), 320

Marr, D. 80 (FN 22)
Marsh, K. L. 345 f., 348

Martin, D. B. 221 (FN 11)

Mattock, K. 167

Maturana, H. R. 25 (FN 6), 77 (FN 13), 82,89 (FN 44)

Mauss, M. 298

Maynard Smith, J. 74

Mayr, E. 80 f. (FN 24, 28)

Mazza, P. P. A. $309,312,325$

McBrearty, S. 309, 313, 324

McCune, L. $151 \mathrm{f}$.

McDougal, I. 308

McDowell, J. 7, 75 f. (FN 6, 9), $78 \mathrm{f}$.

(FN 14, 19), 89 (FN 45), 201

McMullen, E. 120

McNeill, D. 153, 166, 339

Mead, G. H. 100, 122, 178 f. (FN 9), 181 (FN 14)

Meier, B. P. 112

Meixner, U. 13

Mellars, P. 308

Meltzoff, A. N. 114

Menary, R. 1, 4, 35, 43, 50, 53, 79

(FN 16), 296, 315, 321

Menninghaus, W. 355 (FN 3)

Merleau-Ponty, M. 46, 80 f., 83 f. (FN 36), $100,108,111,123,217 \mathrm{f}$. (FN 10)

Merritt, D. J. 64

Mesoudi, A. $292 \mathrm{f}$.

Metzinger, T. 175

Meuter, N. 176 (FN 3)

Michaelian, K. 51

Michaels, A. 256, 261

Mill, J.S. $242 \mathrm{f}$.

Miller, A. R. 298

Miller, H. W. 271

Milligan, K. 319, 323

Millikan, R. 80, 83, 85 (FN 38), 89 (FN 43), 132

Mithen, S. 308, 311 f., 325 f., 358 (FN 11)

Monaghan, P. 167

Moore, G. E. 76

Moore, J. 194

Moore, M. K. 114

Morse, M. A. 309

Muller, G. B. 300

Mumford, S. 33 (FN 2)

Murray, L. 115 (FN 8)

Myin, E. 15, 57-59 (FN 1, 3), 175, 291, 296, 312, 316-318 (FN 3)

Myles, S. 299 
Nagel, T. 75 (FN 3), 93, 177, 299

Nash, A. 313 (FN 1)

Nelson, K. 118

Neth, H. 62

New, J. 312

Nietzsche, F. 75 (FN 7), 217

Noble, W. 307

Noë, A. 80, 85, 175 (FN 2), 296

Nowell, A. $308 \mathrm{f}$.

Núñez, R. E. 62, 69 f.

O’Brien, M.J. 313

Oden, D. L. 323

Odling-Smee, J. 46, 299f., 313, 324

Olsen, B. 298

Olsen, M. 148

Oostenbroek, J. 115

Overmann, K. 60 (FN 5)

Oyama, S. 158, 300

Özyurek, A. 166

Pääbo, S. 356 (FN 5)

Pacherie, E. 337, 340

Pálsson, G. 299

Pannenberg, W. 1

Papagianni, D. 309

Papoušek, H. 115

Papoušek, M. 115

Patel, A. 100

Pauen, M. 206

Pauen, S. 218

Peirce, C.S. 134 f., 175-179 (FN 8), 181-183 (FN 16), 185, 188

Perler, D. 79 (FN 19)

Perniss, P. 166

Persson, T. 48

Pfeifer, R. 18, 27

Philippedes, A. $27 \mathrm{f}$.

Philistion 268 (FN 10)

Piaget, J. 80, 122 (FN 7)

Pigliucci, M. 290 (FN 1), 300

Pinker, S. 205 f., 292, 316

Plessner, H. 1, 256

Pollet, T. V. 300

Popper, K. R. 77 (FN 13), 80

Portmann, A. 1, 5, 197, 207 (FN 3), 213-217, 222

Powell, A. 310

Praxagoras 273

Preucel, R. 307

Preuss, T. M. 356 (FN 5)
Preyer, W. 231

Prinz, J. J. 318

Prinz, W. 114

Proust, J. 80 (FN 21)

Pulvermüller, F. $113 \mathrm{f}$.

Putnam, H. 100, 274

Pylyshyn, Z. W. 107

Quartz, S.R. 300

Quatrefages, J. L. A. 242 (FN 11)

Racine, T. P. $163 \mathrm{f}$.

Ransom, T. G. 287, 296

Ratcliffe, M. $23 \mathrm{f}$.

Read, D. 310

Renfrew, C. 296, 298, $307 \mathrm{f}$.

Rengger, J. R. 237

Revlin, R. 37

Richards, R. 236 (FN 10)

Richardson, M.J. $345 \mathrm{f}$.

Richerson, P.J. 157 f., 292-295, 300 f. (FN 6)

Rizzolatti, G. 121, 211, 249

Roberts, P. 324

Robertson, D. A. $108 \mathrm{f}$.

Rockwell, W.T. $176 \mathrm{f}$.

Roebroeks, W. 309

Rohde, M. 7, 44, 50, 85

Rolle, F. 234 (FN 9)

Rosch, E. 1, 75 (FN 5), 79f. (FN 15), 108f., 296, 299, 301

Rossano, M. 318

Rowlands, M. 31, 44 (FN 4), 79 (FN 16), 315

Rowlands, M.J. 231 (FN 6)

Ruffman, T. 323

Rupert, D. 37-39, 41

Russell, J. 324

Rutz, C. 297 (FN 5)

Saffran, J.R. 120

Sampson, E. E. 64

Sandler, W. 168

Scheler, M. 1

Schiffer, M. B. 298

Schlimm, D. 62

Schmidt, R. C. 345 f.

Schnall, S. 112

Schnegg, M. 3

Schopenhauer, A. 89 (FN 42)

Schroer, M. 205 
Schwarcz, H. P. 309

Schwarz, N. 112

Searle, J. R. 197, 340 f., 346

Segal, C. 271

Sejnowski, T.J. 300

Sellen, D. W. 314

Shalev, I. 112

Shapiro, L. 13, 17, 291

Shea, J.J. 308

Sheets-Johnstone, M. 4

Shennan, S. 310

Sherrington, C.S. 22

Singer, W. 3, 76 (FN 11), 360 (FN 20)

Sinigaglia, C. 211, 249

Skramowsky, B. M. 231 (FN 6)

Smith, A. 120 (FN 12), 195, 240-243

Smith, T. 26

Sober, E. 158, 162

Socrates 75

Soffer, O. 309

Sonesson, G. 152, 166

Soressi, M. 309

Spahn, C. 15 f., 43, 74 (FN 2), 77-82

(FN 13, 18, 23, 26, 29), 84, 90, 207 (FN 4)

Spelke, E.S. 312

Spencer, H. 74 (FN 2), 206 (FN 2), 230

Sperber, D. 155, 311, 313 (FN 1)

Speth, J. 309

Spitz, R. A. 117

Spitzer, M. 356

Stapleton, M. 2, 13f., 19-21 (FN 1, 3), 24, $29,32,40,42$ (FN 3), 44-46

Steinert, U. 220

Sterelny, K. 2, 4, 46, 53, 300, 307, $313-315,324,339$

Stern, D. 49, 108, 115, 249

Stewart, J. 2, 7

Stiner, M. 318

Storch, V. 355 (FN 5)

Stotz, K. 297, 300

Stout, D. 307

Strawson, G. 93

Stringer, C. B. $308 \mathrm{f}$.

Stuckenbruck, L. T. 274 (FN 18)

Stulp, G. 300

Süssmuth, H. 215 (FN 8)

Sutton, J. 33, 40 f., 51, 53, 296

Taglialatela, J.P. 153

Tallis, R. 299, 316
Tanner, J. 215 f., 218

Taoka, M. 324

Tatterstall, I. 152, 157

Taylor, C. 99f., 181, 188 (FN 26), 220

Temkin, O. 266-268 (FN 2, 6), 273

(FN 16, 17), 277

Tewes, C. 14, 78, 90, 92 (FN 49), 100

Thagard, P. 323

Theißen, G. 221

Thieme, H. 325

Thiering, M. 347 (FN 2)

Thomas, M. G. 310

Thompson, A. $27 \mathrm{f}$.

Thompson, E. 1-3, 13, 16, $20 \mathrm{f}$., $24 \mathrm{f}$. (FN 5, 6), 29, 32, 41-46 (FN 3), 73, 75 (FN 5), 79-91 (FN 15, 30, 31, 32, 36, 40, 41, 42, 46, 47), 93, 108 f., 163, 196, 208, 216, 274, 279, 296, 299, 301

Thompson, R. K. R. 323

Thompson, T. 166

Tieleman, T. 267

Tinbergen, N. 74 (FN 2)

Todes, D. 231

Tollefsen, D. 337

Tolman, E. C. 57

Tomasello, M. 1, 4f., 7, 48f., 52, 108, 116-119 (FN 9), 152, 154, 157, 159, 161, 163-165, 168, 176 (FN 7), 179 FN 10), 184-187 (FN 19, 23), 197, 199, 206-210 (FN 7), 212f., 218, 238, 251, 359

Tooby, J. 57 (FN 2), 74 (FN 2), 292, 312

Tosello, G. 308, 325

Trabant, J. 120 (FN 11)

Trevarthen, C. 49, 108, 115 f. (FN 8), 120 (FN 12), $163 \mathrm{f}$.

Trivers, R. L. 74 (FN 2)

Tugendhat, E. 100

Tulving, E. 154

Tuomela, R. 337

Turati, C. 114

Uribe, R. 25 (FN 6)

Umiltà, M. A. 121

Vaesen, K. 155

Valenza, E. 114

van der Eijk, P. 268, 271-273 (FN 13, 14), 276

van der Lely, H. K. J. 316

van Ulzen, N. R. 346 
Varela, F. J. 1f., 25 (FN 6), 75 (FN 5), $79 \mathrm{f}$. (FN 15), 82, 90 (FN 46), 108 f., 296, 299, 301

Vietmeier, K. 278f. (FN 27)

Vigliocco, G. 166

Villa, P. 309, 312

Vogt, C. 234

Vogt, M. 243

Vollmer, G. 78

Wadley, L. 308, 325

Waldenfels, B. 196, 198f., 206 (FN 1)

Walker, P. 167

Wallace, A. R. $234 \mathrm{f} ., 237$

Wandschneider, D. 84 (FN 37), 91 (FN 48)

Watkins, T. 313, 324

Webmoor, T. 298

Wegner, D. M. $51 \mathrm{f}$.

Weick, K.E. 110

Weissenrieder, A. 200, 220-222, 267 (FN 6), 277

Welker, M. 8, 221

Wellman, B. 159

Welsch, U. 355 (FN 5)

Welsch, W. 3, 75 (FN 4), 288, 308, 353 (FN 1), 361 (FN 23)

Werner, H. 151

Westermann, G. 317

Wheeler, M. 14, 19, 25, 35 f., 38f., 291, 296, 300, 311f., 317, 324

White, L. 312

White, T. D. 308

Whiten, A. 154, 209f., 292

Wiessner, P. 157, 161

Wilckens, U. 221
Willems, R. M. 113

Wilson, D. 155

Wilson, D.S. 158, 162, 195

Wilson, E. O. 74 (FN 2), 206

Wilson, M. 17

Wilson, R. A. 311

Wilson, S. M. 114

Wimmer, M. 253

Wink, M. 355 (FN 5)

Winter, B. 113

Witmore, C. L. 298

Wittgenstein, L. 100, 180, 184

Wohlers, M. 267 (FN 6), 277

Wolff, H. W. 220-222

Wright, C. 235

Wulf, C. 48, 198 f., 215, 250f., 253, 256-259, $261 \mathrm{f}$.

Wunder, A. 3

Wylie, A. 312

Wynn, T. 307 f., 311 f., 314, $317 f$., $324 f$.

Yellen, J.E. 309

Young, P. 157

Yule, G. 6

Zahidi, K. 15, 312, 316-318 (FN 3)

Zatorre, R. J. 120

Zeligowski, A. 300

Zhong, C. B. 112

Ziemke, T. 17, $108 \mathrm{f}$.

Zilhão, J. 307, 309f., 313, 318, 320

Zirfas, J. $258 \mathrm{f}$.

Zlatev, J. 48, 101, 103, 108, 151-153, 155, $157 \mathrm{f} ., 164-168,253$

Zwaan, R.A. 109 


\section{Index of Subjects}

Actor-Network-Theory 287, 338, 340

Adaptedness 194, 197

Adaption 288, 355 (FN 3)

Affect 13, 17, 22-24, 49, 115, 120, 138, $154,301,340,346,353$

Agency 73, 76, 80-83, 87, 89, 144, 146, $245,287,296,338,340-342$

Agent 5, 25-27, $33 \mathrm{f}$. (FN 2), 37f., 40, $44 \mathrm{f} ., 48-50,90,107,110$ (FN 2), 123, 132, 200, 210 (FN 7), 278, 286f., 314-316, 319, 322 f., 337 f., 341 f., 344, 348

Alloparenting hypothesis 103, 152, 164

Animal rationale 99, 107

Animality 107

Archaeology 307f., 310-314, $324 \mathrm{f}$.

Articulation 104, 142, 175, 177-181, 184-188, 199, 213

- semiotic 179

- somatic 179

Arts 194, 216, 362

Attention 37, 40, 49, 74, 114, 116-119, 132f., 138, 154, 167, 181 (FN 14), 193, $198,210,212,237,315,345$

- joint $8,102,116,118,124,141,152$, $156,163,165,212,285,287,337,343$, 345,348

- mutual 6

Autonomy $41 \mathrm{f}$. (FN 3), $44 \mathrm{f} ., 75,196 \mathrm{f}$., 342

Awareness 22f., 60-62, 73, 79, 88-91 (FN 41), 93, 109, 215, 232

Axial-Age 104, 188 (FN 24, 25)

Behavior 4, 8, 15, 18, 25, $27 \mathrm{f}$. (FN 7, 8), 35, 42-44, 47 f., 57 f., 62-65, 68 f., $74 \mathrm{f}$. (FN 2), 80, 103, 115, 117-119 (FN 9), $130,132,135,141,143,146-149$, 154, 157 f., 162, 178, 183, 195 f., 199 , 207-209, 211, 213-215 (FN 8), 217, 219, 236, 238, 240, 252 f., 256, 261 f.,
270, 274, 285-288, 293 f., 299 f., 307-313, 315-319 (FN 3), 326, 346, $360 \mathrm{f}$. (FN 24)

Blood 86, 124, 200, 209, 267-269, 271-274, 278f., 356 (FN 5)

Brain 1f., 4, 13f., 16-20, 22, 24f., 27, 31, 35, 40, 44, 47 f., 51, 66 f., 69 f., 73, 79, 81, 85-90 (FN 42, 47), 101 f., 107-109, 113 f., 120, 122-124, 129, 135, 137-139, 142-144, 146-149, 153, 159-163, 175 (FN 1, 2), 177, 181, 197, 200, 214-216, $218,233,235,242,245,249,265$ f., 268-271, 273 f., 277-279, 285 f., 288, 291, 293, 295 f., 299, 301, 307, 309-311, 313 (FN 1), 315-317 (FN 3), 319-325, 338 f., 341, 344, 354-358 (FN 4, 5, 7, 9, 10), 360 (FN 20)

Cartesianism 200 f., 218 (FN 10)

Causality 7, 211, 279, 320

Causal role 33f., 36, 39, 295

Causal mechanism 74

Children 5, 64 f., 67, 108, 119, 123, 133, 141, 152, 154, 157 f.,163 f., 185, $197 \mathrm{f}$., 208-212 (FN 5, 6), 218, 237, 242, $249 \mathrm{f}$., 259f., 262, 273 f., 314, 318 f., 323, 337, 360 (FN 20)

Civilization 188 (FN 25), 194, 359 f. (FN 20)

Circular process (also "circular relation") $2,53,301$

Constitution 2-4, 7f., 14, 34, 48-51, 53, 84, 200, 236, 250, 287 f., 290, 292f., 301, 349,353 f., 360

Construct (see also "construction", "niche construction”), 23, 89 (FN 42), 111, 155, 177, 181 (FN 14), 257, 266, 287, 298, 314f., 319, 323 f., 326

- cultural 3, 205, 310, 313, 316

Conversation 38, 52, 111, 115, 165

- internalized 178 
Co-constitution of organism and environment 3 f., 43, 46

Coevolution 162, 187, 195, 244, 292, 294

Cortex 355-357 (FN 9), 143

- limbic motor 22

- neo- 355 (FN 4), 159, 163

- parietal 137

- prefrontal 137f., 160, 163, 218

- premotor $113 \mathrm{f} ., 121$

Cognition $1 \mathrm{f}$., 8, 13-15, 17-29 (FN 6, 7, 8), 31 f., 34 f., 39-41, 43-45, 48, 50 f., 53, 57-60 (FN 2, 5), 62, 64, 69 f., 73, 77-81 (FN 14), 85-87, 89 (FN 43), 90-93 (FN 47, 49), 99-101, $103 \mathrm{f} ., 109$, 111, 129 f., 132, 138, 144 f., 147 f., 151, 154-156, 160, 175 f., 182 f., 185 f., 188 (FN 26), 200, 209, 211, 251 f., 267, 279, 286f., 290-294 (FN 2), 296, 307, 309-315, 326, 337-340, 342, 344

- extended 19, 37, 300, 315, 322

Cognitive architecture $33,35-38,315$, 319,324

Cognitive mechanism 65, 70

Cognitivism (also cognitivist) 32, $57 \mathrm{f}$, 68f., 290-292 (FN 2), 295-297

Collective action 337,349

Concept 4, 6, 8, 13-16, 23, 25, $32 \mathrm{f}$. (FN 1), 41, 44, 51-53, 61-63 (FN 6), 67, 69f., 73, 78 (FN 14), 85, 88-91 (FN 48), 104, 107, 111 (FN 5), 114, 118, 121 (FN 13), 129, 132, 149, 153, 155, 175, $177 \mathrm{f} ., 182$ (FN 16), $185 \mathrm{f}$. (FN 21), 188 (FN 25), 194-197, 200 f., 206 (FN 1), 220, 228, 230, 234, 243, 252, 255, 257, 274, 290-292 (FN 1), $307 \mathrm{f} ., 311,315 \mathrm{f} ., 318-320,323,338$, $340-342,347$ (FN 2)

Conceptual abilities $62,69,79$

Consciousness 13f., $16 \mathrm{f}$., $21 \mathrm{f}$., 24 (FN 5), 28, 85-89 (FN 42), 91 (FN 48), 175 f., 180f., 184, 186 f., 200 f., 220, 233, 258, 265, 267f., 270-272, 274, 277, 279, 295, 357

Continuity 2 f., 73 f., 78, 87, 130 f., 208, 227, 249, 259-261, 296, 354, 361 (FN 21)

- and change 257,286

- and discontinuity 3,207

- between life and mind $3 \mathrm{f} .$, 7, 43, 279

- ontological 87

- principle of 227,229
Cooperation 53, 101, 152, 158, 187, $196 \mathrm{f}$., 206, 212, 231, 239

Creativity (also "creative") 4, 104, 177, 236, 251 f., 286, 289f., 295-299, 315, 359

Cross-modal mapping 155

Deflationism 58

DNA 131, 293 (FN 4)

Discontinuity (see also "continuity") 4, 74, 152, 308

Double inheritance 251

Emergent features (also "emergent properties”) 4, 34f., 52, 84, 147

Emergentism 89

Emotion (auch "emotional") 6, 13, 19, 22 f., 31 (FN 1), 49f., 52, 100, 102, 104, 115, $120 \mathrm{f} ., 123,129,131,135,137$, 144-146, 152, 160 f., 165, 187, 193 , 196, 199f., 220, 227, 237-240, 242 f., 254, 288, 318, 321, 340, 353, $361 \mathrm{f}$. (FN 25)

Enactivism 2f., 8, $14 \mathrm{f}$., $19 \mathrm{f}$. (FN 2), $31 \mathrm{f}$., 40-46 (FN 3, 4), 50, 52 f., 57, 59, 79, 88f. (FN 41), 175, 207, 217, 299

Enculturation 4, 50, 155, 300, 338

Enculturated human mind 32

Engrams $40,47 \mathrm{f}$.

Enhancement 14, 36, 46, 50, 53, 154, 157, $286 \mathrm{f} ., 307-311,313 \mathrm{f} ., 319,324 \mathrm{f}$.

Environment 1-5, 7, 14f., 18 f., 23, 25-27, 31, 33 f., 36, 41-44, 46, 58f., 62, $67 \mathrm{f}$., $82 \mathrm{f} ., 85 \mathrm{f} ., 89$ (FN 43), 91, 101, 103 f., 107, 109, 116, 119, 148, 175-181, 183, 196 f., 207f., 212-214, 217 f., 221 (FN 11), 229, 231, 236, 249f., 256, 259, 262, 270, 276, 279, 285-287, 293, 299-301 (FN 6), 307, 309f., 312-315 (FN 1, 2), 317 (FN 3), 320, 322, 324, 338-348, 360 (FN 20)

Epilepsy (also "epileptic phenomena") 200f., 265-268 (FN 6), 270, 272-279 (FN 13, 17)

Epistemology 77-80 (FN 13, 14, 18, 23), 89-92 (FN 45)

Exograms 40, 47 f., 50

Explanation 4, 7 f., 14, 26f., 29, 48 f., 53, 57-59, 65-68, 74 (FN 2), 117 (FN 9), $132,138,148,152,158,160,165,167 \mathrm{f}$., 187, 193, 200 f., 228, 234 f., 241, 244, 
266, 270, 273 (FN 14), 285, 301, 308, $310,313,315,348,359,362$

Expression 6, 23, 50, 59, 75, 101, 103, 107 f., 110 (FN 4), 114-116, 119, 121 (FN 13), 144, 146, 153 f., 165 f., 169, 177, 181 (FN 14), 185 (FN 21), 188, 193, 198, 215 (FN 8), 219, 233, 242, 256, 258, 277, 285, 287 f., 299, 311, 321, 326, 349, 356 (FN 5), $361 \mathrm{f}$. (FN 25)

Extended mind (see also “cognition”) 2, 8, 14, 27 (FN 7), 31-35 (FN 1), 41, $43 \mathrm{f} ., 51,79,315,338 \mathrm{f} ., 341$

Externalism 20, 33, 53

- active 33

Exteroception $155 \mathrm{f}$.

Functionalism 18-20, 25, 28, 29 (FN 9), 31, 39, 175 (FN 1)

- extended 14, 32, $34 \mathrm{f}$.

Gaze direction $5 f$.

Genome 130f., 355 (FN 3, 5)

Gestalt 136, 179-181, 183, 185, 187

Gesture 19, 87, 103, 108, 112, $114 \mathrm{f}$., 117-119 (FN 9), 121-123, 142, 153-156, 166f., 169, 177, 179 (FN 12), 182, 185, 187, 212 f., 249, 257 f., 261, $288,299,339,345,361$

- iconic, 107, 118, 156, 213, 258

- pointing (see also "pointing”) 101, 107, 116-119, $121 \mathrm{f} ., 141,212 \mathrm{f}$.

- significant 122

- vocal 101-103,119,121

Hard problem of content 59

Homo 144 f., 158, 286f., 354, 355

- erectus 153, 160, 162, 164, 166, 168, 354 f., 357 (FN 10)

- ergaster 162, 168

- habilis 354, 357 (FN 10)

- heidelbergensis $160 \mathrm{f} ., 169,325$

- helmei 312

- rudolfensis 355

- sapiens 107, 161, 166, 169, 177, 187, 262, 285 f., 309, 312, 322, 325, 353, 355, 358 (FN 11)

Iconism and iconicity $102-105,107$, 118f., 132-135, 137, 139, 141, 151, 156, 165-169, 182 f., 185-187, 213, 258, 362 Icons 102f., 134, 182 f., 213
Illness 265-279

Imitation 5, 48-50, 52, 103, 109 (FN 1), $114 \mathrm{f} ., 118,120$ (FN 12), 143, 147, 153-157, 165, 182, 187, 197-199, 209 (FN 5, 6), 210-212, 214, 232, 235, 237, 252, 255-257, 287, 293, 344, 348

Indexicality 102-105, 132-137, 139-142, 167 f., 182 f., $185-187,259$

Indices $102 \mathrm{f} ., 133-135,141 \mathrm{f} ., 182 \mathrm{f}$.

Intelligence $34,57-59,80,135,165,177 \mathrm{f}$., 180 (FN 13), 228 f., 238f., 269-272, 289f., 296, 299, 312 f., 339, 357 (FN 9)

Intention $5,15,23,32,44 \mathrm{f} ., 48,53,59$, 101, 103, 107-110, 116-119, 121-123, 152 f., 155 f., 159, 161, 163-165, 168, 176 (FN 7), 180, 183-187, 197, 199, 210, 212, 220, 257, 286f., 318, 323, $337 \mathrm{f}, \mathrm{s} 30-347,357$

Interaction $2,5-8,15,23,27,33,36$, 42-44, 49 f., 51 f., 58, 67, 76, 83, 85, 100 f., 103 f., 107-112, 114 f., 118-124, 129-132, 134, 137, 144 f., 152 f., 164, 166, 176f., 179-181, 187, 214, 216, 219, 221, 250, 258 f., 261, 279, 285-287, 292-294, 298, 300f., 310, 314 f., 317, $319 f ., 325,337,339,341-344,346,349$

Intercorporeality (see also "intersubjectivity”) 6, 8, 32, 44, 49f., 52 f., 101, 108, $118 \mathrm{f} ., 122 \mathrm{f}$.

Internalism 1f., 31, 35, 40, 44 (FN 4), 290 (FN 2), 293, 295

Intersubjectivity (also "intersubjective”) 5, 7f., 48-50, 101, 108, 114, 117, 122-124, 151 f., 157, 160, 163-165, 168, 176, 178 (FN 9), 180, 184, 186, 216, $287,337,340,342-344,346$

- primary 101, 114f., 164, 176

- secondary 101,116, 165

Joint action (see also “social action") 287, $337 \mathrm{f} ., 340,342-349$

Joint attention 8, 102, 116, 118, 124, 141, $152,156,163,165,212,285,287,337$, $343,345,348$

Knowledge 4, 22, 46, 48 f., 64, 67, 77, 79 f., 110 (FN 3), 111 (FN 5), 116, 137, 139-142, 144, 146, 148, 237, 249, 251-254, 256-258, 266, 293, 307, 312 f., $316,320,337-339,347$ (FN 2)

- empirical 87,186 
- practical 49, 199, 250f., 253, 255-258, 260-262

- ritual $198 \mathrm{f} ., 249,251,256 \mathrm{f}, 261 \mathrm{f}$.

- scientific 241, 251

Language $6,8,15,33,46-48,50-52$, 58 f., 61 f., 79-80 (FN 24), 84, 99-104, 107-114, 118-124, 132f., $135 \mathrm{f}$., 138-144, 146-148, 151-161, 163-169, 177-187, 212f., 217 f., 220, 227, 233, 235 f., 239 f., 242-244, 253 f., 256, 258, 261, 285, 312, 318 f., 321, 323, 326, 357 (FN 10), $361 \mathrm{f}$.

Learning $4 \mathrm{f}$., $8,26,28,38,44 \mathrm{f}$., $47 \mathrm{f}, 52$, $67 f ., 102,113,115,118 f ., 123,130$, 134, 136-138, 141-143, 147f., 152, 165, 167, 169, 198f., 209-214, 218, 249-253, 255 f., 260, 262, 272, 288, 295 f., 300, $314,341,357-360$ (FN 20)

- social 154, 293

- transfer 48,137f., 142

Life $3 f ., 7 f ., 15 f ., 43,49,60,73-76,79-86$, $88,102,117 \mathrm{f} ., 120,124,130,138,148$, 158-164, 175, 186, 193 f., 196, 198 f., 206, 208, 211, 214f., 218-220, 228-231, 235, 241-244, 249-251, 254f., 257, 260, 262, 272, 279, 285, 291, 293-296, 298, 312, 316, 340 f., 356 f., 359, 360 f.

Mark of the mental 18, 35

Material engagement theory 9, 60 (FN 5), 286f., 289-292, 294, 296-299, 301, 307, 310, 315-317, 319-322, 325, 337f., 340, $344,346,349$

Materiality 9, 200, 217, 292-242, 301, 340,346

Mental faculties (also “mental abilities”) 228, 236-238, 244, $270 \mathrm{f}$.

Mentality 45, 103, 110 (FN 4), 154, 292, 340

Metabolism 35, 42, 82 f., 86, 219

Metaplasticity 286f., 289f., 298f., 307, $311,316,323-326,338,340-342$

Mental 4, 7 f., 13-15, 18, 27 (FN 7), 32-35, 40f., 43, 47, 65 (FN 8), 66 (FN 9), 69, 73, 79, 81, 84 f., 87 f., 90 (FN 47), 93, 116f. (FN9), 129 f., 134-136, 139, 145, 154, 193 f., 200, 207 f., 220, 228, 231, 233 (FN 8), 236-238, 243f., 253, 269-271, 274, 279, 291, 295 f., 300, 311 f., 314-317 (FN 3), 319, 322 f., $348 f$., 362
- formation 8

- operation 7,112 (FN 7)

- process 2, 7,34f., 43, 287, 292, 322

- states 1, 14, 23, 27f., 31-33, $35 \mathrm{f} ., 110$ (FN 4), 220, 293, 319, 341, 344

- symbol system 107

Microfunctionalism (see also "functionalism") 20

Mimesis (also "mimetic processes") 8, 48, 101, 103 f., 151-158, 160, 165-169, 186 f., 198 f., 211, 249-253, 255-262

Mimicry 142f., 198, 250, 255

Mind 1-4, 7f., 13-15, 17-20, 23, 25, 27 (FN 7), 31-35, 37, 40 f., 43, 45 f., 48, 51, 53, 58f., 64, 66 (FN 9), 73-76, 79-81, 84 f., 87-90, 92 f., 99 f., 108 f., 111 (FN 5), 122f., 146, 153 f., 157, 164, 175-179 (FN 19), 183, 196, 200f., 208, 235, 266, 247, 279, 285-287, 289-299, 301, 307, 310-312, 314-316, 319f., 323 f., 337-340, 342, 348

- and brain 70,177, 181

- theory of 6, 20,87, 93,110 (FN 4), 116, 121 (FN 13), 285, 321, 326

Mirror Neurons 101, 114, 121, 211

Morality (also “morals”) 7, 26, 36, 41, 73, 77, 90, 112, 157-159, $164 \mathrm{f} ., 169,175$, 188, 194-196, 227 f. (FN 1), 230, 233 f., 238-245

Moral faculties (also "moral sense") 195-197, 209, 227, 230, 233-235, 239 f., 242-245

Movement (also "to move/moving”) 5 f., 45-47, 49, 53, 58, 65, 80, 88 f., 92 (FN 49), 101, 107-109, 111 (FN 5), 113 f., 117-119, 121 f., 141, 154, 158, 175 f. (FN 3), 179, 185 f., 188 (FN 25), 198, 211 f., 216, 232 f., 249, 251-253, 256, 258-262, 266, 268f., $271 \mathrm{f}$.

(FN 11), 275, 277 f., 285, 287, 290, 295, 298-301, 340f., 343-349, 362 (FN 25)

Multiple realizability 18, 29 (FN 9), 177

Music 115, 120 f., 160, 261, 285, 343, 354, 359

Naturalism 3, 7, 16, $67 \mathrm{f} ., 73,77-79,87$, 89 (FN 44, 45), 91-93, 177, 194 f., 200, 222, 234

Naturalistic fallac 76, 78

Naturalistic monism 74

Natural laws 8, 196, 214, 228 
Nature 1-3, 5, 7f., 15f., 24-26, 31, 37, $42,51,57,59-63,69,73-79,81-84$, 87-89, 91-93, 100, 108, 118, 129, 131 f., 134-136, 140, 145, 147-149, 152 f., 156 f., 165, 168 f., 179 (FN 11), 181, 183-185, 187, 194-201, 205-209, 212-219, 221 f., 227-232, 234-245, 252, 255 f., 260, 266f., 272 f., 276, 285 , 287 f., 290-295, 297-301, 307, 311-313 (FN 1), 316, 318, 324f., 338, 342, 358-363

- and nurture 2,102,130f., $139 \mathrm{f}$.

- human 3, 33, 40, 75, 77f., 102, 129, 131, 139, 144, 149, 152, 193 f., 213-215, 240, 285 f., 288, 297, 300, 316, 324, $360 \mathrm{f}$.

- second 44, 49, 75 (FN 6), 89 (FN 45), 201

- theory of 80

Neanderthals 169, 308f., 312, 318, $320-322,325 \mathrm{f}$.

Neolithic revolution 359 (FN 18)

Neural nets 25

Neural networks 25 f., 274, 320

Neuroconstructivism 286, 300, 307, 311, 317 (FN 3), 319

Niche construction (also "cultural niches”) 4, 46, 164, 196, 199, 208, 299f., 310, 313, 316 f., 338

Normativity 7, 41, 43 f., 73, 76-81, 87, 89 f., 104, 154-157, 176, 182, 184 f., 211, $217,234,285,287,337,340 f$.

Ontogenesis 4-6, 15, 18, 40, 51, $63 \mathrm{f}$., $67 \mathrm{f} ., 101 \mathrm{f} ., 122,151,157,163,166,186$, 212-214, 242, 286, 298, 311, 314

Ontology 16, 77, 83f., 87-89 (FN 42), 91-93, 153, 218 (FN 10), 294, 296

- cartesian 197, 200

- idealistic 92 (FN 49)

- neurocentric 295

- relational 294

- stoic 200

Organism 2-4, 7f., 14-16, 31, 33-35, 41-44, 59, 62, 64, 68, 73-75, 80-90, 99, 101 f., 104 f., 109, 124, 130 f., 163, 175-179, 181, 183, 195f., 198, 207-209, 216, 219, 221, 228 f., 234 f., 241, 244, 272, 292 f., 296, 298-301, 313, 317, 339, 342

- eukaryotic $130 \mathrm{f}$.
Pantomiming 185, $212 \mathrm{f}$.

Perception 6, 15, 17, 22f., 68, 80 (FN 20), $91 \mathrm{f}$. (FN 49), 109, $113 \mathrm{f} ., 119-121$

(FN 13), 123, 195, 199, 217, 219, 221, 256, 258, 271, 286, 288, 296, 318 f., 340, $348,355,357,362$

- direct 6,319

Person 6, 8, 51 f., 84, 88, 110-112, 117, 123, 178 f., 193 f., 196-198, 205-207, 210, 212-215, 217 f., 220-222, 241, 256, 261, 265-267, 273f., 277, 279, 318, 342

Phenomenology 17, 22f., 91, 217, 279, 290

Phylogenesis 8, $212 \mathrm{f}$.

Physicalism 7, 16, 79

Plasticity 4, 44, 101, 129, 146, 197 f., 214, 218, 221 f., 256, 286, 289, 294, $299 \mathrm{f}$., 307, 310f., 314-317, 322-324, 338

Pointing 3, $101 \mathrm{f}$., $107 \mathrm{f} ., 116-119$, 121-124, 133, 141, 145, 156, 163, 182, 185, 205, 212f., 215, 219, 300, 362

Primate (also "primates") 4-6, 22, 69, 116, 130f., 137, 146, 148, 154-156, 159, 161, 164, 187, 199, 209, 213, 238

- non-human 4,155

Proprioception 109, 156

Proto-Cultural Period, 288, 353-355, 357, 359-361, 363

Ratchet effect 7, 187, 209

Rational expressivity $185 \mathrm{f}$. (FN 21, 22)

Rationality 107, 250, 252

Realization 34, 36, 39, 43, 47, 82, 214, 318,342

Reason 7, 41, 75f. (FN 7), 78, 107, 180 (FN 13), 194, 196, 201, 206, 233, 237, 239f., 245, 269, 272

Reciprocity 158, 160, 195, 206

Reduction 41, 45, 49, 53, 74, 99, 147, 201, 206, 239, 355

Reductionism 75 (FN 3), 81, 99, 194, 196

Reference 13, $79 \mathrm{f} ., 100,102-104,116,118$, 129, 132-137, 139-142, 144, 155, 159 f., 167, 177 f., 182 f., 185-187, 234, 237, 255, 259, 261, 273, 276, 278 (FN 25, 27)

- dyadic concepts of 182

- iconic 102-104, 132-135, 139,182f., 186

- indexical 102f., 132-135, 137, 139, 142, 183, 185

- linguistic 79, 132

- symbolic 103f., 129, 132-137, 139f., 144, 159f., 167, $182 \mathrm{f}$. 
Religion 3, 145, 188, 194 f., 216, 234, 241, 244-245, 249, 257, 260, 265, 277, 285, 287

Representation 15, 40, 52, 57-60, $63 \mathrm{f}$., 66-69, 77, 80 (FN 20), 83, 89 (FN 42), 103, $107 \mathrm{f} ., 119,123,151,153,166,169$, 180, 193, 207, 216, 237, 256, 293, 297, 317 (FN 3), 323, 348

- amodal 66f., 69

Resonance 6, 107, 115, 120-122 (FN 13)

- emotional 115

- interbodily 107, 122

Ritual (also "rituals") 33, 103, 115, 133, 139, 154 f., 185, 198 f., 211, 249, 251-253, 256-262, 344

Selection 6-8, 25, $73 \mathrm{f}$. (FN 1), 102, 135, $138,146-148,151,158-162,164,169$, 193-195, 199, 206-208, 228-230, 232, 235 f., 239, 241, 244 f., 254, 285, 287, 290, 282, 299, 301, 307, 312f. (FN 1), 316, $324 \mathrm{f} ., 355$ (FN 3), $357 \mathrm{f}$.

- group 158-162, 195, 244

- kin 158,162, 195

- natural 25, 73 f. (FN 1), 135, 147, 194 f., 199, 206f., 228-230, 235 f., 239, 241, 245, 285, 287, 290, 292, 301, 307, $312 \mathrm{f}$. (FN 1), 316

Selection pressure $6 \mathrm{f} ., 102,206,357 \mathrm{f}$.

Self (also "selfs") 42, 51, 255

- biological 42

- social (also “personal”), 51

Self-awareness $164,187,250$

Self-identity $82-85$

Self-regulation 82

Semiotics 8, 103, 153, 166f., 182, 188 (FN 26), 307

- triadic 182

Sense-making 3, 7, 14, 16, 32 (FN 1), 41, $43,45,49,50,53,73,38,85,89,110 \mathrm{f}$., 124

- participatory 53,85, 111

Sensorimotor brain 101, 108

Sensorimotor system $31,44,86,113$

Sexuality 215, 217, 257, 288, 355

Sign (also “signs”), 62, 99-104, 117-119, 132-135, 137, 144, 152, 155-157, $165 \mathrm{f}$., 167-169, 175, 177 f., 180-186 (FN 16), $185,188,238,318$

Sign-usage 178, 182-184, $186 \mathrm{f}$.

Sign vehicle $132-135$
Signifiers 100, 132, 140, 167

Simulation 6, 25, 27, 121 (FN 13), 208, $287,344,346$

Skillful coping 176

Social action (also "joint action") 161, 239, 253, 256 f., 287, 337-349 (FN 2)

Social instincts 197, 208f., 227, $238 \mathrm{f}$., 242-245

Social life 102, 250, 257, 262, 285, 356f., 360

Soul 198, 200, 220, 233, 242, 274

Space 7, 51, 68, 76 (FN 9), 84 (FN 37), 92 (FN 49), 100, 117, 134, 250, 258f., 274, 285, 287, 296, 323, 339, 341, 343, 347, 349,361

Supervenience 18, 20, 85

Symbolic communication $102 \mathrm{f}$., $107 \mathrm{f}$., 116, 129, 135, 137 f., 145, 149, 151 f., 156, 159, 165 f., 168 f., 182f., 186f., 197 f., 207

Symbolic threshold 183, 185, 187

Symbols (see also "reference") 40, 50, 57, 64f., 92 (FN 49), 102, 104, 110 (FN 3), 122f., 132-136, 138-141, 144, 146, 151, 156, 163, 166-168, 176, 179 (FN 9), $182 \mathrm{f} ., 185,187 \mathrm{f} ., 260,318$

Sympathy $8,115,165,195$ f., 206f., 227, 231, 233, 236f., 239-245

Syntax 110, 123, 142, 357 (FN 10)

Syntactic information 135

System (also “systems") 1f., 4, 14, 17-21, 24, 26-29, 31-37, 39-53 (FN 3), 61, 63-69, 80-84 (FN 22, 29, 30), $85 \mathrm{f}$. (FN 39), 101 f., 107, 109-111, 112-114 (FN 7), 121-123, 136-139, 142-148, 157-159, 162, 166-169, 177f., 188, 197, 211, 255, 265, 268f. (FN 8), 277, 286f., 292 f., 295 f., 299-301 (FN 6), 307, 311, 314f., 317, 320-324, 338f., 342, 345, 256 (FN 7)

- artificial 17-19,24

- autonomous 14,31f., 41-47, 49f., $52 \mathrm{f}$.

- brain-body 321-323

- cognitive $17 \mathrm{f} ., 24,26,28,40,50,80$

- coupled 33-35, 45f., 86

- dynamical 14, 34, 41 f., 345

System identity $43,45,50,53,84$

Time (also "temporality") 27f., 47, 51, 61, 84 (FN 37), 92 (FN 49), 120 (FN 12), 134, 154, 169, 178, 182 f., 188 (FN 25), 193 f., 207, 215 f., 229, 241, 244, 259 f., 292, 297, 317, 321f., 324, 335, 343, 358 
Tool 44, 46, 48, 77, 145, 148, 153, 168, 186, 199, 209, 237, 297, 302, 314, 319, $356 \mathrm{f}$.

- production 153,168, 194, 311,357, 360 (FN 19)

- use 48, 148, 194, 199, 207, 209, $236 \mathrm{f}$., 286, 297, 360 (FN 19)
Universals 139f., 148, 238, 288, 361 (FN 23)

Upper Paleolithic Revolution 288, 308 f., 324 f., 359

Violence 249, $252 \mathrm{f}$.

Vulnerability 205-207, 219f., 236 